
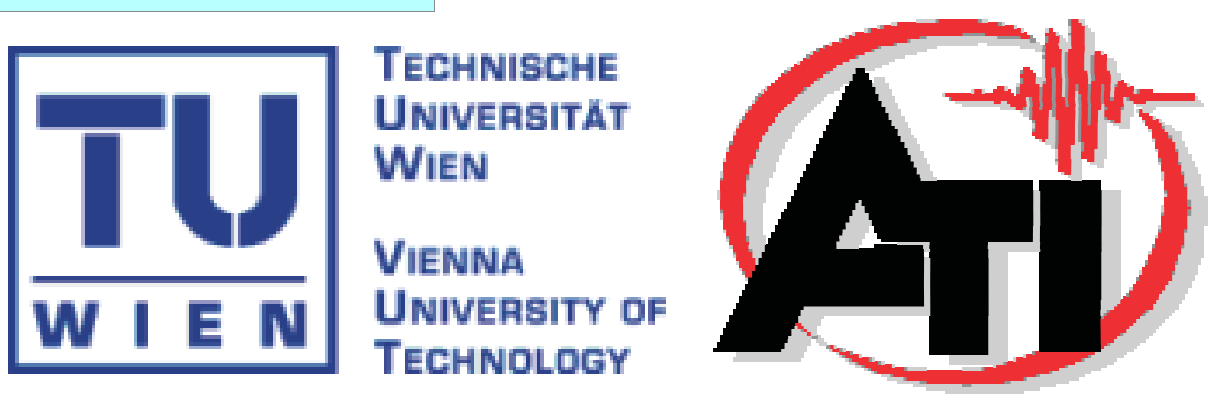

\title{
DISSERTATION
}

\section{Center Vortices and Chiral Symmetry Breaking}

ausgeführt zum Zwecke der Erlangung des akademischen Grades eines

Doktors der technischen Wissenschaften unter der Leitung von

\author{
Ao.Univ.Prof. Dr.techn. Manfried Faber \\ Institutsnummer: E 141 \\ Atominstitut \\ eingereicht an der Technischen Universität Wien \\ Fakultät für Physik \\ von \\ Roman Höllwieser \\ Matrikelnummer: 0127128 \\ Am Brand 10, 2833 Bromberg
}





\section{Zusammenfassung}

Der Gegenstand dieser Dissertation ist die Untersuchung nicht-störungstheoretischer Eigenschaften der Quanten Chromodynamik (QCD) mit Hilfe der Quantenfeldtheorie auf einem vierdimensionalen Raum-Zeit Gitter (Gitter QCD).

Nach einer kurzen Einleitung wird in Abschnitt 2 das Vortexbild, ein Modell zur Erklärung des Quarkeinschlusses (Confinement) in der QCD durch in sich geschlossene Farbwirbel (Vortices), die mit dem Zentrum der Eichgruppe verknüpft sind, vorgestellt. Im 3. Abschnitt werden seine Confinement-Eigenschaften im Rahmen der SU(2) Gittereichtheorie mit (verbesserter) Lüscher-Weisz Wirkung getestet. Danach soll das Vortexmodell auf Phänomene bezüglich chiraler Symmetrie analysiert werden, wobei hier die Relevanz des niedrigen Dirac Spektrums herangezogen wird. Das Atiyah-Singer Index Theorem erklärt topologische Ladung mit Hilfe des Indexes des Dirac Operators während die Banks-Casher Formel die Dichte der nahen Dirac Moden dem chiralen Kondensat, einem Ordnungsparameter der chiralen Symmetriebrechung (SCSB), proportional setzt. Der Dirac Operator wird zuerst in Abschnitt 4 auf dicke, klassische Vortices in Form ebener Flächen (geschlossen durch die Gitterperiodizität) und Kugeloberflächen angewendet und die Position der Nullmoden im Vergleich zur Vortexstruktur wird bestimmt. Es zeigt sich eine interessante Diskrepanz der topologischen Ladung, gemessen an den Nullmoden (Index) und anderen Bestimmungsmöglichkeiten. Danach werden im Abschnitt 5 die Eigenmoden verschiedener Dirac Operatoren in SU(2) Monte Carlo Konfigurationen bestimmt und Eigenschaften wie Verteilung, Dimensionalität und Lokalisation untersucht. Desweiteren werden Korrelationen dieser Moden und der Vortexstruktur gemessen. Das Ergebnis dieser Untersuchungen zeigt, dass der topologischen Untergrund der Eichkonfigurationen deutlichen Einfluss auf die nahen Nullmoden hat. Allgemeine Schlüsse finden sich in Abschnitt 6.

Anhang A gibt einen Überblick aller wichtigen Methoden und Werkzeuge die zum Zwecke der folgenden Untersuchungen verwendet wurden. In Anhang B findet sich eine Zusammenfassung zu statistischen Methoden und Fehleranalyse. Eine Reihe weiterer Abbildungen und Diagramme analysierter Daten sind in Anhang C zusammengefasst. 



\section{Summary}

In this thesis some non-perturbative features of quantum chromodynamics (QCD) are studied in terms of quantum field theory on a four dimensional space-time lattice (LQCD).

The center vortex model, presented in section 2, has been proposed as an explanation of confinement in non-Abelian gauge theories. Some checks of the confinement properties of center vortices in SU(2) lattice gauge theory with improved Lüscher-Weisz action are repeated in section 3 and then phenomena related to chiral symmetry, such as topological charge and spontaneous chiral symmetry breaking (SCSB) are studied within the center vortex model. These non-perturbative features of the QCD vacuum are intimately linked to the properties of the low-lying spectrum of the Dirac operator. The Atiyah-Singer index theorem states that the topological charge of a gauge field equals the index of the Dirac operator, while the Banks-Casher relation sets the spectral density of the nearzero modes proportional to the chiral condensate, the order parameter for SCSB. The Dirac operator is applied to thick classical center vortices in the shapes of planes (closed by lattice periodicity) and spheres in section 4 , and the localization of zero-modes with respect to the position of the thick vortices is investigated. An interesting discrepancy in the topological charge determined by different methods is discussed. In section 5 the eigenmodes of the overlap and asqtad staggered Dirac operators applied on SU(2) Monte Carlo configurations are evaluated and different observables concerning distribution, dimensional characteristics and localization are studied. Further correlations between the low-lying modes and center vortices are investigated. The low-lying modes are apparently sensitive to topological properties of the underlying gauge field configurations. Conclusions are drawn in section 6 .

Appendix A provides an overview of methods and operators used for the purpose of the following studies while appendix B gives some information on statistics and error analysis. Some more plots and figures of analyzed data are collected in appendix C. 

Für meine Familie 



\section{Acknowledgments}

First of all I would like to thank my supervisor Prof. Manfried Faber for his support during the work on this thesis. He had always time to discuss my ideas with him and the most important lesson I have learned from him is that there is often a simple geometrical picture which explains more than a lot of complicated equations. Furthermore, I would like to thank our collaborators, Jeff Greensite, Štefan Olejník and Urs Heller.

I would like to thank my colleague Mario Pitschmann for his collaboration and friendship. He had always an open ear for my problems. Furthermore, I would like to thank Roman Bertle for helping me a lot on questions of computers. I would like to mention Gerald Jordan, who did a lot of preliminary work on section 4 of this thesis, as well as Thomas Layer and Markus Wellenzohn, since we had many interesting discussions.

Special thanks goes to my whole family, since they have always supported me and have helped me through all the minor and major problems which wait for every sailor who goes through the stormy sea of life. 



\section{Contents}

Zusammenfassung $\quad$ i

Summary iii

Acknowledgments $\quad$ vii

1 Introduction 1

2 Vortex Model 3

2.1 Center Vortices . . . . . . . . . . . . . . . . . . . 3

2.2 The vortex picture of quark confinement . . . . . . . . . . . . 4

2.3 Topological Charge . . . . . . . . . . . . . . . . . . . . . . . 7

2.4 Vortices and chiral symmetry breaking . . . . . . . . . . . . 7

3 Numerical Results on Confinement $\quad 11$

3.1 Scaling of vortex density . . . . . . . . . . . . . . . . . . 11

3.2 Center dominance . . . . . . . . . . . . . . . . . . . . 12

3.3 Precocious linearity . . . . . . . . . . . . . . . . . 13

3.4 P-vortices locate thick center vortices . . . . . . . . . . . . . . . . 14

3.5 Casimir scaling . . . . . . . . . . . . . . . . . . . . 14

3.6 Vortices and matter fields . . . . . . . . . . . . . . . . . . . 14

3.7 Finite temperature . . . . . . . . . . . . . . . . 15

4 Topological charge $\quad 19$

4.1 Definitions . . . . . . . . . . . . . . . . . . . . . . . 19

4.2 Plane vortices . . . . . . . . . . . . . . . . . . . 21

4.3 Spherical vortices . . . . . . . . . . . . . . . . . . . . 21

4.4 Topology and Staggered Fermions . . . . . . . . . . . . . . . . . . 29

5 Observables and Results on SCSB $\quad 31$

5.1 Dirac Eigenvalues . . . . . . . . . . . . . . . . . . . . . 31

5.2 Localization and fractal dimension of eigenmodes . . . . . . . . . . 38

5.2 .1 asqtad staggered IPR-Results . . . . . . . . . . . . . . . . 39

5.2 .2 overlap IPR-Results . . . . . . . . . . . . . . . . . 45

5.2 .3 Results of other groups . . . . . . . . . . . . . . . 45

5.3 Peak Analysis . . . . . . . . . . . . . . . . . . . . . 47

5.3 .1 Remaining Norm . . . . . . . . . . . . . . . . . 55

5.3 .2 Peak Fitting . . . . . . . . . . . . . . . 56

5.4 Correlation between vortices and Dirac modes . . . . . . . . . . . . . 59

5.4.1 Correlations and partial projection . . . . . . . . . . . . 64

5.4.2 Correlations and chiral densities . . . . . . . . . . . . . 64

5.4.3 Correlations with partial vortex structures . . . . . . . . . 67

5.5 Eigenmode Correlations . . . . . . . . . . . . . . . . . . 71 
5.6 Chiral Density Peaks and Vortex Structures . . . . . . . . . . . . 77

5.7 Finite Temperature . . . . . . . . . . . . . . . . . . . . . . 88

5.7.1 Vortex correlation in high-temperature configurations . . . . . . . 88

6 Conclusion $\quad 91$

$\begin{array}{ll}\text { A Methods and Operators } & 93\end{array}$

A.1 Lüscher-Weisz Action . . . . . . . . . . . . . . . . . . . . . . . 93

A.2 Fermionic Actions . . . . . . . . . . . . . . . . . . . . 97

A.2.1 Fermion Doubling . . . . . . . . . . . . . . 97

A.2.2 Exact Chiral Symmetry _ . . . . . . . . . . . . . . . 97

A.2.3 The Overlap Operator . . . . . . . . . . . . . . . . . . . . 97

A.2.4 Staggered Fermions . . . . . . . . . . . . . . . . . . . . . . . 99

A.3 Dirac Eigenmodes . . . . . . . . . . . . . . . . . . . 100

A.3.1 Atiyah-Singer index theorem and exact zero modes . . . . . . . 100

A.3.2 Banks-Casher relation and near-zero modes . . . . . . . . . . . 100

A.3.3 Localization of eigenmodes . . . . . . . . . . . . . . . . . . . 101

A.4 Direct Maximal Center Gauge . . . . . . . . . . . . . . . . . . . . 101

A.4.1 Detection of Vortices . . . . . . . . . . . . . . . . . . 102

A.4.2 Smoothing Vortex Configurations . . . . . . . . . . . . . . . 103

A.4.3 Center Vortex Removal . . . . . . . . . . . . . . . . . . . . . . . . 104

A.5 The Wilson Loop and the $q \bar{q}$-potential . . . . . . . . . . . . . . . . . . . 104

A.6 The Polyakov Loop, Free Energy and Center Symmetry . . . . . . . . . . 107

$\begin{array}{ll}\text { B Statistics and Errors } & 109\end{array}$

B.1 Error propagation and correlations . . . . . . . . . . . . 110

B.2 The jackknife error estimate . . . . . . . . . . . . . . . . 112

B.3 Error propagation for the Creutz ratio . . . . . . . . . . . . . . 112

$\begin{array}{ll}\text { C Plots and Figures } & 115\end{array}$ 


\section{List of Tables}

1 Number of links with negative traces and trace values after Landau gauge for the non-orientable spherical vortex of Eq. (18) on a $40^{3} \times 2$-lattice,

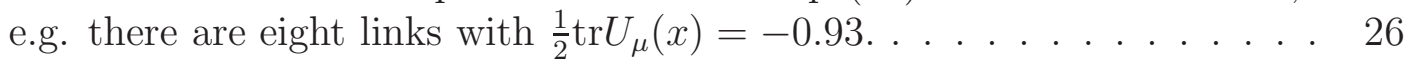

2 Number of links with negative traces and trace values after Landau gauge for the orientable spherical vortex of Eq. (22) on a $40^{3} \times 2$-lattice. . . . . 26

3 Number of zero modes for various smooth field configurations with spherical vortices. The profile functions $\alpha_{ \pm}$are defined in Eq. (20). . . . . . . 28

4 First eight asqtad staggered eigenvalues and their chiralities for positive and negative spherical vortices and HYP-smeared configurations. . . . . .

5 Peak Analysis Results for staggered and overlap fermions: maximum peak height, its FWHM enclosed in brackets, number of peaks higher than $1 / 2$, $1 / 3$ and $1 / 10$ of maximum peak and corresponding averaged FWHM . .

6 Average number of E/B-plaquettes and vortex density (data normalized to total number of possible plaquettes) in a distance $r$ around the maximum eigenmode density peaks . . . . . . . . . . . .

7 Parameters for the Lüscher-Weisz action: coefficients $c_{i}$ and expectation value of the plaquette $u_{0}^{4}=\operatorname{Re} \operatorname{Tr}<U_{p l}>/ 2 \ldots \ldots$. . . . . . . . . 94

8 Lattice string tension $\sigma_{\text {lat }}$, lattice spacing $a$ and corresponding physical volumes, as extracted from linear-plus-Coulomb fits to $V(R, T=$ fixed $)$.

9 Probabilities $P(\bar{x}-n \sigma<x<\bar{x}+n \sigma)$ for measurements from a normal distribution lying in the confidence interval $\mathrm{CI}=[\bar{x} \pm n \sigma]$. The figure illustrates the normal distribution probability function with confidence intervals CI . . . . . . . . . . . . . . . . . . . 110

10 Correlations $\langle X Y\rangle-\langle X\rangle\langle Y\rangle$ between size of the gap or eigenvalue density near zero and Polyakov loop $\left(P(\vec{x})\right.$ or $\left.\mathbb{Z}_{2} P(\vec{x})\right)$, number of electric, magnetic or total number of vortex-plaquettes. Size of gap correlates with Polyakov loops, eigenvalue density near zero correlates with vortex densities.178

11 Correlation between size of the gap and Polyakov loop of center-projected configurations $\left(\mathbb{Z}_{2} P(\vec{x})\right)$, different $\beta_{(L W)} \mathrm{S}(\beta: 2.3-3.0 \rightarrow$ Wilson action, Fig. 161, $\beta_{L W}: 3.3, \ldots \rightarrow$ Lüscher-Weisz action, Fig. 165) and lattice sizes, anti periodic bc. . . . . . . . . . . . . . . . . 178

12 Correlation between eigenvalue density near zero and vortex-density, different $\beta_{(L W)} \mathrm{S}\left(\beta: 2.3-3.0 \rightarrow\right.$ Wilson action, Fig. 160, $\beta_{L W}: 3.3-4.0 \rightarrow$ Lüscher-Weisz action, Fig. 163) and lattice sizes, periodic bc. . . . . . . 178 



\section{Introduction}

\section{Introduction}

The fundamental particles which form the hadronic matter (e.g. protons, neutrons) are "quarks" (fermions) which interact via "gluons" (bosons) due to the strong interaction. Quarks come in different "flavors", namely the "up", "down", "strange", "charmed", "bottom" and "top" quark, but the strong interaction is flavor blind. QCD is a gauge theory based on the unbroken non-Abelian $S U(3)$ group, which assigns every quark one of three "colors". Since there are eight generators of $S U(3)$, there are eight massless gluons carrying a color charge which mediate the strong interactions between quarks. QCD is an asymptotically free theory, forces between quarks become weak for small quark separations (quark-gluon plasma) and therefore perturbative calculations are possible, exploring the short distance structure of QCD.

The asymptotic freedom property of QCD is intimately linked to the non-Abelian structure of the gauge group, which on the other hand causes colored gluons to couple to themselves. These self couplings are believed to be responsible for quark confinement, the fact that color charged particles cannot be isolated and hadrons are colorless. Confinement is the main property of the dynamics at large distances where perturbation theory breaks down due to ultraviolet divergences. The lattice formulation of QCD invented by Kenneth Wilson in 1974 allows to study such non-perturbative phenomena. The gauge field is put on a discrete space-time lattice, which acts like a cutoff in the perturbative integrals, and the path integral formalism of quantum field theory is applied by using numerical methods. With these tools it was possible to study confinement, and already Wilson showed that within the strong coupling approximation QCD confines quarks.

Actually, one of the pioneering papers of Mike Creutz [1] was already showing that the potential between static quarks and antiquarks is asymptotically linearly rising with the distance. The corresponding constant force, the string tension, is incredibly high, around $1 \mathrm{GeV} / \mathrm{fm}$. The origin of this strong force should be found in the properties of the QCD vacuum. This is highly non-trivial, filled with quantum fluctuations and topological excitations which dominate the behavior of the QCD vacuum at long distance scales. On the other hand the color electric field between quarks and antiquarks has regular flux lines and does not like to enter the stochastically fluctuating QCD vacuum. Therefore, it is energetically favorable to compress the electric flux-lines to a small tunnel between quark and antiquark. The distribution of this color magnetic flux was nicely shown in lattice calculations [2]. Despite intensive efforts over three decades there is no derivation of confinement from first principles nor is there a generally accepted explanation. Candidates for topological excitations responsible for confinement were mainly instantons, Abelian monopoles and vortices. Instantons live on a length scale of around $0.2 \mathrm{fm}$ and can therefore contribute only little to the large distance force between heavy quarks [3].

By a transformation to the dual degrees of freedom one can show analytically that confinement in U(1) lattice gauge theory is due to magnetic monopoles. Kronfeld, Schierholz, and Wiese [4] devised a method for non-Abelian gauge theories to detect monopoles by Abelian gauge fixing and Abelian projection (see App. A.4). The property that an 


\section{Introduction}

Abelian component of the color field can explain the full string tension was shown in [5] and was dubbed Abelian dominance. The monopole confinement mechanism leads to a very nice picture, the dual superconductor model of confinement, where magnetic monopoles and antimonopoles form a solenoidal current around the electric flux tube between quark and antiquark. But Del Debbio et al. [6] showed that the hypothesis of Abelian dominance in the maximally Abelian gauge, which was known to work for Wilson loops in the fundamental representation, fails for Wilson loops in higher group representations. Such a problem does not appear in the center vortex picture of confinement.

The center vortex model was first proposed by 't Hooft [7], Mack and Petkova [8] and [9] as an explanation of confinement in non-Abelian gauge theories. The central idea was to filter out the important infrared degrees of freedom responsible for confinement and then to simplify the field configurations by projection. Center vortices, quantized magnetic flux lines, compress the gluonic flux into tubes and cause a linearly rising potential at large separations. Numerical evidence has been produced to support this assumption $[10,11]$ and in addition, simulations have indicated that vortices could also account for phenomena related to chiral symmetry, such as topological charge and spontaneous chiral symmetry breaking (SCSB) [12, 13, 14, 15, 16, 17, 18].

These non-perturbative features of the QCD vacuum are intimately linked to the properties of the low-lying spectrum of the Dirac operator. The Atiyah-Singer index theorem [19, 20, 21] states that the topological charge of a gauge field equals the index of the Dirac operator, while the Banks-Casher relation [22] sets the spectral density of the near-zero modes proportional to the chiral condensate, the order parameter for chiral symmetry breaking. The fundamental problems of investigating chiral symmetry on the lattice have been overcome by the invention of overlap fermions. The overlap operator obeys the Ginsparg-Wilson relation and features an exact chiral symmetry [17]. It further implements a lattice version of the index theorem [18], and may even be used for the definition of a local topological charge density [12].

In the following sections, the vortex model is introduced (2) and its confinement properties are reviewed (3). A summary of previous studies on topological charge is given (4) and results on SCSB are presented (5). 


\section{Vortex Model}

The vortex model was first proposed by 't Hooft [7], Mack and Petkova [8] and [9]. Due to lack of an identification method for vortices, almost no numerical investigations were done for 25 years. Maximal center gauge and center projection (see App. A.4) provided means to identify vortices [10] and led to new investigations using the vortex model. The central idea was to filter out the important infrared degrees of freedom responsible for confinement and then to simplify the field configurations by projection. Other identification methods for vortices were proposed, Laplacian center gauge by de Forcrand and coworkers [15, 23], a method by Langfeld et al. [24] which combines Laplacian center gauge and direct maximal center gauge, and direct Laplacian center gauge [25]. All of the center gauges yield qualitatively similar results.

\subsection{Center Vortices}

Vortices are closed magnetic flux lines forming 2-dimensional world sheets. They carry magnetic flux corresponding to the center of the gauge group ${ }^{1}$, therefore vortices are denoted as center vortices. The flux can only take on a discrete number of values given by the non-trivial center elements, for $S U(2)$, this is only $-\mathbb{1}$. On the lattice, thin center vortices are located by plaquettes with negative center element, the vortex world sheet consists of plaquettes dual to these (negative) P-plaquettes. Such a P-vortex can be realized for example by simply setting all links to $+\mathbb{1}$ except for the time-like links in a 3-dimensional Dirac volume $V$ (see Fig. 1):

$$
U_{t}\left(\vec{x}, t_{0}\right)=-\mathbb{1}, \quad a<z \leq b, \forall x, \forall y
$$

The vortex surface $\partial V$ are the $x y$-planes at $t=t_{0}$ and $z=a, b$. The only plaquettes that are non-trivial are the $z$-plaquettes (corresponding to a field $E_{z}$ ) on these planes. They signal the presence of two vortices carrying a flux of $\pi$ each.

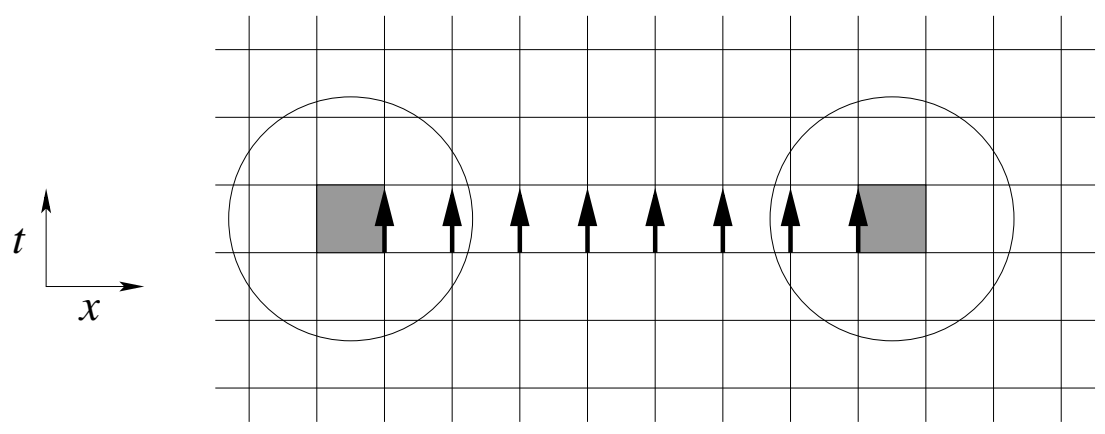

Figure 1: If in some three-volume $V$ the temporal links are multiplied with a nontrivial center element, thin center vortices (indicated by shaded plaquettes) are created at the border of the three-volume. [26]

\footnotetext{
${ }^{1}$ The center of a group is defined as the subgroup of elements commuting with all group elements.
} 
Further, with untwisted boundary conditions it is not possible to create a single plane $S U(2)$ vortex. The planes always come in pairs and therefore form a closed vortex world sheet by definition. A thick vortex is created by smearing out the thin vortex sheet, details of how to construct thick vortices will be discussed in chapter 4. In Monte Carlo configurations one generally finds thick vortices, the corresponding P-vortices have a very branched structure (fluctuating surface) and can be smoothed out (see appendix A.4.2).

\subsection{The vortex picture of quark confinement}

Quark confinement has been introduced as the phenomenon that quarks are never observed as isolated, free particles but are confined in composed particles, the hadrons. A simple phenomenological model for hadrons is a quark and an anti-quark sitting at the ends of a straight line, called string. In the framework of QCD one identifies the string with an electric flux tube of fixed cross-sectional area, in which the field between two quarks is collimated. Suppose this string has a constant energy density $\sigma$ (in its rest system), the string tension, which for an electric flux tube is $\sigma=\frac{1}{2} \int d^{2} x_{\perp} \vec{E}^{a} \cdot \vec{E}^{a}$. Therefore the quark-antiquark potential rises linearly at intermediate distances. But at some distance $r_{0}$, the string breaking distance, the energy of the string is high enough to create a new quark-antiquark pair. Quarks of finite mass are therefore confined only in the sense that after string-breaking they again recombine into hadrons.

The relation of vortices to quark confinement can be presented in an even more simplified phenomenological model. Suppose the vortex world sheet carrying its magnetic flux compresses somehow the electric field diverging from a quark into the mentioned flux tube. A simple illustration of this model is shown in Fig. 2.

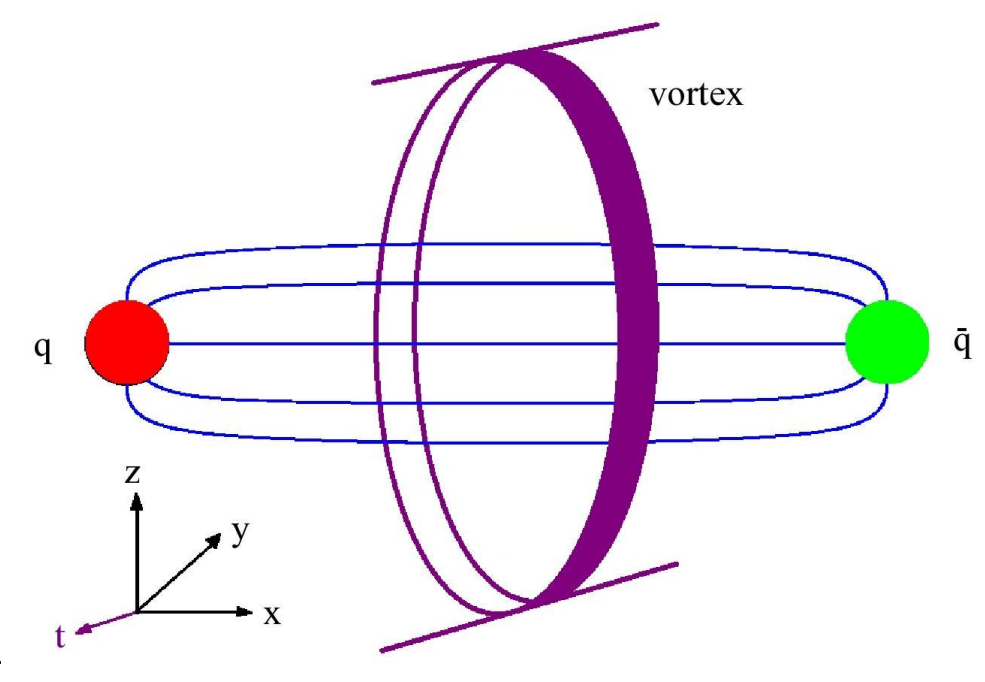

Figure 2: The color-magnetic vortex flux tube (extending in time direction) compresses the color-electric field, the string between quark $q$ and antiquark $\bar{q}$.

The physical relevance of this picture can be seen from the Wilson loop criterion of 
confinement. In appendix A.5 it is shown that the expectation value of the Wilson loop has to follow an area law in the confined phase. The vortex model is able to deduce the area law from independent vortex piercings of the Wilson loop, which can be interpreted as crossings of the static electric flux tube and moving closed magnetic flux. Since a plaquette is pierced by a $\mathrm{P}$-vortex if the product of its center-projected links gives -1 , the expectation value $\langle W(A)\rangle$ of the Wilson loop of area $A=R \times T$ with $f$ denoting the probability that a plaquette has the value -1 , gives (see also Fig. 3 )

$$
\langle W(A)\rangle=[f \cdot(-1)+(1-f) \cdot 1]^{A}=\exp [\underbrace{\ln (1-2 f)}_{-\sigma} A] .
$$

The area law follows from fluctuations in the number of vortices piercing a Wilson loop, which gives the string tension $\sigma \equiv-\ln (1-2 f) \approx 2 f$. Numerical tests for this vortex picture were first presented in $[11,27]$.
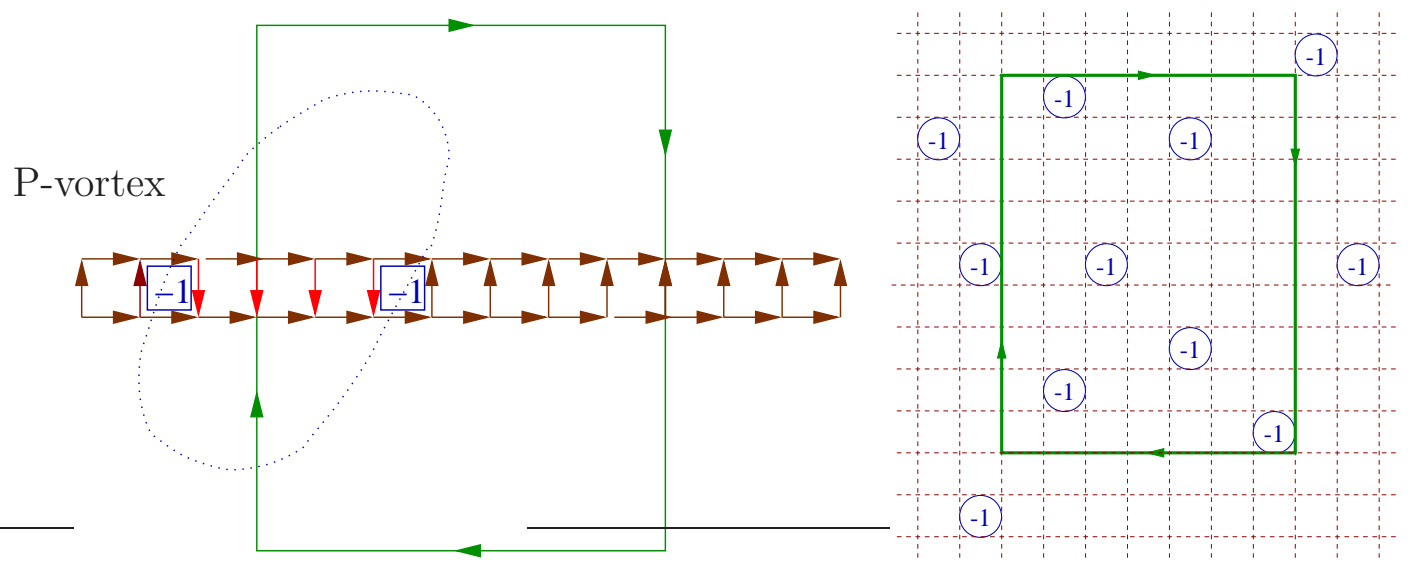

Figure 3: The Wilson loop (green) pierced by P-vortices.

The mathematical approach to the relevance of center degrees of freedom for confinement can be seen from $N$-ality. For $S U(N)$ in general, the $N$-ality classifies representations of a group according to the representation of their center. There is an infinite number of representations of the gauge group $S U(N)$, but only $N$ different representations for the center $Z_{N}$. Every representation of $S U(N)$ falls into one of these $N$ subsets depending on the representation of the $Z_{N}$ subgroup in the given $S U(N)$ representation. The center in a representation of $N$-ality $k=0, \ldots, N-1$ labels the center element $z_{N} \in Z_{N}$. The fundamental representation has $k=1$ (because it is the defining representation), while the adjoint representation has $k=0$, so that every center element is mapped to unity. Now the asymptotic string tension $\sigma$ for static charges in a representation of some $N$-ality only depends on the presence or absence of dynamical fields of this $N$-ality. Therefore fields of zero $N$-ality (e.g. gluons) cannot break the strings of particles in non-zero $N$-ality (e.g. quarks).

There exists a global center symmetry which allows to define an order parameter for confinement and deconfinement in systems at finite temperature. Center symmetry implies gauge transformations which are periodic up to a center element, corresponding 
to a multiplication by a factor $\exp \left(\frac{2 \pi i k n}{N}\right)$. Applying these gauge transformations leaves the Lagrangian invariant since $z_{N}$ is a center element and commutes with the gauge potential $A_{\mu}(\vec{x}, t)$. On the lattice this can be realized by multiplying all time-like link variables in a given time-slice with a center element (see Fig. 4).

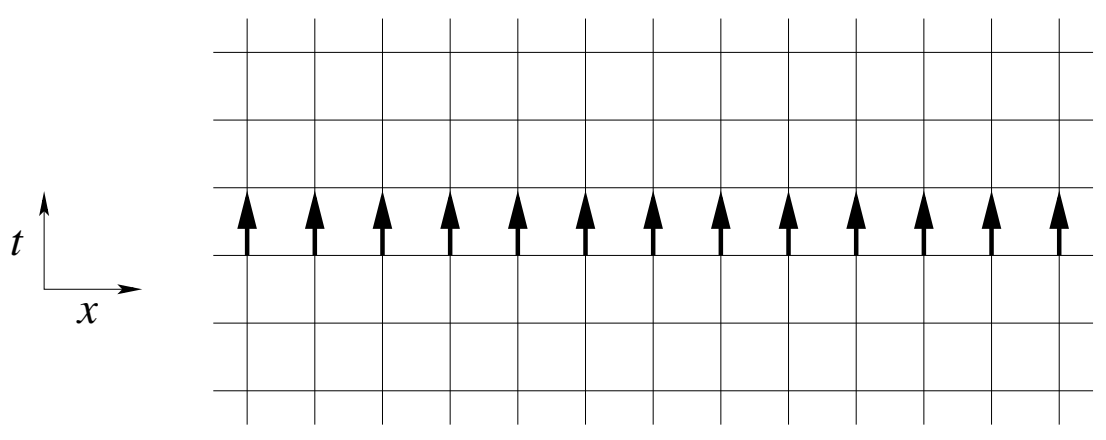

Figure 4: For an aperiodic gauge transformation, all time-like links in a given time-slice are multiplied with a center element. [26]

An observable affected by such a singular (aperiodic) gauge transformation is the Polyakov loop. The Polyakov loop $P(\vec{x})$ is the parallel transporter ("product of link variables") along a curve $C$ extending straightly in time direction, closed by lattice periodicity. It simply involves all time-like links along a single time-line and it can easily be seen that $P(\vec{x})$ is non-invariant under an aperiodic gauge transformation. The only chance for its expectation value $\langle P(\vec{x})\rangle$ to be invariant is to be equal to zero. Therefore it represents an order parameter for center symmetry breaking, finite in the center symmetric phase and zero in the phase of broken center symmetry.

Physically, the Polyakov loop describes a static (infinitely massive) test quark, with a time-like world-line. As shown in App. A.6, the expectation value of the Polyakov loop measures the free energy of an isolated static quark. This impacts confinement: In the confined phase, the free energy of such an isolated quark is infinite. Hence the Polyakov loop vanishes and remains trivially invariant under center transformations. Conversely, in the deconfined phase, the free energy is finite. Thus, the Polyakov loop is non-zero and not invariant under the center transformation. Consequently, confinement is intimately related to the center symmetry, whose excitations are just the center vortices. If the gauge transformation described above does not involve the whole time-slice but covers only a three-dimensional volume (see Fig. 1), the Dirac volume, the plaquettes within the Dirac volume remain unchanged. However, the plaquettes at the boundary of the Dirac volume are multiplied by a center element and simply represent the P-vortex plaquettes.

If fundamental matter fields such as fermions or Higgs field are present, the center symmetry is explicitly broken, this impacts confinement. For observables in the adjoint representation on the other hand, the symmetry always holds, which is again related to $N$-ality. Lattice simulations have provided quite an impressive collection of results which mainly corroborate the vortex model of confinement. Numerical results, based on [28] are presented in section 3, re-measured with improved gauge action. 


\subsection{Topological Charge}

The vortex world-surfaces allow to determine the topological charge of configurations. This was first discussed in the continuum by Cornwall [29, 30], Engelhardt and Reinhardt [31] and then on the lattice in ref. [32]. From the definition

$$
q \propto \epsilon_{\mu \nu \alpha \beta} F_{\mu \nu} F_{\alpha \beta}
$$

it is clear that topological charge arises only where two perpendicular non-trivial plaquettes meet. More precisely, the topological charge arises at lattice sites at which the tangent vectors to the vortex surface span all four space-time directions. Such sites are either (self-)intersection points or writhing points, see Fig. 5. Intersection points contribute by $Q= \pm \frac{1}{2}$ to the topological charge $q$, writhing points by $|Q|<\frac{1}{2}$.

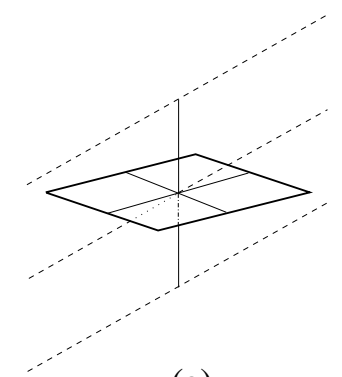

(a)

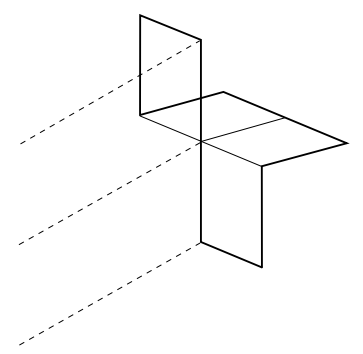

(b)

Figure 5: Intersection points (a) and writhing points (b) which contribute to the topological charge of a P-vortex surface. The full lines are space-like and the dashed lines time-like. [31]

\section{Relation to magnetic monopoles}

Vortices carry color-magnetic flux, after Abelian projection this flux appears as a monopole-antimonopole chain, as indicated schematically in Fig. 6 and discussed in ref. [33].

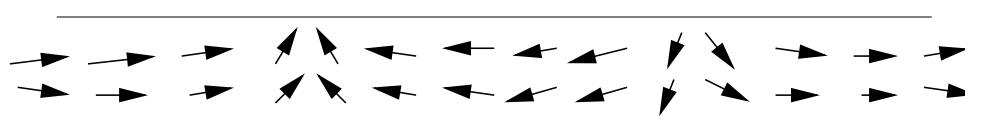

Figure 6: Vortex field strength after maximally Abelian gauge fixing. Vortex strength is mainly in the horizontal $\pm \sigma_{3}$ direction. [33]

\subsection{Vortices and chiral symmetry breaking}

The chiral condensate

$$
\bar{\psi} \psi=\bar{\psi}_{l} \psi_{r}+\bar{\psi}_{r} \psi_{l}
$$


is the order parameter which indicates whether chiral symmetry is present or broken. In the chiral symmetric phase the phase transformations of right and left-handed quarks are independent and average $\bar{\psi} \psi$ to zero. In the chirally broken phase transformations of left handed quarks lead to phase changes of right handed quarks and result in $\bar{\psi} \psi \neq 0$. Lattice calculations indicate that a transition from the confined to the quark-gluon plasma phase is associated with a transition from the chirally broken to the chiral symmetric phase. This indicates that both phenomena, confinement and dynamical chiral symmetry breaking may have the same origin. Due to the strong indications that vortices explain confinement it is very important to investigate the relation of vortices to chiral symmetry breaking. A remarkable result was found by Forcrand and d'Elia [14], removing vortices from lattice configurations leads to restoration of chiral symmetry. Fig. 7 shows the chiral condensate tending to zero for vortex-removed ("Modified") configurations.

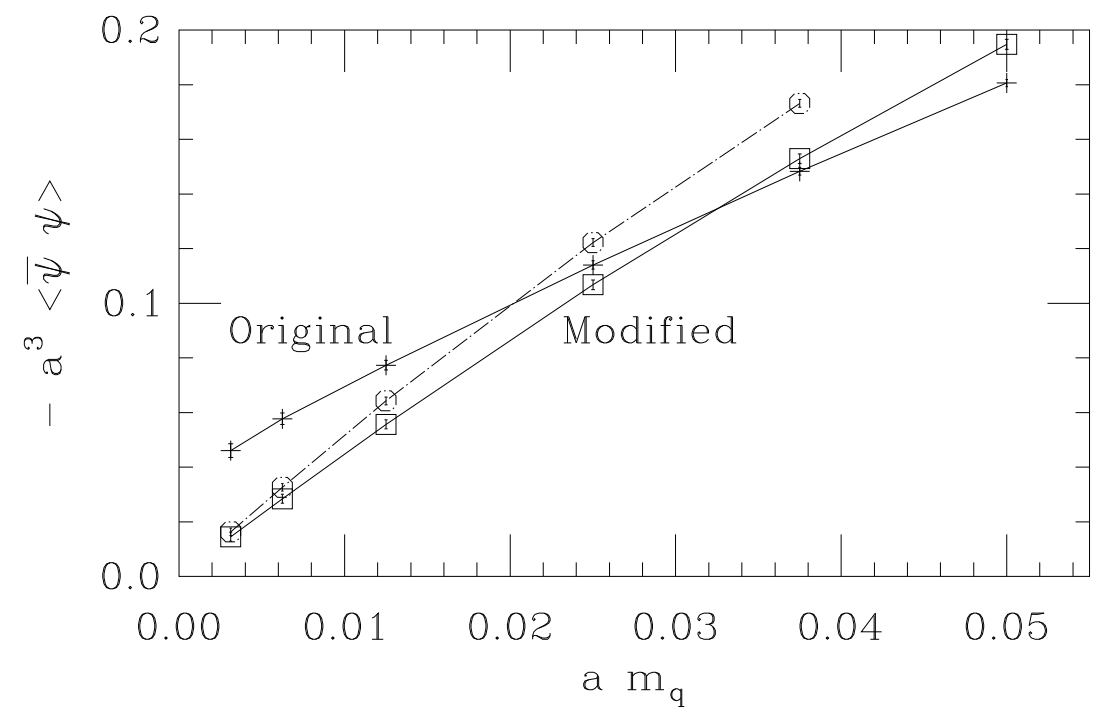

Figure 7: Chiral condensate in quenched lattice configurations before ("Original") and after ("Modified") vortex removal. From de Forcrand and D'Elia [14].

That smooth vortex configurations give rise to zero modes of the Dirac operator was shown first in analytical calculations by the Tübingen group [34]. The zero modes of the Dirac operator tend to peak at the intersections as shown in Fig. 8. The plot shows the probability density of the zero mode in a background of two pairs of intersecting vortex sheets. Using the chirally improved Dirac operator Gattringer and the Tübingen group $[35,36]$ have investigated the influence of center vortices on the properties of the Dirac spectrum. They have shown, see Fig. 9, that the removal of center vortices eliminates the zero modes and near-zero modes of the Dirac operator implying via the Banks-Casher relation the restoration of chiral symmetry. It was not understood why the spectra of the center-projected configuration has developed a large gap indicating chirally symmetric field configurations. This is a very interesting result. It is up to now the only case where confinement does not lead to chiral symmetry breaking. 


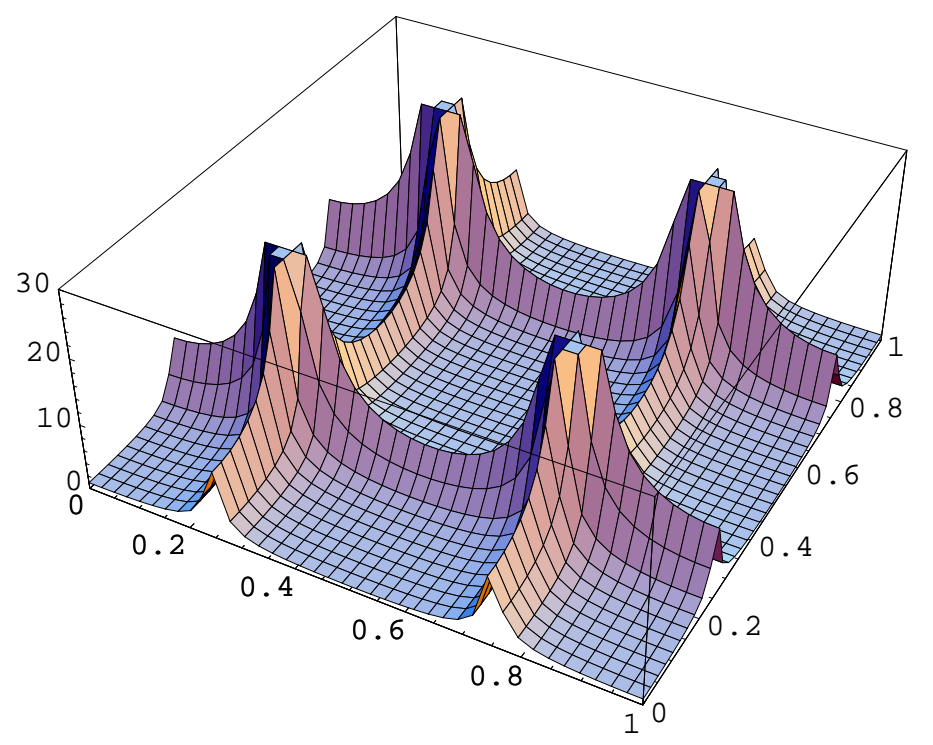

Figure 8: Probability density of the zero mode in the background of four intersecting vortex sheets is shown in the two-dimensional subspace defined by the intersection points. $[35,36]$

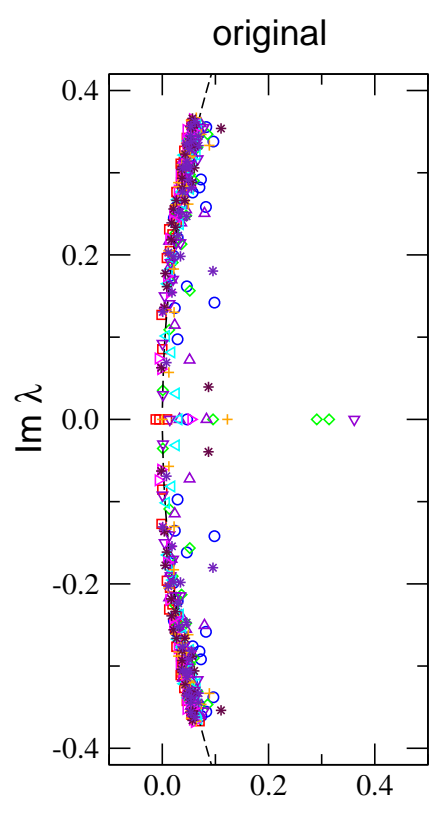

$\operatorname{Re} \lambda$

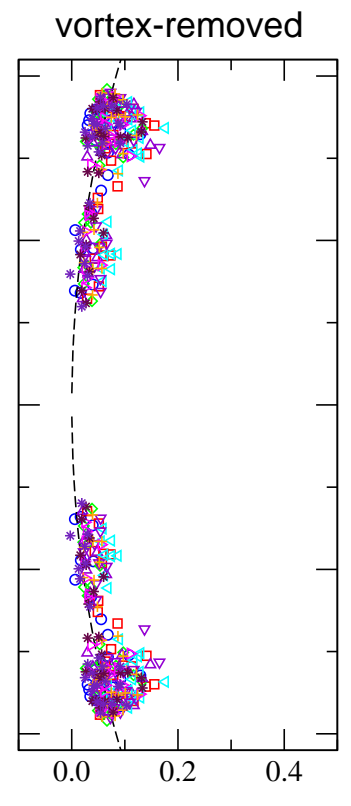

$\operatorname{Re} \lambda$
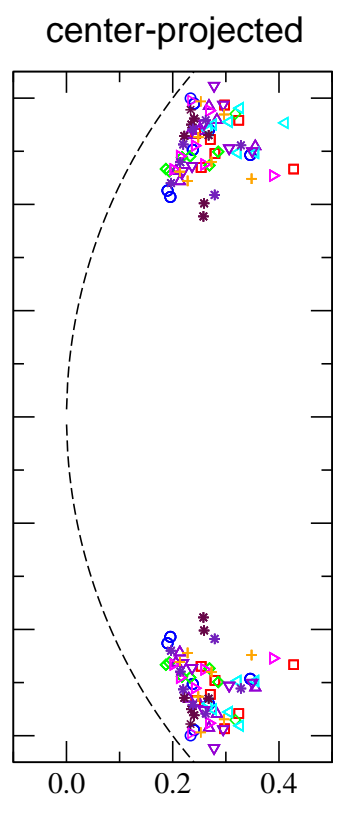

$\operatorname{Re} \lambda$

Figure 9: The 50 smallest Dirac eigenvalues from 10 different configurations are shown in the complex plane. The spectrum for the original ensemble (lhs. plot) are compared to the spectrum for vortex-removed configurations (center) and the spectrum of center-projected configurations (rhs. plot). [35, 36] 



\section{Numerical Results on Confinement}

All previous numerical checks of the vortex confinement mechanism, via maximal center gauge fixing and center projection, have been carried out on lattices generated from Monte Carlo simulations of the Wilson action (a discussion of these tests and their significance can be found in Ref. [28].) It is important to repeat these checks for the Lüscher-Weisz action. It turns out that there are no surprises, and vortex results derived using the new action are consistent with the previous work with the Wilson action. The most important achievements of the vortex picture are listed below.

\subsection{Scaling of vortex density}

Fig. 10 shows the $\mathrm{P}$-vortex density (in lattice units) vs. $\beta_{L W}$. The solid line is the two-loop asymptotic freedom behavior for this quantity, for a choice of vortex density $\rho$ (area per unit volume) in physical units satisfying $\sqrt{\rho / 6 \Lambda^{2}}=50$.

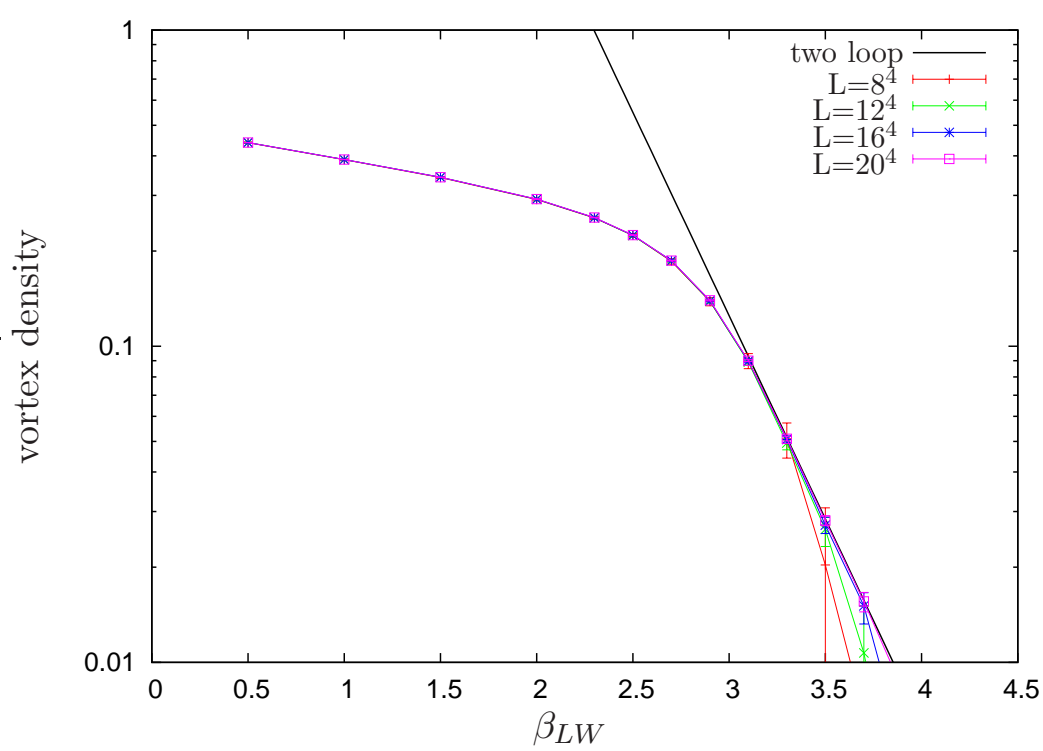

Figure 10: P-vortex surface density vs. coupling constant $\beta_{L W}$. "Two loop" line is the scaling prediction with $\sqrt{\rho / 6 \Lambda^{2}}=50$.

This was first observed by Langfeld et al. [37]. Let $N_{P}$ denote the number of Pplaquettes and $N_{T}$ the total number of plaquettes. The vortex density $p$ is given by

$$
p=\frac{N_{P}}{N_{T}}=\frac{N_{P} a^{4}}{6\left(N_{T} / 6\right) a^{4}}=\frac{1}{6} \frac{\text { Vortex area }}{\text { Lattice volume }} a^{2}=\frac{\rho a^{2}}{6}
$$

If the vortices are physical objects, their density should scale with $\beta\left(\beta_{L W}\right)$ according to renormalization group theory, which predicts (numerical constants specific to $S U(2)$ )

$$
p=\frac{1}{6} \frac{\rho}{\Lambda^{2}}\left(\frac{6 \pi^{2}}{11} \beta\right)^{102 / 121} \exp -\frac{6 \pi^{2}}{11} \beta
$$




\subsection{Center dominance}

The vortices in the projected $\mathrm{Z}(2)$ gauge theory reproduce a good deal of the string tension of the full Yang-Mills theory, see Fig. 11. A removal of the P-vortices from the lattice configuration results in a loss of the confining properties as depicted in Fig. 12.

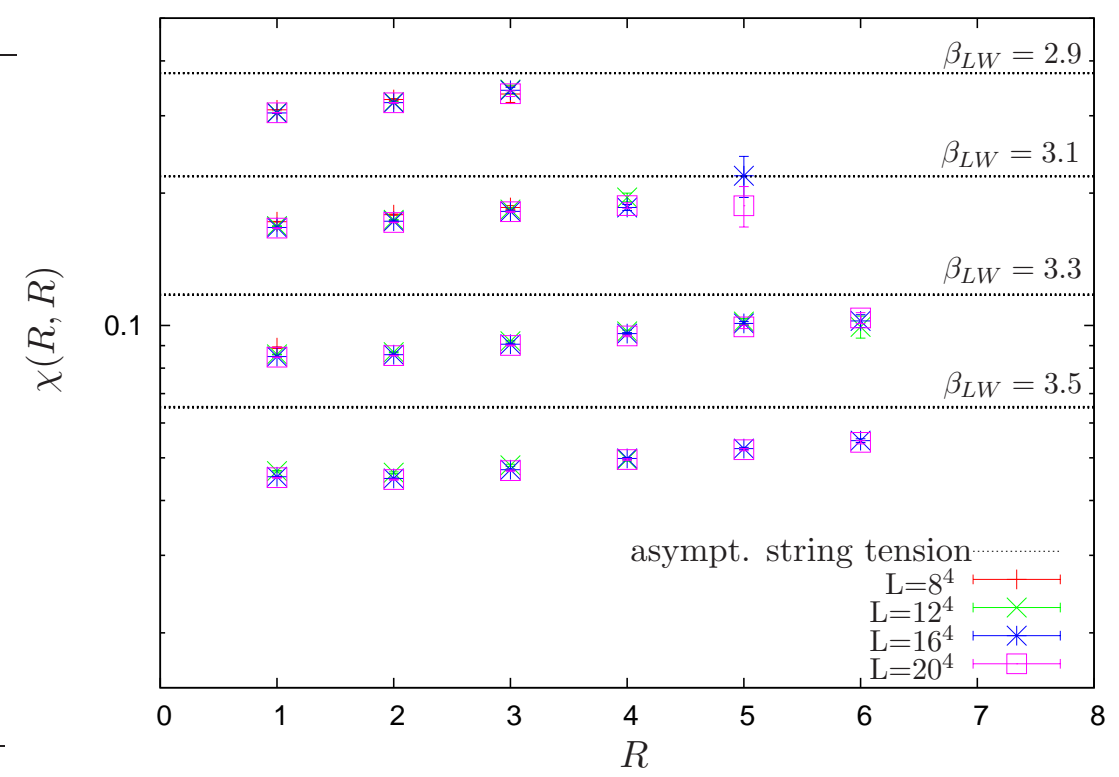

Figure 11: Center-projected Creutz ratios at $\beta_{L W}=2.9-3.5$ obtained after direct Laplacian center gauge fixing. Horizontal bands indicate the asymptotic string tensions on the unprojected lattice, with the corresponding error bars.

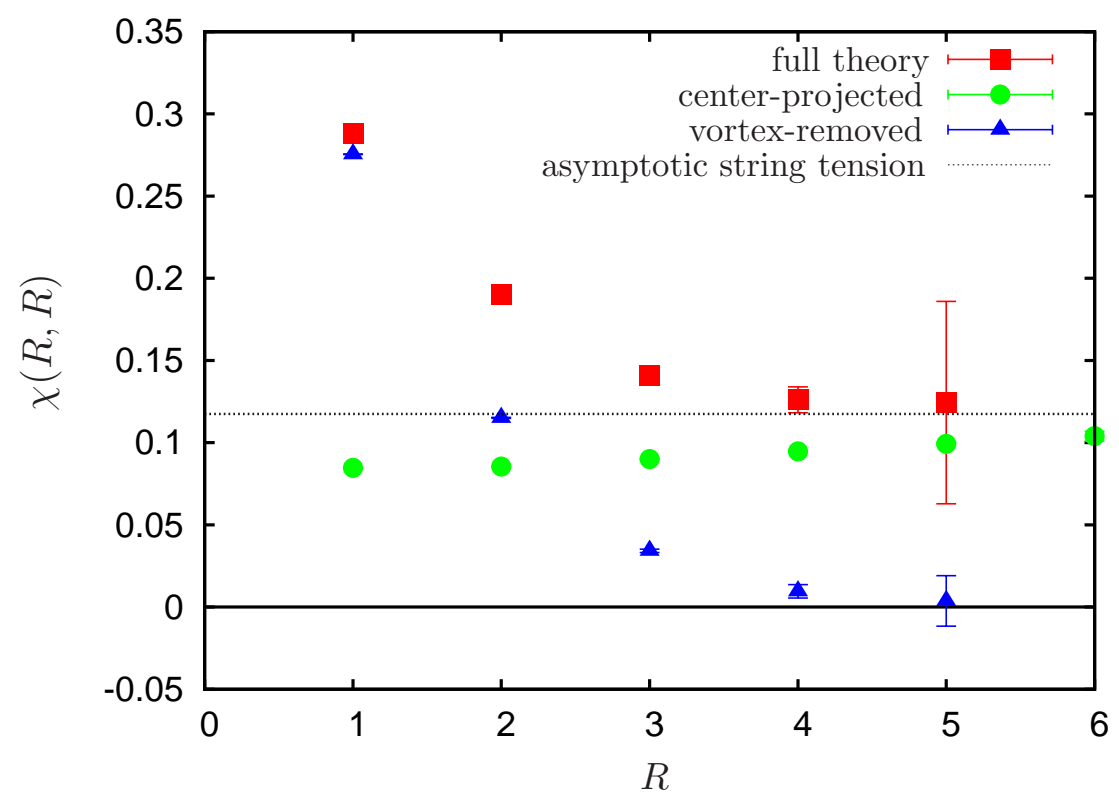

Figure 12: Creutz ratios on $20^{4}$-lattices at $\beta_{L W}=3.3$ for full, center-projected and vortex-removed data. Horizontal bands indicate asymptotic string tension on the unprojected lattice with error bars. 


\subsection{Precocious linearity}

The fact that the Creutz ratios from center-projected Wilson loops are almost independent of $R$ is known as Precocious linearity. It implies that the projected potential is already linear at two lattice spacings [25]. For the Lüscher-Weisz action the centerprojected Creutz ratios coincide with the "two loop" scaling prediction at one lattice spacing already, as can be seen in Fig. 13.

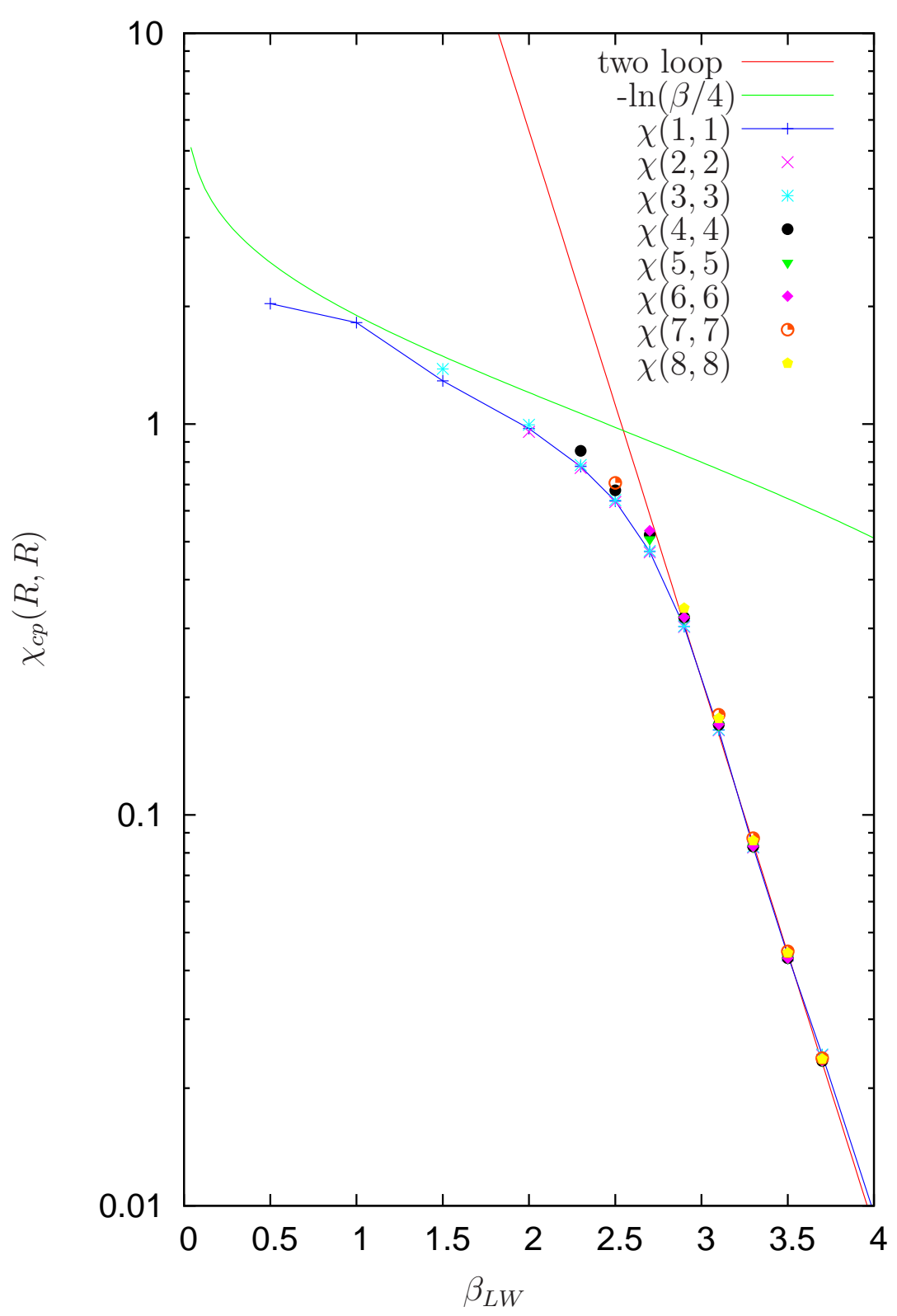

Figure 13: Creutz ratios from center-projected Wilson loops on 204-lattice configurations, in direct Laplacian center gauge. "Two loop" line is again the scaling prediction with $\sqrt{\rho / 6 \Lambda^{2}}=50$. 


\subsection{P-vortices locate thick center vortices}

Vortex limited Wilson loops $W_{n}$ are expectation values of Wilson loops in the sub ensemble of those configurations where the minimal area of the loop is pierced by precisely $n$ P-vortices. As shown in Fig. 14 for large loop area $W_{n}$ approaches the limit $(-1)^{n} W_{0}$.

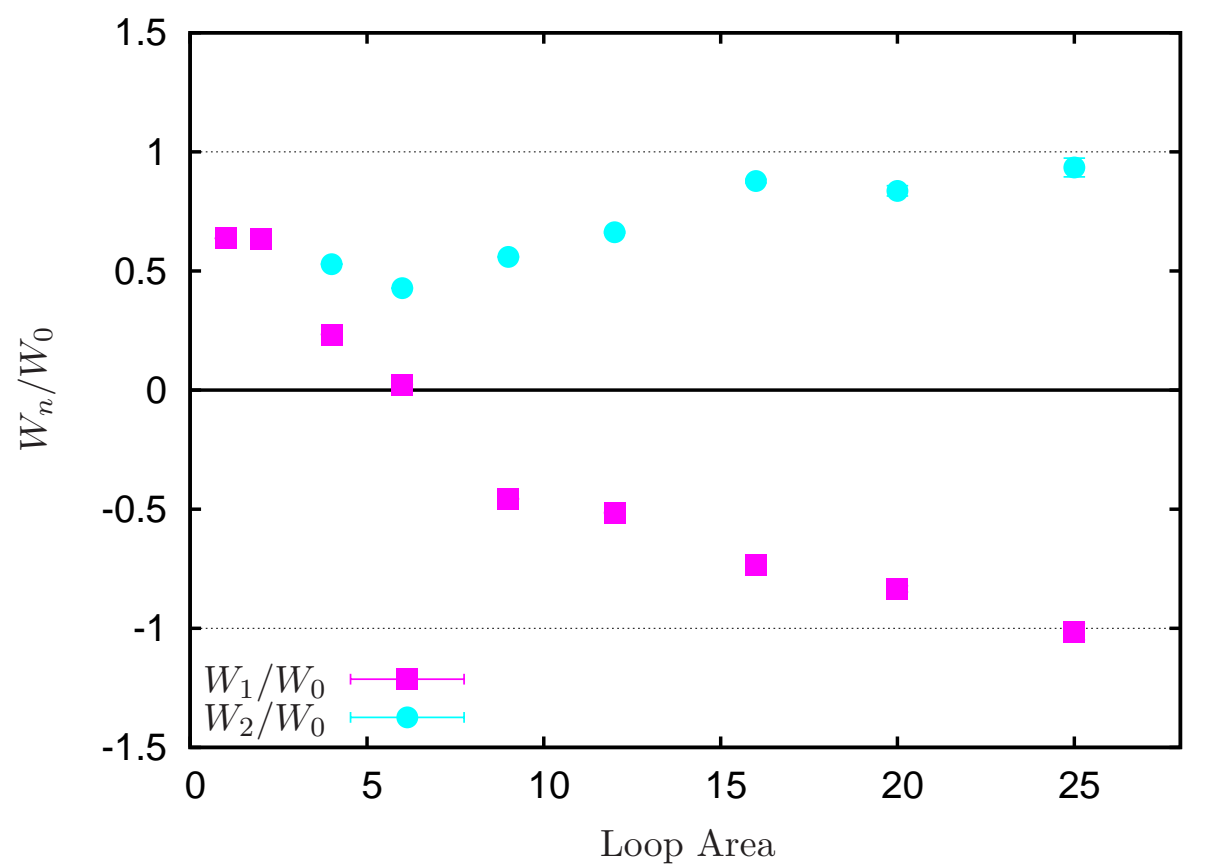

Figure 14: $W_{n} / W_{0}$ Wilson loop ratios on $20^{4}$-lattice configurations at $\beta=3.1$.

\subsection{Casimir scaling}

The asymptotic string tension depends on $\mathrm{N}$-ality of the color charge only, so that for $S U(2) \sigma_{j}=\sigma_{1 / 2}$ for $j$ half-integer $\sigma_{j}=0$ for $j$ integer there is still an intermediate range of distances where Casimir scaling applies (at least approximately), i.e. for $S U(2)$ $\sigma_{j}=\frac{1}{2} j(j+1)$. One could show by a very simple ansatz [33] that for charge distances comparable to the thickness of these vortices the proportionality of the string tensions to the eigenvalue of the quadratic Casimir operator is very natural in a thick vortex model (Fig. 15). For distances large compared to the vortex thickness the string tension reduces to that of the thin vortex model.

\subsection{Vortices and matter fields}

Matter fields lead to a breaking of the gluon string. The interesting question how matter fields influence vortices was first studied in the $S U(2)$-Higgs model in the continuum in refs. [38, 39] and then on the lattice in [40, 41, 42]. 


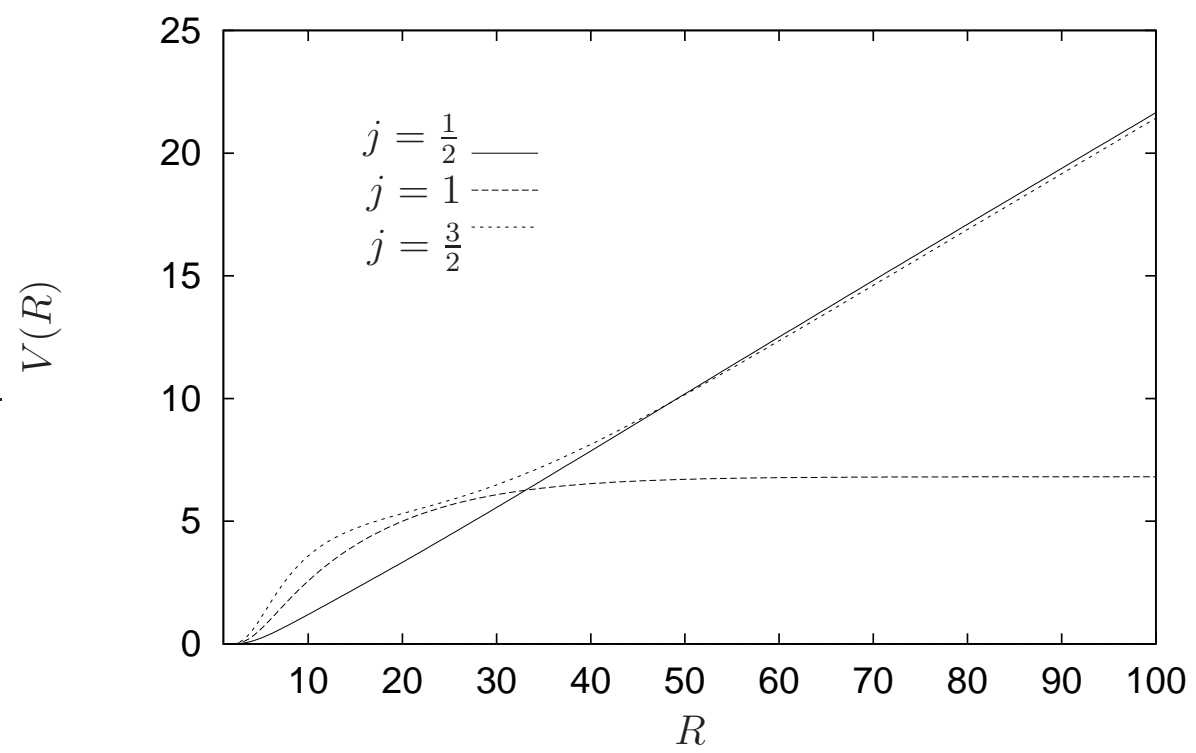

Figure 15: Inter quark potential $V(R)$ induced by center vortices, according to the thick vortex model discussed in the text, for quark charges in the in the fundamental $\left(j=\frac{1}{2}\right)$, adjoint $(j=1)$, and $j=\frac{3}{2}$ representations. [33]

\subsection{Finite temperature}

The P-vortex density across the deconfinement phase transition was first carried out by Langfeld et al. [43] and Chernodub et al. [44]. At zero temperature vortices are unorientable surfaces and percolate through the lattice [45]. At finite temperature Pvortices exist also in the deconfined phase. They form cylindric objects which extend in time direction, see Fig. 16. This explains the area law for space-like Wilson loops and the perimeter law for time-like Wilson loops.

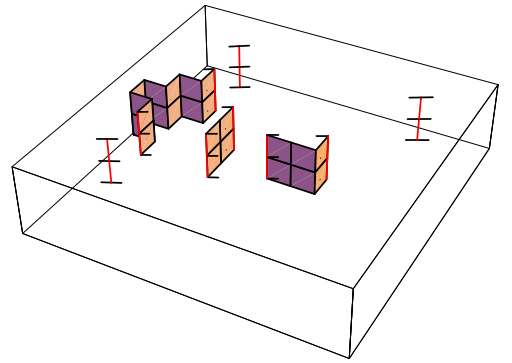

(a)

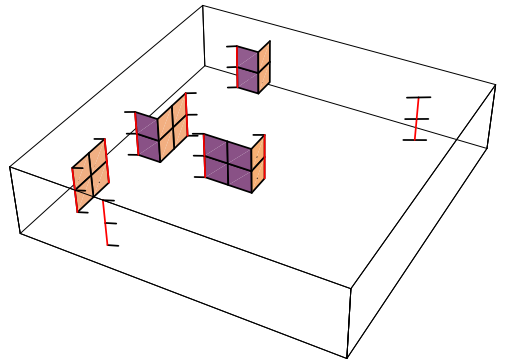

(b)

Figure 16: Dual P-plaquettes in a typical field configuration on a $12^{3} \times 2$-lattice. Two successive z-slices for the xyt-subspace are shown. The amputated lines leaving the left figure towards right arrive in the right figure from the left.[45]

Finally, Figs. 17 and 18 show the deconfinement phase transition for full and centerprojected Polyakov loops on $12^{3} \times N_{t}$-lattices with $N_{t}=2,4,6$ and 12 for comparison with Wilson and Lüscher-Weisz action. 
a)

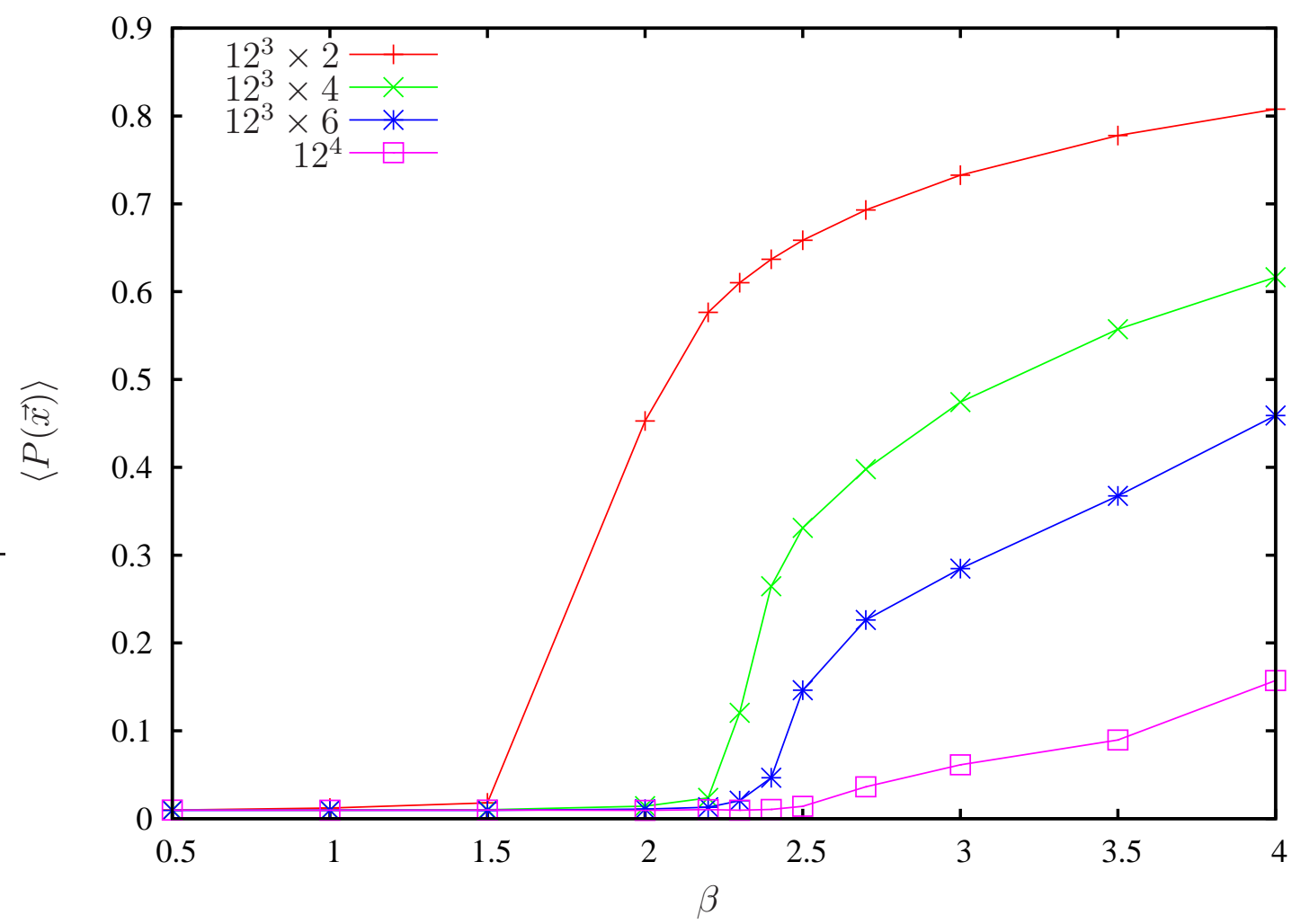

b)

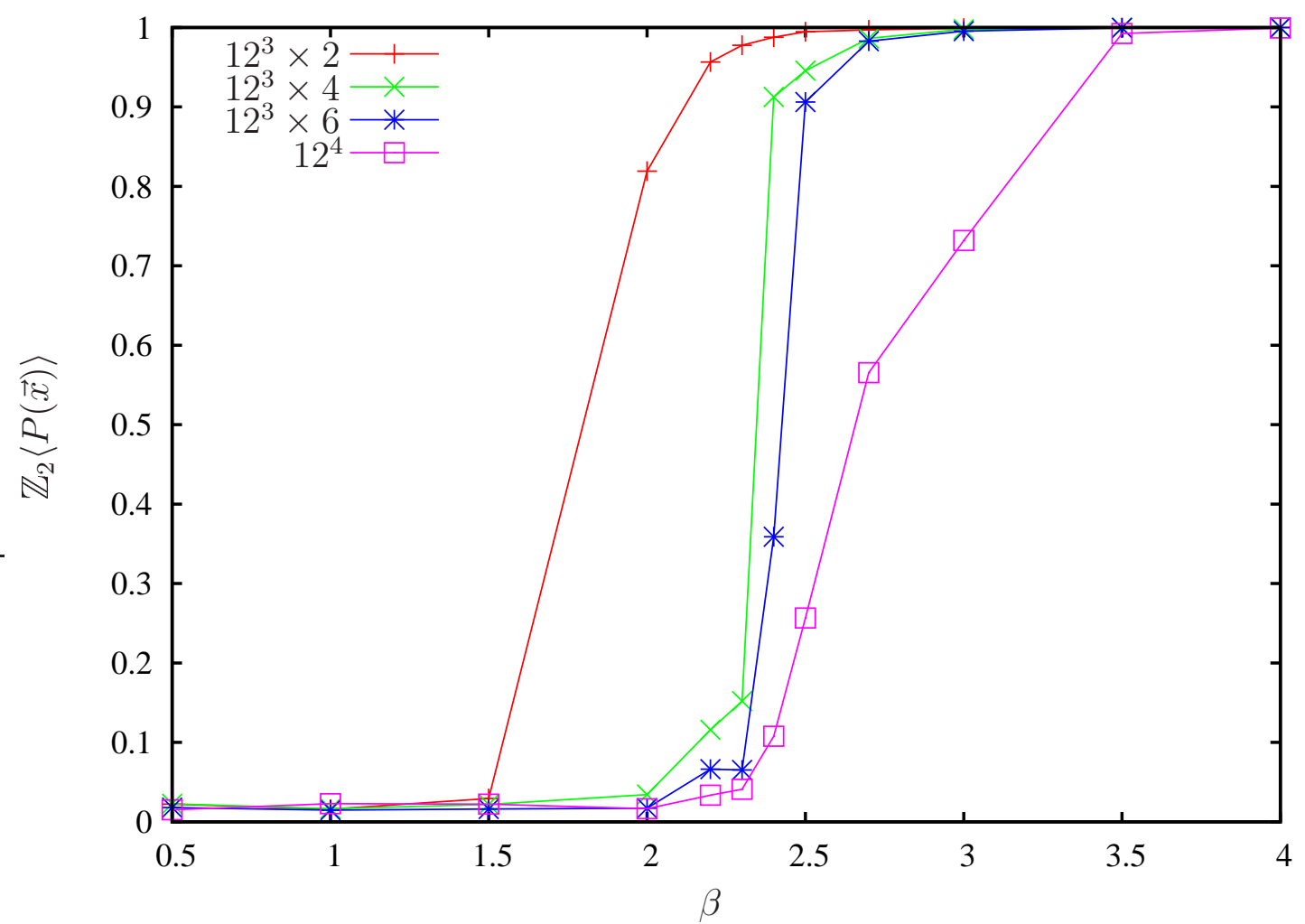

Figure 17: Polyakov loop phase transition of a) full and b) center-projected configurations for different lattice sizes with Wilson action. 
a)

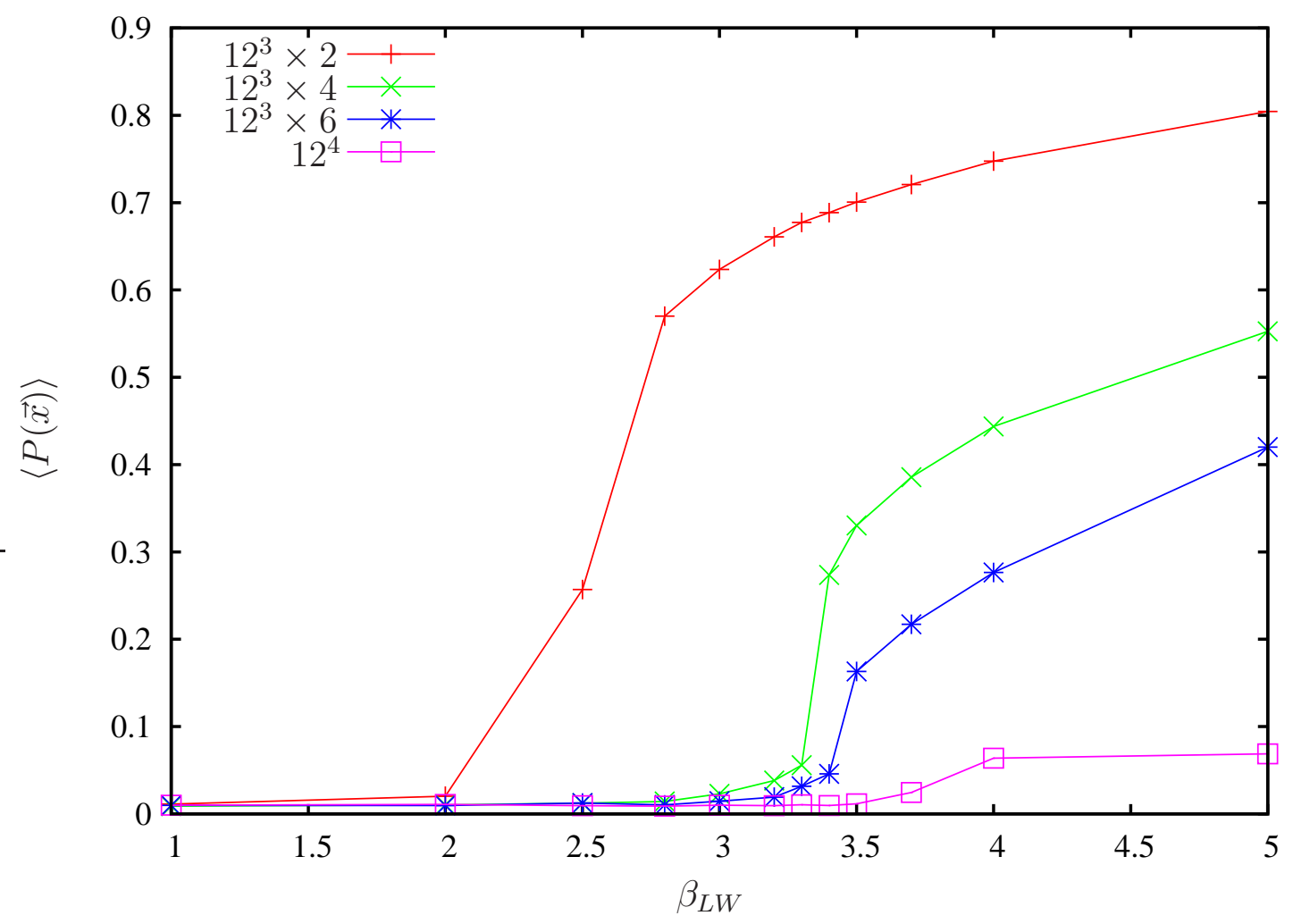

b)

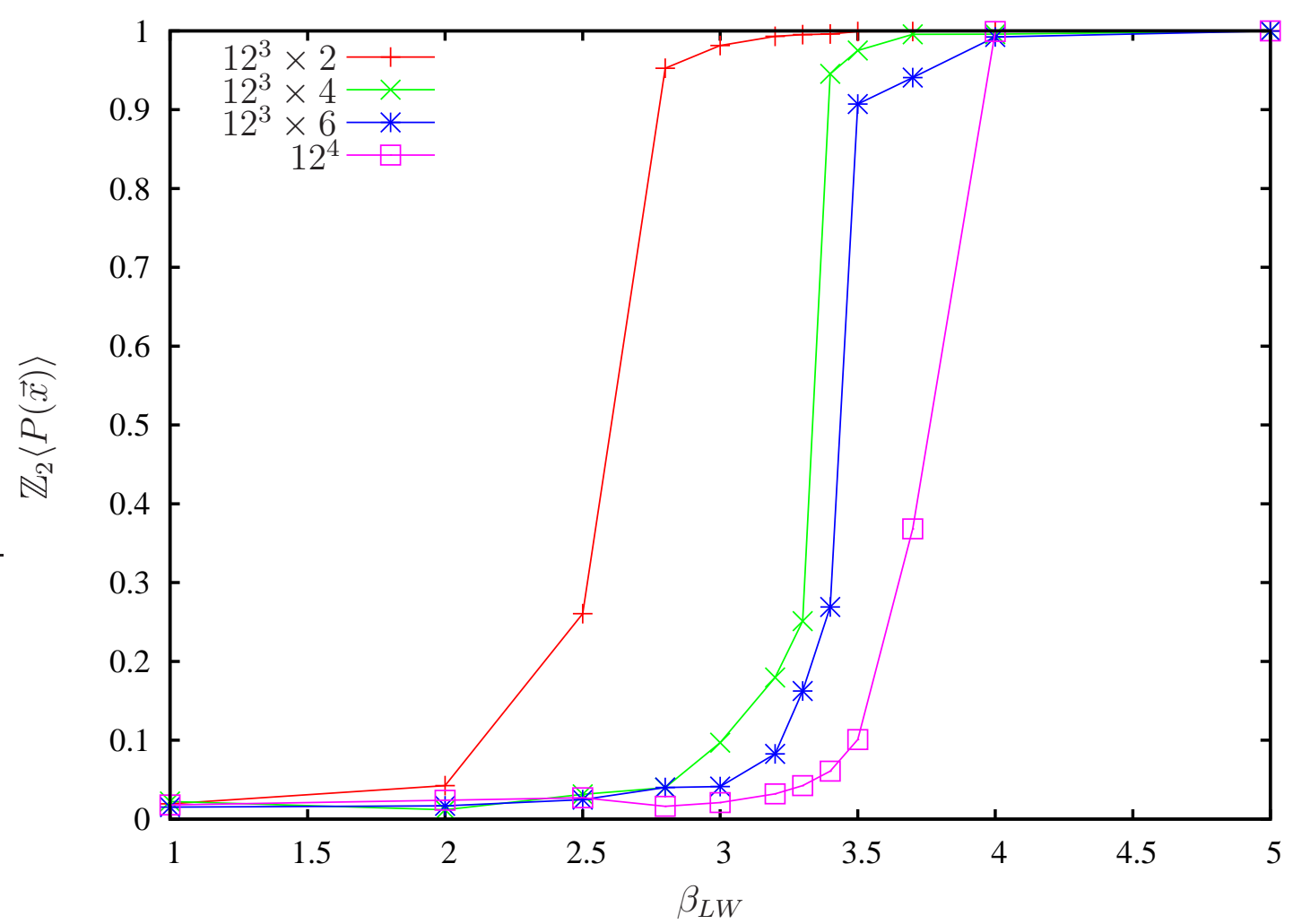

Figure 18: Polyakov loop phase transition of a) full and b) center-projected configurations for different lattice sizes with Lüscher-Weisz action. 



\section{Topological charge}

The correct description of topological charge is very important within a model of quark confinement. This section reports on calculations of the lattice index theorem with the overlap Dirac operator applied to thick classical center vortices in the shapes of planes (closed by lattice periodicity) and spheres. The localization of zero modes with respect to the position of the thick vortices is investigated and an interesting discrepancy in the topological charge determined by different methods is found. The details of the individual vortex types will be discussed along with the results. First, the different definitions of the lattice topological charge are given.

\subsection{Definitions}

(1) The topological charge of the continuum gauge field configuration for the following, rather simple vortex geometries amounts to adding up the contributions from the intersection points, which according to the color orientation carry a topological charge $Q= \pm \frac{1}{2}[46]$.

(2) The index of the overlap Dirac operator $[18,47]$. According to the Atiyah-Singer index theorem the topological charge is given by the index

$$
\text { ind } D[A]=n_{-}-n_{+}=Q
$$

where $n_{-}$and $n_{+}$are the number of left- and right-handed zero modes of the Dirac operator [19, 20, 21]. The overlap Dirac operator is defined by [48]

$$
D=\frac{1}{2}\left[1+\gamma_{5} \epsilon\left(H_{L}^{+}\right)\right]
$$

Here, $\epsilon$ is the sign function, $H_{L}^{+}=\gamma_{5} D_{w}\left(-m_{0}\right)$ and $D_{w}$ is the usual lattice Wilson Dirac operator with mass $-m_{0}$ (here, $m_{0}=1.0$ is used). It has been shown that this fermionic definition of $Q$ coincides with the continuum simple gluonic definition in the continuum limit [49].

The lattice version of the index theorem is only valid as long as the gauge field satisfies a so-called "admissibility" condition. This condition assures that $H_{L}^{+}$has no zero eigenvalues so that the sign-function is well-defined. It requires that the plaquette-values $U_{\mu \nu}$ are bounded close to trivial $\mathbb{1}$. A sufficient, but not necessary bound for the "admissibility" of the gauge field is $[50,51]$

$$
\operatorname{tr}\left(\mathbb{1}-U_{\mu \nu}\right)<0.03
$$

(3) The integral (sum, on the lattice) of the gluonic charge density $q(x)=\frac{1}{16 \pi^{2}} \operatorname{tr}\left(\mathcal{F}_{\mu \nu} \tilde{\mathcal{F}}_{\mu \nu}\right)$ in the "plaquette" and/or "hypercube" definitions on the lattice, see ref. [52, 53]. Since Monte-Carlo configurations are in general too coarse, these definitions are usually only applied after cooling. 
With the field strength tensors $\mathcal{F}_{\mu \nu}(x)=\partial_{\mu} \mathcal{A}_{\nu}-\partial_{\nu} \mathcal{A}_{\mu}+\mathrm{i}\left[\mathcal{A}_{\mu}, \mathcal{A}_{\nu}\right]$ and $\tilde{\mathcal{F}}_{\mu \nu}=$ $\frac{1}{2} \epsilon_{\mu \nu \rho \sigma} \mathcal{F}_{\rho \sigma}$ the quantity which determines the affiliation to a homotopy class, the topological charge $Q$ or (negative) Pontryagin index reads for trivial boundary conditions [54]

$$
\begin{aligned}
Q & \equiv \int d^{4} x q(x)=-\frac{1}{16 \pi^{2}} \int d^{4} x \operatorname{tr}\left(\mathcal{F}_{\mu \nu} \tilde{\mathcal{F}}_{\mu \nu}\right)= \\
& =-\frac{1}{8 \pi^{2}} \epsilon_{\mu \nu \rho \sigma} \int d^{4} x \operatorname{tr}\left\{\left[\partial_{\mu} \mathcal{A}_{\nu}+\mathrm{i} \mathcal{A}_{\mu} \mathcal{A}_{\nu}\right]\left[\partial_{\rho} \mathcal{A}_{\sigma}+\mathrm{i} \mathcal{A}_{\rho} \mathcal{A}_{\sigma}\right]\right\}= \\
& =-\frac{1}{8 \pi^{2}} \epsilon_{\mu \nu \rho \sigma} \int d^{4} x \operatorname{tr}\left\{\partial_{\mu} \mathcal{A}_{\nu} \partial_{\rho} \mathcal{A}_{\sigma}+2 \mathrm{i} \mathcal{A}_{\mu} \mathcal{A}_{\nu} \partial_{\rho} \mathcal{A}_{\sigma}\right\}
\end{aligned}
$$

In the continuum, the topological charge density $q(x)$ is the total derivative of the topological current $k_{\mu}[55]$

$$
q(x)=\partial_{\mu} k_{\mu}, \quad k_{\mu}=-\frac{1}{8 \pi^{2}} \epsilon_{\mu \nu \rho \sigma} \operatorname{tr}\left[\mathcal{A}_{\nu} \partial_{\rho} \mathcal{A}_{\sigma}+\mathrm{i} \frac{2}{3} \mathcal{A}_{\nu} \mathcal{A}_{\rho} \mathcal{A}_{\sigma}\right]
$$

For smooth gauge fields one can apply the Gauss-theorem to transform the expression for $Q$ into a surface integral

$$
Q=\int d^{4} x q(x)=\oint_{S^{3}} d \sigma_{\mu} k_{\mu}
$$

For $x^{2} \rightarrow \infty$ a sufficiently fast decaying field strength is assumed, so that $\mathcal{F}_{\mu \nu}=0$ implies $\epsilon_{\mu \nu \rho \sigma} \partial_{\rho} \mathcal{A}_{\sigma}=-\mathrm{i} \epsilon_{\mu \nu \rho \sigma} \mathcal{A}_{\rho} \mathcal{A}_{\sigma}$. Thus one obtains

$$
Q=\frac{\mathrm{i} \epsilon_{\mu \nu \rho \sigma}}{24 \pi^{2}} \oint_{S^{3}} d \sigma_{\mu} \operatorname{tr}\left[\mathcal{A}_{\nu} \mathcal{A}_{\rho} \mathcal{A}_{\sigma}\right]
$$

By a gauge transformation $\Omega(x)$ the free gauge field at infinity is put to zero

$$
0=\mathcal{A}_{\mu}^{\prime}\left(x^{2} \rightarrow \infty\right)=\Omega^{\dagger}(x)\left[\mathcal{A}_{\mu}(x)-\mathrm{i} \partial_{\mu}\right] \Omega(x) \quad \Leftarrow: \quad \mathcal{A}_{\mu}\left(x^{2} \rightarrow \infty\right)=\mathrm{i} \partial_{\mu} \Omega \Omega^{\dagger} .
$$

The pure gauge potential $\mathcal{A}_{\mu}$ in Eq. (16) maps a 3D volume element $d x_{\nu} d x_{\rho} d_{\sigma}$ at infinity to a volume element

$$
\mathrm{i} \mathcal{A}_{\nu} \mathcal{A}_{\rho} \mathcal{A}_{\sigma} d x_{\nu} d x_{\rho} d_{\sigma}=\partial_{\nu} \Omega \Omega^{\dagger} \partial_{\rho} \Omega \Omega^{\dagger} \partial_{\sigma} \Omega \Omega^{\dagger} d x_{\nu} d x_{\rho} d_{\sigma}
$$

in $S U(2)$ group space. The requirement that the gauge transformation $\Omega(x)$ is smooth implies that the topological charge $Q$ in Eq. (15) is integer, $Q \in \mathbb{Z}$. $Q$ measures the number of times $\Omega(x)$ wraps around the group when $x$ covers the space time surface $S^{3}$ at $x^{2} \rightarrow \infty$ once. Choosing bases in the tangent spaces of the two $S^{3}$ manifolds, one can define an orientation of the map. Thus $Q$ can take positive and negative values. 

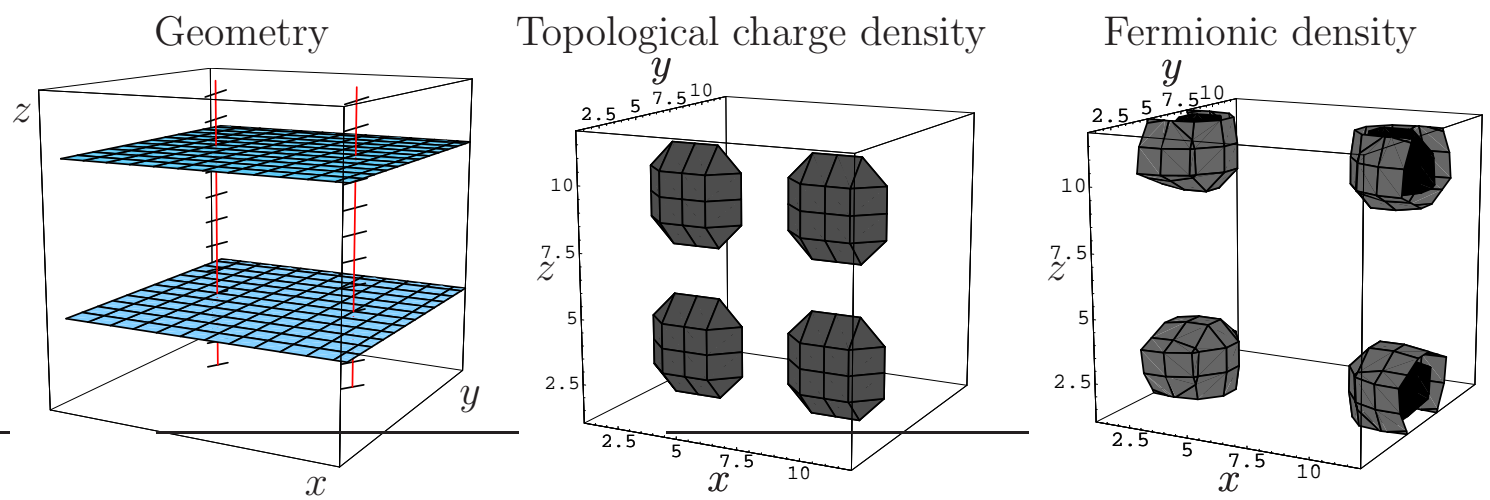

Figure 19: Plane vortices on a $12^{4}$-lattice in xy- and zt-planes intersect in four points giving rise to topological charge and localized fermionic density.

\subsection{Plane vortices}

Planar vortices are constructed as explained in [26]. At periodic boundary conditions they always come in pairs and therefore are automatically closed. All three definitions of $Q$ yield identical results for all configurations containing only plane vortices.

As an example, Fig. 19 shows some diagrams for two orthogonal pairs of plane vortices on a $12^{4}$ lattice, which intersect in 4 places. The left picture shows the position of the P-plaquettes after going to maximal center gauge and center projection. One vortex pair extends in xy-planes, the other pair in zt-planes. The short pieces of lines attached to the vertical line symbolize the extent of the zt-vortices in the time-direction. The diagram in the middle of Fig. 19 shows an equi-density surface of the topological charge density determined in the plaquette definition and the right diagram shows an equidensity surface of the scalar fermionic density.

Each of the four intersection points gives rise to a topological charge $Q= \pm \frac{1}{2}$ [12]. The sign of the contribution at a given intersection point can be changed by a flip of the orientation of the vortex surface, this means by a transition from links $U_{\mu}(x)$ creating one of the vortices to $U_{\mu}^{\dagger}(x)$ in the region of the intersection. After abelian projection a smooth transition of the vortex orientation in color space produces a monopole line. In this way one can produce configurations with two vortex pairs of topological charge $Q=0, \pm 1$ or \pm 2 . In all these cases the lattice index theorem is fulfilled and the results agree with the analytical solution for the zero modes presented in [34].

\subsection{Spherical vortices}

One distinguishes an orientable

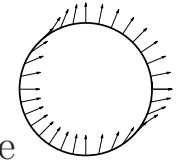

from a non-orientable

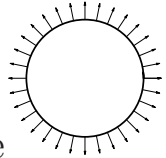

spherical vortex.

1. The non-orientable spherical vortex of radius $R$ and thickness $\Delta$ is constructed with an appropriate "profile"-function $\alpha\left(\left|\vec{r}-\vec{r}_{0}\right|\right)$ by time-like links which form a 
hedgehog in one time-slice of the lattice, where $\vec{r}_{0}$ is the center of a spatial sphere

$$
U_{\mu}(x)= \begin{cases}\exp \left\{\mathrm{i} \alpha\left(\left|\vec{r}-\vec{r}_{0}\right|\right) \vec{n}(x) \cdot \vec{\sigma}\right\} & t=1, \mu=4, \\ \mathbb{1} & \text { elsewhere }\end{cases}
$$

A characteristic property of a hedgehog configuration is the agreement between the color direction $\vec{n}$ and the spatial direction of $\vec{r}-\vec{r}_{0}$

$$
\vec{n}(\vec{r}, t)=\frac{\vec{r}-\vec{r}_{0}}{\left|\vec{r}-\vec{r}_{0}\right|}
$$

see left diagram in Fig. 20. For the choice (18) of the links the time-like Wilson lines (holonomies) agree with the time-like links in the $t=1$ time-slice.

At $\vec{r}=\vec{r}_{0}$ and at the largest possible distances from $\vec{r}_{0}$ on a periodic lattice the direction of the color-vectors (19) are undefined. This does not lead to a singularity of the gauge field if the profile-function $\alpha(r)$ is appropriately chosen such that the Wilson lines at these special points become center elements of the gauge group. A good choice for the profile-function $\alpha$ is either one from $\alpha_{+}, \alpha_{-}$, which are defined as

$$
\begin{aligned}
& \alpha_{+}(r)= \begin{cases}0 & r<R-\frac{\Delta}{2} \\
\frac{\pi}{2}\left(1+\frac{r-R}{\frac{\Delta}{2}}\right) & R-\frac{\Delta}{2}<r<R+\frac{\Delta}{2} \\
\pi & R+\frac{\Delta}{2}<r\end{cases} \\
& \alpha_{-}(r)= \begin{cases}\pi & r<R-\frac{\Delta}{2} \\
\frac{\pi}{2}\left(1-\frac{r-R}{\frac{\Delta}{2}}\right) & R-\frac{\Delta}{2}<r<R+\frac{\Delta}{2} \\
0 & R+\frac{\Delta}{2}<r\end{cases}
\end{aligned}
$$

This means that all links are equal to $\mathbb{1}$ except for the $t$-links in a single time-slice at fixed $t=1$. The phase changes from 0 to $\pi$ from inside to outside (or vice versa). The graph of $\alpha_{-}(r)$ for the largest lattice $40^{3} \times N_{t}$ is shown in Fig. 20 (right diagram). The traces of all plaquettes are close to unity, $\operatorname{tr}\left(\mathbb{1}-U_{\mu \nu}\right) \leq$ $1-\cos \frac{\pi}{18}=0.015$. In the following computations, $R$ is set to half the lattice size, and $\Delta$ is chosen such that only 3 links along any direction are equal to $+\mathbb{1}$ and $-\mathbb{1}$, respectively. The color vector $\vec{n}$ changes according to the spatial direction (see Fig. 20, left).

2. The orientable vortex is constructed in a similar way:

$$
U_{\mu}(x)= \begin{cases}\exp \left\{\mathrm{i} \alpha\left(\left|\vec{r}-\vec{r}_{0}\right|\right)|\vec{n}(x)| \cdot \vec{\sigma}\right\} & t=1, \mu=4 \\ \mathbb{1} & \text { elsewhere }\end{cases}
$$

Notice the absolute value of the coordinates of the color direction vector $\vec{n}$. These links cover only half of the $S U(2)$ group space and this half they cover twice. 

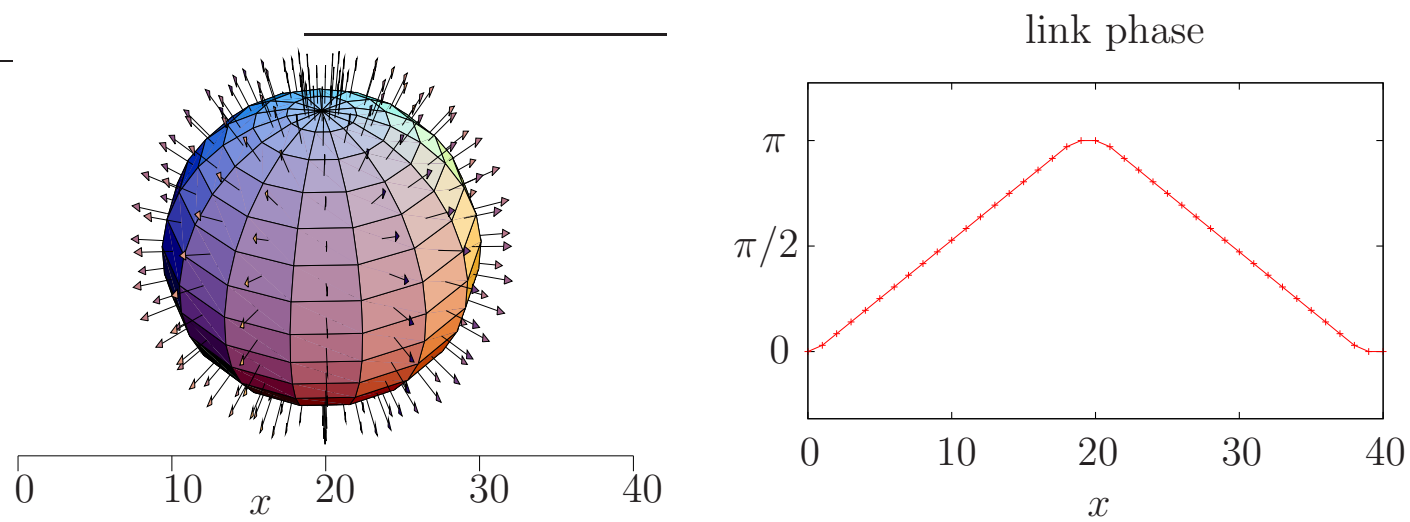

Figure 20: Thick Spherical $S U(2)$-vortex (hedgehog, non-orientable) and change of its link phase $\alpha(r)=\alpha_{-}(r)$ according to Eq. (18).

The distinction non-/orientable [45] refers to the orientation of the vortex surface assigned by abelian projection. While the orientable vortex has a global orientation, the non-orientable vortex consists of 2 patches of opposite orientation separated by a closed monopole world line. This is indicated schematically in Fig. 21 with three colors.

The check that this configuration is a vortex is done with maximal center gauge and center projection and results in a P-vortex forming a lattice representation of a sphere of radius 10 in the first time slice. The color structure of the thick vortex, which is symbolically indicated in the left diagram of Fig. 21, leads to a monopole loop on a great circle of the P-vortex after maximal abelian gauge and abelian projection. The direction of the loop depends on the U(1) subgroup chosen as abelian degrees of freedom. For the subgroup defined by the Pauli matrices $\sigma_{1}, \sigma_{2}$ or $\sigma_{3}$ the monopole loops are in the yz-, zx- and xy-plane, respectively. (Fig. 21)
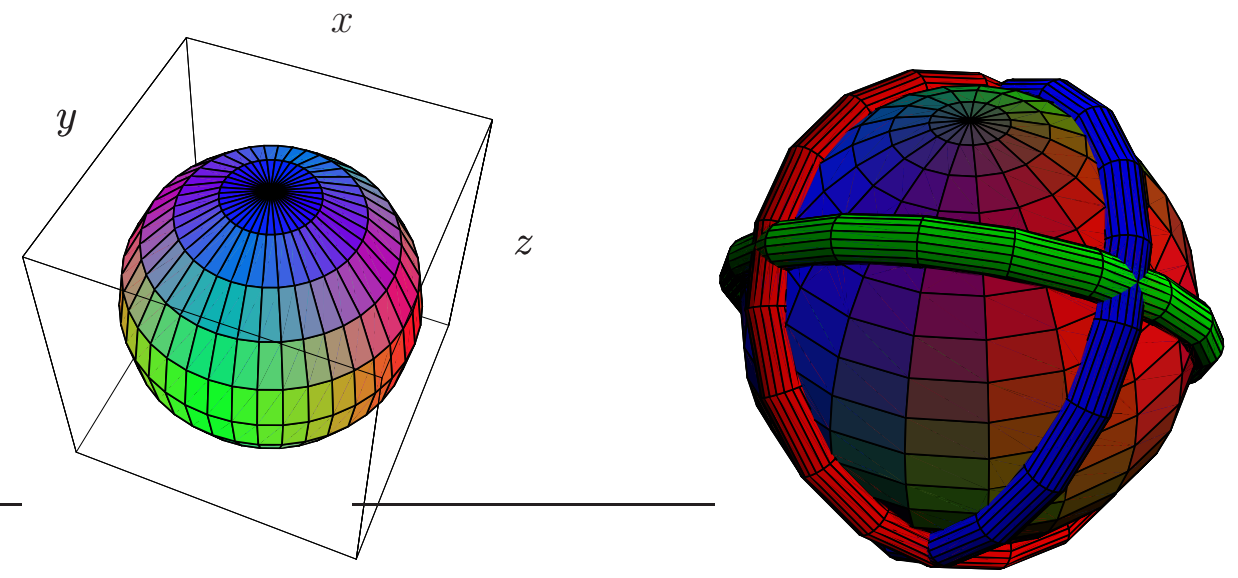

Figure 21: The color structure of a non-orientable vortex surface (1) leads to monopole lines after abelian projection (r) 
orientable vortex

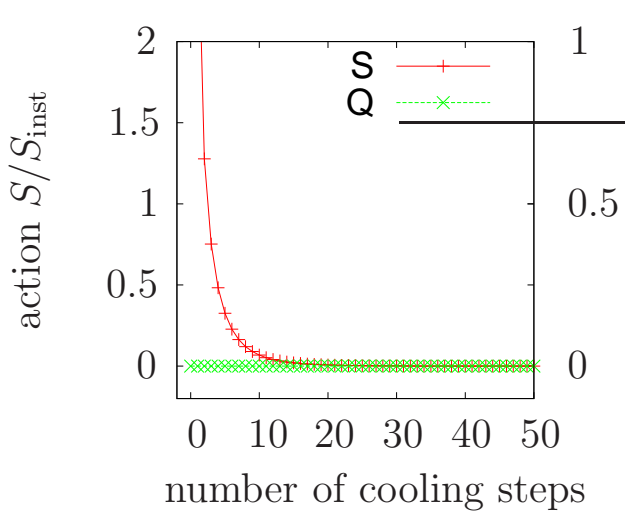

non-orientable vortex

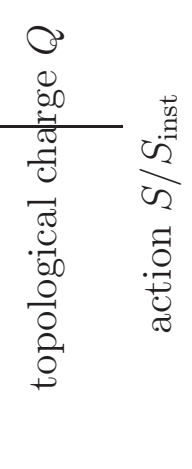

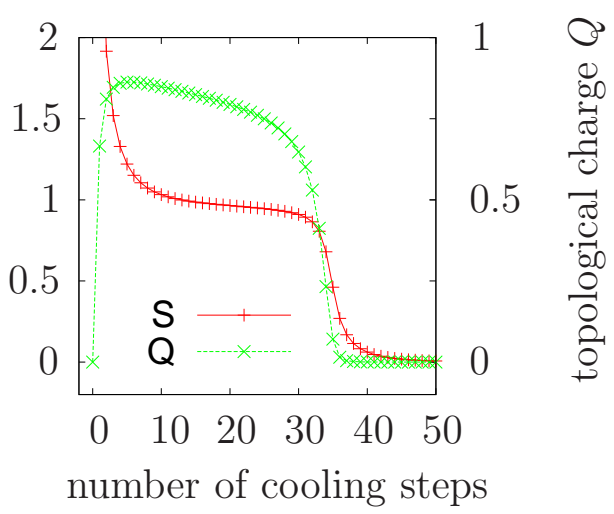

Figure 22: For configurations with an orientable vortex the action vanishes during cooling and no topological charge is measured (1), whereas for non-orientable vortex configurations the topological charge rises to 1 while the action $S$ reaches a (non-zero) plateau (r). The left scale shows the action $S=\sum_{\square}\left(1-\frac{1}{2} \operatorname{Tr} U_{\square}\right)$ in units of the one-instanton action $S_{\text {inst }}$. The data is taken from a $12^{4}$-lattice.

The P-vortex surface which is a closed surface in dual space consists only of the duals of space-time plaquettes. There are no vortex intersections and no writhing points. Therefore, the topological charge $Q$ determined from the P-vortex surface [12] vanishes, $Q=0$. The traces of all plaquettes are close to unity, $\operatorname{tr}\left(\mathbb{1}-U_{\mu \nu}\right) \leq 1-\cos \frac{\pi}{18}=0.015$, compare the link-phases in Fig. 20. The topological charge in the plaquette definition or in the hypercube definition can be used safely without cooling. According to Eq. (18) the only non-trivial links are time-like, therefore all space-space plaquettes are trivial, which confirms the vanishing of the topological charge $Q \sim \epsilon_{\mu \nu \rho \sigma} U_{\mu \nu} U_{\rho \sigma}$. This is independent of the lattice constant and thus holds also in the continuum limit.

For the orientable vortex, the topological charge after cooling and the overlap index are also equal to zero, in keeping with the continuum expectation (see left diagram of Fig. 22). However, a discrepancy in the case of the non-orientable spherical vortex is found, both, for the cooling process and the lattice index theorem.

First, during cooling the topological charge rises near to \pm 1 for $\alpha_{ \pm}$(right diagram of Fig. 22) while the action $S$ reaches a (non-zero) plateau. Further, the index of the overlap operator is also non-zero, ind $D=\mp 1$ for $\alpha_{ \pm}$.

For the spherical vortex with profile-function $\alpha_{-}(r)$ on a $40^{3} \times N_{t}$-lattice the overlap Dirac operator gives three zero modes ${ }^{2}$ of positive chirality and four zero modes of negative chirality. Normalized densities of positive and negative chirality modes have the same spatial distribution.

\footnotetext{
${ }^{2}$ In this work, a "zero mode" denotes any eigenmode of $D^{\dagger} D$ of the overlap Dirac operator (8) with eigenvalue smaller than $10^{-5}$. If such modes exist of both positive and negative chirality they are most likely not exact zero modes, but lifted slightly above zero pairwise. This numerical inaccuracy does not affect the value of the index (7).
} 

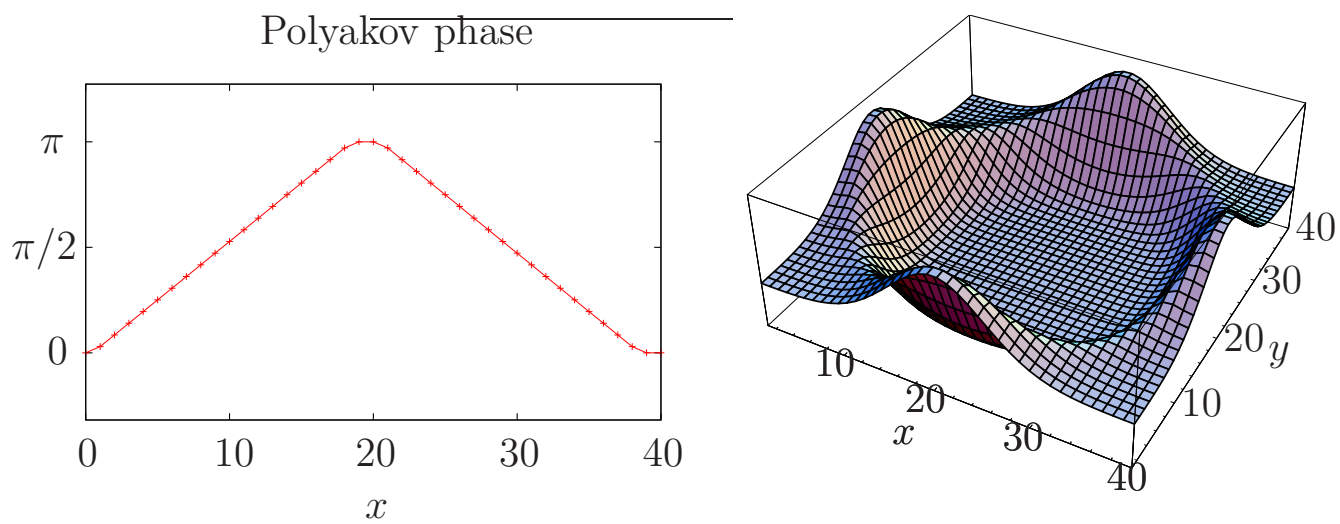

Figure 23: Phases of Wilson lines along a line through the center of a spherical vortex (1), normalized scalar densities for all zero modes of one chirality in the planes with $z=20$ close to the center of the sphere (r). The density vanishes in the region where the phase of the Polyakov loop is $\pi$. Both chiralities and both time-slices on the $40^{3} \times 2$-lattice give the same picture.

Fig. 23 shows the scalar density in the plane $z=20$. The non-trivial links connect $z=20$ and $z=21$, and the densities in both time-slices are identical. The zero modes are localized mainly in the region of trivial Wilson lines (for $\left|\vec{r}-\vec{r}_{0}\right| \sim 20$ ) and seem compressed in the regions where the mirror pictures of the spherical vortex on the periodic lattice approach each other. In the region where the Wilson lines are nontrivial center elements (for $\left|\vec{r}-\vec{r}_{0}\right|$ around 0 ), the density vanishes. According to the index theorem the topological charge is given by $Q=n_{-}-n_{+}=1$.

This value is in disagreement with the values determined above $(Q=0)$. But it agrees with the gluonic result which one gets after cooling. (compare Fig. 22, right). The spherical vortex is not a minimum of the action, i.e. not an instanton. During cooling its action is first rapidly decreasing, whereas its topological charge starts with zero and reaches a value close to one after a few cooling steps. On the $40^{3} \times 2$-lattice the action does not really approach a plateau value. Since a plateau appears for a $12^{4}$-lattice this seems to be related to the strong asymmetry of the lattice. Hence one would expect to see a clear plateau on a symmetric, but finer $40^{4}$ lattice, as well. The number of zero modes is the same for various lattice sizes used: $12^{4}$, and $12^{3} \times N_{t}$ up to $40^{3} \times N_{t}$, increasing the spacial extent $N_{s}$ in steps of 4 and using $N_{t}=2,4$ and 6 . One concludes that cooling and overlap fermions give a different topological charge than the discretized integral $\frac{1}{16 \pi^{2}} \int d^{4} x \operatorname{tr}\left(\mathcal{F}_{\mu \nu} \tilde{\mathcal{F}}_{\mu \nu}\right)$ even for very smooth field configurations.

The hedgehog configuration is characterized by links in the center of the spatial sphere which are non-trivial center elements and Wilson lines $L(\vec{x}), \vec{x}=(x, y, z)$, with a topological winding number $\nu=1$,

$$
\nu(\{L\})=\frac{1}{24 \pi^{2}} \int \mathrm{d}^{3} r \epsilon_{i j k} \operatorname{Tr}\left(V_{i} V_{j} V_{k}\right), \quad V_{i}=\partial_{i} L L^{\dagger} .
$$


The topological charge determined after the cooling process and from the index theorem agrees with this winding number. In the continuum limit, the gauge field at the center of the hedgehog becomes singular. This singularity is due to the topological obstruction and cannot be removed by a gauge transformation. With Landau-gauge for 100 gauge copies on a $40^{3} \times 2$-lattice, it is not possible to get rid of links close to non-trivial center elements. For the lattice spacing $a$ approaching zero, the gauge field for these links, $\mathcal{A}_{0}(x) \approx \pi \sigma_{3} /(g a)$, diverges. In all gauge copies the same links with negative traces are found. The number of negative links and the values of their traces are listed in Table 1.

\begin{tabular}{|c|ccccccc|}
\hline number of links & 8 & 24 & 24 & 32 & 48 & 24 & 48 \\
\hline$\frac{1}{2} \operatorname{tr} U_{\mu}(x)$ & -0.93 & -0.68 & -0.46 & -0.30 & -0.18 & -0.08 & -0.01 \\
\hline
\end{tabular}

Table 1: Number of links with negative traces and trace values after Landau gauge for the non-orientable spherical vortex of Eq. (18) on a $40^{3} \times 2$-lattice, e.g. there are eight links with $\frac{1}{2} \operatorname{tr} U_{\mu}(x)=-0.93$.

For comparison, in the case of an orientable spherical vortex, the time-like Wilson lines have topological winding number zero. This configuration has no zero modes and all ways to determine the topological charge lead to the same result, $Q=0$. With maximal Abelian gauge no monopole lines appear. With Landau-gauge, again for 100 gauge copies on a $40^{3} \times 2$-lattice, one can get rid of links close to non-trivial center elements. Table 2 lists the number of negative links and the values of their traces.

\begin{tabular}{|c|cccc|}
\hline number of links & 8 & 24 & 24 & 24 \\
\hline$\frac{1}{2} \operatorname{tr} U_{\mu}(x)$ & -0.26 & -0.15 & -0.06 & -0.008 \\
\hline
\end{tabular}

Table 2: Number of links with negative traces and trace values after Landau gauge for the orientable spherical vortex of Eq. (22) on a $40^{3} \times 2$-lattice.

For the non-orientable spherical vortex with profile function $\alpha_{+}(r)$ on a $40^{3} \times N_{t}$-lattice the overlap Dirac operator gives only one zero mode of negative chirality.

The phases of the links in the time slice agree again with the phases of the Wilson lines in time direction and are shown in Fig. 24. In the center of the spherical vortex the temporal Wilson lines are trivial and at "infinity" they are non-trivial center elements.

The zero mode is localized in the center of the spatial sphere and in both time-slices occupied by the vortex. Again, the scalar density prefers regions of trivial Wilson lines over those of non-trivial values. According to the index theorem the topological charge is -1 and agrees with the winding number (23) of the hedgehog of Wilson lines. The topological charge computed from plaquettes is $Q=0$ while the plaquette traces differ by less than $1.5 \%$ from trivial ones. Since this configuration is related to the configuration in Fig. 23 by a non-periodic gauge transformation, it is not surprising that again the different determinations of the topological charge do not agree.

The non-orientable vortex also gives extra contributions to the index when it is combined with other vortices, possibly including intersection points which produce "real" 

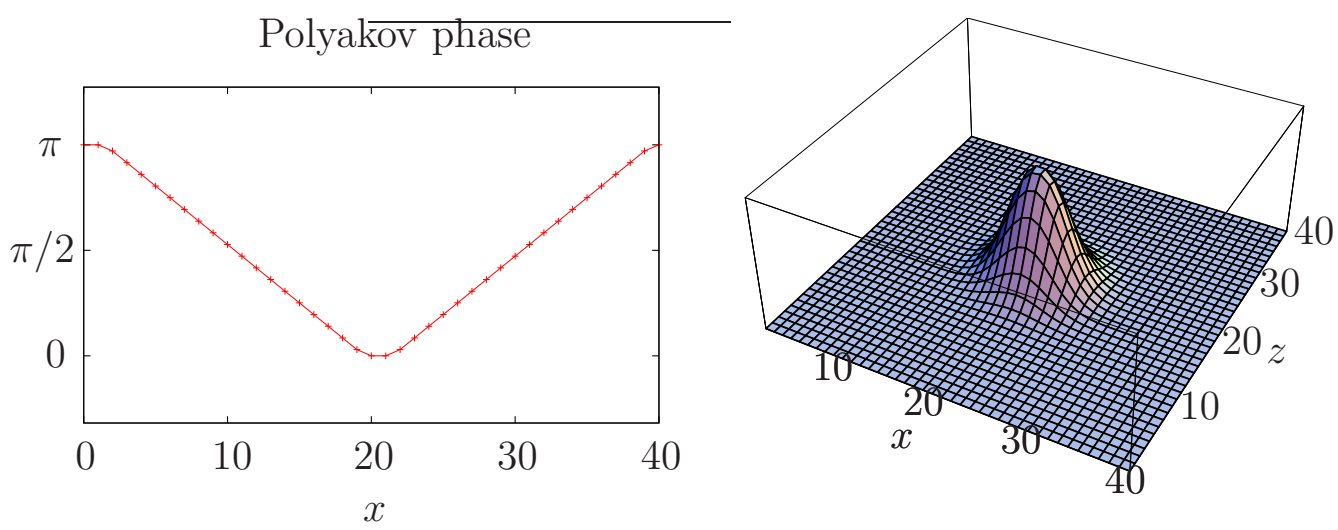

Figure 24: Phase-diagram of the Polyakov loop (1) along a line through the center of a spherical vortex with the profile function $\alpha=\alpha_{+}$(l) and scalar density of the only fermionic zero mode (r) which has positive chirality.

topological charge.

For two equal vortices of this type, $\alpha=\alpha_{+}$, one in time slice $t=1$ and another at $t=2$ of the $40^{3} \times 2$-lattice there are three zero modes of positive chirality with a scalar density like the diagram in Fig. 23 and five of negative chirality with the densities shown in Fig. 25. Since the modes are degenerate, they can mix and the plots display the sum of the densities of all zero modes. The left diagram displays the $t=1$ slice, where only four of these modes contribute significantly, while the fifth mode leads only to the small peak in the middle. Conversely, the density in the $t=2$ slice (shown in the right picture) is dominated by the fifth mode in the center, the other modes being of a magnitude invisible on this scale. The phase of the Polyakov line for this configuration is zero in the center of the spherical vortices and due to the $2 \pi$-periodicity in the region between the mirror pictures on the periodic lattice. Again the zero modes are localized in the region with trivial time-like Wilson lines.

A configuration with two vortices of different type in the two time-slices, one with $\alpha=\alpha_{-}$at $t=1$ and another with $\alpha=\alpha_{+}$at $t=2$ does not show any zero mode. In this case there is no discrepancy in the determinations of the topological charge. The phase of the Wilson line, which is the sum of the phases from the two vortices, is constant and equal to $\pi$.

A summary of the the number of zero modes for the configurations described above with spherical vortices is given in Table 3 .

More generally, the following empirical rule can be formulated: Non-orientable spherical vortices in slices (3D volumes) of the lattice contribute to cooled topological charge and Dirac operator index with an integer given by the "winding number" $[47,56]$ of the corresponding Wilson lines, mapping the 3D volume of the slice to the $S U(2)$ manifold of the Wilson lines. To compute this "winding number", the $t$-links are seen as a map not from $\mathbb{T}^{4}$, but from the compactified time-slice $t=0$, in which the sphere is located, to $S U(2)$. The time-slice can be compactified to $S^{3}$ because the links outside the sphere are all equal to $+\mathbb{1}$. 

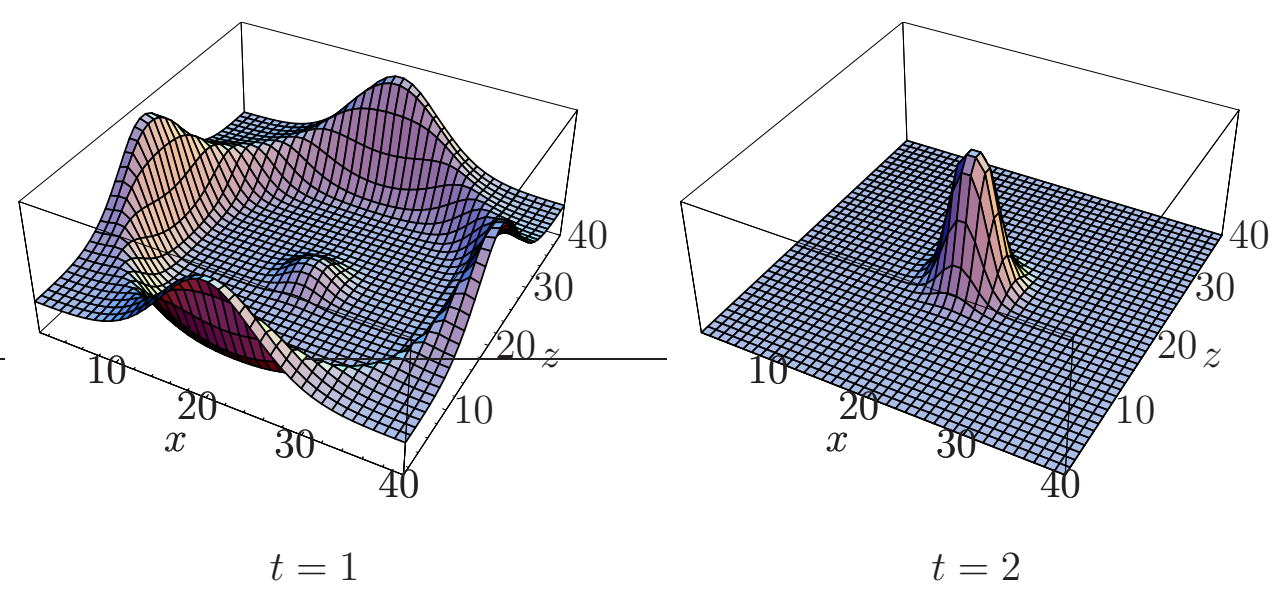

Figure 25: Scalar densities of fermionic zero modes of negative chirality for two equal vortices with the profile function $\alpha=\alpha_{+}$, in the time slices $t=1$ and $t=2$ of the $40^{3} \times 2$-lattice.

\begin{tabular}{|c|cc|}
\hline profile $\alpha$ & $n_{+}$ & $n_{-}$ \\
\hline orientable, $\alpha_{ \pm}$ & 0 & 0 \\
non-orientable, $\alpha_{-}$at $t=1$ & 3 & 4 \\
non-orientable, $\alpha_{+}$at $t=1$ & 1 & 0 \\
non-orientable, $\alpha_{+}$at $t=1$ and $\alpha_{+}$at $t=2$ & 3 & 5 \\
non-orientable, $\alpha_{-}$at $t=1$ and $\alpha_{-}$at $t=2$ & 5 & 3 \\
non-orientable, $\alpha_{-}$at $t=1$ and $\alpha_{+}$at $t=2$ & 0 & 0 \\
\hline
\end{tabular}

Table 3: Number of zero modes for various smooth field configurations with spherical vortices. The profile functions $\alpha_{ \pm}$are defined in Eq. (20).

One concludes that for non-orientable spherical vortices, i.e. configurations with a non-zero winding number of the Wilson lines, the index of the overlap Dirac operator differs from the topological charge in the continuum limit. It is very likely that this non-orientability is related to non-zero winding number of the gauge field and the artificial configurations reflect properties which saturate the path integral. The discrepancy between overlap index and continuum topological charge is not due to the coarse discretization. Lattice sizes with $N_{t}=2,4,6$ and $N_{s}$ ranging from 8 to 40 in steps of 4 have been used [57]. For $N_{s} \geq 40$, the traces of all plaquettes are close to unity and the admissibility condition (9) is satisfied, but the results remain unaltered. The reason for the seeming contradiction is probably the singular nature of the continuum gauge field equivalent to the spherical vortex. The singularities in the gauge field are related to non-trivial mappings of Wilson lines. These singularities invalidate the usual derivation of the index theorem. 


\subsection{Topology and Staggered Fermions}

"It is conventional wisdom that staggered fermions do not feel gauge field topology." [58] However, the cited paper provides a strategy to improve the response of staggered fermions to the topological background. Staggered fermions don't have exact zero modes, but a separation between "would be" zero modes and non-chiral modes is observed for improved staggered quark actions. These results shall be verified first, by comparing standard and asqtad improved staggered fermions and using the HYP (hyper cubic blocking) smearing algorithm. The results are shown in Fig. 26, presenting the first twenty eigenmodes of thirty configurations, generated by lattice Monte Carlo simulation of the tadpole improved Lüscher-Weisz pure-gauge action at coupling $\beta_{L W}=3.7$, since increasing coupling also improves the desired effect. The eigenvalues are plotted against the chirality (pseudo-scalar density) of the modes, which for staggered fermions is determined by $\left\langle\Psi \gamma_{5} \Psi\right\rangle$, where $\gamma_{5}$ corresponds to a displacement along the diagonal of a hypercube. To ensure gauge invariance the product includes gauge field multiplications along all shortest paths connecting opposite corners of the hypercube. Comparing standard (red) and asqtad improved (green) staggered fermions, a slight improvement is observed, "would be" zero modes show a higher chirality and are "closer" to zero. But for these rough configurations it still seems hard to really identify exact zero modes, whereas after five steps of HYP-smearing the chirality of the zero modes gets well defined (close to one, negative chiralities were projected to the positive plane) and the eigenvalues get close to zero. There is no big difference between standard (blue) and asqtad improved (magenta) fermions any more.

These results promise to be able to identify zero modes on rather smooth configurations, as the above examples of spherical vortices provide. The asqtad staggered modes are calculated for a positive and a negative non-orientable spherical vortex made up of time links in a single time sheet of $40^{3} \times 8$ lattices. The results concerning zero modes and the index theorem are exactly the same (see Table 4) as for the overlap Dirac operator, smearing only precises the eigenvalue close to zero. The scalar densities for the single mode of each chirality also distribute similar (Fig. 27, compare to Figs. 23 and 24).

\begin{tabular}{cccccccc}
\multicolumn{4}{c}{ positive spherical vortex } & \multicolumn{5}{c}{ negative spherical vortex } \\
$\lambda$ & $\left\langle\psi \gamma_{5} \psi\right\rangle$ & HYP $\lambda$ & $\left\langle\psi \gamma_{5} \psi\right\rangle$ & $\lambda$ & $\left\langle\psi \gamma_{5} \psi\right\rangle$ & HYP $\lambda$ & $\left\langle\psi \gamma_{5} \psi\right\rangle$ \\
$\mathbf{0 . 0 1 0 6 0}$ & $\mathbf{0 . 9 6 0 9}$ & $7.9710^{-6}$ & 0.7951 & $\mathbf{0 . 0 0 1 3 9}$ & $\mathbf{- 0 . 9 9 7 2}$ & $5.6810^{-6}$ & 0.0304 \\
0.26577 & -0.0333 & 0.28403 & -0.0086 & 0.00168 & 0.9956 & $7.4010^{-6}$ & -0.1729 \\
0.26577 & -0.0385 & 0.28403 & -0.0144 & 0.00168 & 0.9958 & $8.0910^{-6}$ & 0.0395 \\
0.26577 & -0.0401 & 0.28403 & -0.0136 & 0.00168 & 0.9960 & $9.1110^{-6}$ & -0.2421 \\
0.27095 & 0.0096 & 0.28403 & -0.0090 & 0.00245 & -0.9913 & $9.5010^{-6}$ & -0.0661 \\
0.27095 & 0.0140 & 0.28403 & -0.0096 & 0.00245 & -0.9928 & $9.7810^{-6}$ & 0.2491 \\
0.27095 & 0.0116 & 0.28403 & -0.0089 & 0.00245 & -0.9909 & $1.0110^{-5}$ & -0.2829 \\
0.34335 & 0.0128 & 0.35622 & 0.0081 & 0.10450 & 0.0391 & 0.11128 & 0.0091
\end{tabular}

Table 4: First eight asqtad staggered eigenvalues and their chiralities for positive and negative spherical vortices and HYP-smeared configurations. 


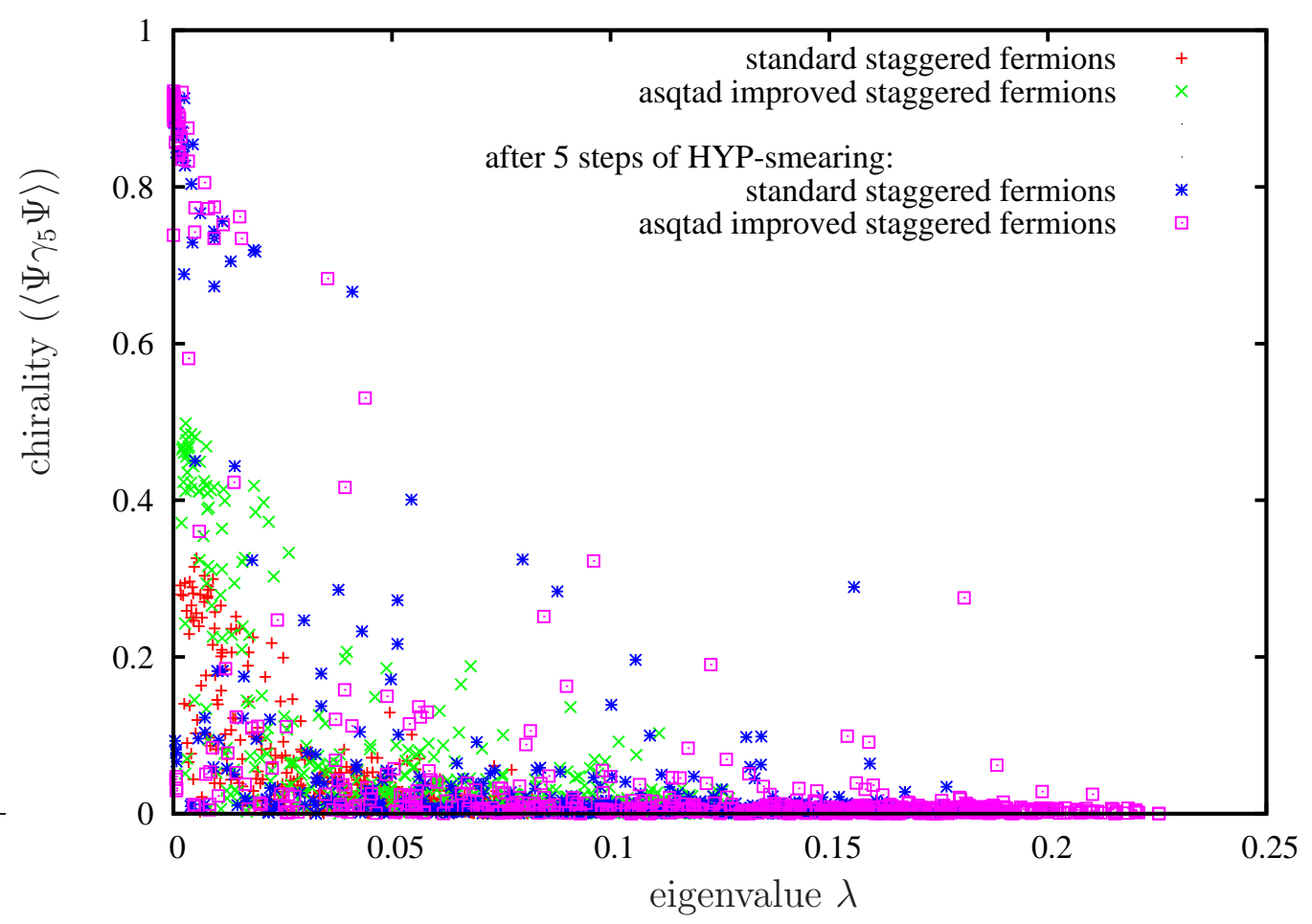

Figure 26: First twenty eigenmodes of thirty Monte Carlo configurations on a $20^{4}$-lattice, for standard and asqtad staggered fermions and smeared gauge fields.
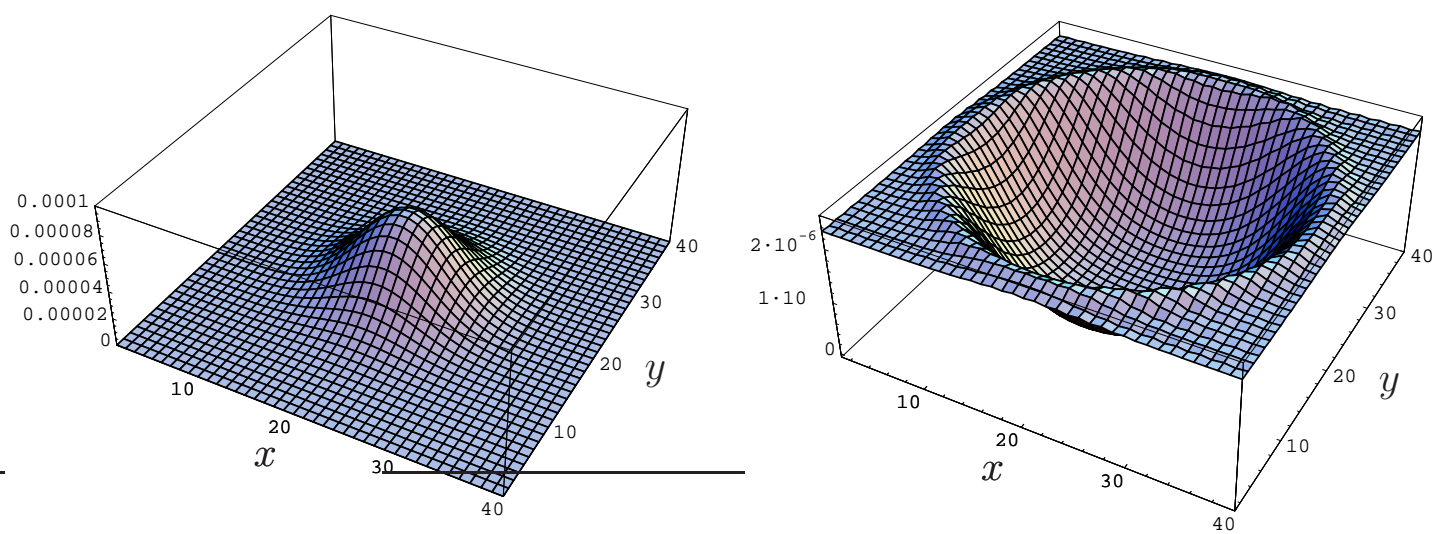

Figure 27: Scalar densities of the single (unpaired) asqtad staggered zero mode of positive chirality for the positive $\left(\alpha_{+}\right.$, left) and negative chirality for the negative $\left(\alpha_{-}\right.$, right) spherical vortex in a time slice of a $40^{3} \times 8$-lattice. 


\section{Observables and Results on SCSB}

The correct description of chiral symmetry breaking within the center vortex model is analyzed in quenched LQCD. $S U(2)$ gauge configurations were produced by Monte Carlo methods using the heat-bath algorithm with improved Lüscher-Weisz action and the Dirac operators (see App. A.2) were applied afterwards. For each set of data, the results presented below underly a statistics of a 100 configurations for the asqtad staggered and 30 configurations for the overlap Dirac operator. For discussions some representative examples were depicted only. The results of all data can be found in appendix C.

\subsection{Dirac Eigenvalues}

In order to solve the question whether the failure to explain the chiral condensate from the center-projected configurations is caused by the approximation which is due the chirally improved fermions or whether the pure P-vortices miss some important information concerning chiral symmetry breaking, the overlap Dirac operator is investigated. Fig. 28 presents the first twenty eigenvalues on the Ginsparg-Wilson circle for a $16^{4}$ lattice at $\beta_{L W}=3.3$. The projected data shows up the same strange behavior. There is a big gap around zero, indicating zero chiral condensate and therefore quark confinement without chiral symmetry breaking. The vortex-removed data shows four near-zero modes for each chirality, which can be interpreted as real zero modes since they disappear in case of anti periodic boundary conditions (see Fig. 29) and therefore are irrelevant to chiral symmetry breaking. Looking closer at the center-projected eigenvalues one spots only five of the twenty eigenvalues. This indicates a degeneracy of four eigenvalues each and also the scalar density of the eigenvectors shows up this degeneracy, see Fig. 30.
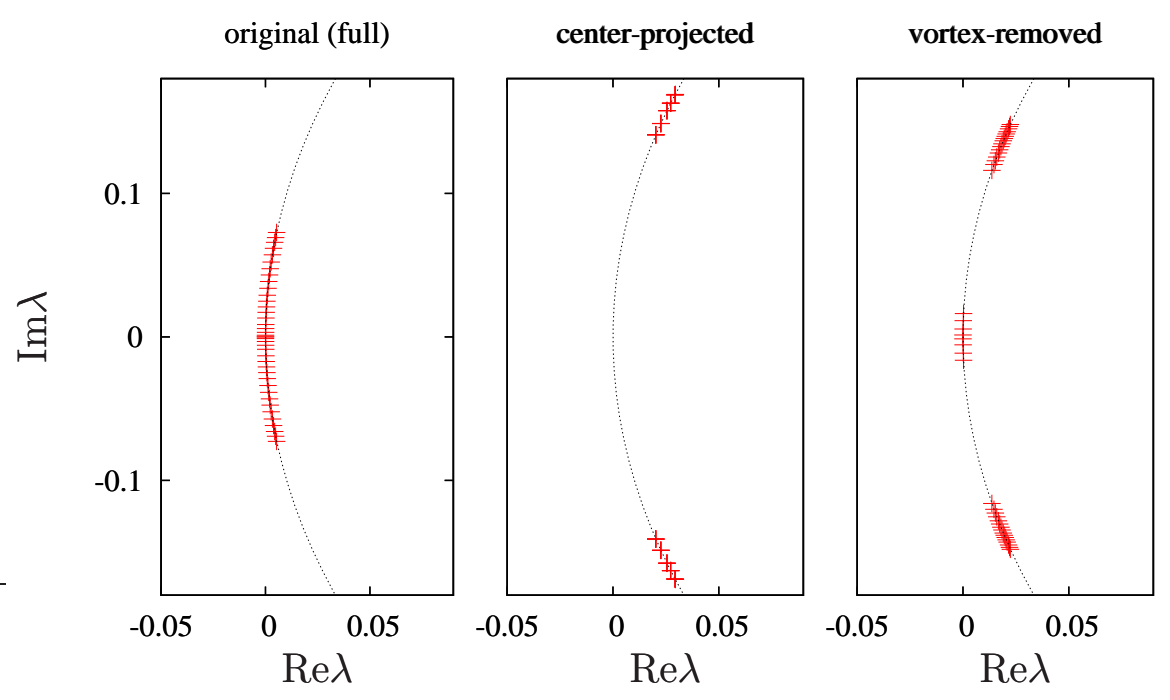

Figure 28: First twenty overlap Dirac eigenvalues on the Ginsparg-Wilson circle for a $16^{4}$ lattice at $\beta_{L W}=3.3$. 

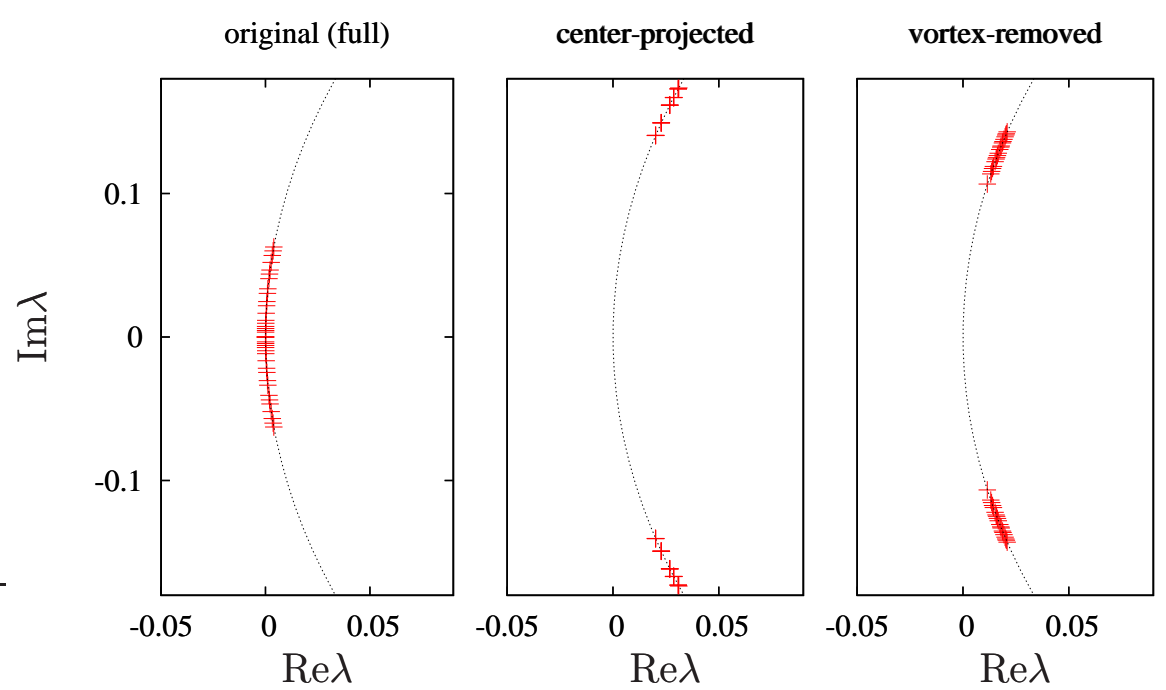

Figure 29: First twenty overlap Dirac eigenvalues on the Ginsparg-Wilson circle for a $16^{4}$ lattice at $\beta_{L W}=3.3$ using anti periodic boundary conditions.
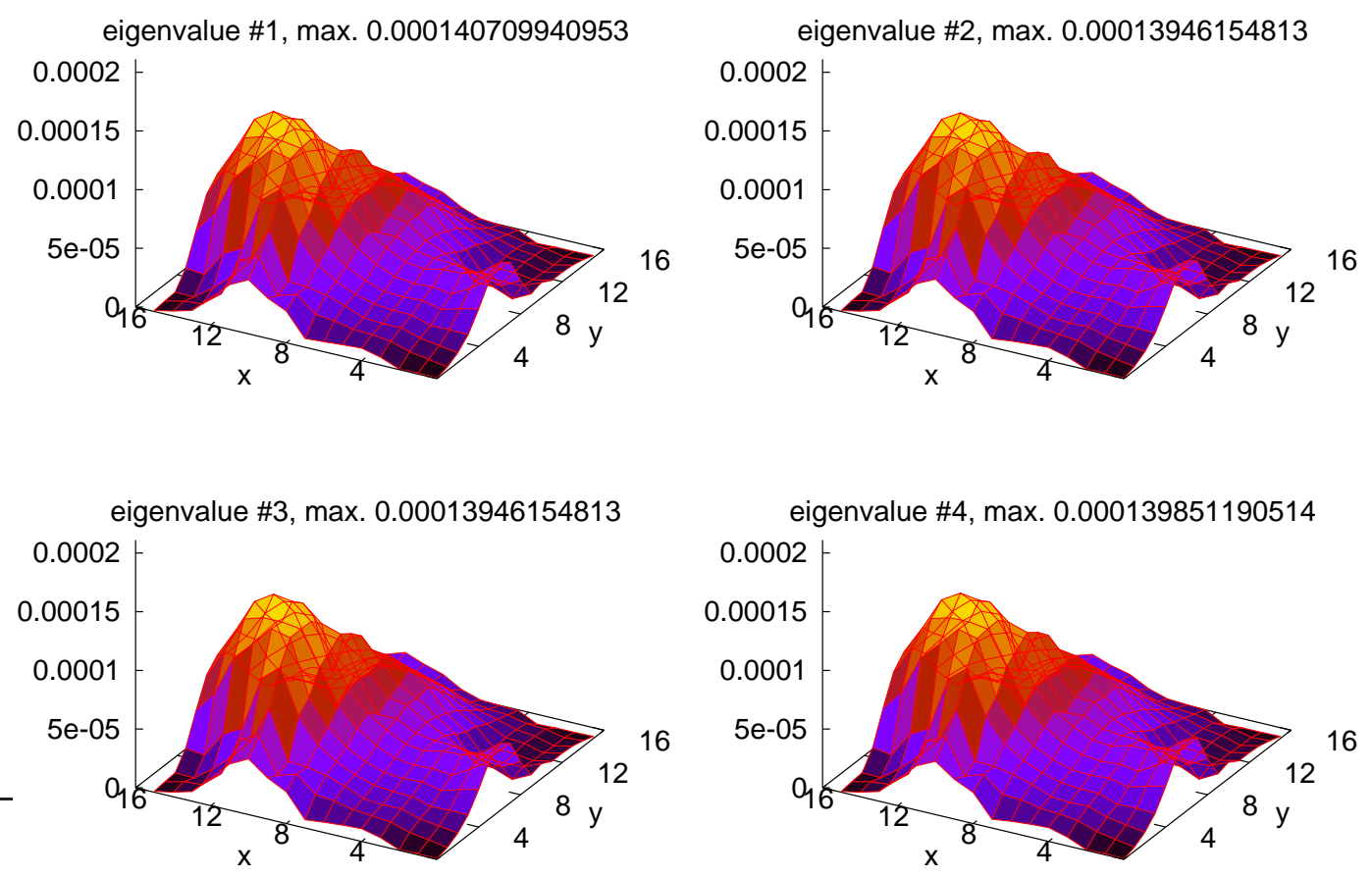

Figure 30: Scalar density of first four eigenmodes of a single projected configuration on a $16^{4}$ lattice at $\beta_{L W}=3.3$, presenting the maximum peak in $z=4, t=9$ plane. 
This factor of four has the following origin: In the first place, when link variables are simply plus or minus the $2 \times 2$ identity matrix, the two colors decouple, and we have a factor of two degeneracy. Secondly, whenever the link variables are real and the Dirac operator has the Wilson or overlap (but not staggered) form, the eigenvalue equation $D \psi_{n}=l_{n} \psi_{n}$ is invariant under charge conjugation. Thus, if $\psi_{n}$ is an eigenstate with eigenvalue $l_{n}$, then $C^{-1} \psi_{n}^{*}$ is also an eigenstate, with the same eigenvalue [59]. This gives another factor of two, resulting in an overall four-fold degeneracy.

The question which comes to mind is whether the large gap found in center-projected overlap spectra, as well as by Gattnar et al. [35, 36] for the chirally improved Dirac operator, is related to the way in which chiral symmetry is realized on the lattice. The Casher argument [60] that confinement implies chiral symmetry breaking is based on the usual $S U\left(N_{f}\right)_{L} \times S U\left(N_{f}\right)_{R}$ symmetry of the continuum theory with massless fermions. However, the chirally improved Dirac operator only approximates this symmetry for gauge-field configurations which vary smoothly at the lattice scale. Center-projected configurations are not even close to smooth; plaquette variables make a sudden transition from the trivial center element outside the thin vortex, to a non-trivial center element inside. The chirally-improved Dirac operator is not necessarily chirally symmetric, even approximately, in such backgrounds. In the absence of a symmetry, there is no reason to expect spontaneous symmetry breaking. If this fact explains why there is a gap in the eigenvalue spectrum of the chirally-improved operator, then it is reasonable to also expect a gap in the spectrum of the overlap operator, when evaluated on center-projected configurations. Of course the overlap operator, in contrast to the chirally-improved operator, does have an exact global symmetry, but the symmetry transformations are gauge-field dependent [17], and only approximate the $S U\left(N_{f}\right)_{L} \times S U\left(N_{f}\right)_{R}$ chiral symmetry transformations of the continuum theory for configurations which vary slowly at the scale of the lattice spacing. While this smoothness condition is expected in the continuum limit, it is never the case for center-projected configurations, and the Casher argument relating confinement to chiral symmetry breaking need not apply.

If the overlap operator yields misleading results on center-projected lattices, because of the lack of smoothness of center-projected configurations, then perhaps the overlap operator would produce a more reasonable answer when applied to a smoother version of the center-projected lattice. Therefore an interpolation between full (gauged) and projected configurations is performed. Given that $S U(2)$ group elements can be represented by unit 4 -vectors $a_{\mu}$, where $U=a_{0} I_{2}+i a_{k} \sigma^{k}$, let $\theta_{\mu}(x)$ denote the angle between the vector representing group element $U_{\mu}(x)$ in maximal center gauge, and the vector representing the $S U(2)$ center element $Z_{\mu}(x)\left(I_{2}\right)$, where $Z_{\mu}(x)$ was defined in Eq. (57). Center projection simply takes this angle to zero, at every link, but one may also consider partial projections in which $\theta_{\mu}(x)$ is everywhere reduced by some fixed percentage. These partial projections interpolate between the unprojected lattice, in maximal center gauge, and the fully center-projected lattice. The original $S U(2)$ elements $U_{\mu}(x)$ are partially turned into the according center element $Z_{\mu}(x)= \pm 1$ (north/south pole) by partial steps of the angle $\theta_{\mu}(x)$ (see Fig. 31).

The low-lying overlap eigenvalues of a single configuration on a $16^{4}$ lattice at $\beta_{L W}=3.3$ during interpolation are shown in Fig. 32. The spectra are shown for a partial projection 


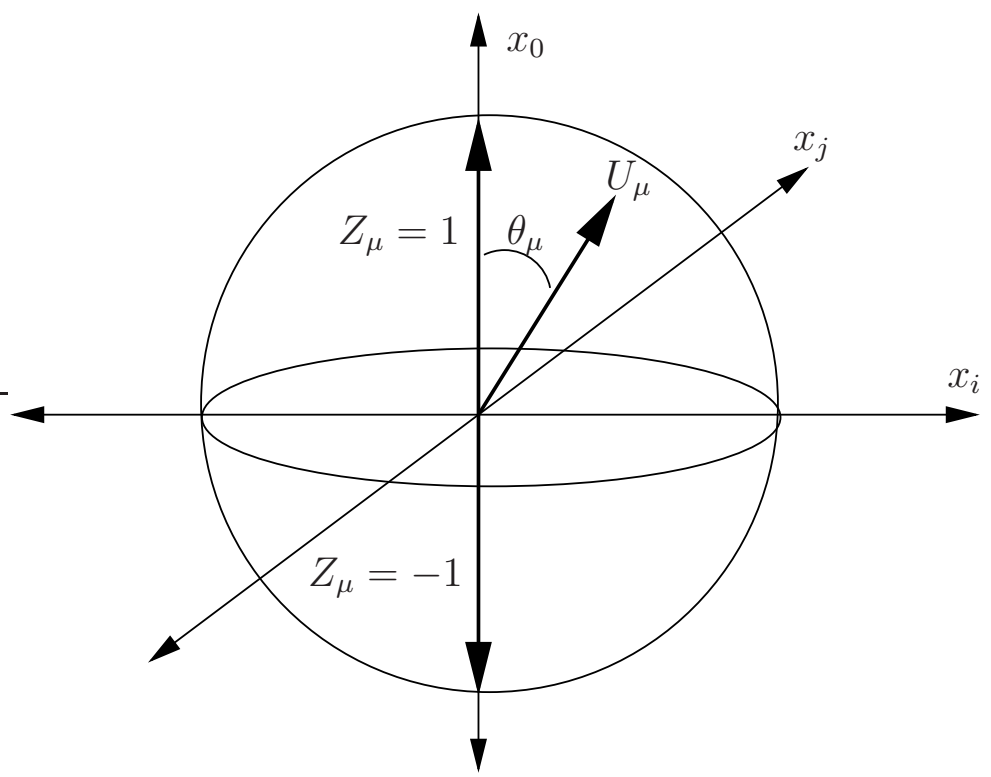

Figure 31: Hyper sphere $S^{3}$ of $S U(2)$ elements $U_{\mu}$, center elements $Z_{\mu}= \pm 1$ (poles)

angle $\theta_{\mu}(x)$ reduced by $50 \%, 75 \%, 85 \%, \ldots$, together with the unprojected $(0 \%)$ and fully $(100 \%)$ center-projected lattices. There is no really obvious gap in the partially-projected lattices, even at $85 \%$ projection. This agrees with the conjecture that applying the overlap operator to a smoother version of the vortex-only vacuum would give a result consistent with chiral symmetry breaking and the Banks-Casher relation.

The development of the first four eigenmodes to a degenerated quartet is shown in Figs. 33 and 34 for a partial projection angle $\theta_{\mu}(x)$ reduced in $25 \%$-steps. The modes only degenerate on the center-projected lattice.

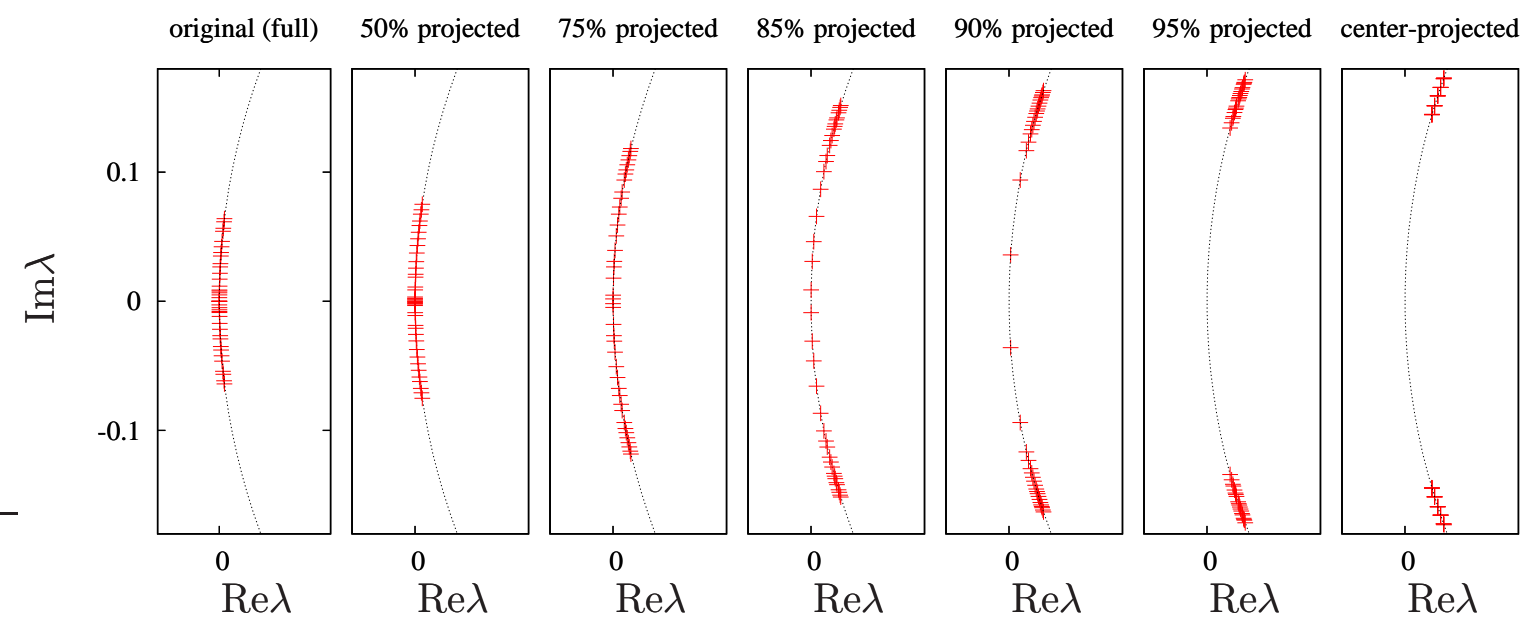

Figure 32: First twenty overlap Dirac eigenvalues of a single configuration on a $16^{4}$ lattice at $\beta_{L W}=3.3$ for interpolated fields. 


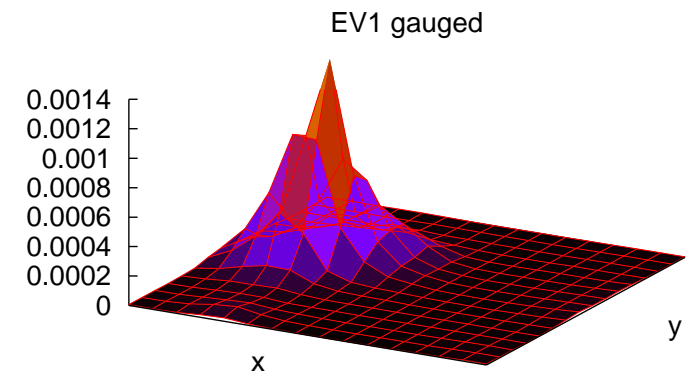

EV1 25\% projected
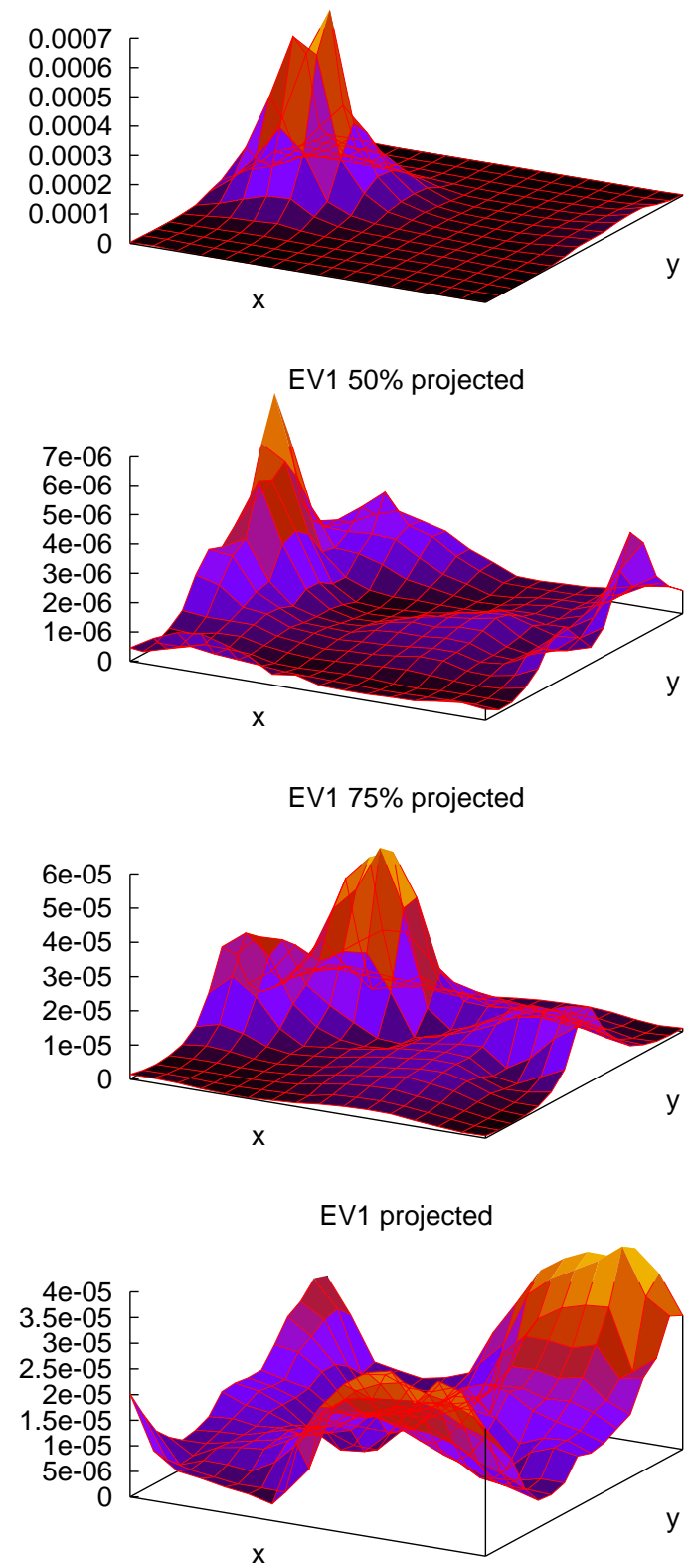

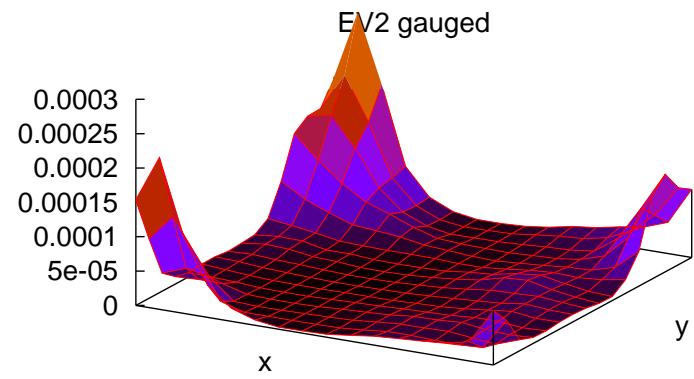

EV2 25\% projected

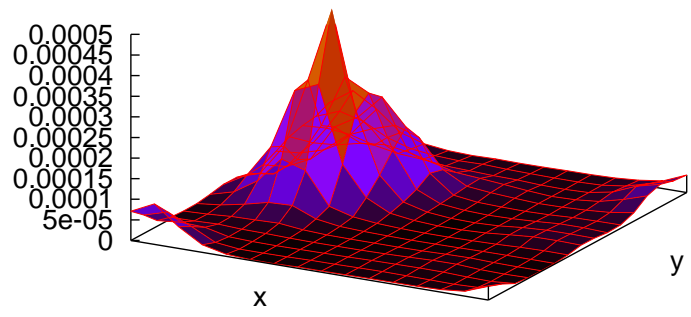

EV2 $50 \%$ projected
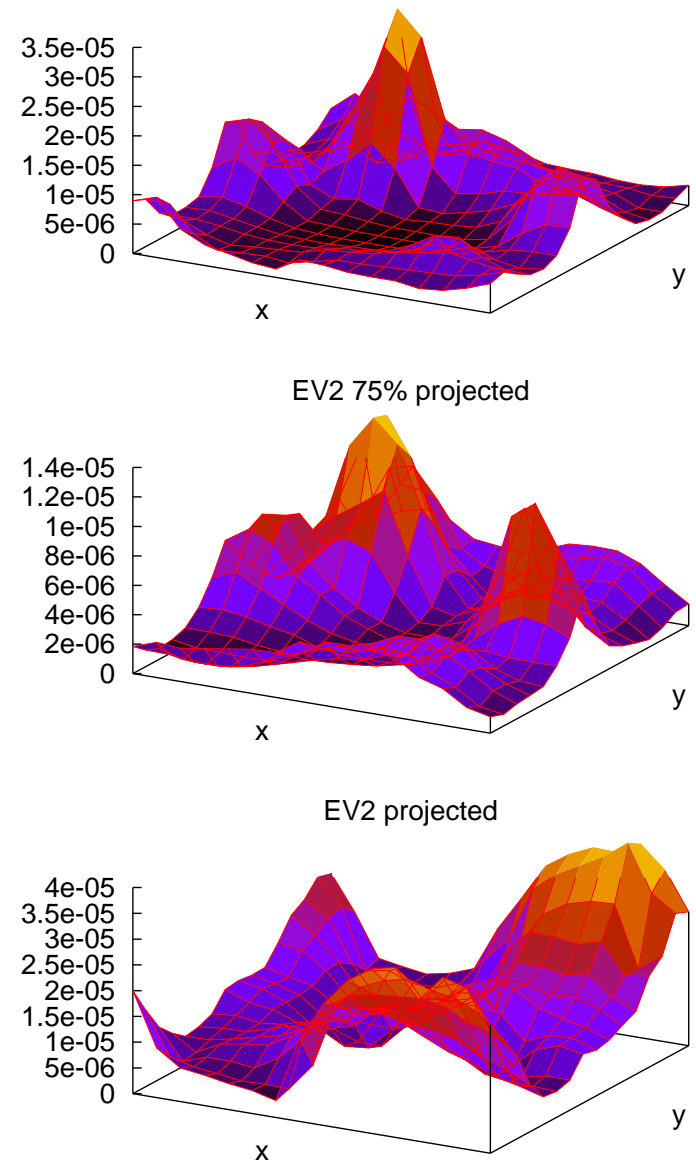

Figure 33: Development of $1^{\text {st }}$ and $2^{\text {nd }}$ eigenmode during interpolation. 


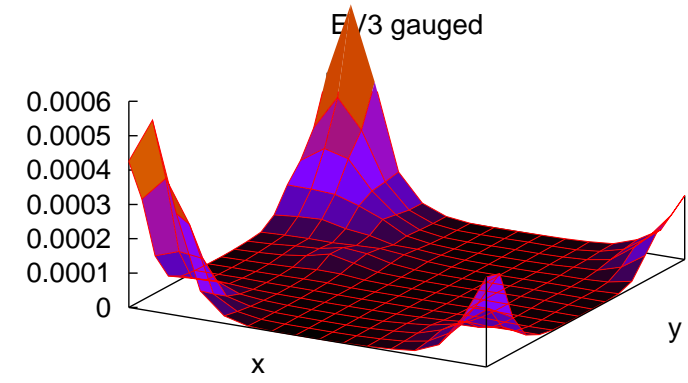

EV3 25\% projected

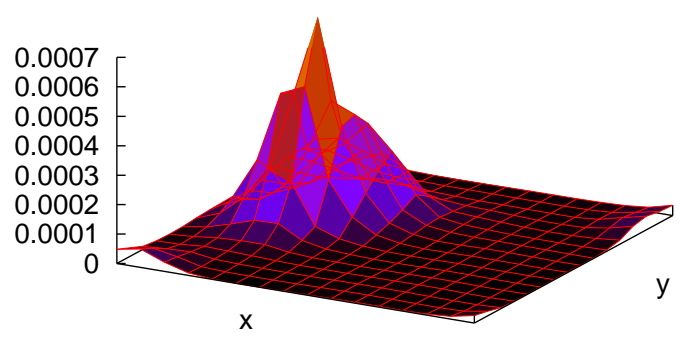

EV3 $50 \%$ projected
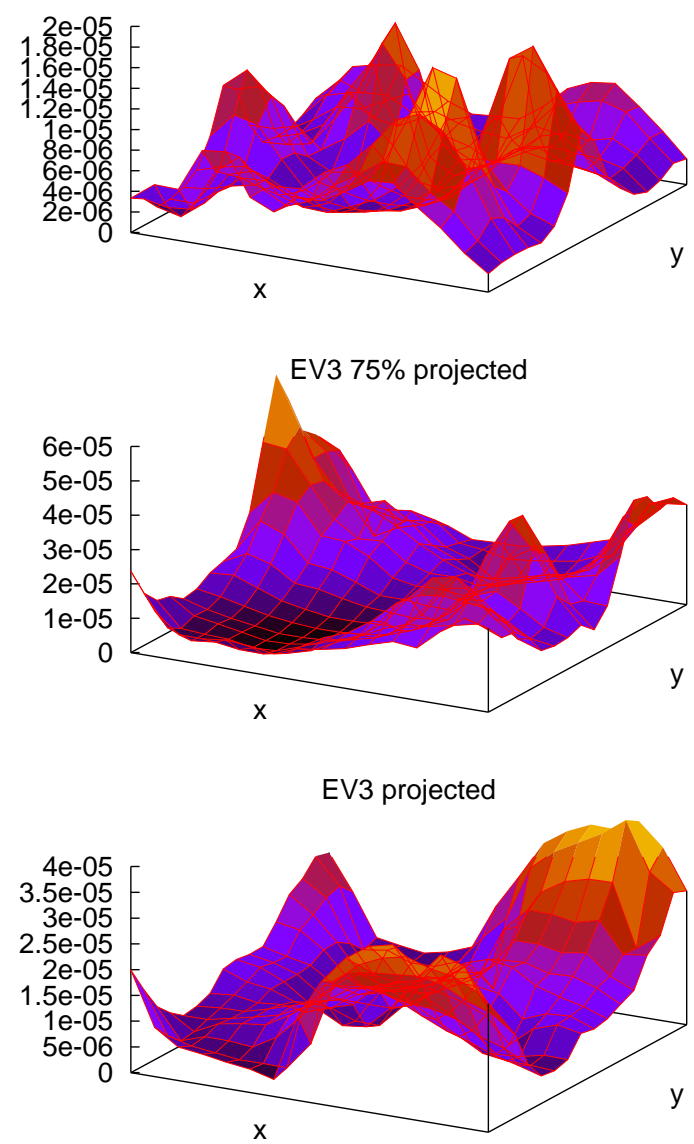
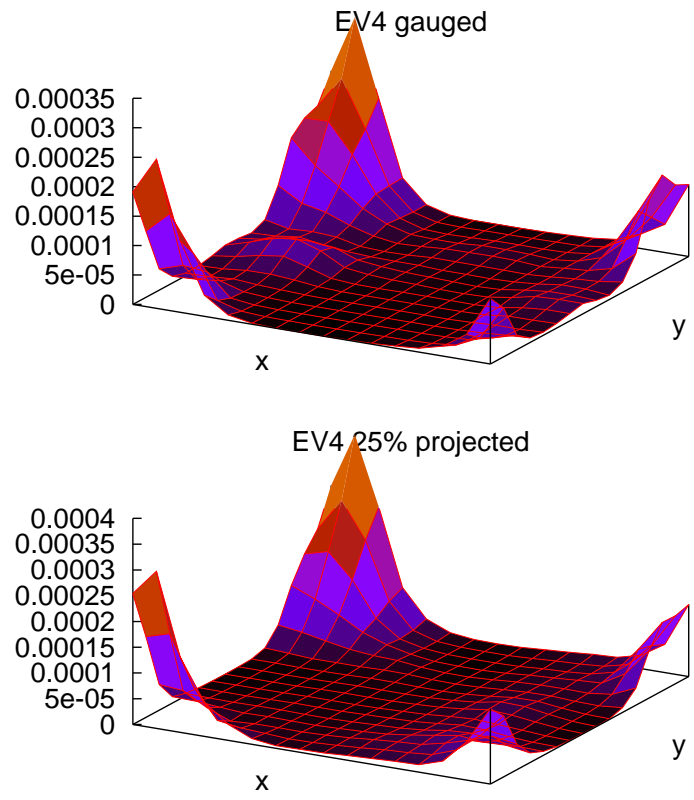

EV4 50\% projected

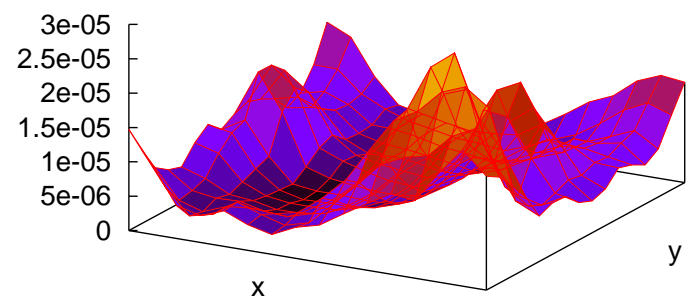

EV4 $75 \%$ projected

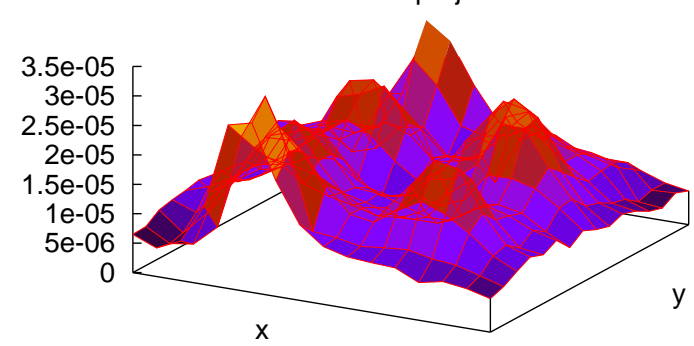

EV4 projected

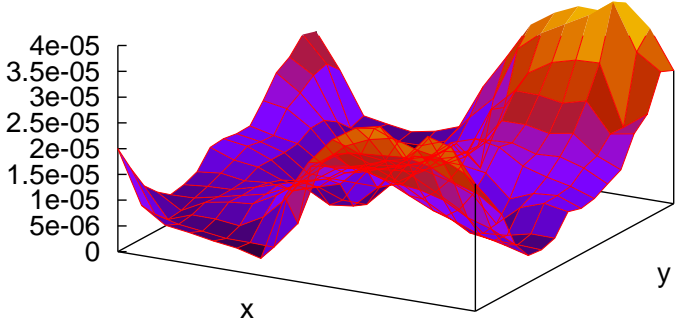

Figure 34: Development of $3^{\text {rd }}$ and $4^{\text {th }}$ eigenmode during interpolation. 
On the other hand, the Lagrangian for staggered fermions (and their asqtad cousins [61, $62,63])$ preserves a continuous $U(1) \times U(1)$ symmetry, which is a remnant of the original chiral symmetry group, irrespective of the smoothness of the configuration. By the Casher argument [60], one would expect this remaining symmetry to be spontaneously broken, on the lattice, by any ensemble of gauge configurations with the confinement property. Then, according to the Banks-Casher relation, there should not be any gap in the vortex-only eigenvalue spectrum. If the puzzling gap in the Dirac eigenvalue spectrum found by Gattnar et al. [35, 36] is a consequence of the roughness of centerprojected lattices, then one might expect this gap to disappear in the spectrum of the staggered or asqtad Dirac operators. Indeed, there is already a relevant result in [15], which reported that $\langle\bar{\psi} \psi\rangle>0$ for staggered fermions on a center-projected lattice.

Therefore, asqtad improved staggered fermions are considered next. The eigenvalues distribute very differently now (see Fig. 35), the low eigenmode density (chiral condensate) increases for projected compared to full (original) data whereas the vortex-removed data develops a central band around $\operatorname{Im} \lambda=0$ of eight doubly degenerate eigenmodes per chirality, which are separated by a gap from the higher modes. Now, the free-field Dirac operator for massless staggered fermions has exactly four zero modes for each of four "tastes", and this number must be multiplied by the number of colors (i.e. two for $S U(2)$ ), for a total of 32 free-field zero modes. So it is reasonable to guess that the 32 eigenmodes in the central band of the vortex-removed spectrum are simply the would-be zero modes of the free staggered theory. In fact, these modes disappear for anti periodic boundary conditions (see Fig. 36), which is sufficient to remove the zero modes of the free theory and therefore can be treated as real zero modes which do not contribute to the chiral condensate via the Banks-Casher relation.
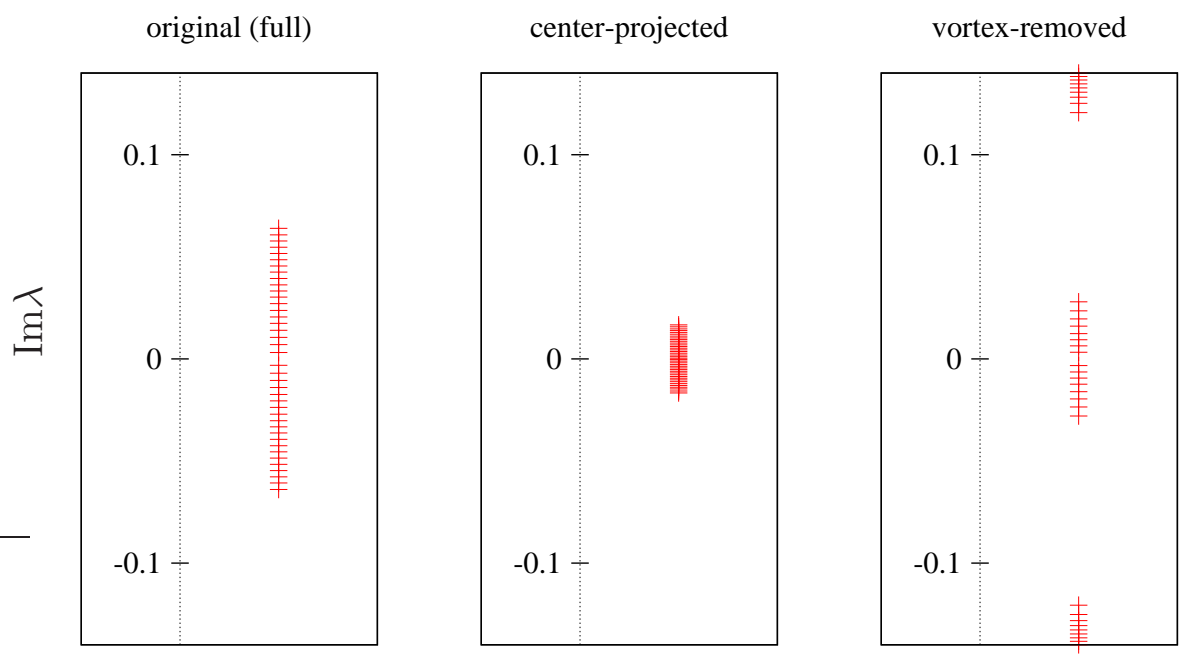

Figure 35: First twenty asqtad staggered Dirac eigenvalues on a $16^{4}$ lattice at $\beta_{L W}=3.3$.

These results seem pretty promising, there is confinement with chiral symmetry breaking for vortex configurations and even increasing effects for center-projected data, whereas both properties vanish for vortex-removed configurations. So the vortex excitations of 

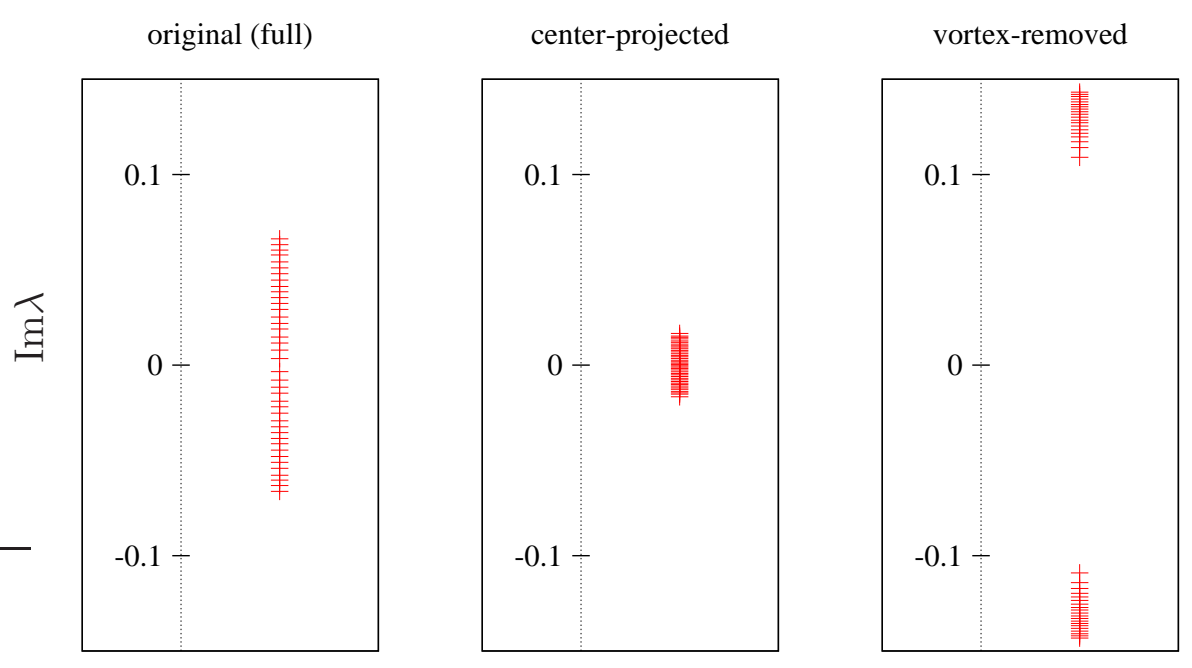

Figure 36: First twenty asqtad staggered Dirac eigenvalues on a $16^{4}$ lattice at $\beta_{L W}=$ 3.3 using anti periodic boundary conditions.

the vortex-only lattice carry not only the information about confinement, but are also responsible for chiral symmetry breaking via the Banks-Casher relation. This result was anticipated in [15], which found a non-zero $\langle\bar{\psi} \psi\rangle$ condensate on center-projected lattices. Chiral symmetry breaking disappears for vortex-removed lattices, as discovered long ago by de Forcrand and D'Elia, in a direct calculation of $\langle\bar{\psi} \psi\rangle$ [14].

The next question is how these processes are related to the vortices themselves and therefore the correlation between the Dirac eigenmodes and the vortices shall be considered. First of all the localization properties of the eigenmodes are determined.

\subsection{Localization and fractal dimension of eigenmodes}

A useful measure to quantify the localization of eigenmodes is the inverse participation ratio (IPR) $[64,65,66,67]$. The IPR of a normalized field $\rho_{i}(x)$ is defined as

$$
I=N \sum_{x=0}^{N} \rho_{i}^{2}(x)
$$

where $\mathrm{N}$ is the number of lattice sites $\mathrm{x}$. Here, $\rho_{i}(x)=\psi_{i}^{\dagger} \psi_{i}(x)$ and $\psi_{i}(x)$ is the $i$-th, normalized $\left(\sum_{x} \rho_{i}(x)=1\right)$, lowest eigenvector of the Dirac operator.

With this definition, I characterizes the inverse fraction of sites contributing significantly to the support of $\rho(x)$. A simple calculation shows that the IPR takes the following values for these simple situations:

$$
\begin{array}{cl}
\text { Unlocalized: } \rho(x)=\text { const. } & I=1 \\
\delta \text {-function: } \rho(x)=\delta\left(x_{0}\right) & I=N \\
\text { localized on fraction } f \text { of sites: } & I=1 / f
\end{array}
$$


The scaling of the IPR with lattice spacing is sometimes used to determine the dimensionality of eigenmode concentration. Suppose the objects responsible for confinement, or indeed any physics governing the lowest Dirac eigenmodes (LDE), localize the LDEs. As the lattice spacing $a$ is reduced, the fraction of sites contributing to the IPR scales as $a^{d} / a^{4}$. Thus the IPR indicates the co-dimension of these objects: $d=4$ for instantons, $d=3$ for monopoles, and $d=2$ for vortices. Gauge dislocations should contribute as $d=0$ objects, however their density diverges as $a^{-4}$ so that they should give a constant contribution: $a^{0} /\left(a^{4} a^{-4}\right)$.

Since the IPR $\sim 1 / f$, reducing the lattice spacing at fixed physical volume gives

$$
a \rightarrow 0 \text { at fixed volume: } I \sim a^{4-d}
$$

On the other hand, increasing the volume at fixed lattice spacing includes proportionately more of the confining objects, whatever their dimension. Thus the IPR is expected to remain constant,

$$
L \rightarrow \infty \text { at fixed } a: I \sim \text { constant. }
$$

This dimensionality, deduced from the IPR by reasoning that if the eigenmode has support mainly on a sub manifold of dimension $d$, with a thickness in the $4-d$ orthogonal directions which is a fixed number of lattice units, then the IPR should scale with lattice spacing as $1 / a^{4-d}$, can lead to incorrect conclusions, because it is not necessarily true that the thickness of the localization region is a constant number of lattice spacings, regardless of coupling. An instructive example is provided by the lowest eigenmode of the covariant Laplacian in the adjoint representation, which was studied in [68]. In that case it was found that the IPR scaled like $1 / a^{2}$, suggesting an eigenmode concentration on surfaces. Instead, it turns out that the lowest eigenmode is sharply concentrated in a point like region. The peculiar scaling of the IPR arises because the volume $b$ of the region of concentration, in lattice units, scales in a peculiar way. If this volume were a constant in physical units, then $b a^{4}$ would be constant. If instead the volume were constant in lattice units, then $b$ itself would be constant. In fact, it is $b a^{2}$ which is constant; the volume of the eigenmode concentration region goes to zero in physical units, but infinity in lattice units, in the continuum limit. The naive deduction of the dimensionality of the concentration region, purely from the scaling of the IPR, leads in this case to an incorrect conclusion.

\subsection{1 asqtad staggered IPR-Results}

The following figures show IPRs for asqtad staggered Dirac eigenmodes on 12,16 and $20^{4}$ lattices for $\beta_{L W}=2.9,3.1,3.3,3.5$ and 3.7 (100 configurations each). The mean value is plotted against the number of the corresponding eigenmode.

The Figures 37-38 show the dependence on lattice-spacing $a\left(\beta_{L W}\right)$ of the IPR for fixed lattice size $\left(20^{4}\right)$ for full (Fig. 37), projected (Fig.38a) and vortex-removed (Fig.38b) configurations. It generally shows an increasing IPR with $\beta_{L W}$ for unprojected data (Fig.37). According to $[66,67]$ the IPR should go like $1 / a$, in which case it should 
roughly double in going from $\beta_{L W}=2.9$ to $3.3, \beta_{L W}=3.1$ to 3.5 or $\beta_{L W}=3.3$ to 3.7 and in fact, that's what about it does. For projected configurations (Fig.38a) the IPR of the lowest modes is roughly 11 at $\beta_{L W}=2.9$, 33 at $\beta_{L W}=3.1,140$ at $\beta_{L W}=3.3$ and 400 at $\beta_{L W}=3.5$. Now, if the eigenmode density has support on point like regions, it means that the IPR should go like $1 / a^{4}$. That means the IPR should increase by a factor of 4 from $\beta_{L W}=2.9$ to 3.1 , from $\beta_{L W}=3.1$ to 3.3 and from $\beta_{L W}=3.3$ to 3.5. Thats not so far off the obtained results. So one figures out that the eigenmodes in the projected case are sharply peaked, maybe at vortex intersections what has to be checked. Therefore peaks in the eigenmode densities are identified in the next section for a better understanding of the IPR-results.

The Figures 39-41 show the IPR as a function of lattice size $L$ for fixed lattice spacing for full (Fig. 39), projected (Fig. 40) and vortex-removed (Fig. 41) configurations, each for $\beta_{L W}=3.1$ and 3.3. There is a fairly modest increase in IPR with lattice volume. The eigenmodes seem to be localized on a few lattice sites (IPR tending to a constant value $\left(1<\mathrm{IPR}<<L^{4}\right)$ for growing $\left.L\right)$ for full (unprojected) and projected data. Therefore the number of eigenmode density peaks is expected to increase with the lattice volume.

The IPR of vortex-removed configurations behaves somehow strange. The eigenmodes of the central band (1-8) seem to be delocalized completely (zero modes) whereas the higher eigenmodes again seem to be localized on a fraction of sites.

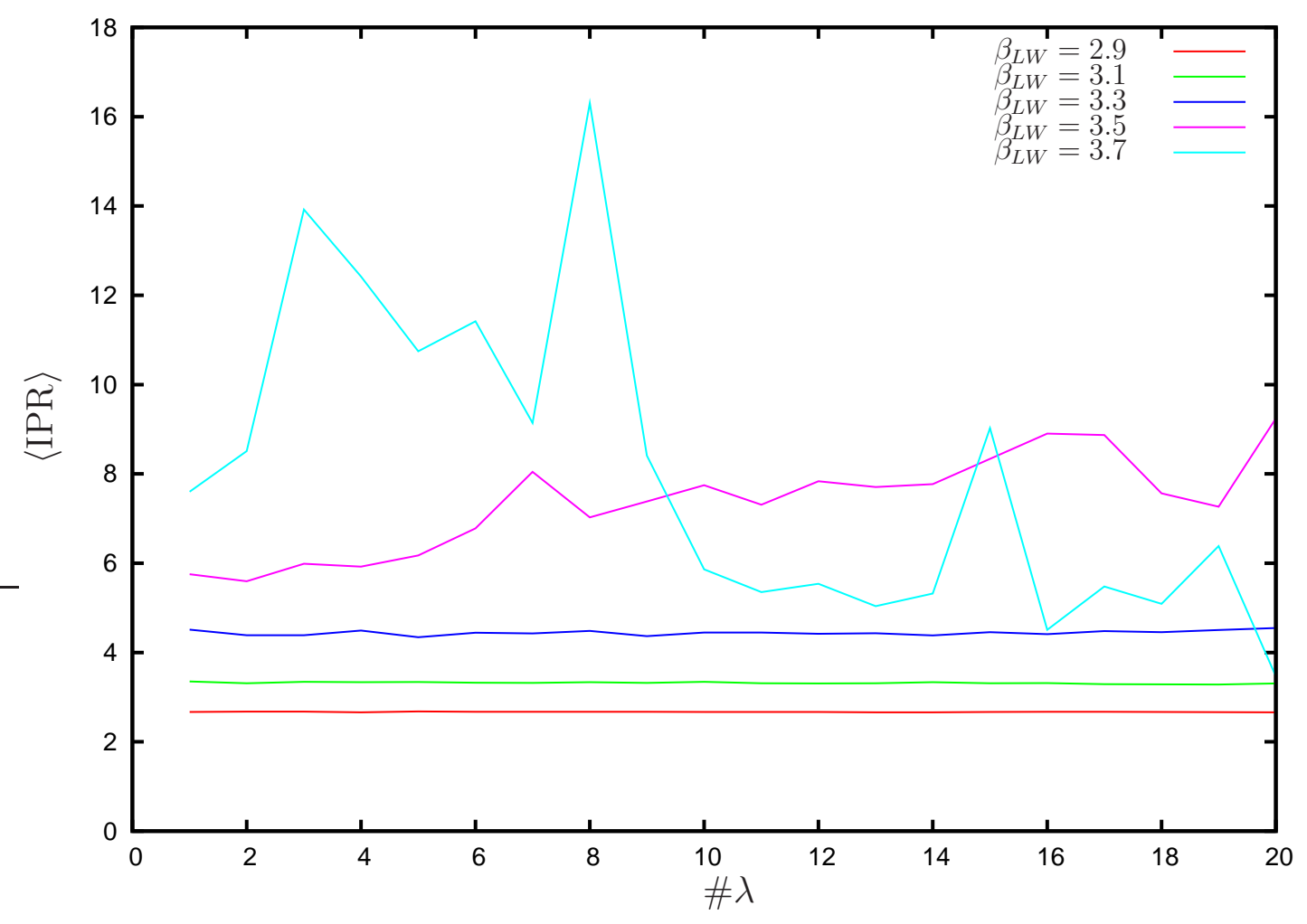

Figure 37: Inverse Participation Ratio for full (unprojected) configurations on a $20^{4}$ lattice and different $\beta_{L W}$ 
a)

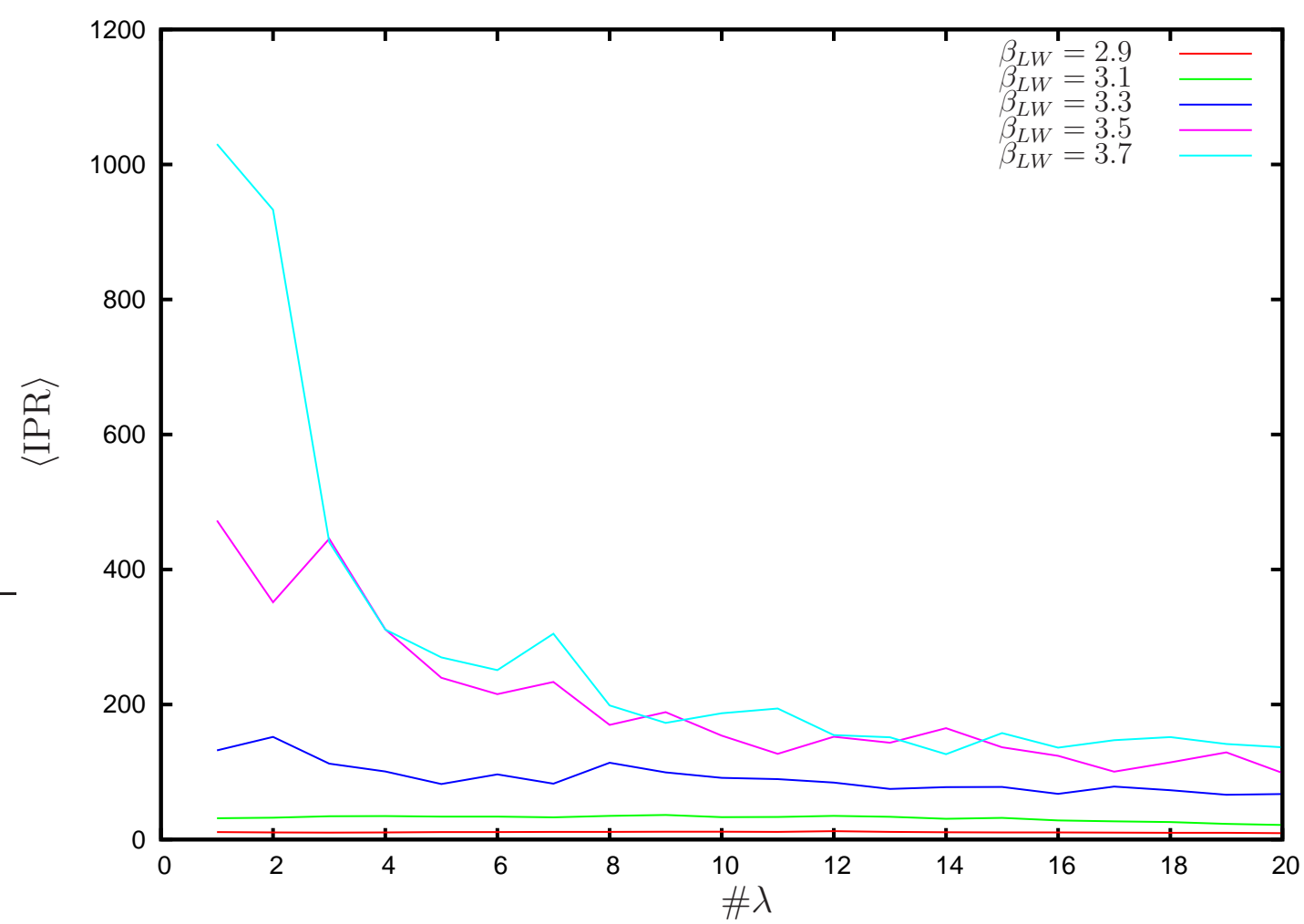

b)

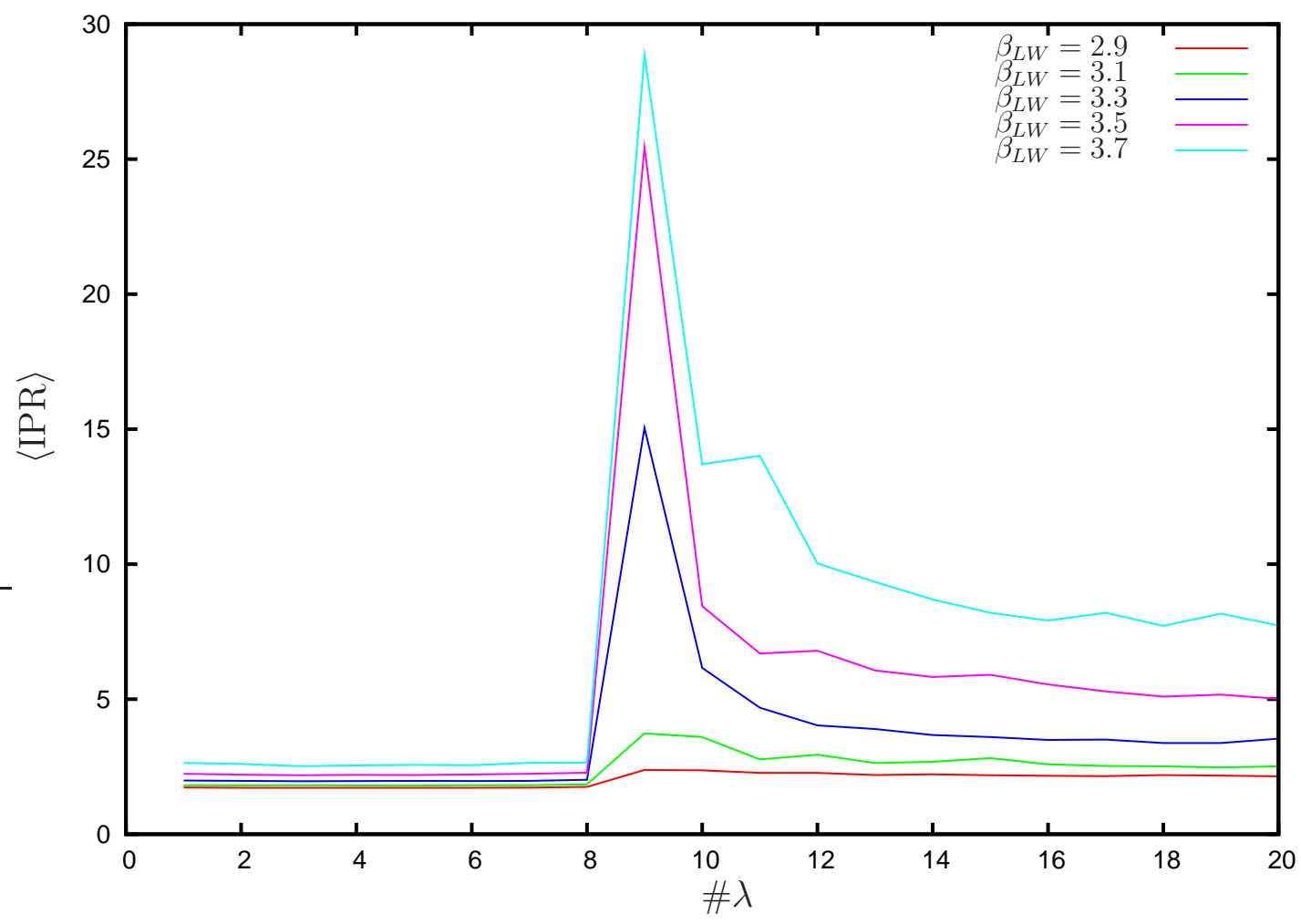

Figure 38: Inverse Participation Ratio for a) center-projected and b) vortex-removed configurations on a $20^{4}$-lattice and different $\beta_{L W}$ 
a) $\beta_{L W}=3.1$

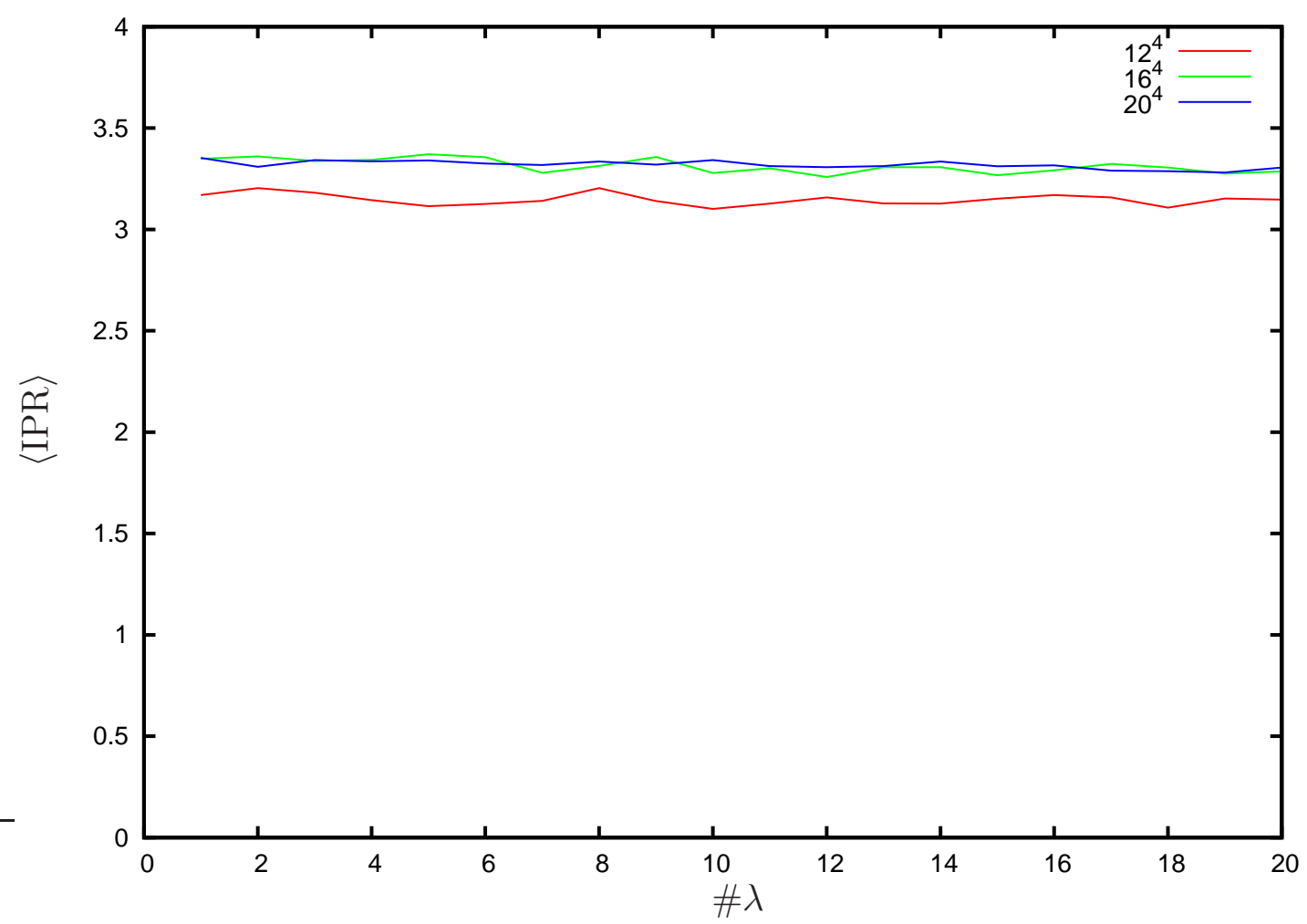

b) $\beta_{L W}=3.3$

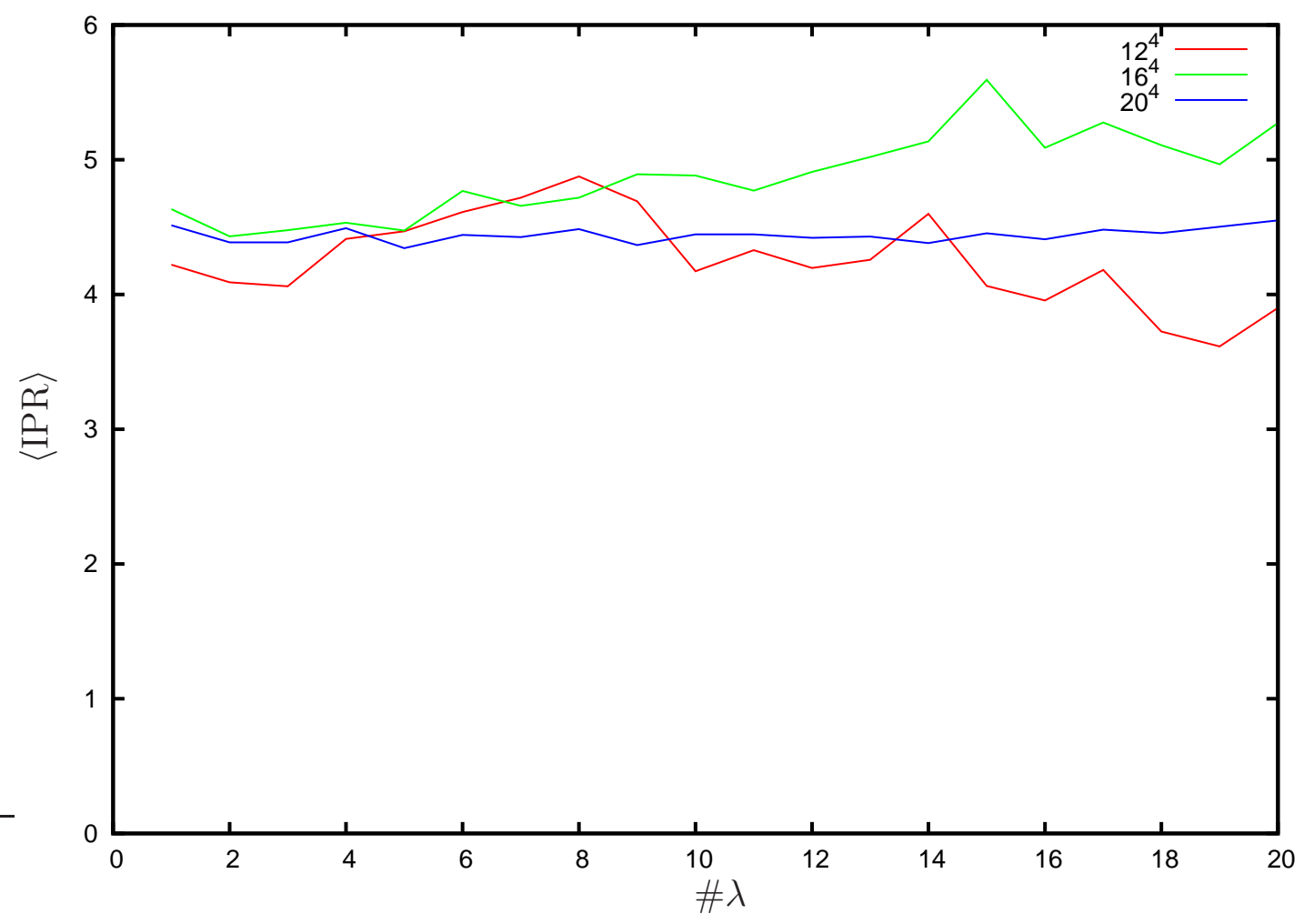

Figure 39: Inverse Participation Ratio for full (unprojected) configurations for a) $\beta_{L W}=$ 3.1 and b) $\beta_{L W}=3.3$ and different lattice sizes 
a) $\beta_{L W}=3.1$

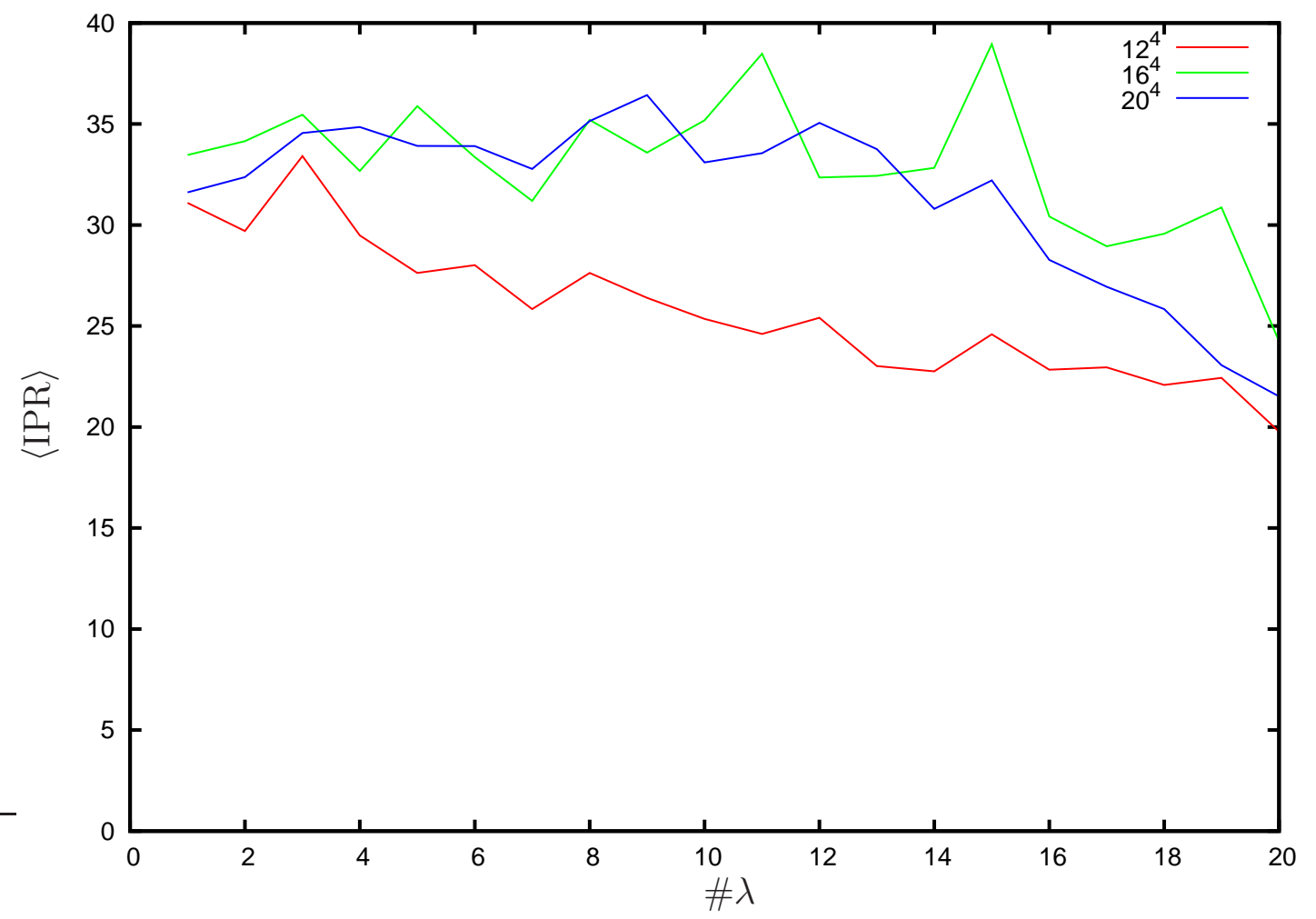

b) $\beta_{L W}=3.3$

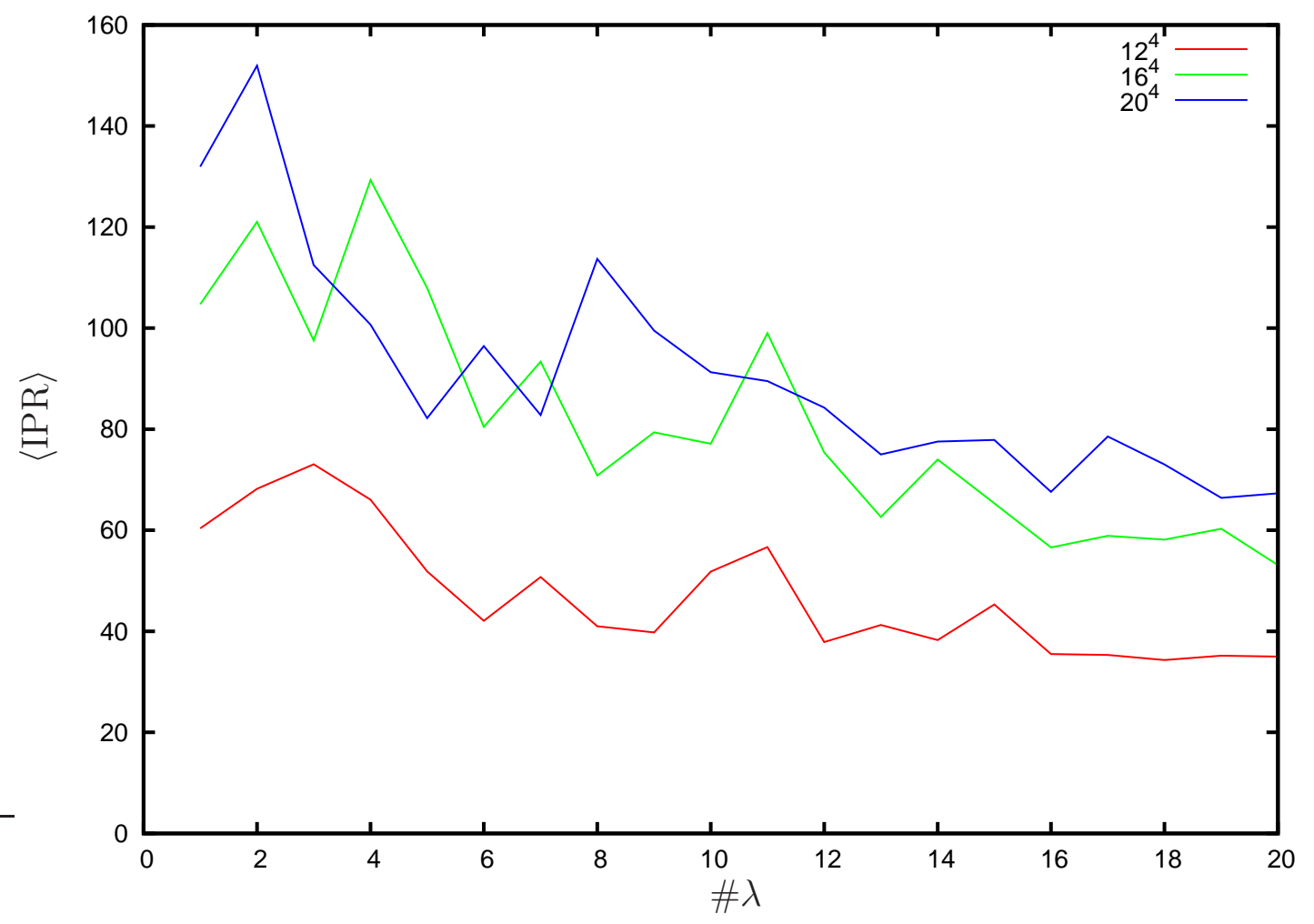

Figure 40: Inverse Participation Ratio for center-projected configurations for a) $\beta_{L W}=$ 3.1 and b) $\beta_{L W}=3.3$ and different lattice sizes 
a) $\beta_{L W}=3.1$

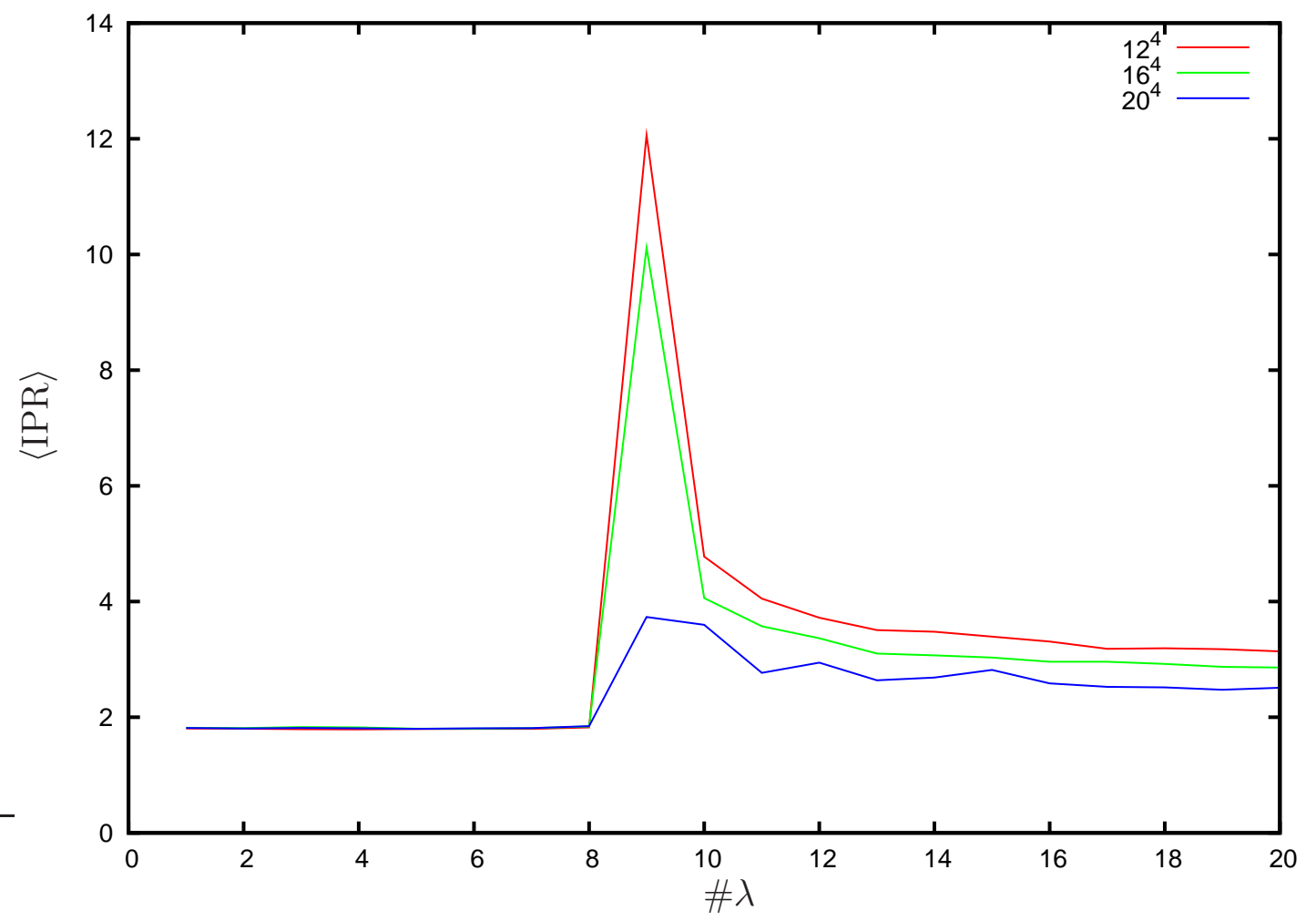

b) $\beta_{L W}=3.3$

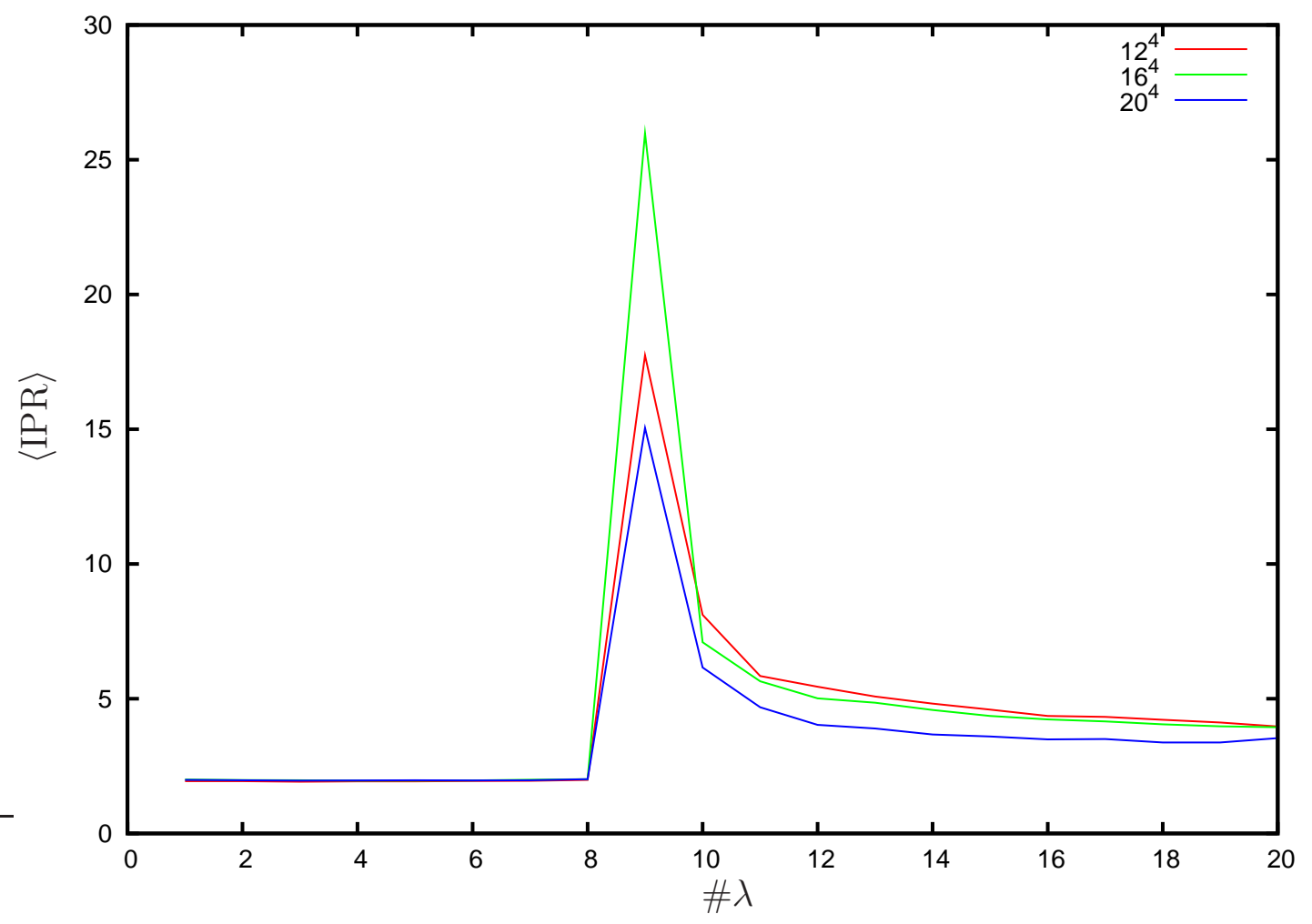

Figure 41: Inverse Participation Ratio for vortex-removed configurations for a) $\beta_{L W}=$ 3.1 and b) $\beta_{L W}=3.3$ and different lattice sizes 


\subsection{2 overlap IPR-Results}

For overlap fermions the IPR-results are shown in Figures 42-43. For full configurations (Fig. 42) the statistics of 20 configurations seems to be too low to give any reasonable messages. The projected data (Fig. 43a) shows the fourfold degeneracy and vortexremoved IPR (Fig. 43b) shows up some interesting plateau for eigenmodes two to four which is not due to zero modes or any degeneracy.

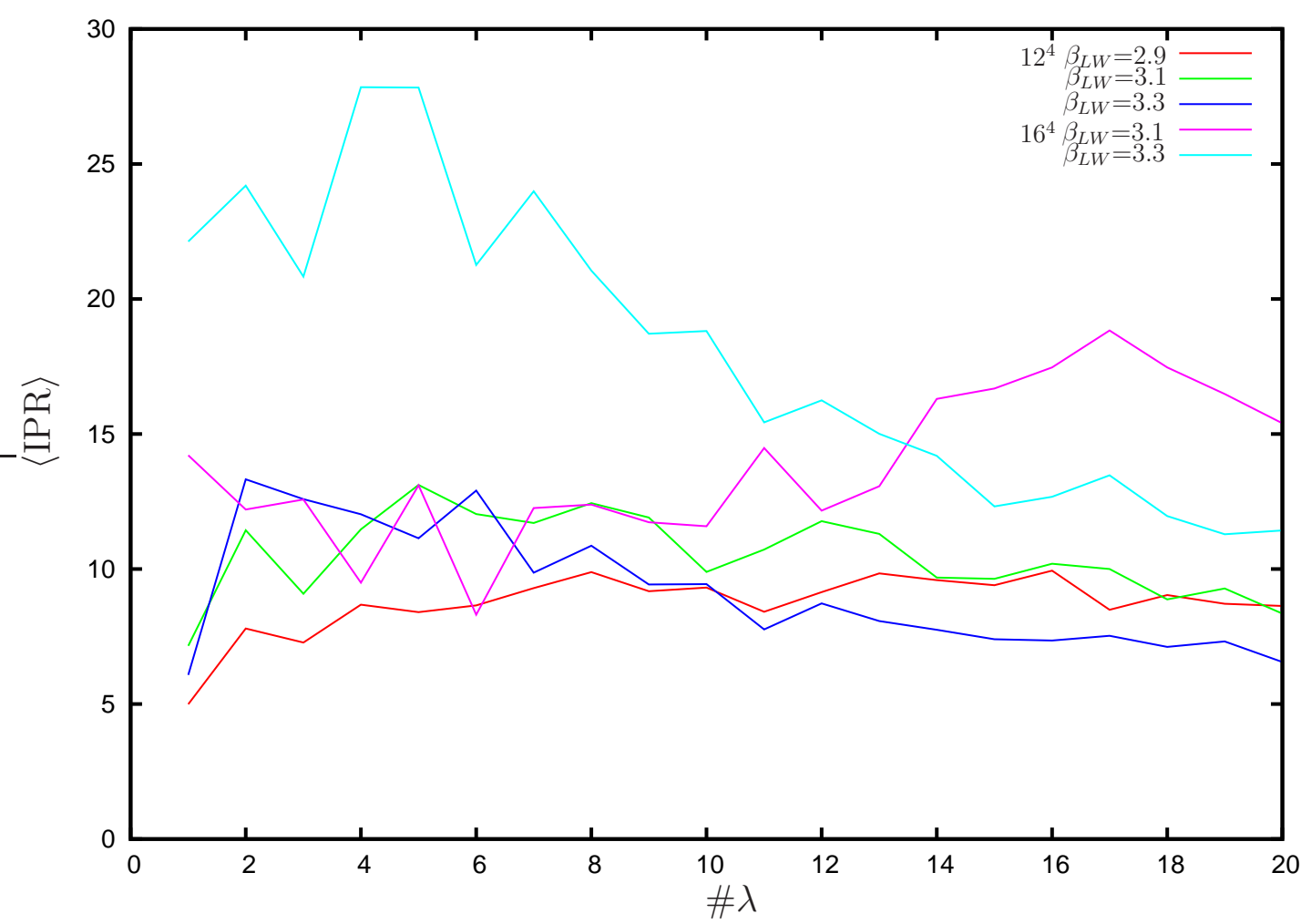

Figure 42: Inverse Participation Ratio for full (unprojected) configurations on $12^{4}$ and $16^{4}$-lattices and different $\beta_{L W}$

\subsubsection{Results of other groups}

For Dirac eigenmodes, conclusions based on the scaling of the IPR have not been entirely consistent with one another (cf. the overview in [69]). Results of the MILC collaboration, with asqtad fermions, indicate a dimensionality $d=3[66,67]$, while the ITEP group has reported results, for overlap fermions and the Wilson action, consistent with $d=0$ [70]. A third study, using overlap fermions and the Lüscher-Weisz action, again indicates $d=3[71,72]$ while the latest study of this group, using generalized IPR's defined using higher powers of $\rho_{i}(x)$, suggest eigenmode concentrations on manifolds of dimension between $d=0$ and $d=1$ [73]. 
a)

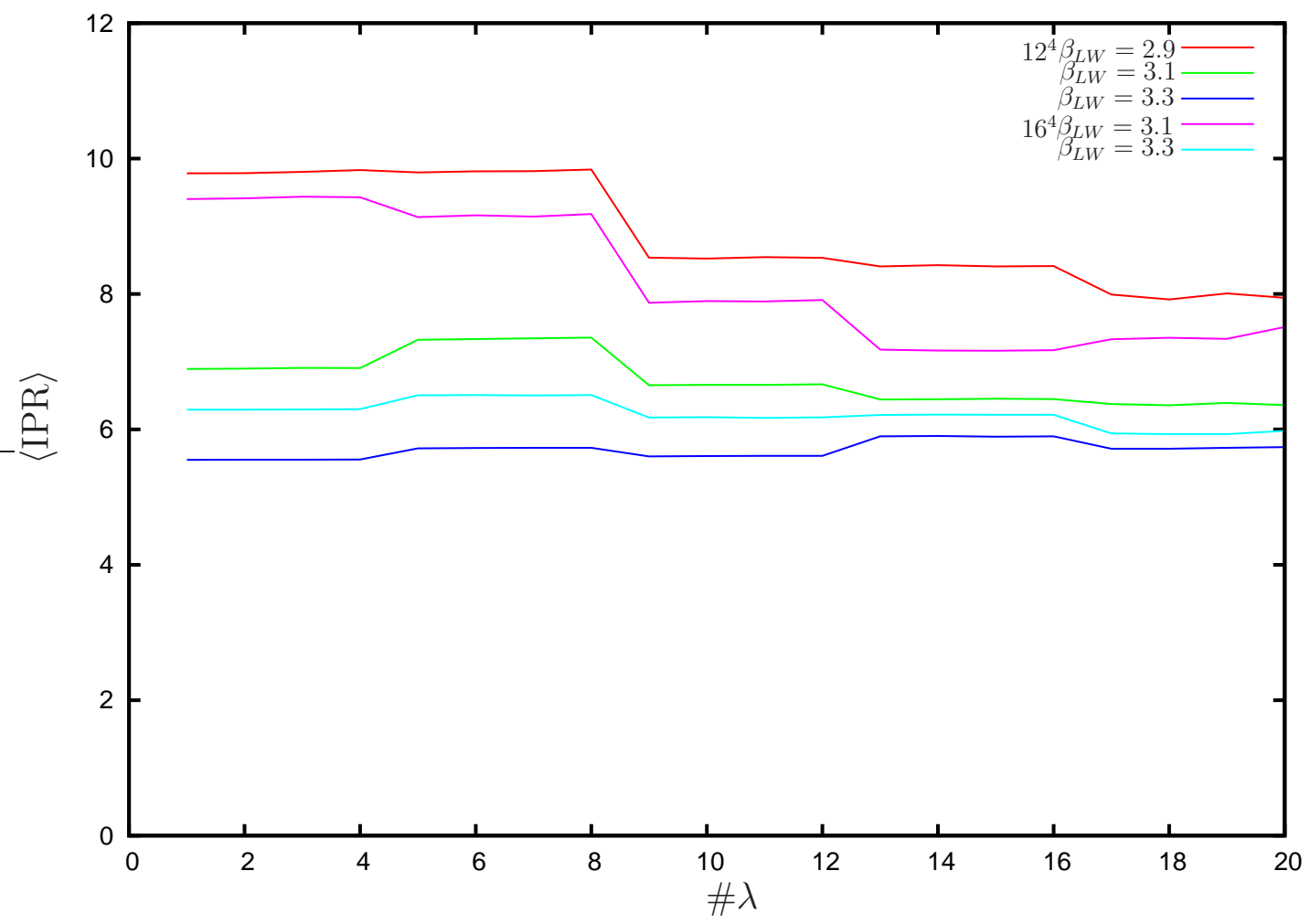

b)

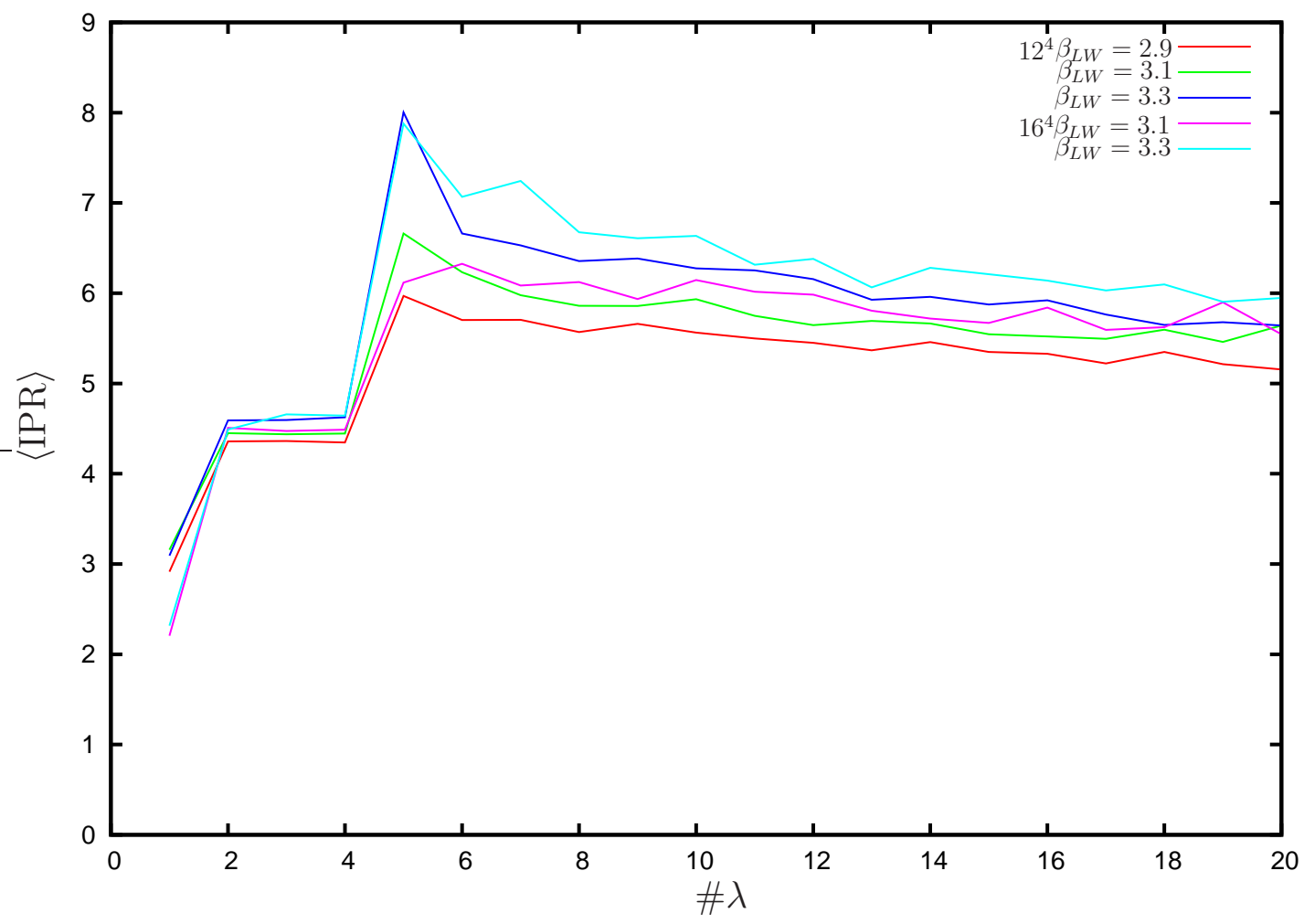

Figure 43: Inverse Participation Ratio for a) center-projected and b) vortex-removed configurations on $12^{4}$ and $16^{4}$-lattices and different $\beta_{L W}$ 


\subsection{Peak Analysis}

The IPR shows that the eigenmodes are rather localized to peaks. To confirm this result a possibly more reliable (if less quantitative) approach is to simply look at sample plots of $\rho_{\lambda}(x)$ throughout the lattice volume. Looking at single configurations the scalar densities of single eigenmodes show up as follows. The figures (44-50) show the maximum density peaks of overlap (44-46) and staggered (47-50) Dirac eigenmodes. Each lattice contains several sharp peaks of this kind; it is obvious that the concentration of eigenmode density is in a point-like region, rather than being spread over a sub manifold of higher dimensionality. The figures display a set of $x y$-plots of $\rho(x, y, z, t)$ at various values of $z$ and fixed $t$, but there is an equally strong falloff of the peak as one moves away from the maximum in the time direction.

In more detail, Fig. 44 shows the eigenmode density of full overlap modes which are peaked rather sharp whereas for projected data the eigenmode concentration is very broad, extending over most of the lattice volume, as seen in Fig. 45 . Since the overlap operator, evaluated on center-projected configurations, does not have any lowlying eigenmodes, let alone a zero mode, it is not surprising that the eigenmode density is qualitatively different from all other configurations. Overlap eigenmodes of vortexremoved configurations show small, large peaks in a noisy background (Fig. 46).

The asqtad staggered modes show even sharper peaks with less noise (Figs. 47, 48 and 50) except the first eight ("real" zero) modes of vortex-removed configurations (Fig. 49). Eigenmode peaks are by far the sharpest for eigenmodes of the asqtad operator on the center-projected lattice. Taking account of the vertical scales in these figures, the peak in the asqtad-center-projected case (Fig. 48), is about an order of magnitude higher than the peak in the asqtad-unprojected case (Fig. 47). This difference is of course reflected in a comparison of the IPRs of asqtad eigenmodes on the full and center-projected lattices, already shown in Fig. 37 and 38b), which indicate a far higher degree of eigenmode concentration in the center-projected case.

It is not too surprising that would-be zero modes of the asqtad operator would be very highly concentrated when evaluated on center-projected lattices. On thin vortices, topological charge is concentrated not just in point-like regions, but in fact at individual lattice sites on the dual lattice, where thin vortex sheets writhe and/or intersect. Since zero modes concentrate on regions of non-zero topological charge density, and topological charge is concentrated at individual sites on the center-projected lattice, the high degree of localization of the lowest-lying modes, in volumes of lattice-scale extension, is to be expected. On unprojected lattices the sources of topological charge, whether vortices, instantons, calorons, or something else, are more spread out, and there is no particular reason to expect that the eigenmode density would concentrate in tiny regions of lattice scale extension. What would be interesting to know, of course, is which of the candidate sources of topological charge density is giving the main effect. The density plots give a strong indication that the charge density concentrates in point-like regions, rather than surfaces or three-volumes, but this fact would be compatible with instanton, caloron, and vortex (intersection/writhing) sources. Therefore eigenmode density-vortex surface correlations are performed in section 5.4. 

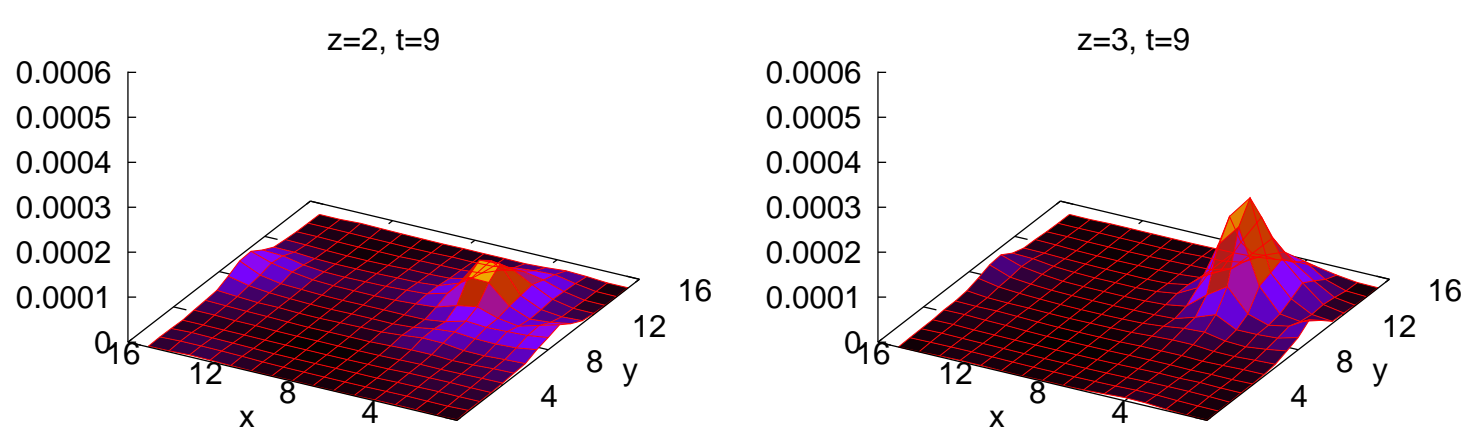

density of eigenvalue \#1, maximum 0.000401170640834 at $x=5, y=12, z=4, t=9$
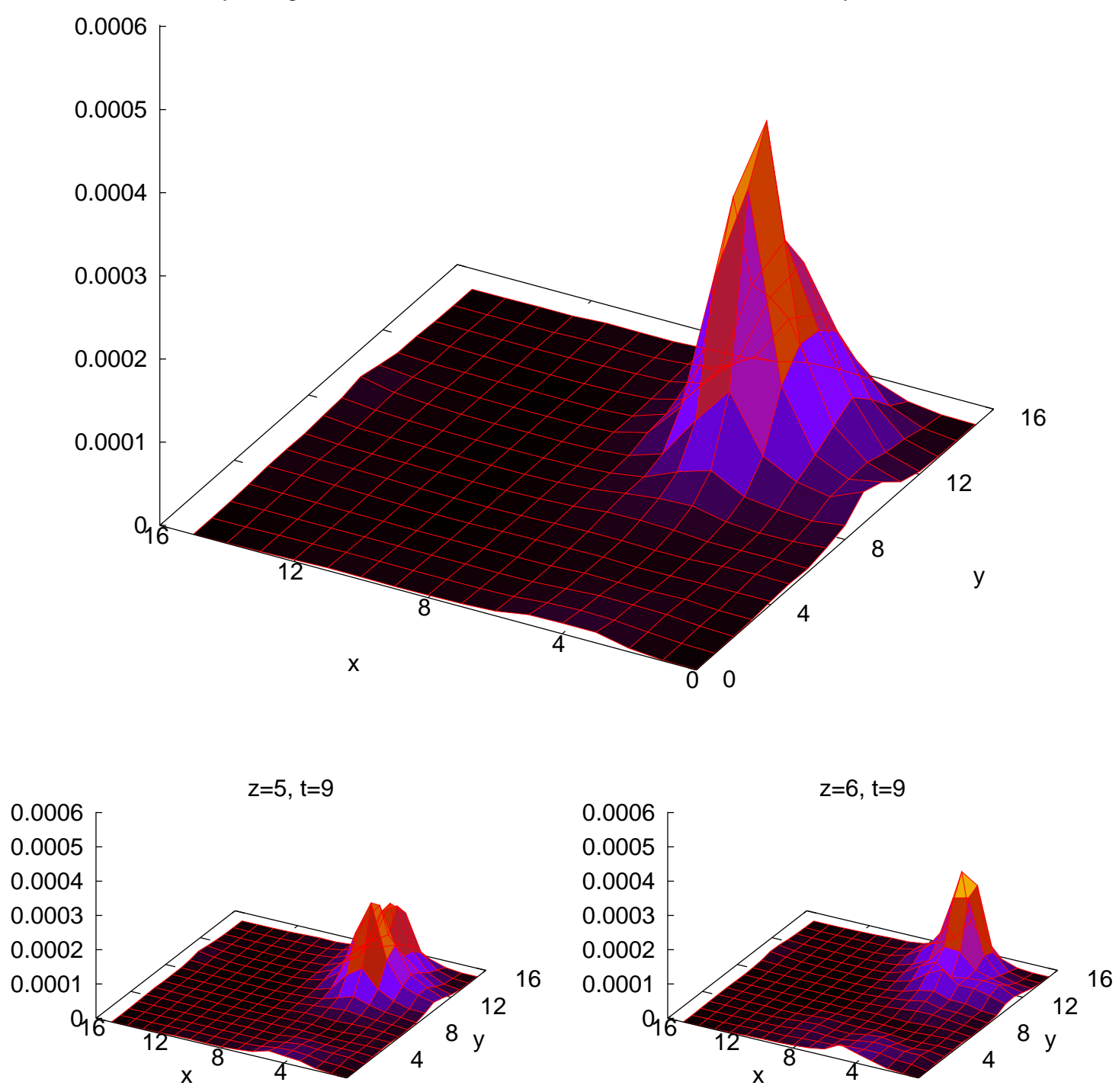

Figure 44: Maximum density peak (center) of first overlap eigenmode for a full configuration on a $16^{4}$-lattice at $\beta_{L W}=3.3$ with upper (above) and lower (below) z-slices of the same t-slice. (rather sharp peak, little noise) 

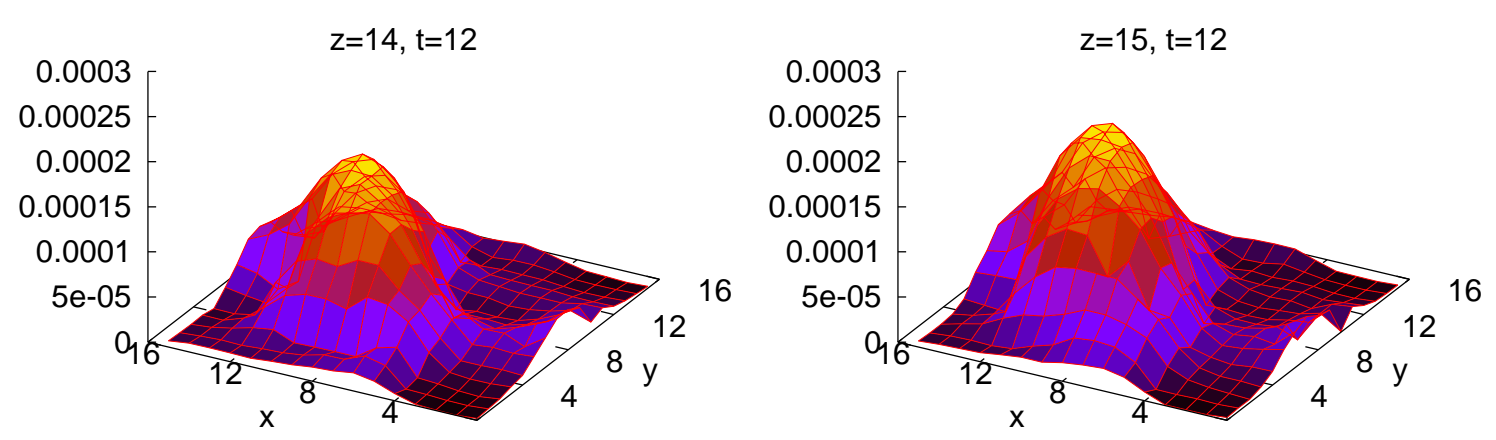

density of eigenvalue \#1, maximum 0.000199916673677 at $x=12, y=9, z=16, t=12$
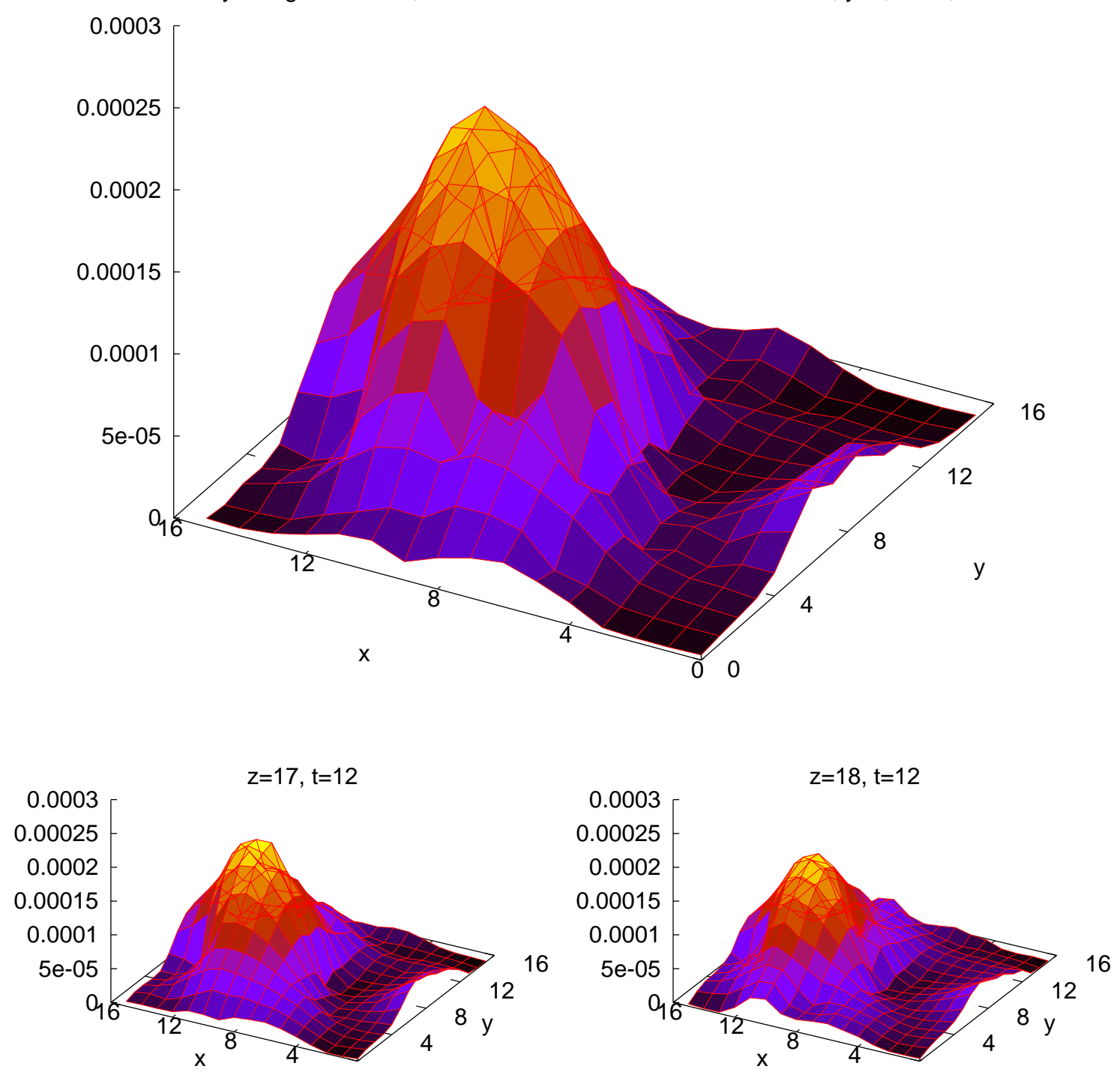

Figure 45: Maximum density peak (center) of first overlap eigenmode for a projected configuration on a $16^{4}$-lattice at $\beta_{L W}=3.3$ with upper (above) and lower (below) z-slices of the same t-slice. (one large peak covering whole configuration) 

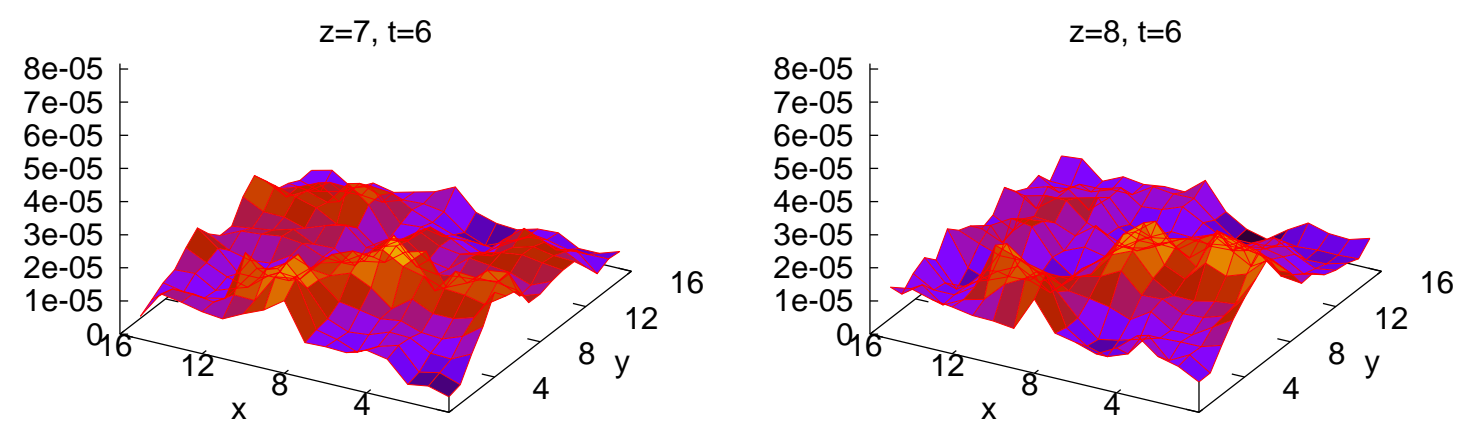

density of eigenvalue \#1, maximum 5.44240973371e-05 at $x=4, y=8, z=9, t=6$
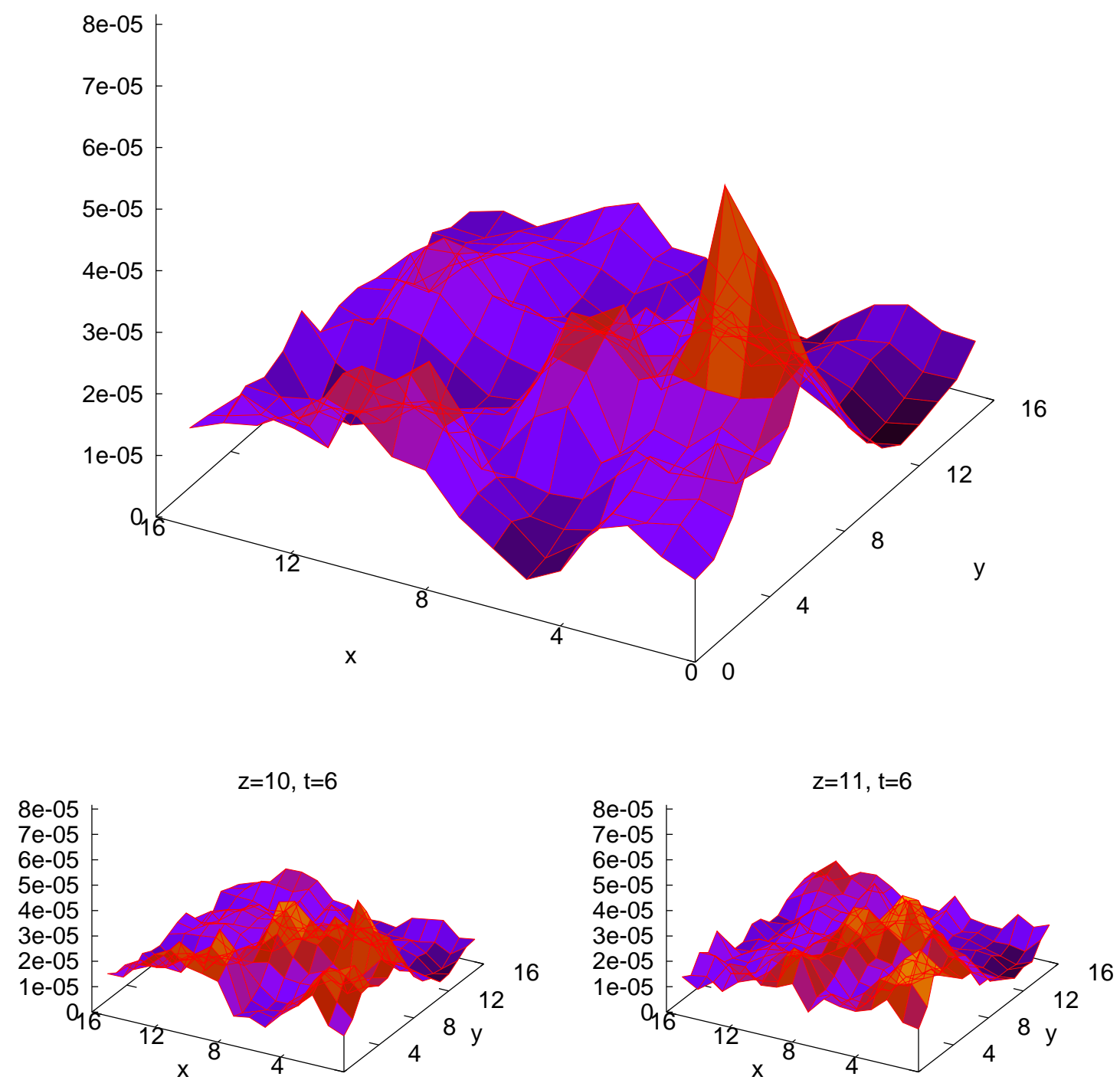

Figure 46: Maximum density peak (center) of first overlap eigenmode for a vortexremoved configuration on a $16^{4}$-lattice at $\beta_{L W}=3.3$ with upper (above) and lower (below) z-slices of the same t-slice. (large peak, much noise) 

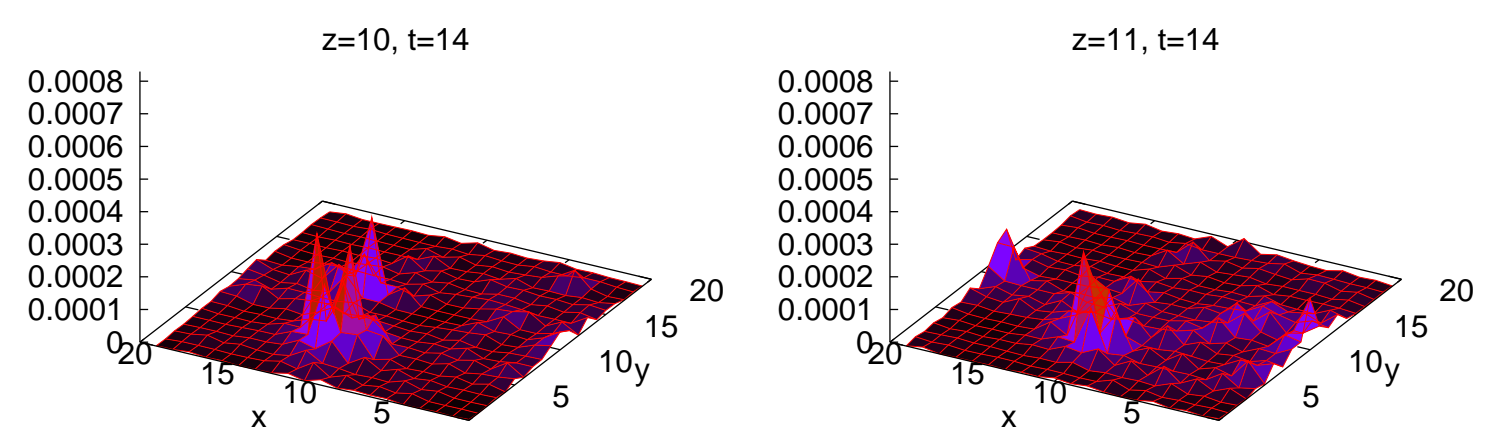

density of eigenvalue \#1, maximum 0.000553011984922 at $x=12, y=7, z=12, t=14$
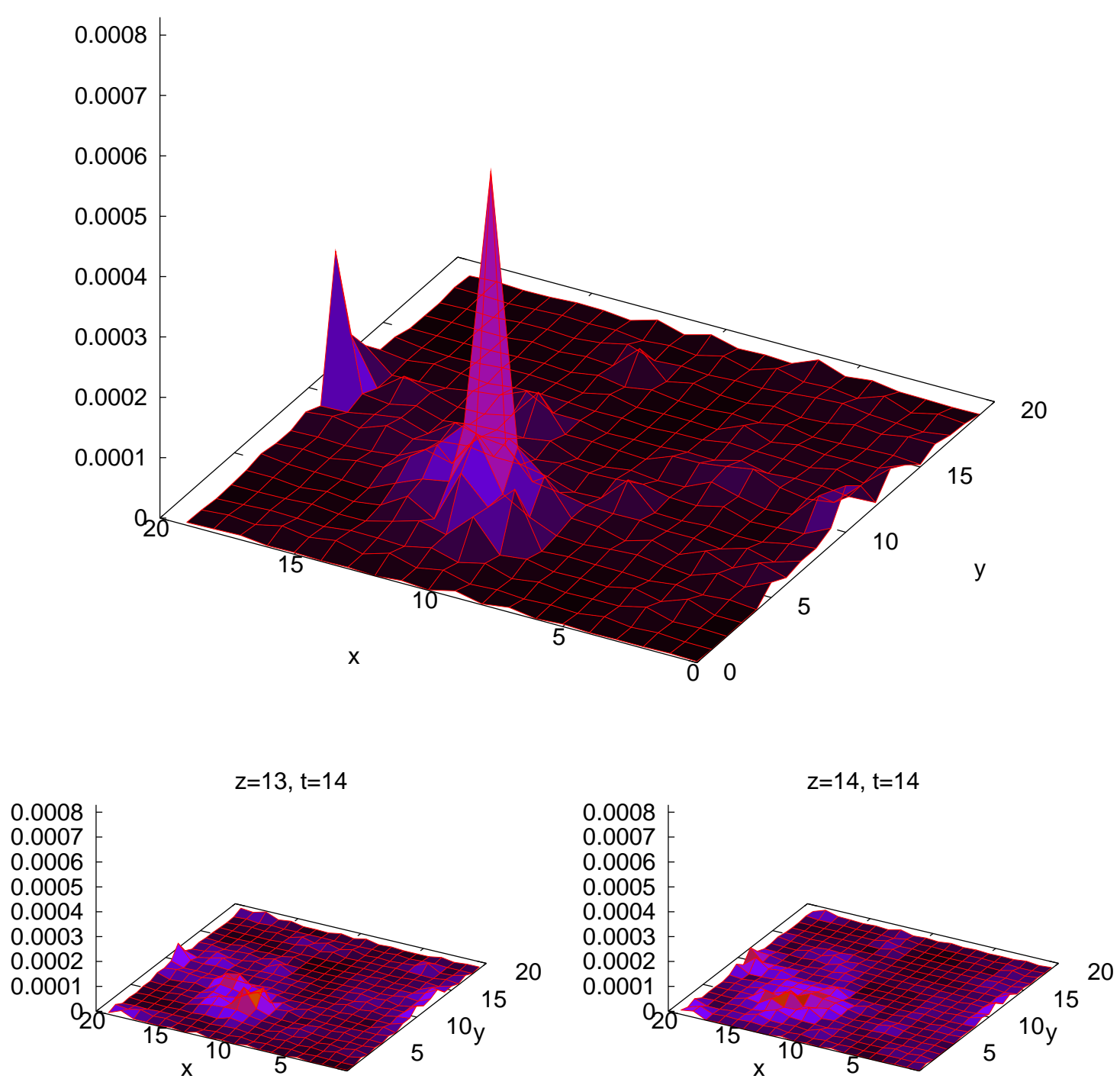

Figure 47: Maximum density peak (center) of first asqtad staggered eigenmode for a full configuration on a $20^{4}$-lattice at $\beta_{L W}=3.3$ with upper (above) and lower (below) z-slices of the same t-slice. (sharp peak, little noise) 

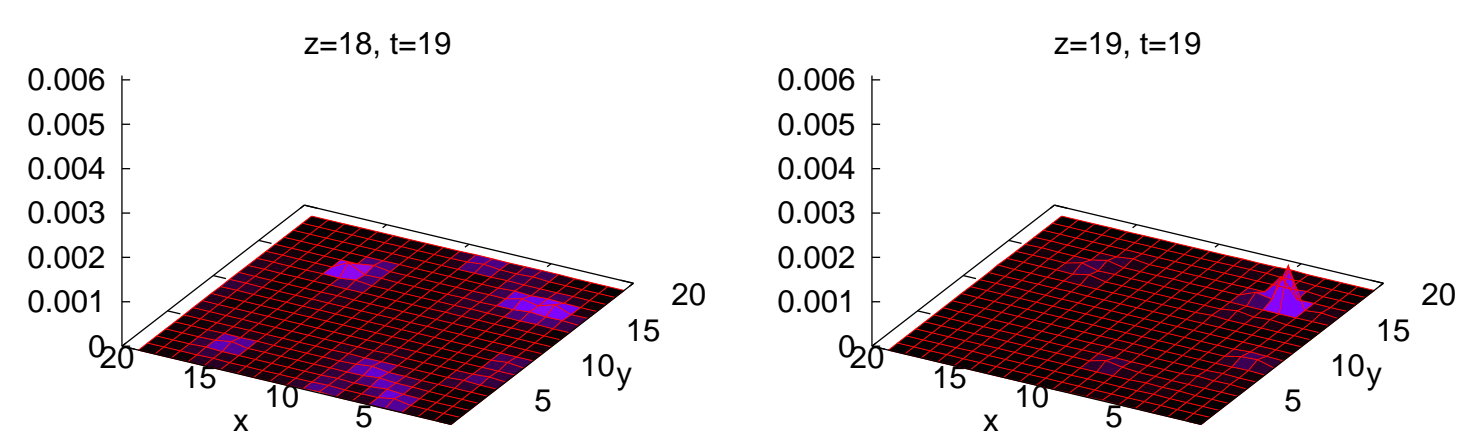

density of eigenvalue \#1, maximum 0.00406513280358 at $x=5, y=16, z=20, t=19$
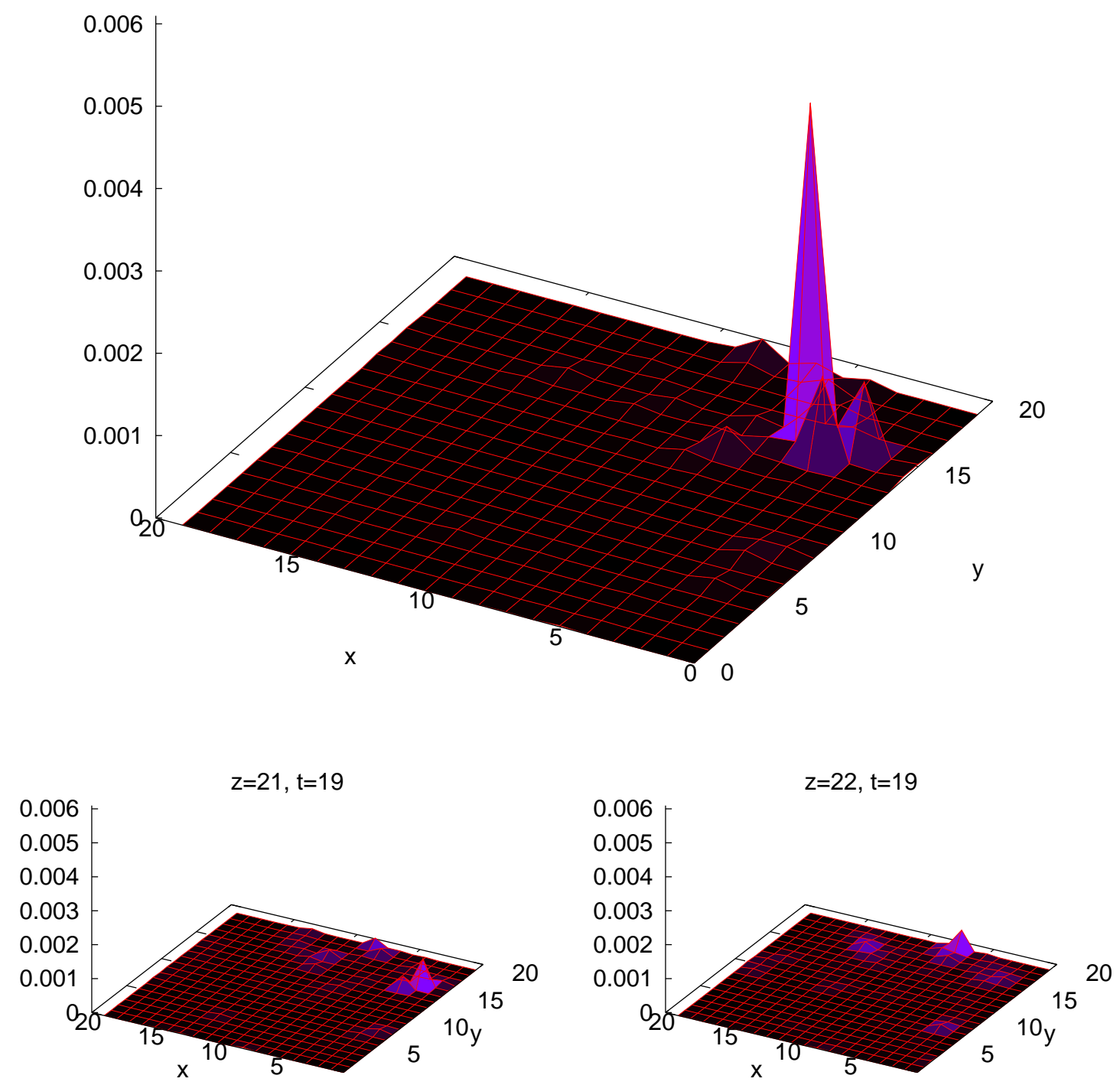

Figure 48: Maximum density peak (center) of first asqtad staggered eigenmode for a projected configuration on a $20^{4}$-lattice at $\beta_{L W}=3.3$ with upper (above) and lower (below) z-slices of the same t-slice. (sharper peak, less noise) 

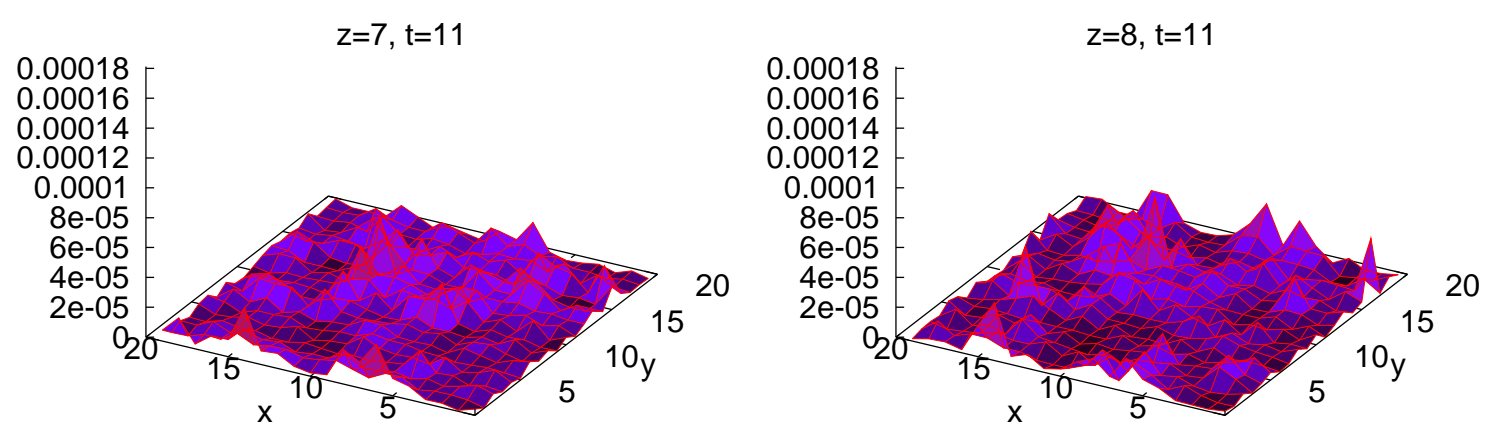

density of eigenvalue \#1, maximum 0.00012080876109 at $x=12, y=15, z=9, t=11$
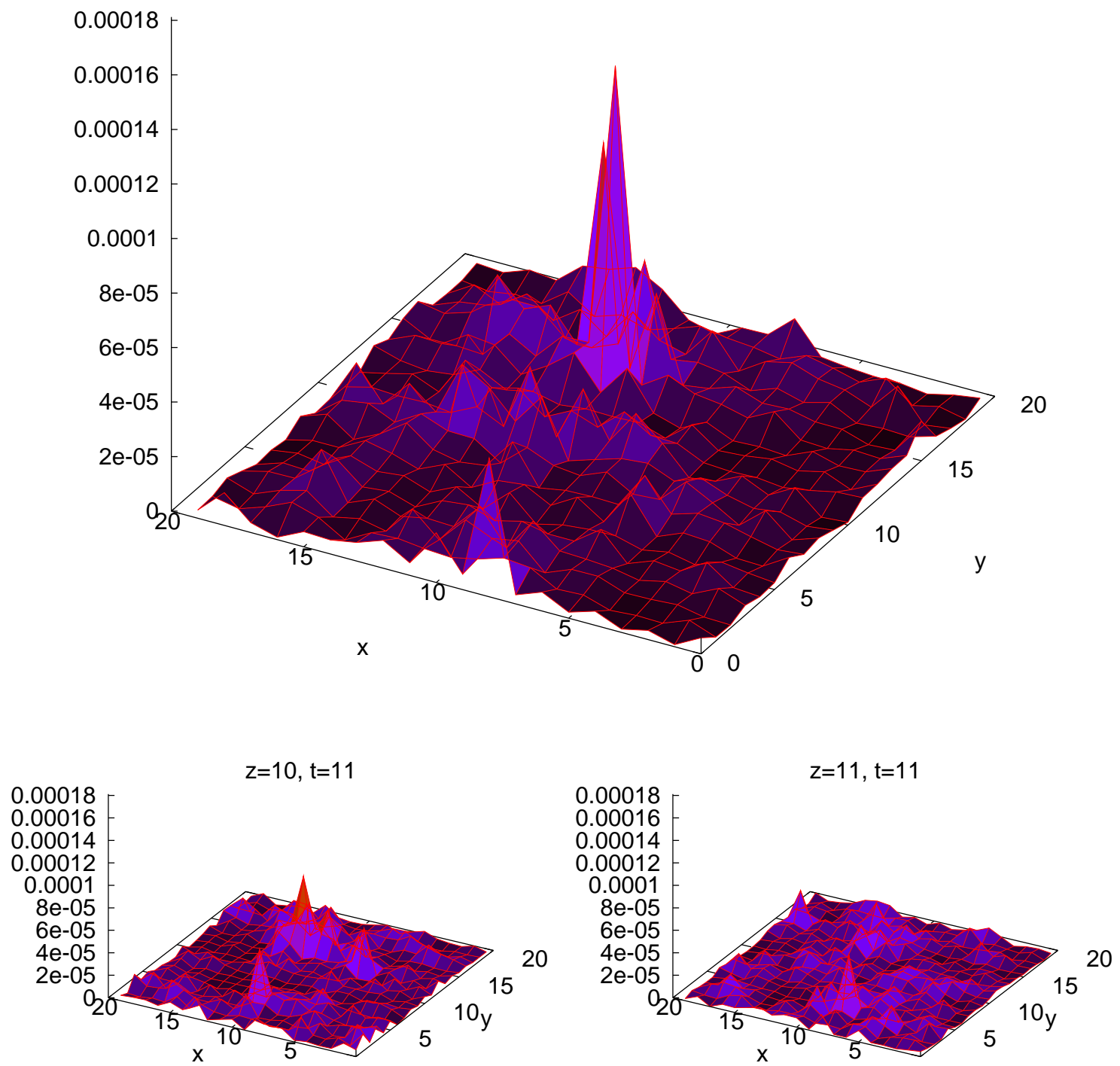

Figure 49: Maximum density peak (center) of first asqtad staggered eigenmode for a vortex-removed configuration on a $20^{4}$-lattice at $\beta_{L W}=3.3$ with upper (above) and lower (below) z-slices of the same t-slice. (small peak, much noise) 

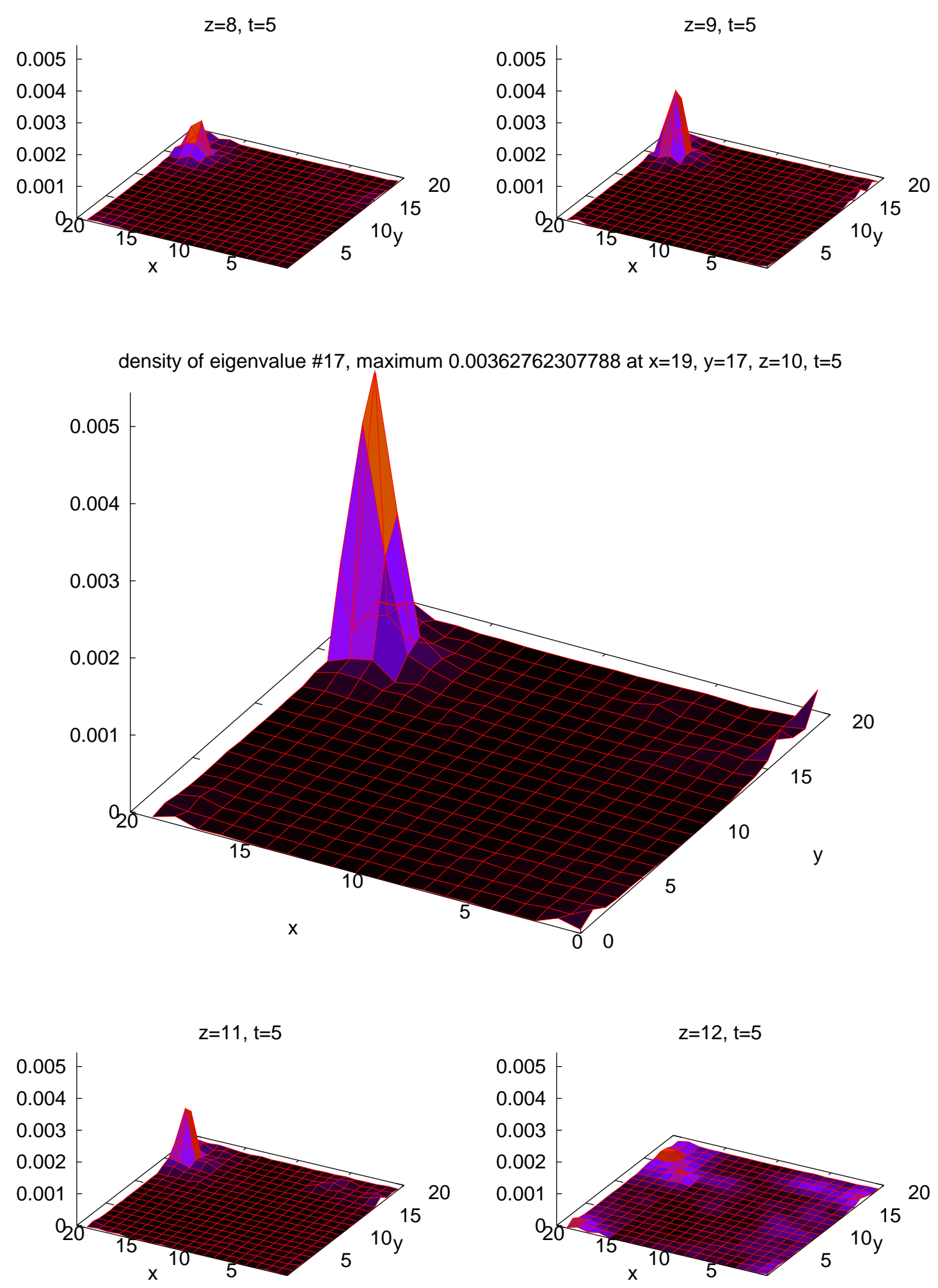

Figure 50: Maximum density peak (center) of ninth asqtad staggered eigenmode for a vortex-removed configuration on a $20^{4}$-lattice at $\beta_{L W}=3.3$ with upper (above) and lower (below) z-slices of the same t-slice. (sharp peak, little noise) 


\subsubsection{Remaining Norm}

The remaining norm $\mathrm{R}$, defined as

$$
R(n)=1-\sum_{i=1}^{n} \rho_{i}(x) \quad \ldots \quad 1 \leq n \leq L^{4},
$$

where $\rho_{i}(x)$ are the eigenmode densities of all $x \in L^{4}$, the lattice volume, sorted from highest to lowest by index $i$ gives some information about the background in the eigenmode densities. A steep slope indicates a low background, a flat slope a higher one.

Fig. 51 shows the $R(n)$-plot for overlap fermions of full, projected and vortex-removed configurations on a $12^{4}$-lattice and different $\beta_{L W}$.

Fig. 52 shows the $R(n)$-plot for staggered fermions of full, projected and vortexremoved configurations on a $20^{4}$-lattice and different $\beta_{L W}$. The background noise always decreases with increasing $\beta_{L W}$, except for projected overlap data, whose background is mostly due to its broad peaks. The projected staggered data has a very low background. Comparing overlap and asqtad staggered data, the remaining norm for full and vortexremoved configurations are about the same, vortex-removed data always shows the most background noise.

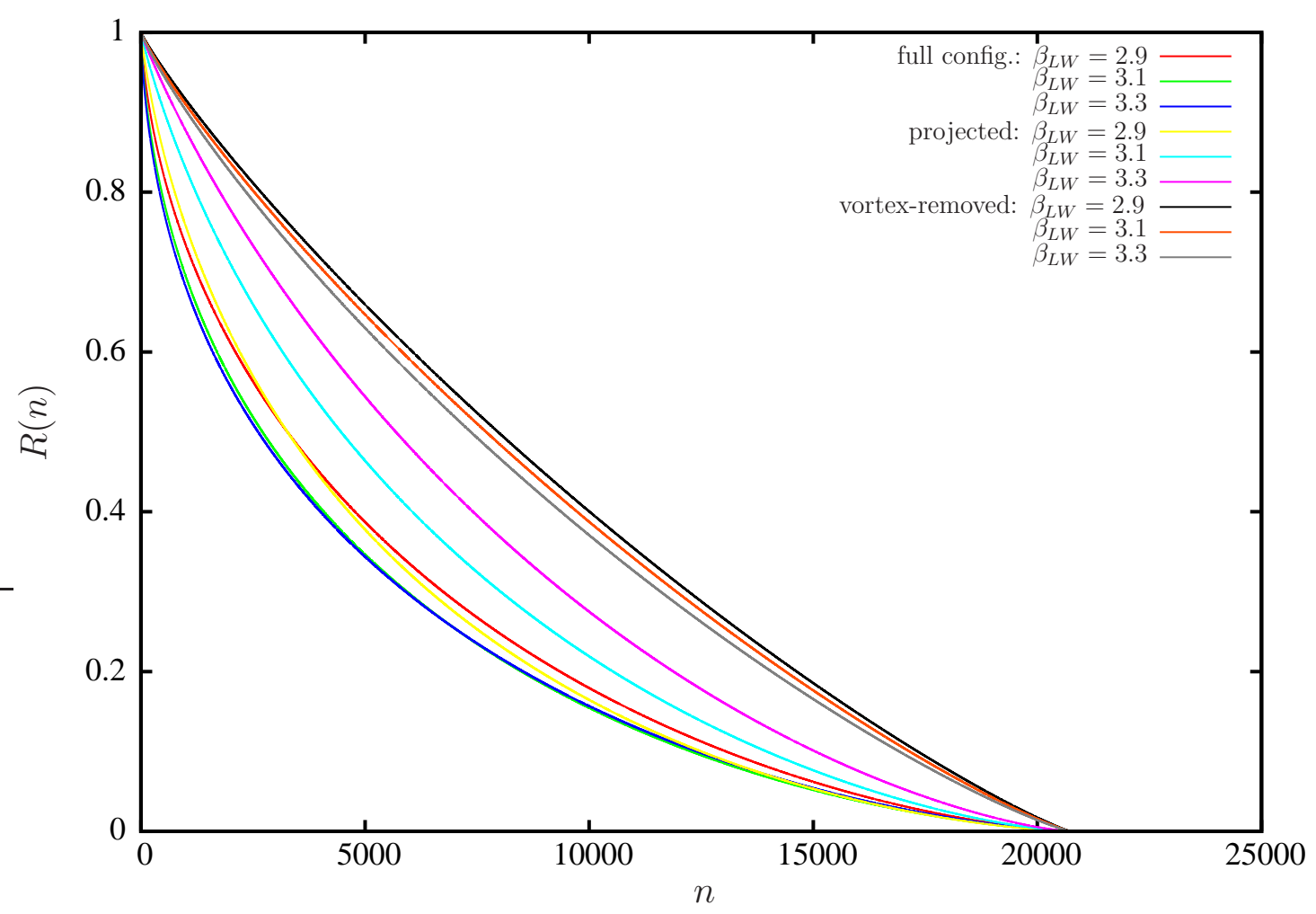

Figure 51: Remaining Norm of first non-zero overlap eigenmode of full, projected and vortex-removed configurations for different $\beta_{L W}$ on a $12^{4}$-lattice. 


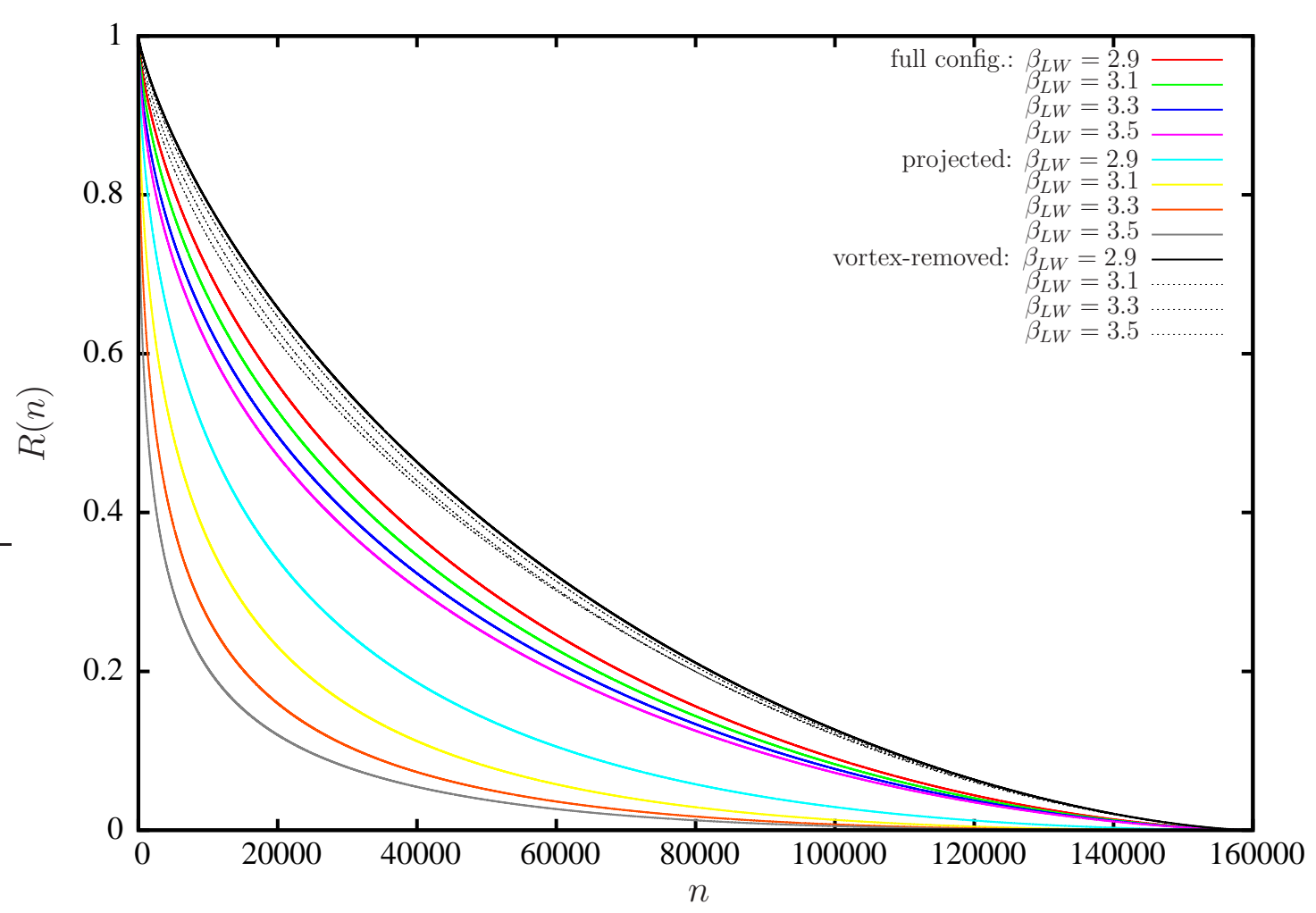

Figure 52: Remaining Norm of first asqtad staggered eigenmode of full, projected and vortex-removed configurations for different $\beta_{L W}$ on a $20^{4}$-lattice.

\subsubsection{Peak Fitting}

Next, some peak scaling in dependence of $\beta_{L W}$ considering the full width half maximum (FWHM) is performed. The FWHM is determined by fitting a distribution function into the peaks. Therefore the average density at points on a hyper sphere around the maximum with radii $1, \sqrt{2}, \sqrt{3}, 2, \sqrt{7}$ and 3 are taken, normalizing their volume to one. The standard distribution function

$$
f(x)=\frac{1}{\sigma \sqrt{2 \pi}} \cdot \exp \frac{-\left(x-x_{0}\right)}{2 \sigma^{2}},
$$

where $x_{0}$ is the maximum peak position, is fitted by some least squares algorithm in order to get the $\sigma$, which gives the FWHM $=2 \sqrt{2 \ln 2} \sigma$. The quality of the fits is shown in Fig. 53, which is quite good except for overlap projected data due to its broad peaks. The FWHM is used to identify further peaks which are at least in a distance of $2 \cdot \mathrm{FWHM}$ of each other. Table 5 lists the results for every lattice and $\beta_{L W}$, full and projected configurations, asqtad staggered and overlap data. For every case the maximum, its FWHM, the number of peaks higher than $1 / 2,1 / 3$ and $10 \%$ of the maximum and the average FWHM of these peaks are listed. All data seems to make sense and looks quite well except for overlap projected configurations. It is somehow interesting that 
the FWHM increases with $\beta_{L W}$. The peak height increases drastically with $\beta_{L W}$ and therefore the peaks look much sharper. But also the FWHM increases slightly and therefore the peak volume (in lattice units) increases strongly. This can also be seen very nicely from decreasing number of peaks for increasing $\beta_{L W}$.
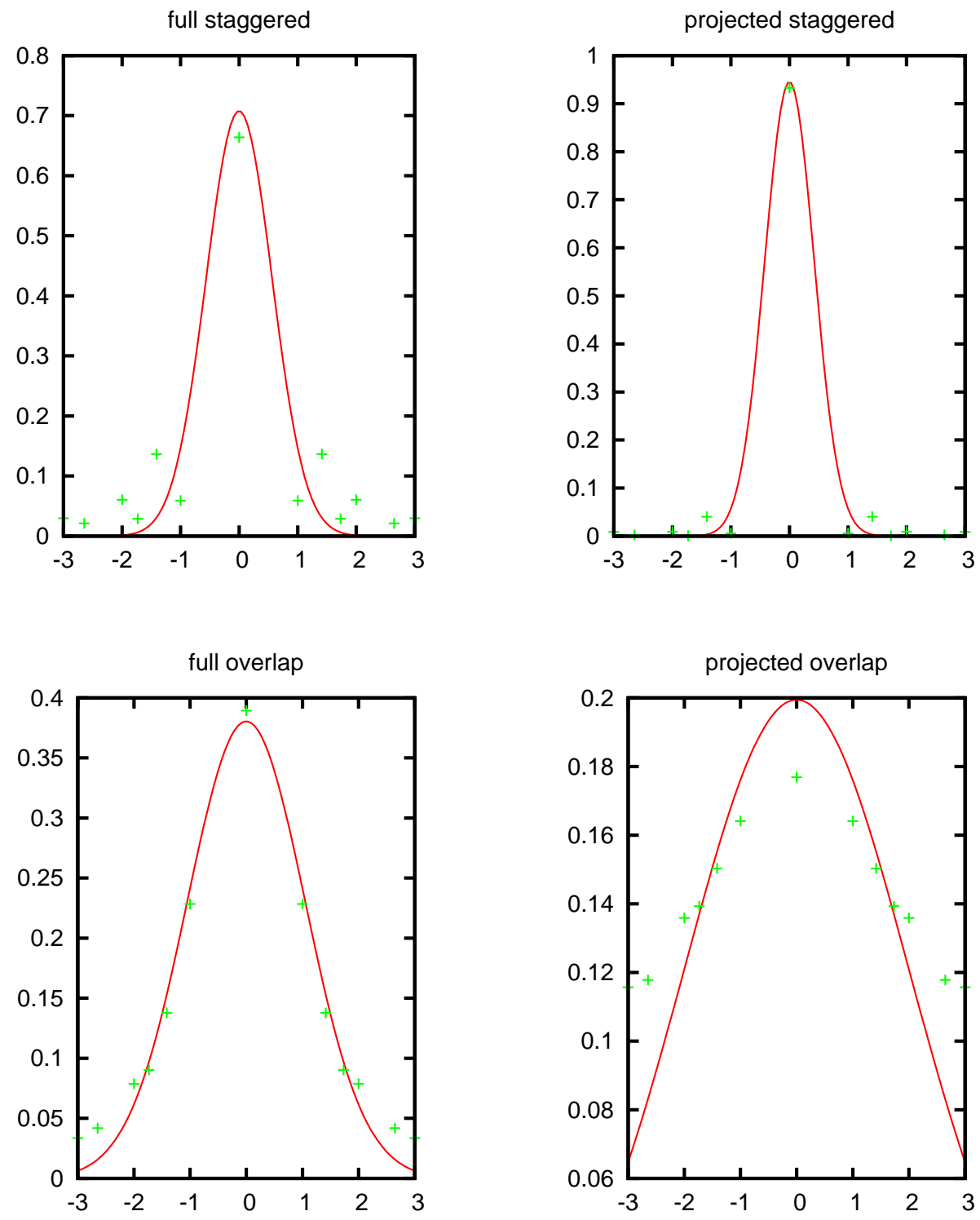

Figure 53: Quality of fits for all configurations on $16^{4}$ lattice at $\beta_{L W}=3.3$. The green crosses give the average density normalized to one (ordinate) at points around the maximum in a certain radius (abscissa), whereas the red line gives the fitted distribution function $f(x)$. 


\begin{tabular}{|c|c|c|c|c|c|c|}
\hline$L^{4}$ & $\beta_{L W}$ & peak & stag.full & projected & ovl.full & projected \\
\hline \multirow[t]{12}{*}{$12^{4}$} & 2.9 & $\max .:$ & $0.0015(1.438)$ & $0.0105(1.025)$ & $0.0014(2.534)$ & $0.0005(4.341)$ \\
\hline & & $>\max / 2:$ & $10.95(1.655)$ & $3.41(1.075)$ & $3.4(2.976)$ & $6.85(4.514)$ \\
\hline & & $>\max / 3$ & $565.46(1.664)$ & $154.32(1.078)$ & $38.1(2.99)$ & $82.85(4.54)$ \\
\hline & & $>\max / 10$ & $19260.24(1.672)$ & $5616.08(1.082)$ & $340.4(3.133)$ & $711.0(4.56)$ \\
\hline & 3.1 & $\max .:$ & $0.0019(1.542)$ & $0.0183(1.022)$ & $0.0018(2.533)$ & $0.0003(4.709)$ \\
\hline & & $>\max / 2:$ & $6.8(1.679)$ & $2.95(1.083)$ & $2.5(2.855)$ & $21.95(4.709)$ \\
\hline & & $>\max / 3$ & $356.03(1.68)$ & $137.48(1.078)$ & $27.9(2.82)$ & $258.5(4.709)$ \\
\hline & & $>\max / 10$ & $12322.24(1.684)$ & 4958.11(1.078) & $235.35(2.923)$ & $2139.15(4.709)$ \\
\hline & 3.3 & $\max .:$ & $0.0029(1.784)$ & $0.0275(1.067)$ & $0.0021(2.565)$ & $0.0002(4.709)$ \\
\hline & & $>\max / 2:$ & $3.09(1.889)$ & $2.41(1.123)$ & $2.25(2.952)$ & $51.55(4.709)$ \\
\hline & & $>\max / 3$ & $158.11(1.889)$ & $93.06(1.117)$ & $25.25(2.993)$ & $574.95(4.709)$ \\
\hline & & $>\max / 10$ & $5649.35(1.903)$ & $3278.59(1.118)$ & $202.05(3.155)$ & 4377.4(4.709) \\
\hline \multirow[t]{12}{*}{$16^{4}$} & 2.9 & max.: & $0.0006(1.38)$ & $0.0049(1.005)$ & & \\
\hline & & $>\max / 2:$ & $14.42(1.555)$ & $4.3(1.052)$ & & \\
\hline & & $>\max / 3$ & $747.4(1.562)$ & $210.82(1.055)$ & & \\
\hline & & $>\max / 10$ & 26199.61(1.58) & $7350.42(1.058)$ & & \\
\hline & 3.1 & max.: & $0.0009(1.435)$ & $0.0096(1.012)$ & & \\
\hline & & $>\max / 2:$ & $10.11(1.583)$ & $3.28(1.065)$ & & \\
\hline & & $>\max / 3:$ & $527.61(1.588)$ & $144.4(1.066)$ & & \\
\hline & & $>\max / 10$ & $18340.62(1.597)$ & $4635.4(1.068)$ & & \\
\hline & 3.3 & $\max .:$ & $0.0015(1.707)$ & $0.0195(1.033)$ & $0.0019(2.389)$ & $0.0001(4.709)$ \\
\hline & & $>\max / 2:$ & $4.68(1.782)$ & $2.47(1.08)$ & $1.45(2.686)$ & $71.15(4.709)$ \\
\hline & & $>\max / 3:$ & $260.63(1.796)$ & $90.7(1.082)$ & $17.6(2.739)$ & $874.7(4.709)$ \\
\hline & & $>\max / 10$ & $9673.35(1.811)$ & $3034.79(1.086)$ & $154.85(2.91)$ & $7230.0(4.709)$ \\
\hline \multirow[t]{12}{*}{$20^{4}$} & 2.9 & $\max .:$ & $0.0003(1.304)$ & $0.0023(0.989)$ & & \\
\hline & & $>\max / 2:$ & $17.17(1.476)$ & $5.0(1.033)$ & & \\
\hline & & $>\max / 3$ & $927.22(1.488)$ & $245.91(1.035)$ & & \\
\hline & & $>\max / 10$ & $33150.75(1.515)$ & $8692.08(1.039)$ & & \\
\hline & 3.1 & $\max .:$ & $0.0005(1.383)$ & $0.0054(1.007)$ & & \\
\hline & & $>\max / 2:$ & $10.38(1.507)$ & $3.59(1.037)$ & & \\
\hline & & $>\max / 3$ & $555.9(1.519)$ & $170.37(1.034)$ & & \\
\hline & & $>\max / 10$ & $19756.87(1.534)$ & $5757.93(1.034)$ & & \\
\hline & 3.3 & max.: & $0.0008(1.536)$ & $0.0135(1.001)$ & & \\
\hline & & $>\max / 2:$ & $6.95(1.636)$ & $2.59(1.041)$ & & \\
\hline & & $>\max / 3$ & $371.67(1.642)$ & $105.77(1.045)$ & & \\
\hline & & $>\max / 10$ & $13351.86(1.647)$ & $3516.07(1.05)$ & & \\
\hline
\end{tabular}

Table 5: Peak Analysis Results for staggered and overlap fermions: maximum peak height, its FWHM enclosed in brackets, number of peaks higher than $1 / 2$, $1 / 3$ and $1 / 10$ of maximum peak and corresponding averaged FWHM 
The results roughly confirm conclusions and assumptions in the previous section. First, the number of peaks within the 10\%-barrier isn't linearly rising with lattice volume but it rises proportionally to the IPR data. Secondly, the peaks for projected configurations are much higher and sharper than all the others and third, the peaks found for eigenmodes of the central band in vortex-removed data are only small maxima in a noisy background while for the 9th and higher eigenmodes the data roughly look like those for full or even projected configurations again. In the following section correlations between the density of Dirac modes and vortices resp. topological charge determined from vortices are measured.

\subsection{Correlation between vortices and Dirac modes}

In order to clarify the role of the vortices in the topological structure of the vacuum, the correlator $C_{\lambda}$ between the density of the eigenmode $\lambda$ and the vortex surface is investigated. The correlator depends on the eigenvalue and on the local geometry of the vortex. The vortex points $P_{i}$ live on the dual lattice and they are correlated to the averaged scalar eigenmode density $\rho_{\lambda}(x)$ over the vertices $x$ of the 4 d hypercube, $H$, dual to $P_{i}$. [74]

$$
C_{\lambda}=\frac{\sum_{P_{i}} \sum_{x \in H}\left(V \rho_{\lambda}(x)-\left\langle V \rho_{\lambda}(x)\right\rangle\right.}{\sum_{P_{i}} \sum_{x \in H} 1}
$$

This correlator strongly depends on the number of the vortex plaquettes, attached to a point $P_{i}$. Numerical results for asqtad staggered and overlap eigenmodes are shown in Figs. 54 and 55 respectively. The more vortex plaquettes are attached to a point $P_{i}$, the larger the correlator gets in general. Fig. 54a shows the correlations for full configurations, for more than ten plaquettes attached to a point $P_{i}$ the error bars are very large because of low statistics. For zero attached plaquettes by a trivial reason the correlator gets even negative, one and two plaquettes do not give any contribution since at least three vortex plaquettes are necessary to attach one point in order to form a closed vortex surface. The correlation for projected data is much higher (Fig. 54b) since the vortex surface is determined from the P-vortices. There is not much difference between first and twentieth mode for asqtad staggered data. For full overlap data the correlation is smaller and decreasing for higher modes (Fig. 54a), in agreement with the general picture that vortices are related to chiral symmetry breaking, which is due to the low lying eigenmodes. The overlap eigenmodes for center-projected configurations show an anti correlation, the modes do not peak at vortex structures (Fig. 54b).

Determining the topological charge after [46] and again using the same correlator of equation (27), where $P_{i}$ now denotes the point with topological charge $c$, gives the correlation of vortices and topological charge. The results are presented in Figs. 56 and 57 for asqtad staggered and overlap eigenmodes respectively. Again, there are nice correlations except for overlap projected data, but errors are very big. Nevertheless, the results of this section provide a modest degree of evidence in support of a vortex origin of topological charge density. There is also the possibility, of course, that topological charge density may come from more than one type of source. 
a)

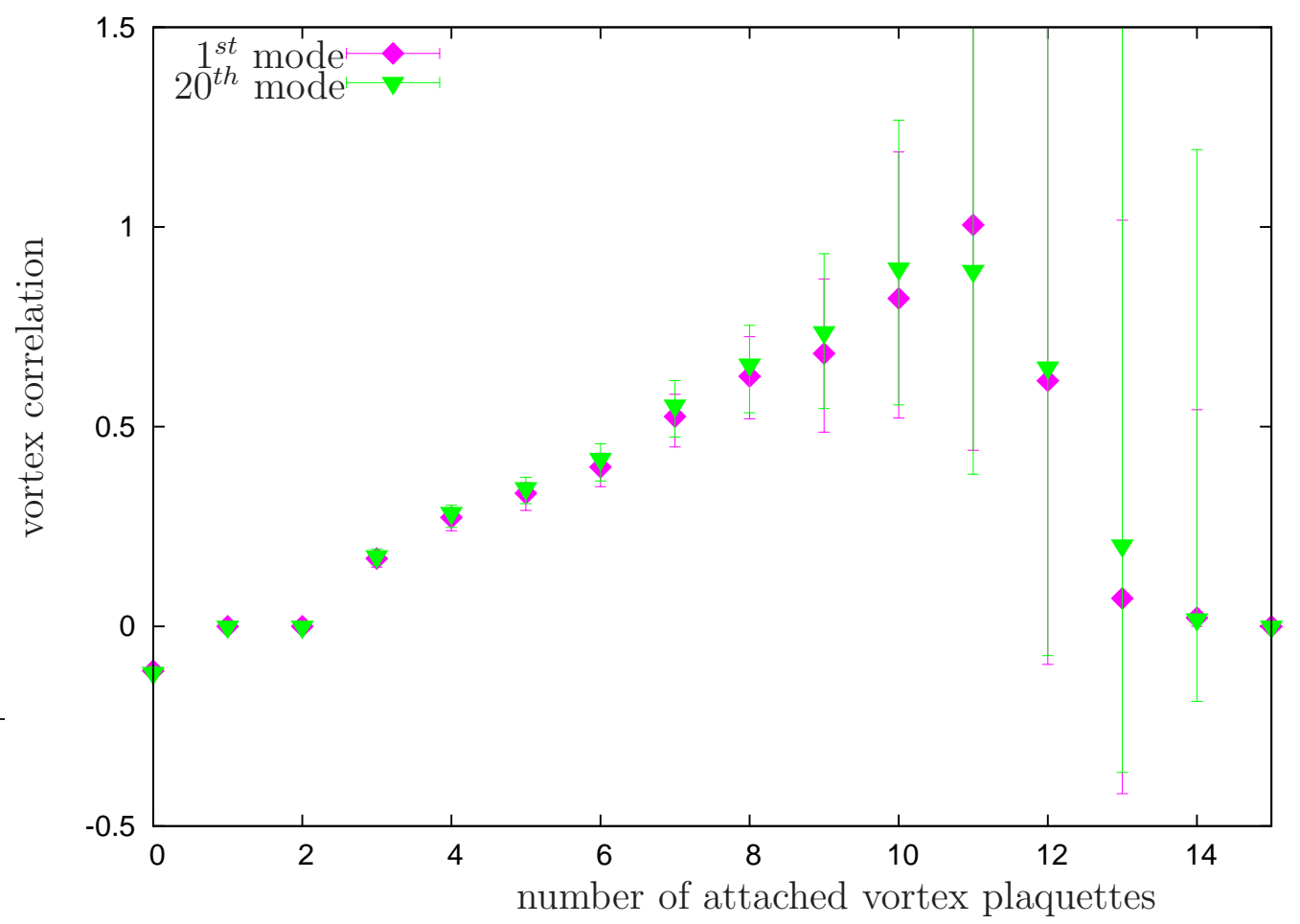

b)

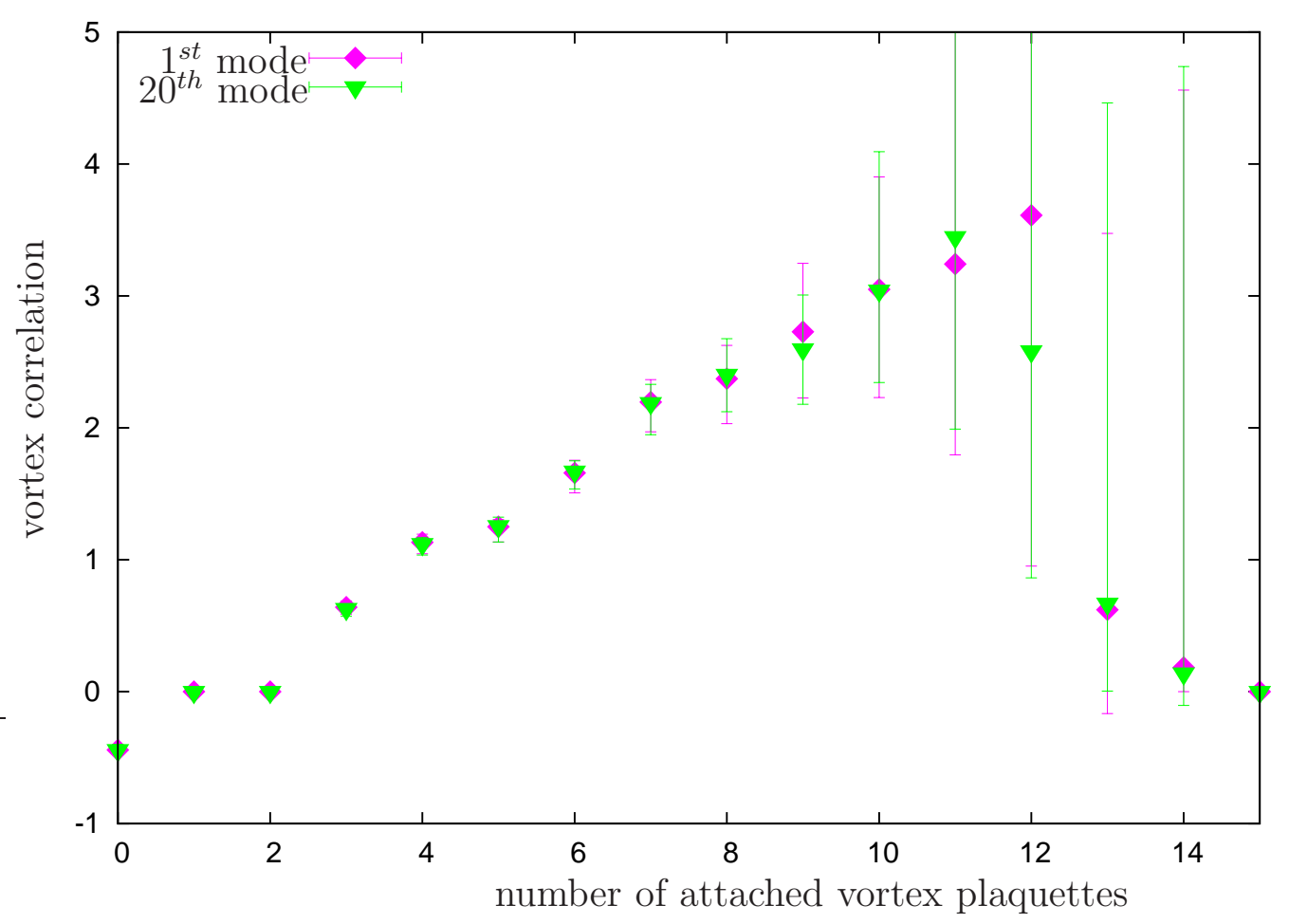

Figure 54: Vortex Correlation for asqtad staggered eigenmodes on a $20^{4}$ lattice at $\beta_{L W}=$ 3.3 , a) full and b) center-projected configuration. 
a)

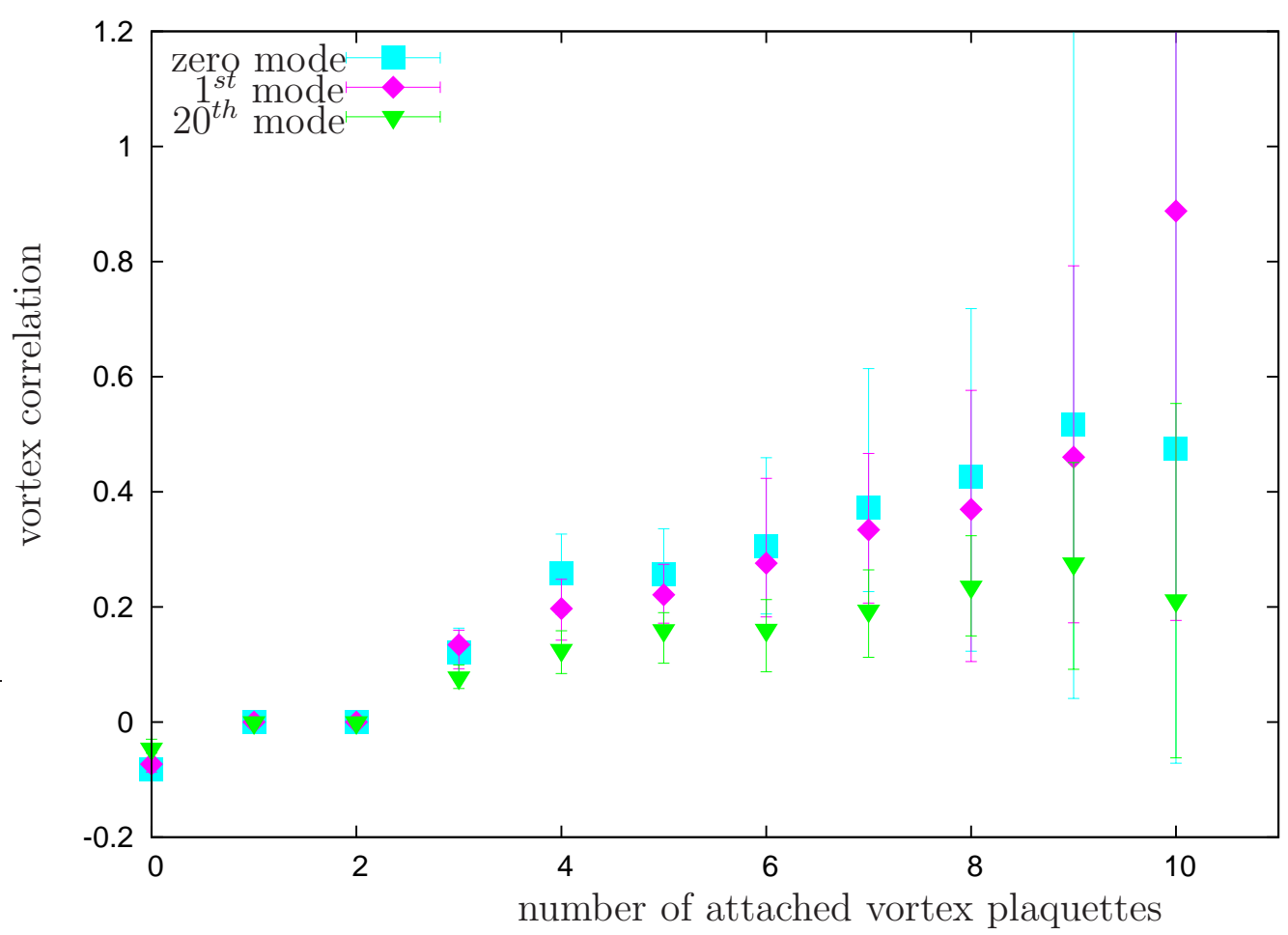

b)

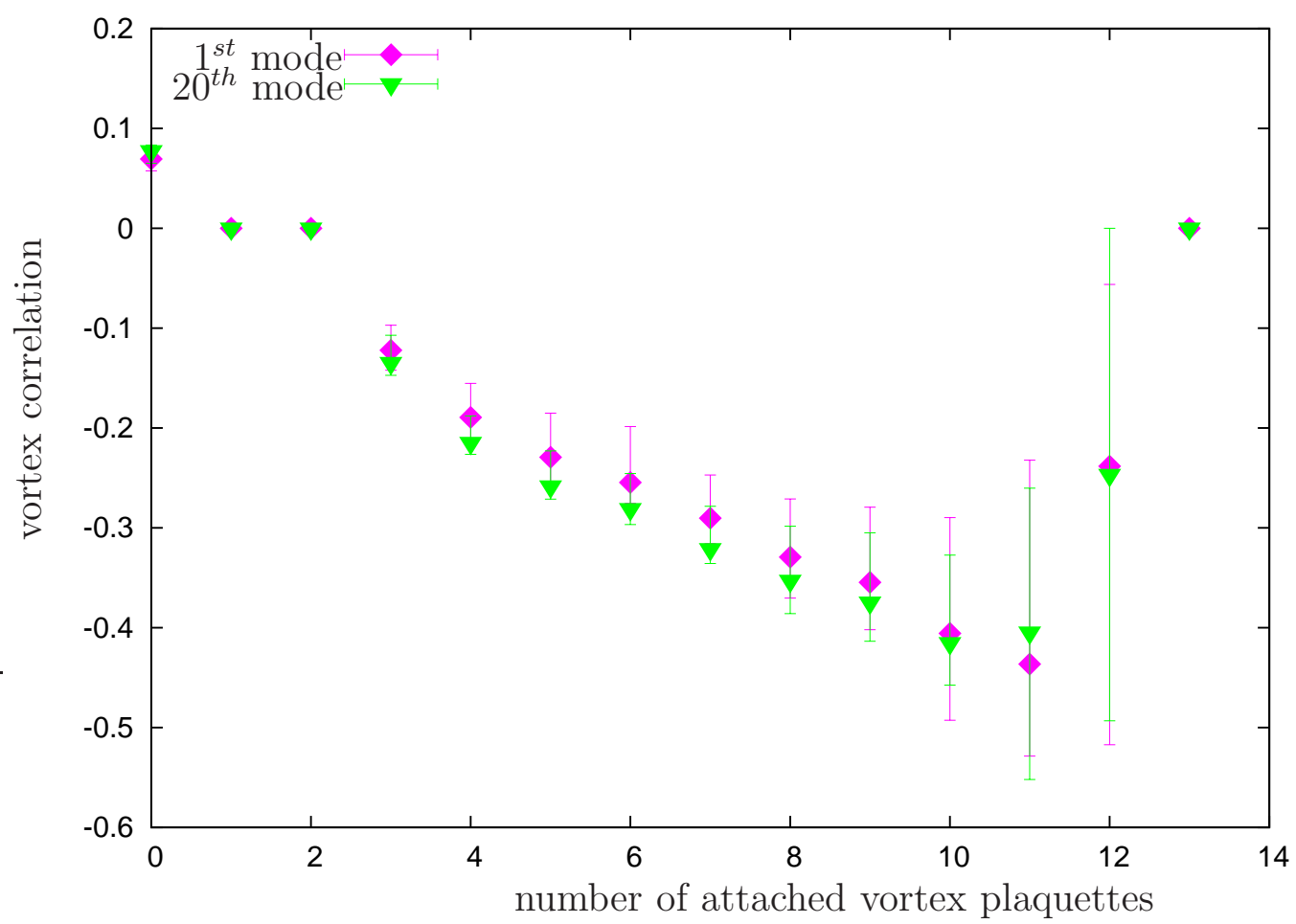

Figure 55: Vortex Correlation for overlap eigenmodes on a $16^{4}$ lattice at $\beta_{L W}=3.3$, a) full and b) center-projected configuration. 
a)

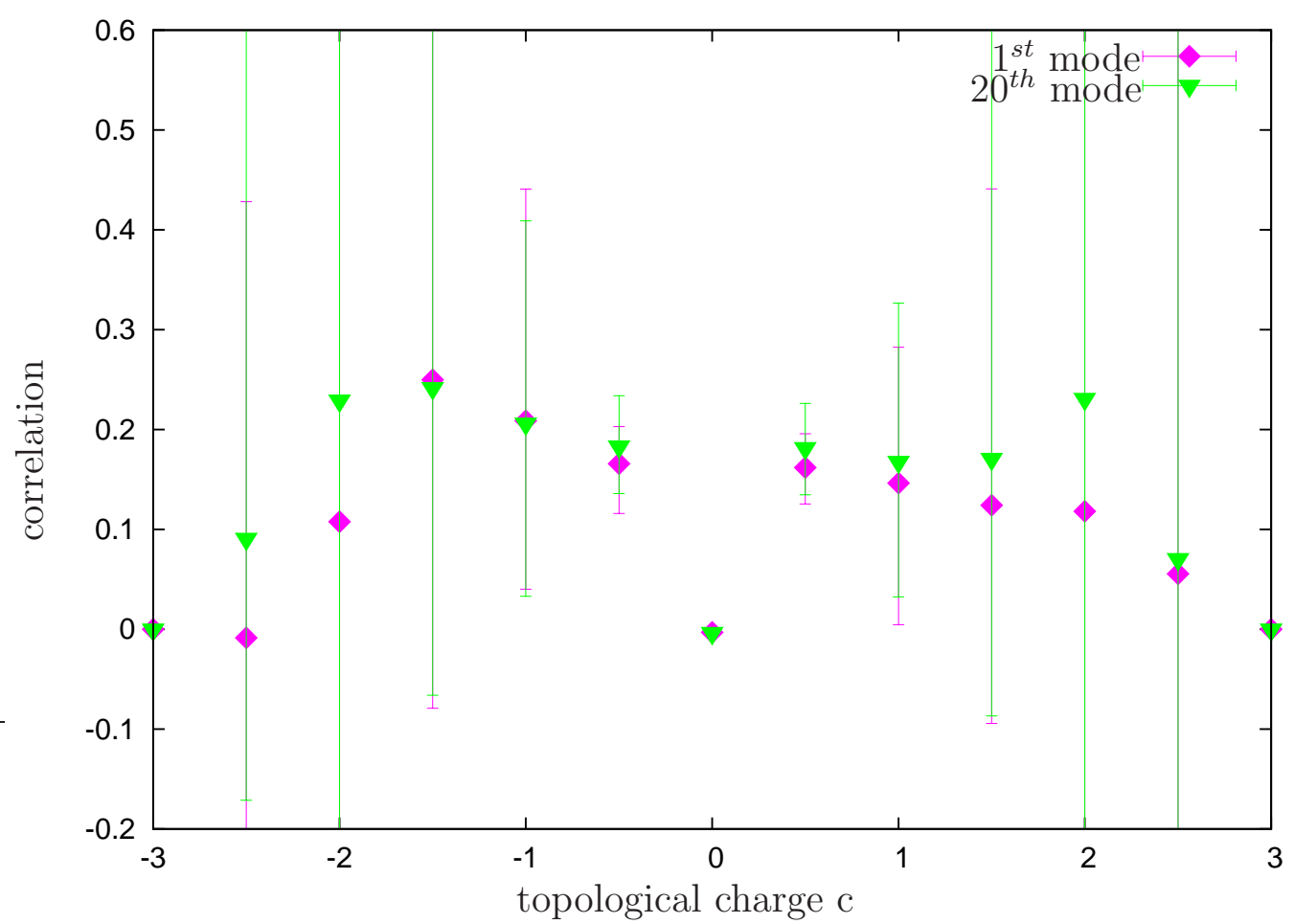

b)

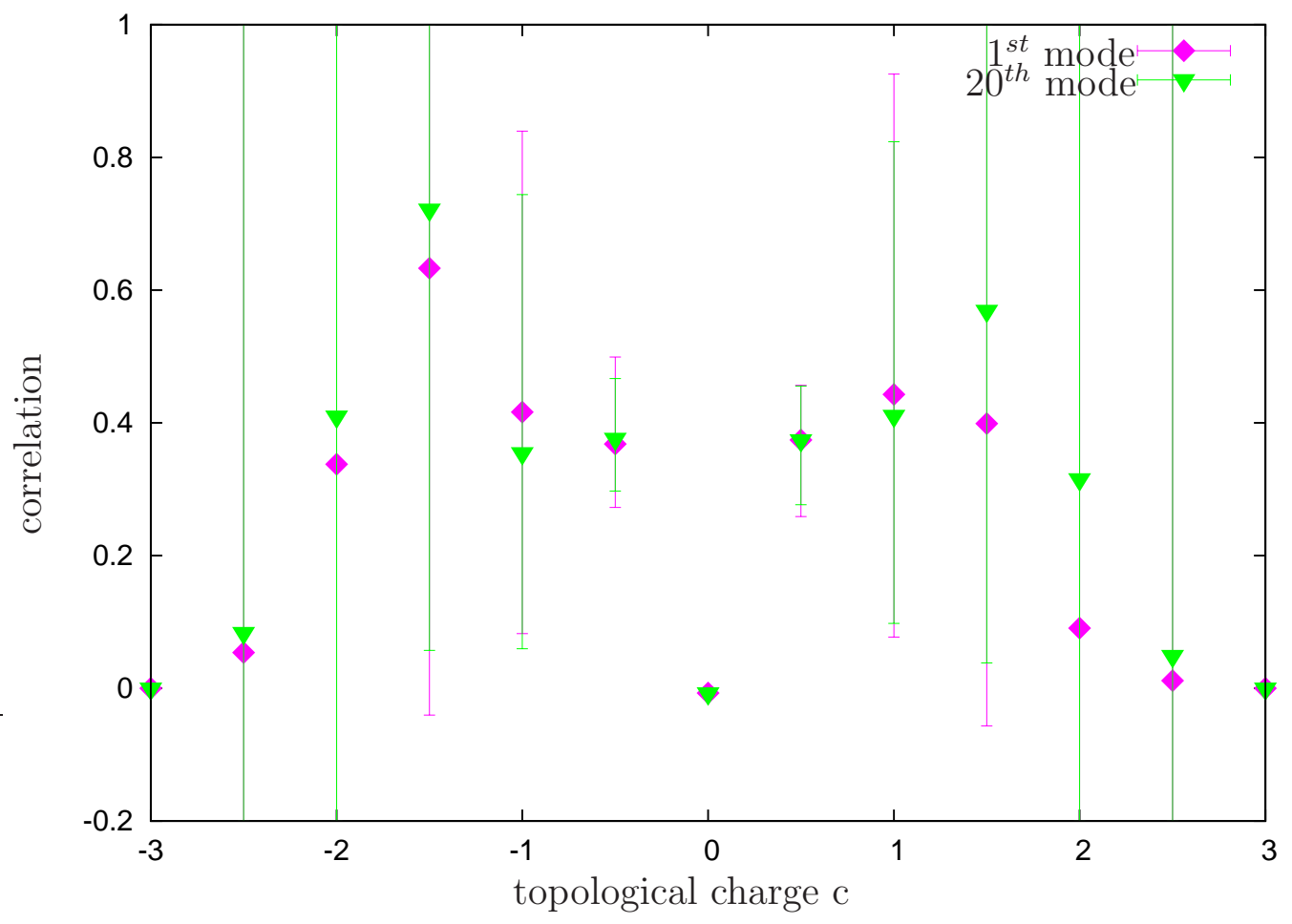

Figure 56: Correlation between topological charge and asqtad staggered eigenmodes on a $20^{4}$ lattice at $\beta_{L W}=3.3$, a) full and b) center-projected configuration. 
a)

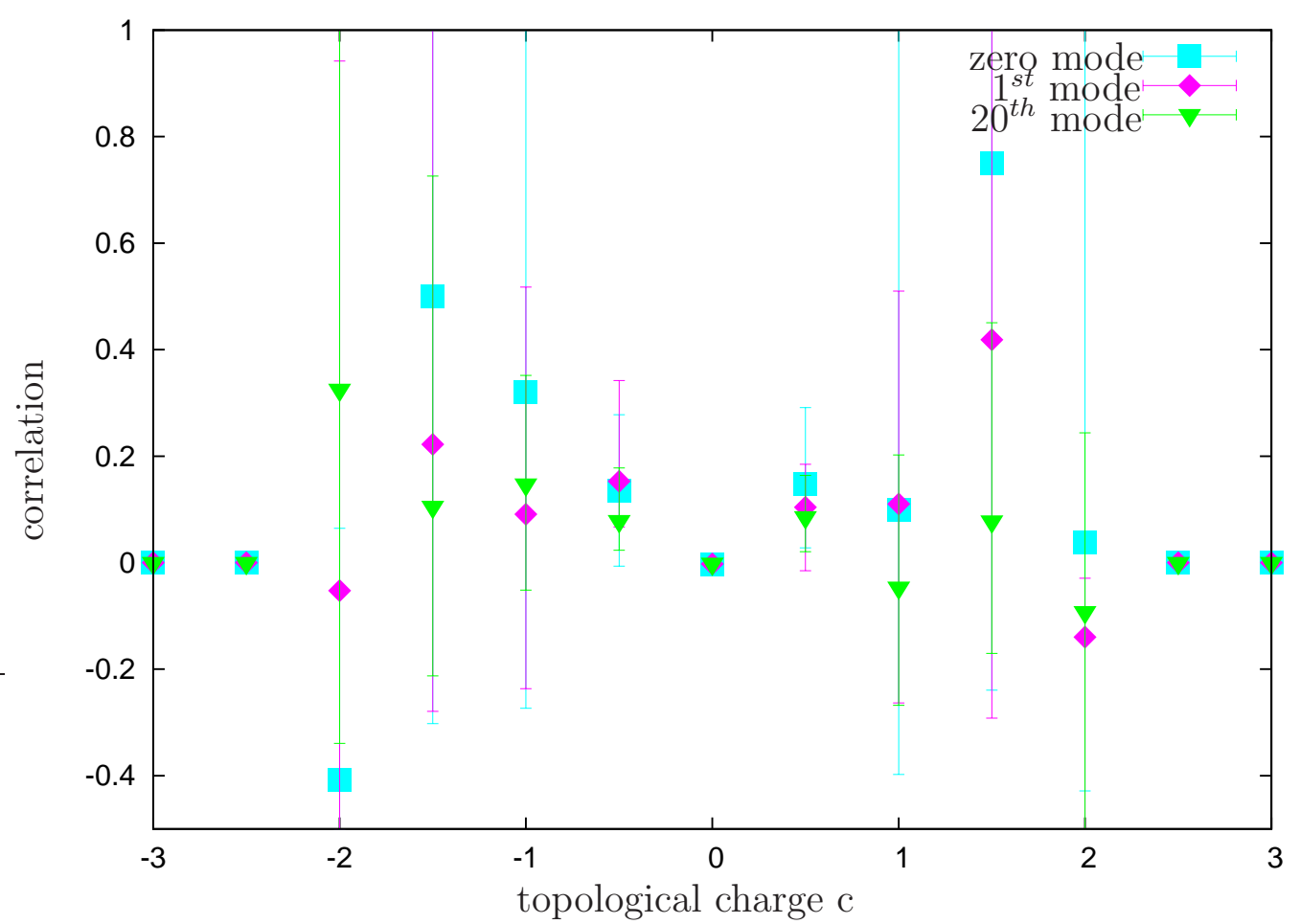

b)

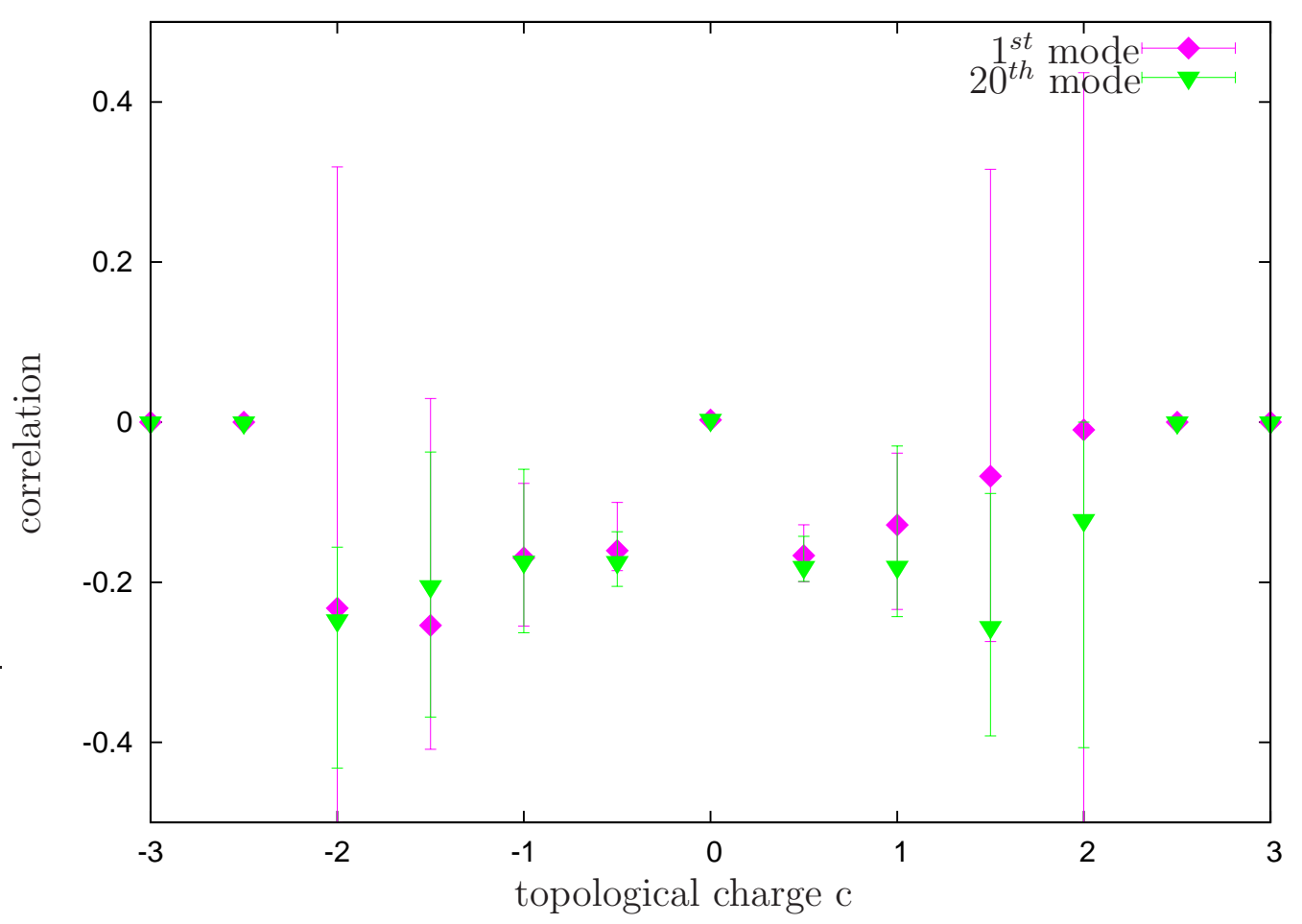

Figure 57: Correlation between topological charge and overlap eigenmodes on a $16^{4}$ lattice at $\beta_{L W}=3.3$, a) full and b) center-projected configuration. 


\subsubsection{Correlations and partial projection}

In order to analyze where the strong anti correlation of overlap modes and P-vortices in center-projected configurations comes from, again the interpolation between full and center-projected lattices is performed, as described in section 5.1. The vector of the full $S U(2)$ element $U_{\mu}(x)$ is partially turned into the corresponding $S U(2)$ center element $Z_{\mu}(x)= \pm 1$. Determining the vortex structure and overlap modes of these interpolated fields and again calculating their correlation from equation 27 gives an interesting result. Fig. 58 shows that full and $25 \%$ projected modes correlate quite well with the corresponding vortex surfaces, but rather quickly vanishes and for $50 \%$ projection already the anti correlation dominates. This suggests two interpretations: First there is again the reasoning that the overlap Dirac operator has some troubles with rather unsmooth partially projected configurations or secondly the vortex structure determined on center-projected fields is quite different from partially projected configurations. Since eigenvalue spectra on partially projected configurations seemed pretty reasonable even for $85 \%$ projection, the second reasoning seems preferable.

\subsubsection{Correlations and chiral densities}

Next, the vortex correlations shall be determined for different eigenmode densities. When up to now the scalar density

$$
\rho_{0}(x)=\sum_{c, d}\left|\vec{v}(x)_{c d}\right|^{2}
$$

of the eigenvector $\vec{v}(x)_{c d}$ with color and Dirac indices, was used, the chiral densities $\rho_{5}(x), \rho_{+}(x)$ (left-handed) and $\rho_{-}(x)$ (right-handed), defined as

$$
\begin{gathered}
\rho_{5}(x)=\sum_{c, d, d^{\prime}} \vec{v}(x)_{c d}^{*} \gamma_{5}^{c, d^{\prime}} \vec{v}(x)_{c d^{\prime}} \\
\rho_{ \pm}(x)=\sum_{c, d, d^{\prime}} \vec{v}(x)_{c d}^{*} \frac{1 \pm \gamma_{5}^{c, d^{\prime}}}{2} \vec{v}(x)_{c d^{\prime}}
\end{gathered}
$$

shall be considered next. The results show up in Fig. 59 for first and $20^{\text {th }}$ overlap eigenmodes together with the previous results for the scalar density. By definition, the correlations of left- and right-handed chiral density average to the scalar density correlation, whereas the chiral density $\rho_{5}(x)$ gives even higher correlation. This increasing of correlation has its origin, of course, in higher chiral density because of removal of any background noise density of the eigenmodes, but also gives a first hint that the peaks of left- and right-handed modes appear at different positions. These peaks shall be analyzed in section 5.6 in more detail. 
a)

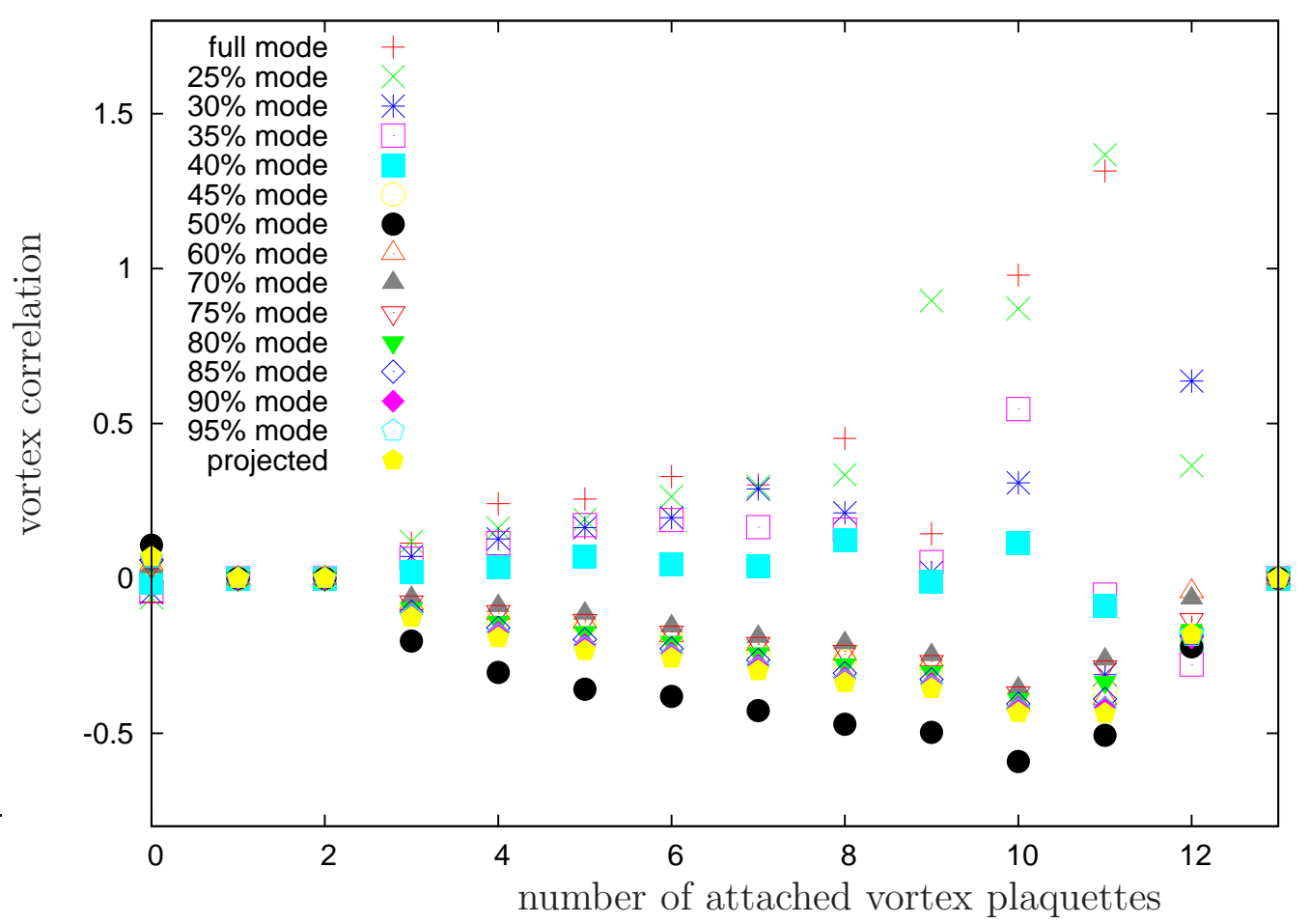

b)

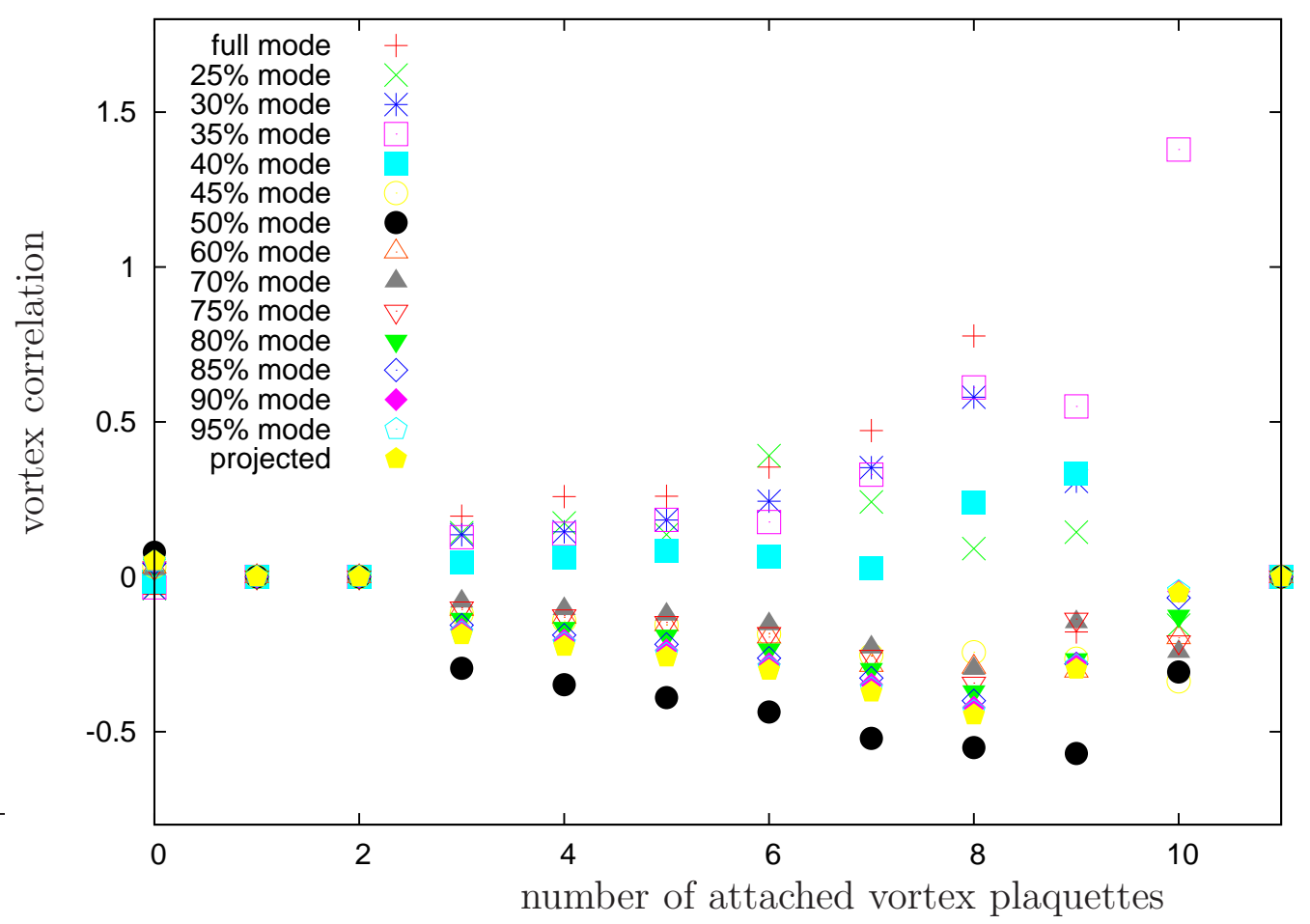

Figure 58: Correlation of overlap eigenmodes and a) original or b) smoothed vortex surface of partially projected configurations on a $16^{4}$-lattice at $\beta_{L W}=3.3$. 
a)

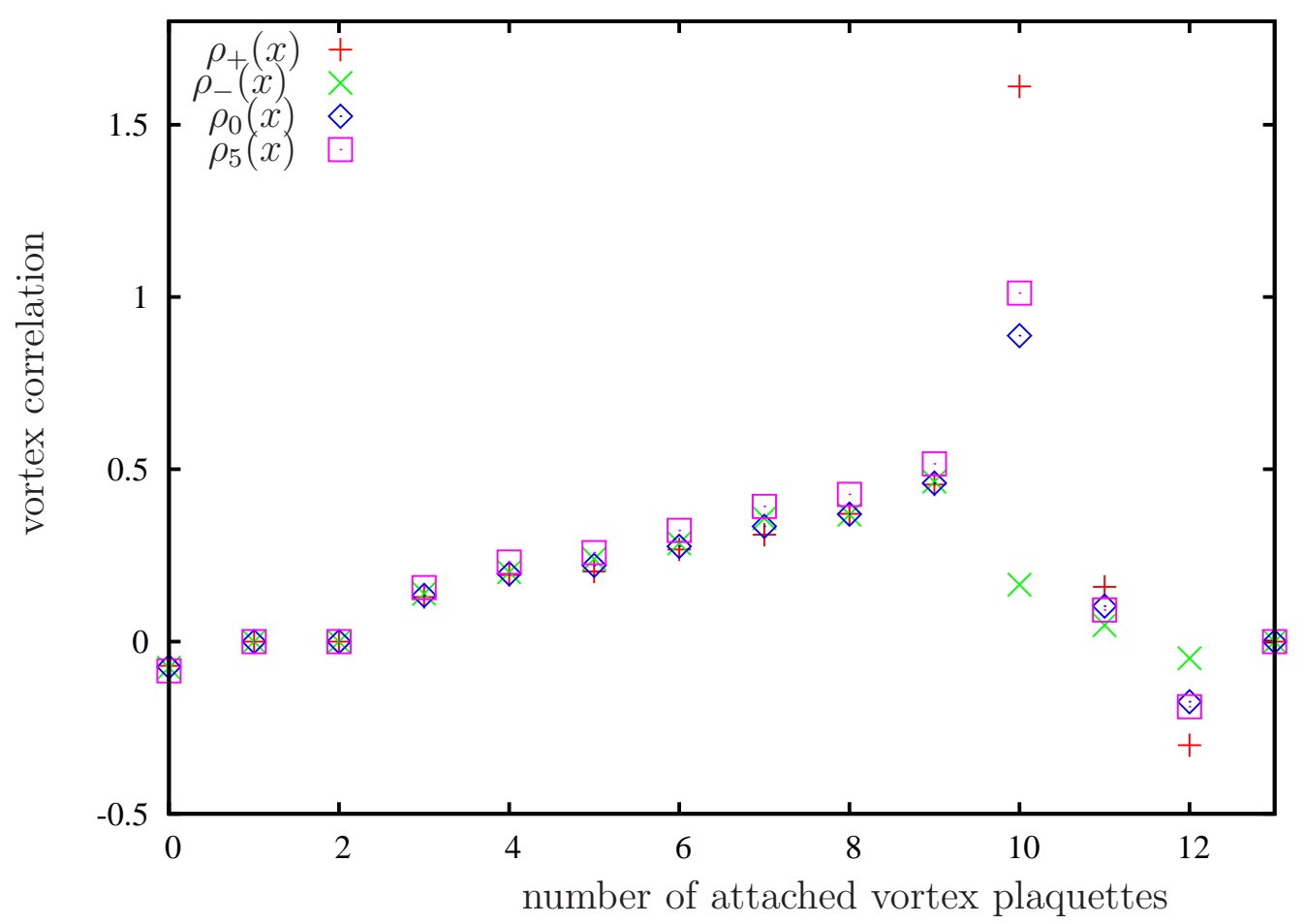

b)

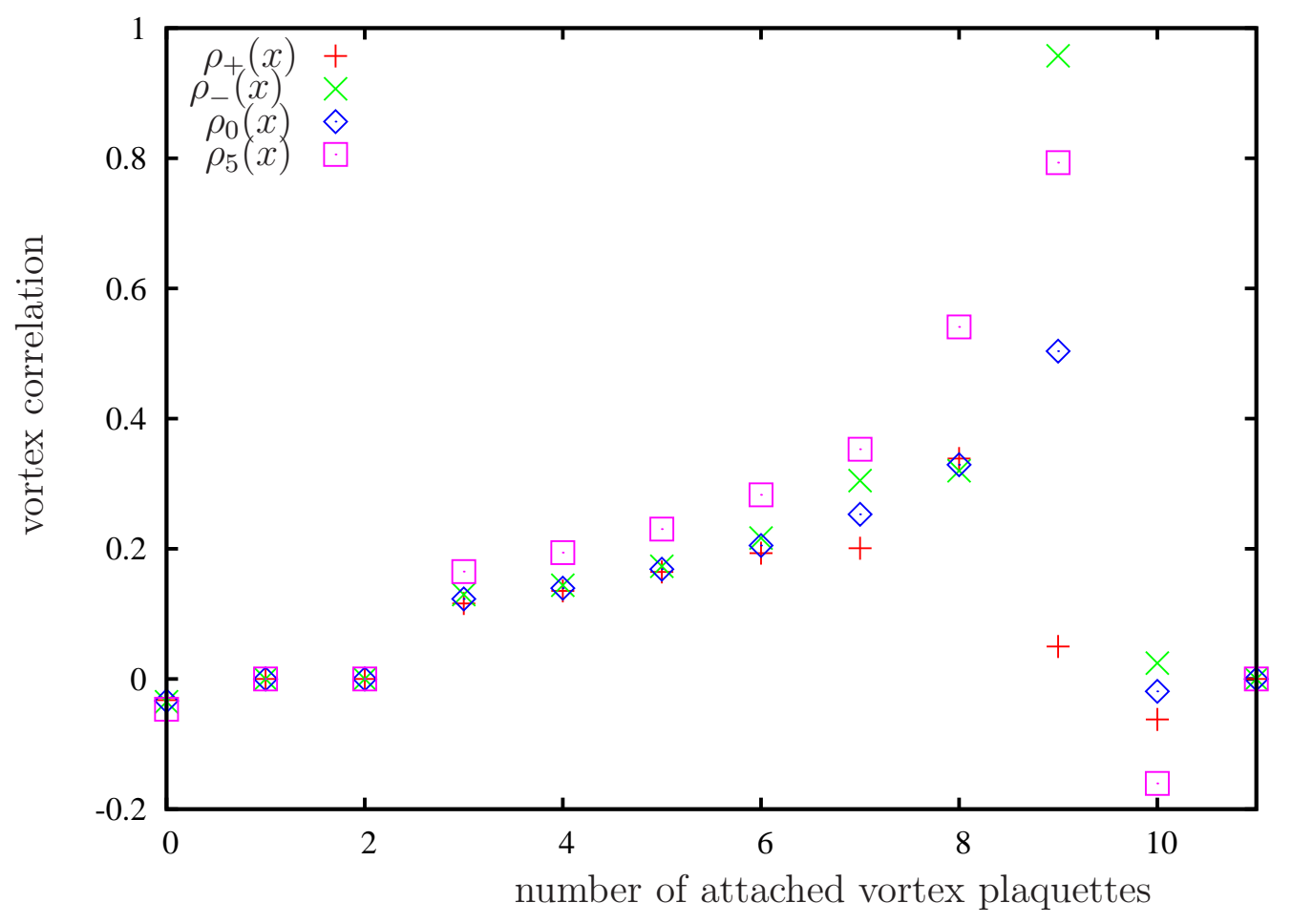

Figure 59: Vortex Correlation for a) first and b) $20^{\text {th }}$ overlap eigenmode on a $16^{4}$-lattice at $\beta_{L W}=3.3$, using scalar $\left(\rho_{0}(x)\right)$, chiral $\left(\rho_{5}(x)\right)$ and right-/left-handed $\left(\rho_{ \pm}(x)\right)$ eigenmode densities. 


\subsubsection{Correlations with partial vortex structures}

In this section, once again the correlator $C_{\lambda}(27)$ between Dirac eigenmodes and vortex structures is analyzed. First, the correlations of overlap and asqtad staggered eigenmodes to only E- ("electric", space-time-) or B- ("magnetic", space-space-) plaquettes of the vortex surface shall be analyzed. Taking only one type of plaquettes at single vortex points also allows to get correlations to vortex points with only one B-plaquette, or two plaquettes of a certain type attached. This causes no problems since the vortex surface is closed at these points by the other type of plaquettes. On the other hand it is not possible to form a closed surface with an odd number of E-plaquettes (space-time-plaquettes) attached to a vortex point and therefore only even numbers contribute to the correlation. But apart from that fact, there is not much difference between the two correlations, at least for overlap eigenmodes (Fig. 60) on full configurations. The error bars are rather big (doubled, compared to full vortex structure correlations) since the statistics decreased when dividing the vortex structure into E- and B-plaquettes. For asqtad staggered modes it seems that the correlations are slightly different, the correlation to vortex points with six and eight B-plaquettes attached is clearly lower, it seems that the correlation to Eplaquettes at these points balances the overall correlation-loss due to the fact that there is no correlation to an odd number of E-plaquettes attached. The E- and B-plaquette vortex correlations to asqtad staggered eigenmodes are presented in Fig. 61.

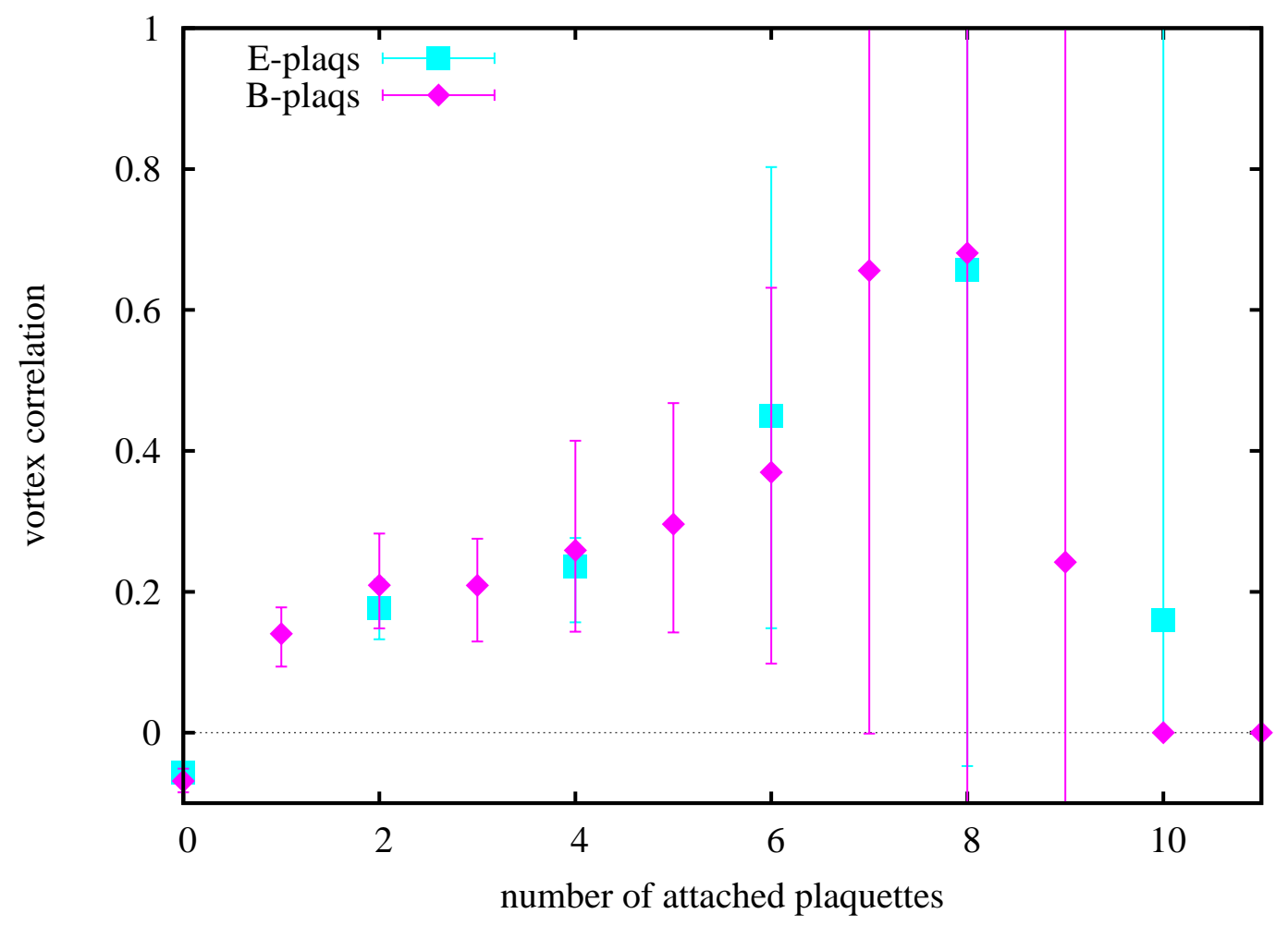

Figure 60: Correlation between eigenmode density and E- respectively B-plaquettes of full configurations at $\beta_{L W}=3.3$ on $16^{4}$ lattices. 
a)

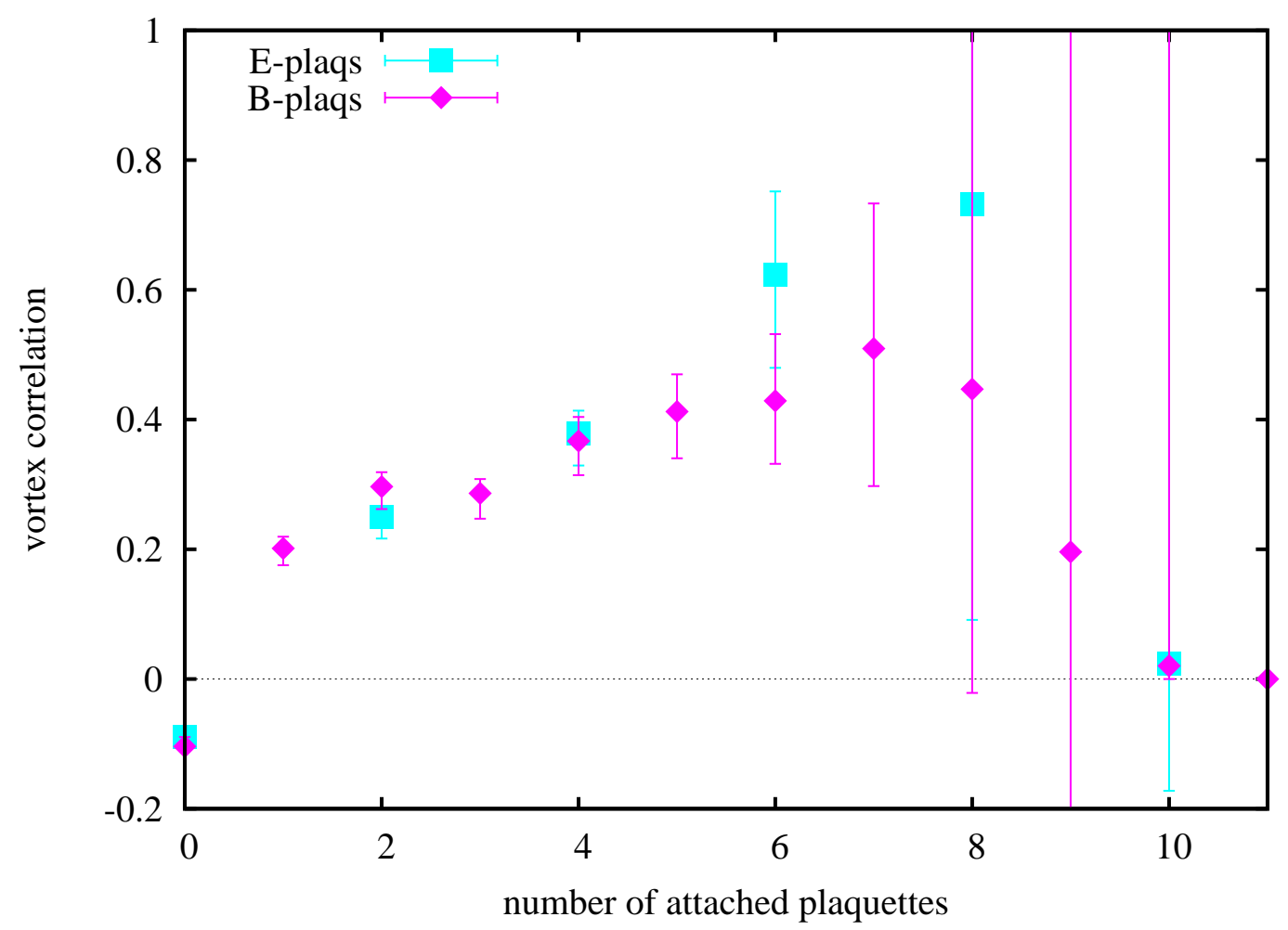

b)

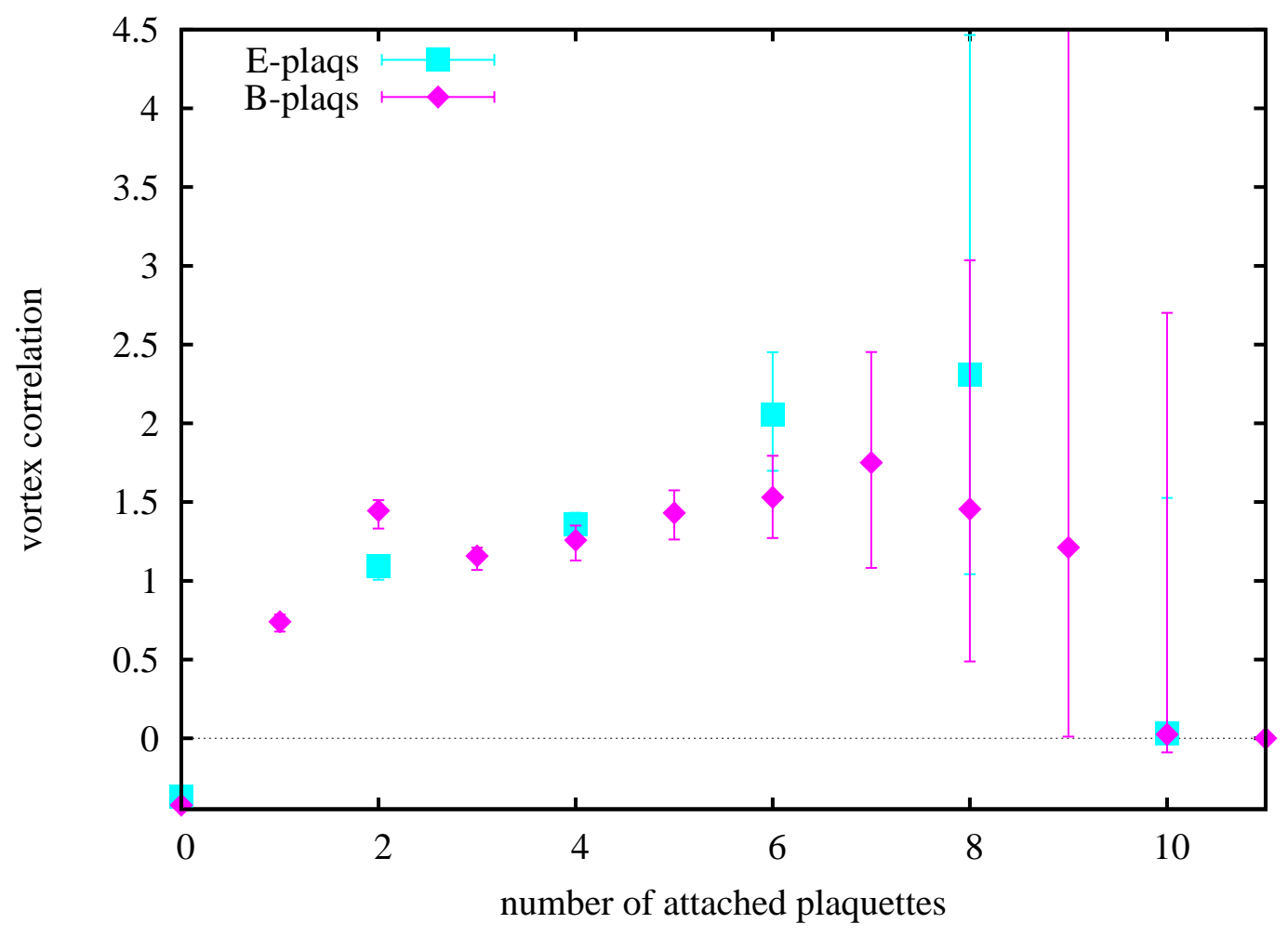

Figure 61: Correlation between eigenmode density and E- respectively B-plaquettes of a) full and b) projected configurations at $\beta_{L W}=3.3$ on $20^{4}$ lattices. 
Next, the smoothing procedure of rough vortex structures is performed, and its effect on the vortex-eigenmode correlations analyzed. This procedure is explained in App. A.4.2 and what it mainly does, is to remove so called vortex fluctuations, e.g. single negative links in the center-projected gauge field. Since smoothing the vortex surface also reduces the number of vortex points, statistics decrease and error bars increase, especially for overlap modes, where the number of configuration is already lower than for asqtad staggered modes. In Fig. 64 the correlation of overlap modes with the rough and smooth vortex structure is shown. There is not much difference between the two correlations for vortex points with up to seven plaquettes attached. Above the signal is too low to give a trust-able analyze. For asqtad staggered modes, Fig. 65, one finds a breakdown of correlation for vortex points with many vortex plaquettes attached, since these points are removed in the smoothing procedure. Especially for center-projected configurations it seems that the correlation loss for points with more vortex points attached reappears at points with less attached vortex plaquettes. The correlation growth for the smoothed vortex structure can also be seen for overlap eigenmodes and the similarity between both fermion formulations on full configurations shall be stressed.

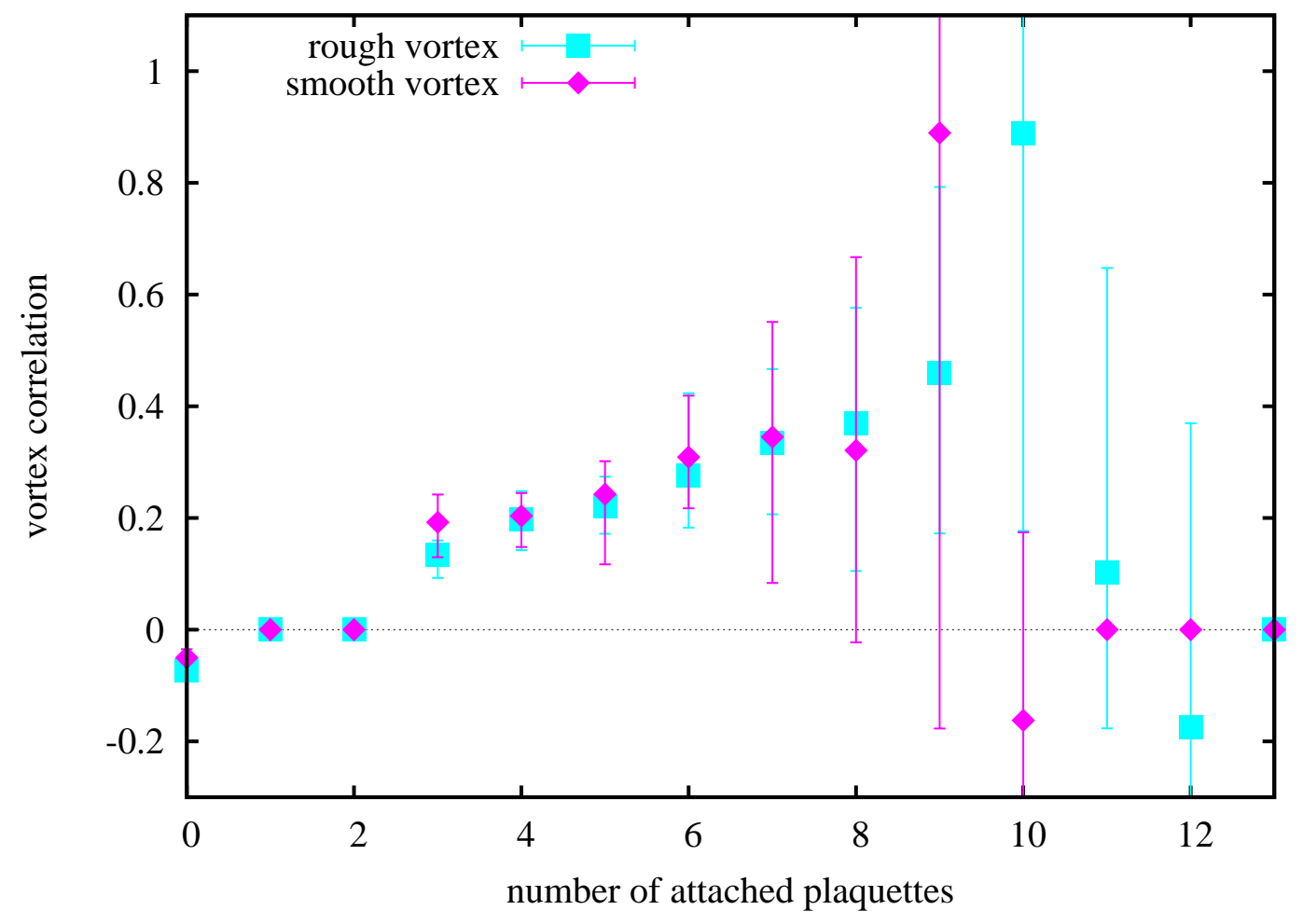

Figure 62: Correlation between overlap eigenmode density and rough respectively smooth vortex structure of full configurations at $\beta_{L W}=3.3$ on $16^{4}$ lattices.

Finally, for each configuration one big main vortex cluster, containing about $95 \%$ of the vortex plaquettes which form one big closed surface, is identified in the vortex structure. The eigenmode densities can be correlated to the main cluster or the rest of the vortex 
a)

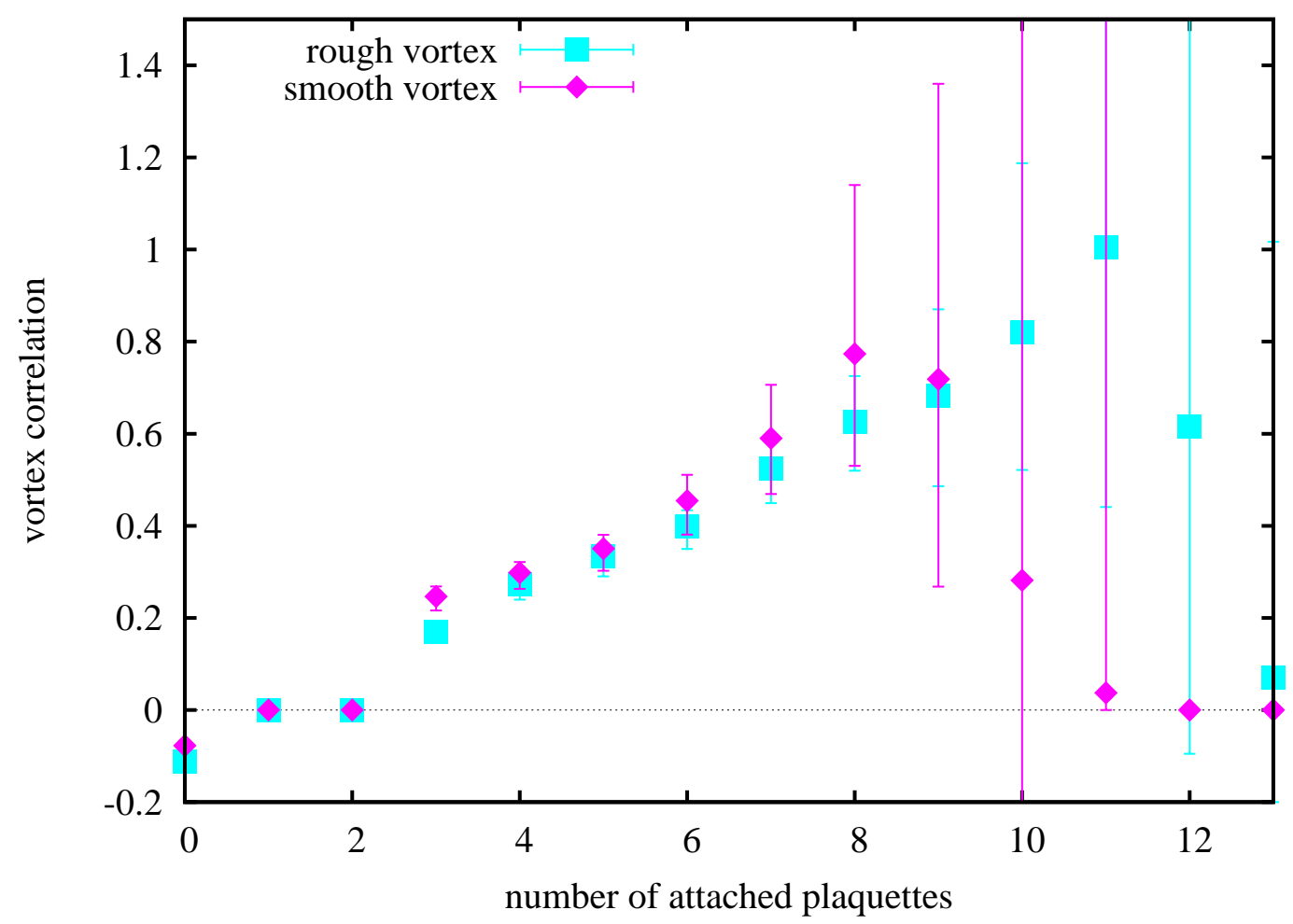

b)

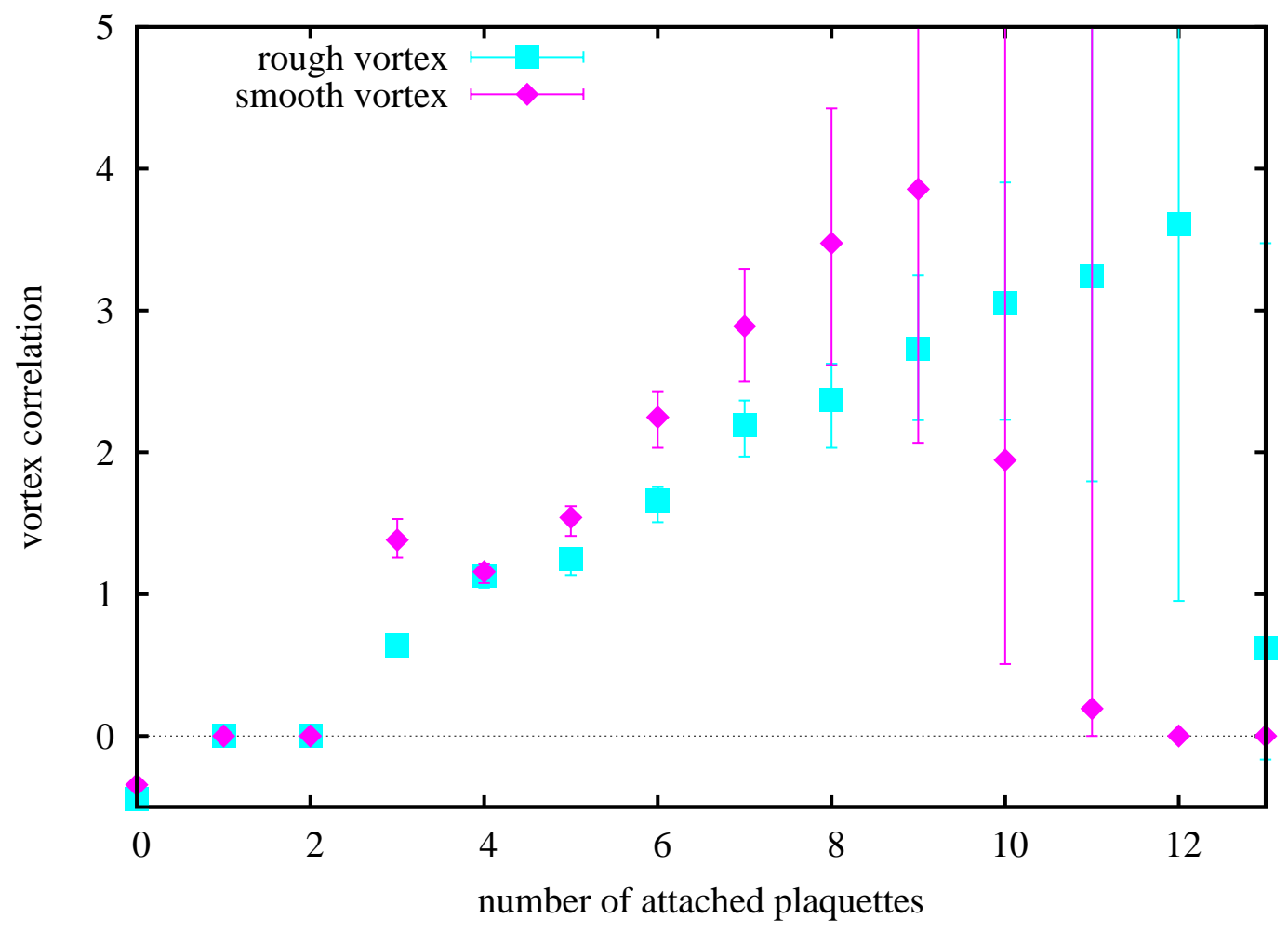

Figure 63: Correlation between eigenmode density and rough respectively smooth vortex structure of a) full and b) center-projected configurations at $\beta_{L W}=3.3$ on $20^{4}$ lattices. 
structure (small fluctuations, e.g. single negative links, forming vortices closed around one hypercube, which are removed during vortex smoothing). Again, in Fig. 64 the correlation signal of the small fluctuations is very noisy, but one can see that most of the correlation is due to the main vortex structure of course, by comparing with Fig. 55 for example. Concerning asqtad staggered eigenmodes (Fig. 65) on full and centerprojected configurations the correlation behaves similar, but there is a better signal of correlation to the vortex fluctuations. Especially for center-projected configurations a good correlation of asqtad staggered modes to vortex fluctuation points with four, which might form closed vortex hypercubes, and more attached plaquettes can be seen in Fig. 65b.

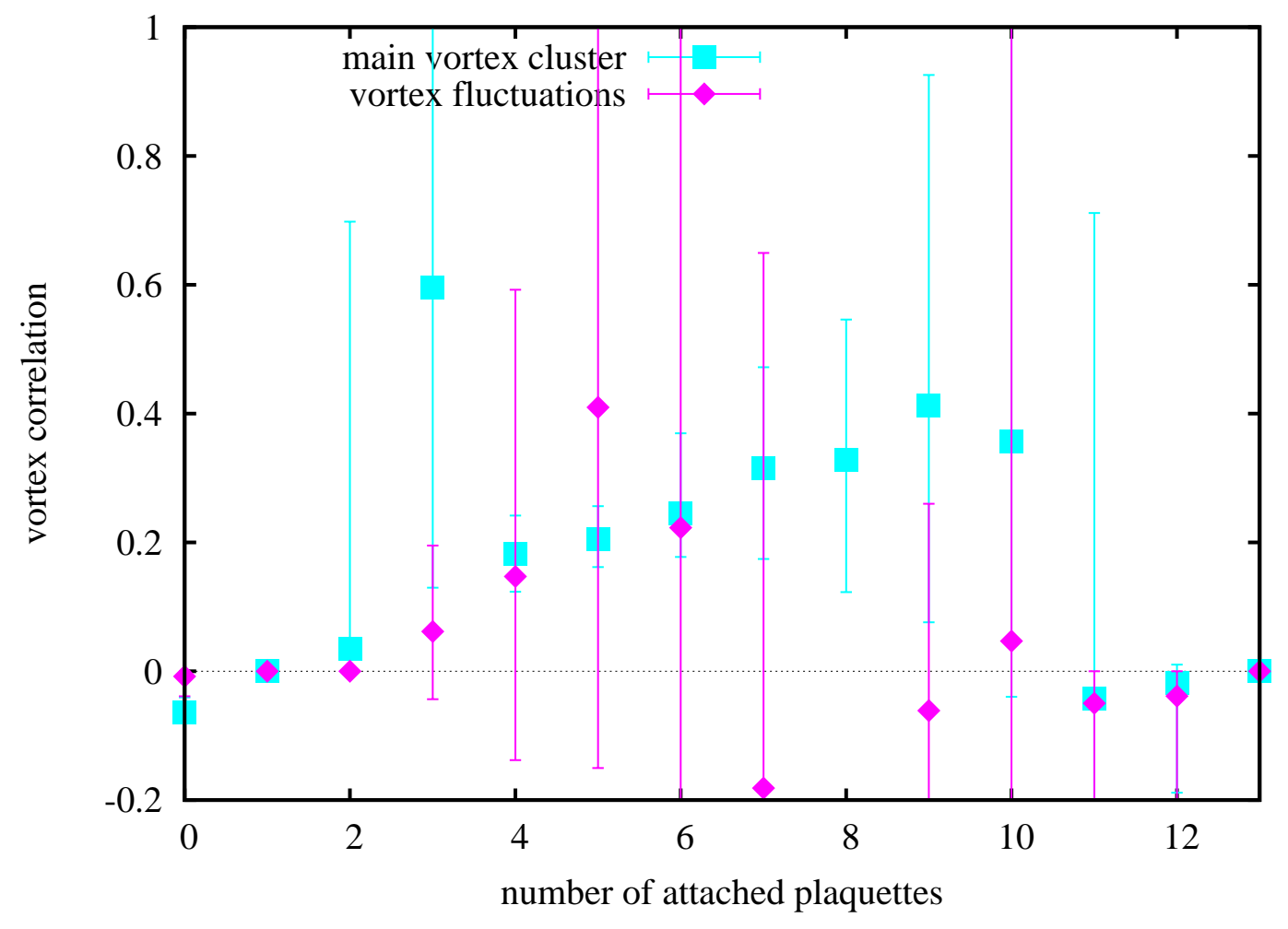

Figure 64: Correlation between eigenmode density and the main vortex cluster or vortex fluctuations of full configurations at $\beta_{L W}=3.3$ on $16^{4}$ lattices.

\subsection{Eigenmode Correlations}

All these vortex correlations in the last section showed similar behavior for overlap and asqtad modes, evaluated on full configurations. Therefore, the correlation of the different fermionic eigenmodes is analyzed here. The first overlap mode corresponds not necessarily to the first asqtad staggered mode, since the energies of eigenmodes do not have to be the same for the two fermion discretizations. In order to overcome this discrepancies the correlation is performed as follows: The scalar densities of the first 
a)

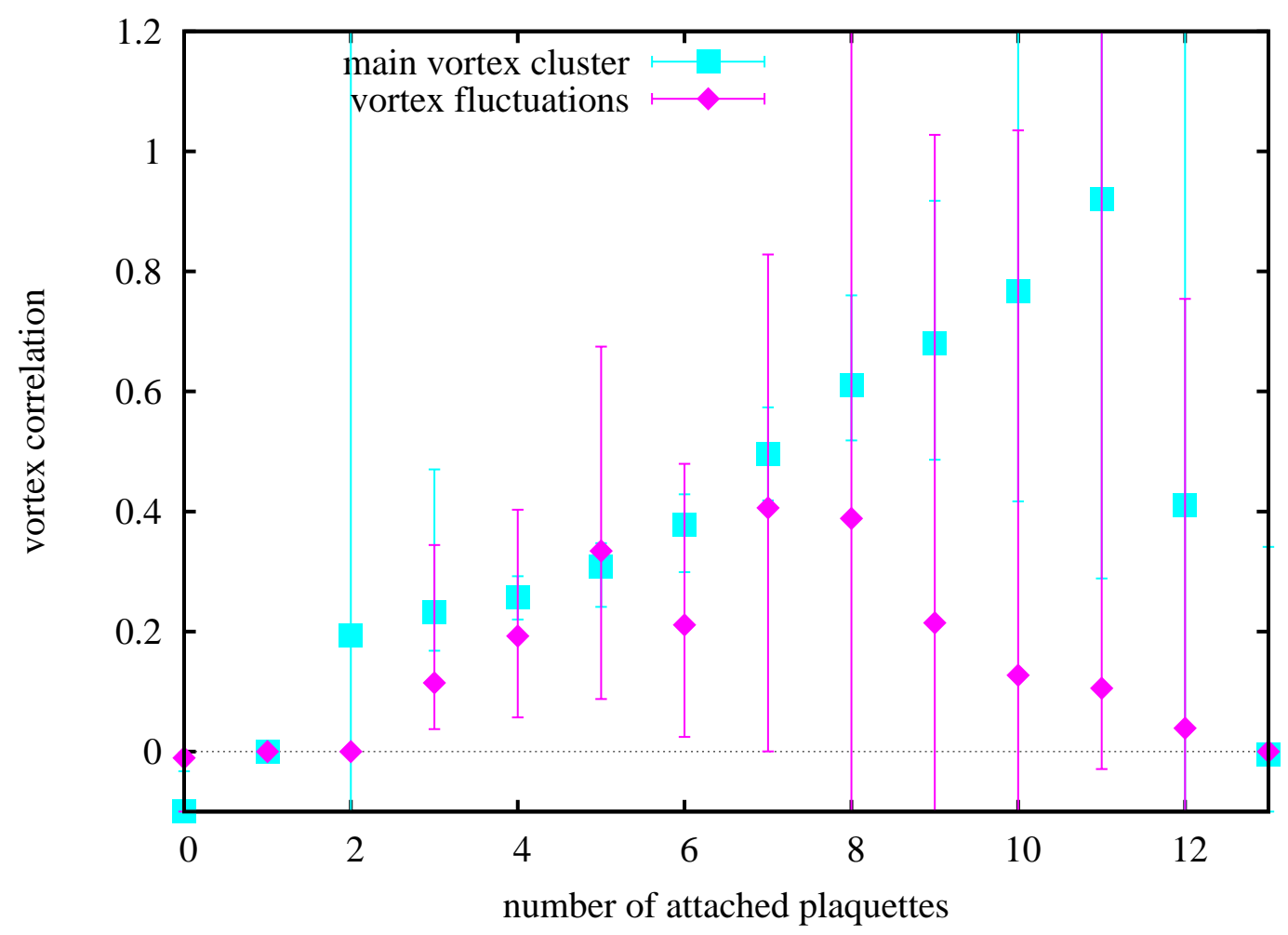

b)

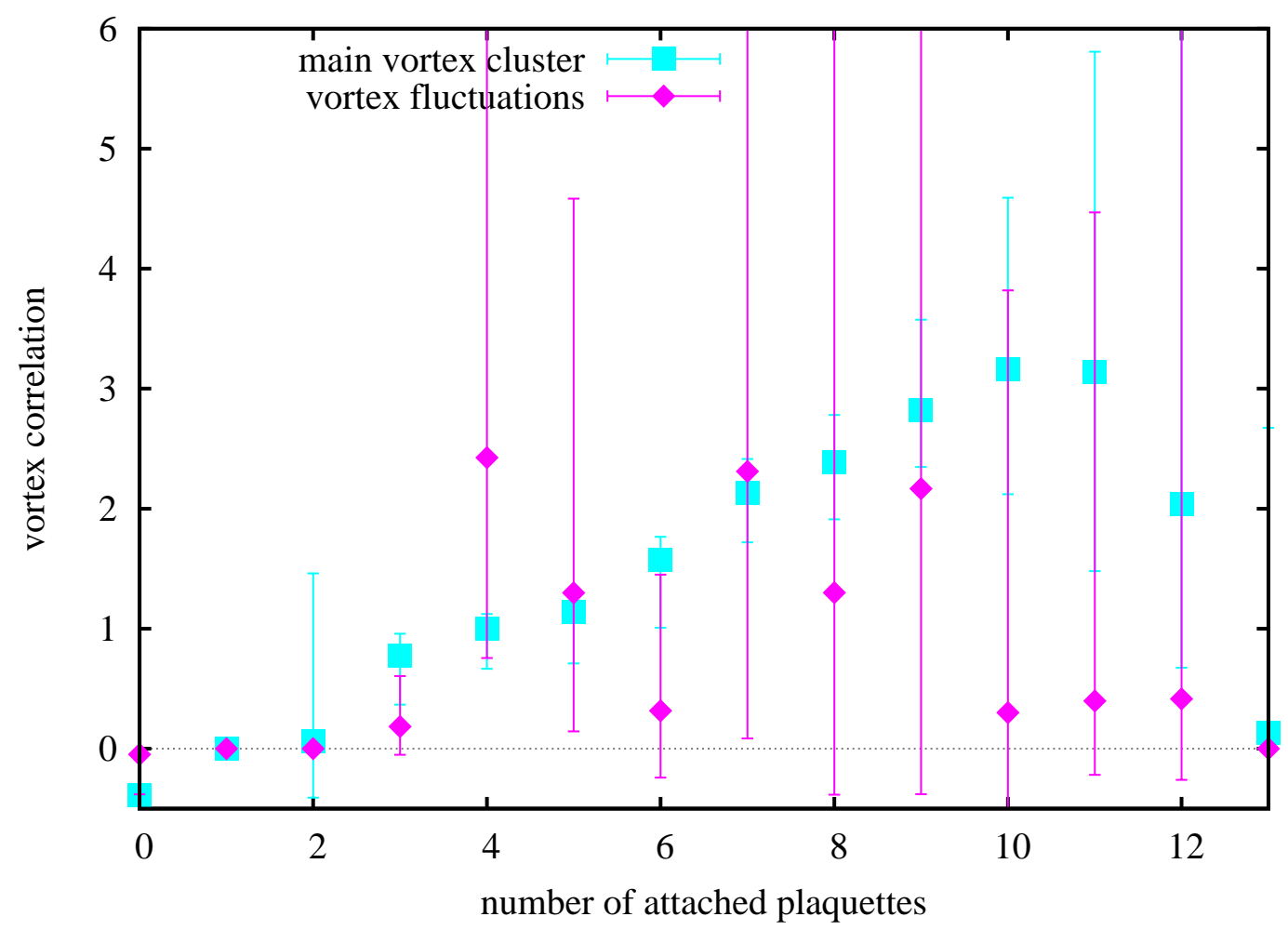

Figure 65: Correlation between eigenmode density and the main vortex cluster or vortex fluctuations of a) full and b) center-projected configurations at $\beta_{L W}=3.3$ on $20^{4}$ lattices. 
twenty modes, overlap and asqtad staggered, are summed up for one single configuration, normalized and correlated as follows:

$$
C=\sum_{x=0}^{L^{4}} \frac{1}{20} \sum_{\lambda=1}^{20} \psi_{\lambda}^{\text {ovl }}(x) \frac{1}{20} \sum_{\lambda=1}^{20} \psi_{\lambda}^{\text {asqtad }}(x) .
$$

This gives a 95\% correlation for overlap and asqtad eigenmodes on full configurations, whereas the correlation between asqtad eigenmodes on full and center-projected configurations is only about $70 \%$. Correlating different configurations gives a signal of about $83 \%$, so this type of correlator seems to mostly correlate the background of the modes and not their maxima. Therefore the correlation of maximum peaks is considered next, where the summation over lattice sites $x$ in (31) now only runs over the sites $x$ within a $4^{4}$ hypercube around the maximum of each eigenmode $\psi_{\lambda}$ :

$$
C=\frac{1}{40} \sum_{\lambda=1}^{20} \sum_{x_{i}=x_{i}^{\max }\left(\psi_{\lambda}^{\text {ovl }}\right)-2}^{x_{i}^{\max }\left(\psi_{\lambda}^{\text {ovl }}\right)+2} \psi_{\lambda}^{\text {ovl }}(x) \sum_{\lambda=1}^{20} \sum_{x_{i}=x_{i}^{\max }\left(\psi_{\lambda}^{\text {asqtad }}\right)-2}^{x_{i}^{\max }\left(\psi_{\lambda}^{\text {asqtad }}\right)+2} \psi_{\lambda}^{\text {asqtad }}(x) .
$$

This correlator now gives almost no signal for different configurations $(0.1 \%)$, very low correlation between full and center-projected asqtad modes (6.8\%) and quite good correlation for overlap and asqtad modes on full configurations $(37.4 \%)$. Considering the chirality of the different modes, increases the correlation of overlap and asqtad modes on full configurations (41.85\%), since the maxima of both chiralities of overlap modes were included in the correlation. Correlating now only asqtad staggered modes of one chirality with the corresponding chiral part of overlap modes for one single configuration gives $34.6 \%$ correlation for positive chirality and $33.6 \%$ for negative chirality. Considering that modes of different configurations do not correlate and even asqtad modes of full and center-projected configurations correlate very little, the correlation of overlap and asqtad staggered eigenmodes on full configurations seems quite impressive. One further can look at correlations of single modes and find different modes (with different energy for different fermion formulations) which peak at same positions. In Fig. 66 the overlap and asqtad staggered modes of two representative configurations are plotted with their eigenvalue and joined when the maximum peak of a certain chiral component of an overlap mode corresponds to the maximum peak of an asqtad staggered mode with same chirality (correlations about 90\%). Lower correlations are identified with close maximum peaks. In the first example, the two overlap zero modes do not correlate to asqtad modes, but overlap modes three and five, which have their maximum peak at the same position, correspond to asqtad staggerd modes two, four and five. In the second example one overlap zero mode corresponds to asqtad staggered modes five and six. Allowing only single pairing, i.e. one overlap mode is identified with one asqtad mode only, of course reduces the joining lines but is maybe a more physical way of presentation (see Fig. 67). Concerning asqtad staggered modes on center-projected configurations, only few correlations are found to either overlap or asqtad staggered modes on the corresponding full configurations (see Fig. 68). 
a)

b)
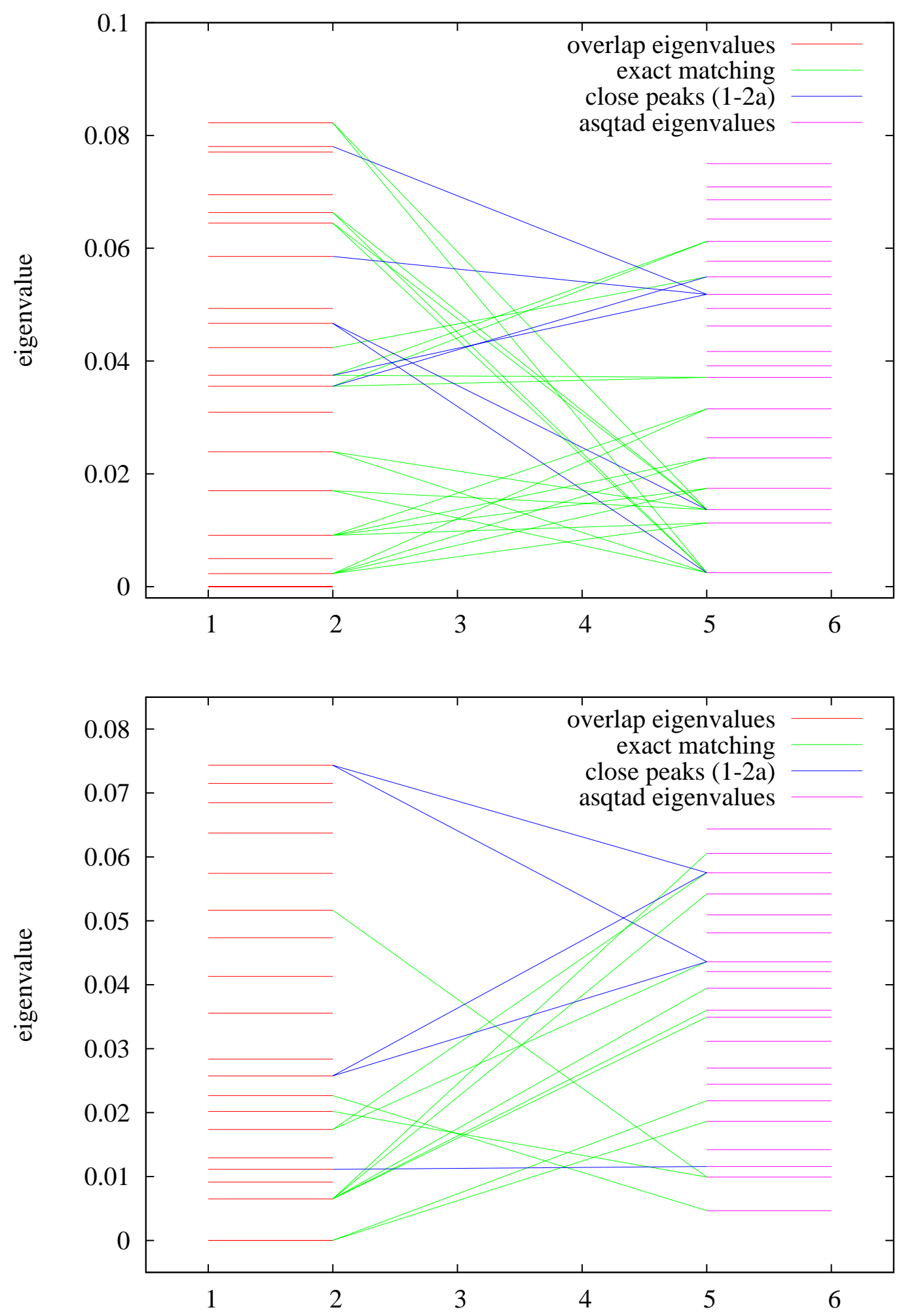

Figure 66: Overlap (left) and asqtad staggered (right) eigenmodes, plotted with their eigenvalues and joined for good correlation (about 90\%) of their maximum eigenmode peaks of certain chirality. Two representative configurations at $\beta_{L W}=3.3$ on $16^{4}$ lattices. 
a)

b)
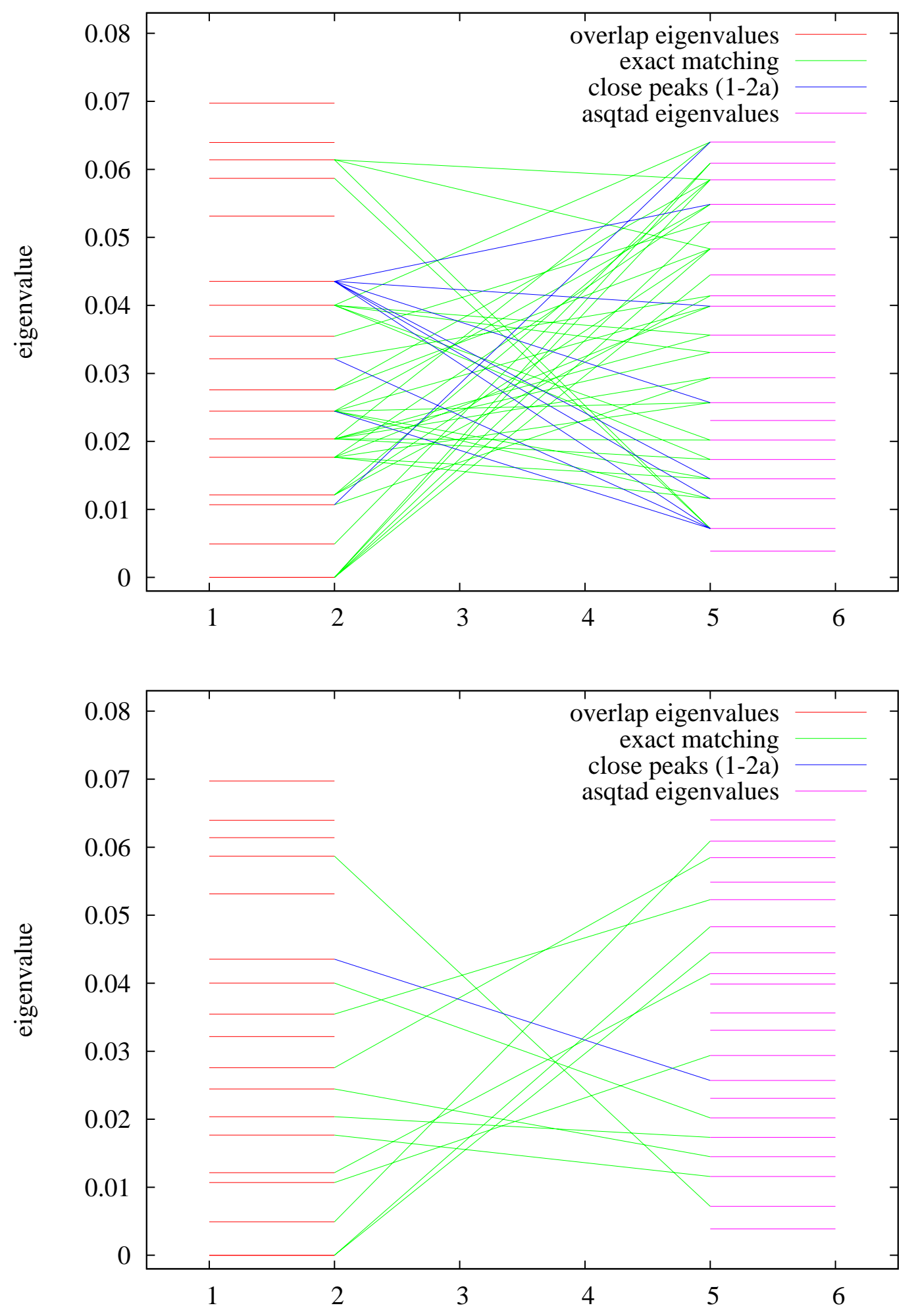

Figure 67: a) same as in Fig. 66. In b) one overlap mode is identified with one asqtad mode only, therefore all multiple lines from one eigenmode (two zero modes!) are removed from a). 
a)

b)
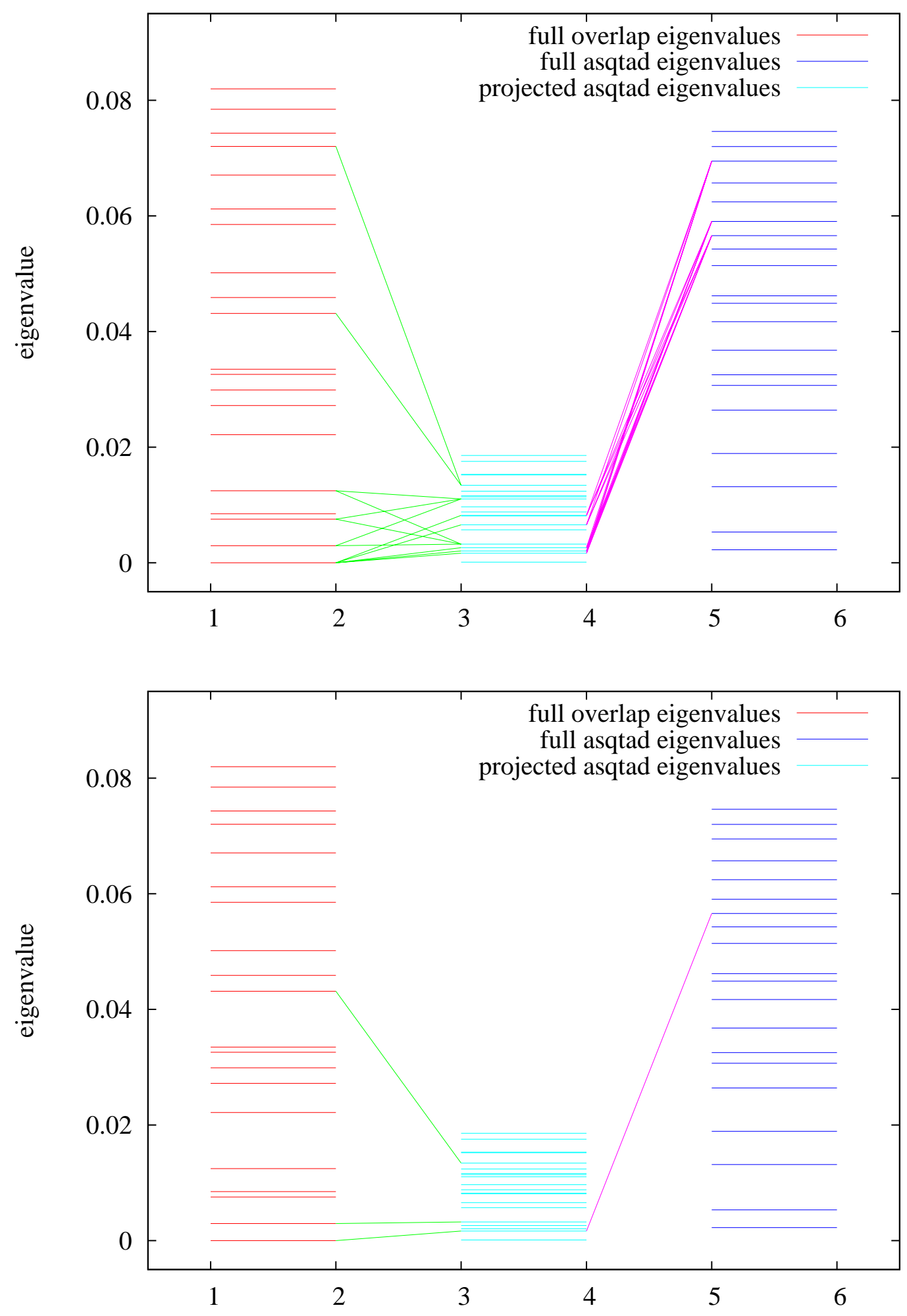

Figure 68: a) Correlations between eigenmodes on a center-projected and the corresponding full configuration. In b) again all multiple lines from one eigenmode are removed from a). 


\subsection{Chiral Density Peaks and Vortex Structures}

Vortex correlations with chiral densities of the overlap Dirac eigenmodes show that the density peaks concentrate near vortex surfaces but left- and right-handed densities behave somehow differently. Since the eigenmode densities are peaked rather sharply (section 5.3), it might be again worthwhile to look at these peaks and where they are located exactly. For a first glance the "Interactive Visualization Package for 4D Lattice Field Theories" by Ivan Hip [75] shall be used. Fig. 69 shows the chiral densities of first and $20^{\text {th }}$ overlap Dirac (non-zero) eigenmodes of a representative configuration.

a)
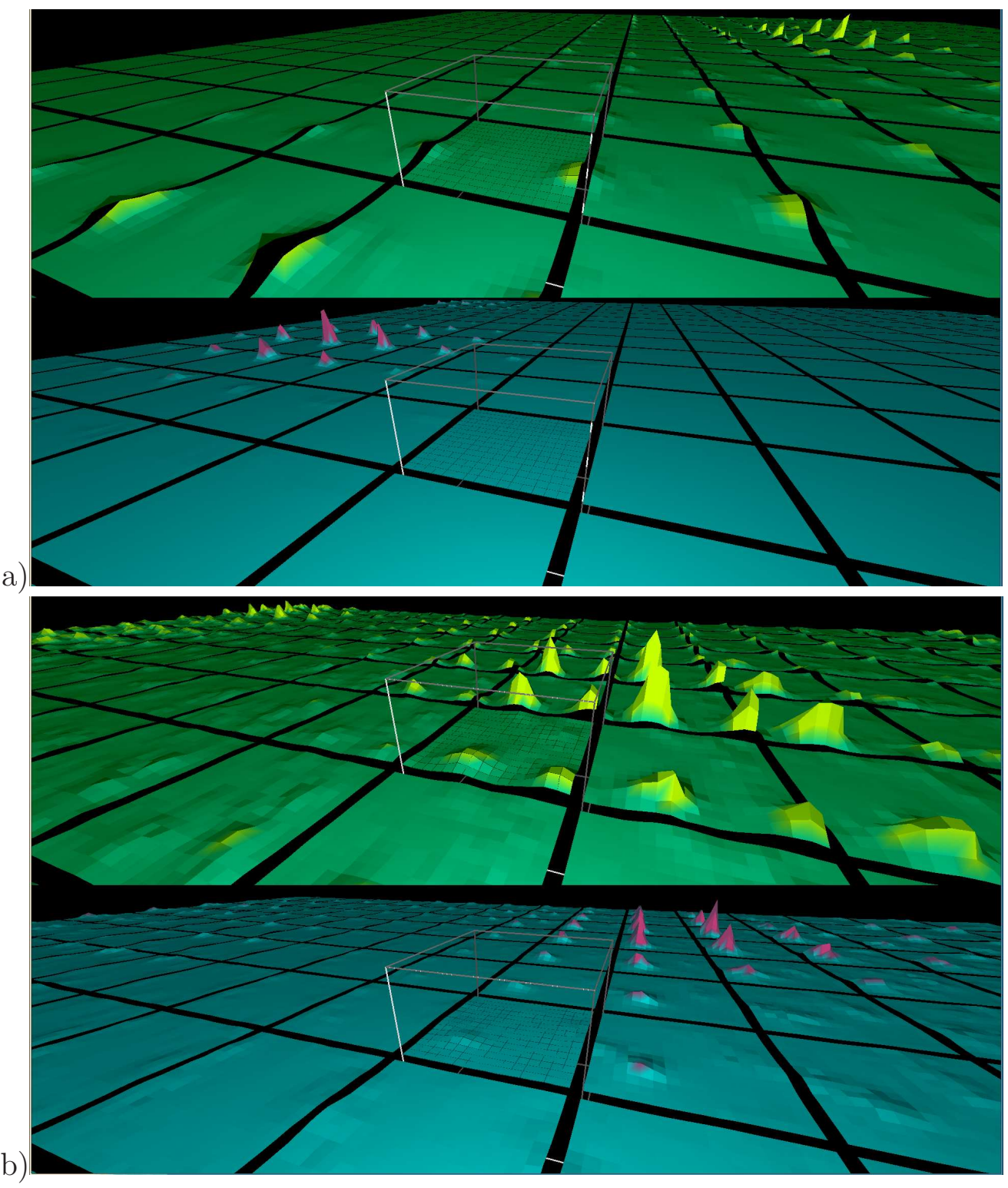

Figure 69: Left- (yellow) and right-handed (magenta) chiral density peaks of a) first and b) $20^{\text {th }}$ overlap Dirac eigenmodes. 
The first (non-zero) mode behaves similar to zero modes, it is located in one main peak and shows no density elsewhere. But differently from real zero modes, it shows one peak for each chirality. These peaks are located in very different positions on the lattice. Higher modes show more and more peaks in their eigenmode densities, but again, the peaks for different chiralities do not completely coincide, but seem to be located closer to each other.

Next, these peaks shall be compared with the vortex structures. Therefore, the eigenmode density in a certain xy-slice, including chiral density peaks is plotted together with the vortex plaquettes in this region. Fig. 70 shows the density of $8^{\text {th }}$ eigenmode and the vortex structure in xy-slice at $z=1$ and $t=1$ of a representative configuration. In Fig. 71 the same plot is split up in three sub-figures, showing right- (up), left-handed (below) chiral densities and the vortex structure (center).

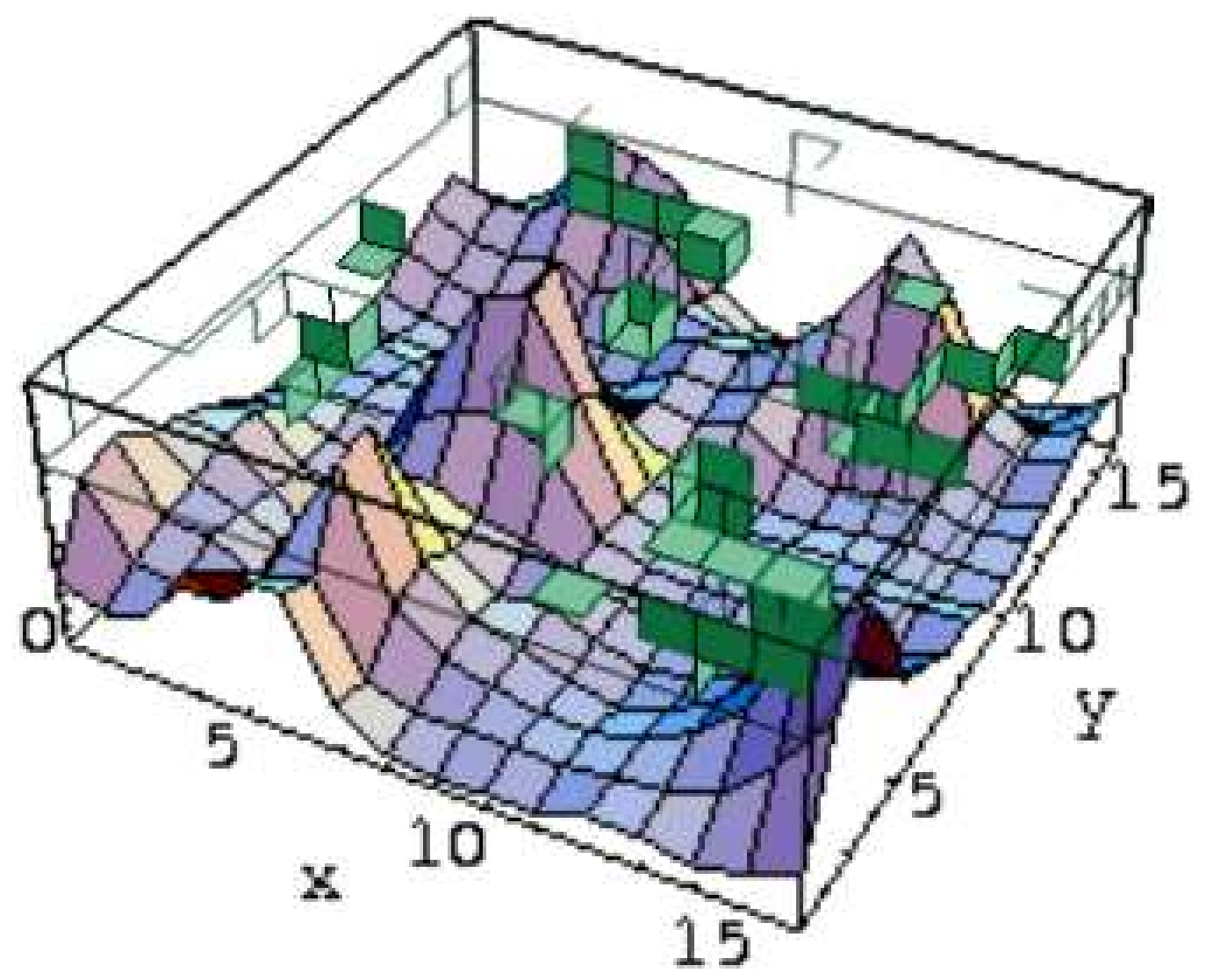

Figure 70: $x y$-plot of $8^{\text {th }}$ eigenmode density and vortex structure at $z=1, t=1$ (vortex plot shows $z=1$ and $z=2$ slices).

The plots show eigenmode density peaks located not exactly at vortex structures, but rather close to. Peaks of different chirality seem to be located at opposite sides of the vortex surface, this should be analyzed in more detail next. 

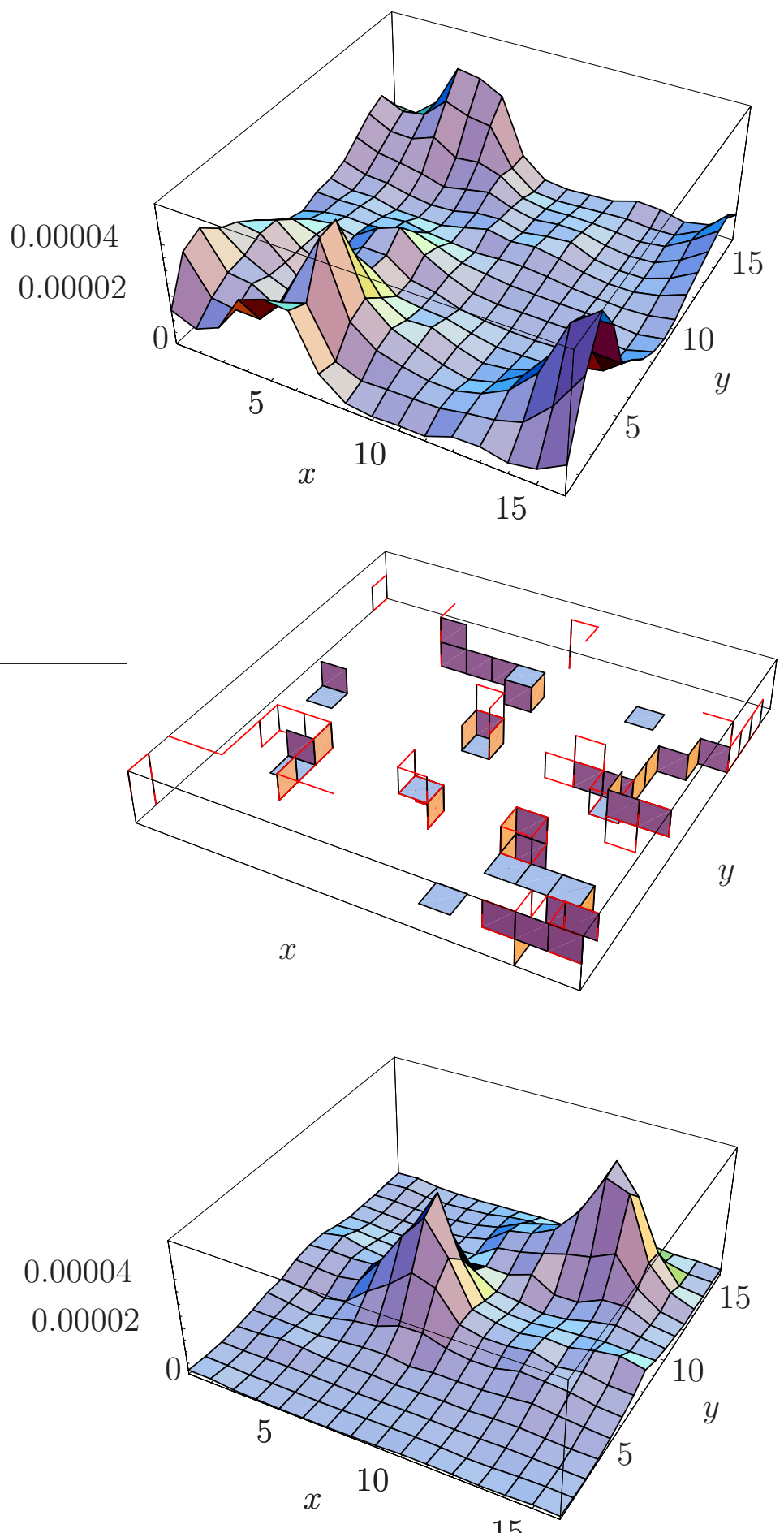

15

Figure 71: $x y$-plots for eigenmode densities of positive (above) resp. negative chirality (below) and vortex structure (center) at $z=1, t=1$ (vortex plot shows $z=1$ and $z=2$ slices). 
Concentrating on low-lying eigenmodes and considering the results of section 5.5, the vortex structures where overlap and/or asqtad staggered eigenmodes show their (chiral) maxima are presented in the following figures.

In Fig. 72 three examples of vortex structures where only overlap eigenmodes of left(above) and right-handed (below) chirality peak on full configurations are shown. The vortices extend in all four space-time directions and all plaquette-types contribute. For the first example in Fig. 72a, respectively example two in Fig. 72b, no plaquette of the (dual) hypercube around the exact maximum contributes to the vortex structure. A difference between left- and right-handed chiral peaks is not obvious.

Fig. 73 presents three examples of vortex structures where overlap and asqtad staggered eigenmode density peaks of left- (above) and right-handed (below) chirality are found. The structures look pretty similar but the plaquette distribution seems to be denser. In fact, almost all examples show all types of vortex plaquettes at the center of the structures, the exact maxima of the peaks. It seems that at structures of righthanded chiral peaks more time-extending plaquettes contribute, i.e. there are more space-space vortex plaquettes in left-handed chiral peak structures.

In Fig. 74 three examples of vortex structures where only asqtad staggered eigenmodes of left- (above) and right-handed (below) chirality peak on full configurations are presented. The plots look pretty similar to the examples in Fig. 73. It seems that there are a few less vortex plaquettes contributing in total, but again, at the exact maximum peak position, all types of plaquettes are found which form partially closed hypercubes. The difference between vortex structures at left- and right-handed chiral peaks from above (space-space vs. space-time plaquettes) cannot be established.

Finally, Fig. 75 shows three examples of vortex structures where only asqtad staggered eigenmodes of left- (above) and right-handed (below) chirality peak on center-projected configurations. The vortex structures are smaller but denser. Here only the surrounding hypercubes of the chiral peak maxima are drawn, in order to show that all types of vortex plaquettes contribute. The second example of left- and first example of right-handed chiral peak structures form closed hypercubes, in all other cases only few plaquettes are missing. These structures are often vortex fluctuations, e.g. single negative links in the center-projected configurations.

To summarize, overlap and asqtad staggered peaks are found at locations with much vortex structure. Only overlap peaks are found with less vortex structure at the precise maximum, but a lot of vortex plaquettes in close neighborhood (at one lattice constant and further) whereas asqtad modes show many plaquettes at the precise point where they peak maximally. On center-projected configurations asqtad staggered modes often peak on vortex fluctuations. This result is not surprising, considering the form of the different eigenmode peaks (e.g. full width half maximum or Figs. 44-48) - overlap peaks seem to spread over a few (3-4) lattice spacings whereas asqtad staggered peaks are much sharper, for center-projected configurations by far the sharpest - but it is interesting that it can be observed so clearly at the vortex structure. For eigenmode density peaks of different chirality the vortex structure pretty looks the same. It is also found that modes of different chirality peak at the same structure, e.g. at the first example in Fig. 75b) three modes of positive and two of negative chirality peak. 
a)
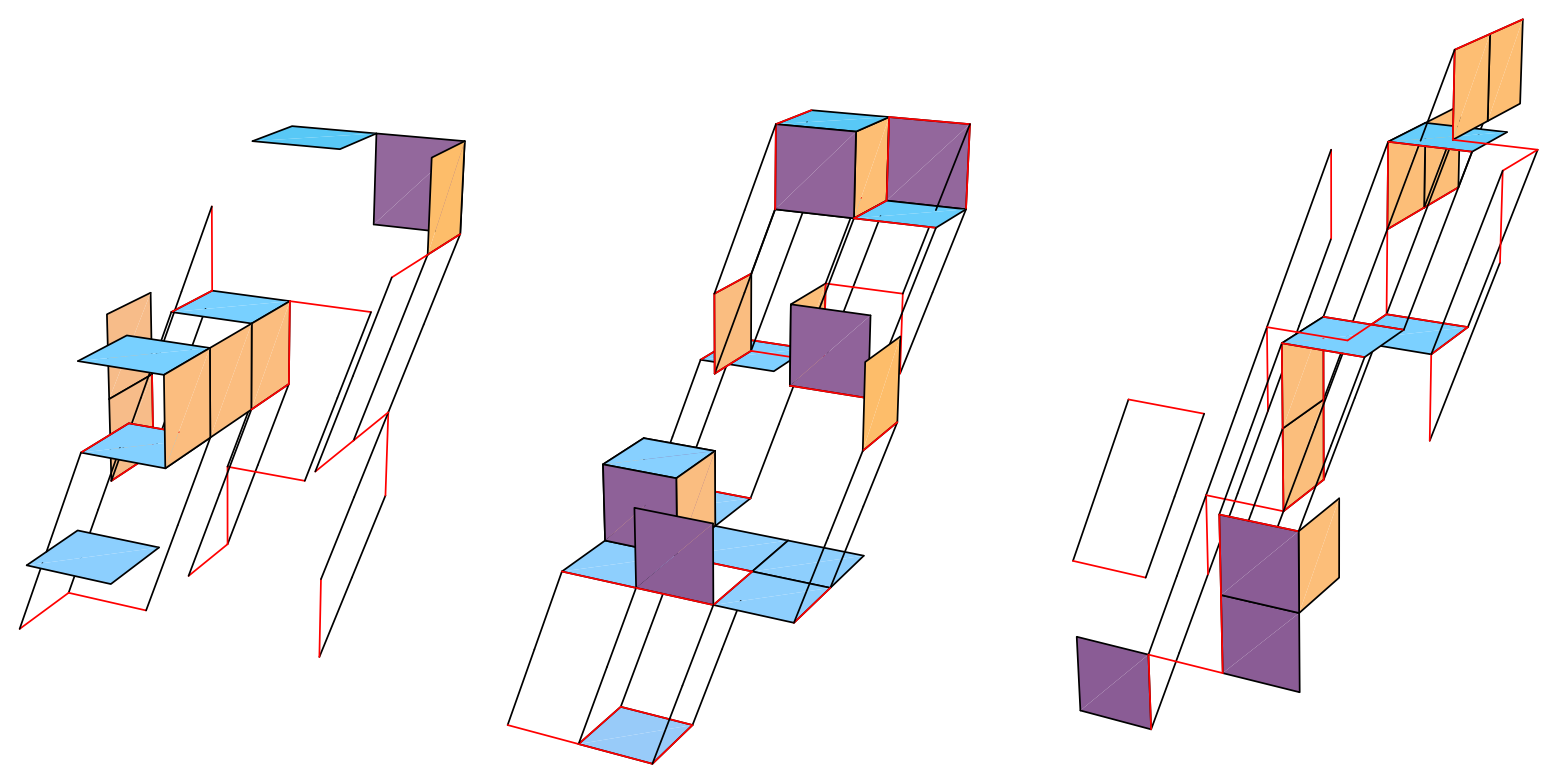

b)
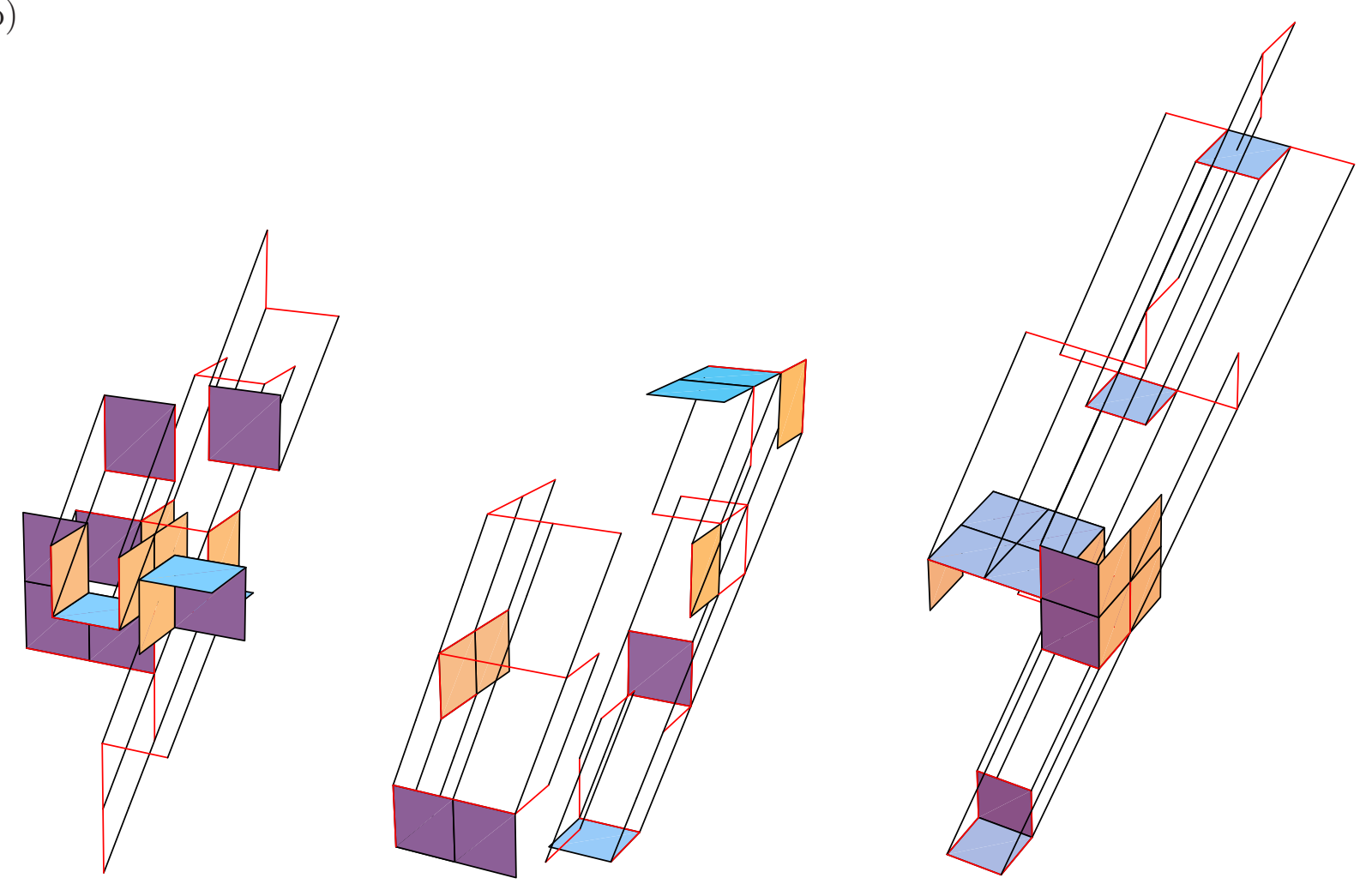

Figure 72: Vortex structures where only overlap eigenmodes of a) left- and b) righthanded chirality peak on full configurations. 
a)
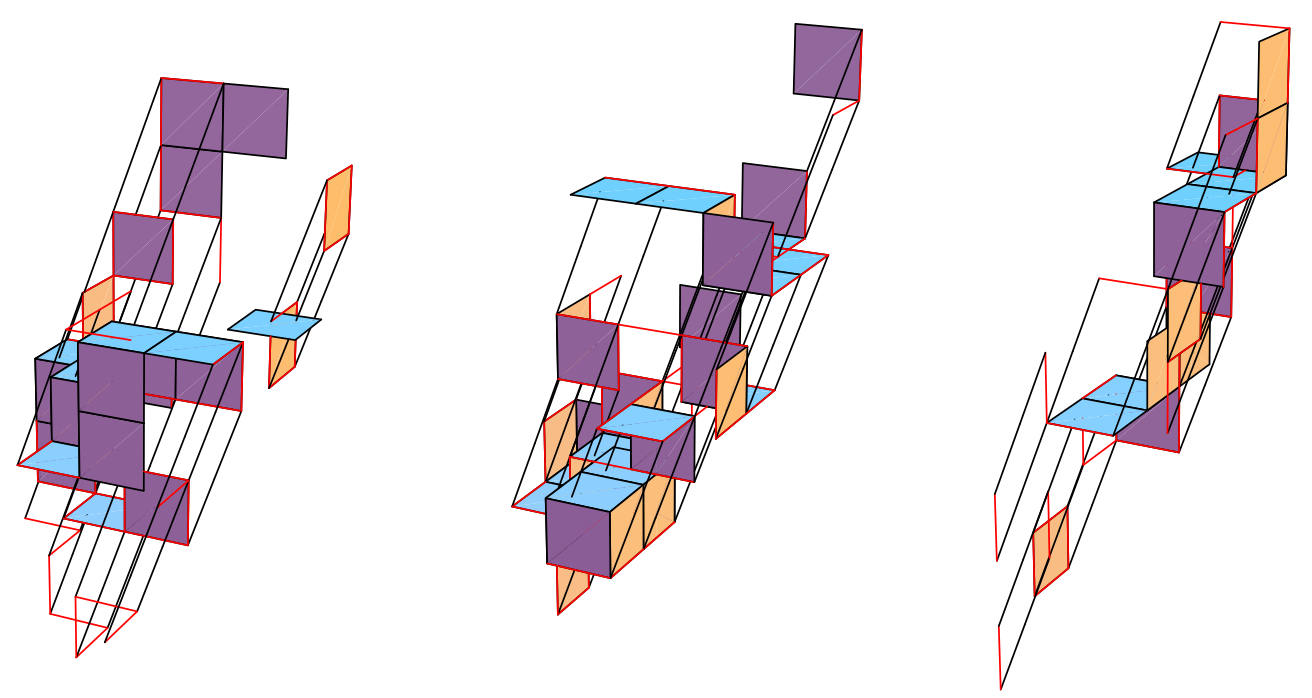

b)
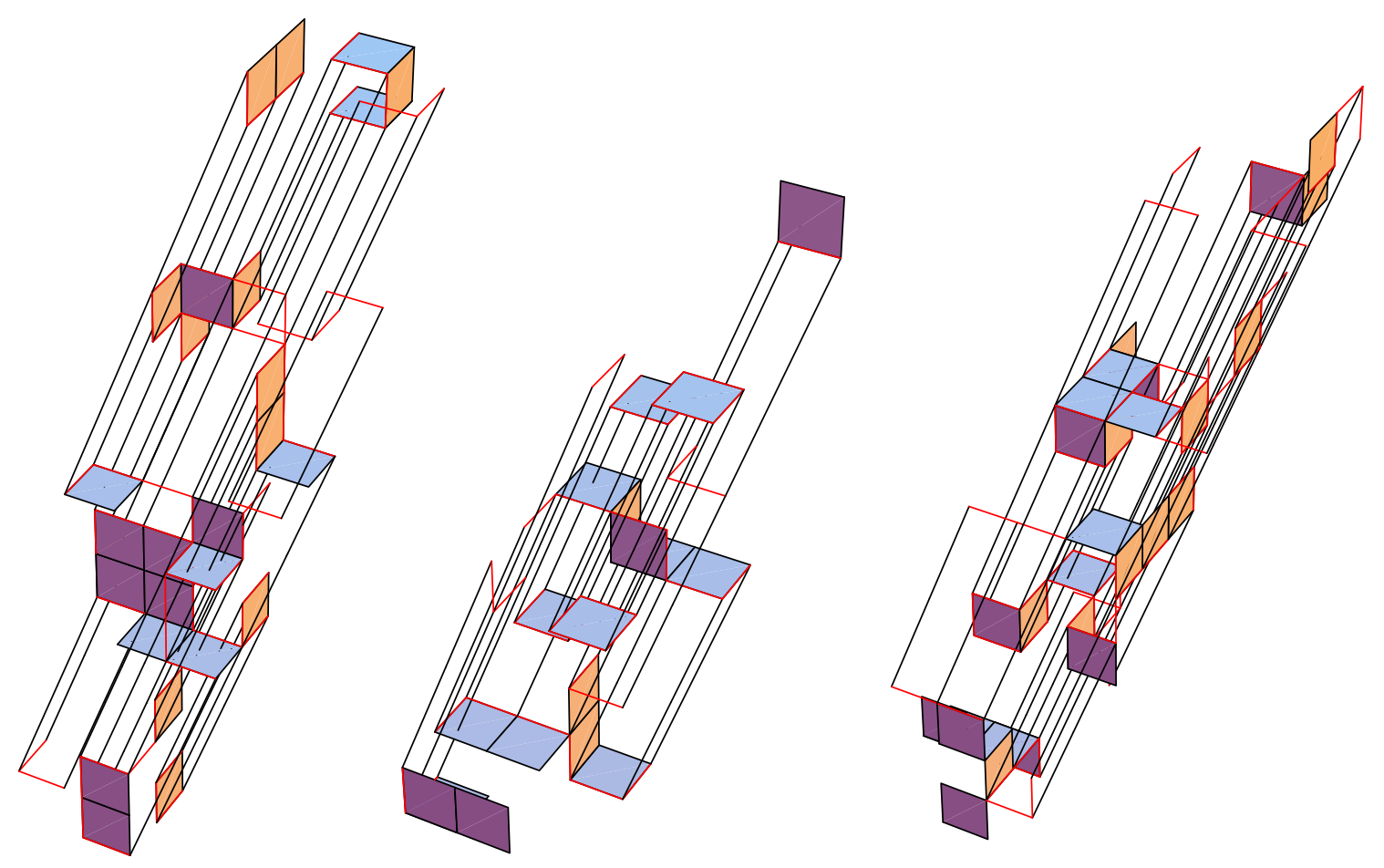

Figure 73: Vortex structures where overlap and asqtad staggered eigenmodes of a) leftand b) right-handed chirality peak on full configurations. 
a)
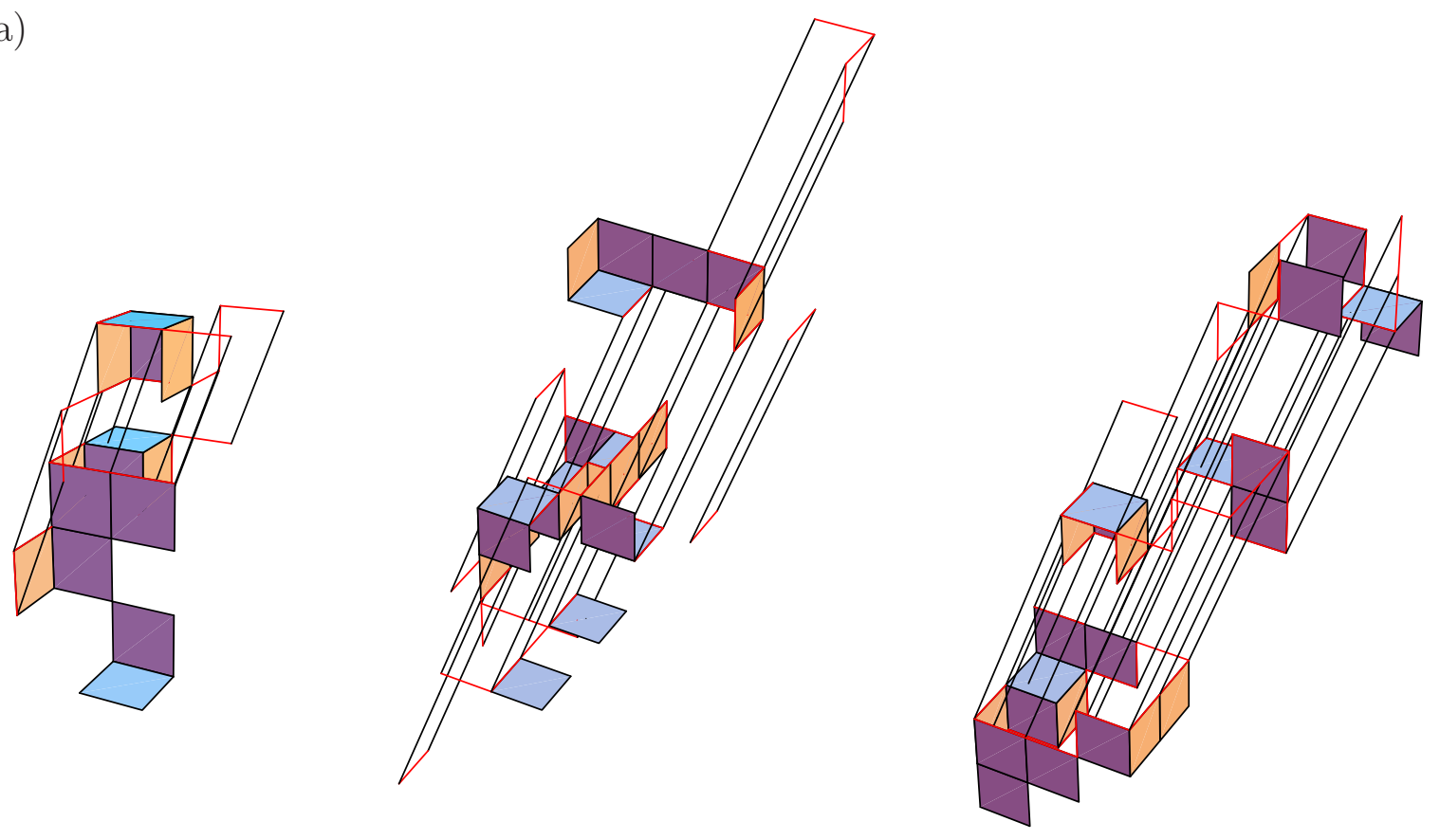

b)
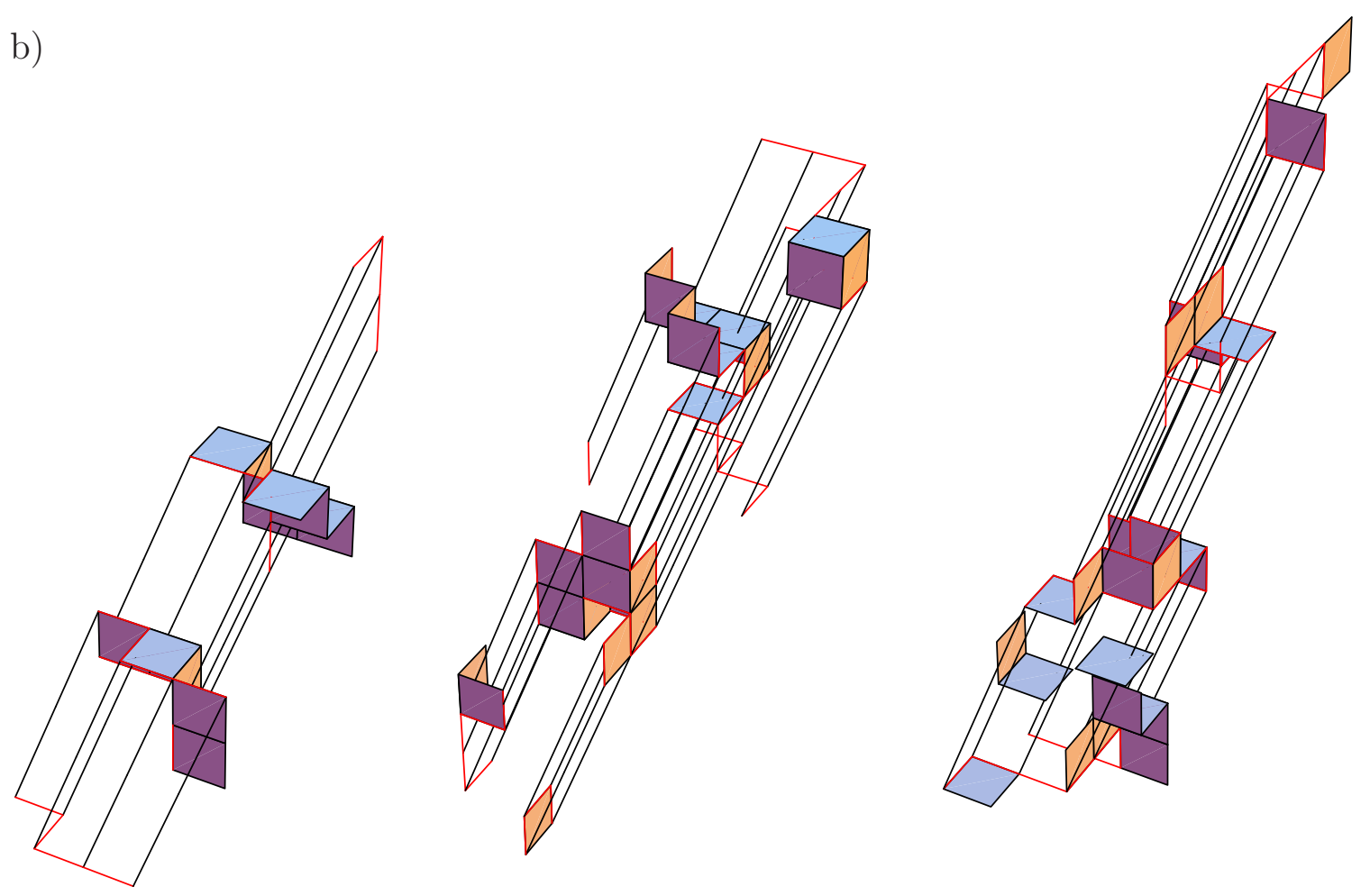

Figure 74: Vortex structures where only asqtad staggered eigenmodes of a) left- and b) right-handed chirality peak on full configurations. 
a)
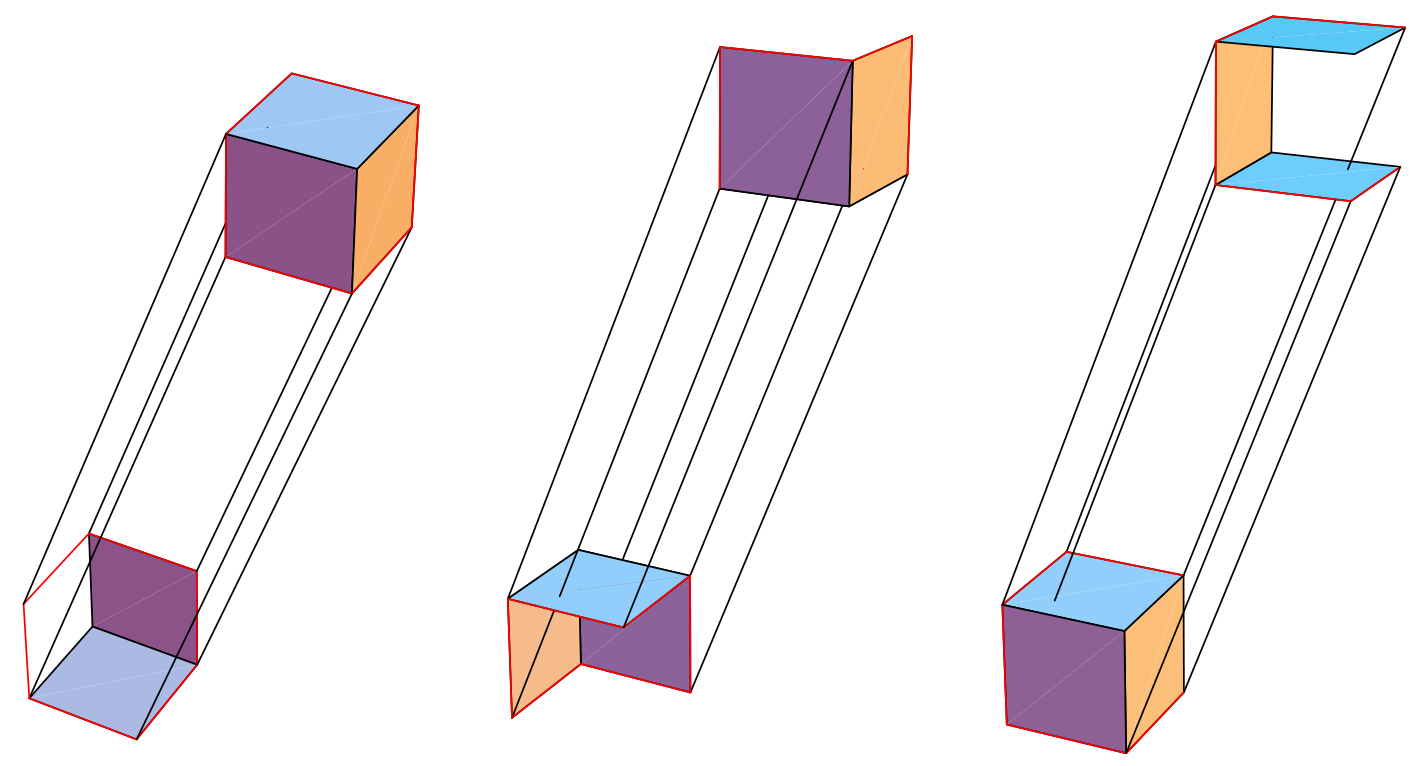

b)
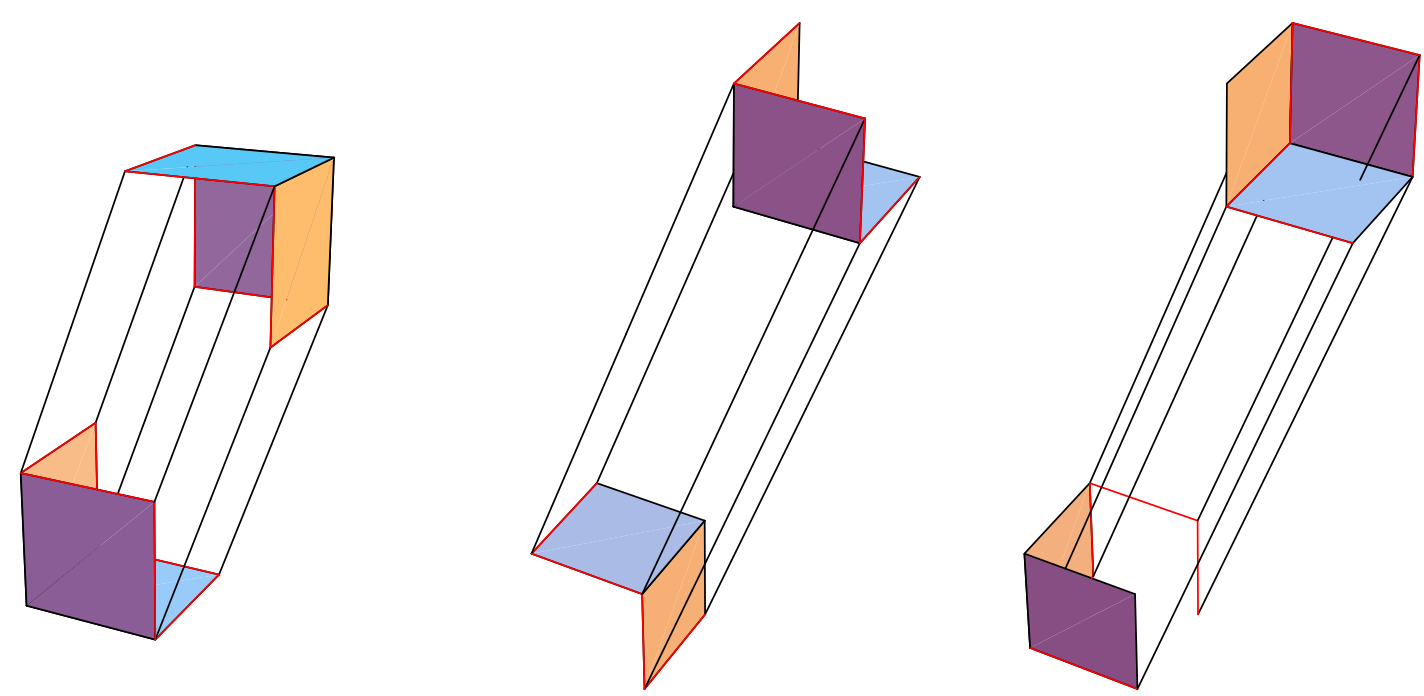

Figure 75: Vortex structures where only asqtad staggered eigenmodes of a) left- and b) right-handed chirality peak on center-projected configurations. 

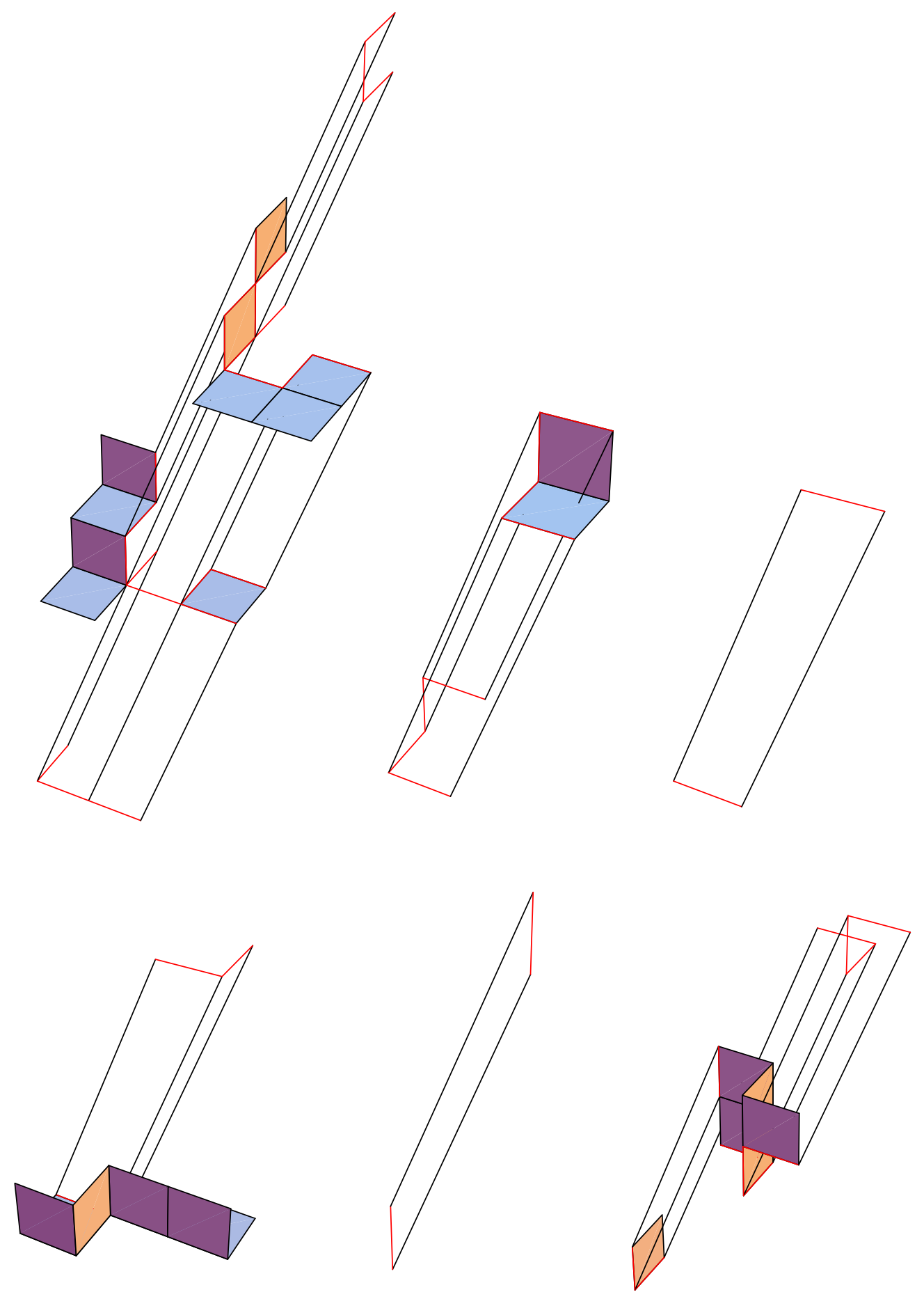

Figure 76: For comparison, six vortex structures at randomly chosen points are plotted, in about one third of chosen random points, no vortex structure was found. 
In order to analyze these issues more globally, the number of E- respectively Bplaquettes in the neighborhood of eigenmode density maxima is analyzed. Therefore the number of vortex plaquettes $(\mathrm{E} / \mathrm{B})$ in a distance $d$ of individual maxima peaks is counted. The eigenmode density maximum peak lies in the center of a hypercube on the dual "vortex-lattice". Vortex plaquettes appear at discrete distances $d$ from this center, the 24 plaquettes of the hypercube for example have $d=\sqrt{0.5} \approx 0.71$. Therefore the plaquettes on spherical shells with radius $r=d$, distances where plaquettes appear, are considered. The average number over all maximum peaks of overlap and/or asqtad staggered eigenmodes on full and asqtad staggered modes on center-projected configurations is listed in Table 6 together with the total number of possible plaquettes at a considered distance and the normalized data (E+B-plaquettes/total number of plaquettes), which of course gives some sort of correlation or simply the vortex density around the eigenmode density peak maxima.

\begin{tabular}{|c|c|c|c|c|c|c|c|c|c|c|}
\hline distances $r$ : & 0.71 & 1.23 & 1.59 & 1.88 & 2.13 & 2.35 & 2.55 & 2.74 & 2.92 & 3.09 \\
\hline plaquettes total & 24 & 96 & 144 & 192 & 312 & 288 & 336 & 576 & 432 & 480 \\
\hline \multicolumn{11}{|l|}{ E-plaquettes: } \\
\hline only overlap peaks: & 1.41 & 4.29 & 5.31 & 6.51 & 9.07 & 7.96 & 8.75 & 15.02 & 10.79 & 11.95 \\
\hline overlap \& asqtad: & 1.45 & 4.45 & 5.77 & 6.46 & 9.15 & 7.96 & 8.99 & 14.76 & 10.61 & 11.56 \\
\hline only asqtad peaks: & 1.58 & 5.07 & 5.49 & 6.36 & 9.37 & 7.74 & 8.68 & 15.09 & 10.27 & 12.27 \\
\hline projected asqtad: & 2.09 & 5.36 & 5.5 & 5.99 & 8.7 & 6.94 & 8.51 & 13.77 & 10.38 & 11.77 \\
\hline \multicolumn{11}{|l|}{ B-plaquettes: } \\
\hline only overlap peaks: & 1.42 & 4.37 & 5.55 & 6.55 & 9.54 & 8.44 & 9.28 & 14.93 & 10.88 & 12.09 \\
\hline overlap \& asqtad: & 1.44 & 4.64 & 5.69 & 7.01 & 9.6 & 7.97 & 8.97 & 14.94 & 11.16 & 11.94 \\
\hline only asqtad peaks: & 1.37 & 4.68 & 5.37 & 6.32 & 9.65 & 7.75 & 9.4 & 15.38 & 11.15 & 11.4 \\
\hline projected asqtad: & 2.02 & 5.58 & 5.72 & 5.96 & 8.45 & 6.92 & 8.78 & 14.27 & 10.55 & 11.48 \\
\hline \multicolumn{11}{|l|}{ vortex density: } \\
\hline only overlap peaks: & 11.8 & 8.9 & 7.4 & 6.8 & 5.8 & 5.5 & 5.2 & 5.2 & 5.0 & 5.0 \\
\hline overlap \& asqtad: & 12.1 & 9.3 & 8.0 & 6.7 & 5.9 & 5.5 & 5.4 & 5.1 & 4.9 & 4.8 \\
\hline only asqtad peaks: & 13.2 & 10.6 & 7.6 & 6.6 & 6.0 & 5.4 & 5.2 & 5.2 & 4.8 & 5.1 \\
\hline projected asqtad: & 17.5 & 11.2 & 7.6 & 6.2 & 5.6 & 4.8 & 5.1 & 4.8 & 4.8 & 4.9 \\
\hline
\end{tabular}

Table 6: Average number of E/B-plaquettes and vortex density (data normalized to total number of possible plaquettes) in a distance $r$ around the maximum eigenmode density peaks

The data confirms the conjectures from above. The number of plaquettes in closest neighborhood increases from overlap to asqtad eigenmodes, only the number of Bplaquettes seems a bit too low for only asqtad peaks on full configurations. The values are maximal for center-projected asqtad modes, but for higher distances they seem to be lower. The normalized data tends to a value of about $5 \%$ at higher distances, which is the average vortex density. This vortex density is plotted in Fig. 77, the discrete block diagrams are smoothed with gnuplot for better perceptibility. 


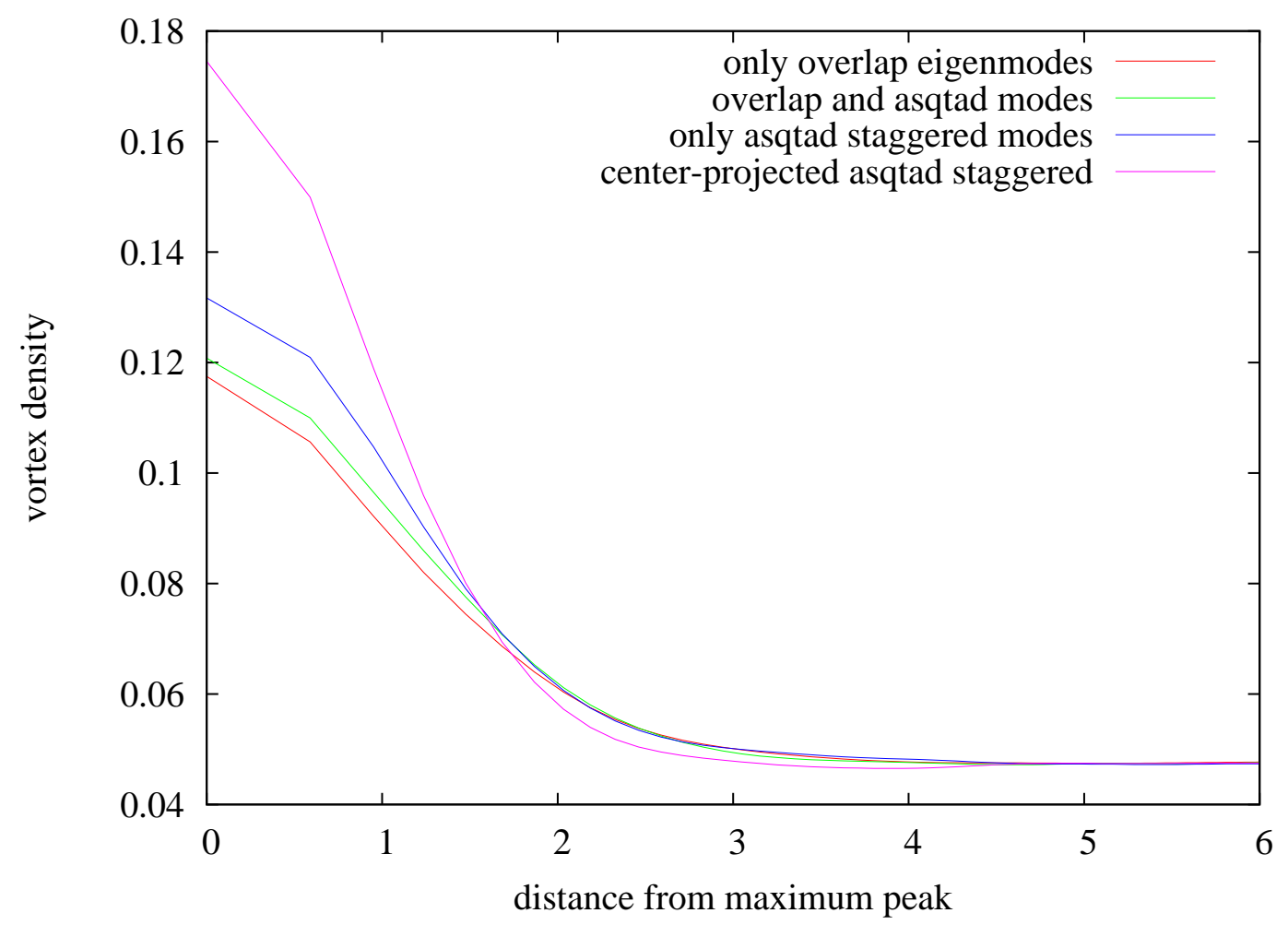

Figure 77: Vortex density at a distance $r$ (see discrete values from above tables) from the maximum eigenmode density peaks (smoothed block digram plot).

The plots of course show the same results as obtained before. For center-projected configurations it should be mentioned that six plaquettes (out of the 24 in closest neighborhood) are necessary to form a closed cube or twelve plaquettes to form a closed hypercube. From Table 6 one can see that in average a total number of more than four plaquettes is present in the closest neighborhood. So, the hypothesis that asqtad staggered eigenmodes on center-projected configurations peak mainly on vortex fluctuations only seems not to be confirmed. Nevertheless, in Fig. 77 one finds the vortex density decreasing from a maximal value (compared to overlap and staggered eigenmodes on full configurations) at the exact eigenmode density peak maximum to a vortex density in close neighborhood (two to four lattice constants) which is even lower than the overall vortex density. Asqtad staggered eigenmodes on center-projected configurations therefore seem to find somehow isolated, very dense vortex structures, whereas on full configurations the peaks are located at simply dense structures within the main vortex cluster.

Taken together, the results of this whole section provide an impressive indication that center vortices are in fact able to discribe chiral symmetry breaking. Still better, they are even necessary for chiral symmetry breaking, whether due to their confining force or some other, still unrevealed properties. 


\subsection{Finite Temperature}

The chiral symmetry section shall be concluded with a high-temperature result. Chiral symmetry is restored at high temperatures, and this fact should also hold for centerprojected lattices. Therefore, at sufficiently high- $T$, a gap should open in the eigenvalue spectrum. This can be seen in Fig. 78, where the low-lying eigenvalues from $12^{3} \times N_{T}$ center-projected lattices at $\beta_{L W}=3.5$ for time-extensions $N_{T}=2,4,6,12$ are displayed. In this case, the theory is certainly in the deconfined phase at $N_{T}=6$, where there is, however, no obvious gap in the eigenvalue spectrum, so it may be that on the projected lattice the chiral transition occurs at a higher temperature than the deconfinement transition. This is also consistent with [15], which found a non-vanishing $\bar{\psi} \psi$ condensate at a temperature somewhat above the Wilson action deconfinement temperature. It should be stressed, however, that there is no reason that the chiral and deconfinement temperatures need coincide on the center-projected lattice. Confinement is a sufficient but not a necessary condition for chiral symmetry breaking, and, while the center-projected lattice is expected to get the static quark potential about right asymptotically, this fact certainly does not hold true at intermediate scales, where the finite thickness of real vortices is crucial. If the static potential on the center-projected lattice is strong enough, chiral symmetry breaking will be realized, and this symmetry breaking may persist somewhat above the actual deconfinement temperature. The point is that if one views Yang-Mills configurations as being in some sense factorizable into vortices $\times$ perturbative fluctuations at short distances, then the thickness and internal structure of vortices is important for certain non-perturbative phenomena (such as Casimir scaling, and perhaps the precise chiral transition point) which are sensitive to the static quark potential at intermediate scales. ${ }^{3}$

\subsubsection{Vortex correlation in high-temperature configurations}

The correlation between the vortex surface and eigenmode density is analyzed with the correlator from equation (27), for asqtad staggered fermions in high-temperature configurations, Fig. 79 shows that the vortex correlation vanishes for high temperatures. As described in section 3.7, at finite temperature P-vortices exist also in the deconfined phase. They form cylindric objects which extend in time direction only, and therefore can't be well located by Dirac modes anymore.

Further figures of spectra and vortex-eigenmode correlations for full and center-projected configurations with Wilson and Lüscher-Weisz action, using different boundary conditions and $\beta_{L W}$, including some correlation studies on Polyakov loops, eigenmode and vortex densities and number of E- (space-space), B- (space-time) and P- (vortex) plaquettes for finite temperature can be found in Appendix C, page $170 \mathrm{ff}$.

\footnotetext{
${ }^{3}$ Cf. [76] for a discussion of vortex thickness and Casimir scaling.
} 

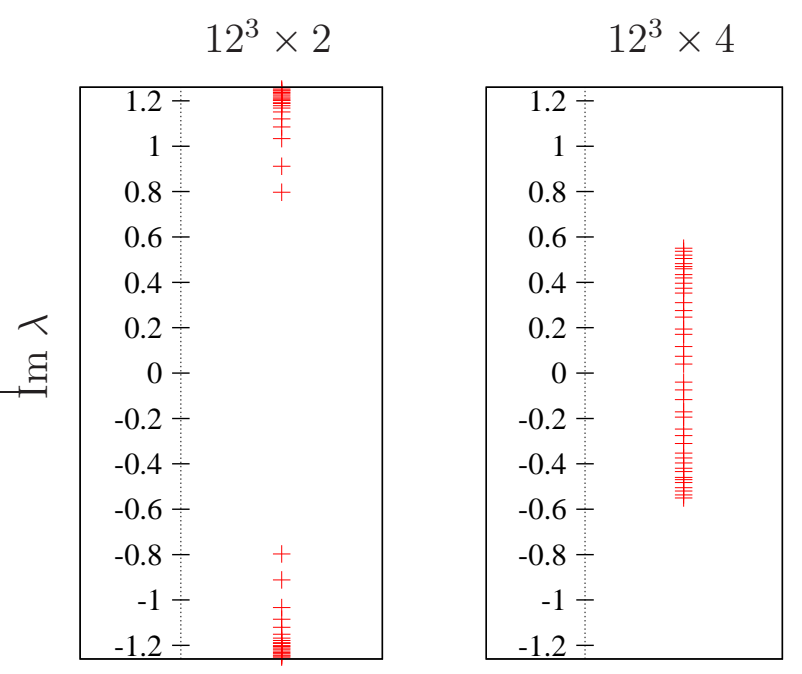

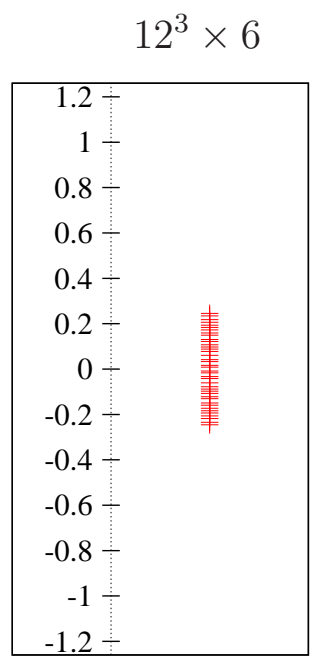

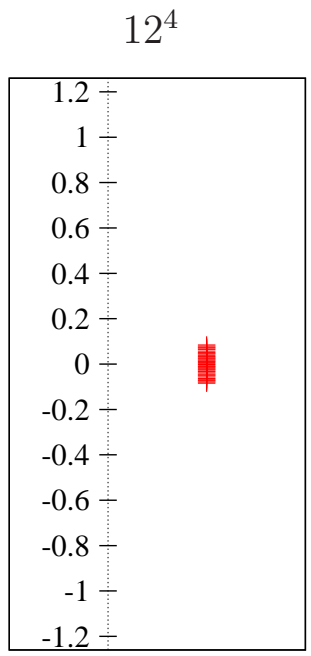

Figure 78: Finite temperature and center projection. The first twenty asqtad Dirac eigenvalue pairs from $12^{3} \times N_{T}$ center-projected lattices at $\beta_{L W}=3.5$ and $N_{T}=2,4,6,12$ lattice spacings, using anti periodic boundary conditions.

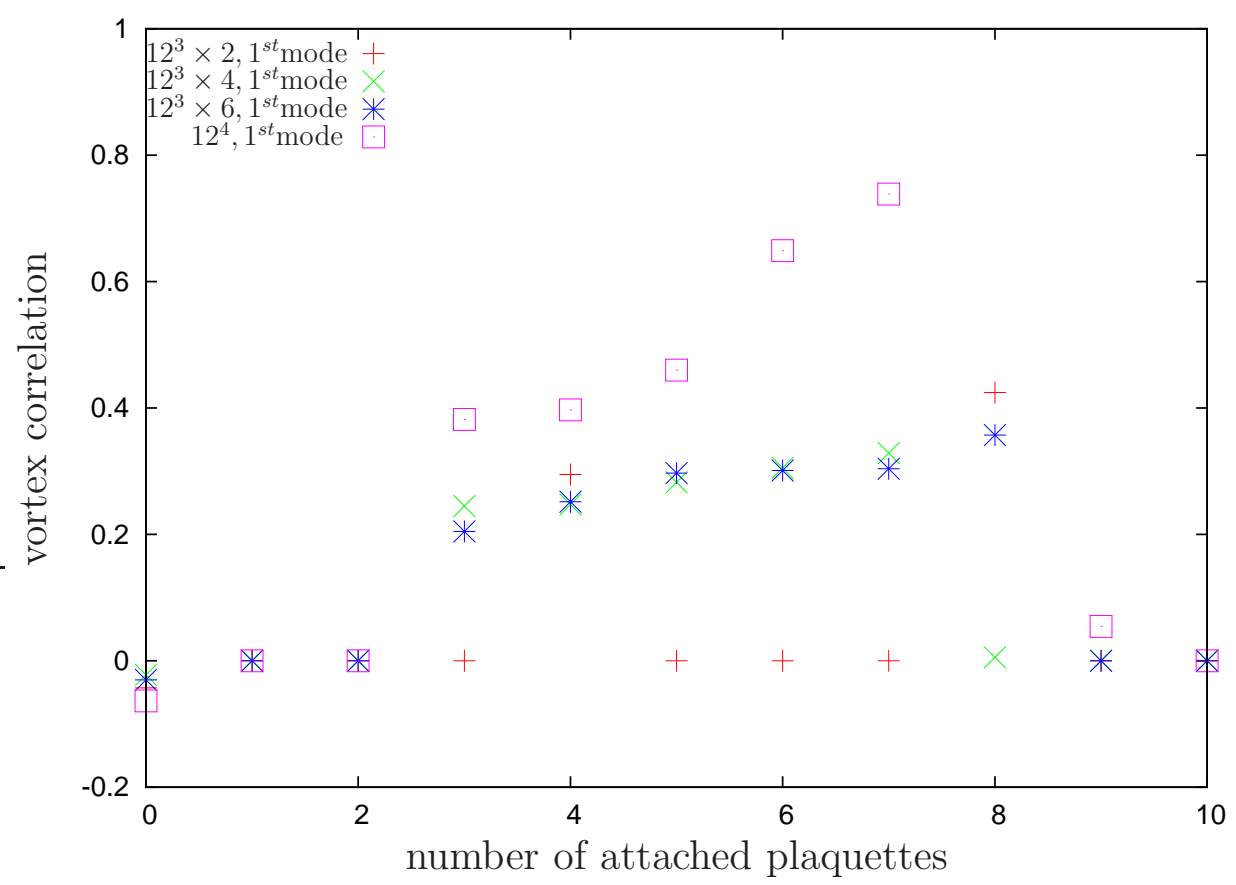

Figure 79: Correlation between eigenmode density and vortex surface for finite temperature configurations at $\beta_{L W}=3.5$ 



\section{Conclusion}

In this thesis the center vortex model of quark confinement was introduced and its confinement properties in $S U(2)$ lattice gauge theory with improved Lüscher-Weisz action were analyzed. The main goal of this work was to study the role of center vortices within the mechanism of chiral symmetry breaking. First, thick classical center vortices in the shape of planes (closed by lattice periodicity) and spheres were analyzed in terms of topological charge. The center vortex model explains topological charge with intersections of vortex sheets and so-called writhing points, regions where the vortex sheet twists around itself. By the Atiyah-Singer index theorem the topological charge of a gauge field is equal to the index of the Dirac operator, the difference of left- and right-handed chiral zero modes. Finally the gluonic charge in plaquette and hypercube definition, a lattice version of $F \bar{F}$, is measured after cooling. For non-orientable spherical vortices, the index of the overlap Dirac operator differs from the topological charge in the continuum limit. The reason for the seeming contradiction is the singular nature of the continuum gauge field equivalent to the spherical vortex. This singularity invalidates the usual derivation of the index theorem. These results were published in [77]. Furthermore, the same discrepancies are found for asqtad staggered fermions, implying that staggered fermions in fact feel the topological background of the gauge field.

The Dirac operators were next applied on $S U(2)$ Monte Carlo configurations generated with improved Lüscher-Weisz gauge action. In particular, the influence of center vortices on the low-lying eigenmodes of the Dirac operator, in both the overlap and asqtad staggered formulations are studied. By the Banks-Casher formula the density of lowlying Dirac modes is proportional to the chiral condensate, an order parameter for chiral symmetry breaking. For center-projected, i.e. vortex-only configurations, one finds that the low-lying near-zero modes are present in the asqtad staggered formulation, but not in the overlap and "chirally-improved" formulations. It is argued in [78] that this is due to the absence of a field-independent chiral symmetry in the latter formulations, when the Dirac operator is evaluated on the very rough configurations generated by center projection. Thus, the vortex excitations of the vortex-only lattice carry not only the information about confinement, but are also responsible for chiral symmetry breaking via the Banks-Casher relation. Furthermore, localization and scaling properties of the Dirac eigenmodes, with respect to center vortices were analyzed. Strong correlations between center vortex locations and the scalar density of low-lying Dirac eigenmodes were found. The low-lying eigenmodes have their largest concentration in point-like regions, rather than on sub manifolds of higher dimensionality. These eigenmode density peaks are located at regions with clearly enhanced vortex structure density. Taken together, these results also support the picture of a center vortex origin of topological charge, and indicate that center vortices have a strong effect on the properties of lowlying eigenmodes of the Dirac operator, i.e. chiral symmetry breaking via the BanksCasher relation. 



\section{A Methods and Operators}

The aim of the present work is to establish the mechanism of chiral symmetry breaking and its possible connection to center vortices. Therefore the following methods and tools are applied in $S U(2)$ lattice gauge field theory.

\section{A.1 Lüscher-Weisz Action}

The gauge action used in this work is a tadpole improved version of the one-loop continuum limit improved $S U(2)$ action of Lüscher and Weisz [79, 80, 81].

a)

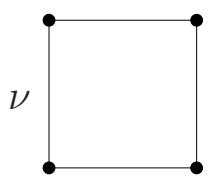

$\mu$ b)

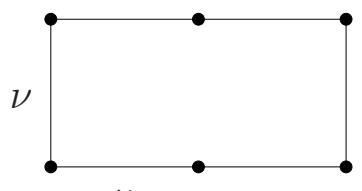

c)

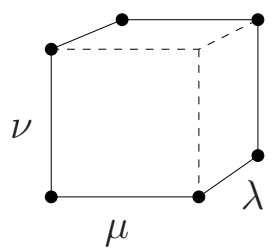

Figure 80: Lüscher-Weisz action Wilson loops: a) standard plaquette, b) $2 \times 1$ rectangle and c) $1 \times 1 \times 1$ parallelogram

The standard Lüscher-Weisz action removes leading ultra-violet cutoff effects by adding a few next-to-nearest neighbor terms to Wilson's action. In addition to the standard plaquette (labeled " $p l$ ") term, it includes a sum over all $2 \times 1$ (planar) rectangle (labeled " $r t$ ") and over all $1 \times 1 \times 1$ parallelogram (labeled " $p g$ ") Wilson loop terms (see Fig. 80). For $S U(N)$ lattice gauge fields $U_{\mu}(x)$ living on a four-dimensional $(\mu=0,1,2,3)$ hyper cubic lattice with sites $x$ and lattice spacing $a$, the improved action reads

$$
S[U]=\beta \sum_{x}\left\{c_{p l} \sum_{p l} S_{p l}+c_{r t} \sum_{r t} S_{r t}+c_{p g} \sum_{p g} S_{p g}\right\}
$$

where $\beta$ denotes the (inverse) coupling constant and $S_{i}=\frac{1}{N} \operatorname{Re} \operatorname{Tr}\left(1-U_{i}\right)$ with $U_{i}$ the corresponding Wilson loops. The coefficients $c_{i}=c_{i}^{0}+4 \pi \alpha_{0} \Delta_{i}$ for one-loop corrections $\Delta_{i}$ have been computed by Lüscher and Weisz for both $S U(2)$ and $S U(3)$ (Table 1 in Ref. [79]). Redefining $\beta \equiv \beta c_{p l}$ the perturbatively renormalized couplings $\beta_{i}=\beta c_{i} / c_{p l}$ for given $\beta$ read [80]

$$
\beta_{r t}=-\frac{\beta}{20}\left[1-\left(\frac{3}{5} \Delta_{p l}+12 \Delta_{r t}\right) 4 \pi \alpha_{0}\right], \quad \beta_{p g}=\frac{3}{5} \beta \Delta_{p g} 4 \pi \alpha_{0}
$$

The continuum limit behavior of the Lüscher-Weisz action can be further improved by making the lattice links more "continuum like". At the mean field level this entails setting $U_{\mu} \rightarrow u_{0}^{-1} U_{\mu}$, where one possible choice for the mean field (or "tadpole") factor 


\section{A Methods and Operators}

$u_{0}$ is using the expectation value of the average plaquette

$$
u_{0}=<\frac{1}{N} \operatorname{Re} \operatorname{Tr} U_{p l}>^{1 / 4} .
$$

The Lüscher-Weisz action can now be tadpole improved by explicitly pulling a $u_{0}^{-1}$ factor out of each link and replacing $\alpha_{0}$ in the one-loop perturbatively renormalized coefficients $c_{i}$ with a non-perturbatively renormalized coupling $\alpha_{s}$ defined through [80]

$$
\alpha_{s}=-4 \frac{\ln u_{0}}{\xi_{N}} \quad \text { with } \quad \xi_{N}=0.366262 \pi \frac{N^{2}-1}{N}= \begin{cases}1.72597, & \text { for } \mathrm{N}=2 \\ 3.06839, & \text { for } \mathrm{N}=3\end{cases}
$$

Defining $\beta_{L W} \equiv u_{0}^{-4} \beta$ (since $U_{p l}$ involves 4 links) the improved action reads for $S U(2)$ [80]

$$
S=\beta_{L W} \sum_{p l} S_{p l}-\frac{\beta_{L W}}{20 u_{0}^{2}}\left[1+0.2227 \alpha_{s}\right] \sum_{r t} S_{r t}-0.02224 \frac{\beta_{L W}}{u_{0}^{2}} \alpha_{s} \sum_{p g} S_{p g} .
$$

The tadpole factor $u_{0}$ is determined during thermalization and then kept fixed. One starts with an initial guess, simulates until the plaquette starts stabilizing, computes a new guess for $u_{0}$ by averaging over the last few iterations and iterates this procedure. Fortunately, the plaquette stabilizes quite fast and can be measured quite accurately (about 4 digits) after only a few iterations. In Table 7 the expectation value of the plaquette $u_{0}^{4}=\operatorname{Re} \operatorname{Tr}\left\langle U_{p l}\right\rangle / 2$ and the values of the new couplings for the treated ensembles are listed. The sample size at each value of $\beta$ is 100 configurations on $20^{4}$ lattices. The update was done with the heat-bath algorithm.

\begin{tabular}{c|ccccc}
$\beta_{L W}$ & 2.9 & 3.1 & 3.3 & 3.5 & 3.7 \\
\hline$u_{0}^{4}$ & $0.5606(2)$ & $0.6018(8)$ & $0.6206(8)$ & $0.6622(5)$ & $0.6722(9)$ \\
$c_{r t}$ & -0.1064 & -0.1017 & -0.0983 & -0.0959 & -0.0942 \\
$c_{p g}$ & -0.0289 & -0.0261 & -0.0242 & -0.0228 & -0.0218
\end{tabular}

Table 7: Parameters for the Lüscher-Weisz action: coefficients $c_{i}$ and expectation value of the plaquette $u_{0}^{4}=\operatorname{Re} \operatorname{Tr}<U_{p l}>/ 2$.

In a next step, in view of extracting physical quantities, the lattice spacing $a$ has to be determined. This is done by fitting the time-dependent potential $V(R, T)=$ $-\log (W[R, T-1] / W[R, T])$ with $W[R, T]$ the Wilson loop (see also section A.5) of size $R \times T$ in space-/time-direction respectively at some fixed $T$, to an ansatz $V(R)=$ $\sigma_{T} R-c / R+v_{0}$ (linear-plus-Coulomb fit). In order to obtain an asymptotic lattice string tension $\sigma_{\text {lat }}$ the extracted string tensions $\sigma_{T}$ for several $T$ values are fitted to some stabilizing function $f(T)=\exp (-k T+d)+\sigma$ with $\sigma$ giving the asymptotic $(T \rightarrow \infty)$ value. All fits were done by least-square routines. To set the scale the physical string tension, $a \sqrt{\sigma_{\text {lat }}}=\sqrt{\sigma_{\text {phys }}} \approx 0.44 \mathrm{GeV}$ [2], is used to determine the lattice spacing $a$. Since natural units, $\hbar=c=k_{B}=1$, are used throughout this work, energy and mass 


\section{A Methods and Operators}

have the dimension of inverse length $1 \mathrm{fm}^{-1}=197.327 \mathrm{MeV}$. Table 8 lists the data for runs of string tension determination on $20^{4}$-lattices with a 1000 thermalization steps, 1000 measurements separated by 200 iterations each, determined lattice spacings $a$ and corresponding physical volumes treated in this work. Since a vortex has a physical scale of about $1 \mathrm{fm}$, the physical extent of the lattice should not fall below 1.5fm. Fig. 81 shows the measured potential-data for different $\beta_{L W}$ 's at fixed $T$ and the fitted curves $V(R)$. Fig. 82a shows the measured potential-data for $\beta_{L W}=3.5$ for different $T$ s and the fitted curves $V(R)$. Fig. 82b shows the measured (fitted) $\sigma_{T}$ values for different $\beta_{L W} \mathrm{~S}$ and fitted curves $f(T)$.

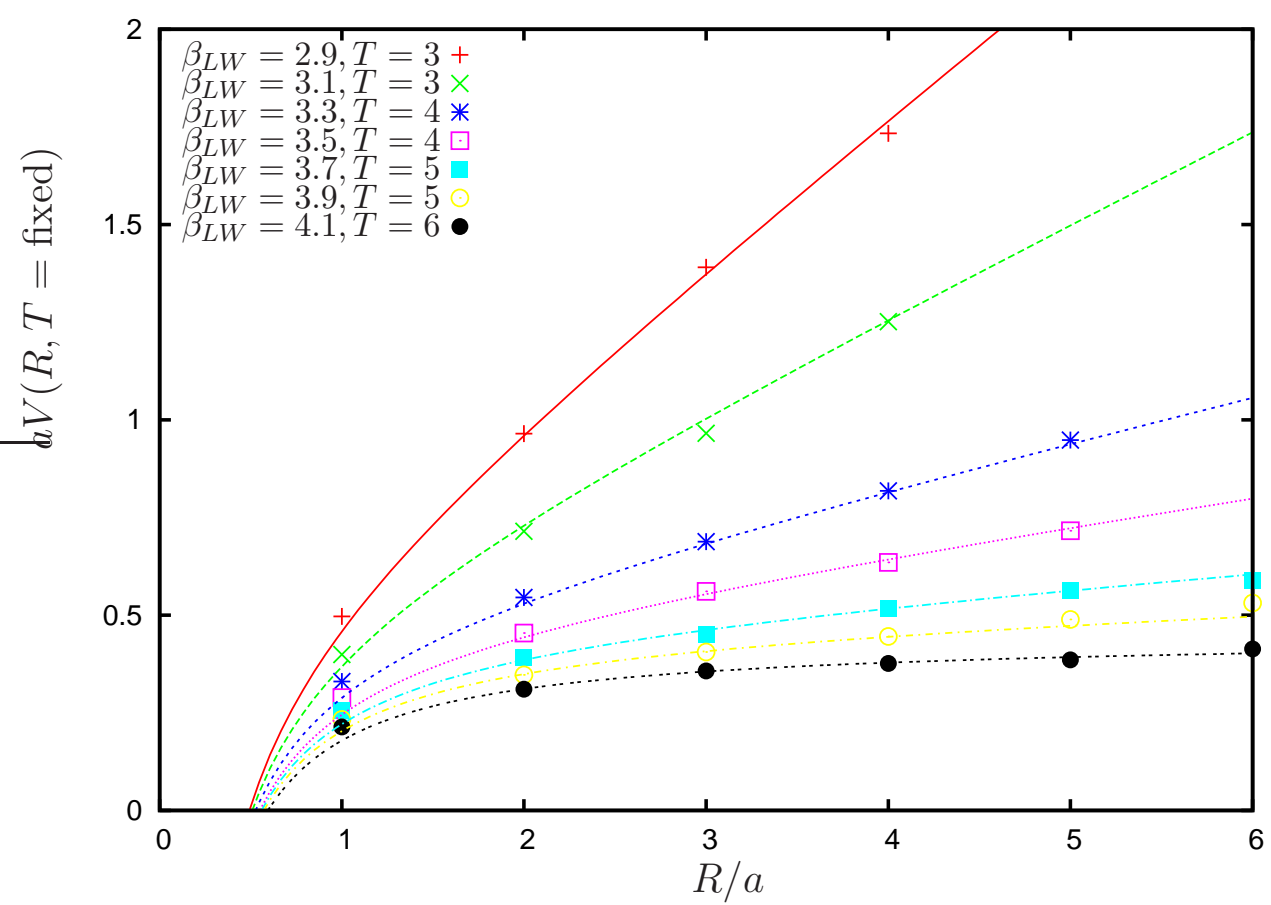

Figure 81: The static potential: data for different $\beta_{L W} \mathrm{~s}$ at fixed $T$ and fitted curves (linear-plus-coulomb fits to $V(R, T=$ fixed $)$ ) on a $20^{4}$-lattice

\begin{tabular}{c|cc|cc|c|c|c|}
$\beta_{L W}$ & $\sigma_{\text {lat }}$ & & $a[\mathrm{fm}]$ & & $L=12$ & $L=16$ & $L=20$ \\
2.9 & 0.3756 & \pm 0.0053 & 0.2749 & \pm 0.0019 & $3.27^{4}$ & $4.36^{4}$ & $5.45^{4}$ \\
3.1 & 0.2254 & \pm 0.0033 & 0.2129 & \pm 0.0016 & $2.58^{4}$ & $3.44^{4}$ & $4.29^{4}$ \\
3.3 & 0.1112 & \pm 0.0017 & 0.1495 & \pm 0.0012 & $1.78^{4}$ & $2.38^{4}$ & $2.97^{4}$ \\
3.5 & 0.0635 & \pm 0.0007 & 0.1138 & \pm 0.0006 & & $1.85^{4}$ & $2.31^{4}$ \\
3.7 & 0.0401 & \pm 0.0003 & 0.0898 & \pm 0.0003 & & & $1.63^{4}$ \\
4.0 & 0.0225 & \pm 0.0002 & 0.0673 & \pm 0.0002 & & &
\end{tabular}

Table 8: Lattice string tension $\sigma_{l a t}$, lattice spacing $a$ and corresponding physical volumes, as extracted from linear-plus-Coulomb fits to $V(R, T=$ fixed $)$ 
a)

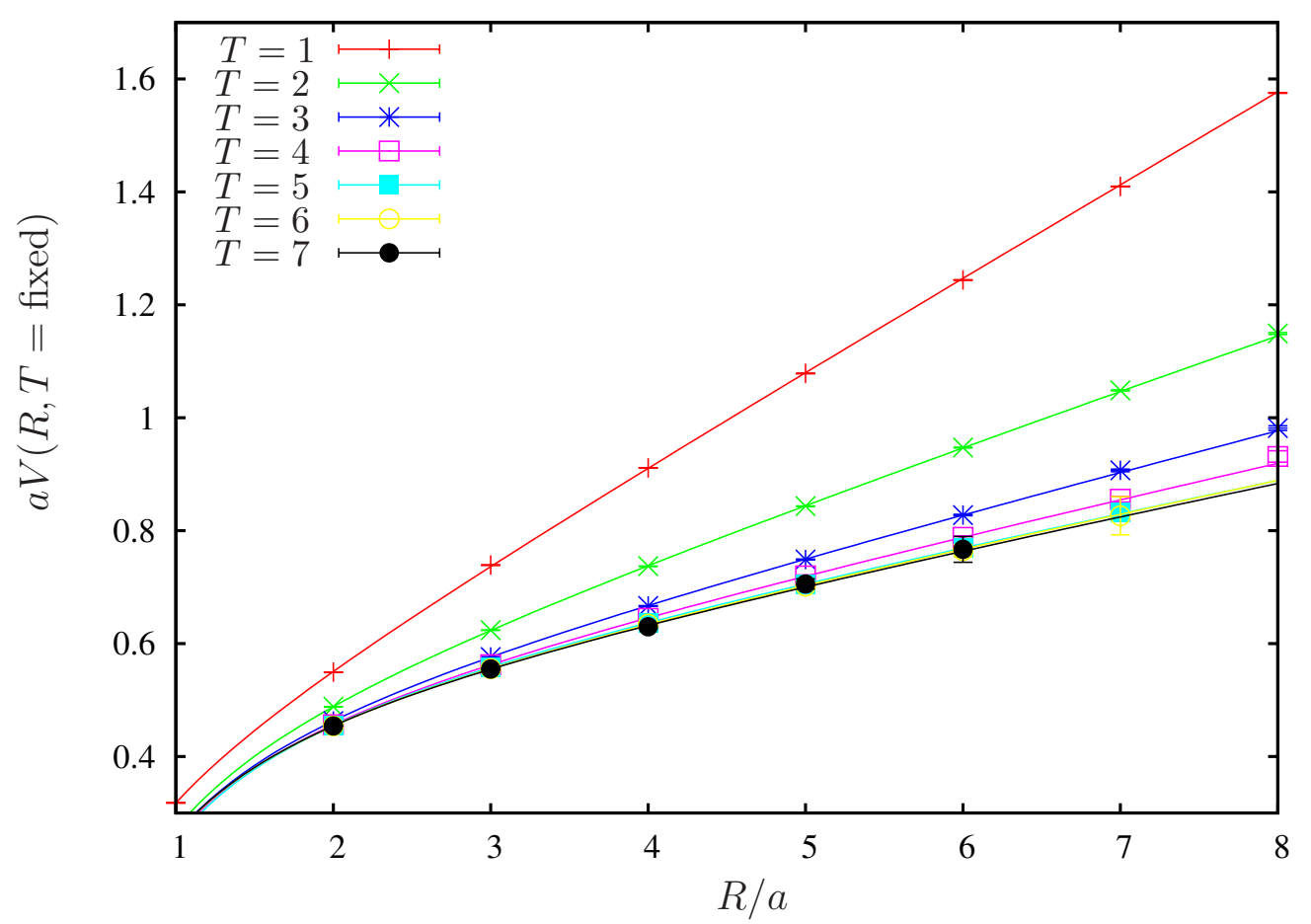

b)

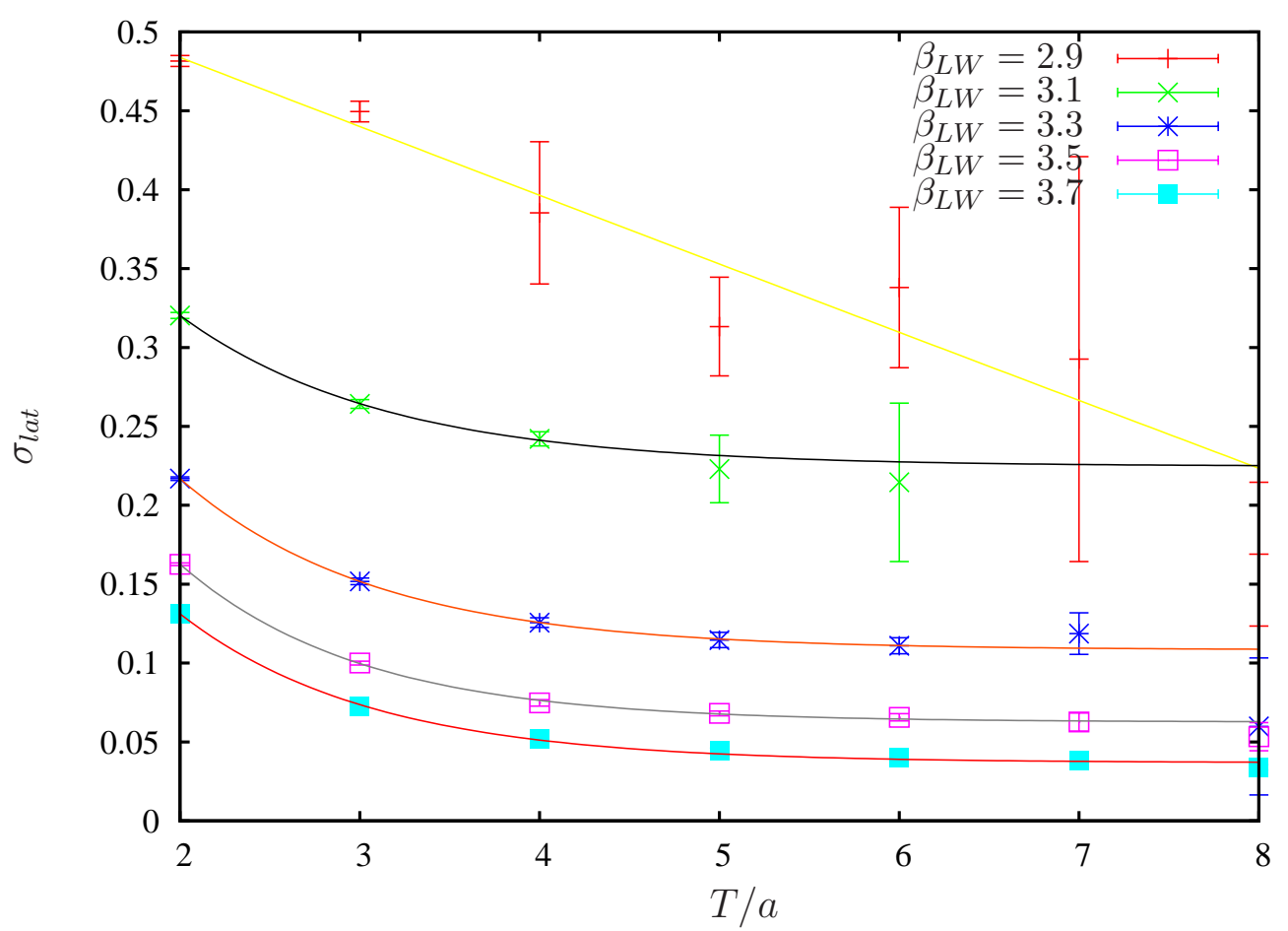

Figure 82: The static potential: a) data for $\beta_{L W}=3.5$ for different $T$ s and the fitted curves $V(R)$ (linear-plus-coulomb fits to $V(R, T=$ fixed)), b) measured (fitted) $\sigma_{T}$ values for different $\beta_{L W} \mathrm{~s}$ and fitted curves $f(T)$ on a $20^{4}$-lattice 


\section{A.2 Fermionic Actions}

\section{A.2.1 Fermion Doubling}

A naive discretization of the continuum Dirac operator leads to unphysical particle content. Fermion fields experience (at least) a doubling of the number of particle types on a discrete space-time lattice. Since fermion functions are defined on lattice points only, the momentum $k$ can be restricted to the first Brillouin zone $B=\left(-\frac{\pi}{a}, \frac{\pi}{a}\right]^{d}$ in $d$ dimensions. On the fourier transformed lattice, functions have to be periodic in $k$.

The eigenvalue of the Dirac operator goes like

$$
\lambda \propto \frac{\sin (k a)}{a}
$$

In the low eigenvalue limit, there are two different regions: one about $k=0$ and another about $k=\pi / a$. They behave like two different particles. In $d$ dimension this leads to $2^{d}$ different types (tastes/flavors) of particles (quarks). To bypass the fermion doubling problem, Wilson suggested to add a term to the action with the effect that the extra fermions acquire an infinite mass in the continuum limit $a \rightarrow 0$.

\section{A.2.2 Exact Chiral Symmetry}

Investigating the chirality of the fermion doublers, half of them turn out to be left- and the others to be right-handed. Nielsen and Ninomiya $[82,83]$ showed that one cannot solve the fermion doubling problem without breaking chiral symmetry in the continuum limit $m \rightarrow 0$. The Nielsen-Ninomiya theorem requires $\left\{D, \gamma_{5}\right\}=0$ to make the fermion action invariant under usual chiral rotations. Instead, the Ginsparg-Wilson relation [84]

$$
\left\{D, \gamma_{5}\right\}=2 a D \gamma_{5} D
$$

in the continuum limit $a \rightarrow 0$ reduces to the usual chiral symmetry, so that actual physics are not affected.

\section{A.2.3 The Overlap Operator}

The overlap Dirac operator $[18,47,85]$ is a special way to define the determinant of the chiral Dirac operator

$$
\operatorname{det} C[A]=\int \mathcal{D} \psi \mathcal{D} \bar{\psi} e e^{-S(\psi, \bar{\psi}, A)} \Leftrightarrow\langle 0-\mid 0+\rangle_{A}
$$

as an overlap of the ground states $|0 \pm\rangle$ of two many-body Hamiltonians $H^{ \pm}$with

$$
H^{-}=\gamma_{5}=\left(\begin{array}{cc}
1 & 0 \\
0 & -1
\end{array}\right) ; \quad H^{+}=\gamma_{5}\left(\gamma_{\mu} D_{\mu}-m\right)=\left(\begin{array}{cc}
-m & C(A) \\
C^{\dagger}(A) & m
\end{array}\right)
$$




\section{A Methods and Operators}

The massless lattice overlap Dirac operator is defined by

$$
D_{o v}=\frac{1}{2}\left[1+\gamma_{5} \epsilon\left(H_{L}^{+}\right)\right]
$$

where on the lattice $H^{-} \rightarrow H_{L}^{-}=\gamma_{5}$ remains unchanged, $\epsilon(x)$ denotes the sign function and $H^{+} \rightarrow H_{L}^{+}=\gamma_{5} D_{w}(-m)$ with $D_{w}$ the usual lattice Wilson Dirac operator for $r=1$

$$
D_{w x, y}(m)=-\frac{1}{2} \sum_{\mu}\left[\left(1+\gamma_{\mu}\right) U_{\mu}(x) \delta_{\hat{\mu}, y}+\left(1-\gamma_{\mu}\right) U_{\mu}^{\dagger}(x-\hat{\mu}) \delta_{x-\hat{\mu}, y}-(m+4) 2 \delta_{x, y}\right]
$$

The numerical implementation of the overlap Dirac operator $[48]^{4}$ provides eigenvalues $|\lambda| \in[0,1]$. Since this operator obeys the Ginsparg-Wilson relation all eigenvalues are restricted to the so-called Ginsparg-Wilson circle, a circle with radius $\frac{1}{2}$ and center $\left(\frac{1}{2}, 0\right)$ in complex plane. Therefore the two corresponding eigenvalues $\lambda$ and $\lambda^{*}$ are constructed as follows (see also Fig. 83).

$$
\begin{array}{ll}
\lambda=x+i y, \quad \lambda^{*}=x-i y & \\
x^{2}+y^{2}=\lambda^{2}, & (1 / 2-x)^{2}+y^{2}=1 / 4 \quad \text { (Fig.83) } \\
\rightarrow \quad x=\lambda^{2}, & y=\sqrt{\lambda^{2}-\lambda^{4}}
\end{array}
$$

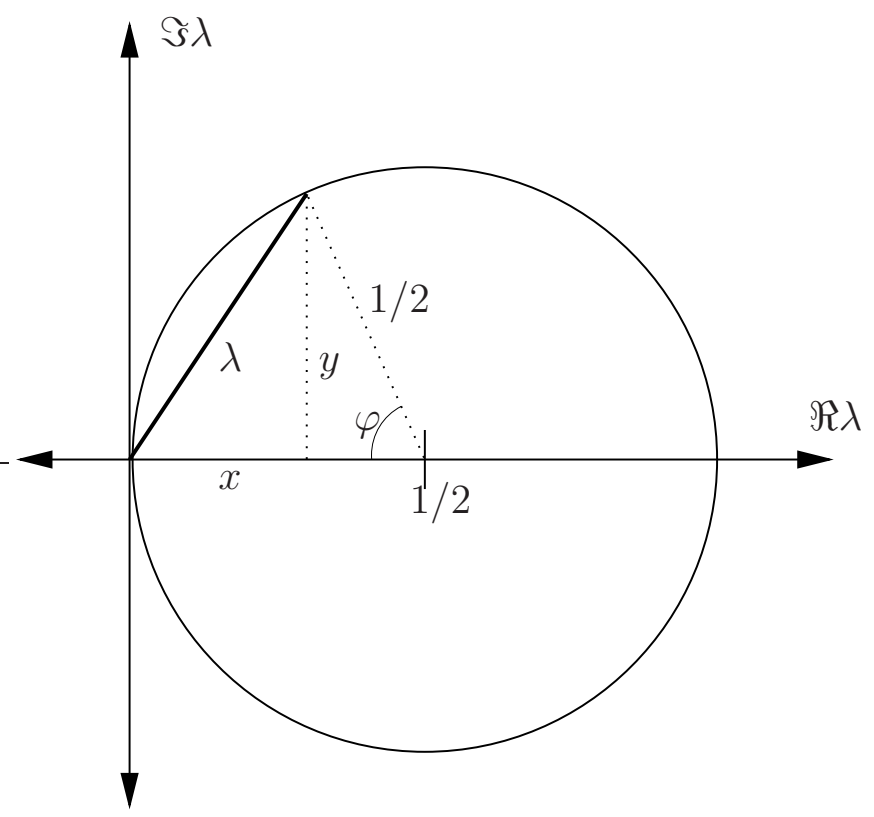

Figure 83: Ginsparg-Wilson circle with eigenvalue $\lambda$

\footnotetext{
${ }^{4}$ The numerical implementation of all Dirac operators were provided by Urs M. Heller, School of Computational Science \& Information Technology, the Florida State University
} 


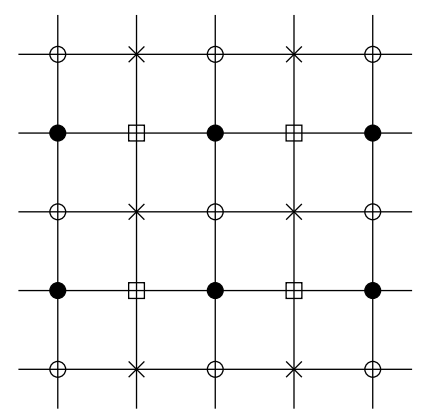

Figure 84: Distributing four degrees of freedom on a two dimensional lattice

\section{A.2.4 Staggered Fermions}

Another possibility to bypass the fermion doubling problem is to construct a nonlocal action where the Dirac operator is treated as a square root. In order to eliminate the unwanted fermion modes one can reduce the Brillouin zone, i.e. double the effective lattice spacing $a$. [86] This is done by distributing the fermionic degrees of freedom over the lattice in such a way that the effective lattice spacing for each type of Grassmann variable is twice the fundamental lattice spacing, i.e. putting different fermionic tastes on every other lattice site (see Fig. 84). On a four dimensional lattice one therefore needs four different tastes which can be interpreted as four different quark flavors (i.e. "up", "down", "strange", etc.). The basic term of the staggered fermion action is the so-called "Kogut-Susskind" term

$$
\bar{\psi} D \psi=\frac{1}{2 a} \bar{\psi}(x) \sum_{\mu} \gamma_{\mu}\left[U_{\mu}(x) \psi(x+\hat{\mu})-U_{\mu}^{\dagger}(x-\hat{\mu}) \psi(x-\hat{\mu})\right]
$$

which is illustrated in Fig. 85. In order to reduce the action in the naive continuum limit to the desired continuum form one adds a couple of next-to-nearest neighbor and staple terms ("asqtad" - "a-squared tadpole" - improvement [61, 62, 63]), namely the Naik term, 3-, 5- and 7-staple terms and the Lepage term. The prefactors for the single terms are derived perturbatively again, using the above tadpole factor $u_{0}$ as well. Finally, in

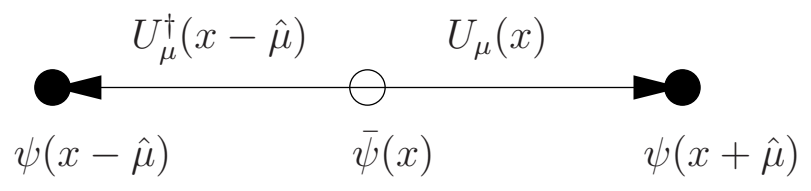

Figure 85: Kogut-Susskind term combines $\bar{\psi}$ and nearest neighbored $\psi \mathrm{s}$

order to reduce the number of tastes from four to one, one can take the fourth root of the Dirac determinant. So far it is not clear, if this "mixing" of tastes and chiralities (the four tastes have pairwise opposite chiralities), still produces physically correct results. 


\section{A Methods and Operators}

\section{A.3 Dirac Eigenmodes}

\section{A.3.1 Atiyah-Singer index theorem and exact zero modes}

In the continuum, integrating the anomaly equation gives a relation for the topological charge $Q=m \operatorname{tr}\left(\gamma_{5} S_{F}\right)$, where $S_{F}$ is the fermion propagator. Resolving the propagator in a sum over eigenmodes of the Dirac operator $\not D$, and noting that all modes with nonzero eigenvalue come in conjugate pairs, one gets the Atiyah-Singer index theorem relating the analytical index, the number of exact zero modes to the topological index, the topological charge: $[18,47,56]$

$$
\operatorname{ind} \not D[A]=n_{-}-n_{+}=Q[A],
$$

with $n_{-}, n_{+}$number of left-/right-handed zero modes [19,20,21]. On the lattice, both sides of the index equation are slightly distorted. The flavor singlet $\gamma_{5}$ does not exactly anti commute with $\not D$, so the trace of $\gamma_{5} S_{F}$ cannot be collapsed into a trace in the zero mode sector alone. Further, there are no exact zero modes, nor is there an exact definition of the topological charge. Nevertheless, the lattice version of the index theorem should hold as long as the gauge field is sufficiently close to the continuum. It has been shown for the overlap Dirac operator that this fermionic definition of $Q$ coincides with the continuum simple gluonic definition in the continuum limit [49].

\section{A.3.2 Banks-Casher relation and near-zero modes}

The Banks-Casher relation [22] gives a connection between chiral symmetry breaking and low-lying eigenmodes of the Dirac operator. The chiral condensate is given by

$$
\int d^{4} x\langle\bar{\psi} \psi\rangle=-\left.\frac{\partial}{\partial m} \ln Z(m)\right|_{m \rightarrow 0}
$$

where $Z(m)$ is the functional integral in Euclidean space

$$
Z(m)=\int \mathcal{D} A_{\mu} \mathcal{D} \psi \mathcal{D} \bar{\psi} \exp \left[-\frac{1}{4} \int F_{\mu \nu} F_{\mu \nu}-\sum_{f}^{N_{f}} \int \bar{\psi}(\not D+m) \psi\right]
$$

On the lattice

$$
-\frac{\partial}{\partial m} \ln Z(m)=\left\langle\operatorname{Tr} \frac{m}{D+m^{2}}\right\rangle \rightarrow \int_{-\infty}^{\infty} d \lambda \rho(\lambda) \frac{m}{\lambda^{2}+m^{2}}
$$

with $\rho(\lambda)$ the spectral density of the eigenvalue $\lambda$. Since $\lim _{m \rightarrow 0} \frac{2 m}{\lambda_{n}^{2}+m^{2}} \longrightarrow \pi \rho(0)$, one obtains the Banks-Casher relation

$$
\langle\bar{\psi} \psi\rangle=\frac{\pi \rho(0)}{V}
$$

which relates the chiral condensate to the spectral density of near-zero modes. 


\section{A.3.3 Localization of eigenmodes}

In order to localize the eigenvectors $\vec{v}$ appropriate observables are the scalar density

$$
\rho(x)=\sum_{c, d}\left|\vec{v}(x)_{c d}\right|^{2}
$$

where the summation indices $c$ and $d$ refer to color and Dirac indices and further the chiral densities $\rho_{5}(x), \rho_{+}(x)$ (left-handed) and $\rho_{-}(x)$ (right-handed)

$$
\begin{gathered}
\rho_{5}(x)=\sum_{c, d, d^{\prime}} \vec{v}(x)_{c d}^{*} \gamma_{5}^{c, d^{\prime}} \vec{v}(x)_{c d^{\prime}} \\
\rho_{ \pm}(x)=\sum_{c, d, d^{\prime}} \vec{v}(x)_{c d}^{*} \frac{1 \pm \gamma_{5}^{c, d^{\prime}}}{2} \vec{v}(x)_{c d^{\prime}}
\end{gathered}
$$

\section{A.4 Direct Maximal Center Gauge}

[26] Direct maximal center gauge (DMCG) is the common name for lattice Landau gauge in the adjoint representation. This gauge is used to locate center vortices in thermalized lattice configurations, which are condensates of thick vortices and other fluctuations and excitations. Since vortices carry magnetic color flux quantized in terms of elements of the center $Z_{N}$ of the gauge group $S U(N)$, most vortex detection methods try to center project such a field configuration, changing all link variables to their nearest center element as will be described in section A.4.1, revealing a thin vortex configuration. Therefore, maximal center gauge (MCG) was the first gauge used to identify vortices, because it gauges links such that they are already as close as possible - on average - at the center elements.

Historically the first type of MCG was an indirect version (IMCG), which builds on an older gauge, the maximal Abelian gauge (MAG). This gauge moves the link variables as close as possible to some chosen Abelian subgroup $U(1)$ of the gauge group $S U(N)$. After Abelian projection the IMCG is fixed and Abelian monopoles can be identified. It has been found that the world lines of Abelian monopoles are located mostly on thin vortex world sheets [27].

DMCG tries to find the absolute maximum of the gauge functional

$$
R=\sum_{\mu, x} \operatorname{Tr}\left[U_{\mu}^{a g}(x)\right]=\sum_{\mu, x} \operatorname{Tr}\left[S_{a g}^{-1} U_{\mu}^{a g}(x) S_{a g}\right]
$$

with links $U_{\mu}^{a g}(x)$ and the gauge matrix $S_{a g}$ in the adjoint representation of gauge group elements $g(x)$. For $S U(2)$, finding the maximum of (53) is equivalent to find the maximum of

$$
R^{\prime}=\sum_{\mu, x}\left|\operatorname{Tr} U_{\mu}^{g}(x)\right|^{2}
$$

with links $U_{\mu}^{g}(x)$ in the fundamental representation. 
In order to maximize a functional $R[\Sigma]$ of a field configuration $\Sigma$ one uses iterative methods, generating a sequence of configurations, each created by a gauge transformation from the previous configuration, which approach a maximum of $R$.

The over-relaxation method [87] changes iteratively, locally the field to some combination of the original field, and of a field which maximizes the functional locally. Over-relaxation finds quite effectively a (local) maximum of $R[\Sigma]$.

Because of the adjoint representation, DMCG is center blind and after gauge fixing there remains a residual gauge freedom stemming from the ambiguous mapping from the gauge matrices $S_{g}$ to the fundamental gauge elements $g$. But apart from this there can be some more ambiguities in gauge fixing because the iterative methods might find not the global, but some local maximum of $R[\Sigma]$. The existence of such Gribov copies will emerge to be important for the detection of vortices.

\section{A.4.1 Detection of Vortices}

[57] Extracting the vortex content of an arbitrary gauge field configuration is accomplished by a procedure known as center projection [88]. It proceeds in two steps:

1. Adjoint Gauge: First, all link variables are uniquely fixed in the DMCG, the adjoint representation of Landau gauge. For the $S U(2)$ group, adjoint links $U_{\mu}^{a g}(x)$ in this gauge satisfy the condition

$$
\operatorname{Tr} \sum_{\mu} L_{i}\left(U_{\mu}^{a g}(x)-U_{\mu}^{a g}(x-\hat{\mu})\right)=0
$$

at every point $x$ (the $L_{i}$ are the $S U(2)$ group generators in the adjoint representation). Since this representation maps all center elements to unity, the gauge leaves a residual center symmetry.

2. Center projection proper: The link in the fundamental representation is decomposed as a product

$$
U_{\mu}^{a g}(x)=Z_{\mu}(x) V_{\mu}(x)
$$

where $Z_{\mu}(x)$ is the center element $Z_{N}$ which is on the $S U(N)$ manifold closest to $U_{\mu}^{a g}(x)$, in the sense of the distance $\frac{1}{2} \operatorname{tr}\left(Z_{\mu}^{*} U_{\mu}^{a g}\right)$. For $S U(2)$, the nearest center element can be found by choosing the sign of the trace

$$
Z_{\mu}(x)=\operatorname{sign} \operatorname{Tr}\left[U_{\mu}^{a g}(x)\right]
$$

Center projection consists in replacing $U_{\mu}^{a g}(x)$ by $Z_{\mu}(x)$, giving the so-called centerprojected field configuration.

Upon creation of a thin center vortex by a "singular gauge transformation", the links are multiplied by a center element $Z^{\prime}$. As the adjoint representation is blind to the center, this change carries over to $U^{a g}$ and therefore

$$
U_{\mu}^{\prime a g}(x)=Z_{\mu}^{\prime}(x) Z_{\mu}(x) V_{\mu}(x)
$$


in a 3-dimensional volume bordered by the vortex surface. Since center projection extracts $Z^{\prime} Z$, thin vortices appear as discontinuity sheets in the resulting $Z_{N}$ configuration at the same points where they were originally created. Thick vortices are collapsed into thin ones, but their location is not uniquely determined. The thin vortices in the projected configuration are known as P-vortices and the plaquettes they pierce are called $\mathrm{P}$-plaquettes. Hence a P-plaquette is always equal to $-\mathbb{1}$. The rationale just presented suggests that $\mathrm{P}$-vortices represent the vortex content of the original configuration.

Problems with this interpretation arise from the finite thickness of center vortices and the occurrence of Gribov copies in gauge fixing. The latter usually consists in the minimization of the gauge functional of the links. Gribov copies are distinct local minima of this functional. Usually it is not possible to track down the global minimum, which would be the correct solution. In order to somehow avoid the Gribov problem, the easiest way is to average over all gauge copies in the Gribov region. For a more detailed discussion of the Gribov Problem in DMCG see [89].

Finally, there exist several procedures for center-projection which may differ in their results. It is not entirely clear why one should be more meaningful than the other. However, any method should meet a minimum requirement, dubbed the "vortex-finding property" [88]: the ability to locate thin vortices inserted "by hand" into an arbitrary gauge field configuration.

\section{A.4.2 Smoothing Vortex Configurations}

An example for a P-vortex, extracted from a Monte-Carlo configuration, is shown in Fig. 86. The vortices form closed surfaces of random structure.

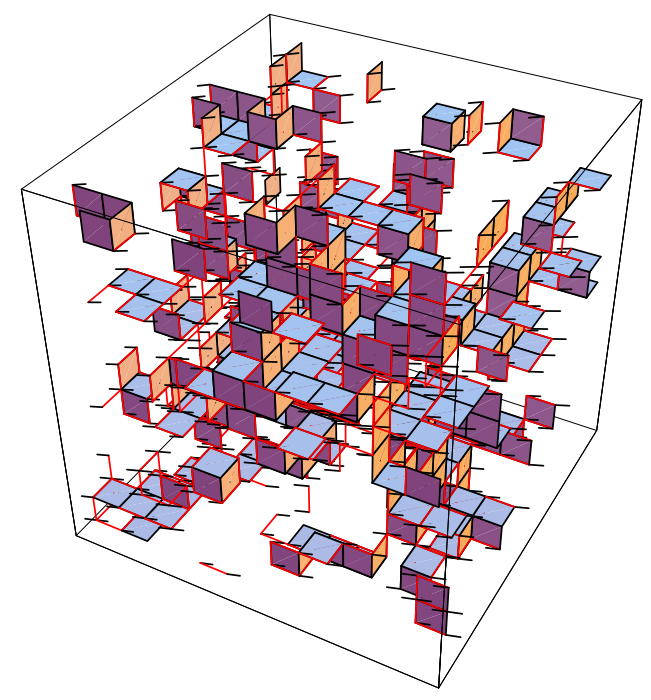

Figure 86: 3-dimensional cut through the dual of a $12^{4}$-lattice.

In order to reduce the small fluctuations of the P-vortices several smoothing steps as depicted in Fig. 87 are performed. This vortex smoothing procedure [26] iteratively scans for cubes of the lattice and transforms them according to the following rules: 


\section{A Methods and Operators}

a) remove single isolated $\mathrm{P}$-vortex cubes

b) identify cubes covered by five P-vortex plaquettes and substitute them by one complementary plaquette

c) substitute cubes with four P-plaquettes which are positioned opposite on the cube by the two missing plaquettes (disconnects two surfaces)

d) substitute cubes with four P-plaquettes by the two missing plaquettes joining a common link

These smoothing steps consist of elementary cube transformations and preserve the closeness of the P-vortex surface. The result of applying repeatedly all smoothing steps a)-d) on the configuration of Fig. 86 is presented in Fig. 88.

\section{A.4.3 Center Vortex Removal}

It was suggested by de Forcrand and D'Elia [14] that one could remove center vortices from a given lattice configuration by multiplying the original links $U_{\mu}(x)$ with the corresponding center-projected links $Z_{\mu}(x)$. Applying the vortex finding procedure to the modified configuration $U_{\mu}^{\prime}(x)$, one can easily check that there are no P-vortices obtained from center projection, since

$$
Z_{\mu}^{\prime}(x)=\operatorname{sign} \operatorname{Tr}\left[U_{\mu}^{\prime a g}(x)\right]=Z_{\mu}^{2}(x)=1
$$

One can therefore say that center vortices have been removed from the lattice configuration, which is denoted as vortex removed configuration. More precisely, what the modification does is to place a thin vortex (one plaquette thickness) in the middle of each thick center vortex core, whose locations are identified by center projection. At large scales, the effects of the thin and thick vortices on Wilson loops will cancel out.

\section{A.5 The Wilson Loop and the $q \bar{q}$-potential}

For large distance $R$ a simple ansatz for the continuum potential $V(R)$ between two infinitely heavy $\left(m_{q} \rightarrow \infty\right)$ quarks $(q \bar{q})$ is given by

$$
V(R)=\sigma R+v_{0}+\alpha R^{-1}+O\left(R^{-2}\right),
$$

with the string tension $\sigma$ and the Coulomb coefficient $\alpha$. In order to measure this potential, one creates a quark-antiquark pair, separated by a distance $R$, propagating for a time interval $t=[0, T]$. In the absence of gauge fixing, the expectation value of a color non-singlet state will average out to zero, so it is necessary to include a parallel transporter between the quarks in order to form a gauge-invariant creation operator $Q(t)$. The parallel transporter along a curve $C$ in the background of the gauge field $A_{\mu}(x)$ is given by

$$
U(C)=\mathcal{P} \exp \left[i g \int_{C} A_{\mu}(x) d x_{\mu}\right],
$$

where $\mathcal{P}$ denotes the path ordering operator. On the lattice, $U(C)$ is represented by the product of link variables $U_{\mu}$ along the curve $C$ (a "Wilson line"). 


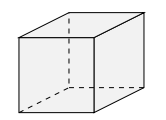

a) 6 plaquettes $\rightarrow 0$ plaquettes
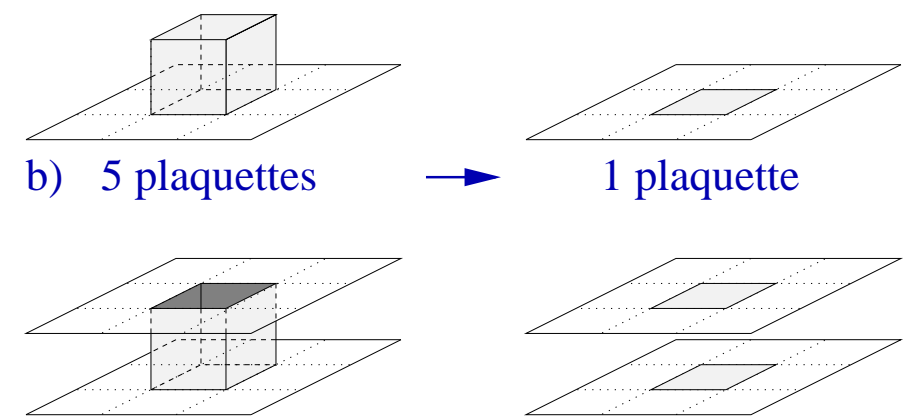

c) 4 plaquettes
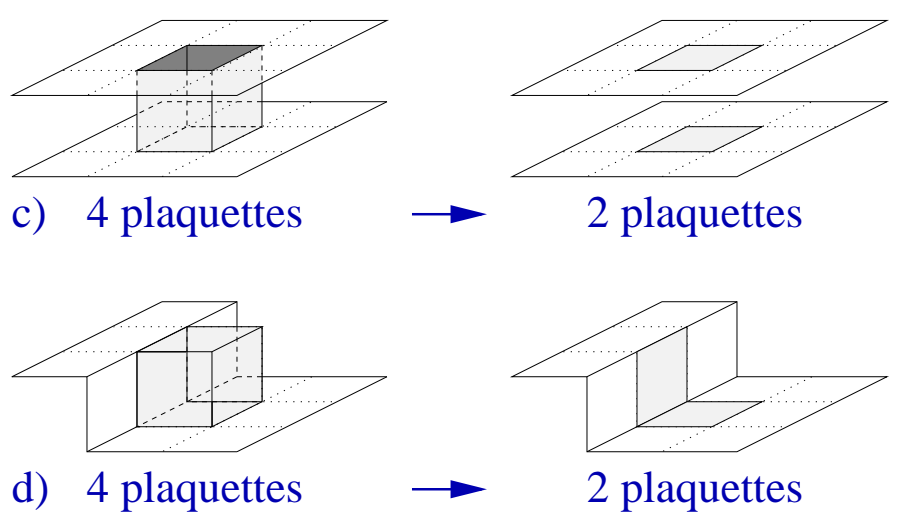

d) 4 plaquettes

Figure 87: Various smoothing steps for vortices

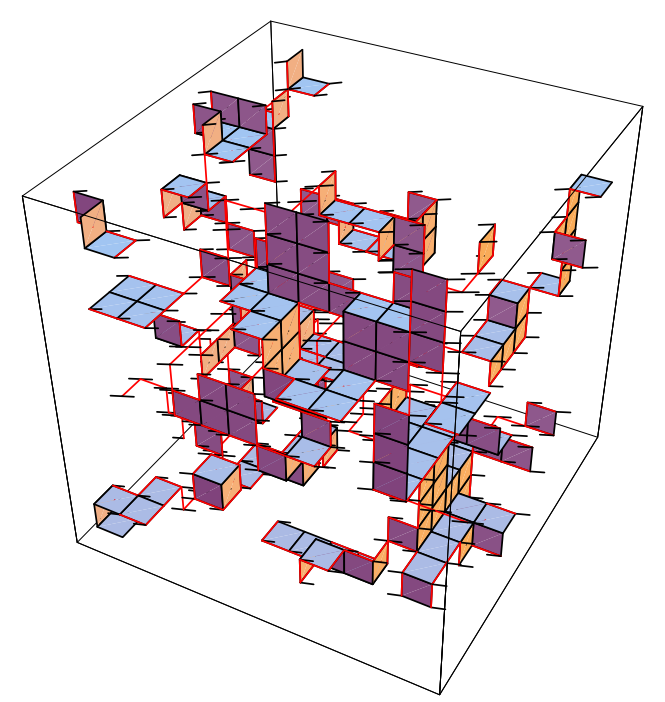

Figure 88: Result of applying all smoothing steps a)-d) on the configuration of Fig. 86. 


\section{A Methods and Operators}

Choosing a closed loop as the path $C$ and taking the trace, one gets a gauge invariant expression

$$
W(C)=\operatorname{Tr} U(C)=\operatorname{Tr} \prod_{\mu \in C} U_{\mu}
$$

\section{the Wilson loop.}

Rectangular $R \times T$ Wilson loops $W(R, T)$ in the fundamental representation can be used to extract the lattice potential. One can regard the expectation value of the Wilson loop as the creation $Q(0)$ of a quark-antiquark pair at a time $t=0$, and its annihilation $Q^{\dagger}(T)$ at a time $t=T$, propagating in time at a static separation by a distance $R$ (including the shortest Wilson lines joining the quark operators in $Q$ and $Q^{\dagger}$, giving the Wilson loop around the rectangle $R \times T$ ). Then, following the usual rules of quantum mechanics and integrating out the quark fields in the path integral [28] one gets

$$
\left\langle Q^{\dagger}(T) Q(0)\right\rangle \propto e^{-V(R) T} \propto\langle W(R, T)\rangle .
$$

Therefore, the static potential follows from the Wilson loop (up to a constant) as

$$
V(R) \propto-\lim _{T \rightarrow \infty} \frac{1}{T} \log \langle W(R, T)\rangle
$$

or without the integration constant

$$
V(R)=-\lim _{T \rightarrow \infty} \log \left[\frac{\langle W(R, T+1)\rangle}{\langle W(R, T)\rangle}\right]
$$

Hence, the expectation value of the Wilson loop provides an order parameter for confinement. In the strong-coupling phase (confinement) the potential is asymptotically rising linearly: $V(R) \approx \sigma R$, with the string tension $\sigma$. The Wilson criterion implies an area law for the Wilson loop

$$
\langle W(R, T)\rangle \propto \exp (-\sigma R T) \propto \exp (-\sigma A) \quad \rightarrow \quad \text { confinement }
$$

with area $A=R T$, whereas in the weak-coupling (deconfined) phase the potential tends to a constant $V(R) \rightarrow \mu$, which leads to a perimeter law

$$
\langle W(R, T)\rangle \propto \exp (-\mu T) \propto \exp (-\mu P) \quad \rightarrow \quad \text { deconfinement }
$$

with perimeter $P \approx T$ for $T \gg R$ since the limit $T \rightarrow \infty$ is required.

Only for large areas $A$ the Wilson loop is governed, if confinement is present, by the area law. In order to extract the string tension $\sigma$ already from smaller loops, the Creutz ratio

$$
\chi(R, T)=-\log \left[\frac{\langle W(R, T)\rangle\langle W(R+1, T+1)\rangle}{\langle W(R, T+1)\rangle\langle W(R+1, T)\rangle}\right]
$$

can be used. In this expression perimeter law and constant factors cancel, and already for moderate areas the Creutz ration approaches the string tension $\chi(R, T) \stackrel{A \rightarrow \infty}{\rightarrow} \sigma$. 


\section{A.6 The Polyakov Loop, Free Energy and Center Symmetry}

The Polyakov loop $P(\vec{x})$ is just a Wilson loop which is periodic in Euclidean time direction. It is defined as the holonomy for a curve extending straightly in time direction. On the lattice it is composed of link variables in time direction and reads

$$
P(\vec{x}):=\frac{1}{N} \operatorname{Tr} \prod_{x_{4}=1}^{N_{T}} U_{4}\left(\vec{x}, x_{4}\right) .
$$

A Polyakov loop can be thought of as the world-line of a massive static quark at spatial position $\vec{x}$, propagating only in the periodic time direction. The static quark potential can be extracted from the correlator of two Polyakov loops at a distance $R=|\vec{x}-\vec{y}|$

$$
\left\langle P(\vec{x}) P^{\dagger}(\vec{y})\right\rangle-\langle P(\vec{x})\rangle\left\langle P^{\dagger}(\vec{y})\right\rangle \propto \exp \left(-V(R) N_{T}\right)
$$

with $N_{T}$ the lattice extension in time direction. For large distances one can obtain from the correlators the free energy of a single static charge

$$
\lim _{|\vec{x}-\vec{y}| \rightarrow \infty}\left\langle P(\vec{x}) P^{\dagger}(\vec{y})\right\rangle=|\langle P\rangle|^{2} .
$$

Therefore, the expectation value of the modulus of $P(\vec{x})$, averaged over the position $\vec{x}$, can be related to the free energy of an isolated quark, measured relatively to the absence of the quark:

$$
\langle|P|\rangle=\left\langle\left|\frac{1}{N_{S}^{3}} \sum_{\vec{x}} P(\vec{x})\right|\right\rangle \stackrel{N_{S} \rightarrow \infty}{=} e^{-F N_{T}}
$$

In the confinement phase, the free energy of an isolated quark is infinite, hence the Polyakov loop vanishes $(\langle P(\vec{x})\rangle=0)$, while in the deconfinement phase the free energy of an isolated quark is finite and $\langle P(\vec{x})\rangle \neq 0$. This behavior reflects a global symmetry under $\mathbb{Z}_{N}$ central conjugations (center symmetry). Suppose to multiply all temporal links which are located in a hyper surface at fixed time $t_{0}$ by a non-trivial center element $z$

$$
U_{4}\left(\vec{x}, t_{0}\right) \rightarrow z U_{4}\left(\vec{x}, t_{0}\right) \quad \forall \vec{x} .
$$

It is straightforward to see that the space-time plaquettes are left unchanged, but the Polyakov loops pick up a factor $z$. So if this $\mathbb{Z}_{N}$ symmetry is spontaneously broken, at the phase transition the expectation value of the Polyakov loop should jump from zero to a non-zero value and this is exactly what happens in lattice computations.

The Polyakov loop is a true order parameter for confinement and center symmetry: zero in confinement phase and therefore trivially invariant under center transformations, non-zero in deconfinement phase, which associates the breaking of global center symmetry from the confinement to the deconfinement phase

$$
\langle P(\vec{x})\rangle \begin{cases}=0 & \text { confinement } \Leftrightarrow \text { unbroken } \mathbb{Z}_{N} \text { symmetry } \\ \neq 0 & \text { deconfinement } \Leftrightarrow \text { broken } \mathbb{Z}_{N} \text { symmetry }\end{cases}
$$





\section{B Statistics and Errors}

$[90,91]$ In probability theory and statistics the expected value $\langle x\rangle$ (expectation or mean value) of a discrete random variable $x$ (probability distribution or sample) is the sum of the probability of each possible value (e.g. outcome of an experiment) multiplied by the (outcome) value (or payoff). Measuring a single variable $x N$-times, e.g on $N$ (hopefully independent) configurations, one gets values $x_{i}, i=1,2, \ldots, N$ with probability $1 / N$ for the outcome of each value. Then the unbiased estimate of the mean value of this variable is

$$
\bar{x}=\langle x\rangle=\sum_{i=1}^{N} \frac{1}{N} x_{i}=\frac{x_{1}+x_{2}+\ldots+x_{N}}{N}=\frac{1}{N} \sum_{i=1}^{N} x_{i} .
$$

The standard deviation $\sigma$ of a probability distribution is defined as the square root of the variance $\sigma^{2}$,

$$
\sigma=\sqrt{\left\langle x^{2}\right\rangle-\langle x\rangle^{2}}=\sqrt{\mu_{2}^{\prime}-\mu^{2}}
$$

where $\mu=\bar{x}=\langle x\rangle$ is the mean and $\mu_{2}^{\prime}=\left\langle x^{2}\right\rangle$ is the second raw moment ${ }^{5}$. The variance $\sigma^{2}$ is therefore equal to the second central moment $\mu_{2}$ (i.e., moment about the mean).

The sample variance of a random variable $x$ is one measure of statistical dispersion, averaging the squared distance of its possible values $x_{i}$ from the expected value $\langle x\rangle=\bar{x}$.

$$
\sigma_{N}^{2}(x)=\left\langle(x-\bar{x})^{2}\right\rangle=\frac{1}{N} \sum_{i=1}^{N}\left(x_{i}-\bar{x}\right)^{2} .
$$

In physical sciences one generally uses the bias-corrected variance

$$
\sigma_{N-1}^{2}(x)=\frac{1}{N-1} \sum_{i=1}^{N}\left(x_{i}-\bar{x}\right)^{2},
$$

where the denominator $N-1$ takes into account the reduction of degrees of freedom in the vector $\left(x_{1}-\bar{x}, x_{2}-\bar{x}, \ldots, x_{N}-\bar{x}\right)$. Anyways, for $N \gg 1$ there is practically no difference between the two definitions.

The sample standard deviation of a random variable $x$ is a measure of the spread of its values $x_{i}$. It is known as the root mean square deviation of the values from their (arithmetic) mean, i.e., the square root of the sample variance, and often simply called standard deviation

$$
\sigma_{N-1}(x)=\sqrt{\left\langle(x-\langle x\rangle)^{2}\right\rangle}=\sqrt{\frac{1}{N-1} \sum_{i=1}^{N}\left(x_{i}-\bar{x}\right)^{2}} .
$$

The standard deviation is commonly used to define a confidence interval CI, an

\footnotetext{
${ }^{5}$ The $n^{t h}$ moment of a probability function $P(x)$ about a value $c$ is given by $\mu_{n}^{\prime}=\int(x-c)^{n} P(x) d x$. For a discrete random variable $x \mu_{n}^{\prime}=\left\langle(x-c)^{n}\right\rangle$. The raw moment is generated about $c=0$.
} 


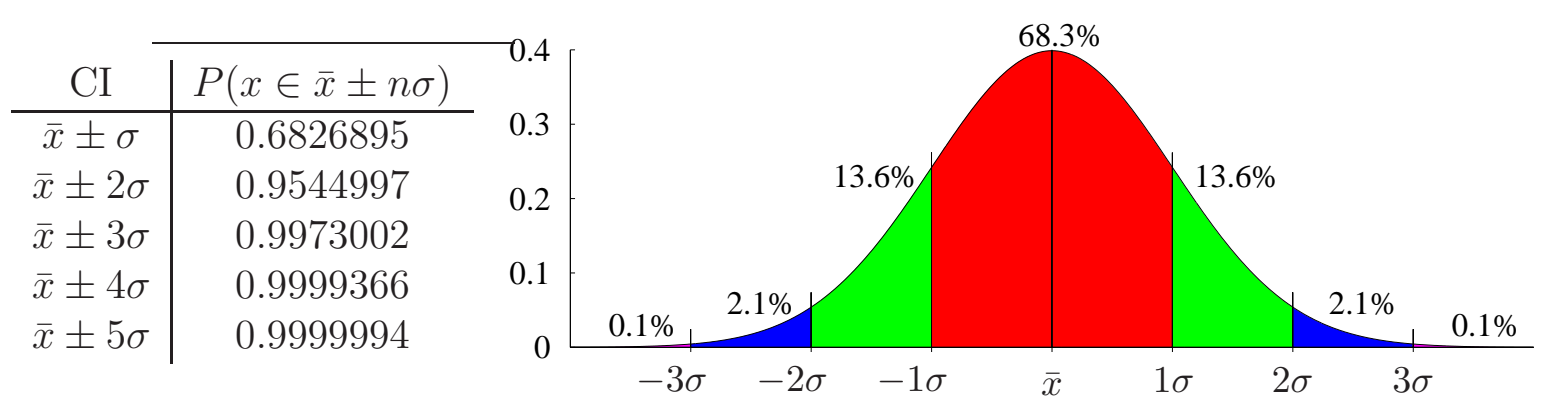

Table 9: Probabilities $P(\bar{x}-n \sigma<x<\bar{x}+n \sigma)$ for measurements from a normal distribution lying in the confidence interval $\mathrm{CI}=[\bar{x} \pm n \sigma]$. The figure illustrates the normal distribution probability function with confidence intervals CI.

interval estimate of a random variable. For a normal distribution ${ }^{6}$ the probability that a measurement falls within $n$ standard deviations $(n \sigma)$ of the mean $\bar{x}$ (i.e., within the confidence interval $[\bar{x}-n \sigma, \bar{x}+n \sigma])$ is given by

$P(\bar{x}-n \sigma<x<\bar{x}+n \sigma)=\frac{1}{\sigma \sqrt{2 \pi}} \int_{\bar{x}-n \sigma}^{\bar{x}+n \sigma} e^{-(x-\bar{x})^{2} /\left(2 \sigma^{2}\right)} d x=\frac{2}{\sigma \sqrt{2 \pi}} \int_{\bar{x}}^{\bar{x}+n \sigma} e^{-(x-\bar{x})^{2} /\left(2 \sigma^{2}\right)} d x$.

Now let $u \equiv(x-\bar{x}) / \sqrt{2} \sigma$, so $d u=d x / \sqrt{2} \sigma$. Then

$$
P(\bar{x}-n \sigma<x<\bar{x}+n \sigma)=\frac{2}{\sqrt{\pi}} \int_{0}^{n / \sqrt{2}} e^{-u^{2}} d u=\operatorname{erf}\left(\frac{n}{\sqrt{2}}\right),
$$

where $\operatorname{erf}(x)$ is the so-called error function

$$
\operatorname{erf}(x)=\frac{2}{\sqrt{\pi}} \int_{0}^{x} e^{-t^{2}} d t
$$

Table 9 lists the probabilities $P(\bar{x}-n \sigma<x<\bar{x}+n \sigma)$ for measurements from a normal distribution lying in the confidence interval $[\bar{x} \pm n \sigma]$ for the first few multiples $n$ of the standard deviation.

\section{B.1 Error propagation and correlations}

Error propagation (or propagation of uncertainty) is the effect of variables' errors on the uncertainty of a function $f$ based on these variables. The uncertainty of a variable $x$ is usually defined by the absolute error $\Delta x$, which is commonly given as the standard deviation $\sigma$. If the variables are correlated, then the covariance must be taken into

\footnotetext{
${ }^{6} \mathrm{~A}$ normal distribution in a variable $x$ with mean $\bar{x}$ and variance $\sigma^{2}$ is a statistic distribution with probability function

$$
P(x)=\frac{1}{\sigma \sqrt{2 \pi}} e^{-(x-\bar{x})^{2} /\left(2 \sigma^{2}\right)}
$$
}


account. Correlation indicates the strength and direction of a linear relationship between two random variables. The correlation coefficient $\rho_{x, y}$ between two random variables $x$ and $y$ with expected values $\bar{x}$ and $\bar{y}$ and standard deviations $\sigma_{x}$ and $\sigma_{y}$ is defined as

$$
\rho_{x, y}=\frac{\operatorname{cov}(x, y)}{\sigma_{x} \sigma_{y}}=\frac{\langle(x-\bar{x})(y-\bar{y})\rangle}{\sigma_{x} \sigma_{y}}=\frac{1}{N \sigma_{x} \sigma_{y}} \sum_{i=1}^{N}\left(x_{i}-\bar{x}\right)\left(y_{i}-\bar{y}\right),
$$

where $\operatorname{cov}(x, y)$ is the covariance of $x$ and $y$.

Let $f\left(x_{1}, x_{2}, \ldots, x_{n}\right)$ be a single function which is a linear combination of $n$ variables $x_{i}$ with combination coefficients $a_{1}, a_{2}, \ldots, a_{n}$.

$$
f_{k}=\sum_{i}^{n} a_{i} x_{i}: \mathbf{f}=\mathbf{a}^{\mathbf{T}} \mathbf{x}
$$

and let the variance-covariance matrix on $x$ be denoted by

$$
\mathbf{M}^{\mathbf{x}}=\left(\begin{array}{cccc}
\sigma_{1}^{2} & \operatorname{cov}_{12} & \operatorname{cov}_{13} & \ldots \\
\operatorname{cov}_{12} & \sigma_{2}^{2} & \operatorname{cov}_{23} & \ldots \\
\operatorname{cov}_{13} & \operatorname{cov}_{23} & \sigma_{3}^{2} & \ldots \\
\cdots & & &
\end{array}\right)
$$

Then the variance of $f$ is given by

$$
\sigma_{f}^{2}=\sum_{i}^{n} \sum_{j}^{n} a_{i} M_{i j}^{x} a_{j}=\mathbf{a}^{\mathbf{T}} \mathbf{M}^{\mathbf{x}} \mathbf{a}=\sum_{i}^{n}\left(a_{i}^{2} \sigma_{i}^{2}+\sum_{j \neq i}^{n} a_{i} a_{j} \operatorname{cov}_{i j}\right) .
$$

When the variables $x_{i}$ are uncorrelated this simplifies to

$$
\sigma_{f}^{2}=\sum_{i}^{n} a_{i}^{2} \sigma_{i}^{2}
$$

In the case of a non-linear function $f$ of the variables $x_{i}$, it must usually be linearized by approximation to a first-order Maclaurin series ${ }^{7}$ expansion

$$
f \approx f_{0}+\sum_{i}^{n} \frac{\partial f}{\partial x_{i}} x_{i}
$$

Since $f_{0}$ is a constant it does not contribute to the error on $f$. Therefore, the propagation of error follows the linear case, but replacing the linear coefficients $a_{i}$ by the partial derivatives $\frac{\partial f}{\partial x_{i}}$.

A simple version of error propagation for uncorrelated variables $x_{i}$ is sometimes given

\footnotetext{
${ }^{7}$ Taylor series using the derivatives at zero
} 
by

$$
\triangle f=\sum_{i}^{n} \frac{\partial f}{\partial x_{i}} \triangle x_{i}
$$

Here, all terms of the sum are taken with a positive sign, even if the partial derivative gives a negative one.

\section{B.2 The jackknife error estimate}

Jackknifing is a statistical re-sampling method to estimate the precision of a sample by using subsets of available data. The basic idea behind the jackknife estimator lies in systematically recomputing the statistic estimate leaving out one observation at a time from the sample set. From this new set of "observations" for the statistic, an estimate for the bias and an estimate for the variance can be calculated.

Again using a sample of values $x_{i}, i=1,2, \ldots, N$ with mean $\bar{x}$ and variance $\sigma^{2}$. The jackknife approach takes sub ensembles with $(N-1)$ points by always leaving out one measured value (jackknife samples). Then the jackknife sample means and the jackknife error in the mean are given by

$$
\bar{x}_{j}=\frac{1}{N-1} \sum_{i \neq j}^{N} x_{i}, \quad \sigma_{j}^{2}=\frac{N-1}{N} \sum_{i=1}^{N}\left(\bar{x}_{j}-\bar{x}\right)^{2} .
$$

The reason for the difference of the placement of the factor $(N-1)$ is that the jackknife means are distributed $(N-1)$-times closer to the mean than the original values $x_{i}$, therefore a correction factor of $(N-1)^{2}$ is needed. In fact, for a single variable, it is easy to show that $\sigma_{j}=\sigma$ coincide.

The benefit of jackknifing lies in implicitly taking into account correlations within functions of correlated variables. Considering a function $f(x, y)$ of random variables $x$ and $y$, in a first step the jackknife means $\bar{x}_{j}$ and $\bar{y}_{j}$ are determined. Then the jackknife function means are given by $\bar{f}_{j}=f\left(\bar{x}_{j}, \bar{y}_{j}\right)$ and finally the jackknife error estimate of the function $f$ is given by

$$
\sigma_{J}^{2}(f)=\frac{N-1}{N} \sum_{j=1}^{N}\left(\bar{f}_{j}-\bar{f}\right) \quad \text { with } \quad \bar{f}=f(\bar{x}, \bar{y}) .
$$

\section{B.3 Error propagation for the Creutz ratio}

The Creutz ratio $\chi$ combines Wilson loops $W(R, T)$ of different size

$$
\chi(R, T)=-\log \left[\frac{\langle W(R+1, T+1)\rangle\langle W(R, T)\rangle}{\langle W(R+1, T)\rangle\langle W(R, T+1)\rangle}\right] .
$$

Denote values, on $N$ individual configurations, of Wilson loops appearing in this expression as $a_{i}, b_{i}, c_{i}, d_{i}(i=1,2, \ldots, N)$, respectively. Then, mean and errors are given 
by

$$
\langle a\rangle=\bar{a}=\frac{1}{N} \sum_{i=1}^{N} a_{i}, \quad \triangle a=\sigma(a)=\sqrt{\frac{1}{N-1} \sum_{i=1}^{N}\left(a_{i}-\bar{a}\right)^{2}}
$$

(plus similar for other quantities). Now, four different methods of determining the error of the Creutz ratios are compared:

a) The simple error propagation of equation 88, which of course is not appropriate because Wilson loops of different size are not uncorrelated, gives error bars as shown in Fig. 89a:

$$
\begin{aligned}
\Delta \chi & =\frac{\Delta a}{\bar{a}}+\frac{\Delta b}{\bar{b}}+\frac{\Delta c}{\bar{c}}+\frac{\Delta d}{\bar{d}} \\
& =\frac{\Delta W(R+1, T+1)}{\bar{W}(R+1, T+1)}+\frac{\Delta W(R, T)}{\bar{W}(R, T)}+\frac{\triangle W(R+1, T)}{\bar{W}(R+1, T)}+\frac{\triangle W(R, T+1)}{\bar{W}(R, T+1)} .
\end{aligned}
$$

b) Standard error propagation of equation 86, still neglecting correlations, gives error bars as shown in Fig. 89b:

$$
\sigma^{2}(\chi)=\frac{\sigma^{2}(a)}{\bar{a}^{2}}+\frac{\sigma^{2}(b)}{\bar{b}^{2}}+\frac{\sigma^{2}(c)}{\bar{c}^{2}}+\frac{\sigma^{2}(d)}{\bar{d}^{2}}
$$

c) Error propagation by taking into account the correlation of Wilson loops of different size (equation 85) gives error bars as shown in Fig. 89c:

$$
\begin{aligned}
\sigma^{2}(\chi)= & \frac{\sigma^{2}(a)}{\bar{a}^{2}}+\frac{\sigma^{2}(b)}{\bar{b}^{2}}+\frac{\sigma^{2}(c)}{\bar{c}^{2}}+\frac{\sigma^{2}(d)}{\bar{d}^{2}}+2 \frac{\operatorname{cov}(a, b)}{\bar{a} \bar{b}} \\
& -2 \frac{\operatorname{cov}(a, c)}{\bar{a} \bar{c}}-2 \frac{\operatorname{cov}(a, d)}{\bar{a} \bar{d}}-2 \frac{\operatorname{cov}(b, c)}{\bar{b} \bar{c}}-2 \frac{\operatorname{cov}(b, d)}{\bar{b} \bar{d}}+2 \frac{\operatorname{cov}(c, d)}{\bar{c} \bar{d}}
\end{aligned}
$$

It is interesting to mention that correlations can also reduce the errors, compared to the (wrong) case above.

d) Finally, jackknife errors are shown in Fig. 89d:

$$
a_{j}=\frac{1}{N-1} \sum_{i \neq j}^{N} a_{i}, \ldots \quad \chi_{j}=-\log \left[\frac{a_{j} b_{j}}{c_{j} d_{j}}\right] \quad \text { and } \quad \sigma^{2}(\chi)=\frac{N-1}{N} \sum_{j=1}^{N}\left(\chi_{j}-\bar{\chi}\right)^{2} .
$$

First, the simple error propagation method (a) strongly underestimates the errors. The growth of error bars with growing radius $R$ seems correct, because Wilson loops of growing size have lower statistics. Standard error propagation, neglecting correlations (b), drastically increases error bars, especially for center-projected configurations, whose errors were the lowest in case a). 


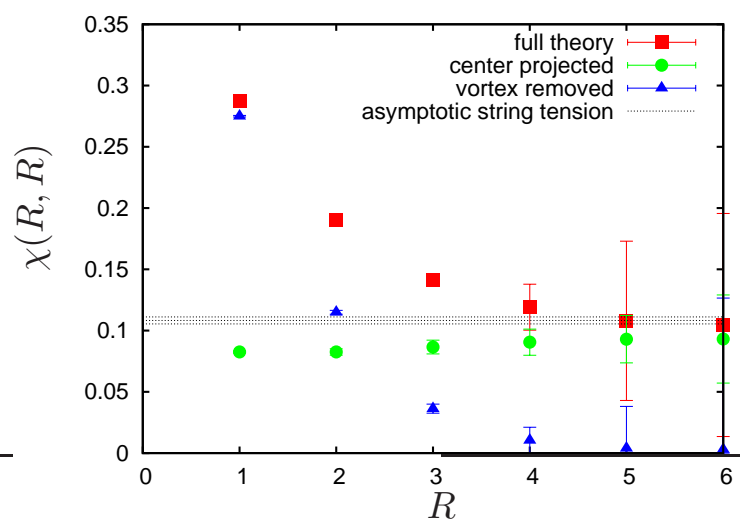

a

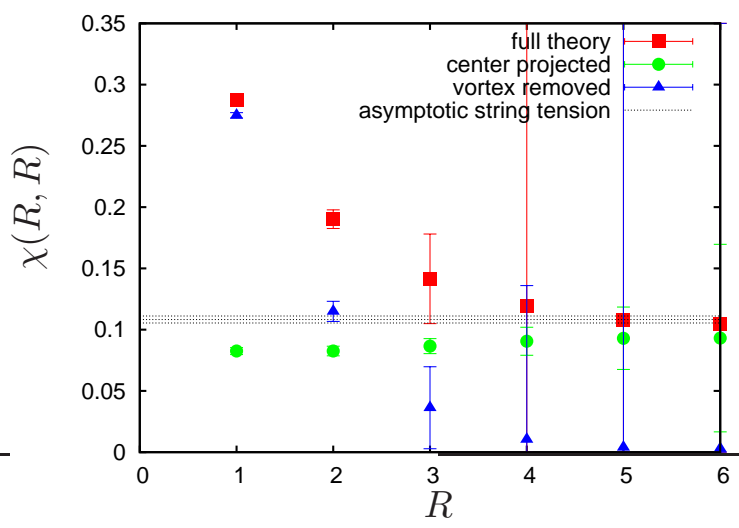

$\mathrm{c}$

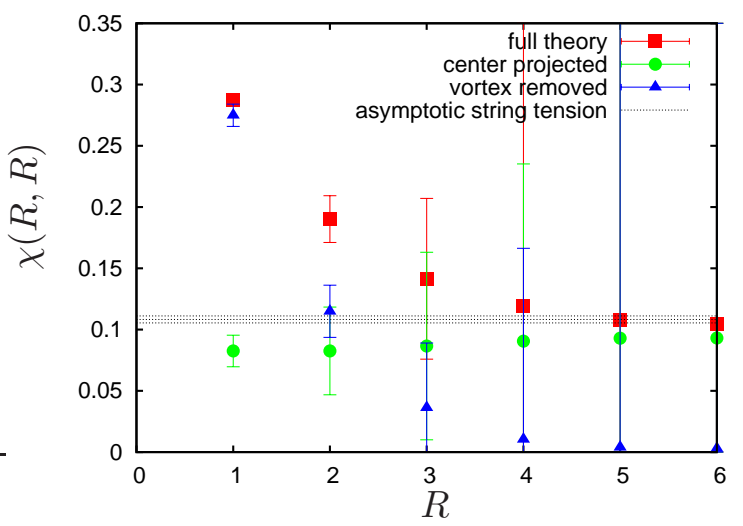

$\mathrm{b}$

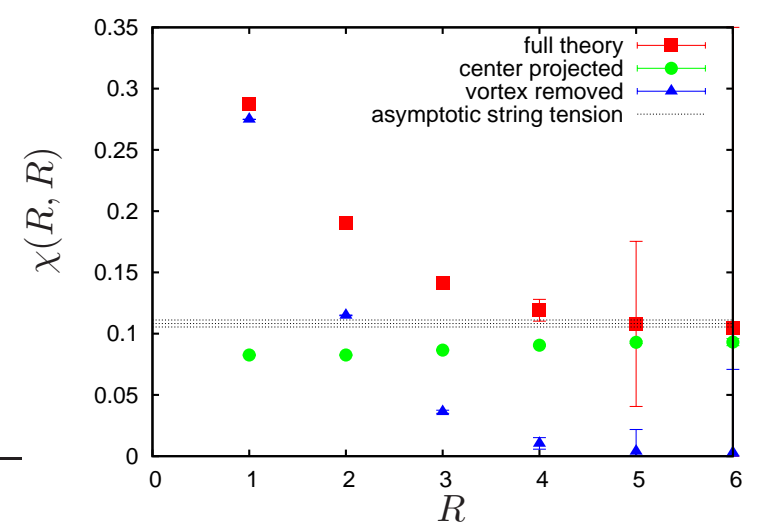

$\mathrm{d}$

Figure 89: Symmetric Creutz ratios $\chi(R, R)$ from measuring Wilson loops on 700 full, center-projected and vortex-removed configurations on a $20^{4}$-lattice at $\beta_{L W}=$ 3.3 , using different methods to determine the error bars.

Taking into account the correlations of Wilson loops (c), lowers error bars again, especially for center-projected configurations. This needs some more detailed discussion: The Wilson loops $W[R+1, R] \equiv c$ and $W[R, R+1] \equiv d$ of course are fully correlated $(\rho(c, d)=1)$, and its covariance $(\operatorname{cov}(c, d))$ contributes positively to the error bars. The second positive correlation term contributing to the error bars is in fact the smallest, the Wilson loops $W[R+1, R+1] \equiv a$ and $W[R, R] \equiv b$ differ most in their sizes. All other correlation terms lower the error bars with comparable covariances because of comparable size differences. Interpreting now Fig. 89c, the Wilson loops of centerprojected correlations seem to correlate equally, independently of their size differences, whereas for full and vortex removed configurations the correlations seem to scale as derived above. Finally, jackknife errors are comparable to those from method a), but implicitly including correlations. 


\section{Plots and Figures}

\section{INDEX:}

- 116...Dirac Spectra

- 116...Overlap Modes

- 118...Asqtad Staggered Modes

- 130...Interpolated Fields

- 132...Eigenmode Density Peaks

- 132...Overlap Modes on full configurations

- 134...Overlap Modes on center-projected configurations

- 136...Asqtad Staggered Modes on full configurations

- 138...Asqtad Staggered Modes on center-projected configurations

- 140...Vortex Correlations

- 155...Topological Charge Correlations

- 170...Finite Temperature

- 170...Polyakov Loops

- 172...Dirac Spectra

- 178...Correlations

- 179...Vortex Correlations 

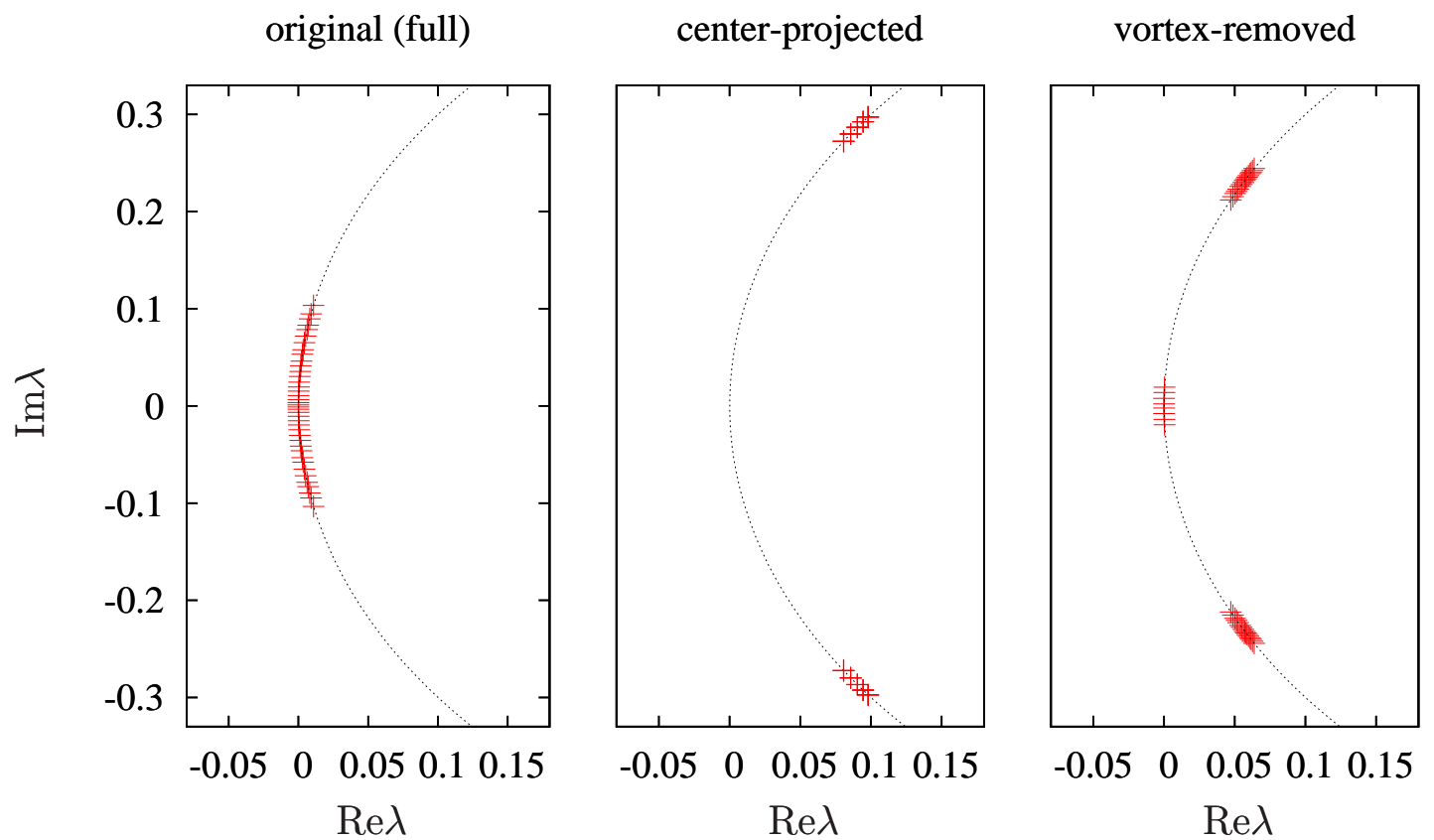

Figure 90: First twenty overlap Dirac eigenvalues on the Ginsparg-Wilson circle for a $12^{4}$ lattice at $\beta=2.9$.
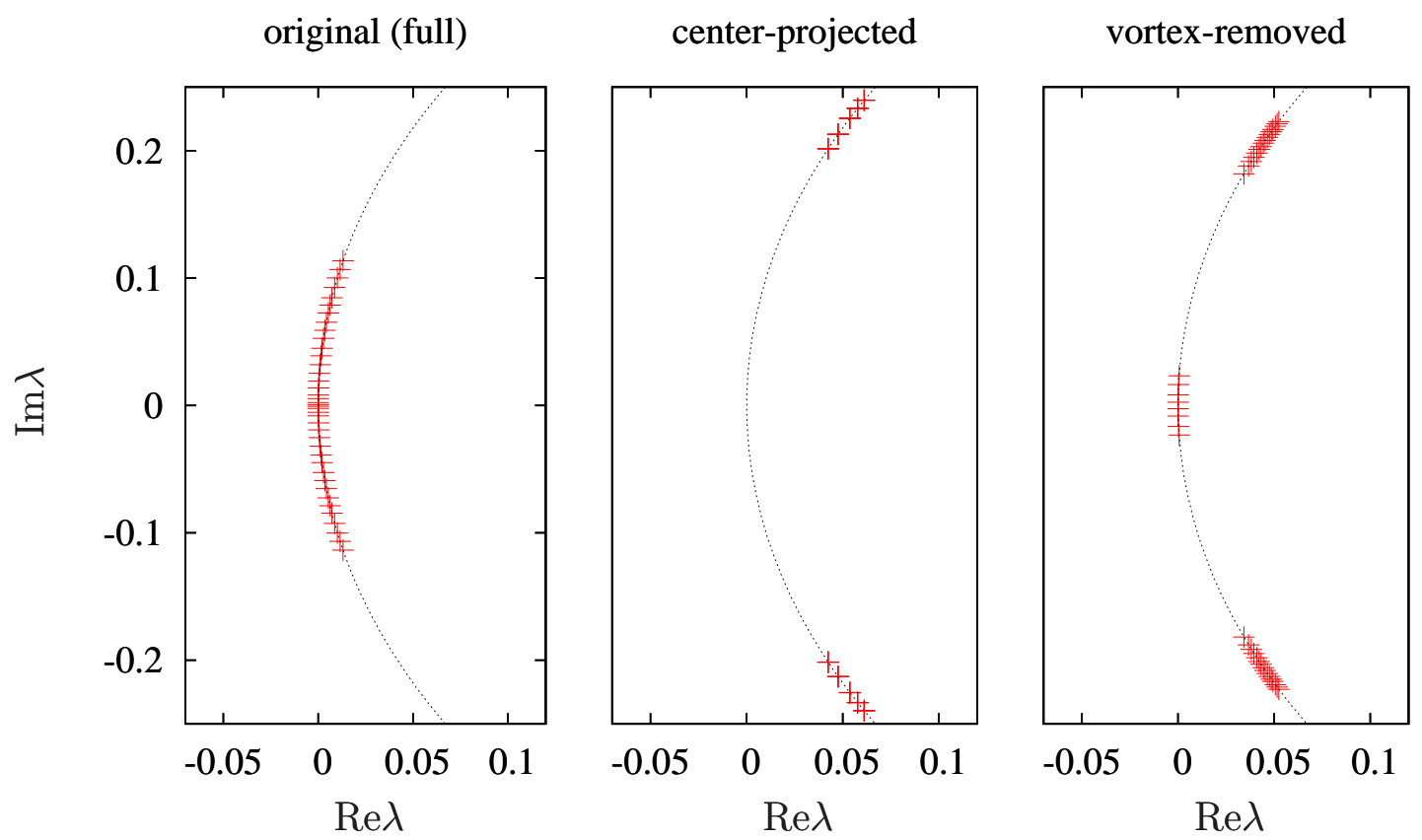

Figure 91: First twenty overlap Dirac eigenvalues on the Ginsparg-Wilson circle for a $12^{4}$ lattice at $\beta=3.1$. 

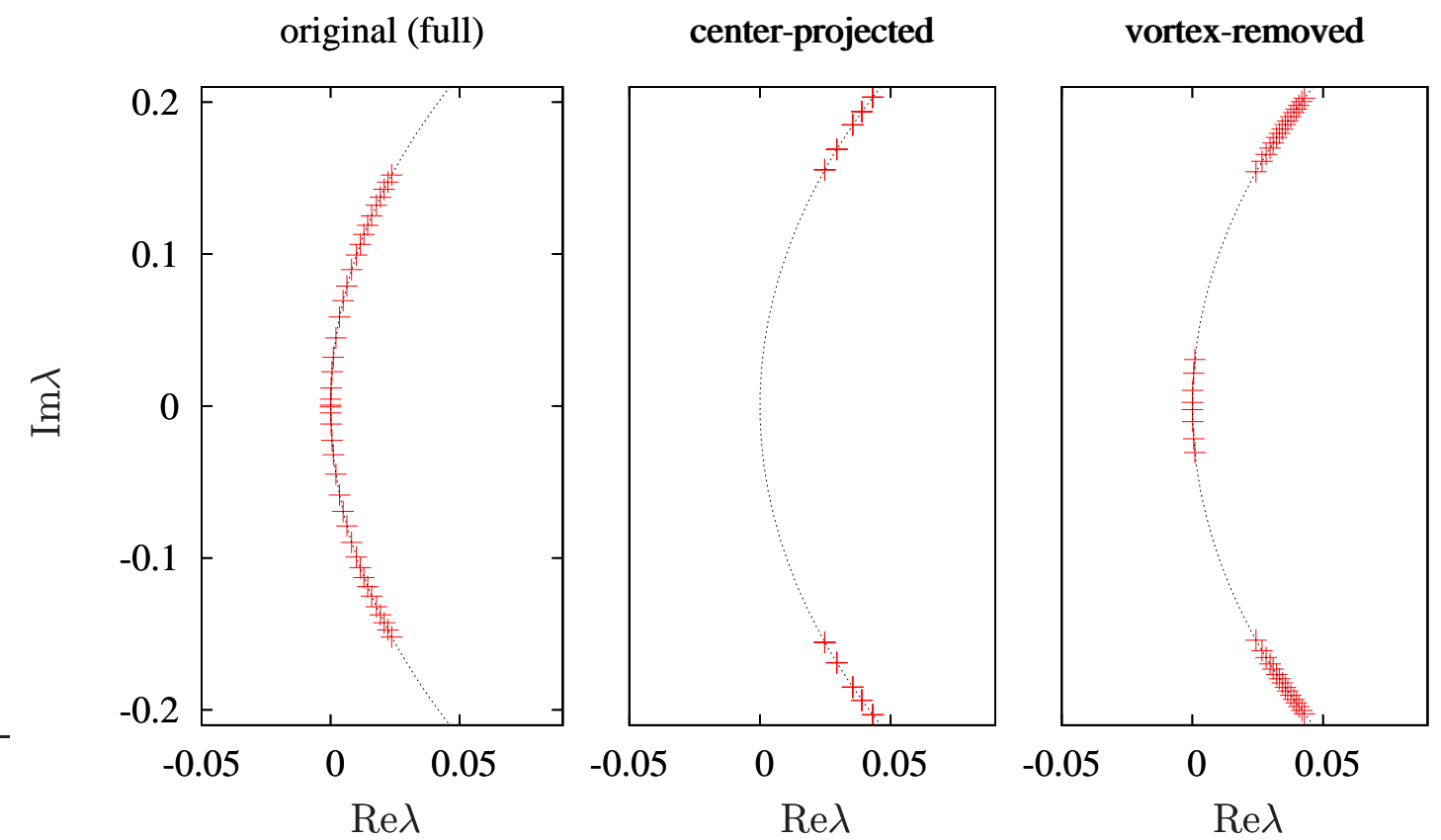

Figure 92: First twenty overlap Dirac eigenvalues on the Ginsparg-Wilson circle for a $12^{4}$ lattice at $\beta=3.3$.
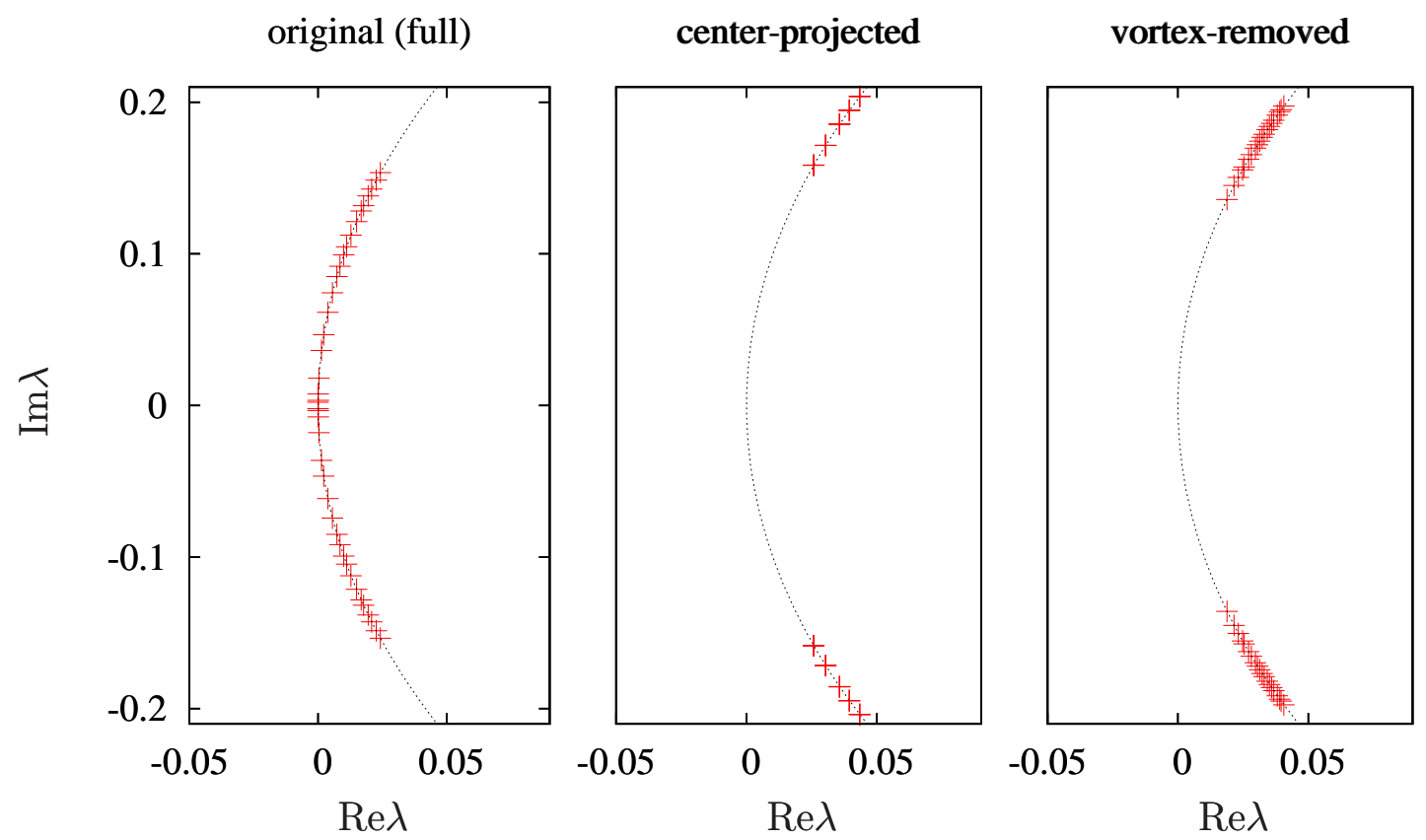

Figure 93: First twenty overlap Dirac eigenvalues on the Ginsparg-Wilson circle for a $12^{4}$ lattice at $\beta=3.3$ using anti periodic boundary conditions. 
C Plots and Figures
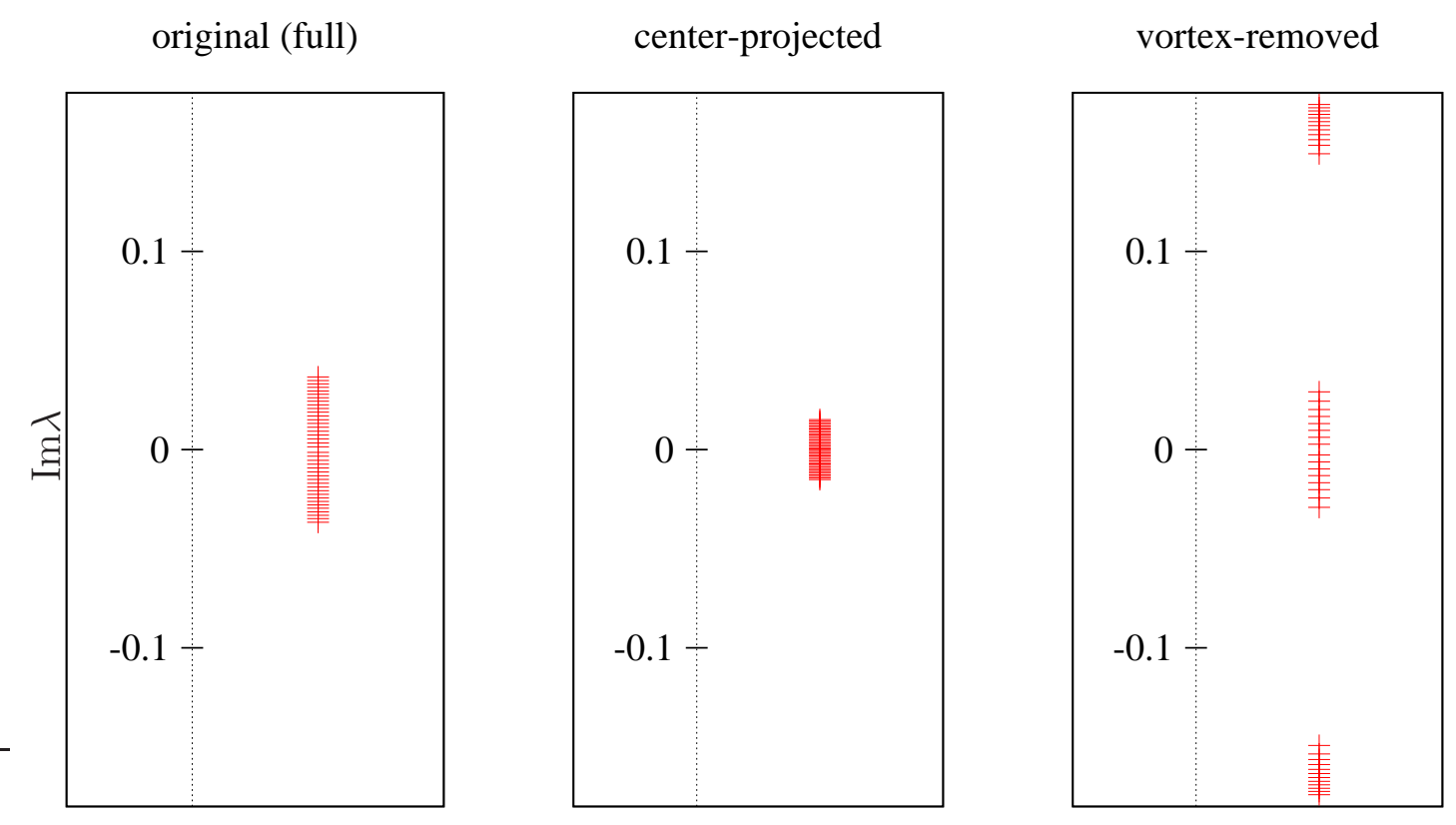

Figure 94: First twenty asqtad staggered Dirac eigenvalues on a $12^{4}$ lattice at $\beta=2.9$.
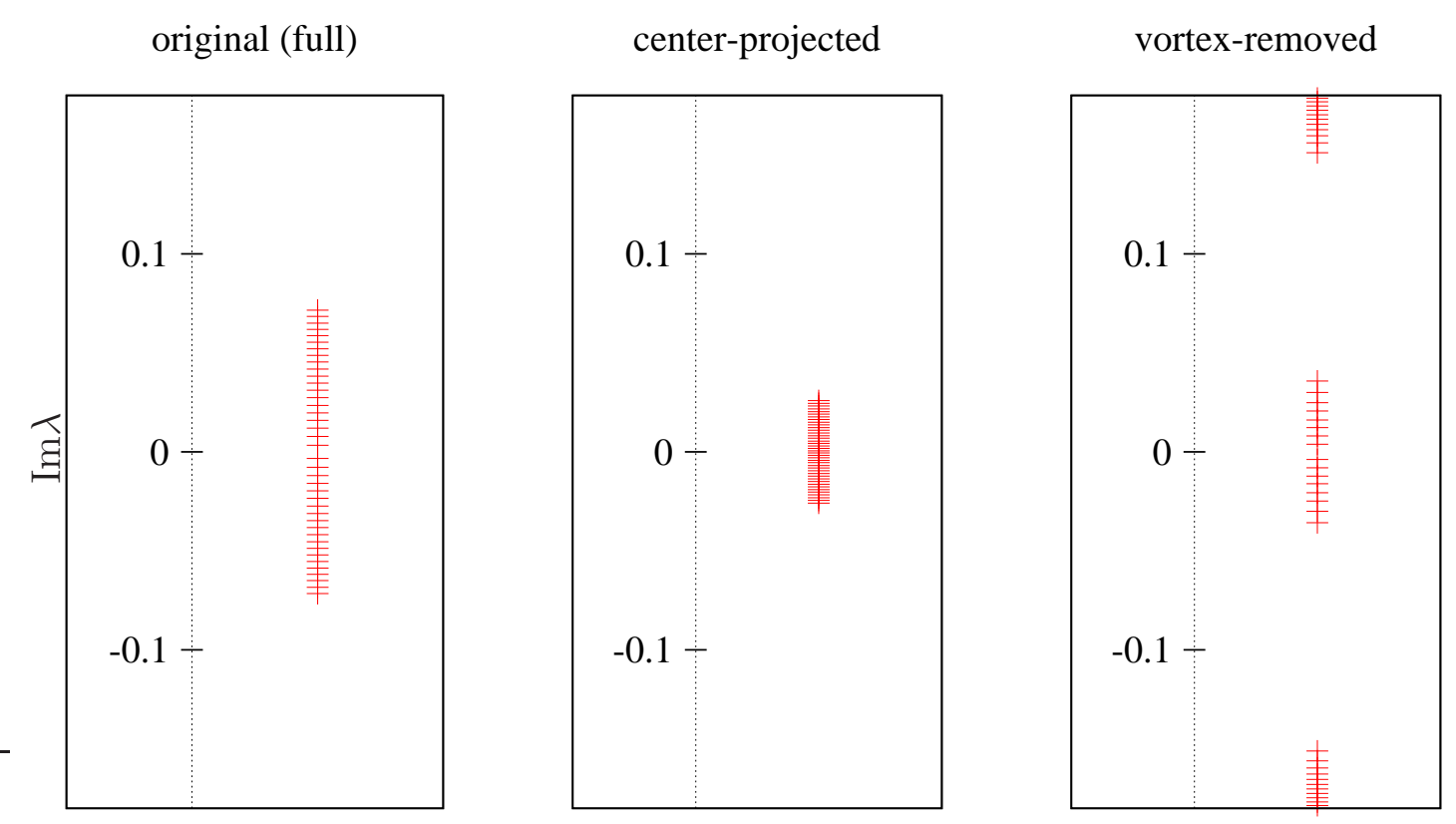

Figure 95: First twenty asqtad staggered Dirac eigenvalues on a $12^{4}$ lattice at $\beta=3.1$. 
C Plots and Figures
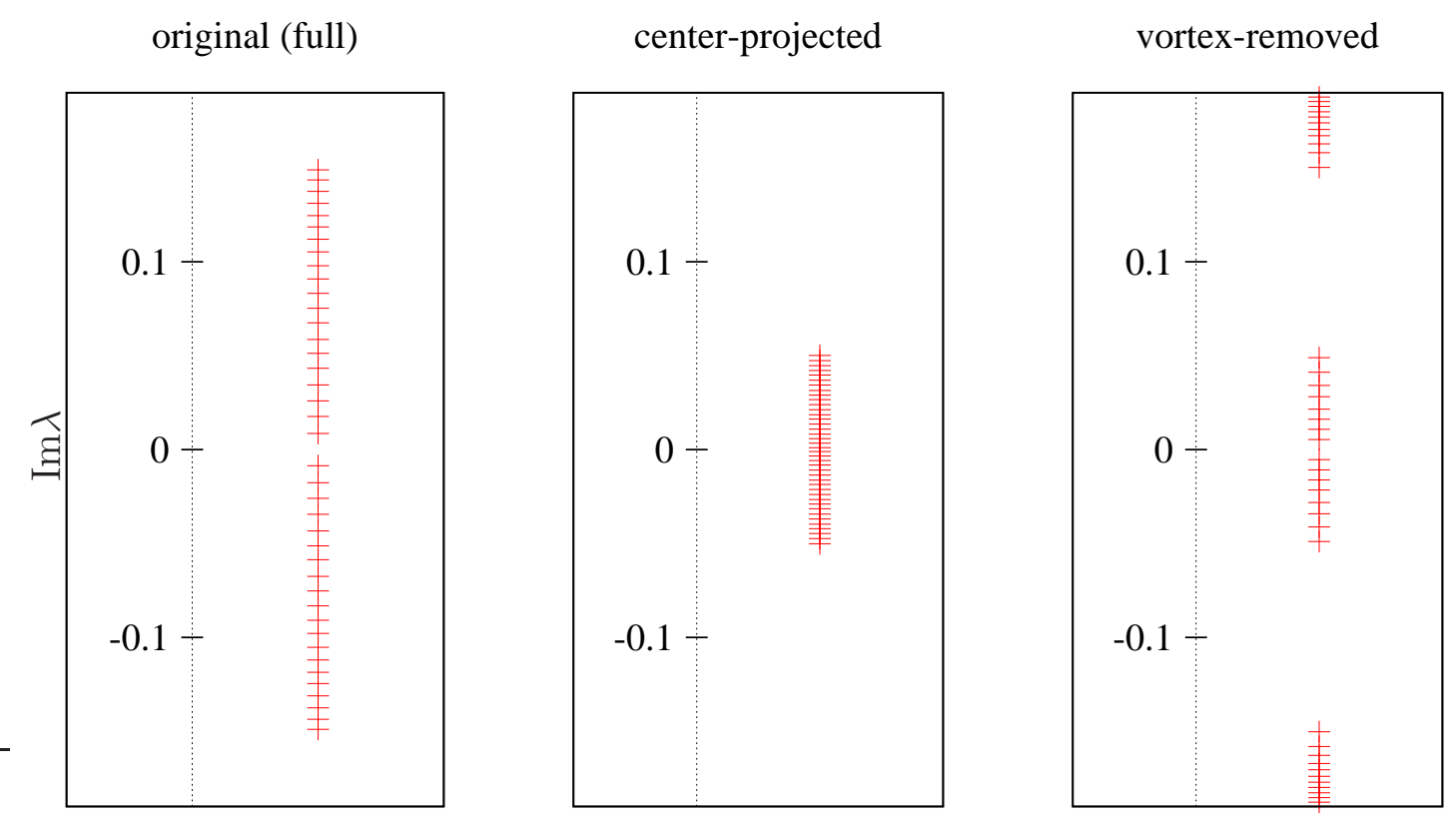

Figure 96: First twenty asqtad staggered Dirac eigenvalues on a $12^{4}$ lattice at $\beta=3.3$.
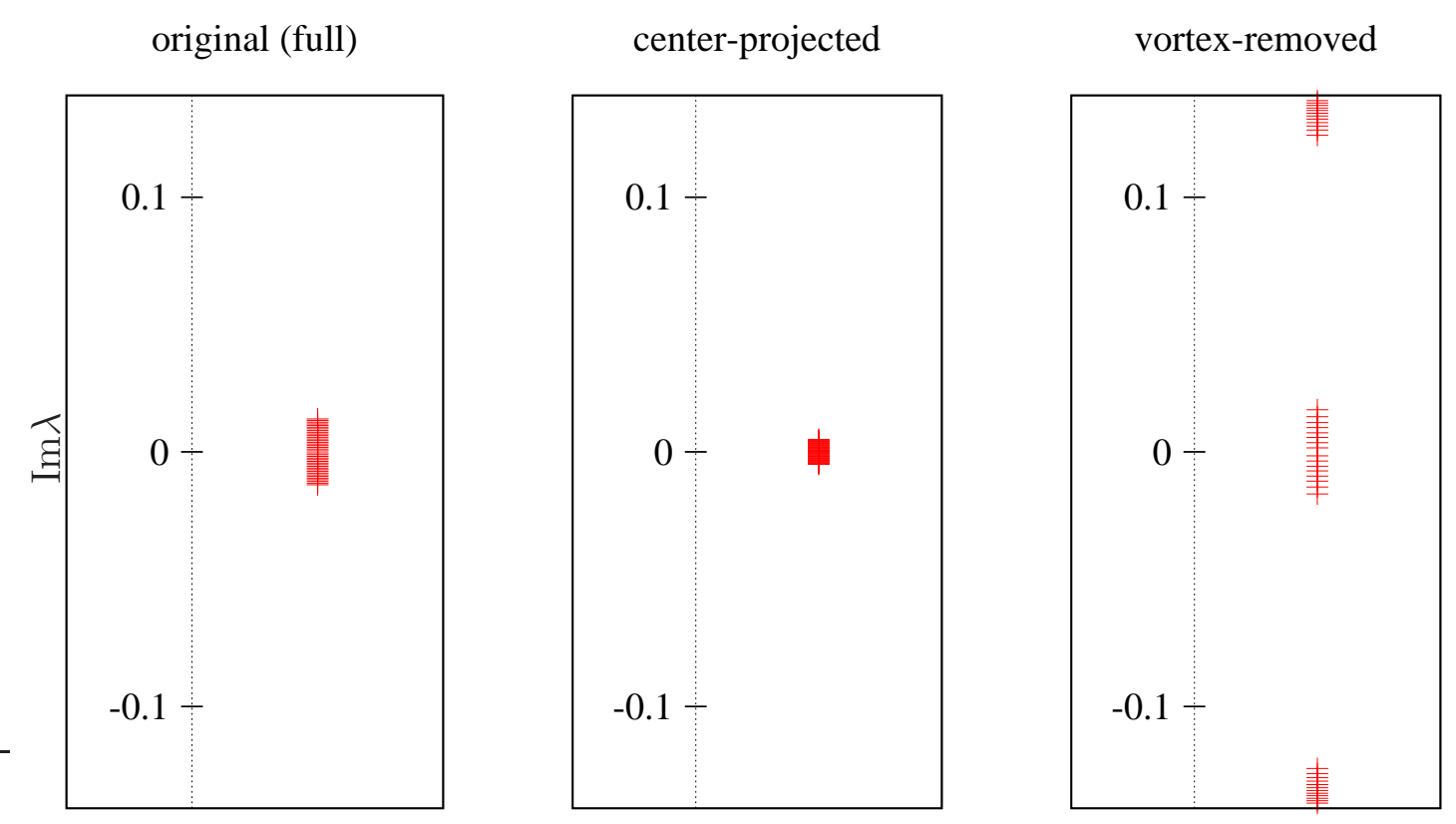

Figure 97: First twenty asqtad staggered Dirac eigenvalues on a $16^{4}$ lattice at $\beta=2.9$. 
C Plots and Figures
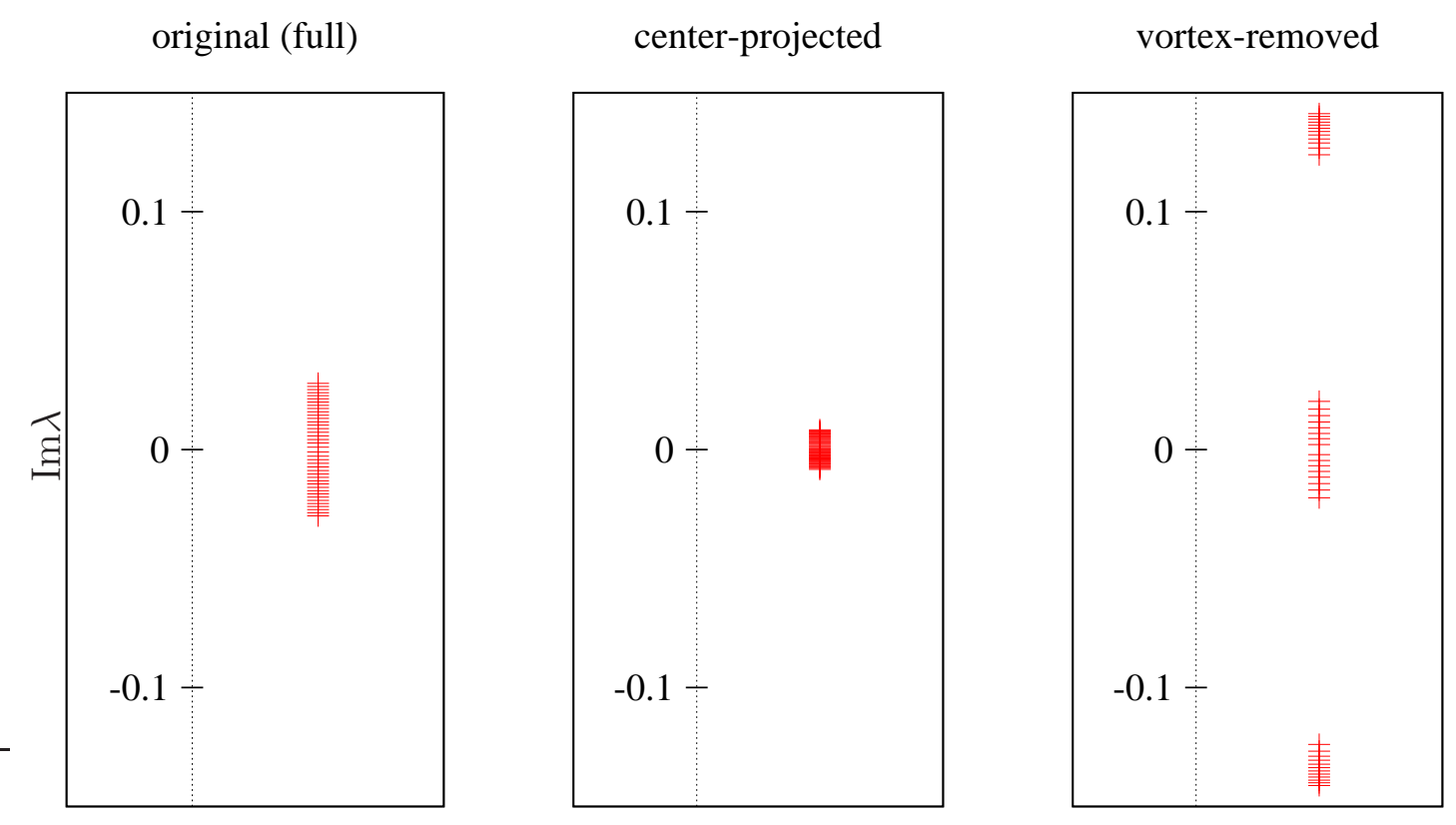

Figure 98: First twenty asqtad staggered Dirac eigenvalues on a $16^{4}$ lattice at $\beta=3.1$.
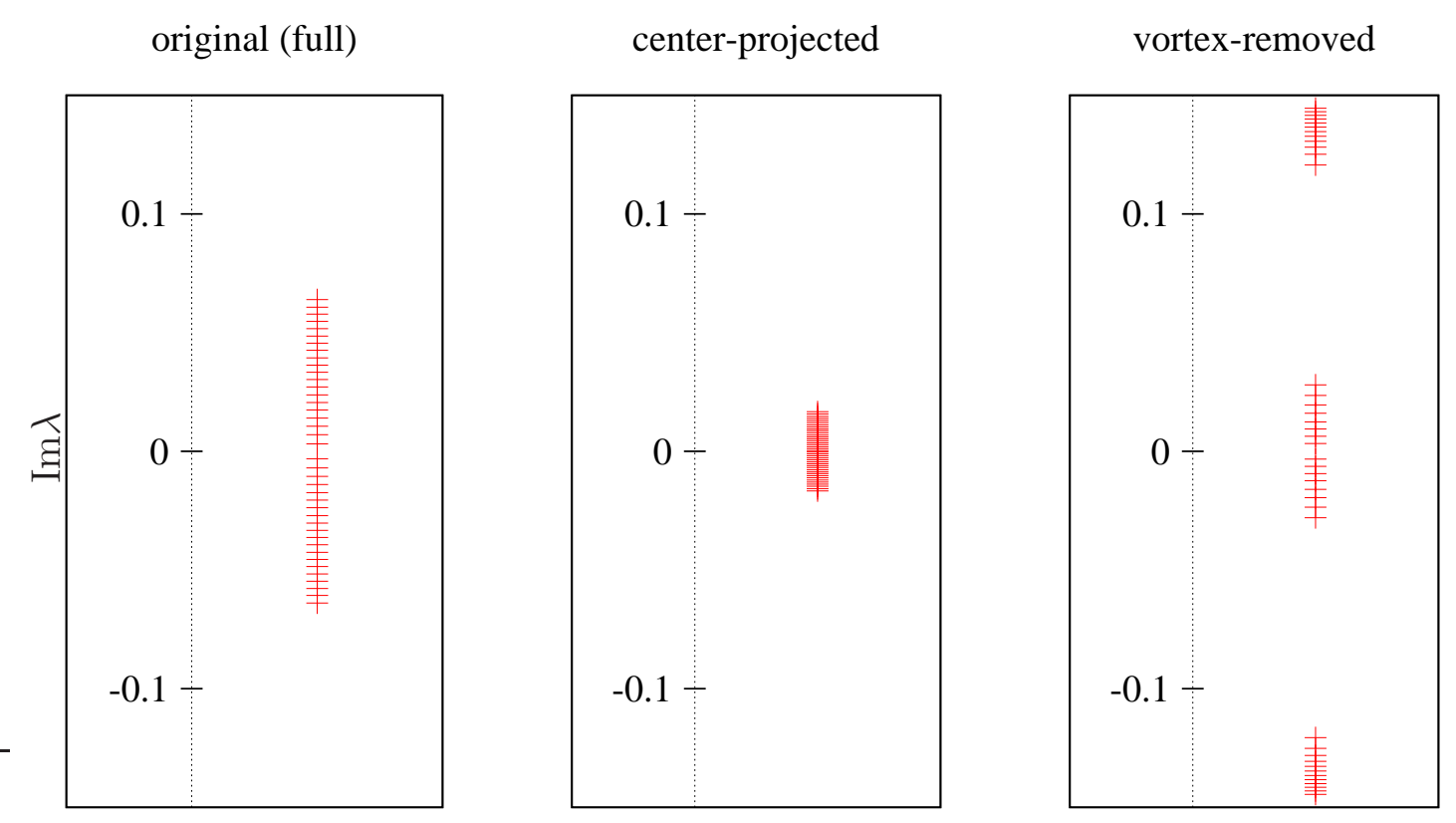

Figure 99: First twenty asqtad staggered Dirac eigenvalues on a $16^{4}$ lattice at $\beta=3.3$. 
C Plots and Figures
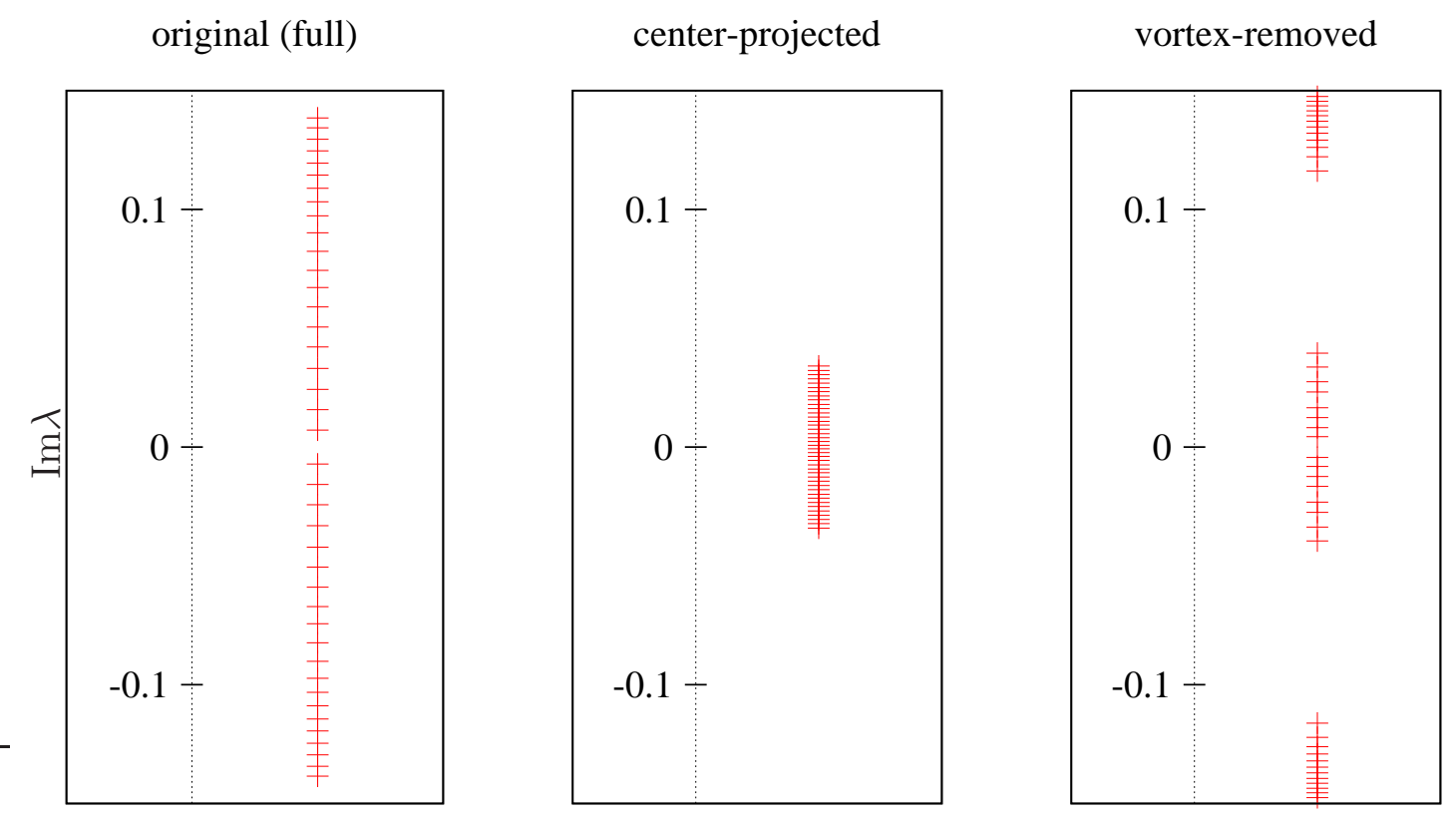

Figure 100: First twenty asqtad staggered Dirac eigenvalues on a $16^{4}$ lattice at $\beta=3.5$.
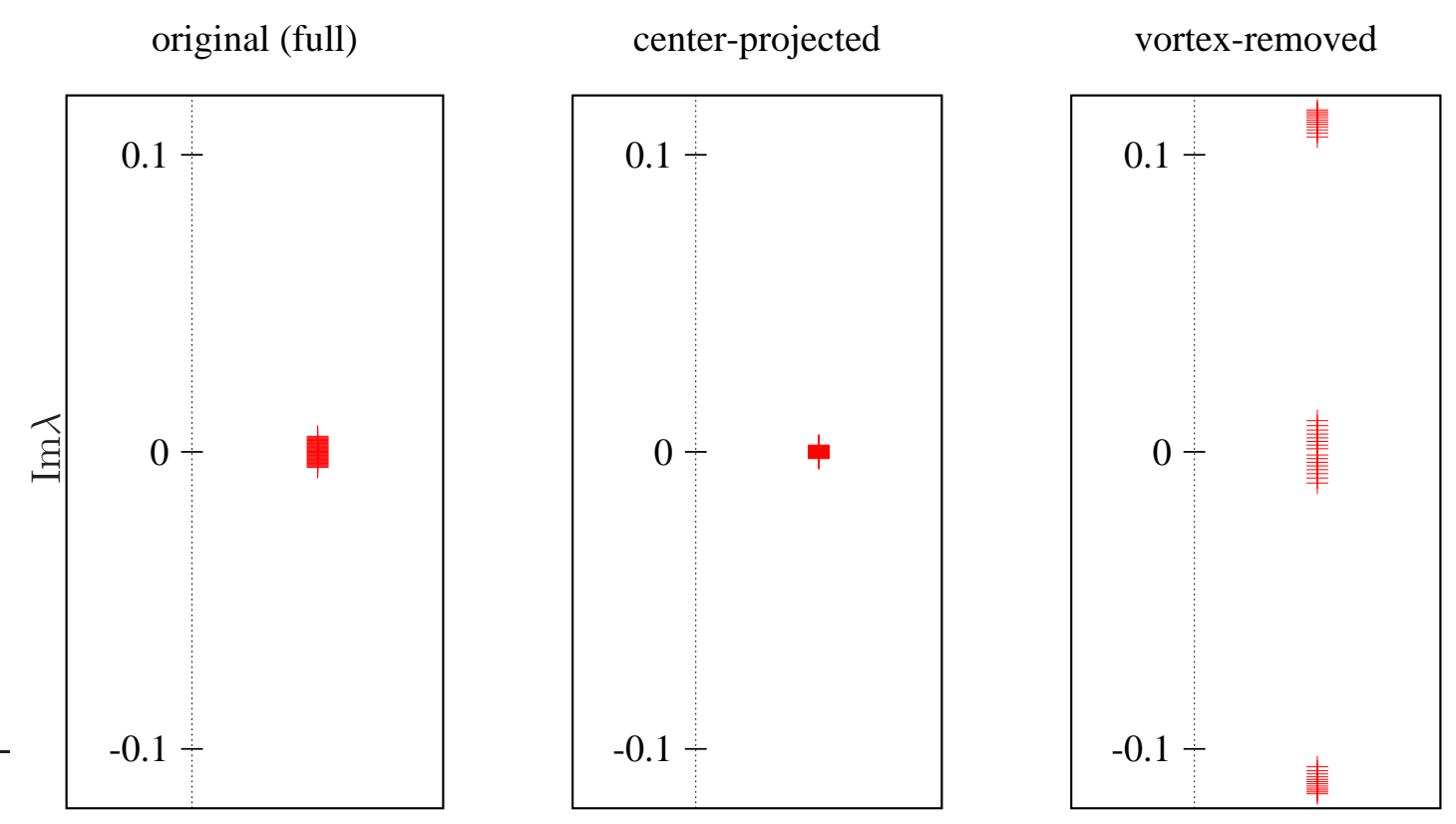

Figure 101: First twenty asqtad staggered Dirac eigenvalues on a $20^{4}$ lattice at $\beta=2.9$. 

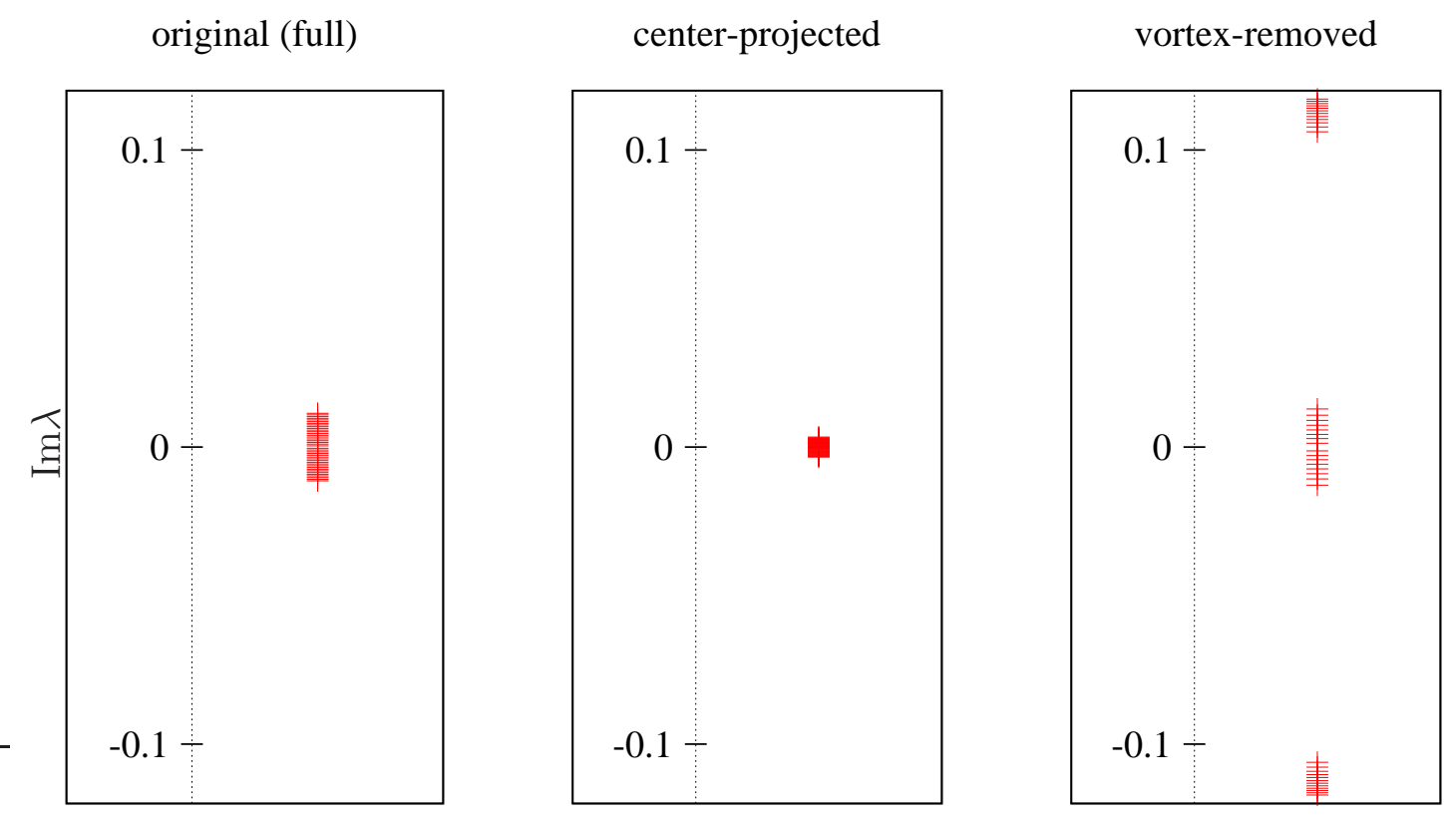

Figure 102: First twenty asqtad staggered Dirac eigenvalues on a $20^{4}$ lattice at $\beta=3.1$.
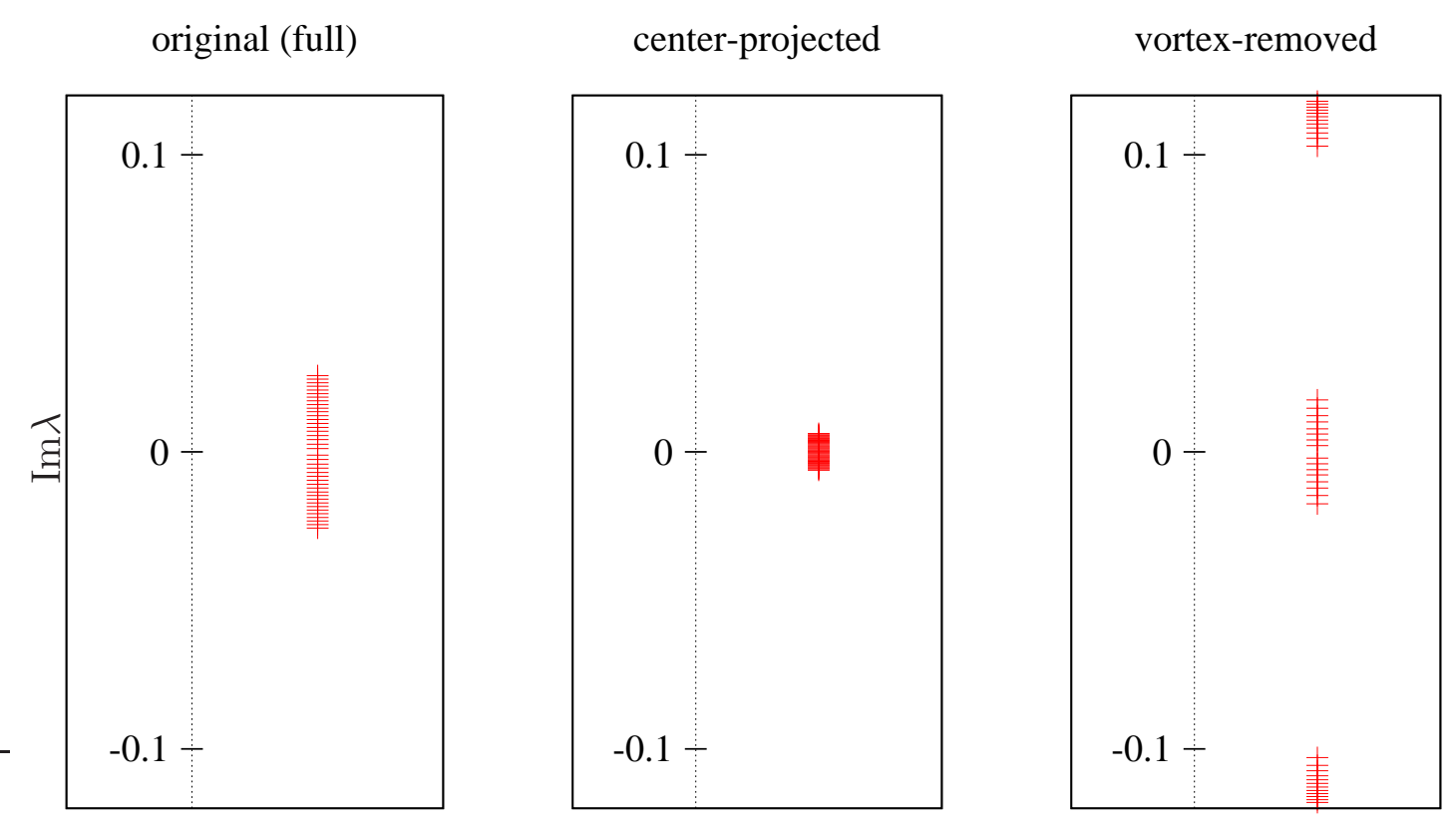

Figure 103: First twenty asqtad staggered Dirac eigenvalues on a $20^{4}$ lattice at $\beta=3.3$. 
C Plots and Figures
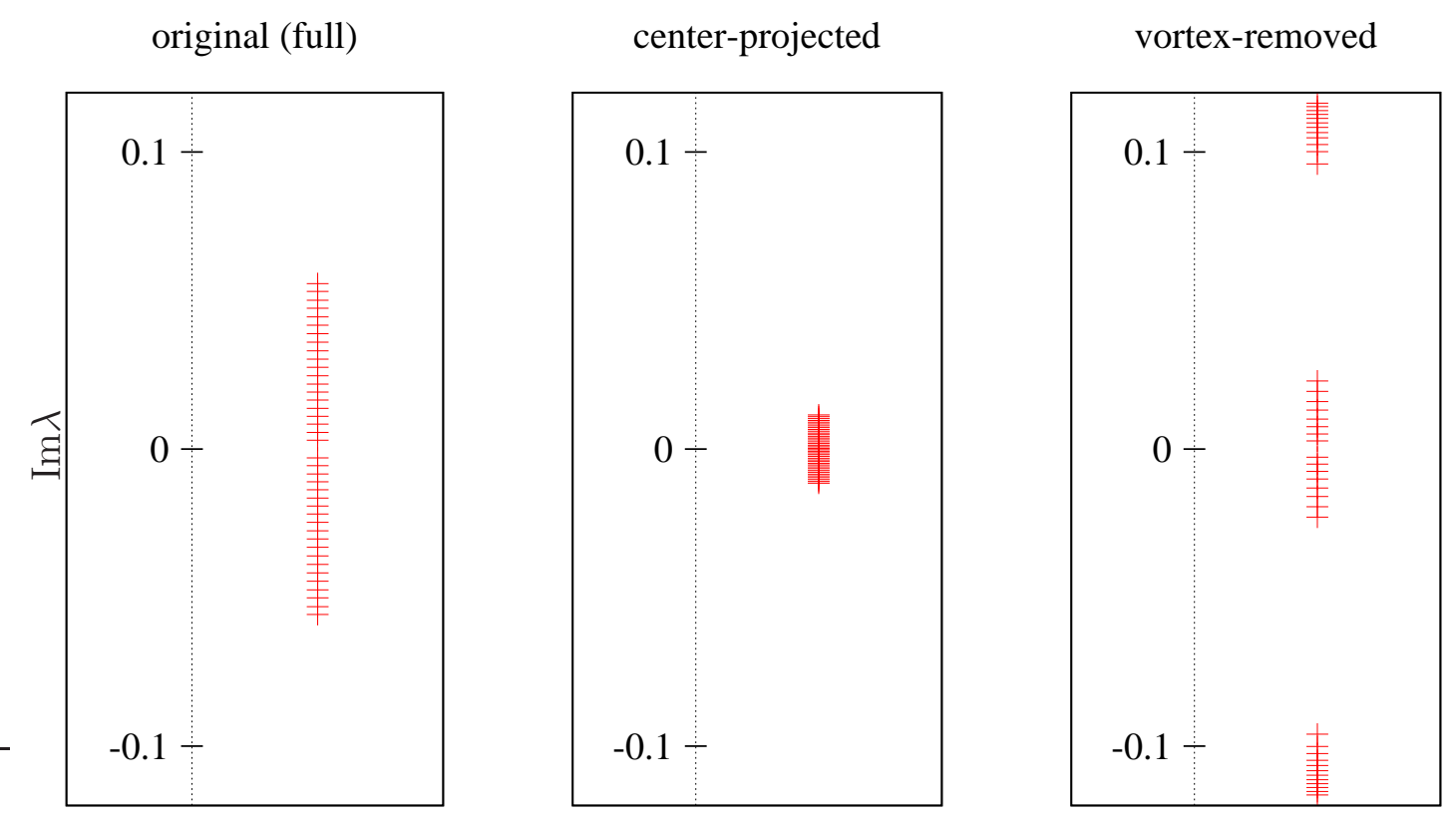

Figure 104: First twenty asqtad staggered Dirac eigenvalues on a $20^{4}$ lattice at $\beta=3.5$.
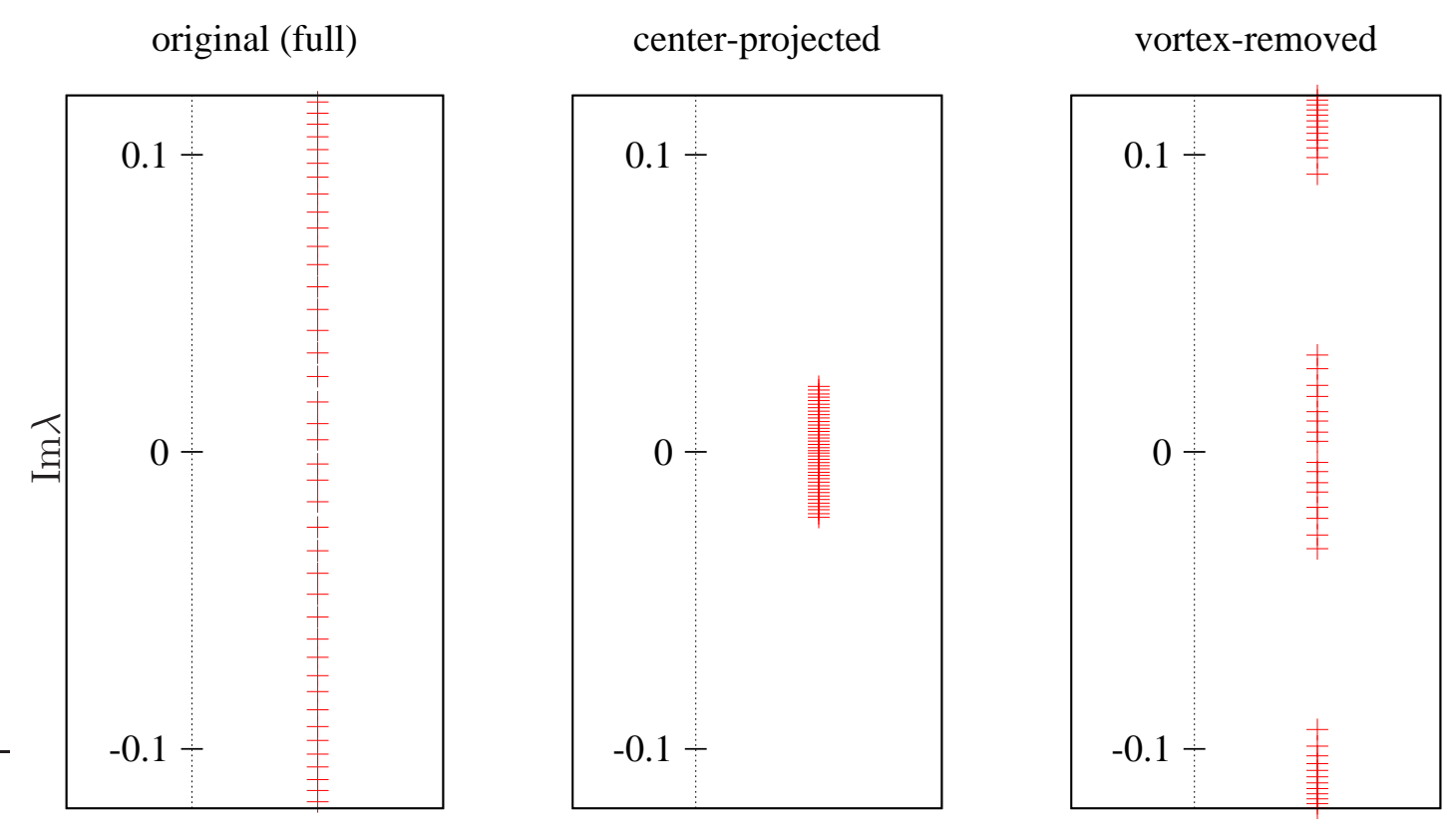

Figure 105: First twenty asqtad staggered Dirac eigenvalues on a $20^{4}$ lattice at $\beta=3.7$. 


\section{Plots and Figures}
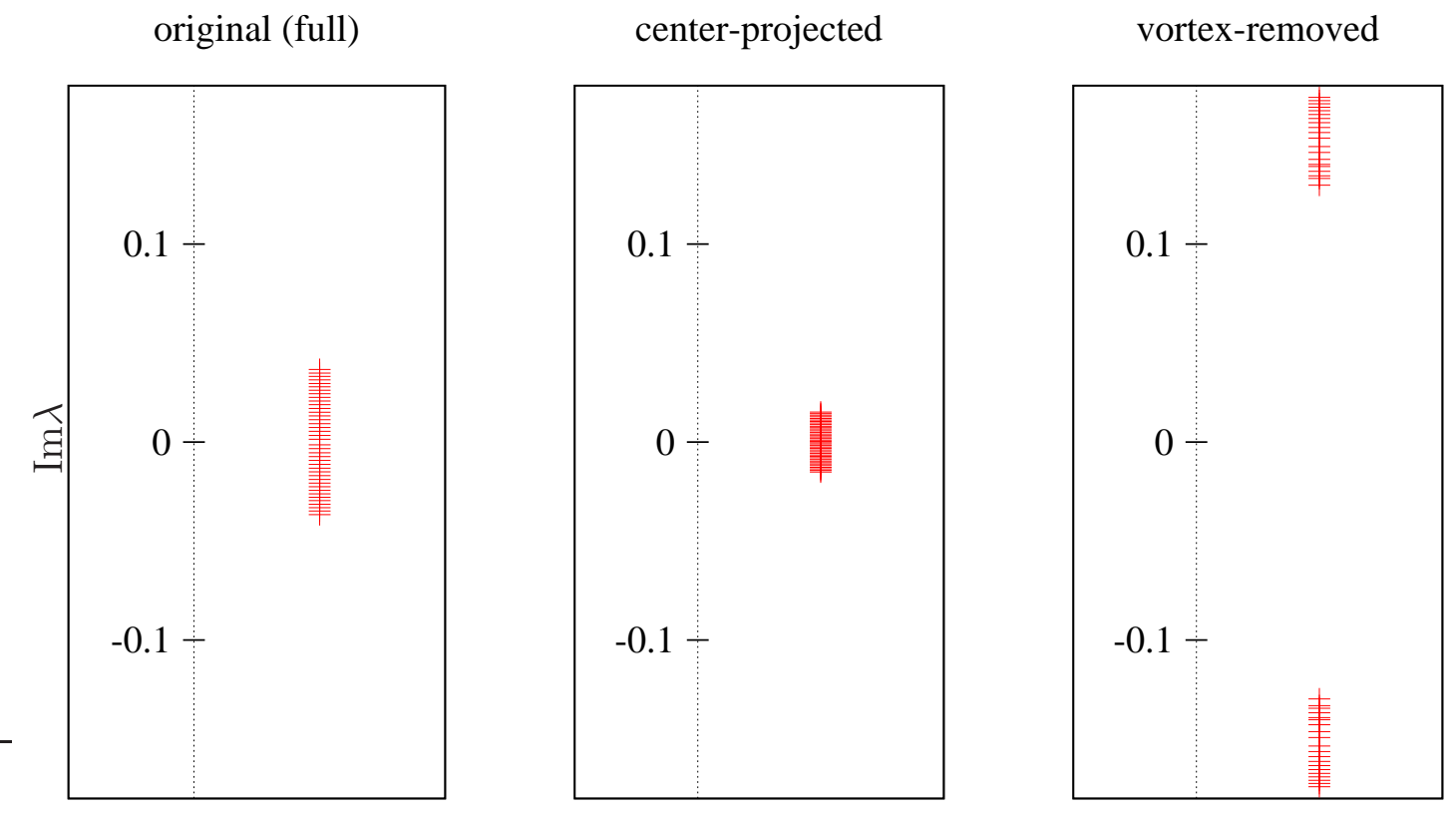

Figure 106: First twenty asqtad staggered Dirac eigenvalues on a $12^{4}$ lattice at $\beta=2.9$ using anti periodic boundary conditions.
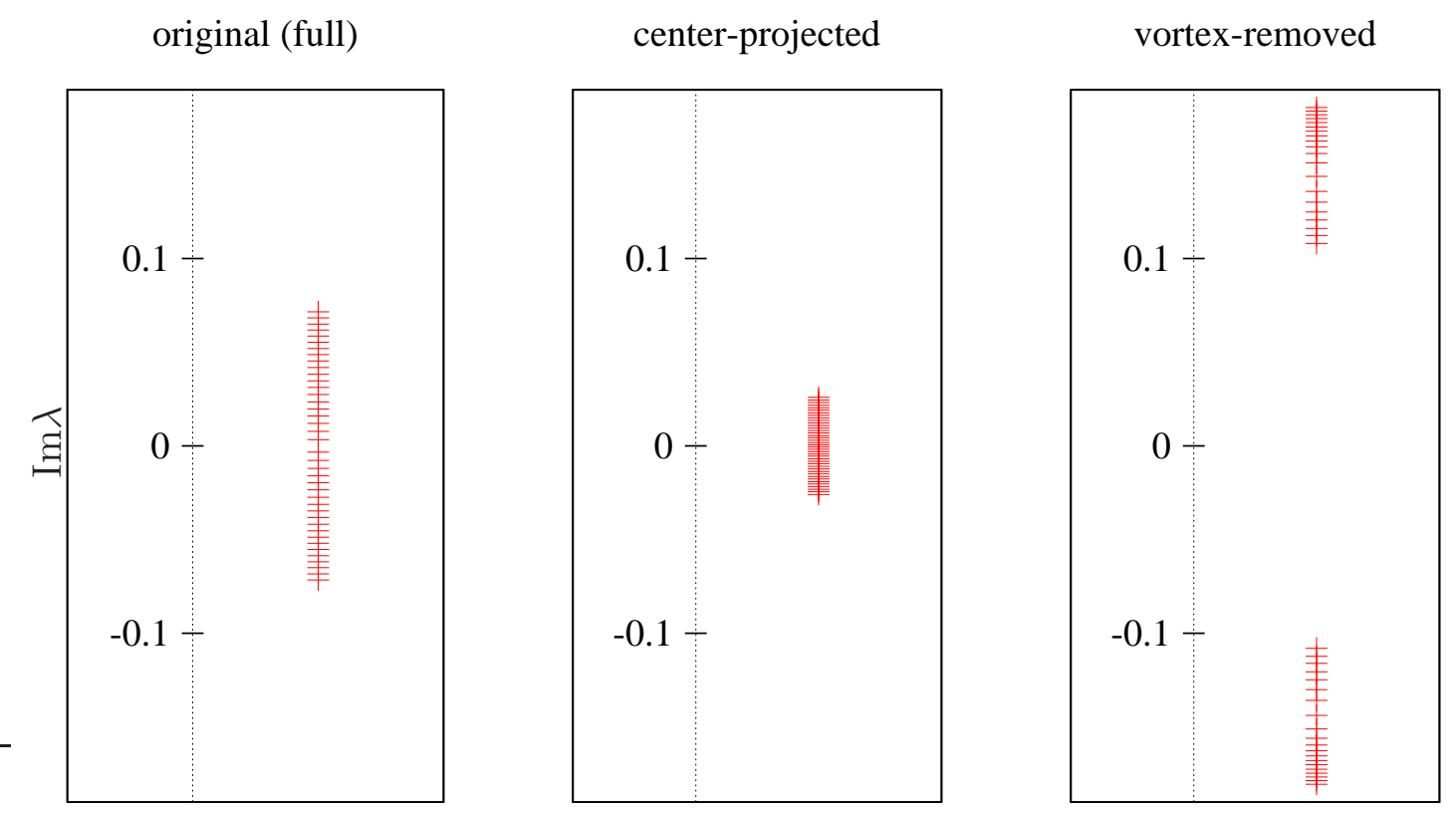

Figure 107: First twenty asqtad staggered Dirac eigenvalues on a $12^{4}$ lattice at $\beta=3.1$ using anti periodic boundary conditions. 


\section{Plots and Figures}
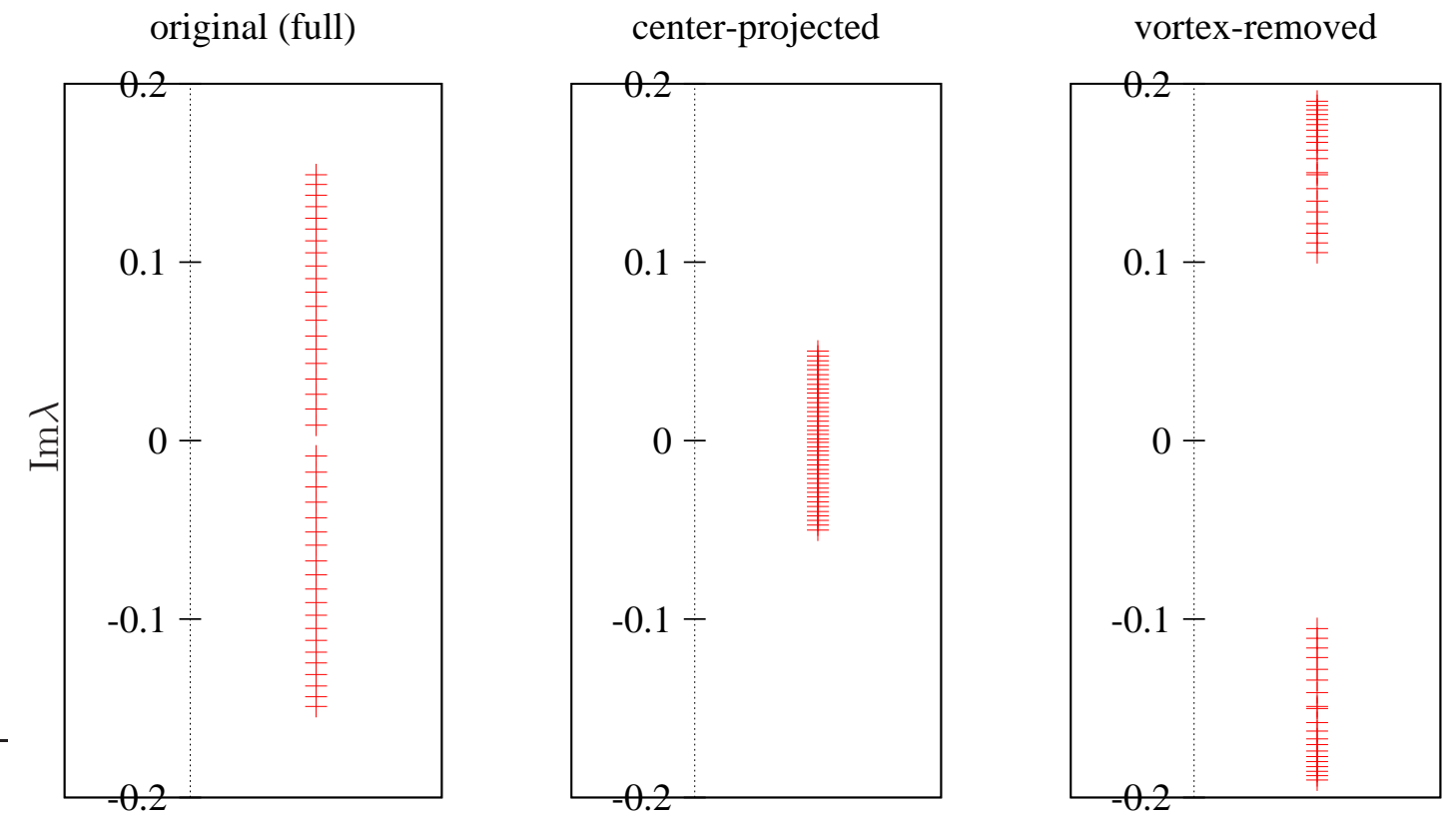

Figure 108: First twenty asqtad staggered Dirac eigenvalues on a $12^{4}$ lattice at $\beta=3.3$ using anti periodic boundary conditions.
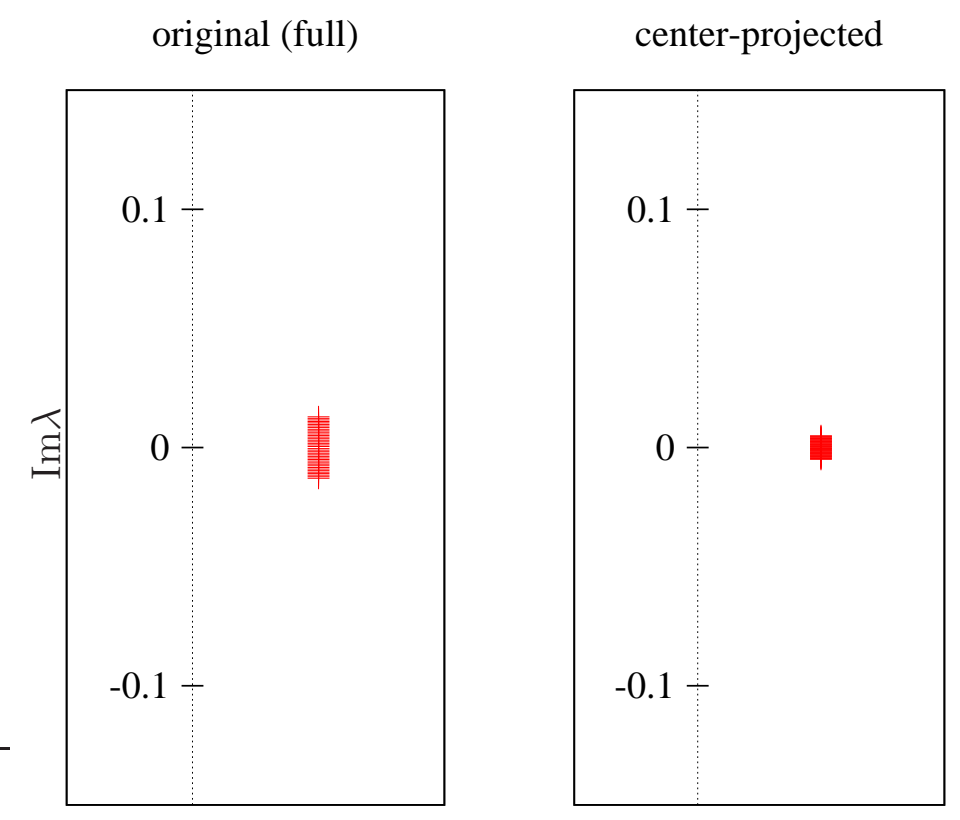

vortex-removed

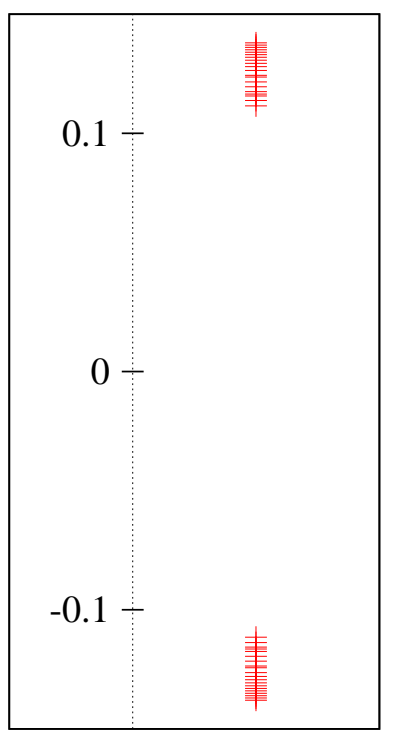

Figure 109: First twenty asqtad staggered Dirac eigenvalues on a $16^{4}$ lattice at $\beta=2.9$ using anti periodic boundary conditions. 

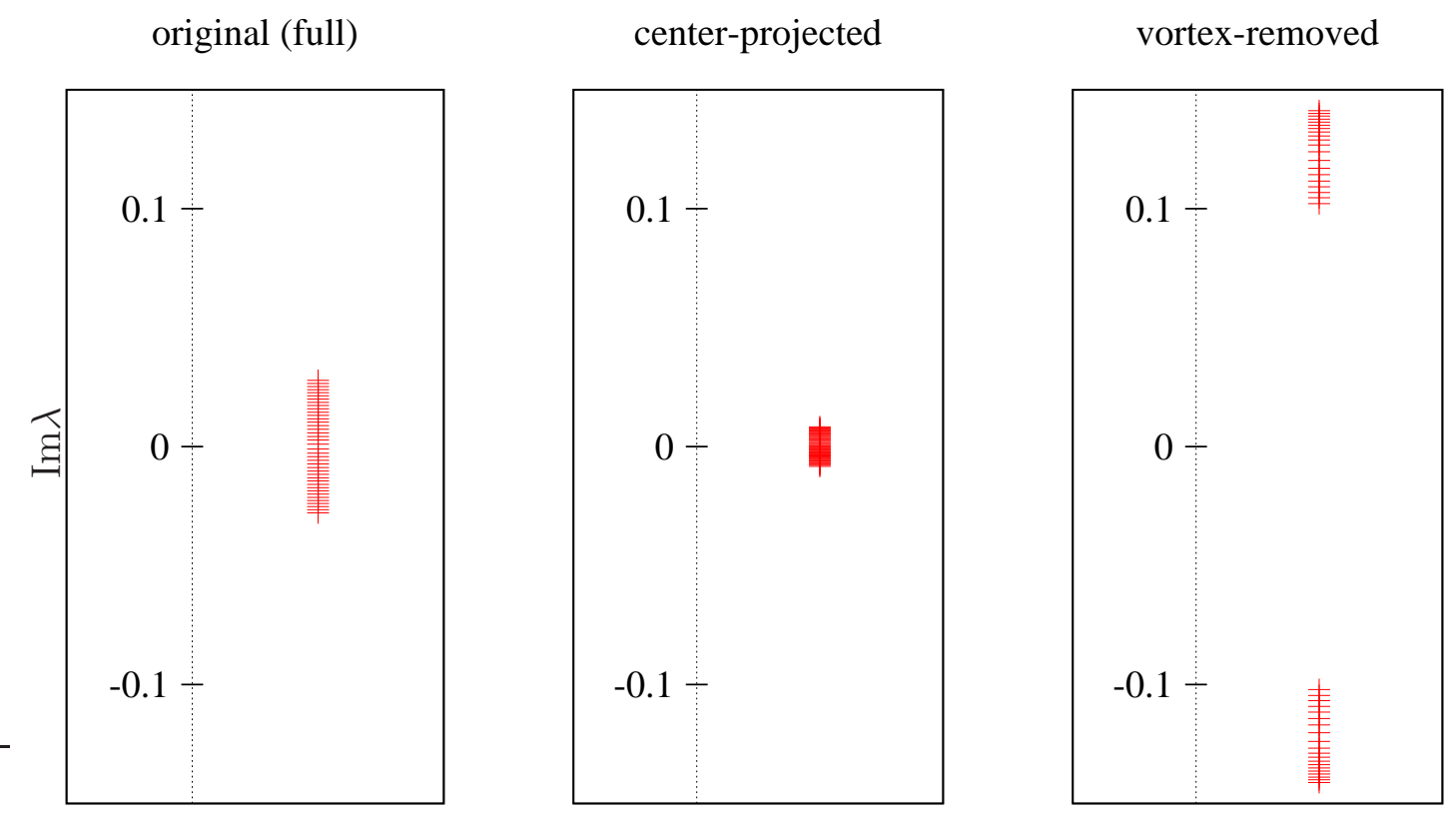

Figure 110: First twenty asqtad staggered Dirac eigenvalues on a $16^{4}$ lattice at $\beta=3.1$ using anti periodic boundary conditions.
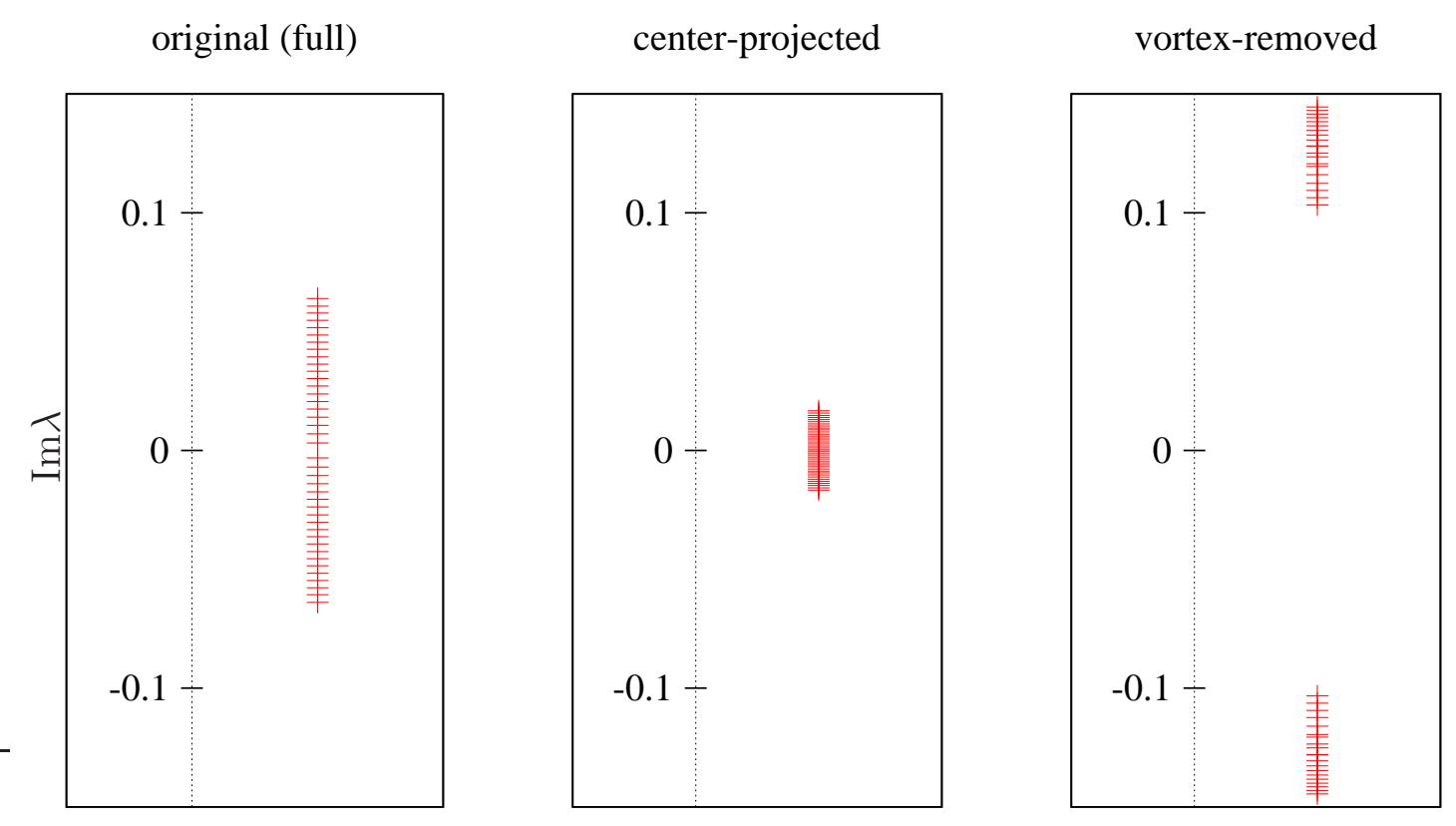

Figure 111: First twenty asqtad staggered Dirac eigenvalues on a $16^{4}$ lattice at $\beta=3.3$ using anti periodic boundary conditions. 


\section{$C$ Plots and Figures}
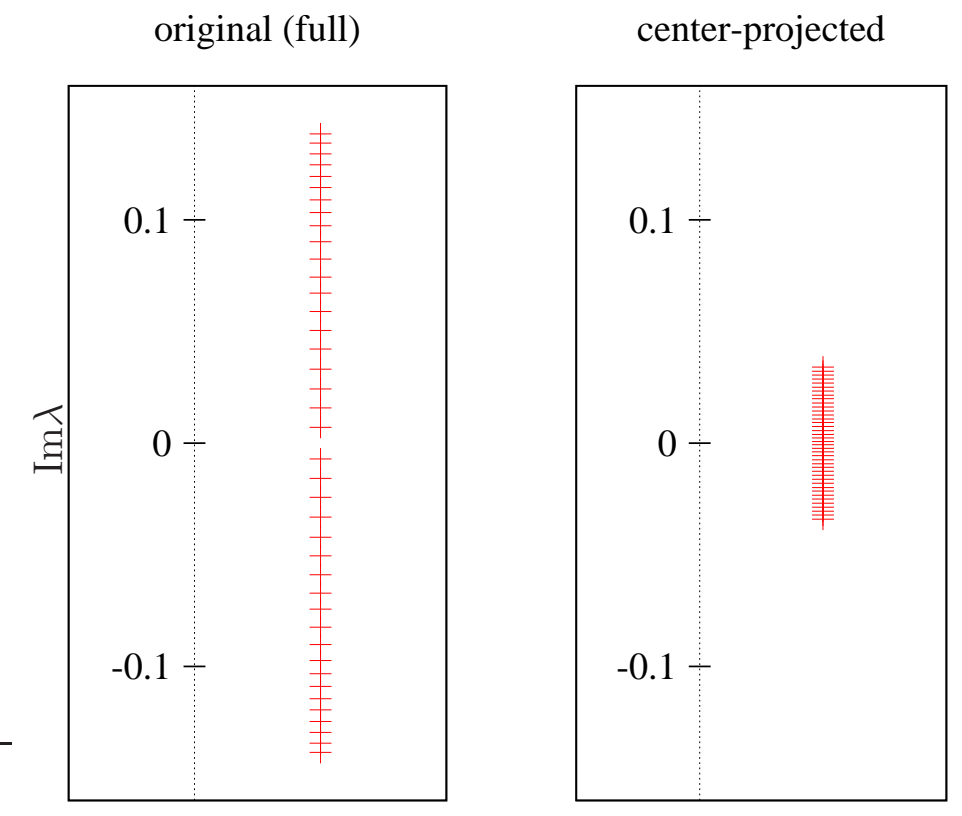

vortex-removed

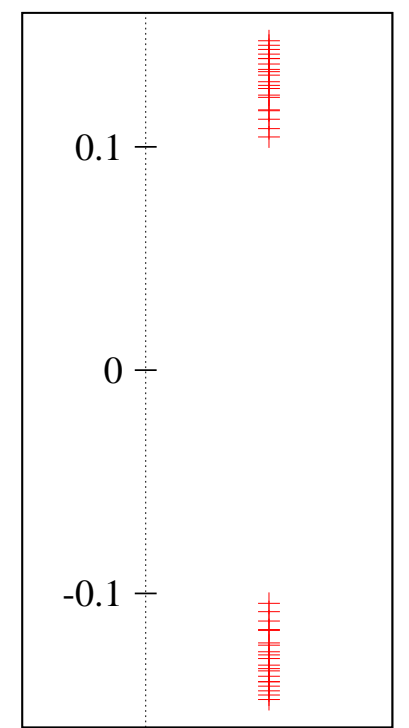

Figure 112: First twenty asqtad staggered Dirac eigenvalues on a $16^{4}$ lattice at $\beta=3.5$ using anti periodic boundary conditions.
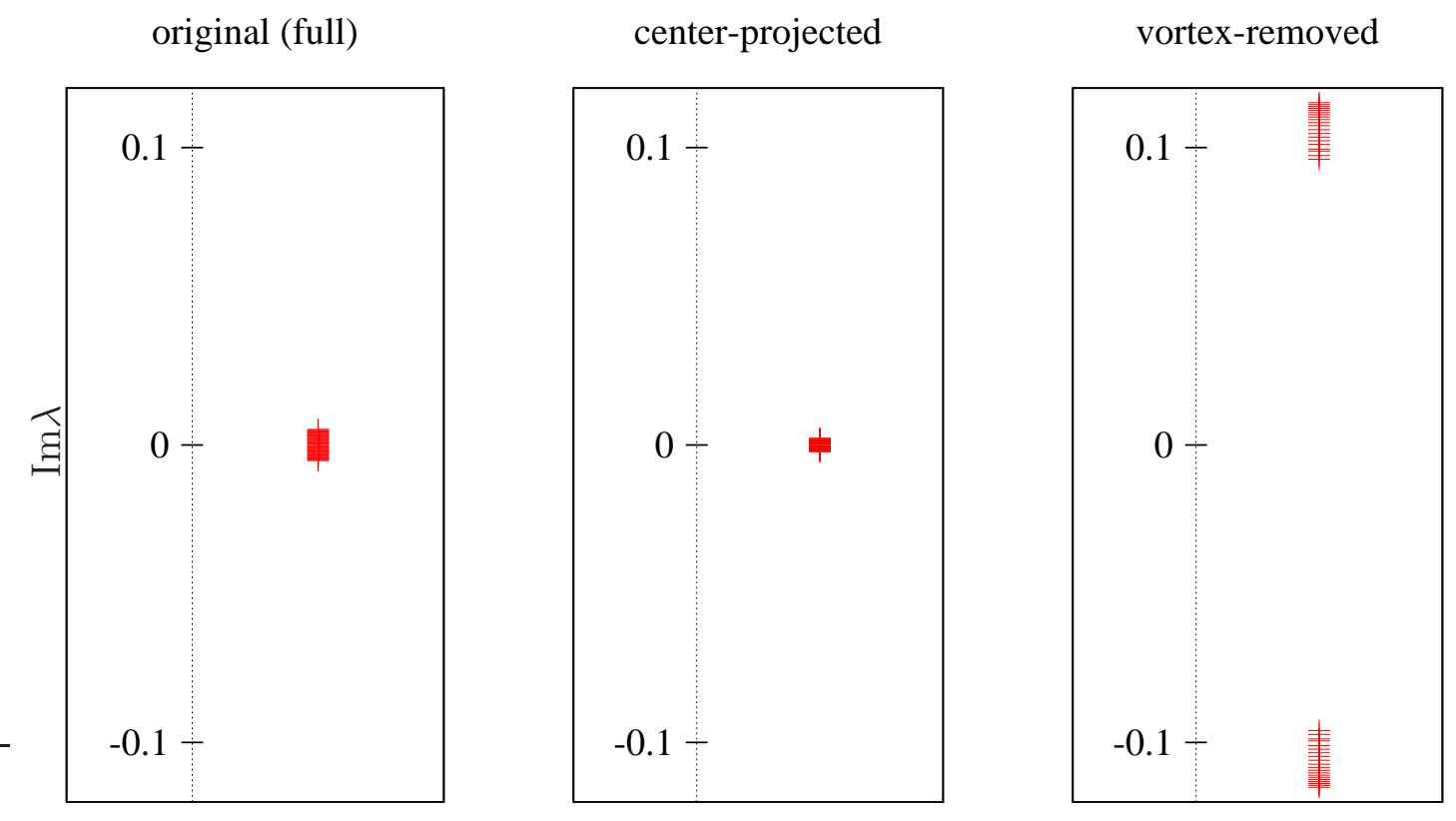

Figure 113: First twenty asqtad staggered Dirac eigenvalues on a $20^{4}$ lattice at $\beta=2.9$ using anti periodic boundary conditions. 

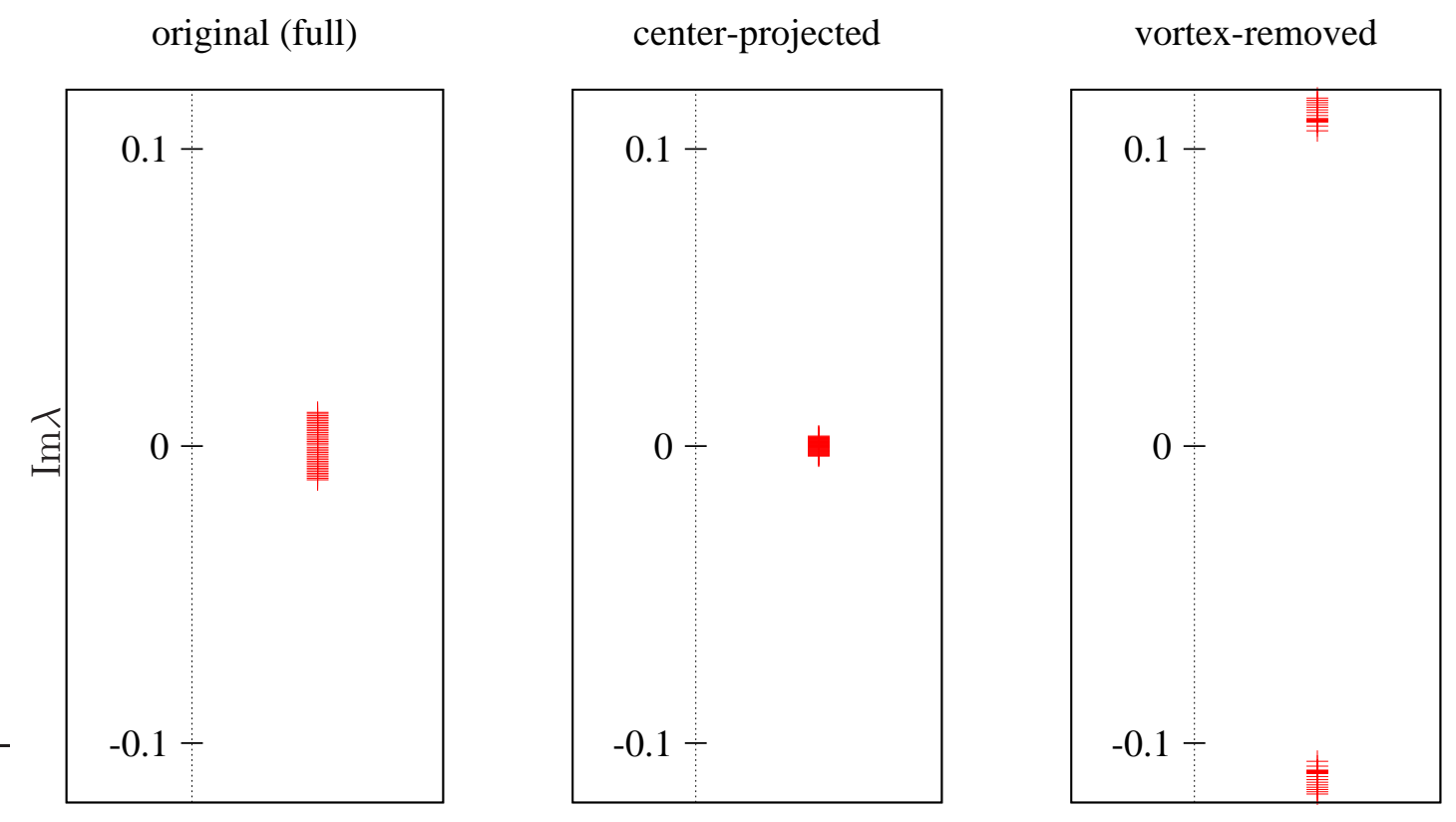

Figure 114: First twenty asqtad staggered Dirac eigenvalues on a $20^{4}$ lattice at $\beta=3.1$ using anti periodic boundary conditions.
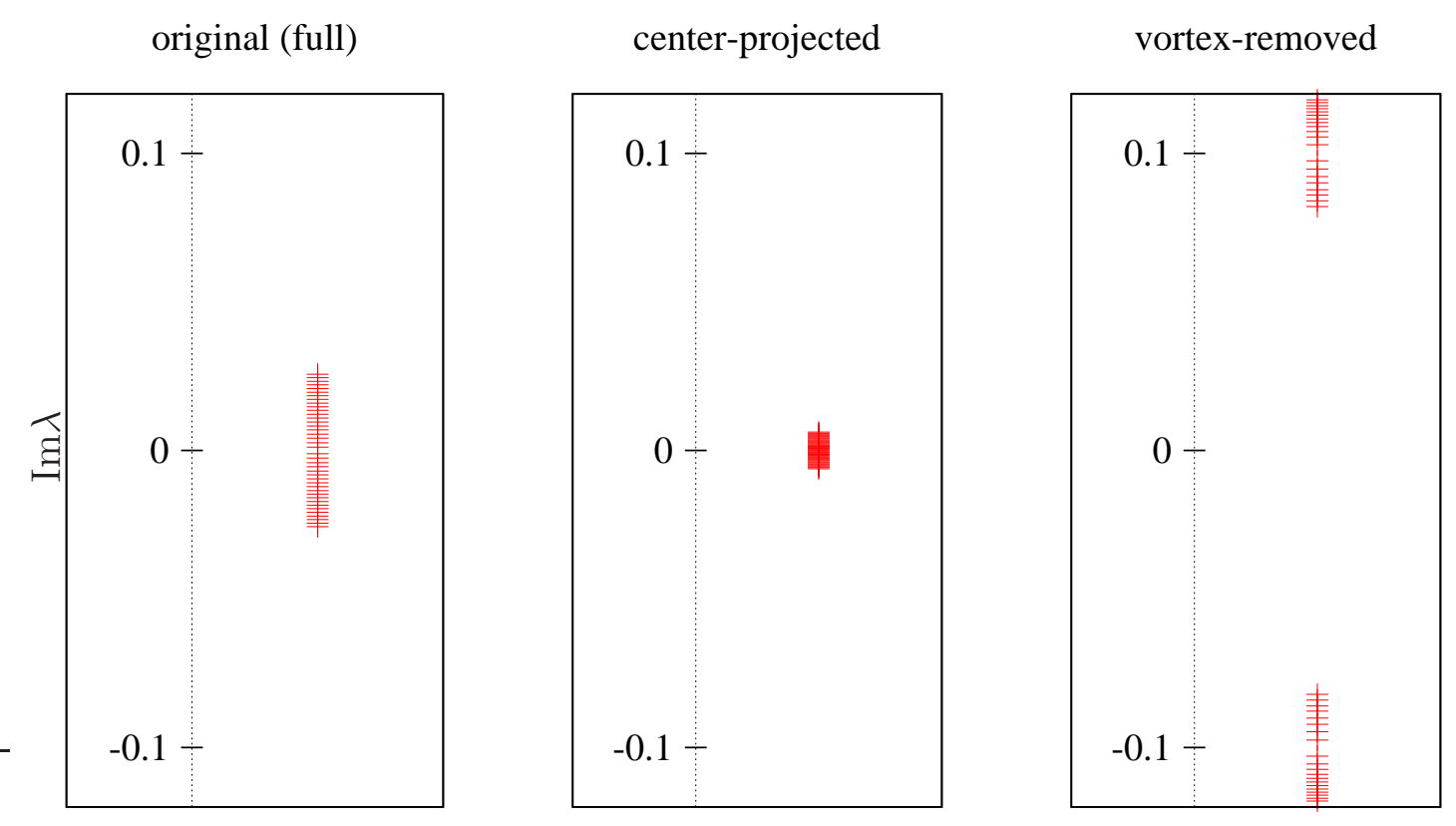

Figure 115: First twenty asqtad staggered Dirac eigenvalues on a $20^{4}$ lattice at $\beta=3.3$ using anti periodic boundary conditions. 

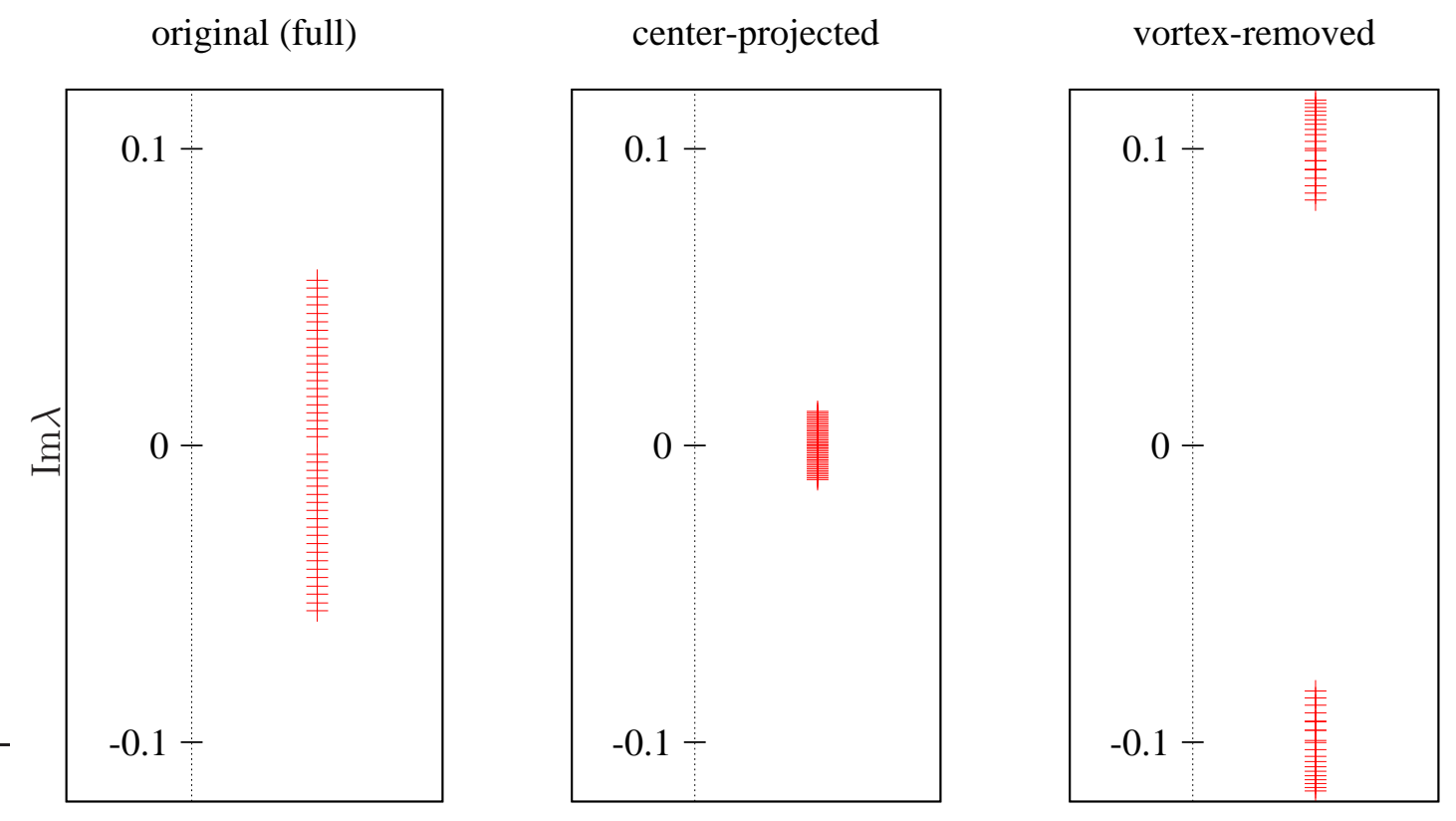

Figure 116: First twenty asqtad staggered Dirac eigenvalues on a $20^{4}$ lattice at $\beta=3.5$ using anti periodic boundary conditions.
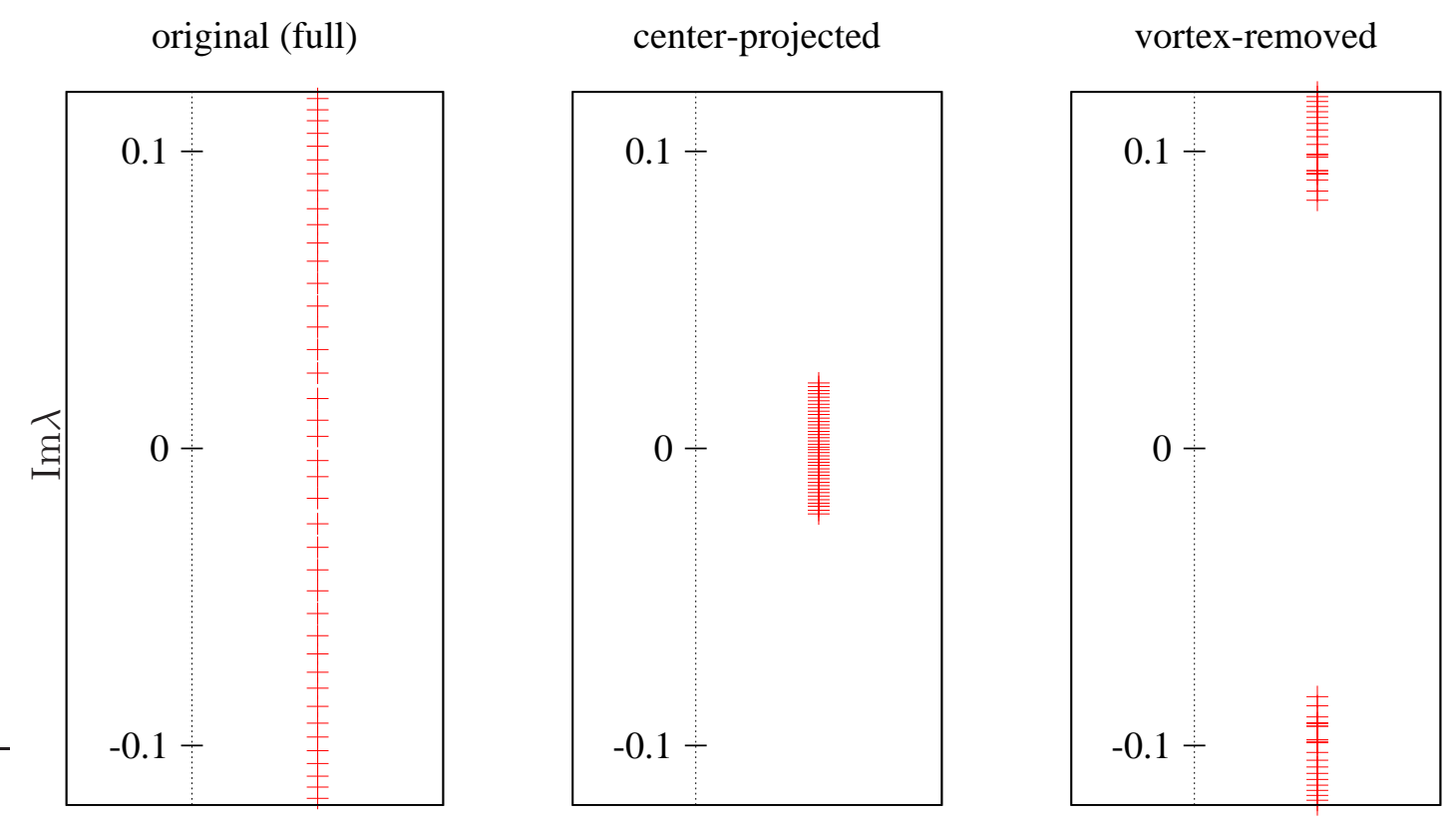

Figure 117: First twenty asqtad staggered Dirac eigenvalues on a $20^{4}$ lattice at $\beta=3.7$ using anti periodic boundary conditions. 


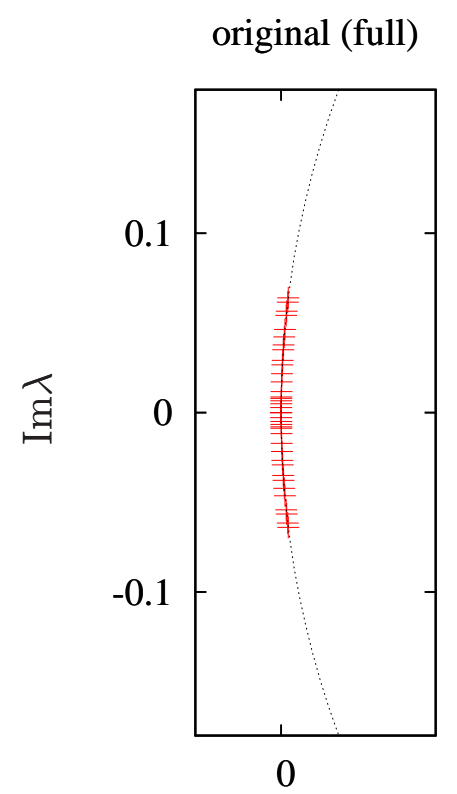

$\operatorname{Re} \lambda$

$80 \%$ projected

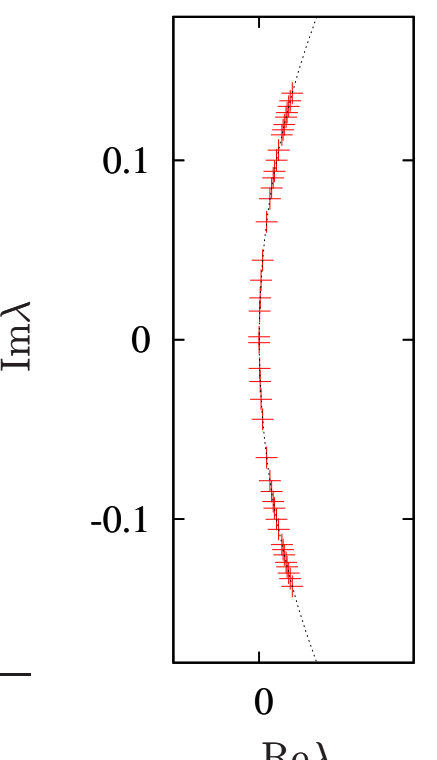

$25 \%$ projected

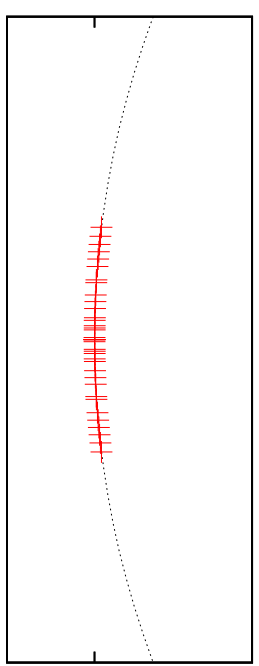

0

$\operatorname{Re} \lambda$

$85 \%$ projected

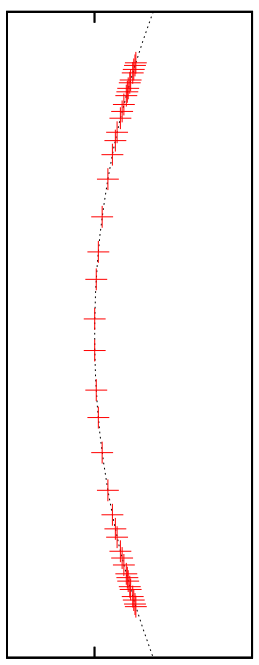

0

$\operatorname{Re} \lambda$
$50 \%$ projected

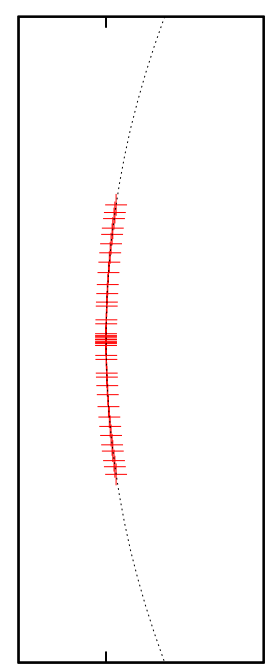

0

$\operatorname{Re} \lambda$

$90 \%$ projected

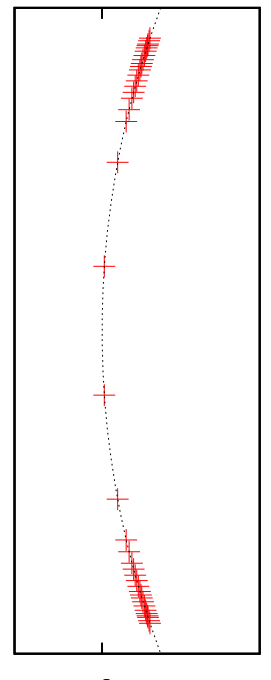

$\operatorname{Re} \lambda$

$70 \%$ projected

$75 \%$ projected

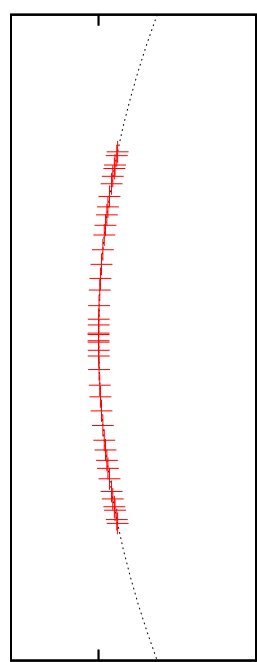

0

$\operatorname{Re} \lambda$

95\% projected

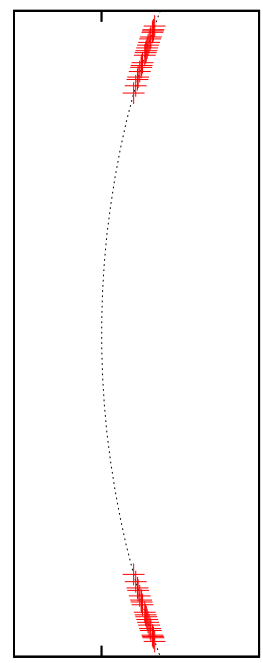

0

$\operatorname{Re} \lambda$

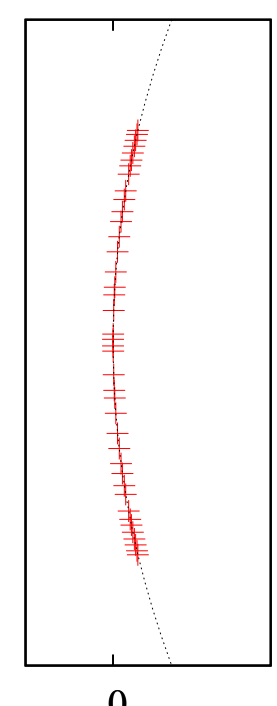

$\operatorname{Re} \lambda$

center-projected

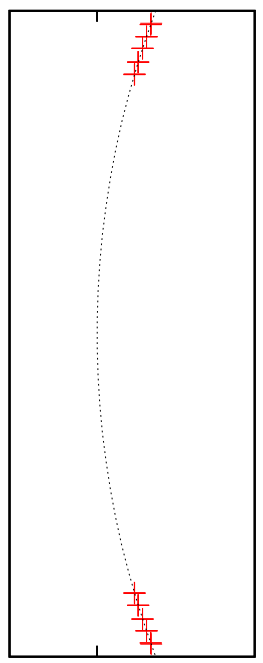
0

Figure 118: First twenty overlap Dirac eigenvalues of a single configuration on a $16^{4}$ lattice at $\beta=3.3$ for interpolated fields. 

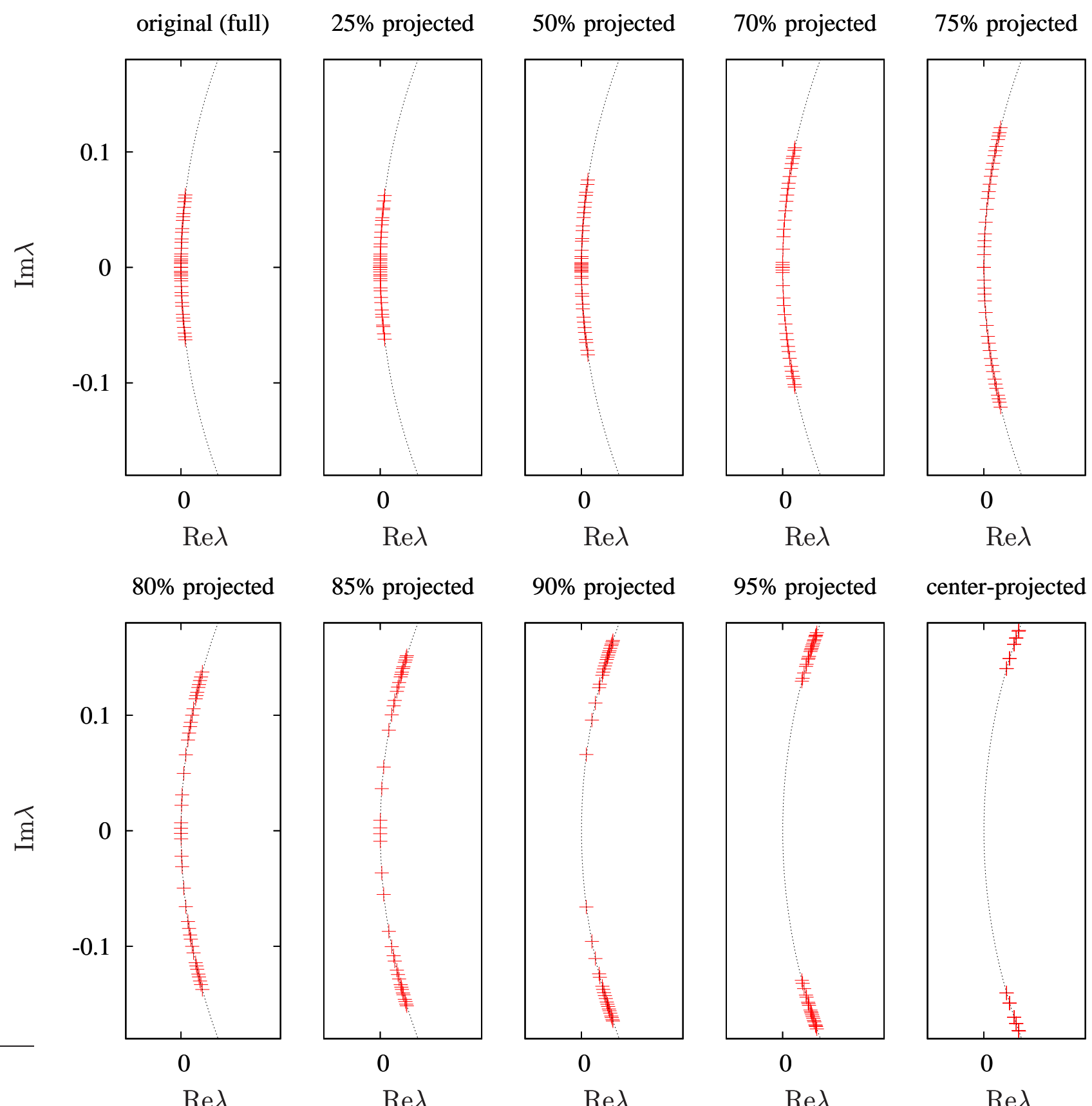

$85 \%$ projected

90\% projected

95\% projected

center-projected

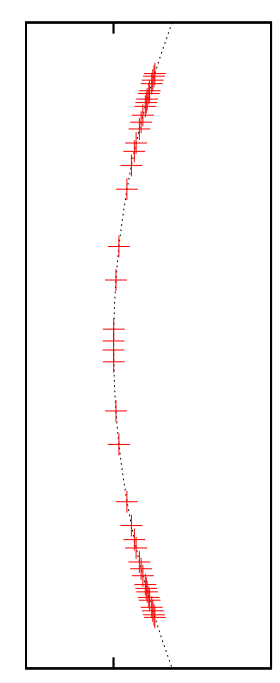

0

$\operatorname{Re} \lambda$

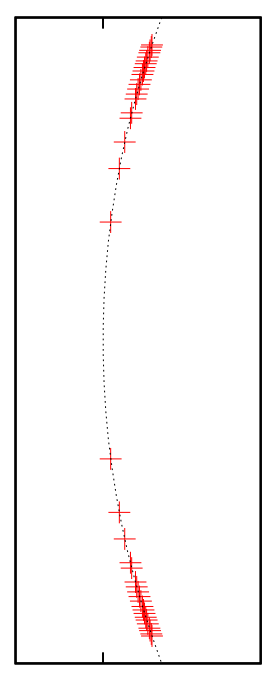

0

$\operatorname{Re} \lambda$

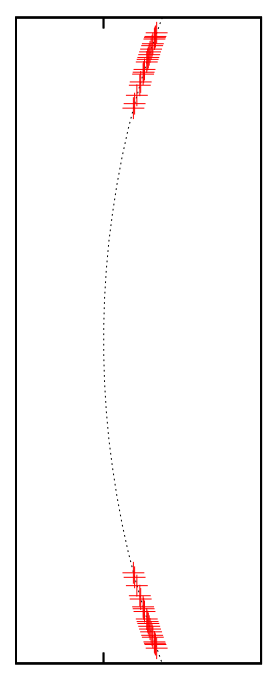

0

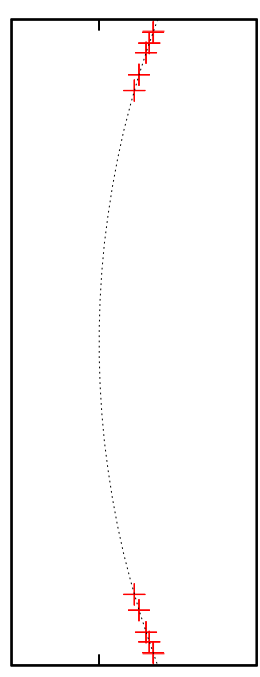

0

Figure 119: First twenty overlap Dirac eigenvalues of a single configuration on a $16^{4}$ lattice at $\beta=3.3$ for interpolated fields using anti periodic boundary conditions. 
$z=3, t=2$

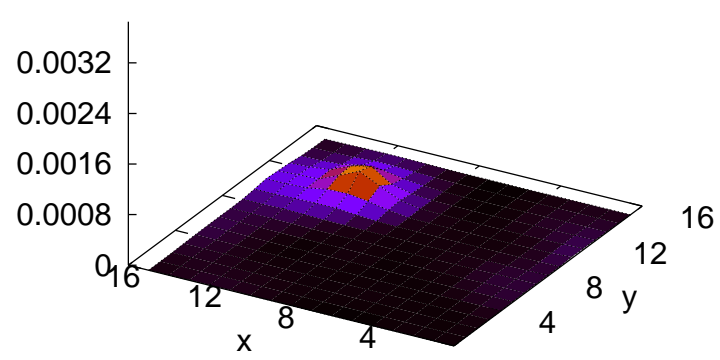

$\mathrm{z}=4, \mathrm{t}=2$

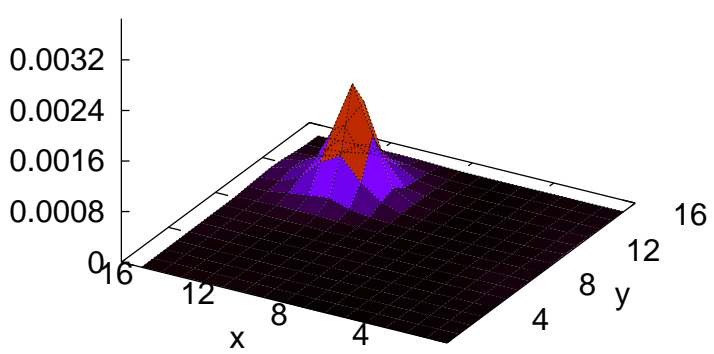

density of eigenvalue $\# 1$, maximum 0.0032086919732 at $x=11, y=12, z=5, t=2$

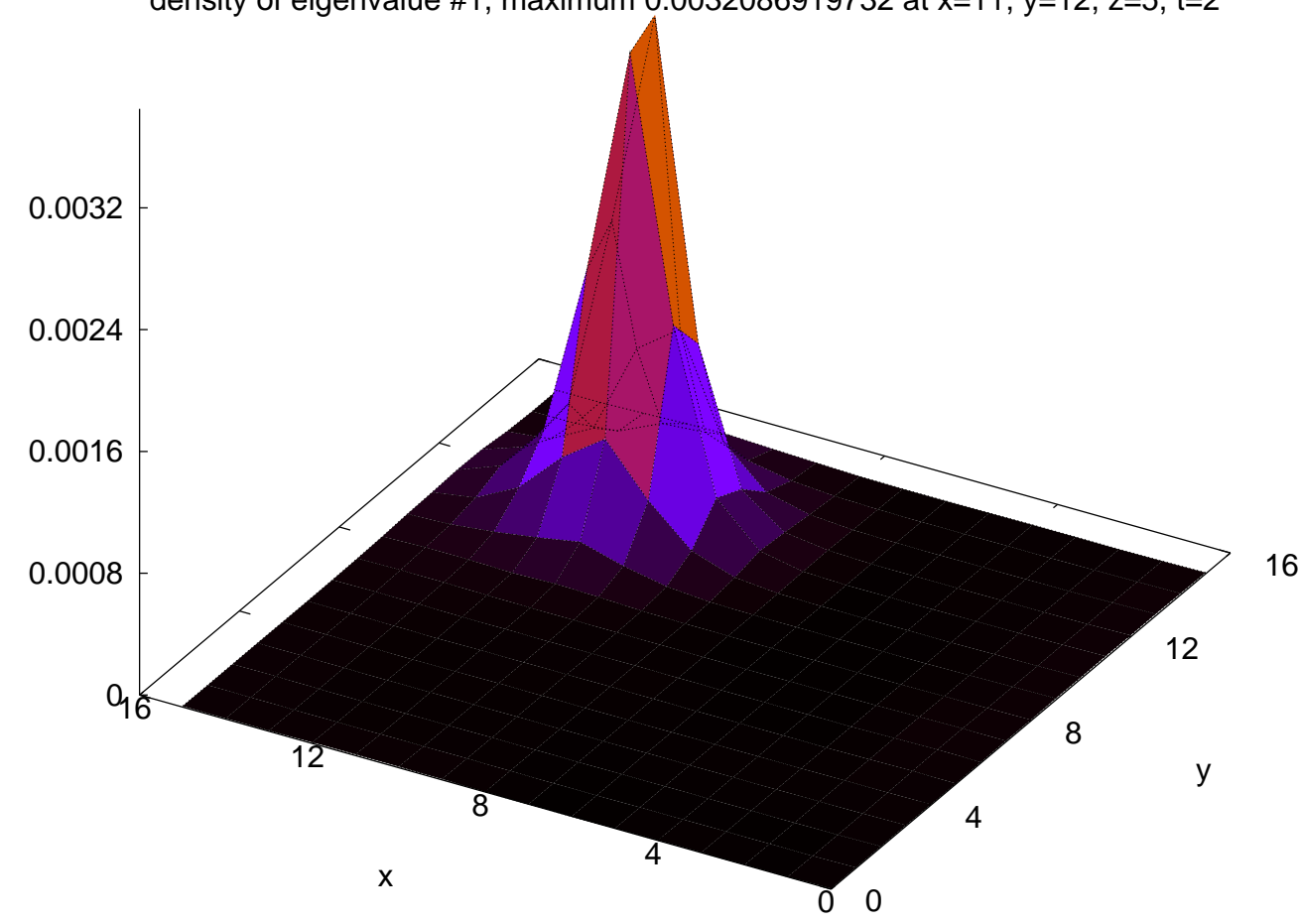

$\mathrm{z}=6, \mathrm{t}=2$

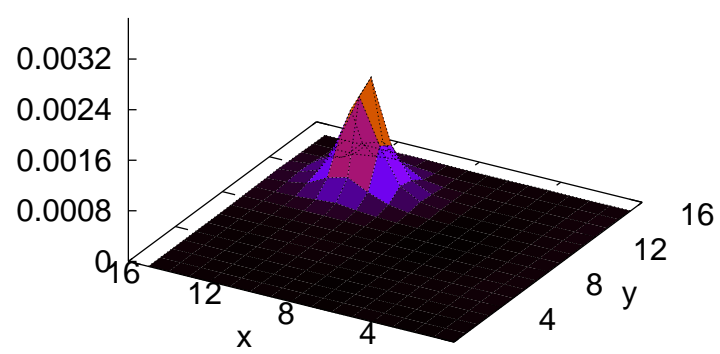

$\mathrm{z}=7, \mathrm{t}=2$

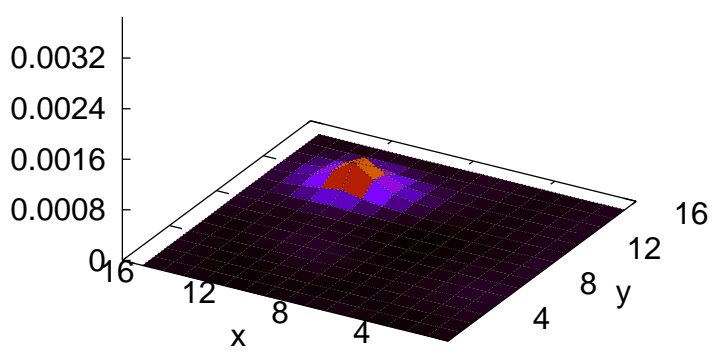

Figure 120: Maximum density peak (center) of first overlap eigenmode for a full configuration on a $16^{4}$-lattice at $\beta_{L W}=3.3$ with upper (above) and lower (below) Z-slices of the same t-slice. 132 
$z=12, t=7$

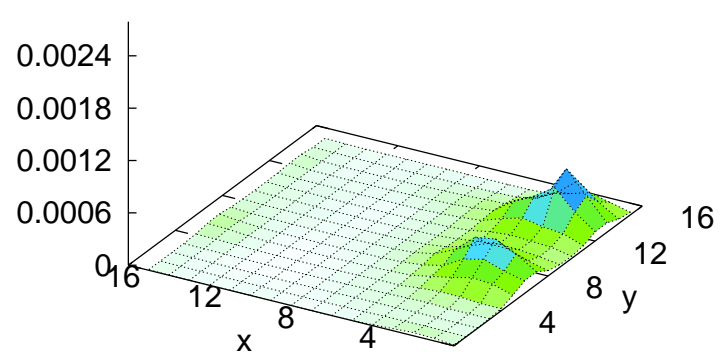

$z=13, t=7$

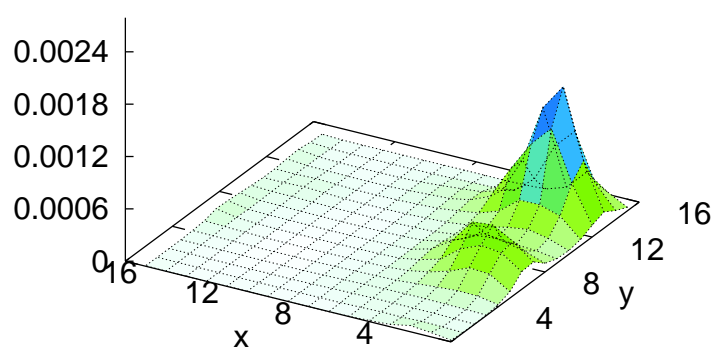

density of eigenvalue $\# 1$, maximum 0.00232418839707 at $x=3, y=13, z=14, t=7$

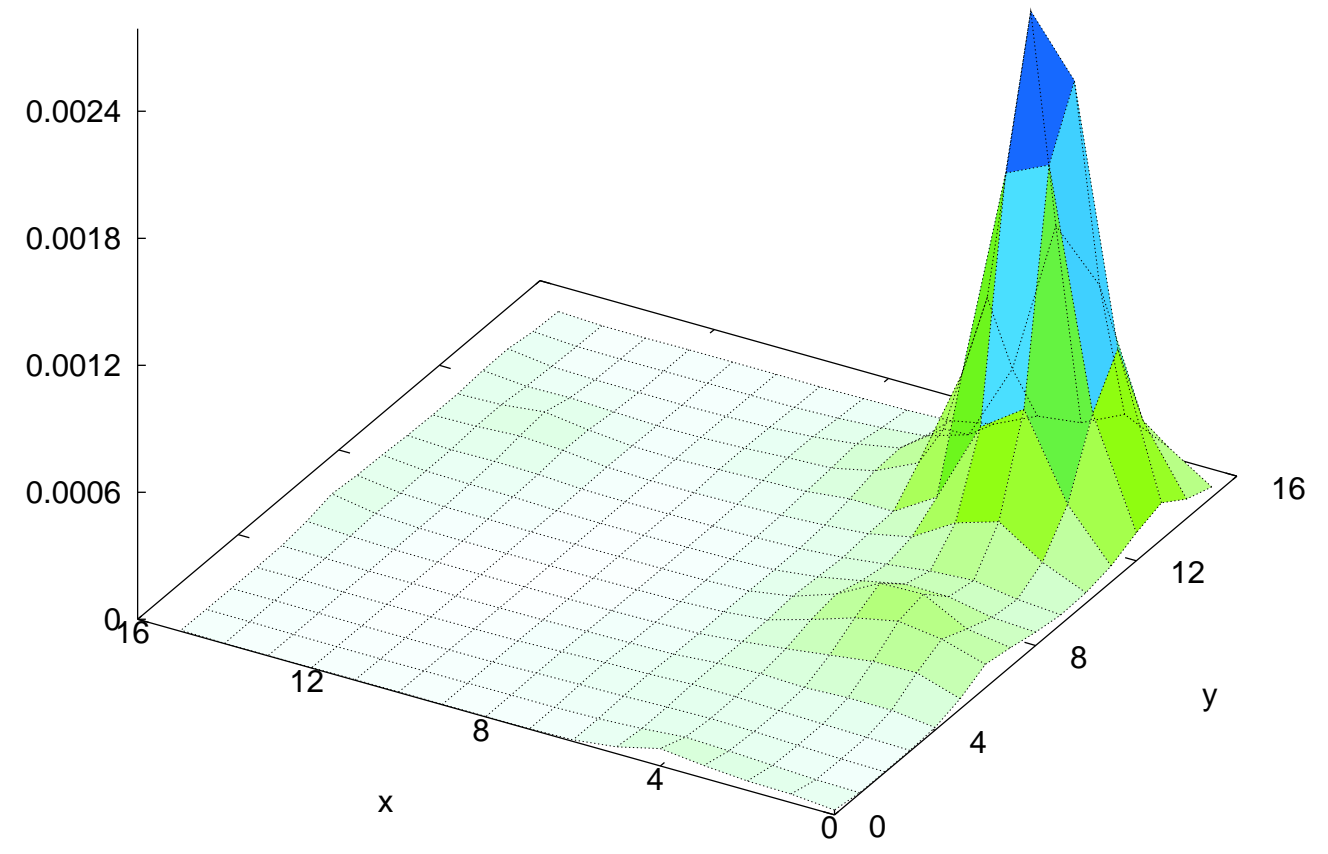

$\mathrm{z}=15, \mathrm{t}=7$

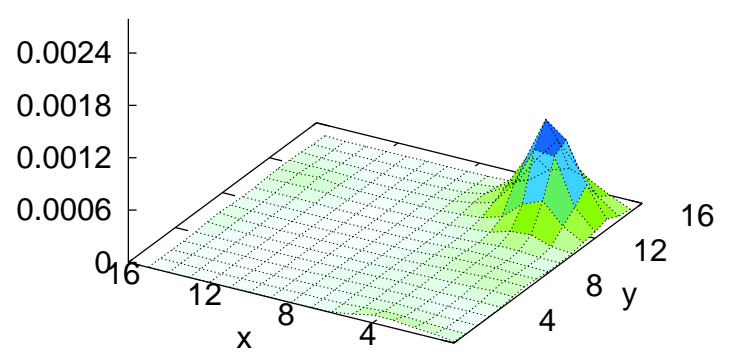

$\mathrm{z}=0, \mathrm{t}=7$

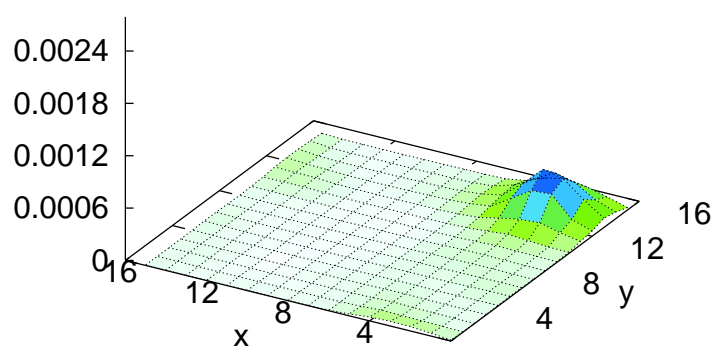

Figure 121: Maximum density peak (center) of first overlap eigenmode for a full configuration on a $16^{4}$-lattice at $\beta_{L W}=3.3$ with upper (above) and lower (below) z-slices of the same t-slice. 133 

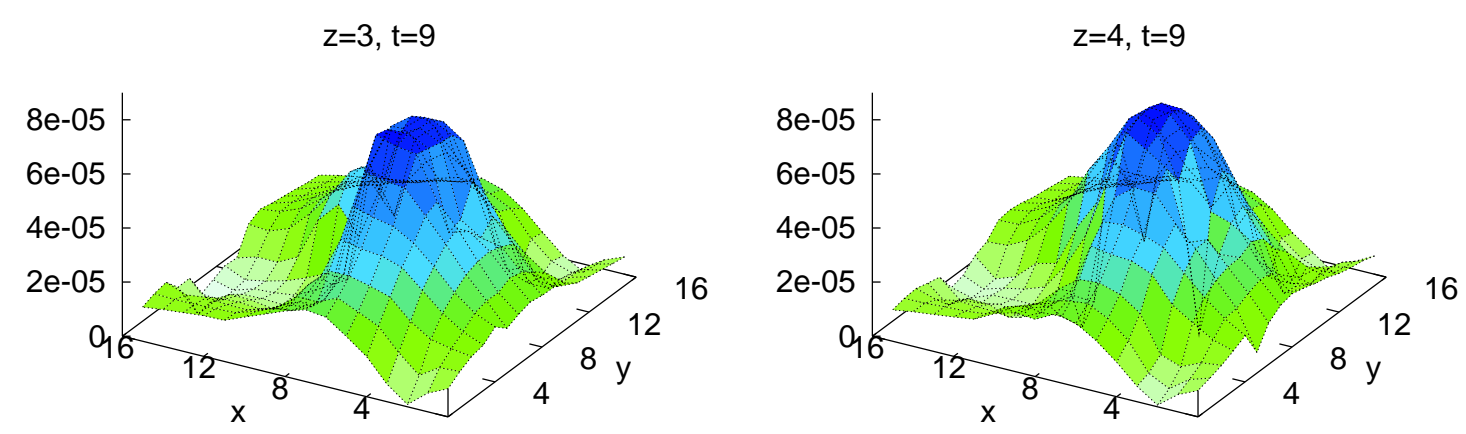

density of eigenvalue $\# 1$, maximum $7.78572660201 \mathrm{e}-05$ at $x=6, y=8, z=5, t=9$

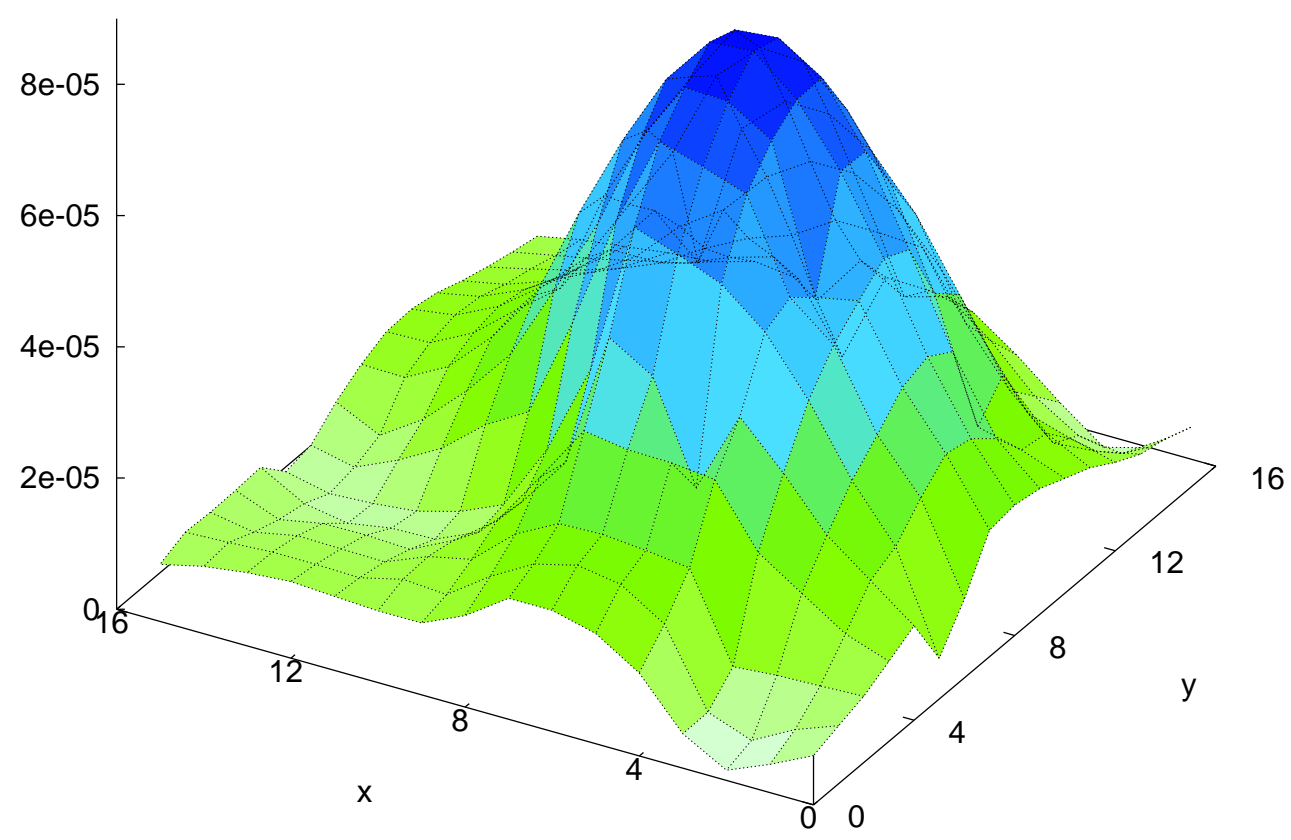

$z=6, t=9$

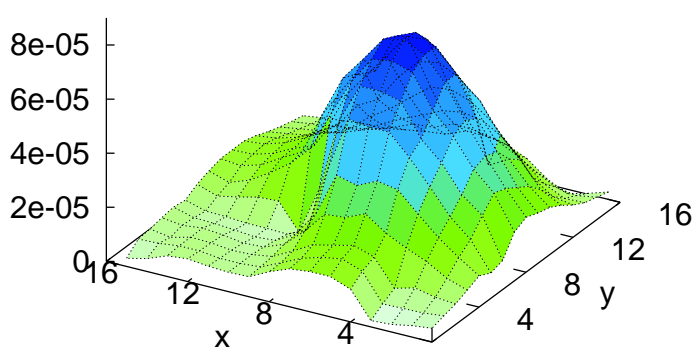

$\mathrm{z}=7, \mathrm{t}=9$

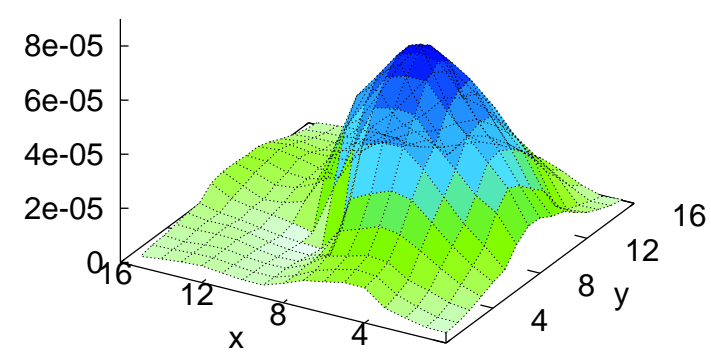

Figure 122: Maximum density peak (center) of first overlap eigenmode for a centerprojected configuration on a $16^{4}$-lattice at $\beta_{L W}=3.3$ with upper (above) and lower (below) z-slices of the same t-slice. 

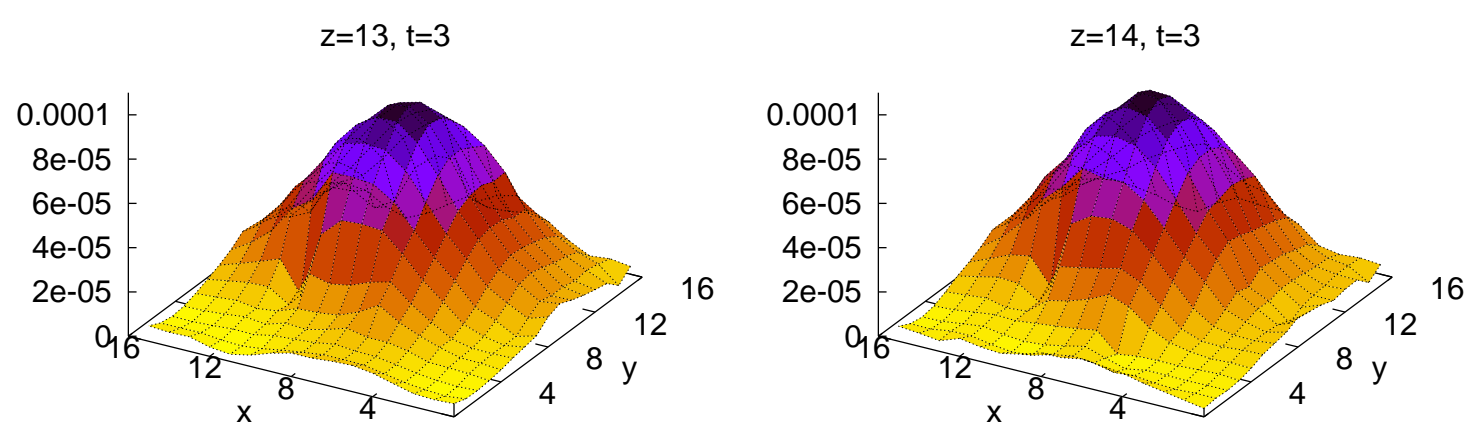

density of eigenvalue \#1, maximum $8.84376963119 \mathrm{e}-05$ at $x=9, y=10, z=15, t=3$
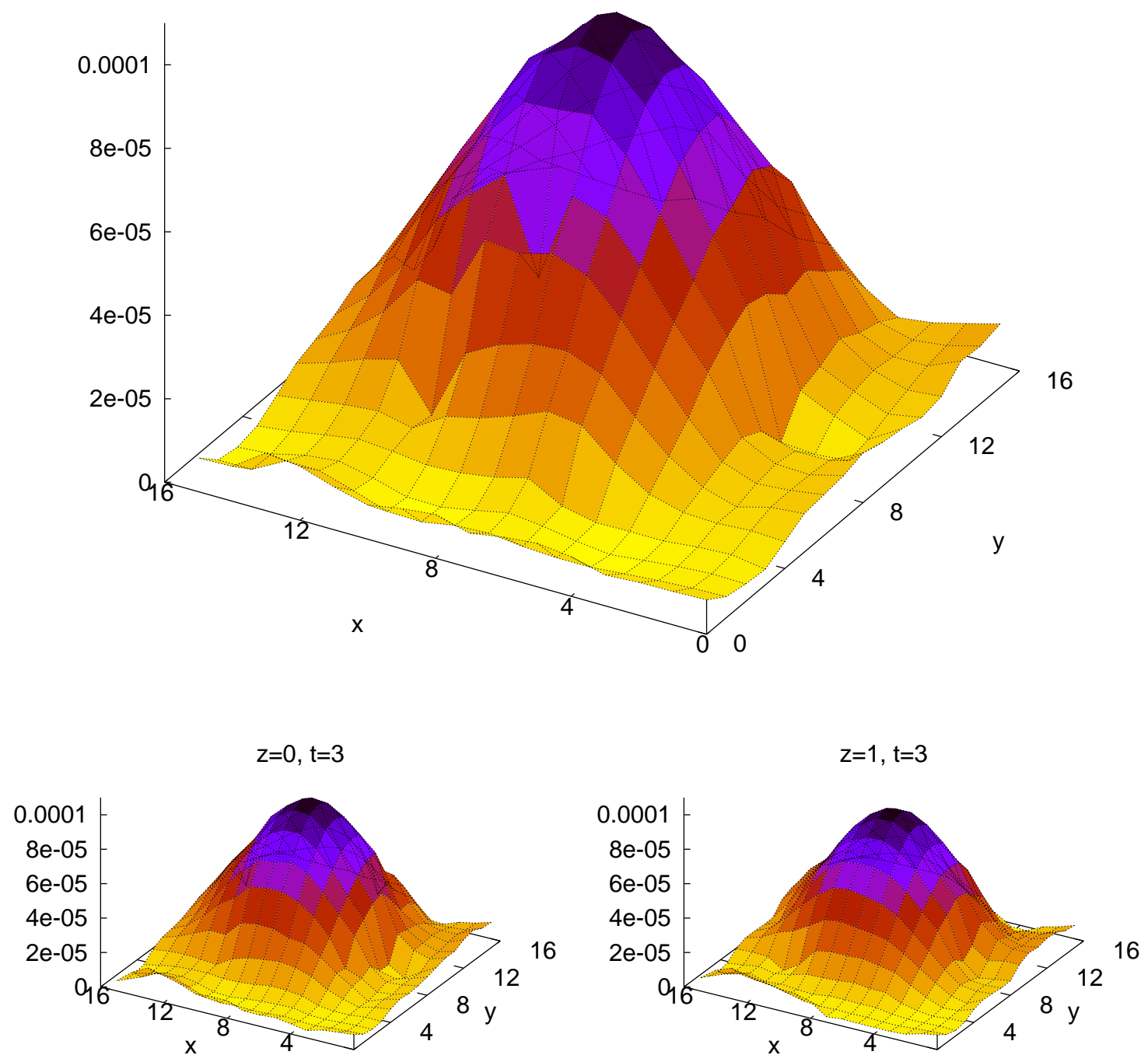

Figure 123: Maximum density peak (center) of first overlap eigenmode for a centerprojected configuration on a $16^{4}$-lattice at $\beta_{L W}=3.3$ with upper (above) and lower (below) z-slices of the same t-slice. (rather sharp peak, little noise) 
$z=10, t=14$

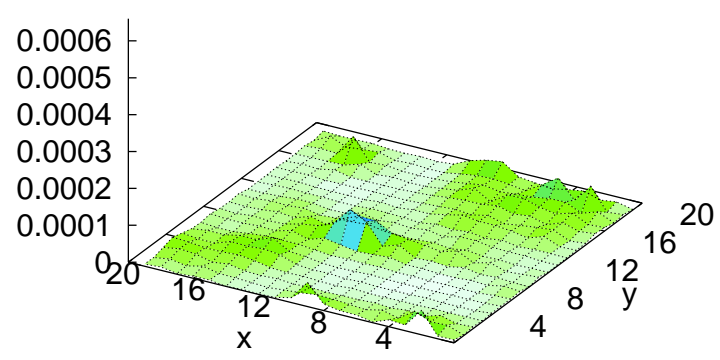

$\mathrm{z}=11, \mathrm{t}=14$

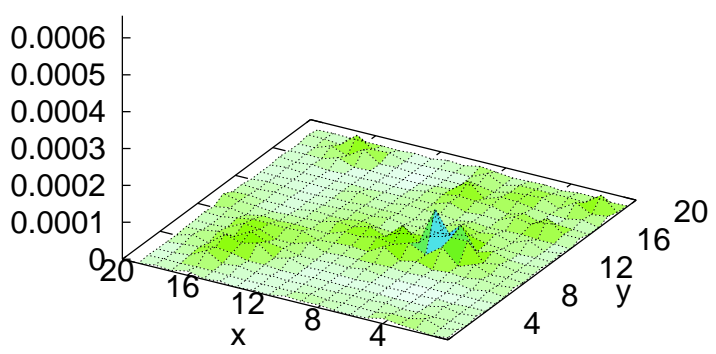

density of eigenvalue \#1, maximum 0.000553522856787 at $x=5, y=9, z=12, t=14$

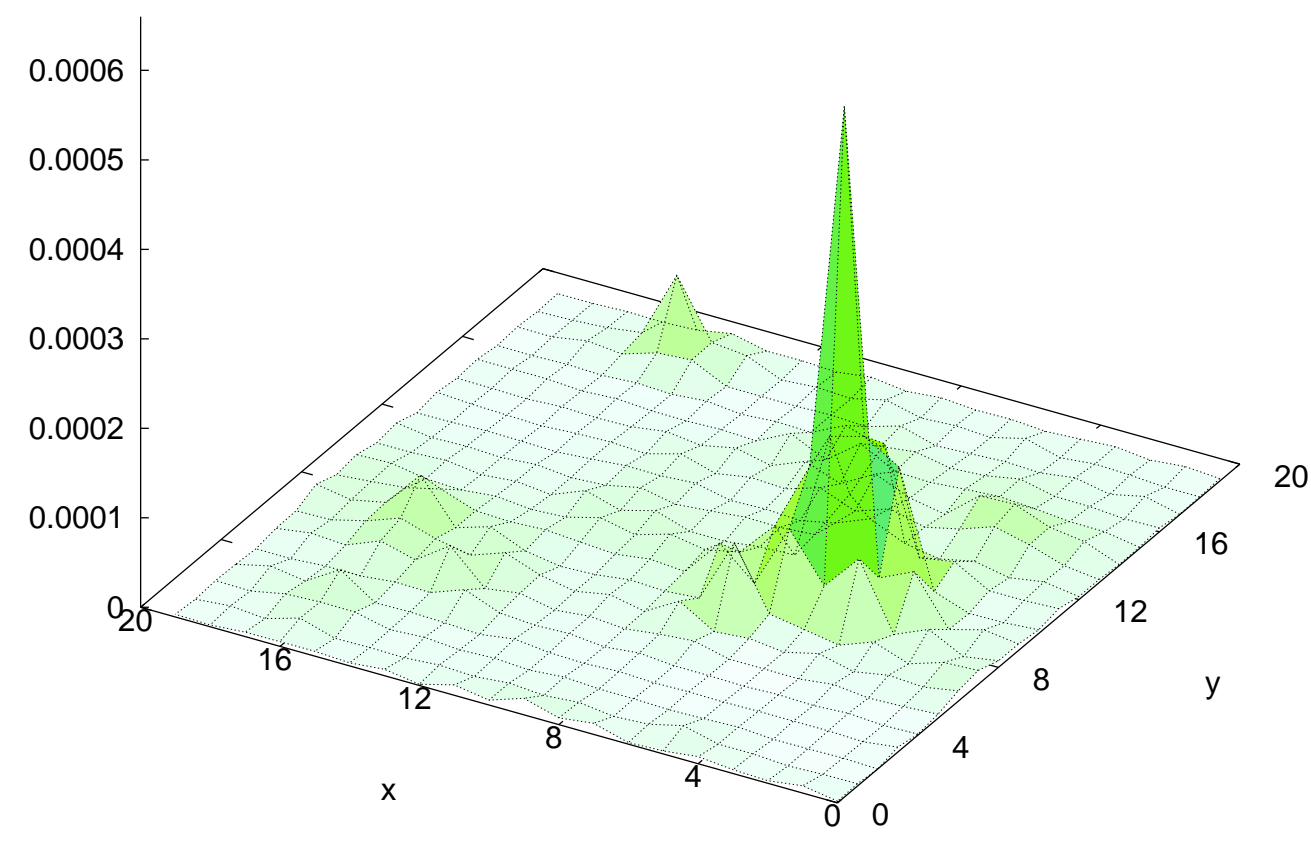

$z=13, t=14$

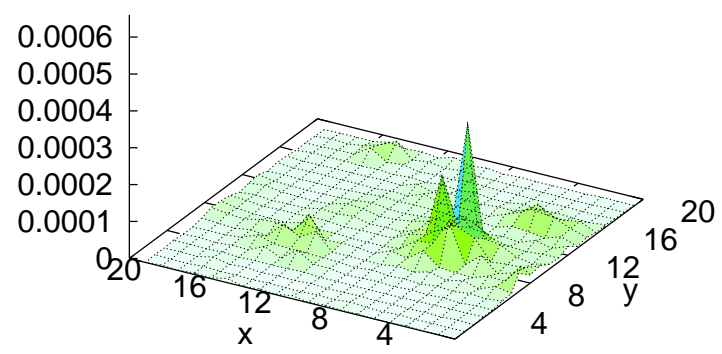

$z=14, t=14$

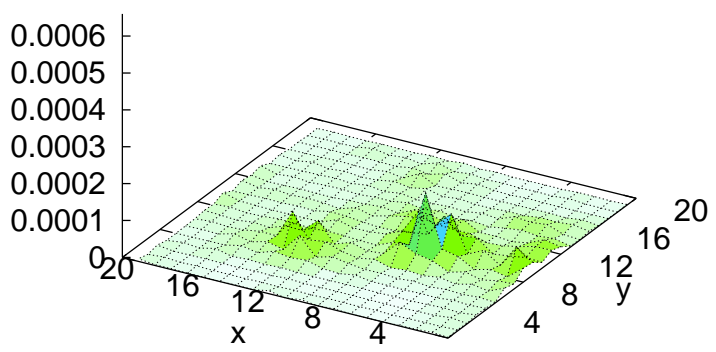

Figure 124: Maximum density peak (center) of first asqtad staggered eigenmode for a full configuration on a $20^{4}$-lattice at $\beta_{L W}=3.3$ with upper (above) and lower (below) z-slices of the same t-slice. 
$z=10, t=14$

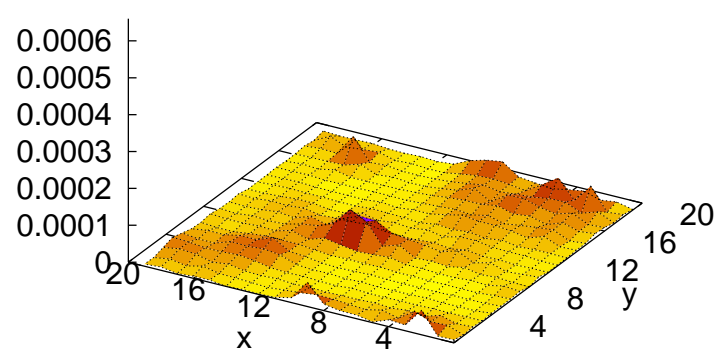

$\mathrm{z}=11, \mathrm{t}=14$

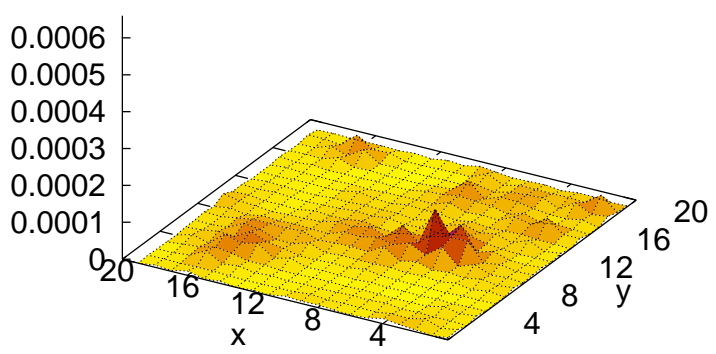

density of eigenvalue \#1, maximum 0.000553522856787 at $x=5, y=9, z=12, t=14$

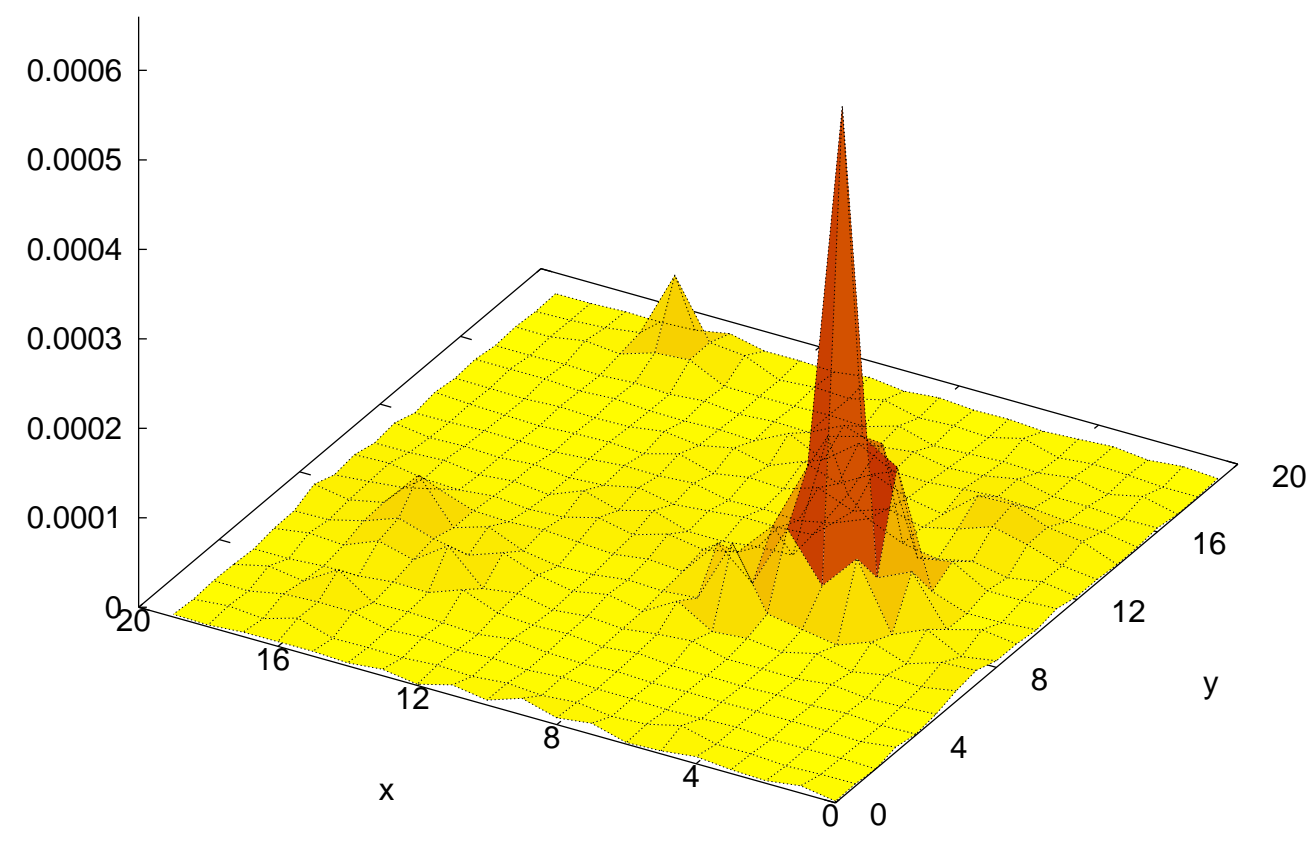

$z=13, t=14$

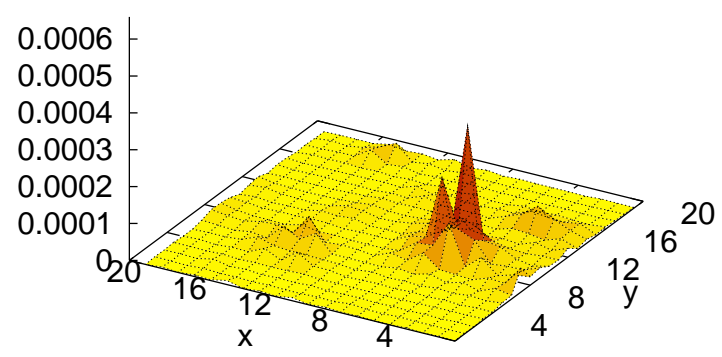

$z=14, t=14$

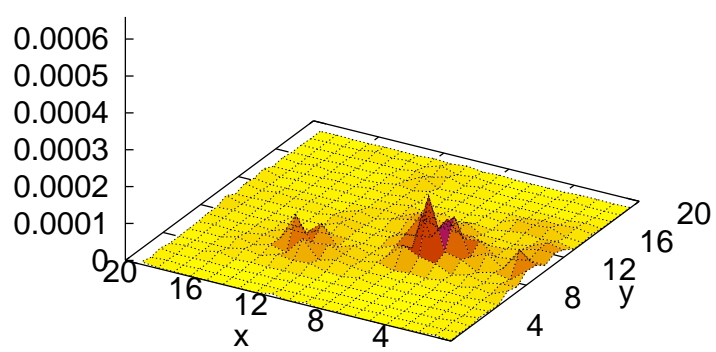

Figure 125: Maximum density peak (center) of first asqtad staggered eigenmode for a full configuration on a $20^{4}$-lattice at $\beta_{L W}=3.3$ with upper (above) and lower (below) z-slices of the same t-slice. 
$z=5, t=13$

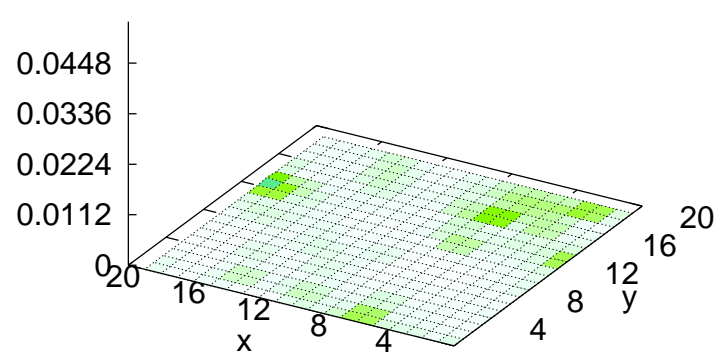

$\mathrm{z}=6, \mathrm{t}=13$

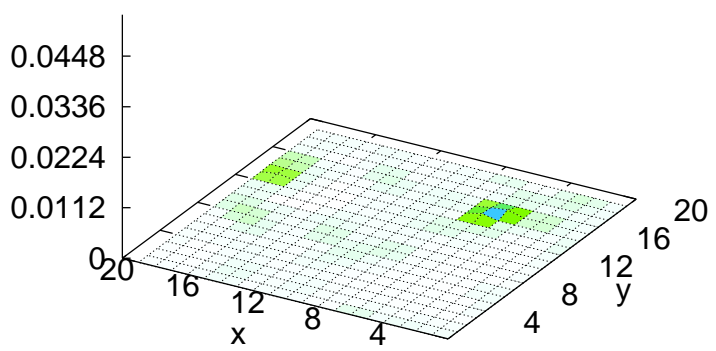

density of eigenvalue \#1, maximum 0.0449931538897 at $x=6, y=15, z=7, t=13$

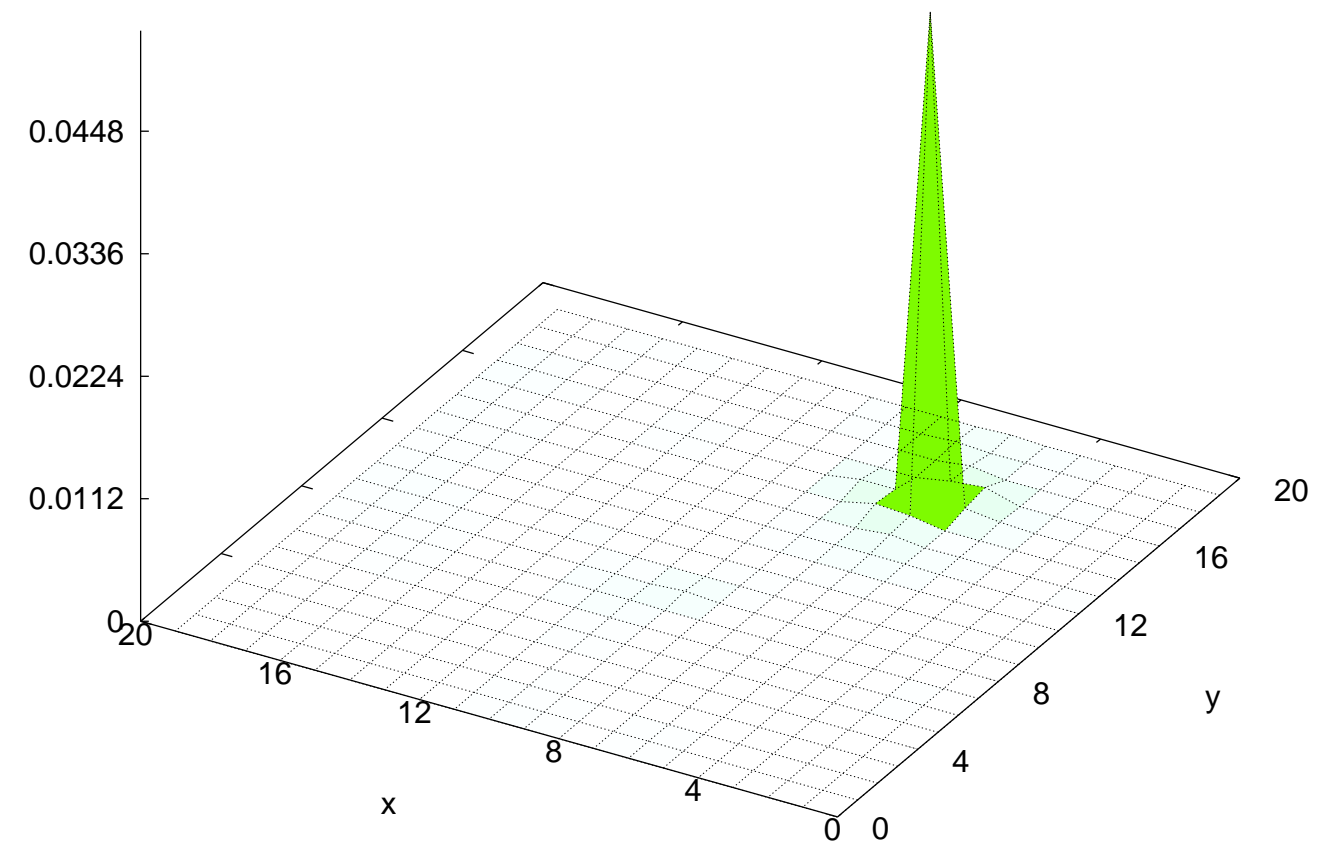

$z=8, t=13$

$z=9, t=13$
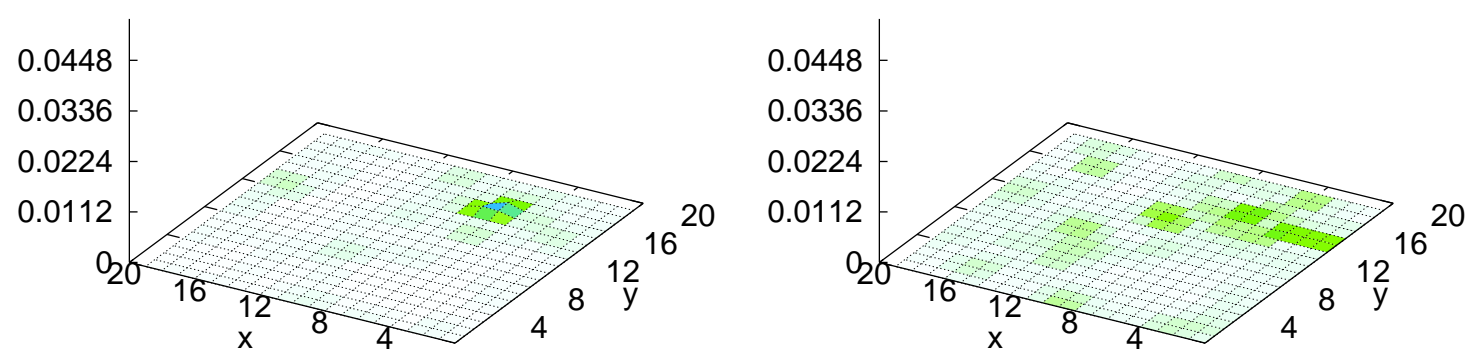

Figure 126: Maximum density peak (center) of first asqtad staggered eigenmode for a center-projected configuration on a $20^{4}$-lattice at $\beta_{L W}=3.3$ with upper (above) and lower (below) z-slices of the same t-slice. 
$\mathrm{z}=2, \mathrm{t}=7$

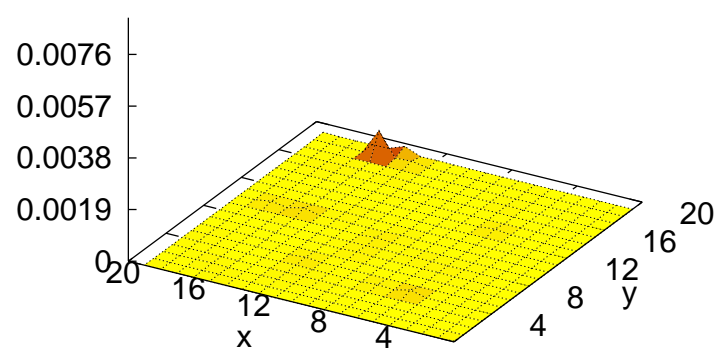

$\mathrm{z}=3, \mathrm{t}=7$

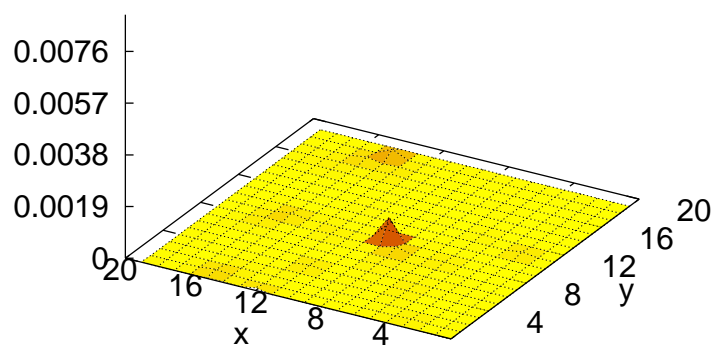

density of eigenvalue \#1, maximum 0.00745768618163 at $x=9, y=8, z=4, t=7$

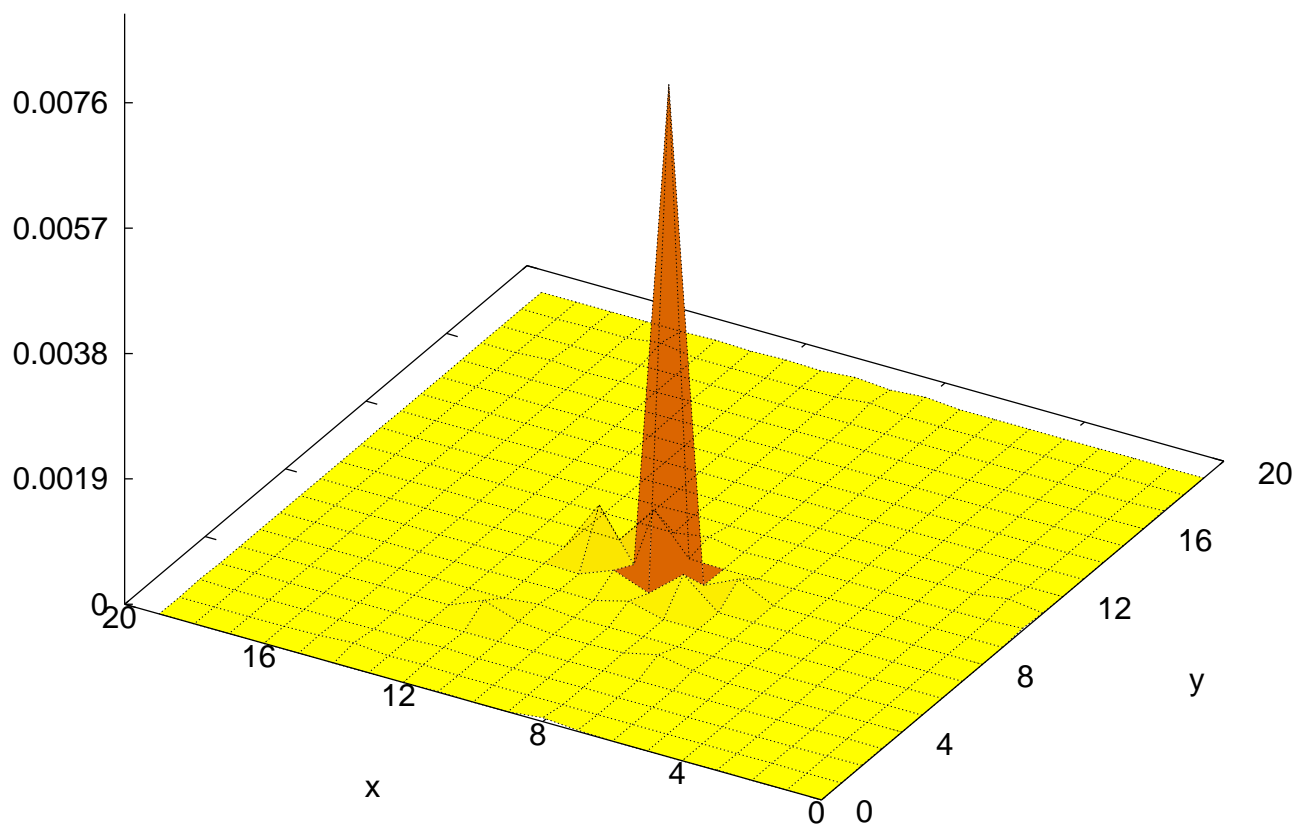

$\mathrm{z}=5, \mathrm{t}=7$

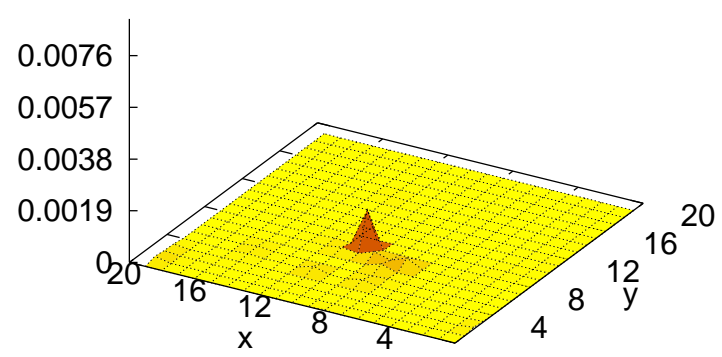

$\mathrm{z}=6, \mathrm{t}=7$

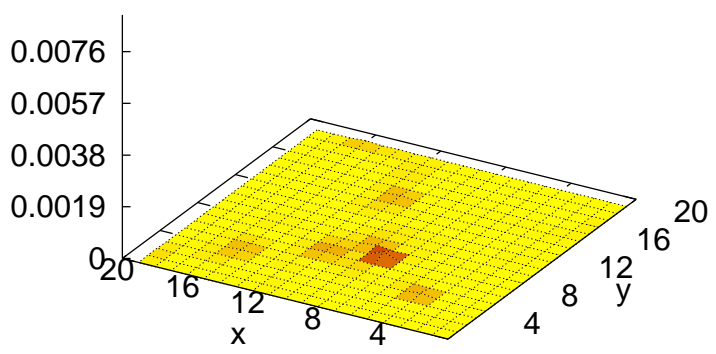

Figure 127: Maximum density peak (center) of first asqtad staggered eigenmode for a center-projected configuration on a $20^{4}$-lattice at $\beta_{L W}=3.3$ with upper (above) and lower (below) z-slices of the same t-slice. 
a)

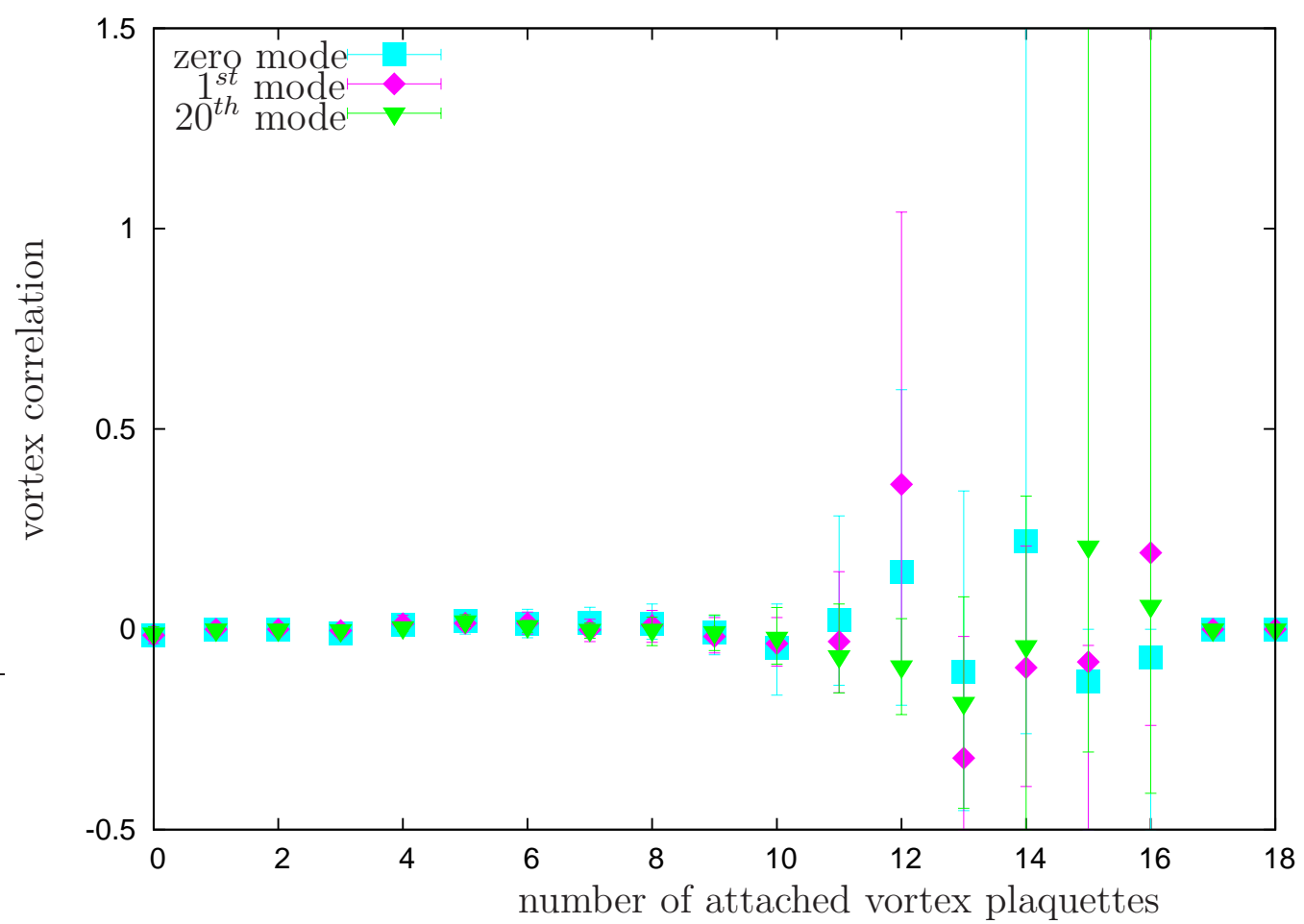

b)

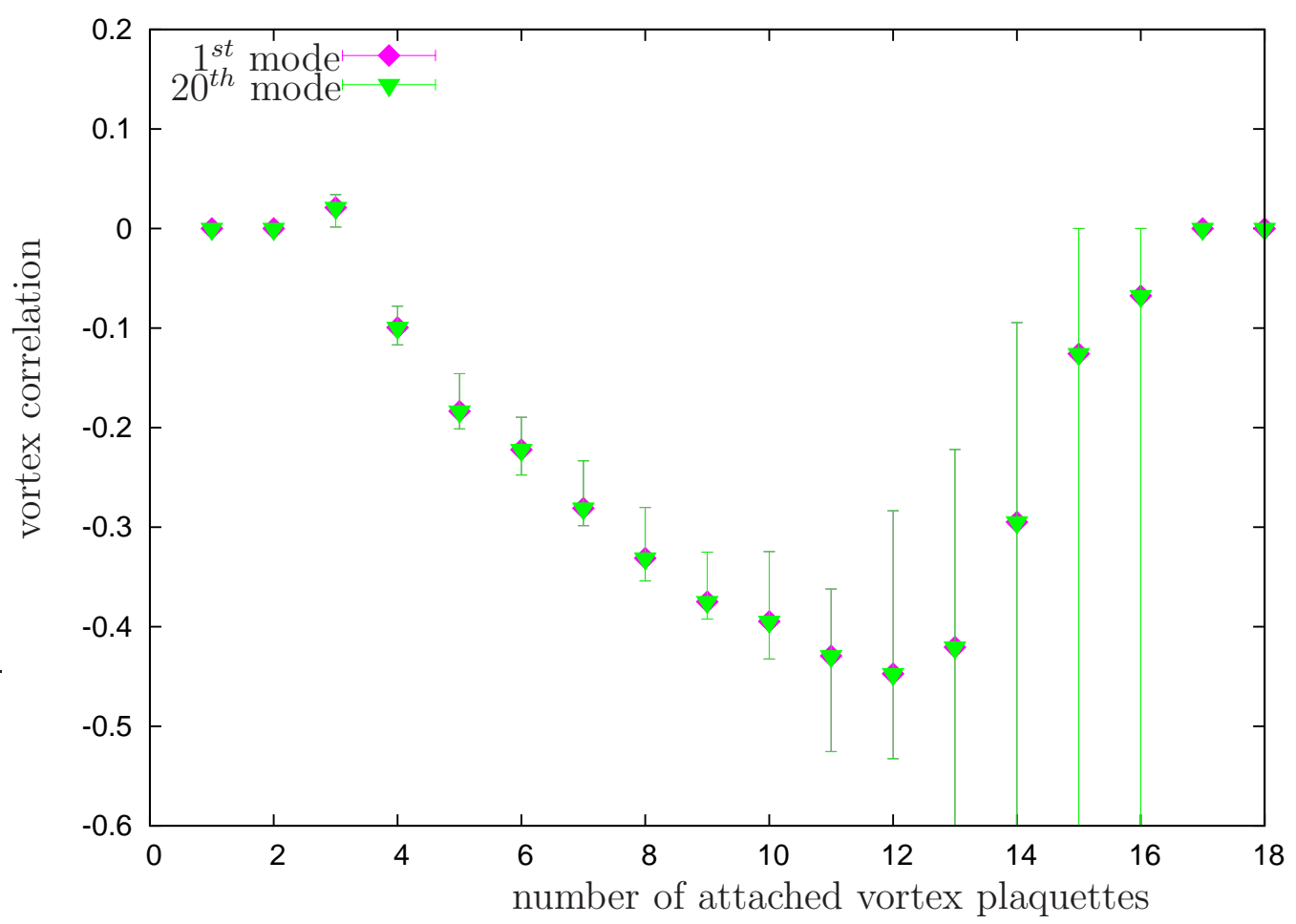

Figure 128: Vortex Correlation for overlap eigenmodes on a $12^{4}$ lattice at $\beta=2.9$, a) full and b) projected configuration. 
a)

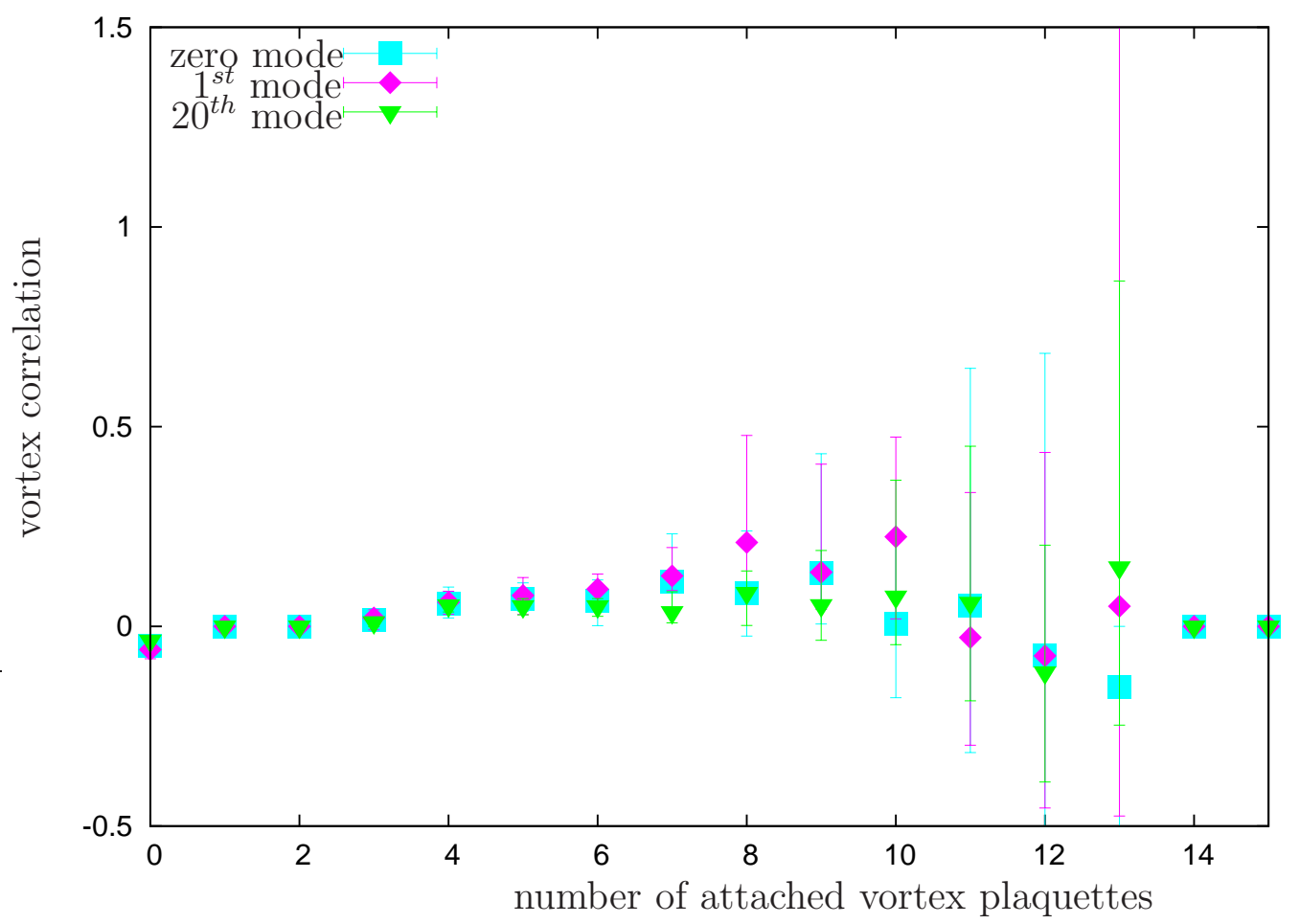

b)

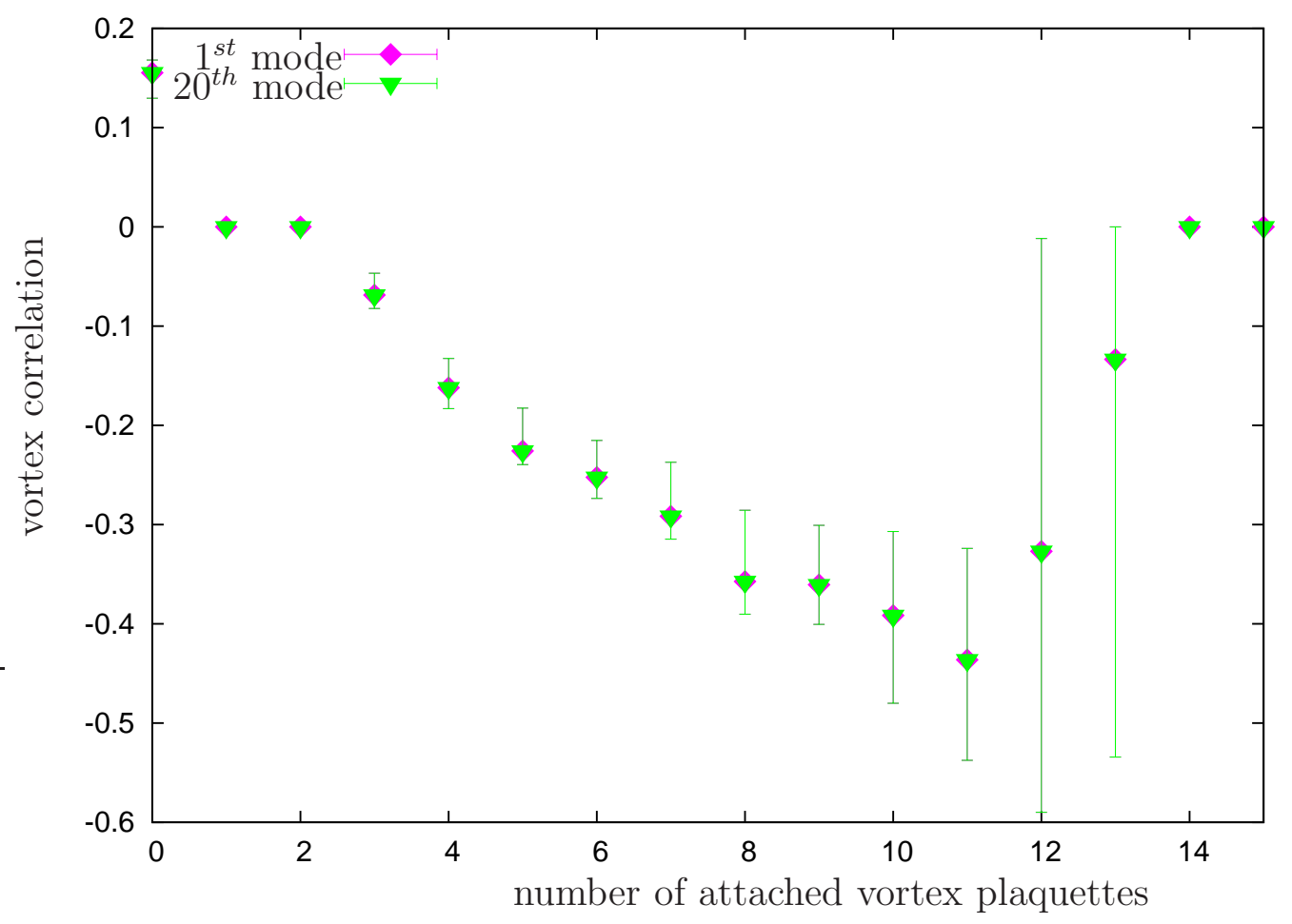

Figure 129: Vortex Correlation for overlap eigenmodes on a $12^{4}$ lattice at $\beta=3.1$, a) full and b) projected configuration. 
a)

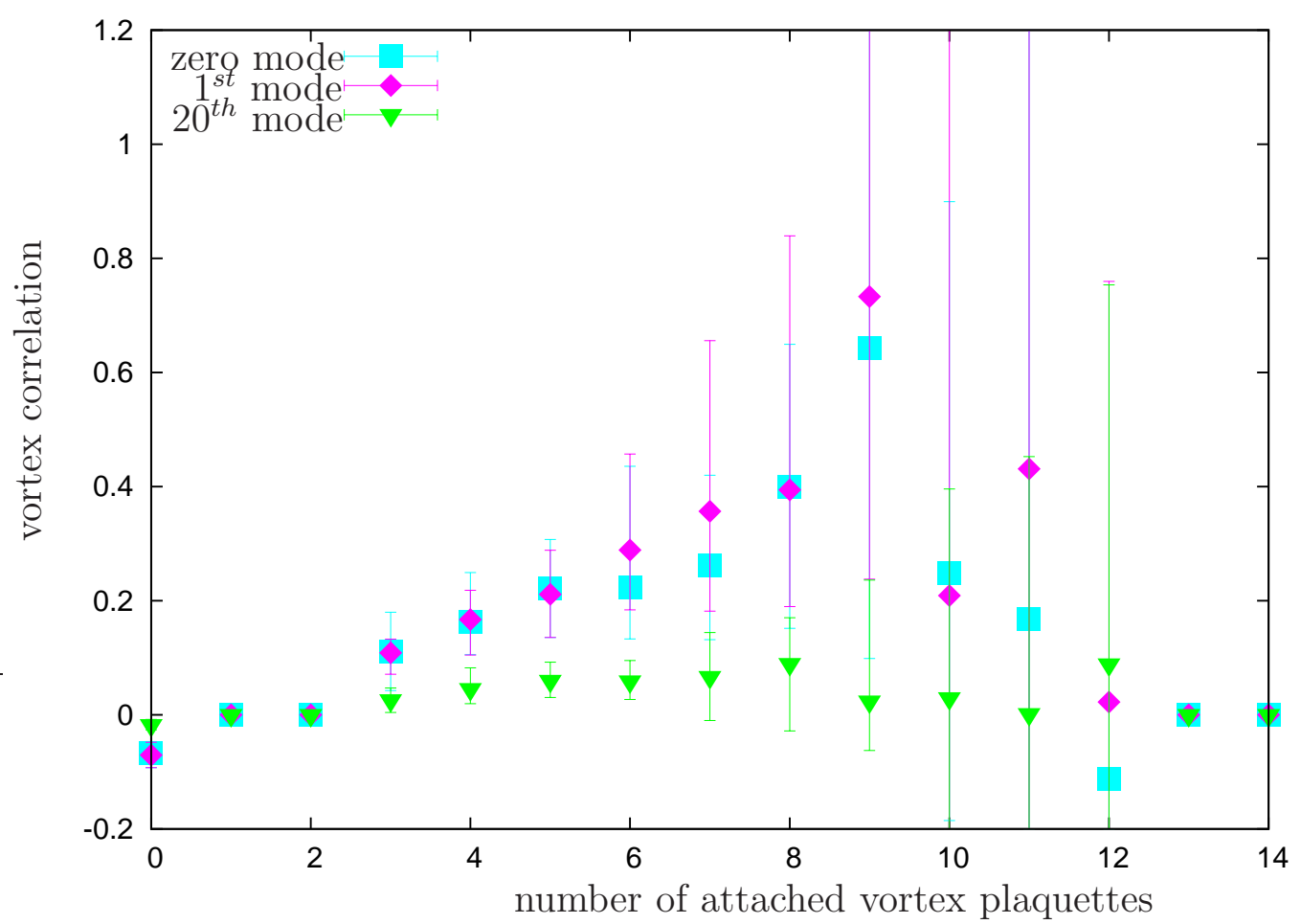

b)

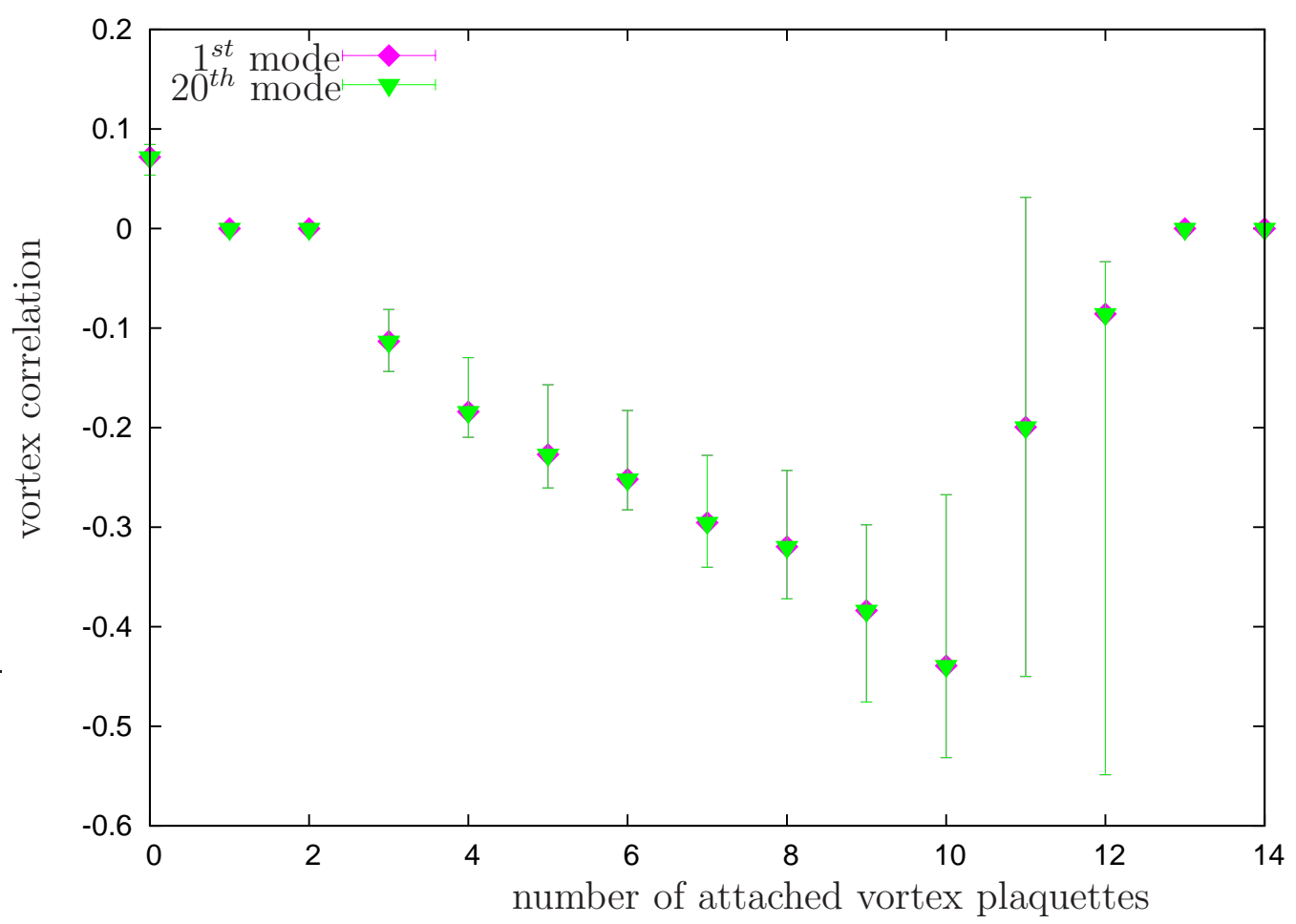

Figure 130: Vortex Correlation for overlap eigenmodes on a $12^{4}$ lattice at $\beta=3.3$, a) full and b) projected configuration. 
a)

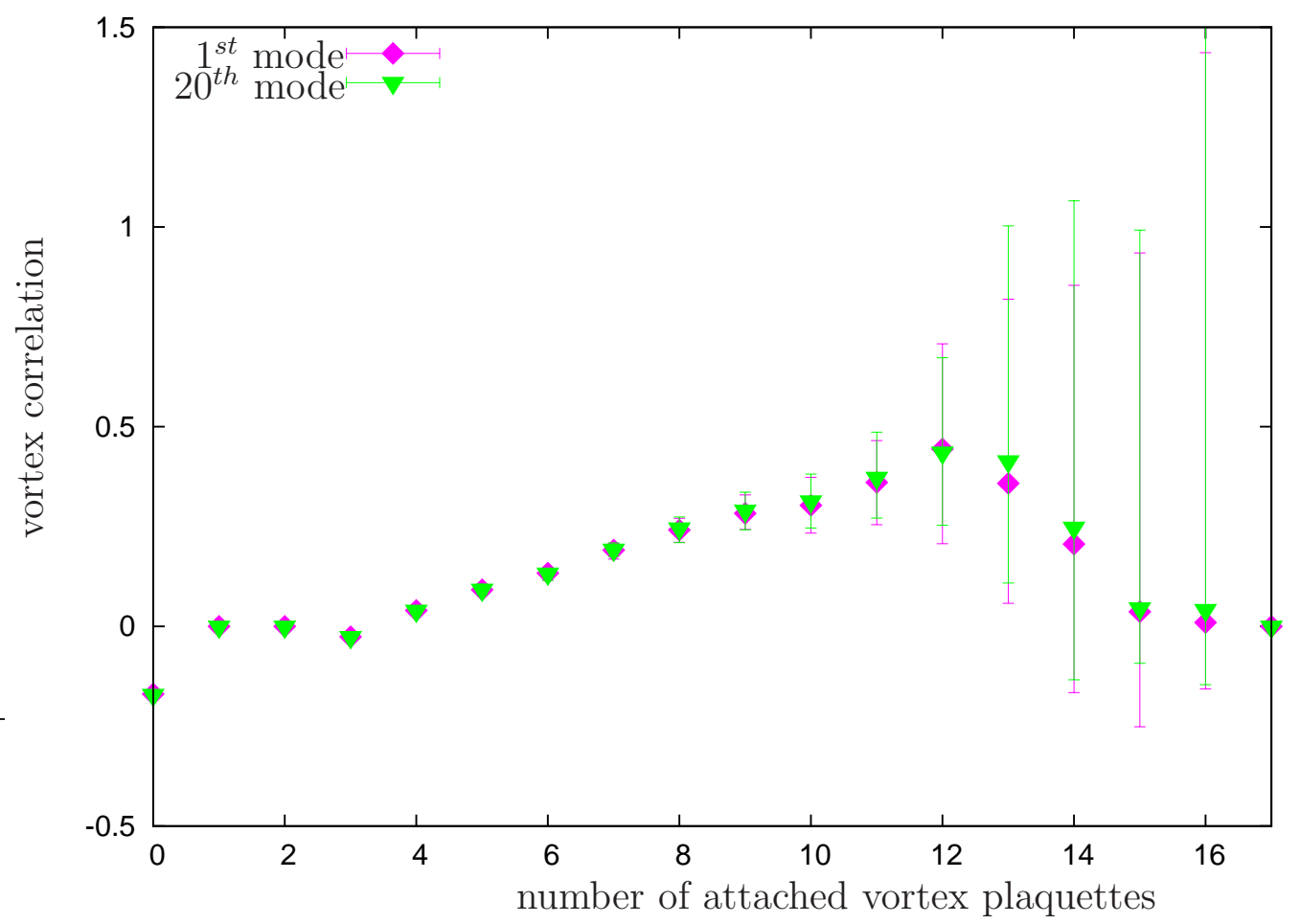

b)

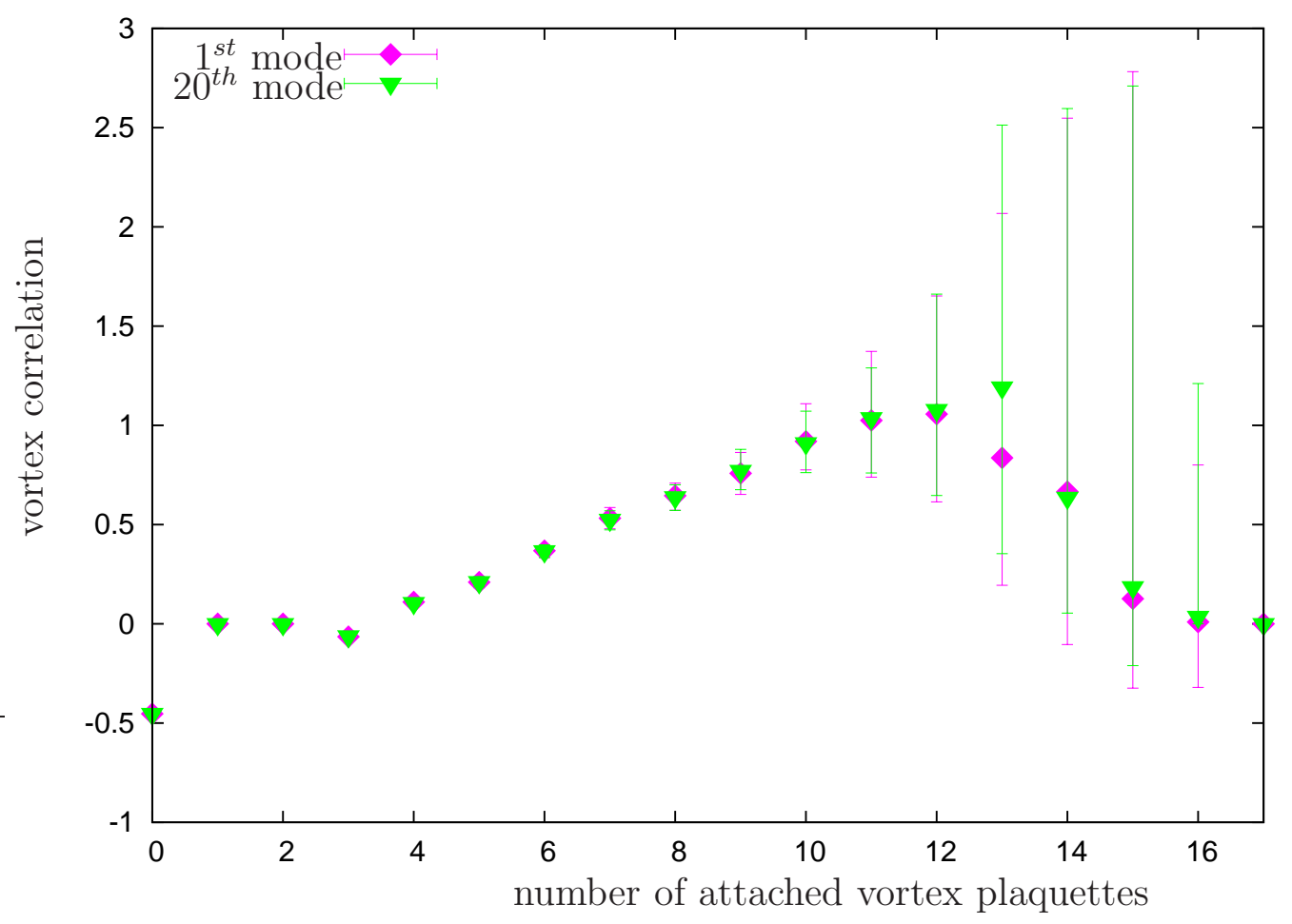

Figure 131: Vortex Correlation for asqtad staggered eigenmodes on a $12^{4}$ lattice at $\beta=$ 2.9 , a) full and b) projected configuration. 
a)

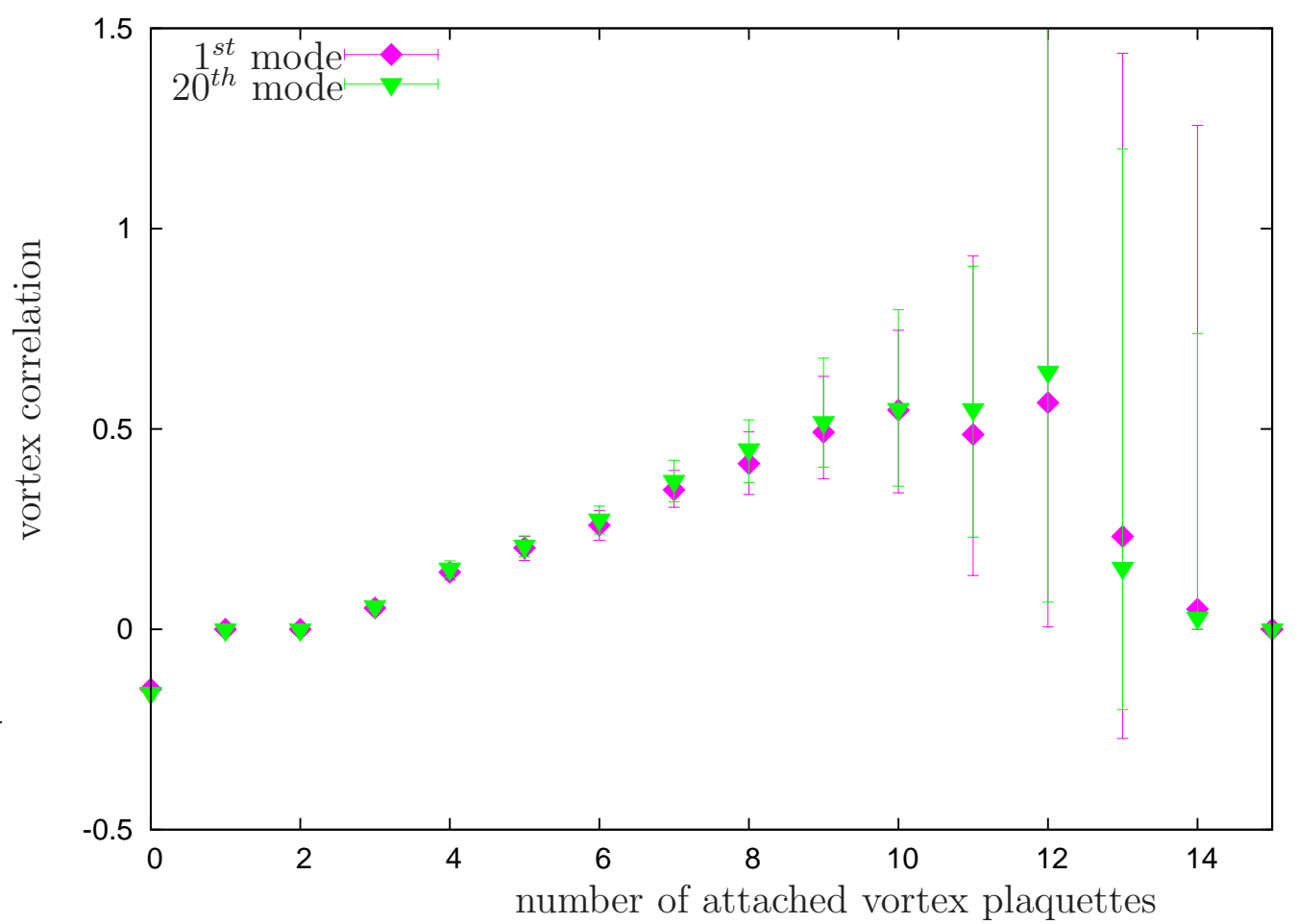

b)

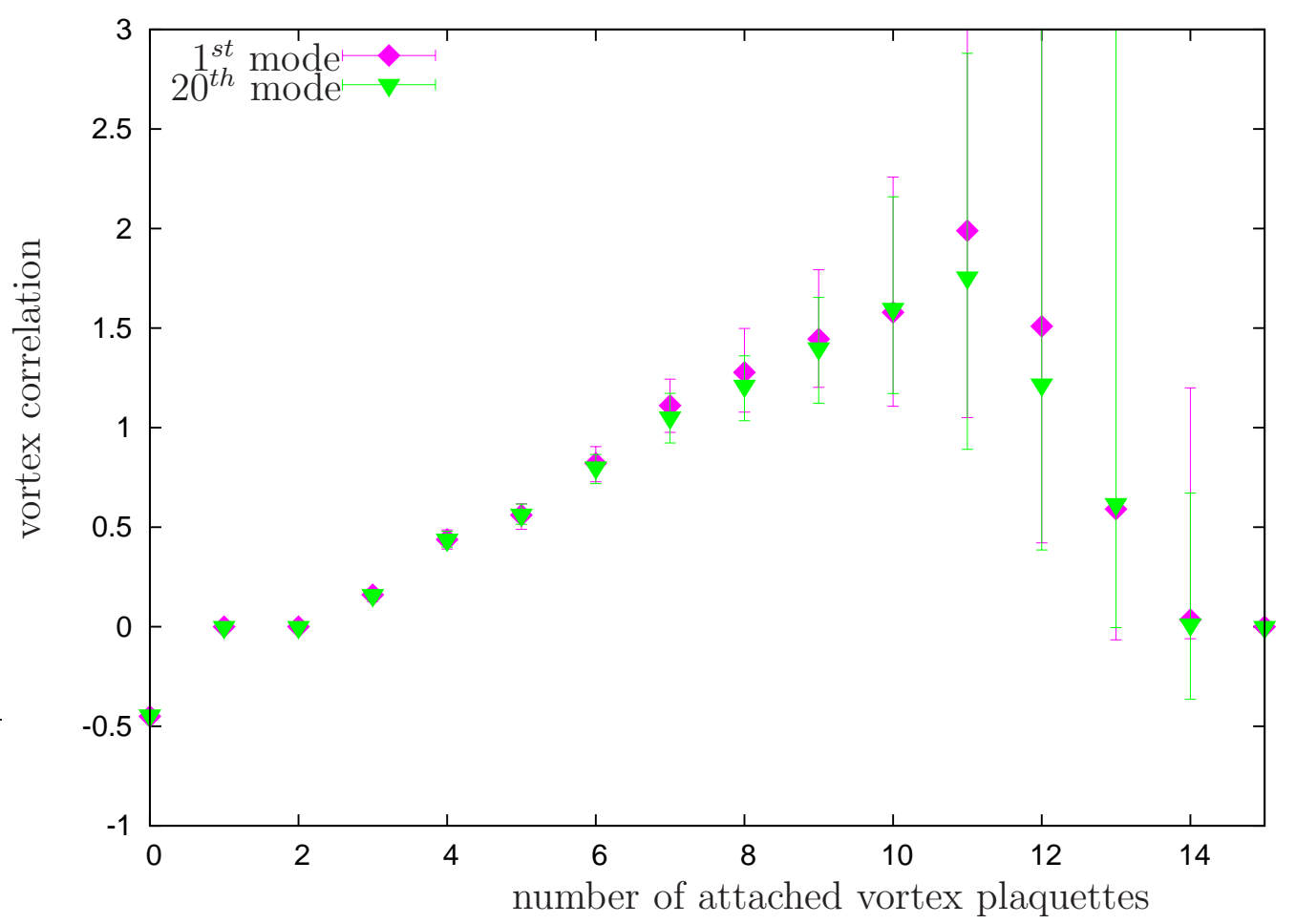

Figure 132: Vortex Correlation for asqtad staggered eigenmodes on a $12^{4}$ lattice at $\beta=$ 3.1 , a) full and b) projected configuration. 
a)

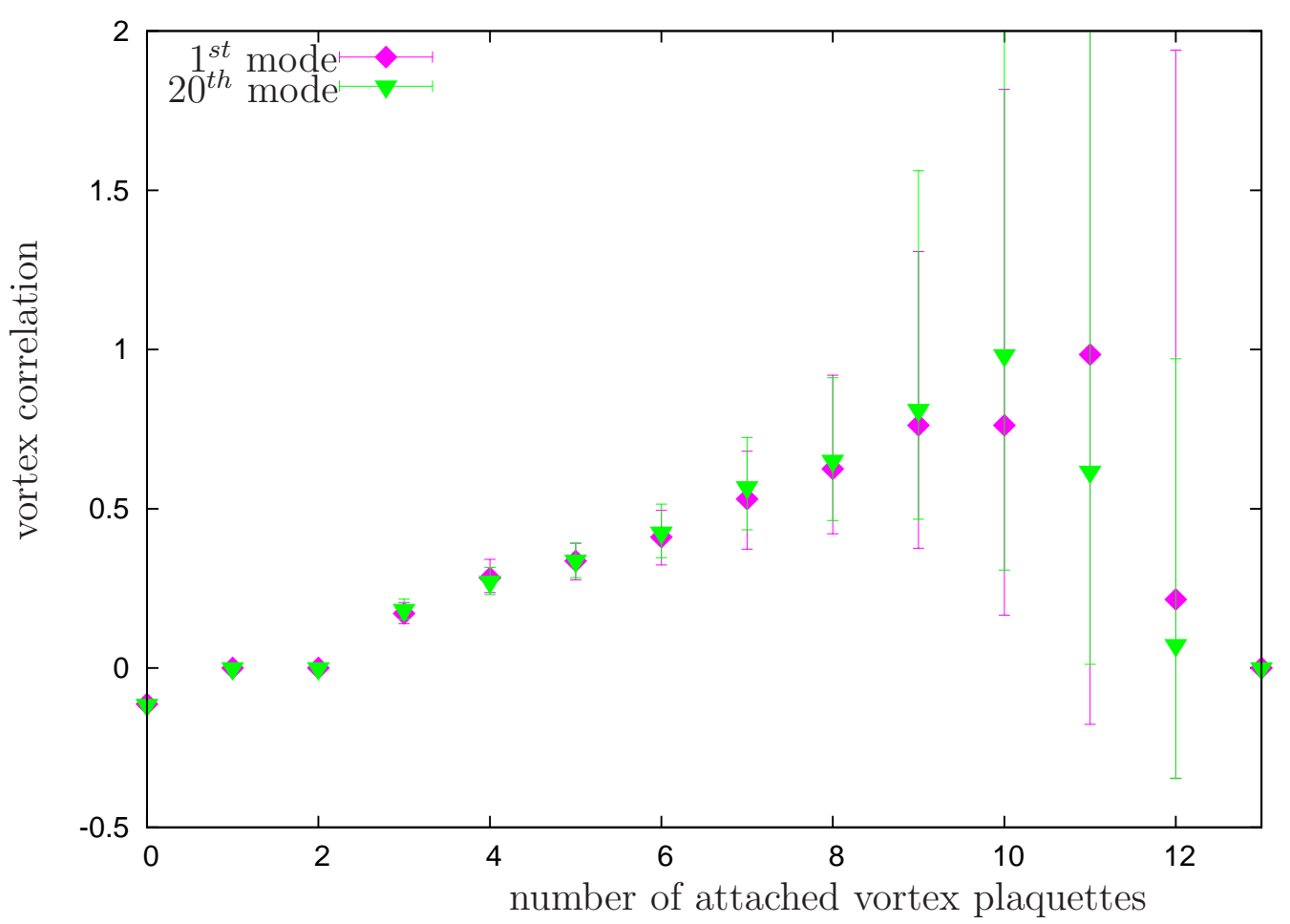

b)

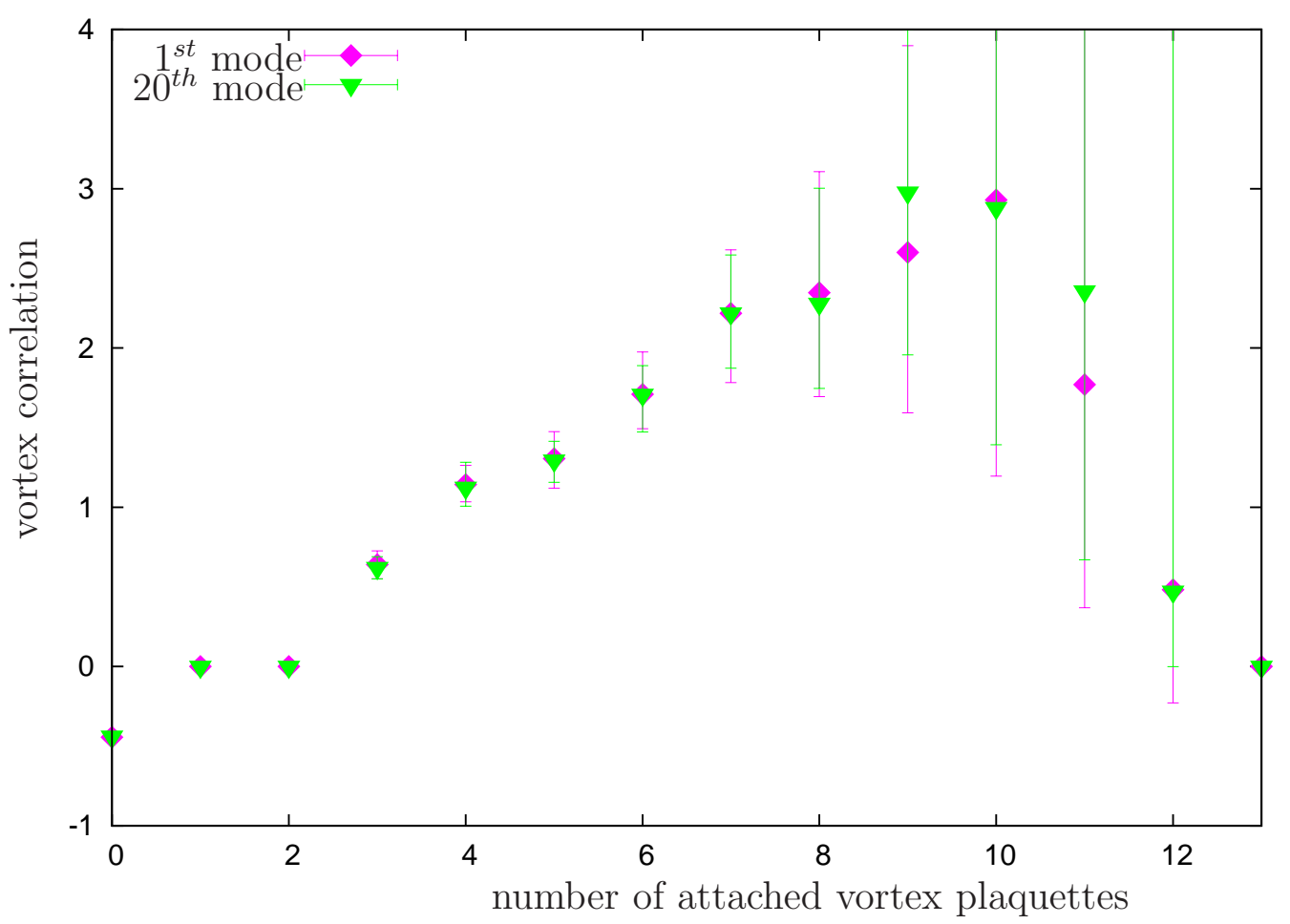

Figure 133: Vortex Correlation for asqtad staggered eigenmodes on a $12^{4}$ lattice at $\beta=$ 3.3 , a) full and b) projected configuration. 
a)

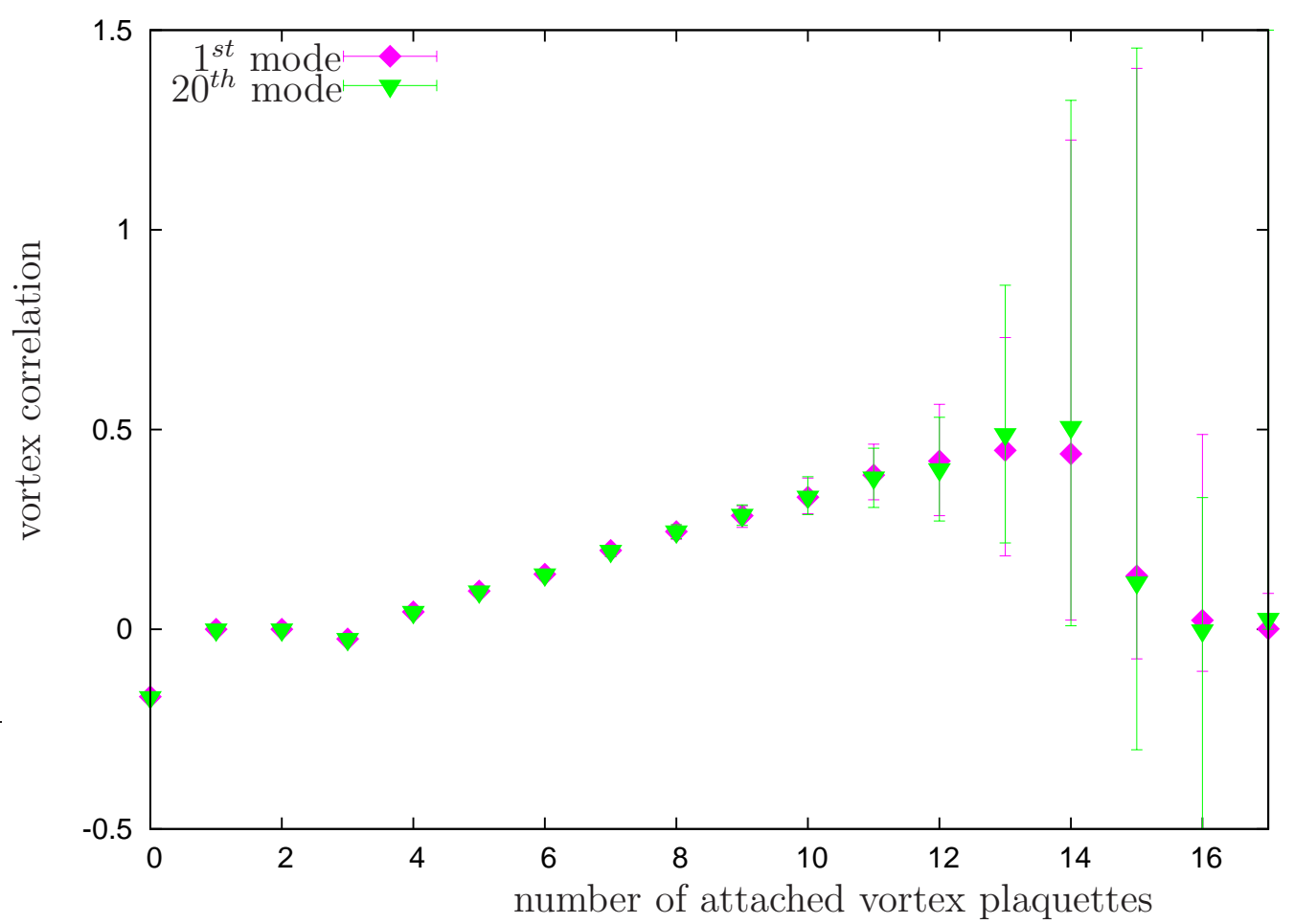

b)

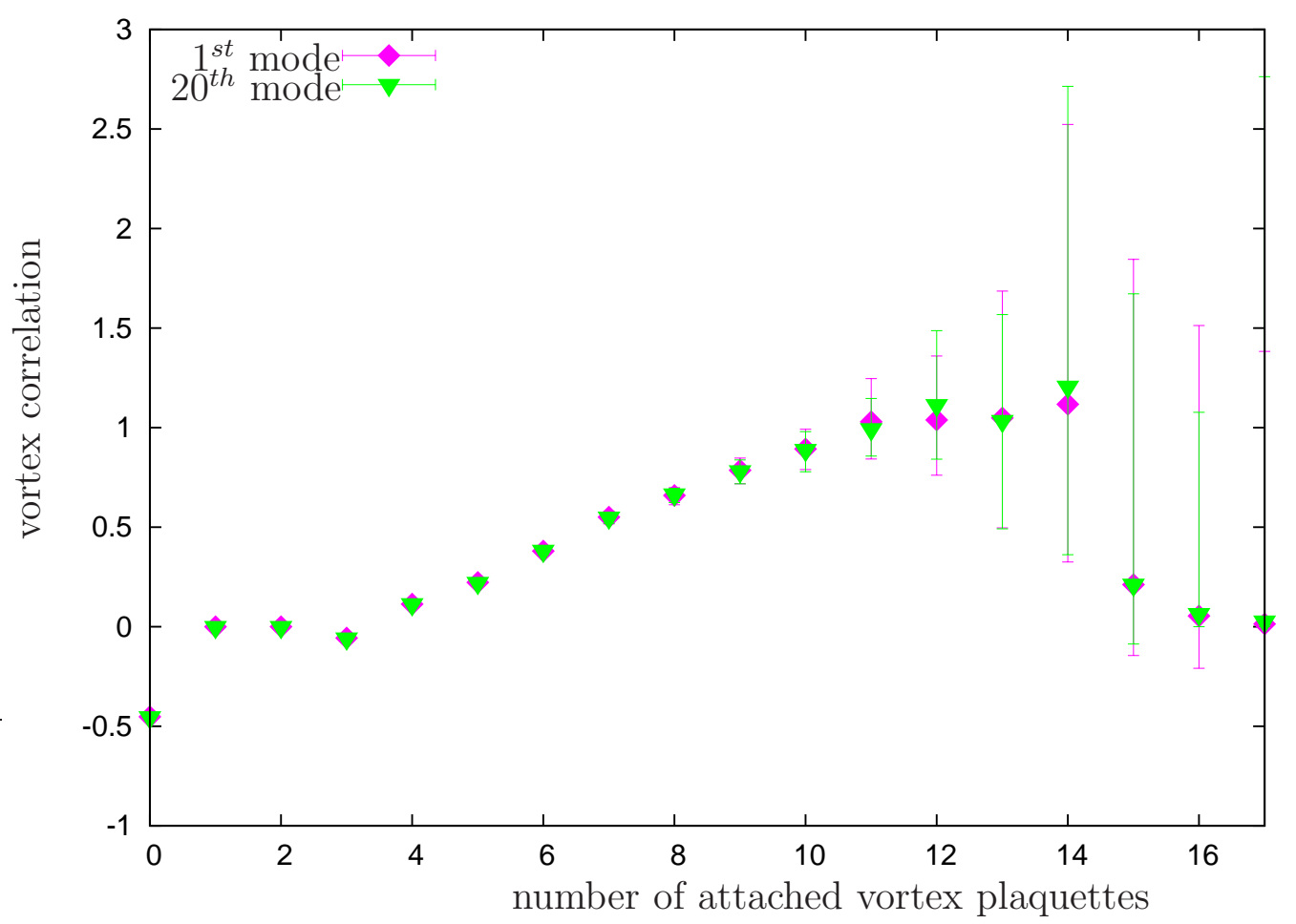

Figure 134: Vortex Correlation for asqtad staggered eigenmodes on a $16^{4}$ lattice at $\beta=$ 2.9 , a) full and b) projected configuration. 
a)

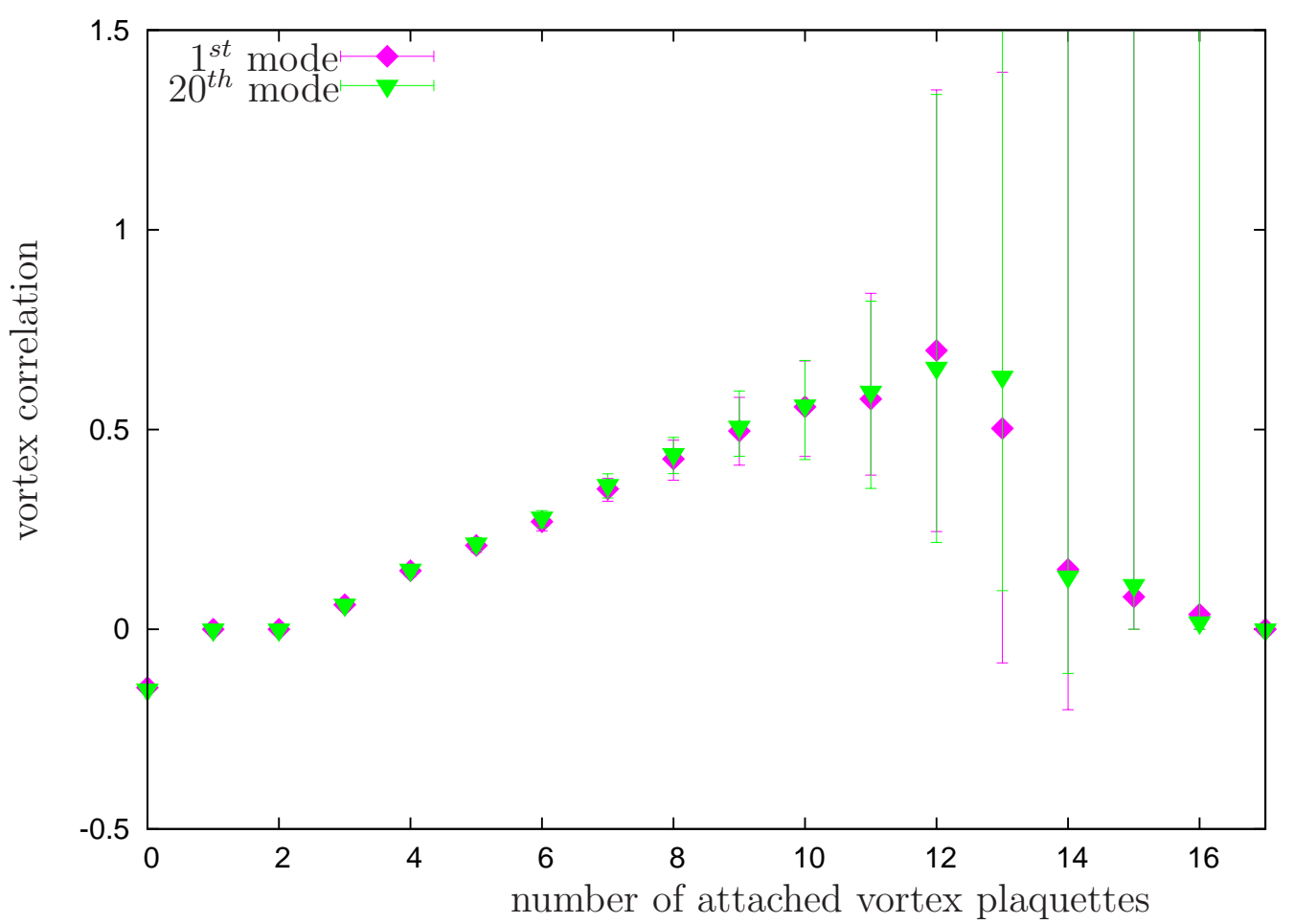

b)

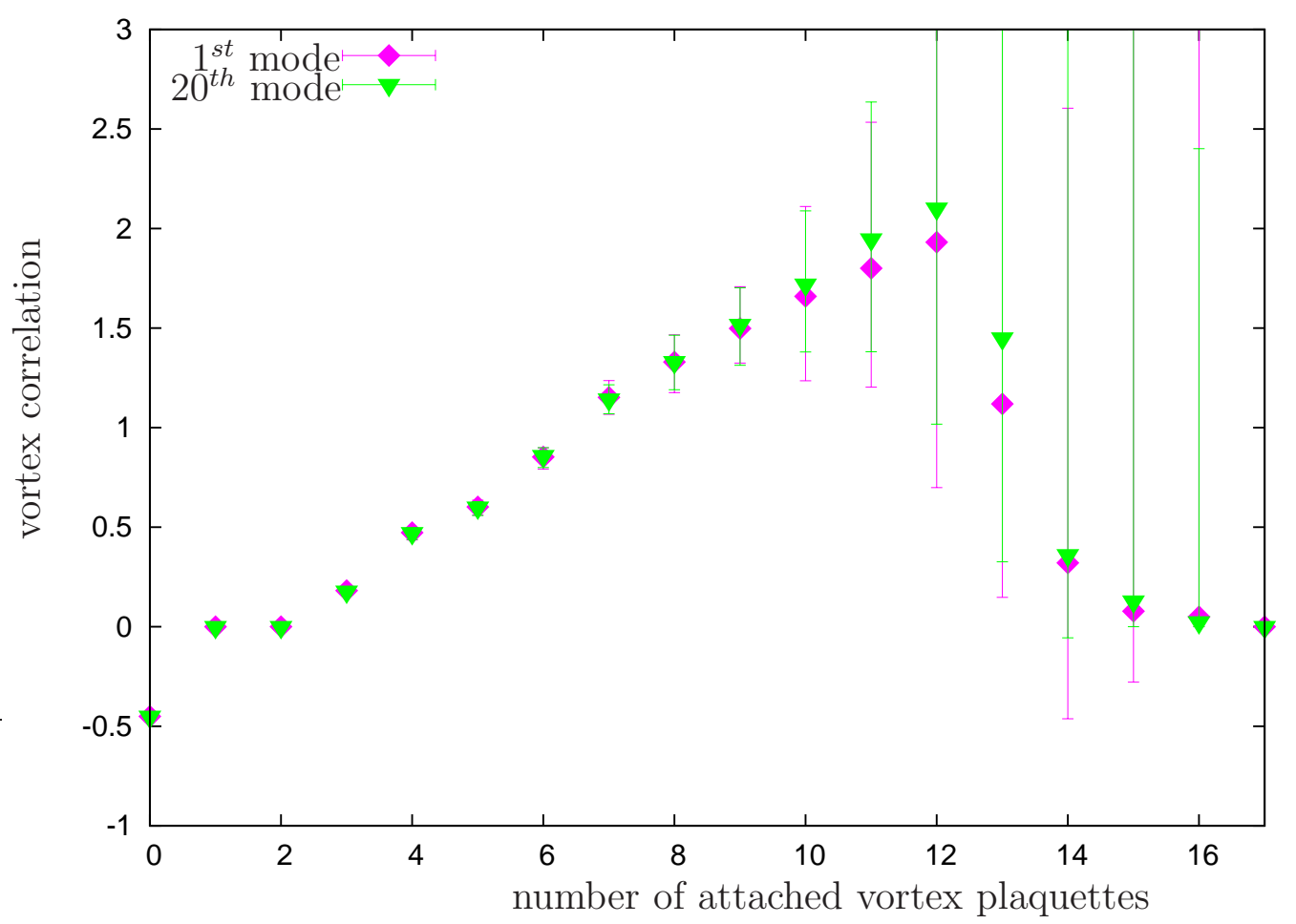

Figure 135: Vortex Correlation for asqtad staggered eigenmodes on a $16^{4}$ lattice at $\beta=$ 3.1 , a) full and b) projected configuration. 
a)

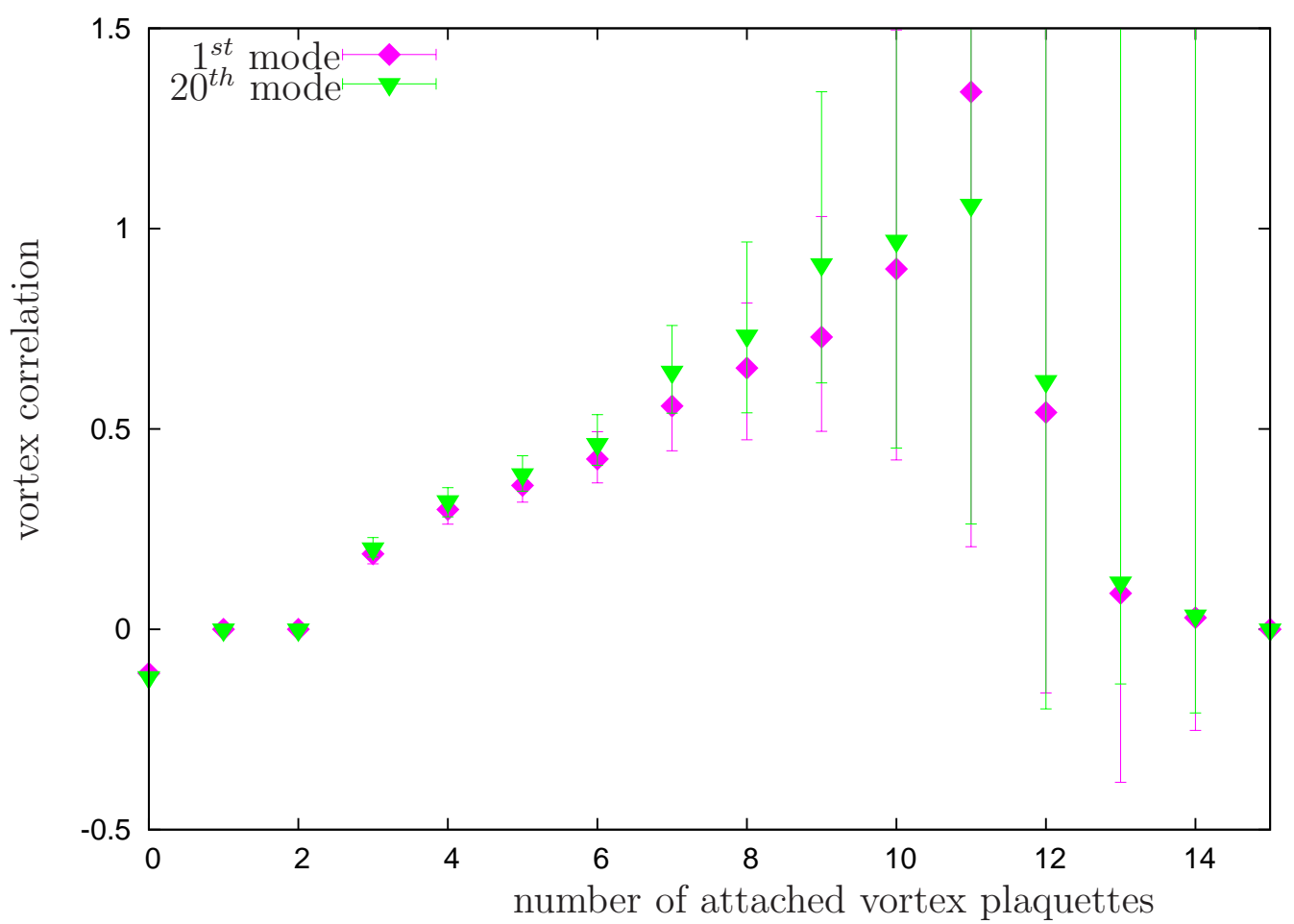

b)

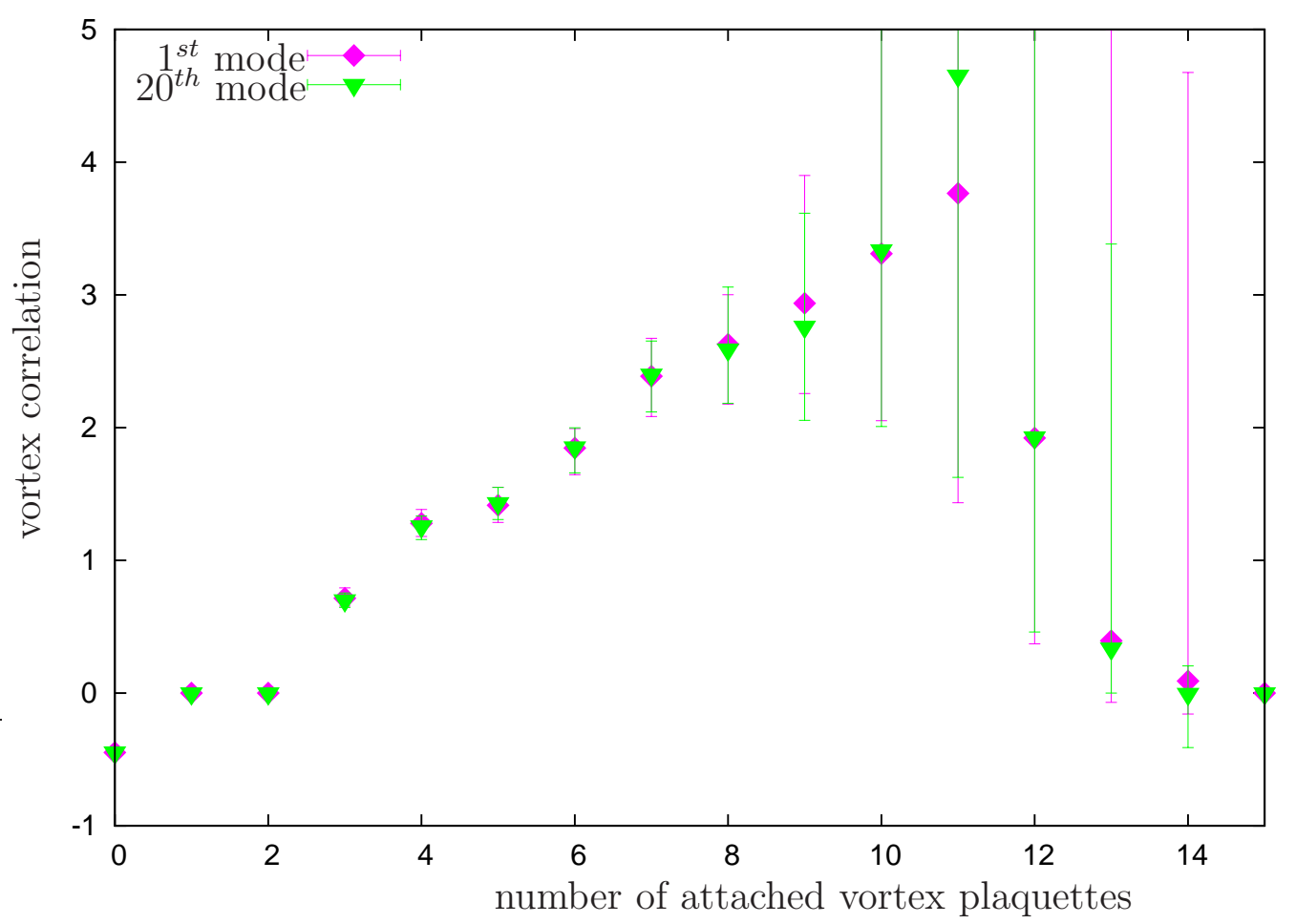

Figure 136: Vortex Correlation for asqtad staggered eigenmodes on a $16^{4}$ lattice at $\beta=$ 3.3 , a) full and b) projected configuration. 
a)

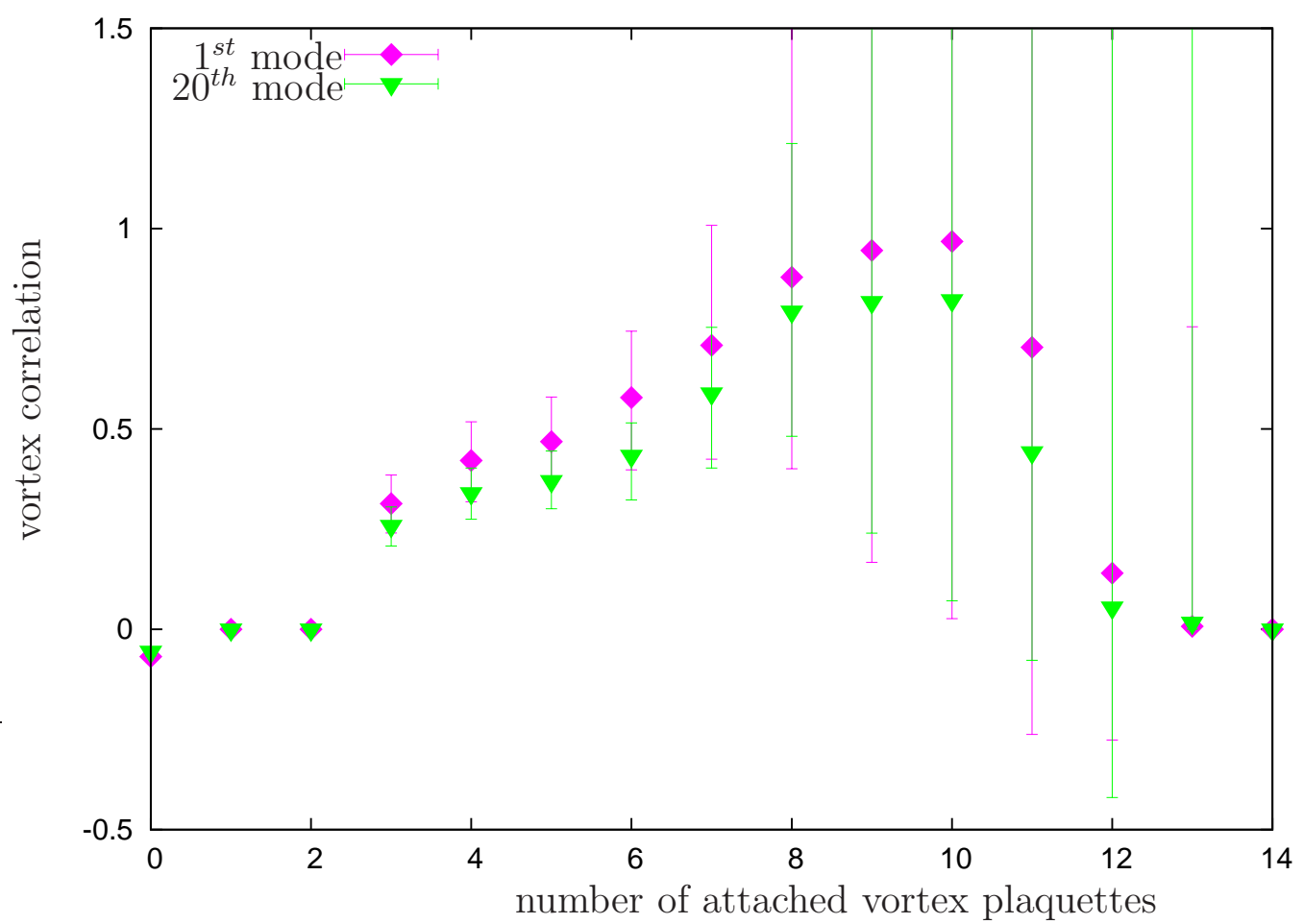

b)

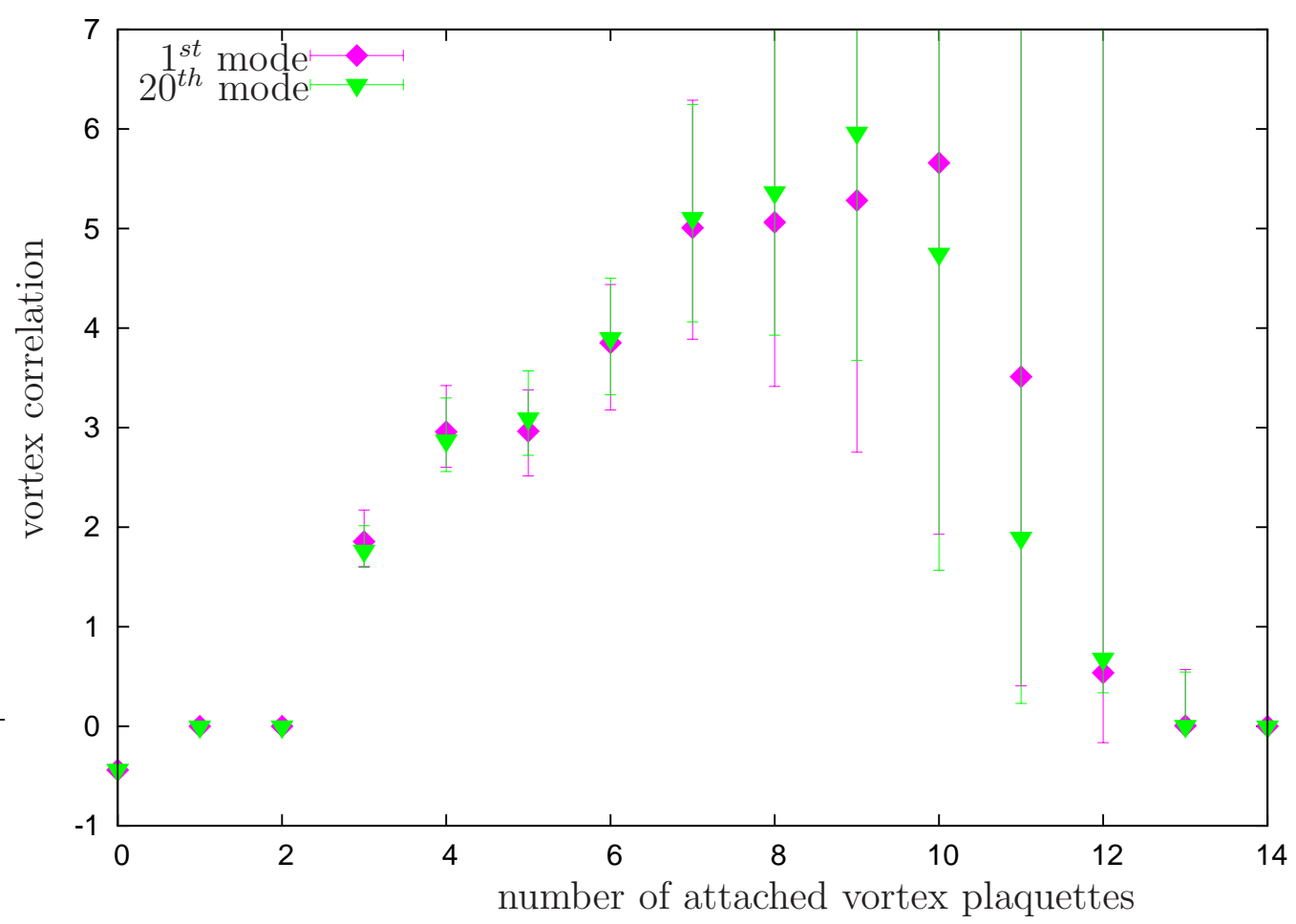

Figure 137: Vortex Correlation for asqtad staggered eigenmodes on a $16^{4}$ lattice at $\beta=$ 3.5 , a) full and b) projected configuration. 
a)

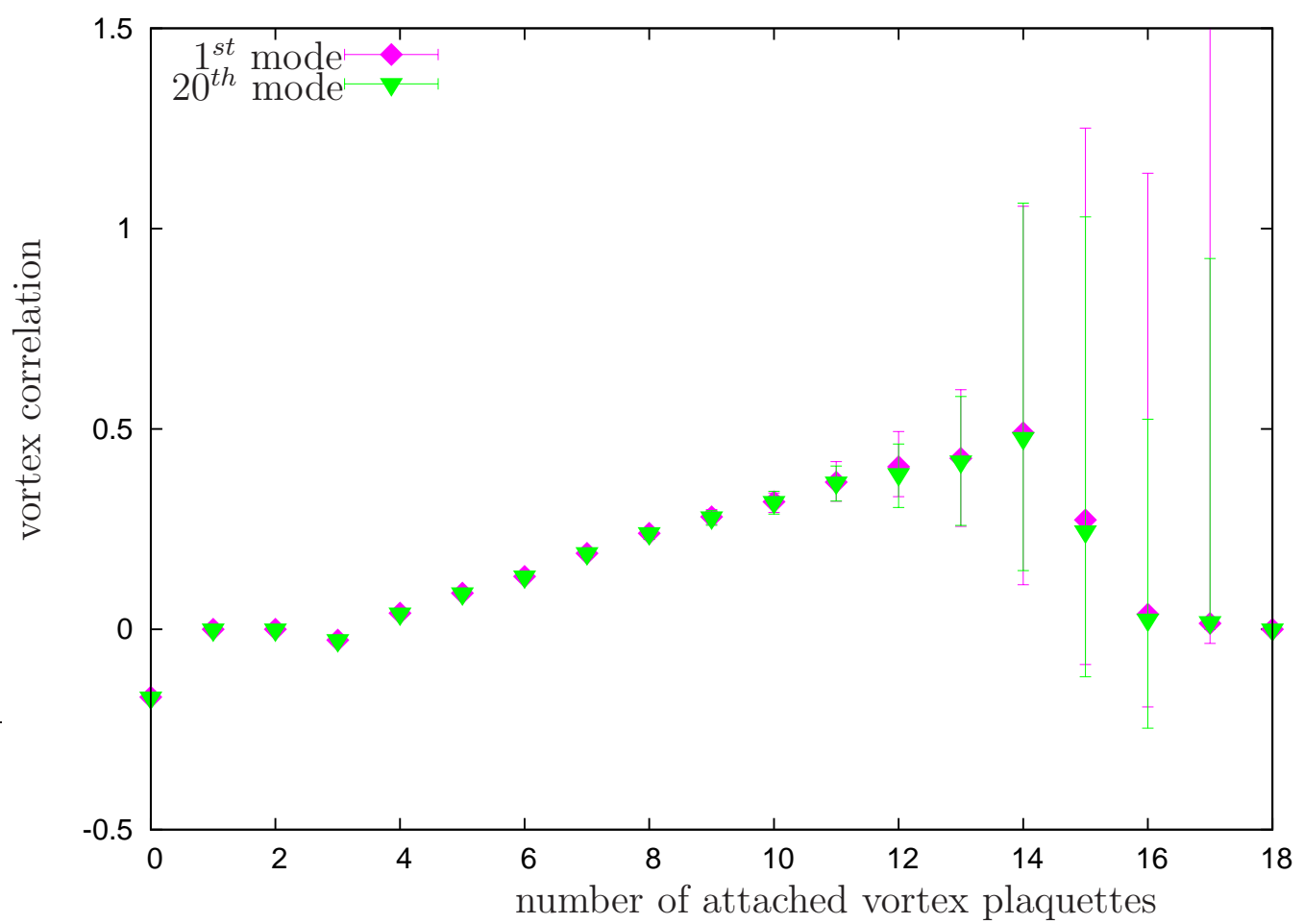

b)

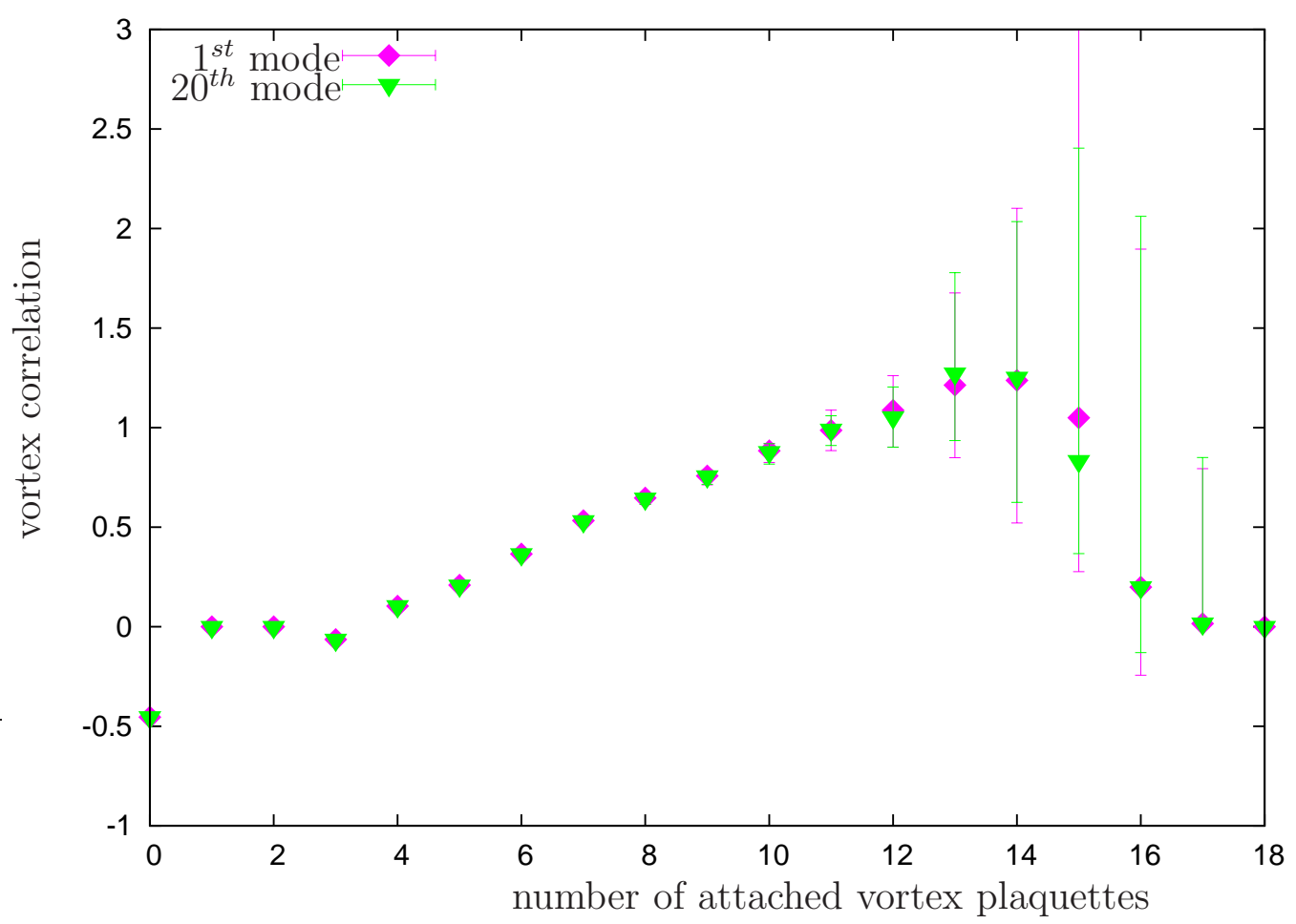

Figure 138: Vortex Correlation for asqtad staggered eigenmodes on a $20^{4}$ lattice at $\beta=$ 2.9 , a) full and b) projected configuration. 
a)

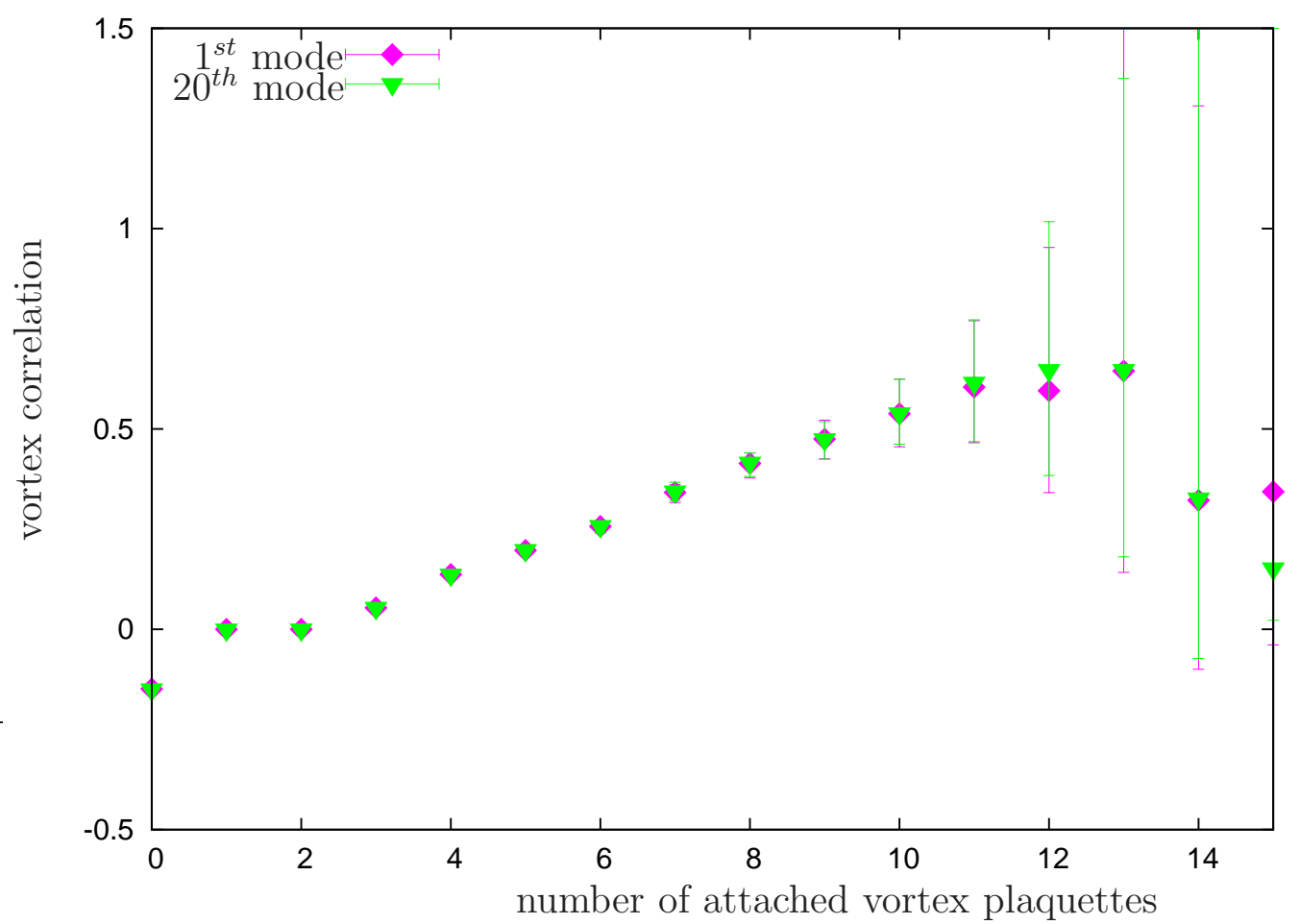

b)

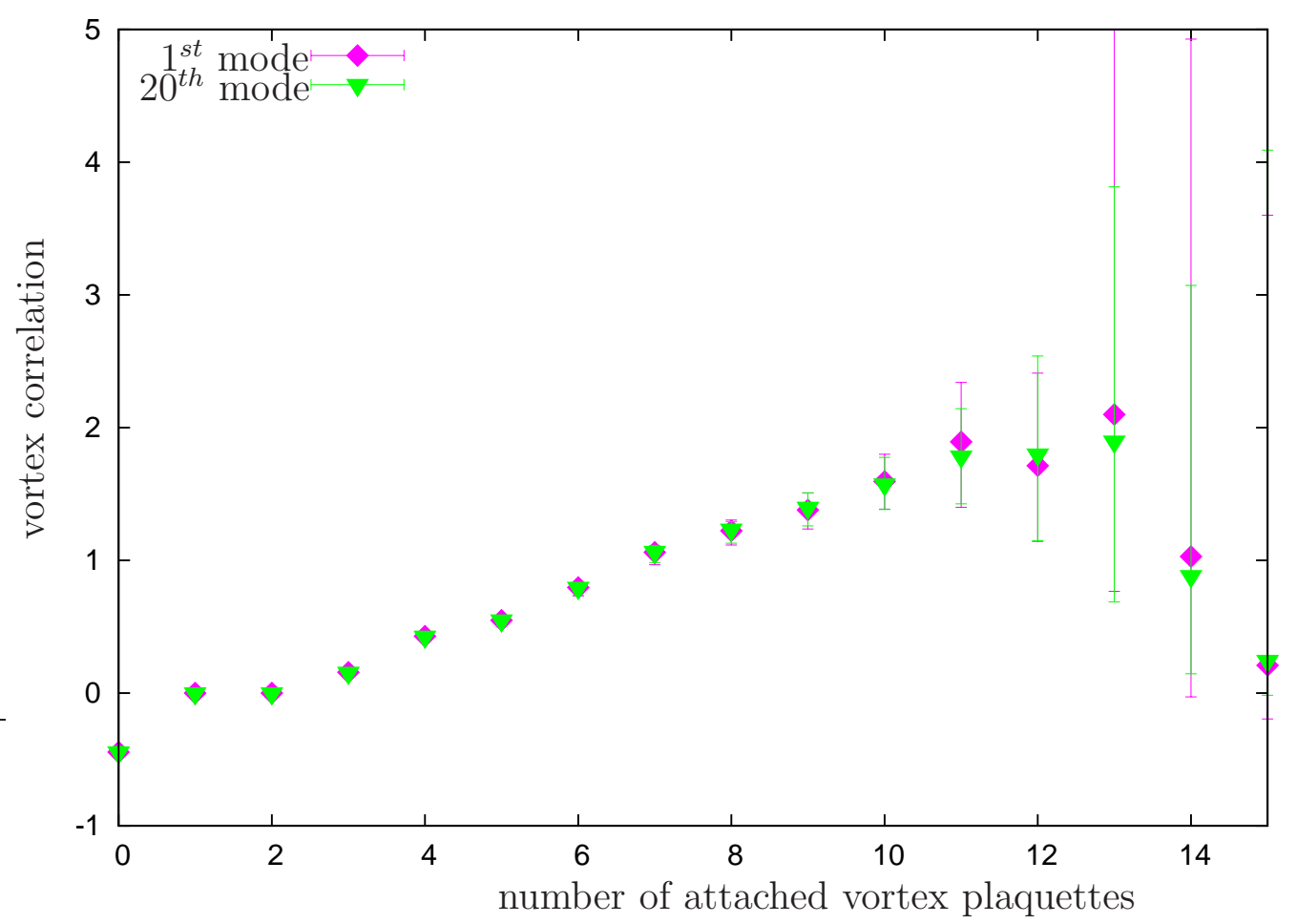

Figure 139: Vortex Correlation for asqtad staggered eigenmodes on a $20^{4}$ lattice at $\beta=$ 3.1 , a) full and b) projected configuration. 
a)

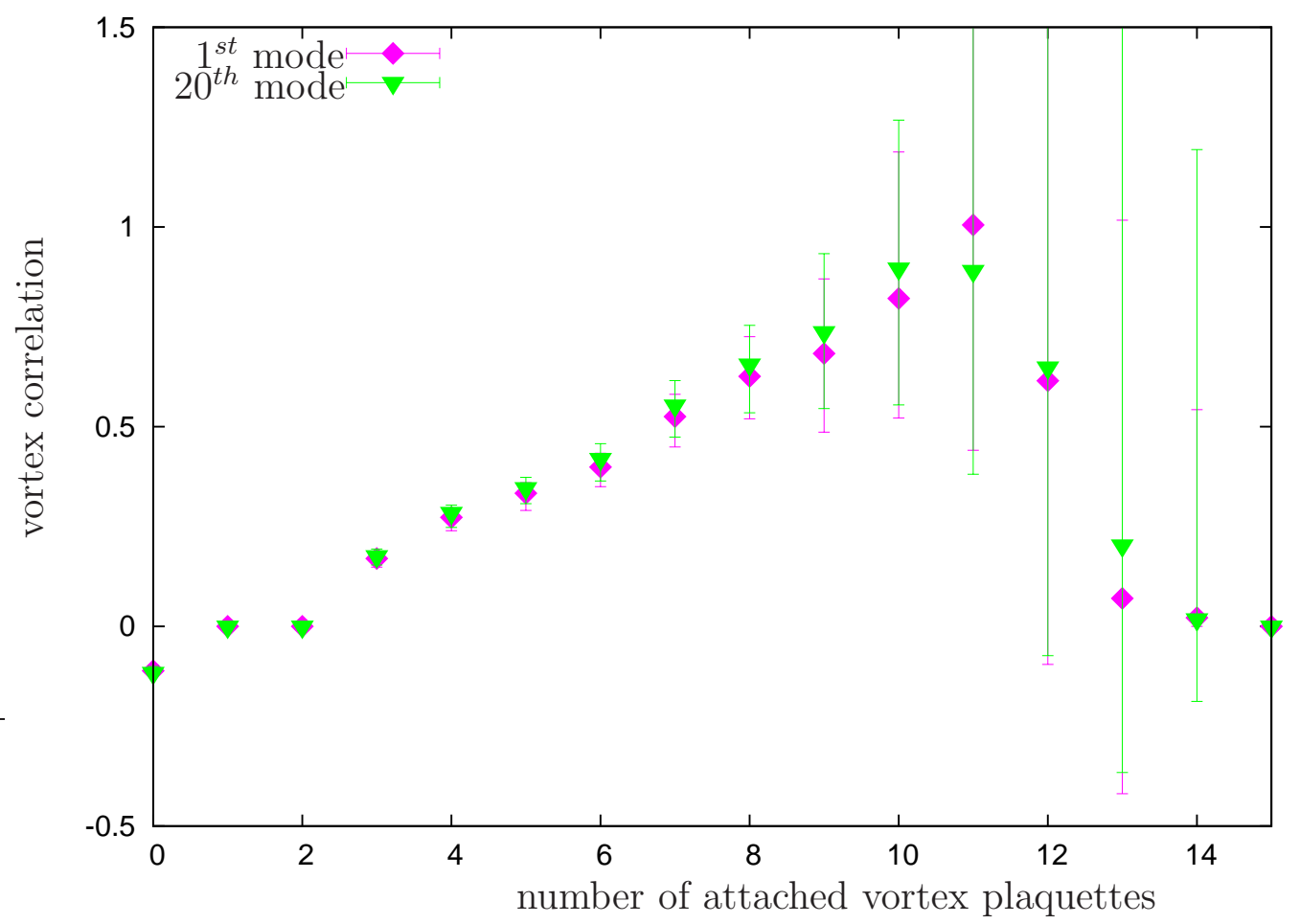

b)

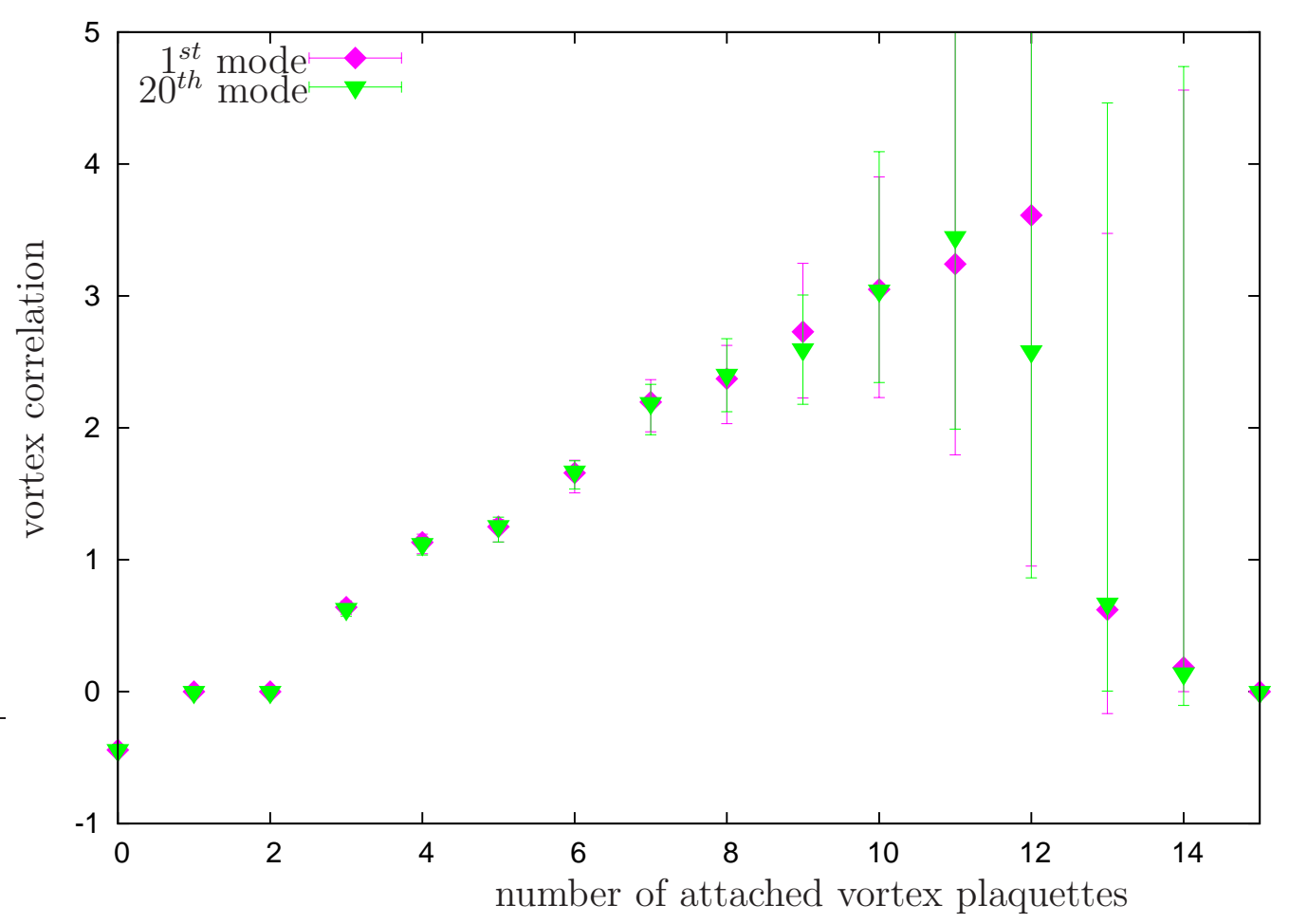

Figure 140: Vortex Correlation for asqtad staggered eigenmodes on a $20^{4}$ lattice at $\beta=$ 3.3 , a) full and b) projected configuration. 
a)

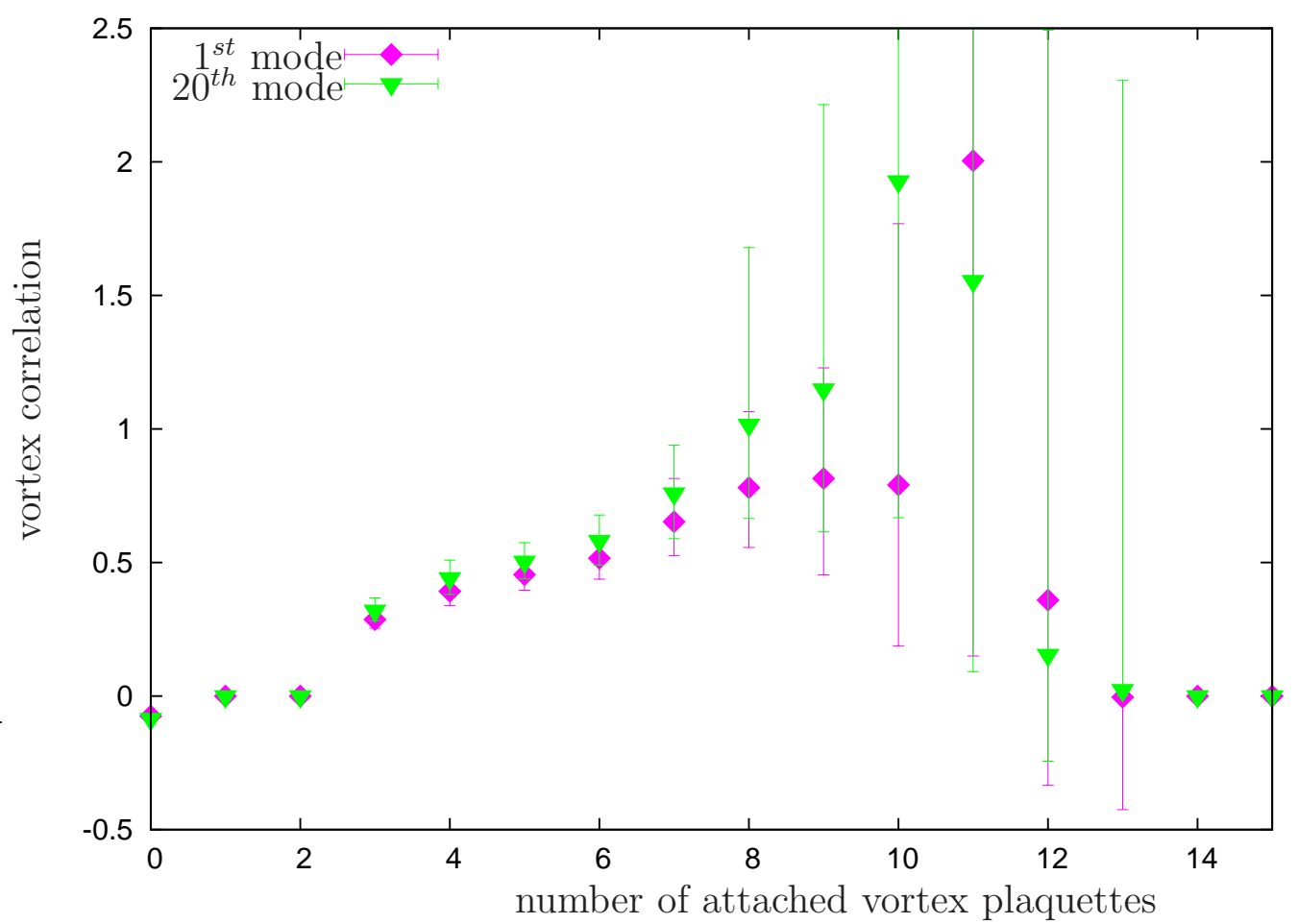

b)

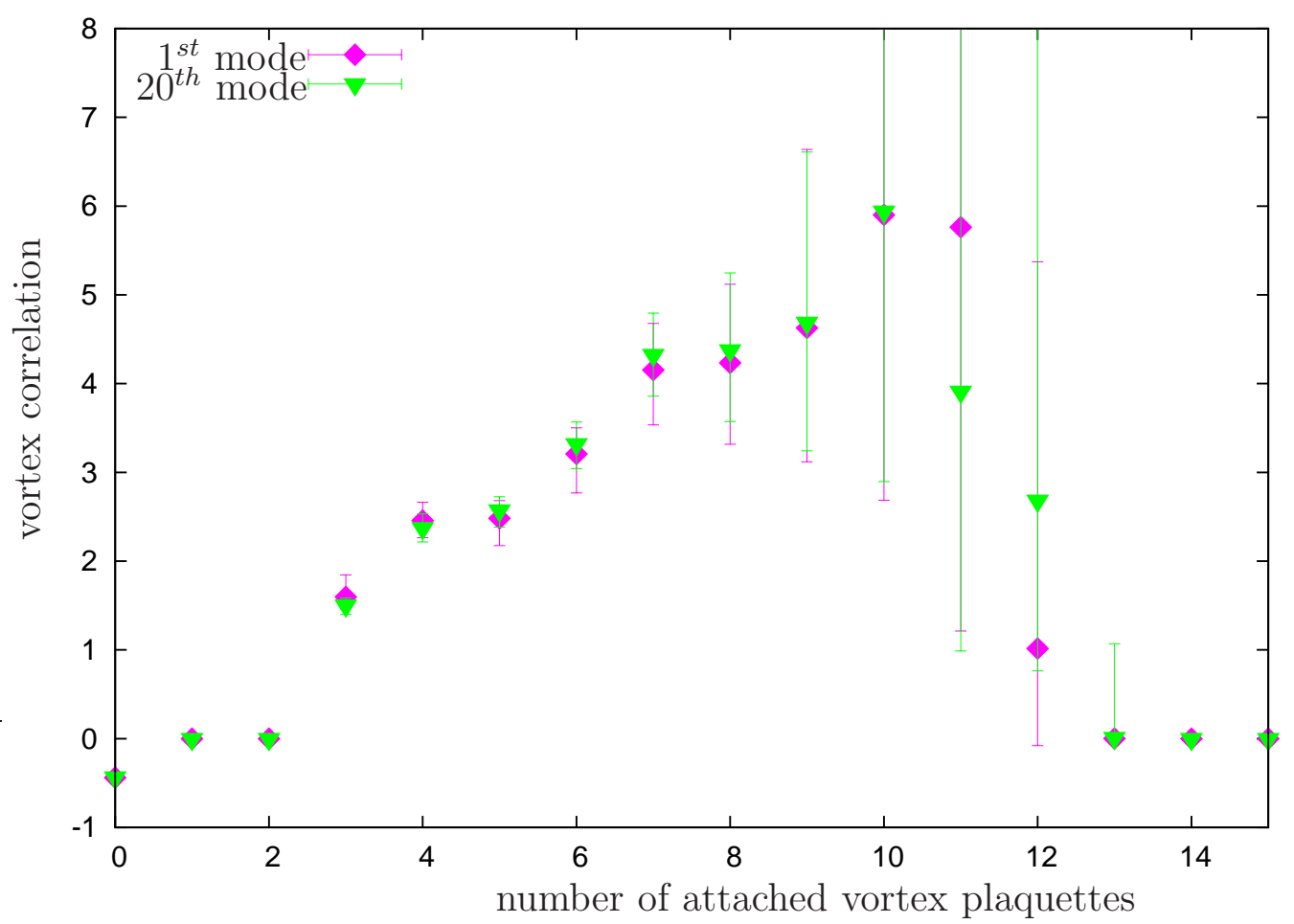

Figure 141: Vortex Correlation for asqtad staggered eigenmodes on a $20^{4}$ lattice at $\beta=$ 3.5 , a) full and b) projected configuration. 
a)

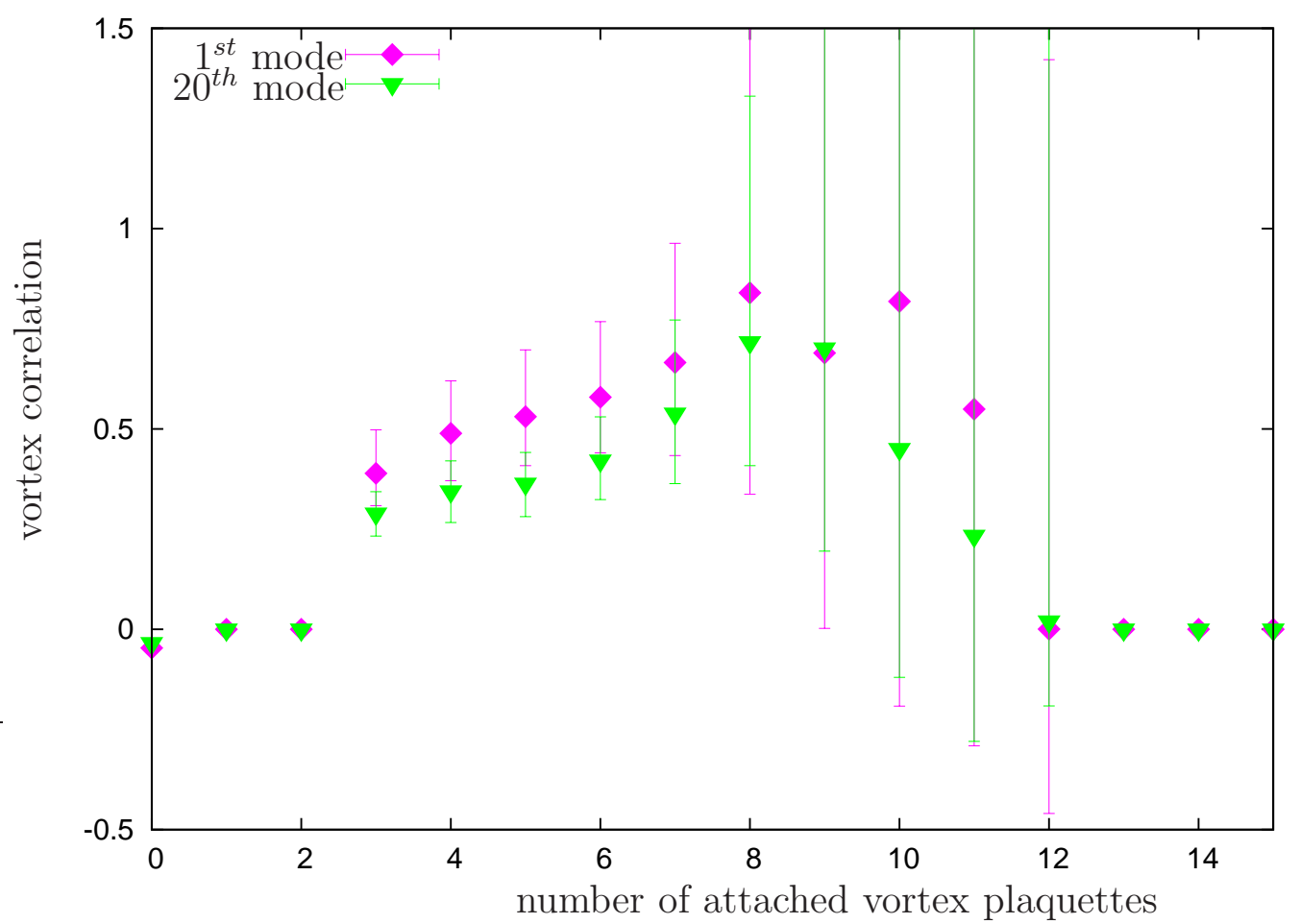

b)

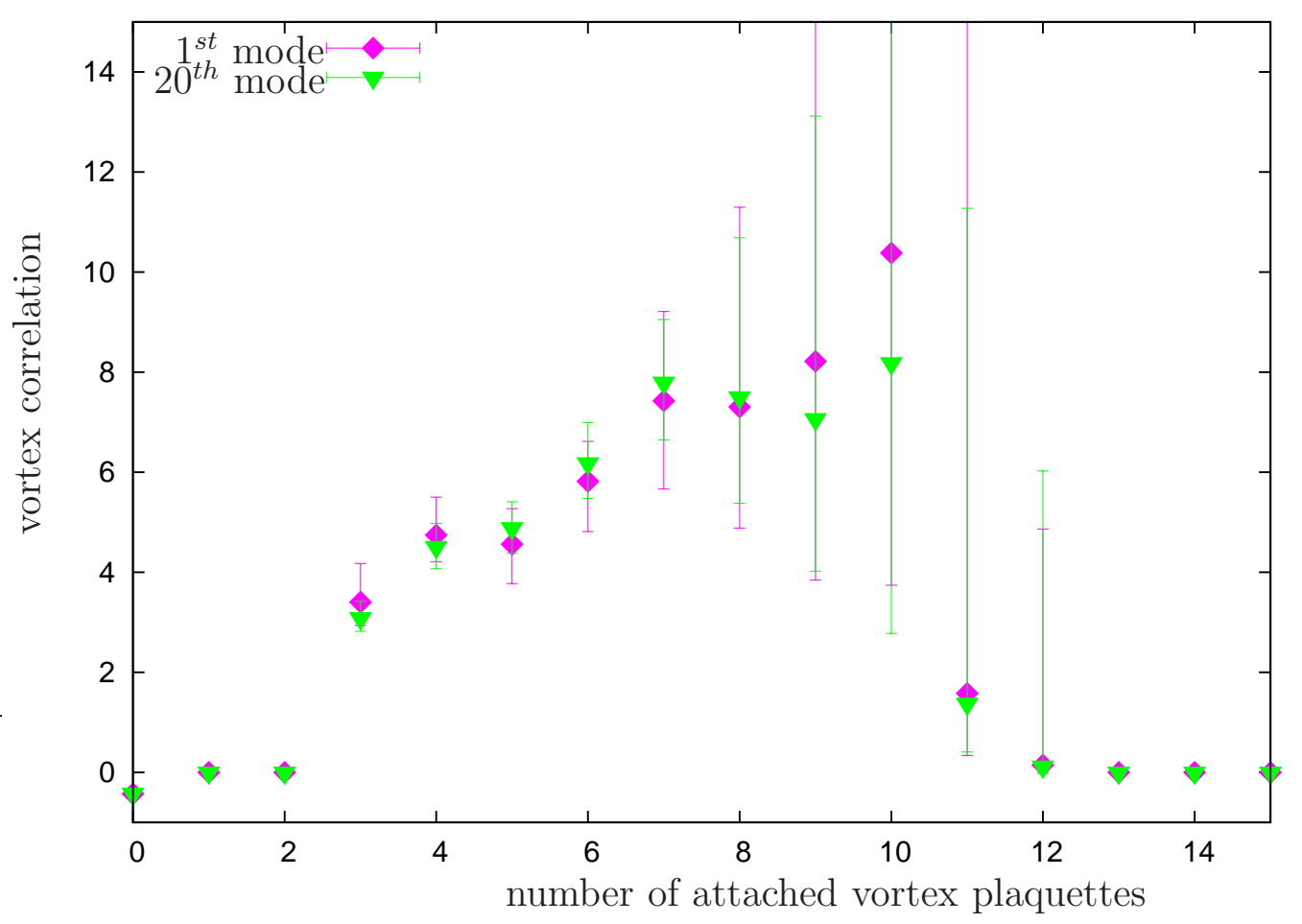

Figure 142: Vortex Correlation for asqtad staggered eigenmodes on a $20^{4}$ lattice at $\beta=$ 3.7 , a) full and b) projected configuration. 
a)

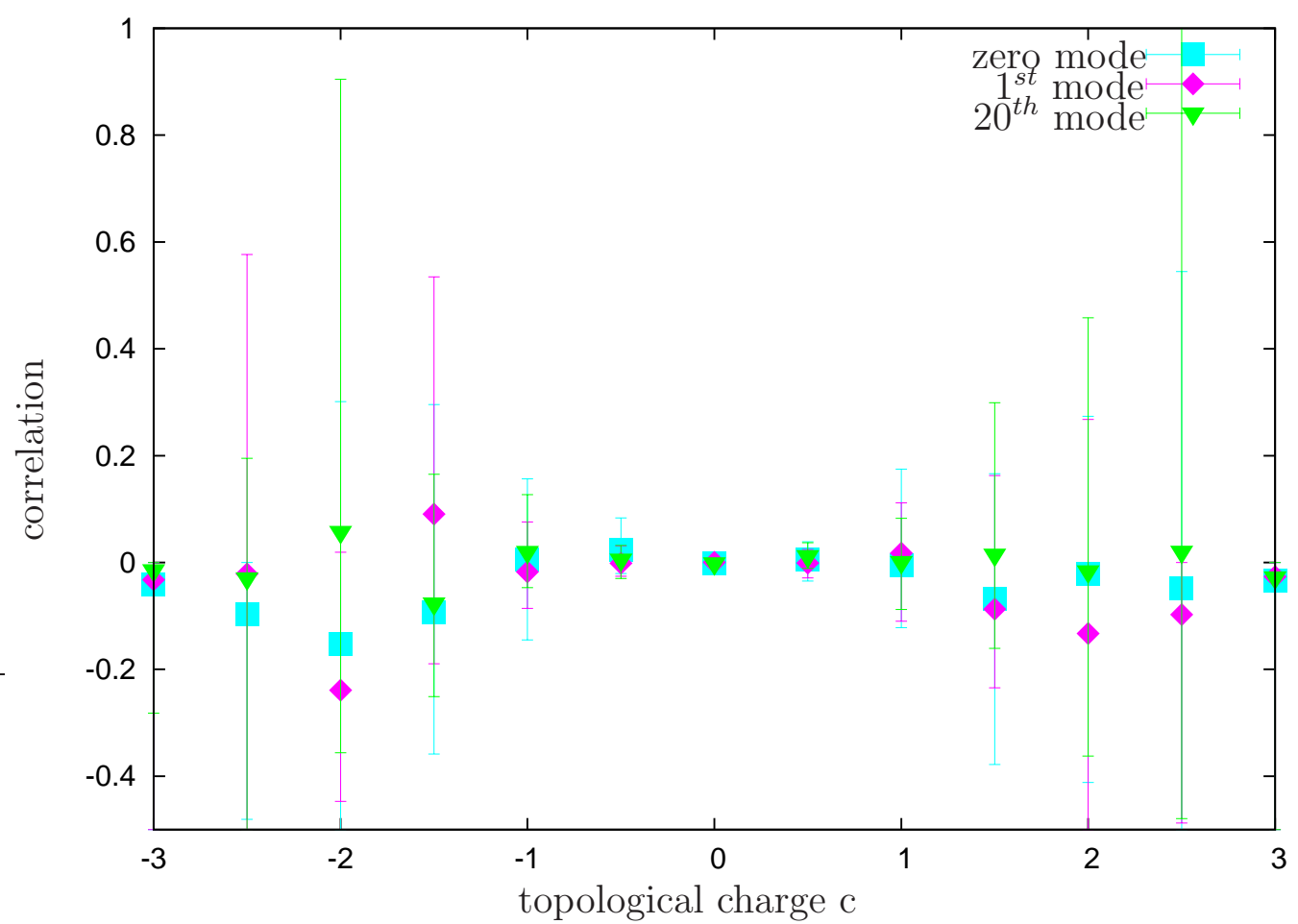

b)

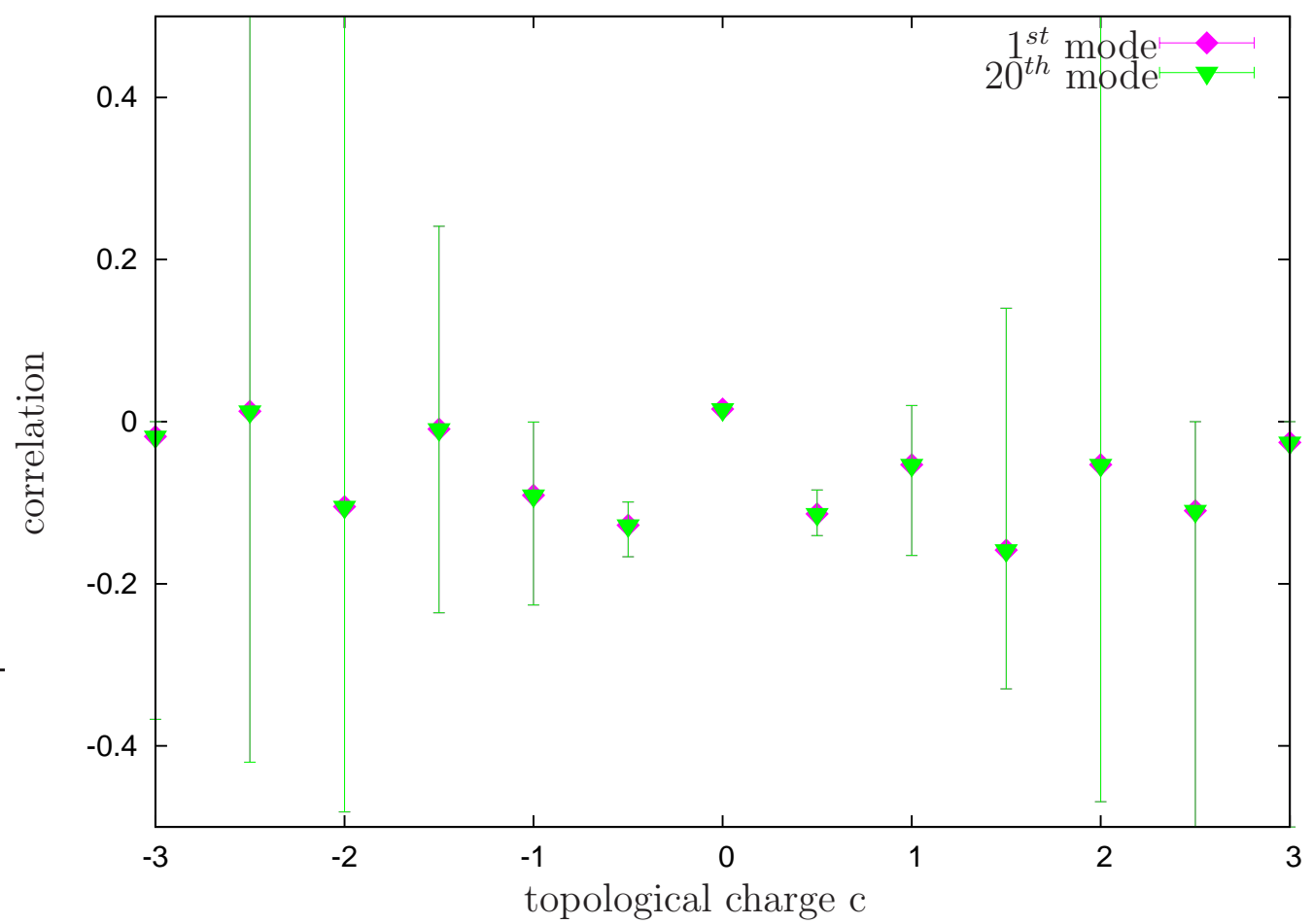

Figure 143: Correlation between topological charge and overlap eigenmodes on a $12^{4}$ lattice at $\beta=2.9$, a) full and b) projected configuration. 


\section{$C$ Plots and Figures}

a)

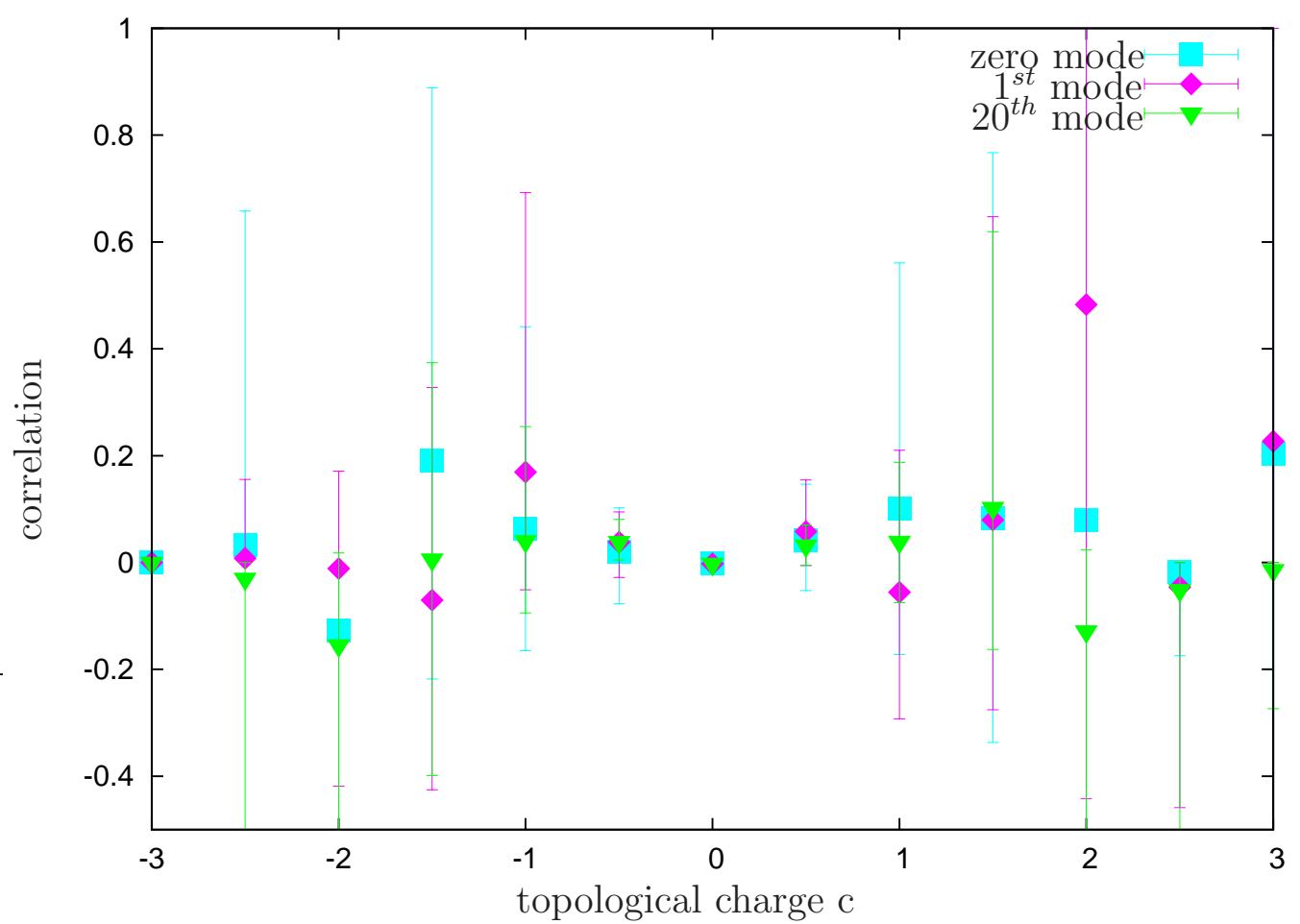

b)

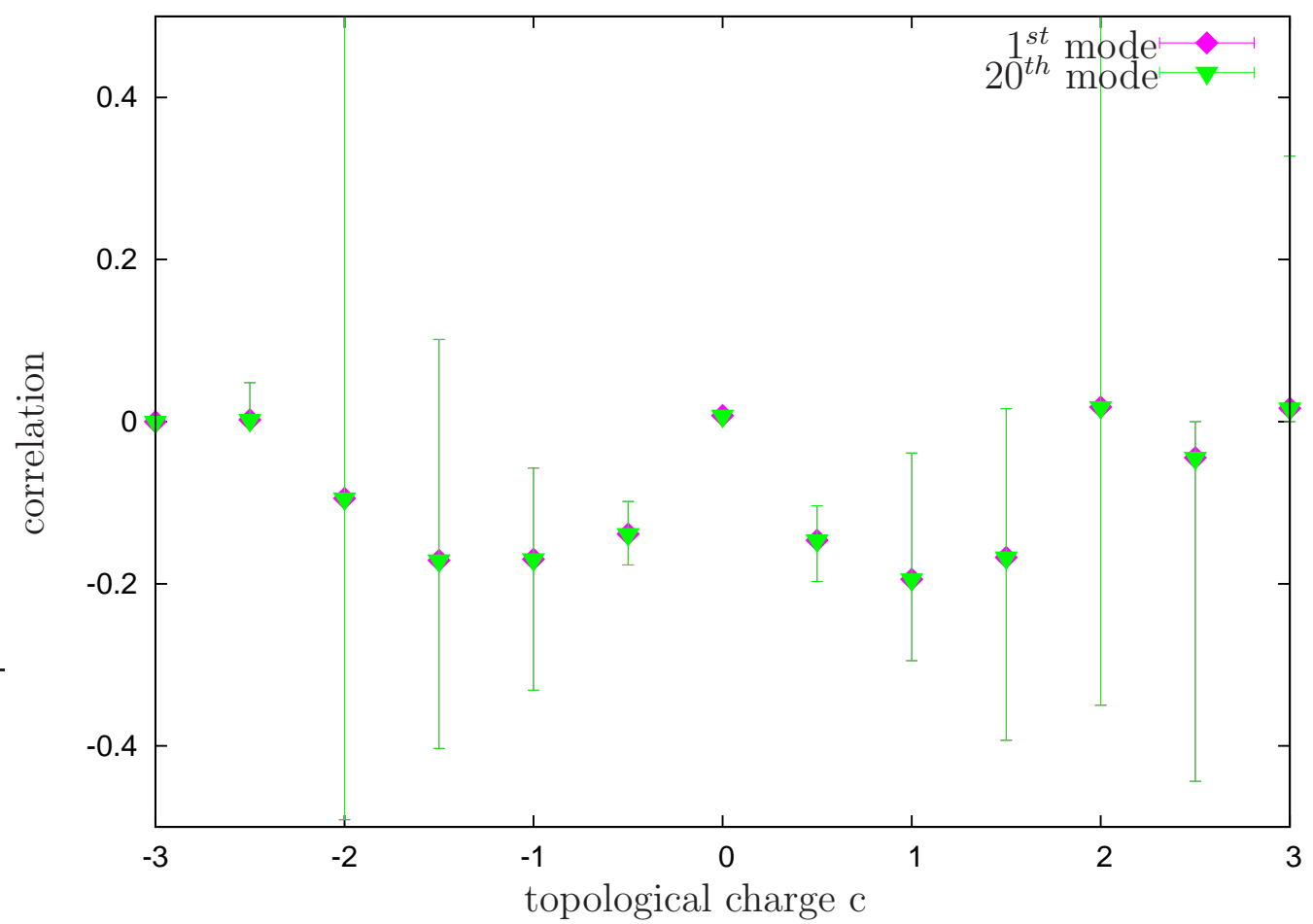

Figure 144: Correlation between topological charge and overlap eigenmodes on a $12^{4}$ lattice at $\beta=3.1$, a) full and b) projected configuration. 


\section{$C$ Plots and Figures}

a)

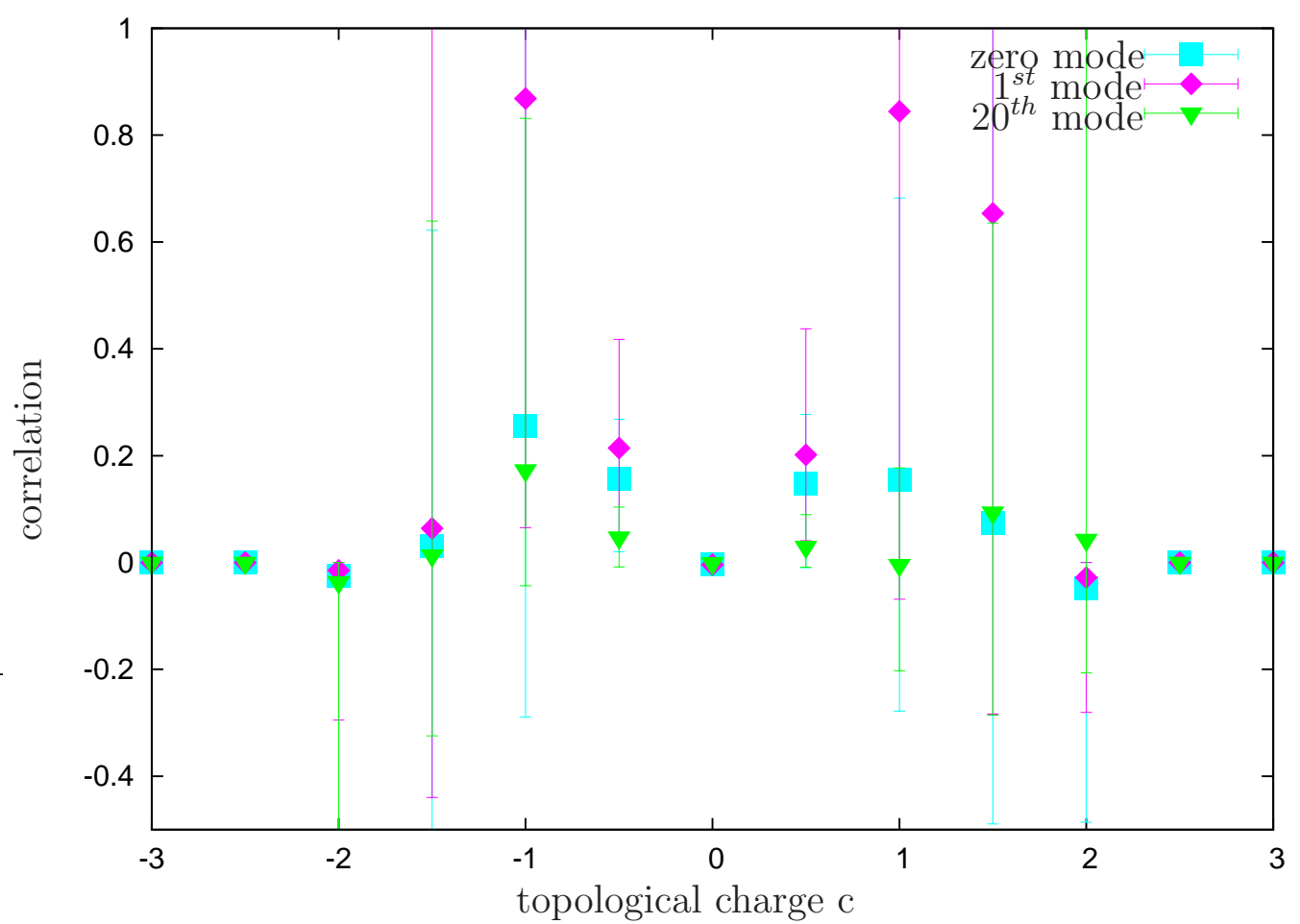

b)

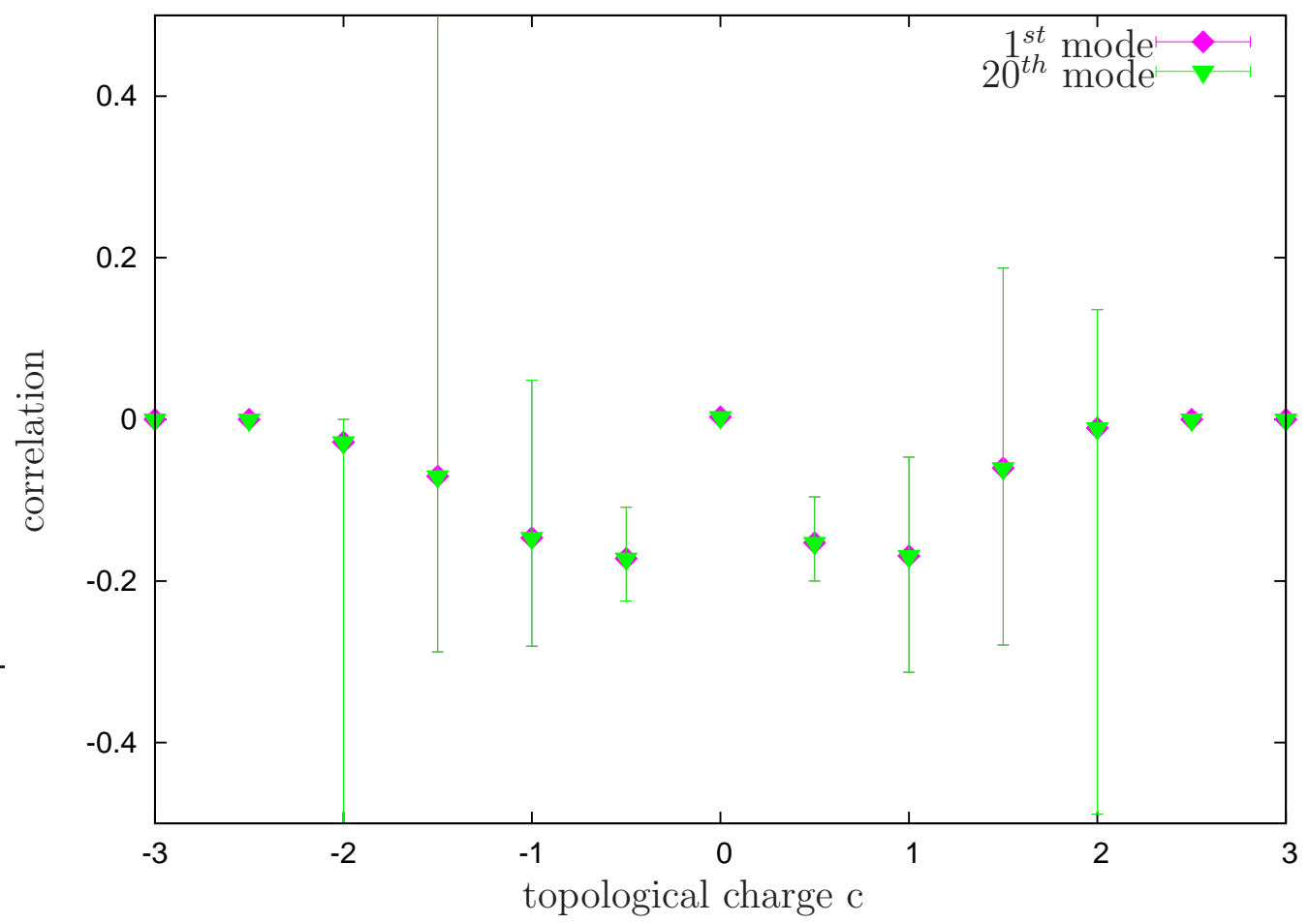

Figure 145: Correlation between topological charge and overlap eigenmodes on a $12^{4}$ lattice at $\beta=3.3$, a) full and b) projected configuration. 
a)

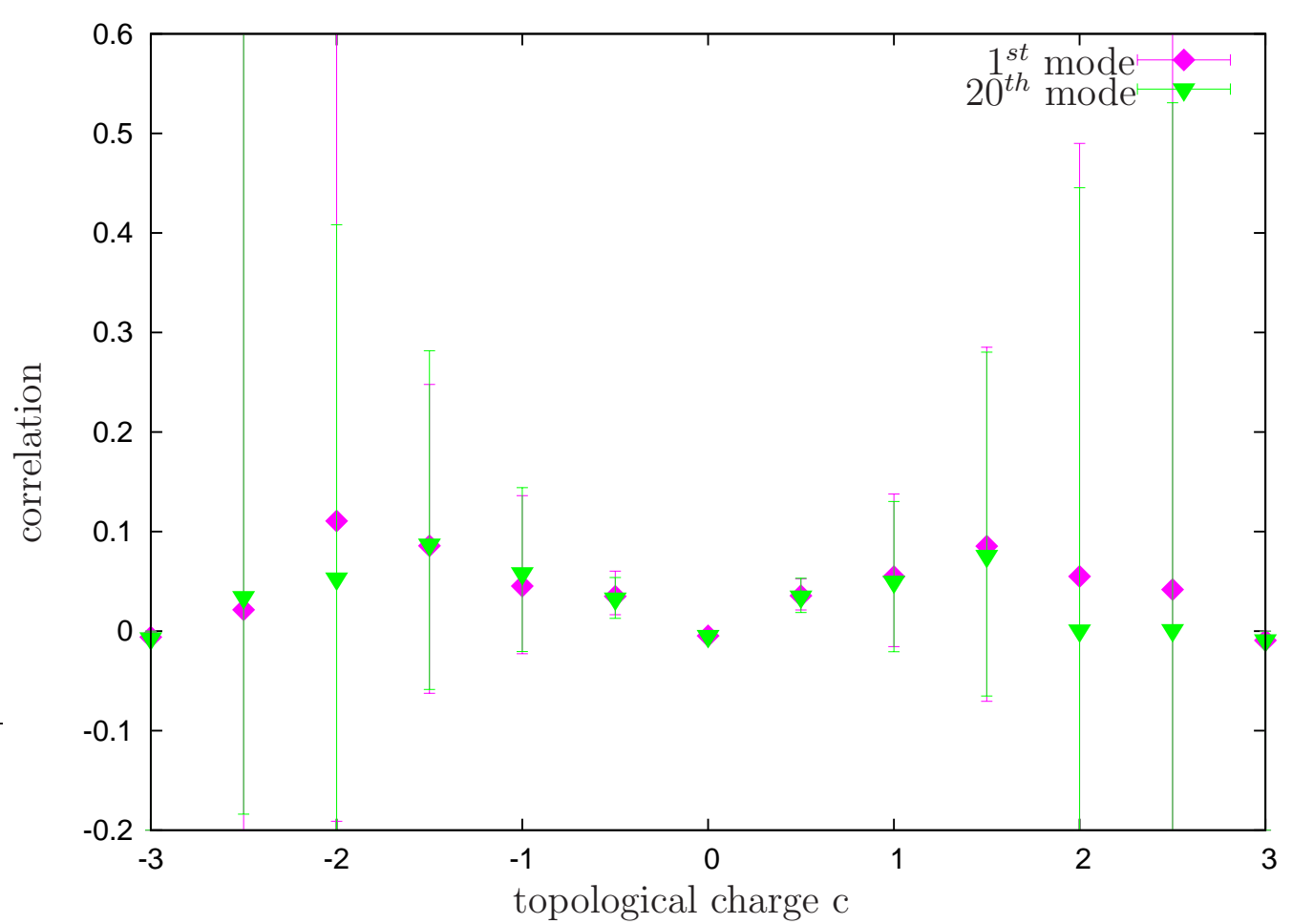

b)

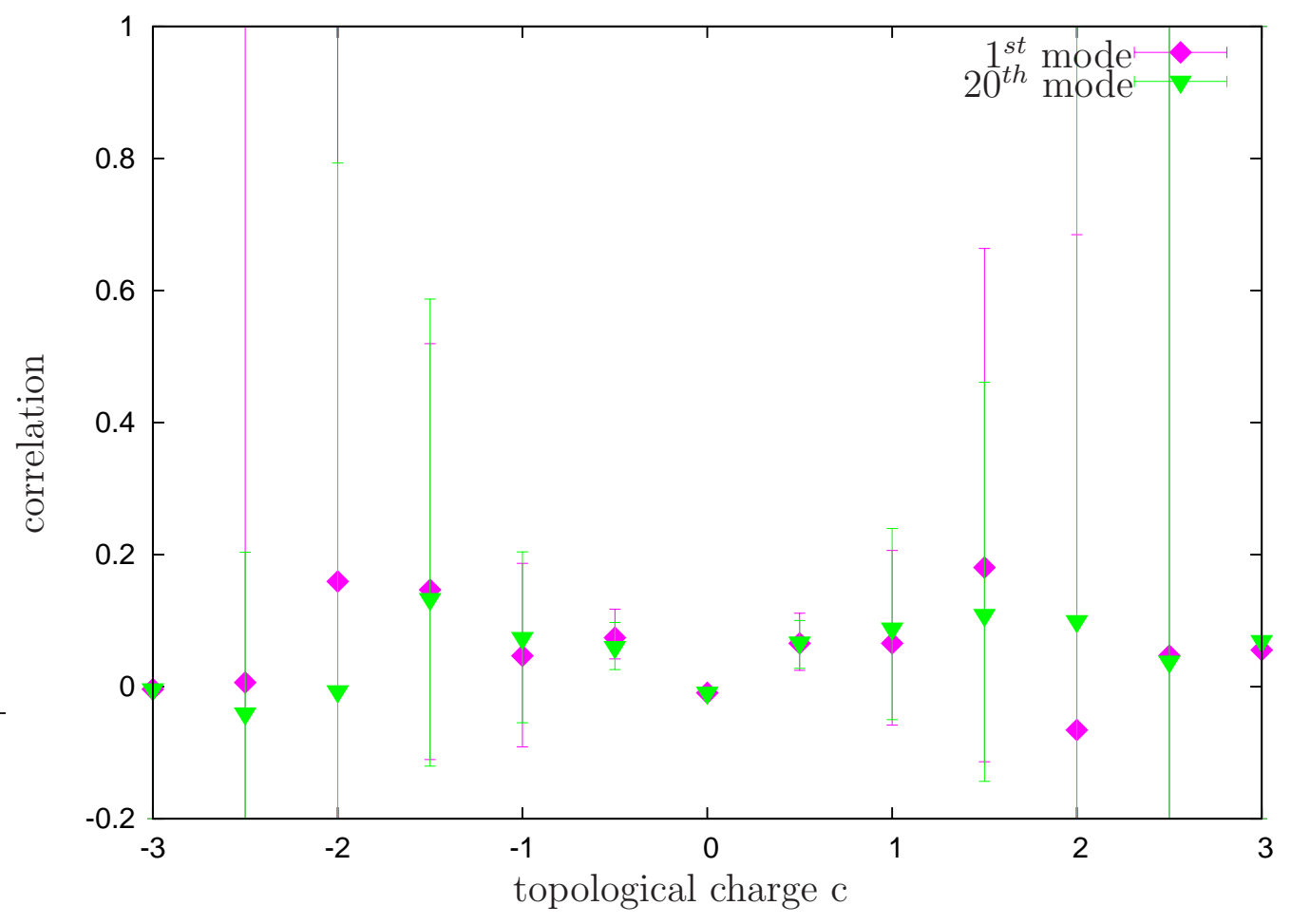

Figure 146: Correlation between topological charge and asqtad staggered eigenmodes on a $12^{4}$ lattice at $\beta=2.9$, a) full and b) projected configuration. 
a)

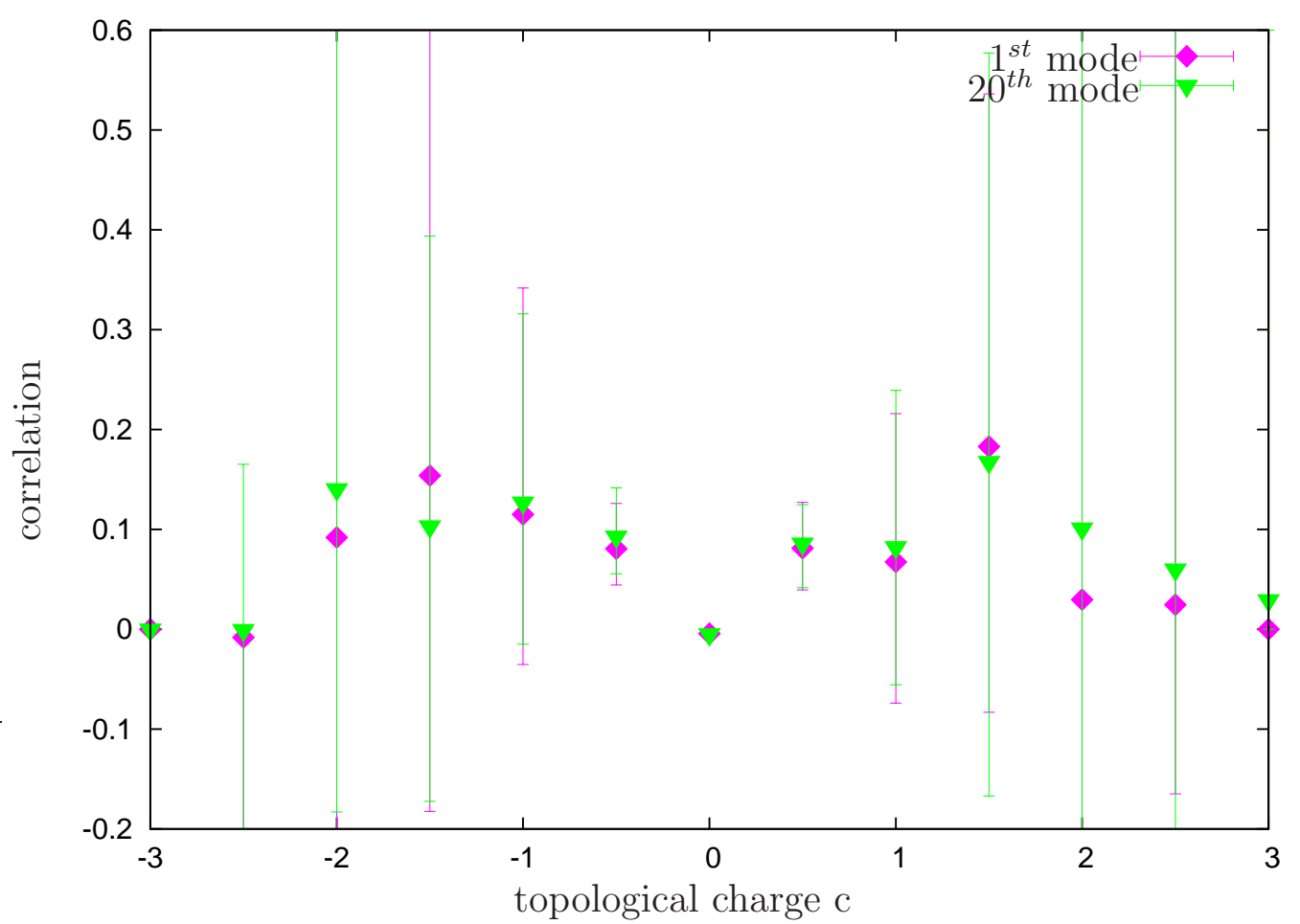

b)

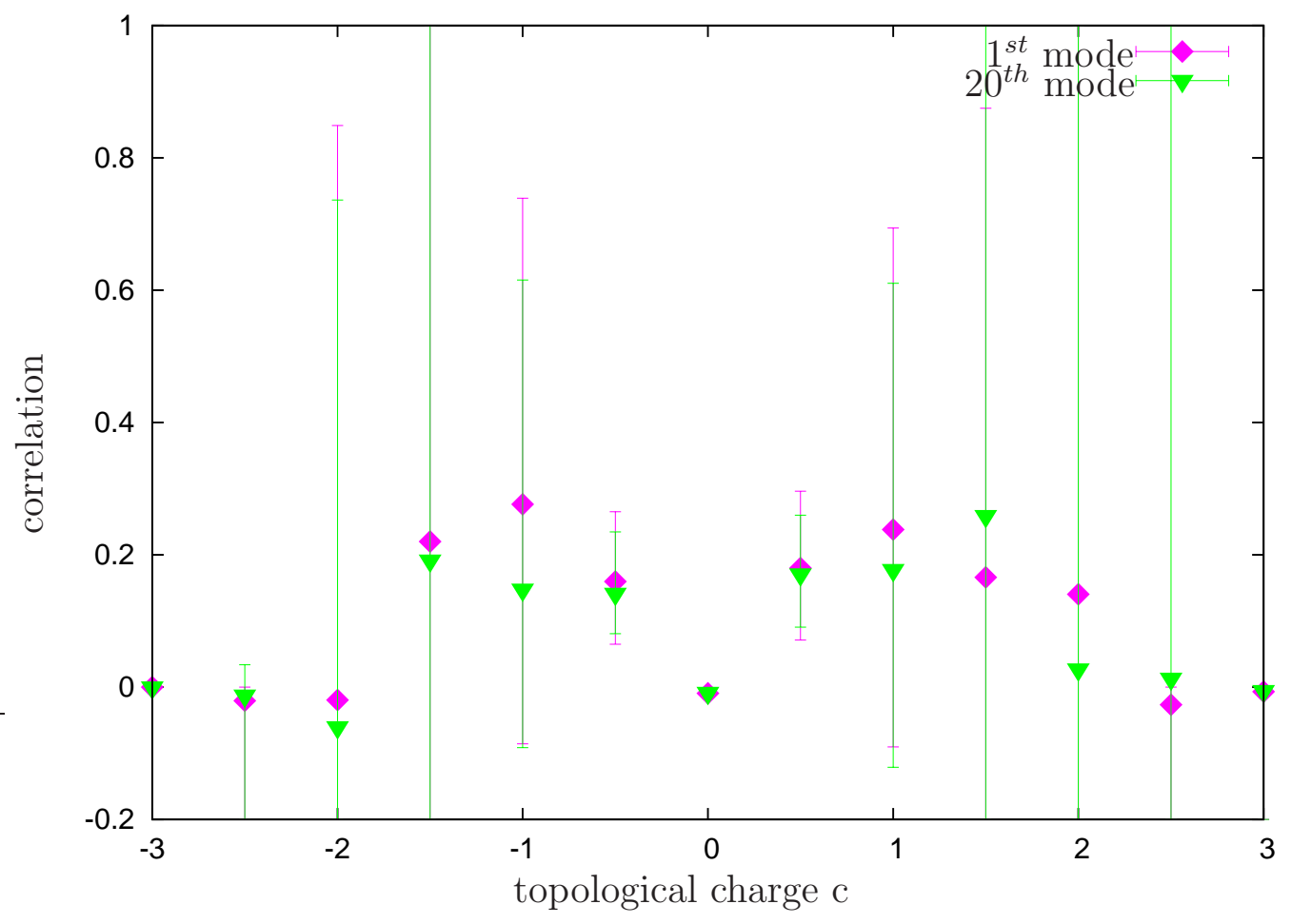

Figure 147: Correlation between topological charge and asqtad staggered eigenmodes on a $12^{4}$ lattice at $\beta=3.1$, a) full and b) projected configuration. 


\section{$C$ Plots and Figures}

a)

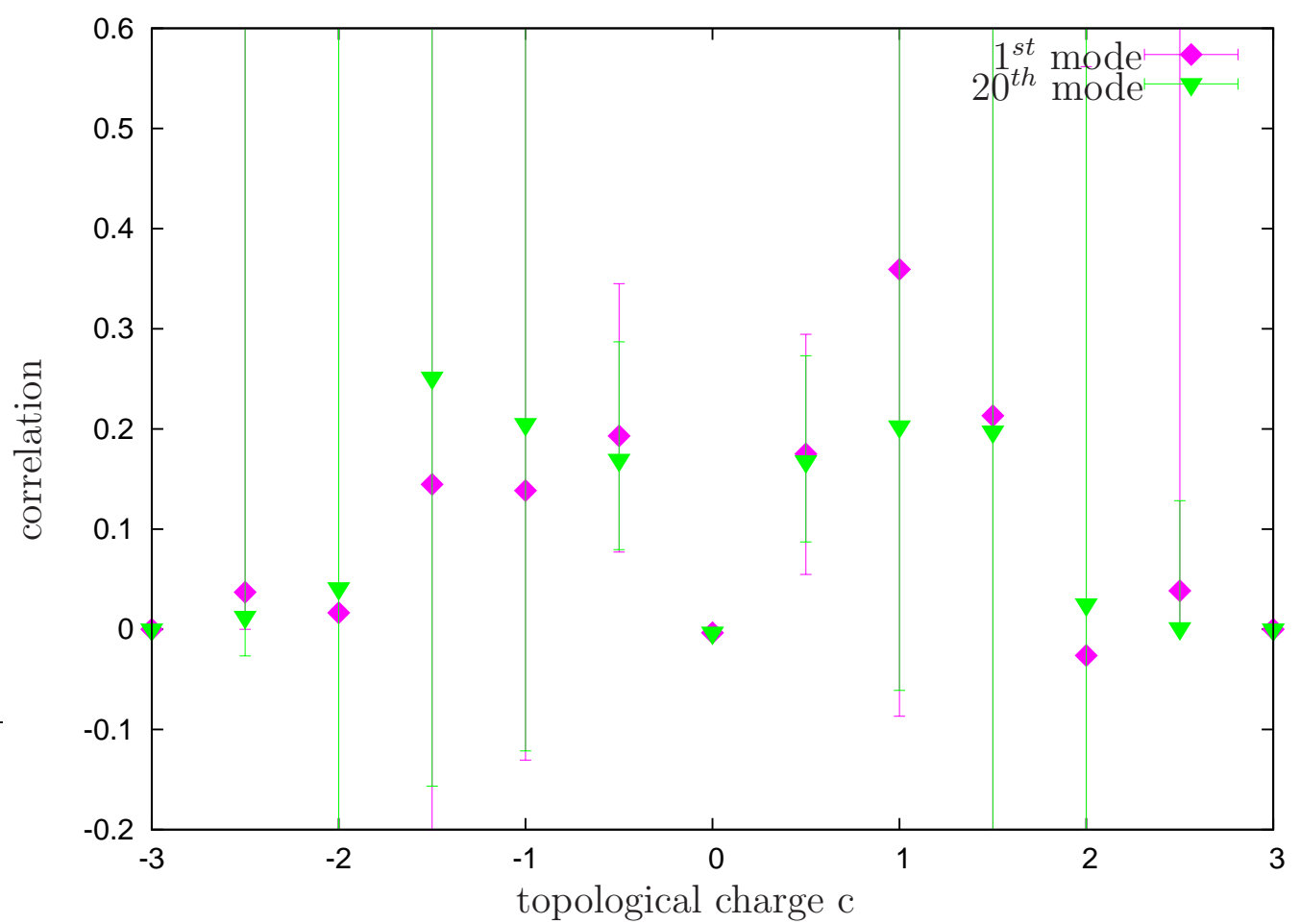

b)

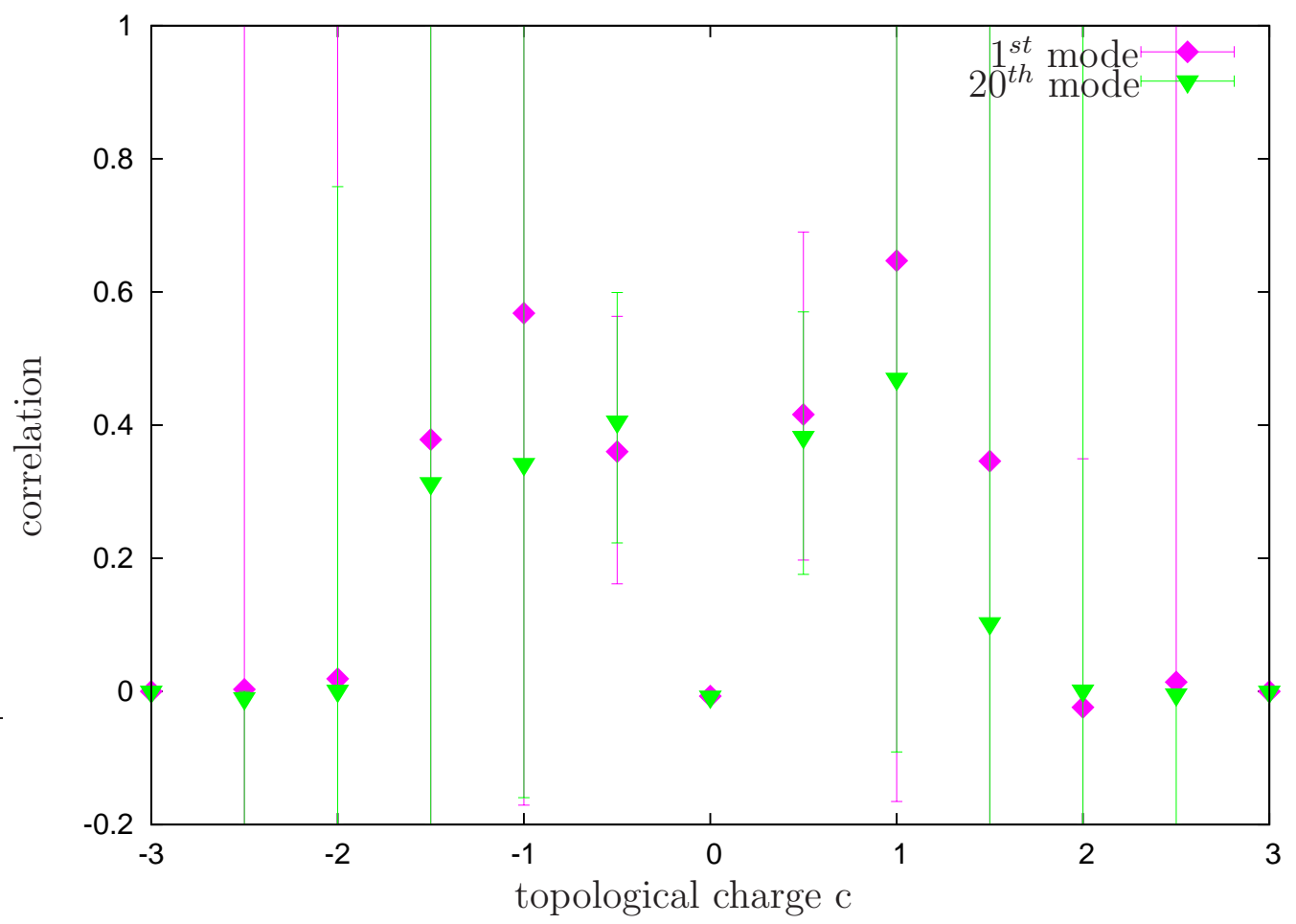

Figure 148: Correlation between topological charge and asqtad staggered eigenmodes on a $12^{4}$ lattice at $\beta=3.3$, a) full and b) projected configuration. 
a)

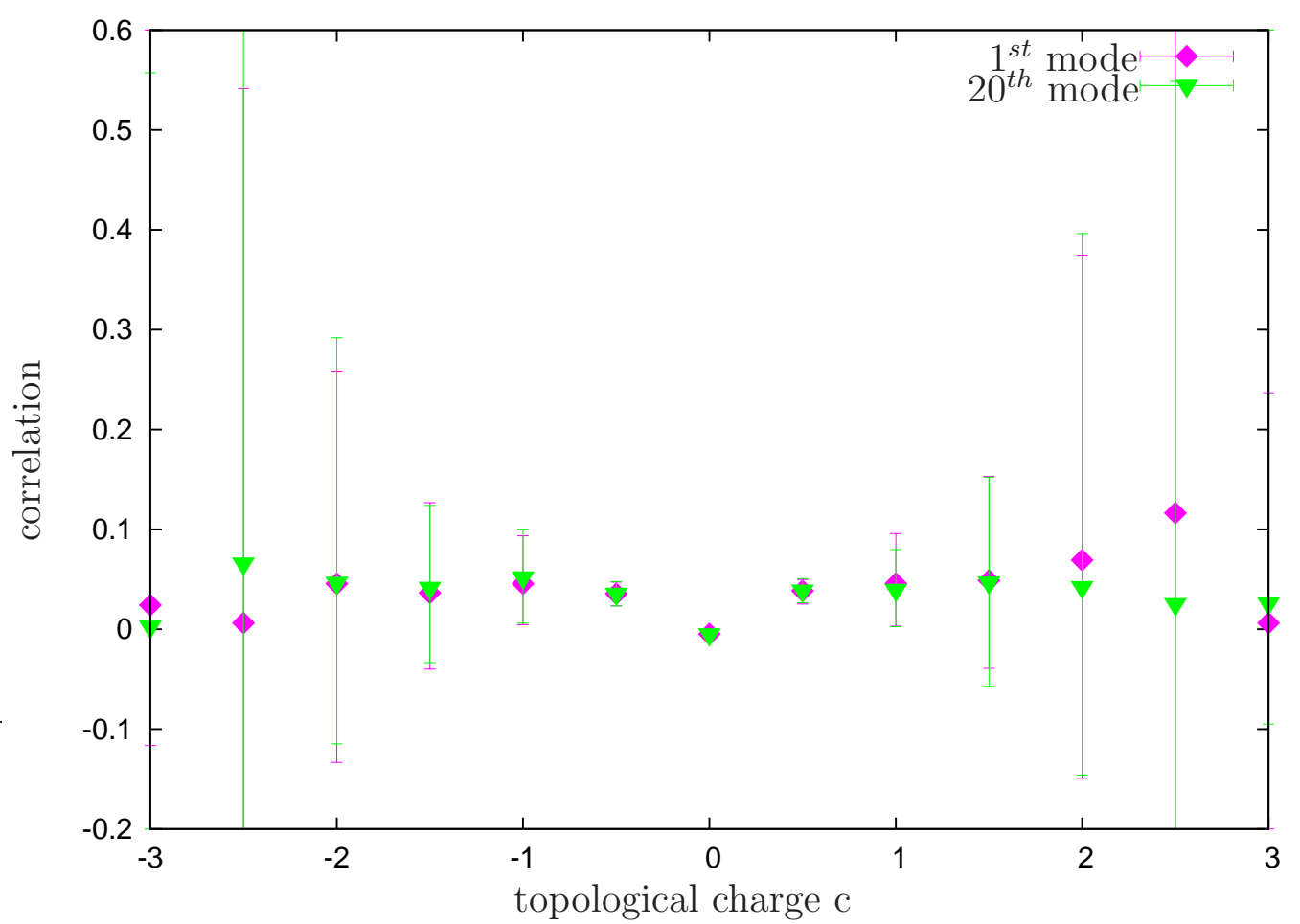

b)

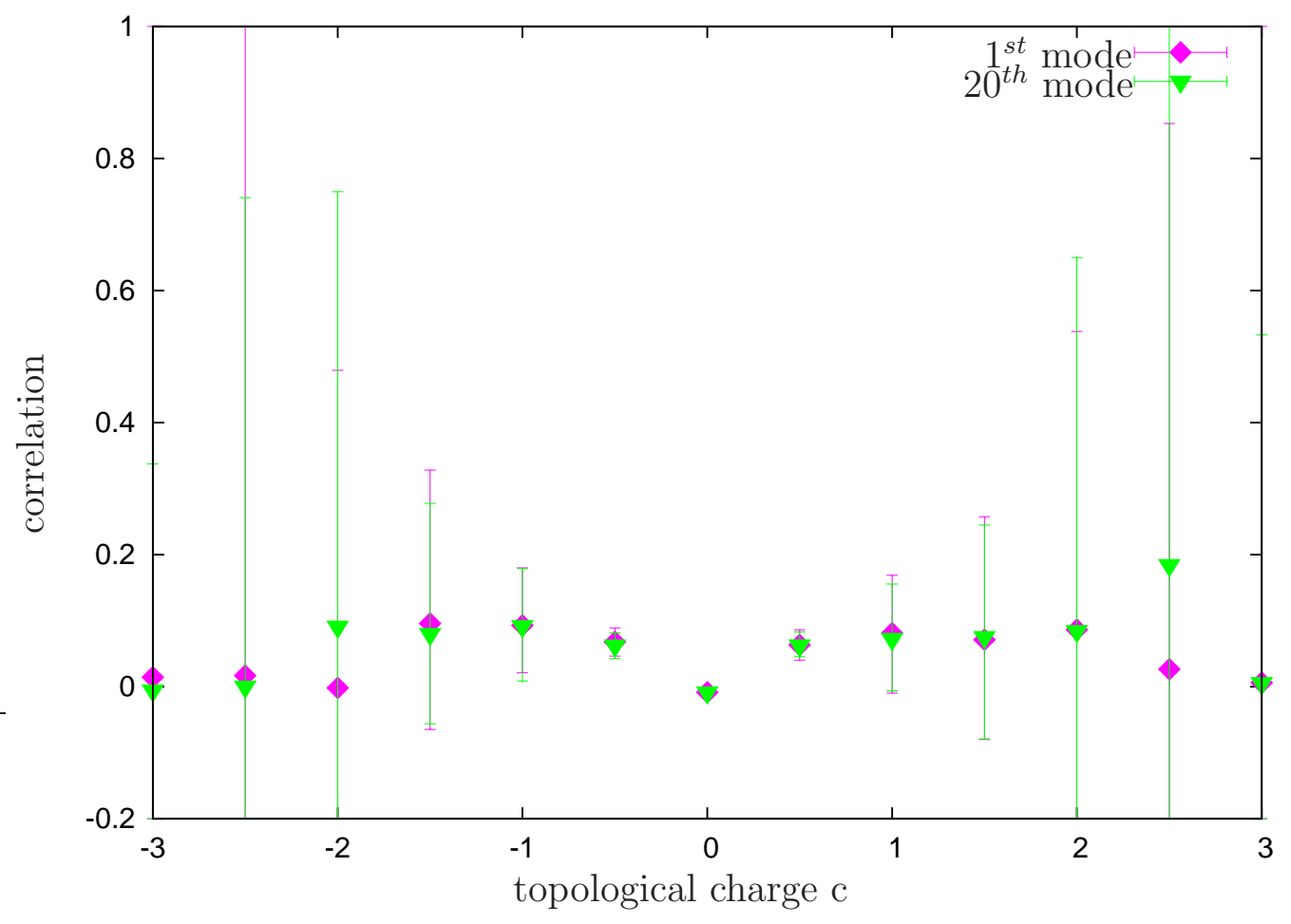

Figure 149: Correlation between topological charge and asqtad staggered eigenmodes on a $16^{4}$ lattice at $\beta=2.9$, a) full and b) projected configuration. 
a)

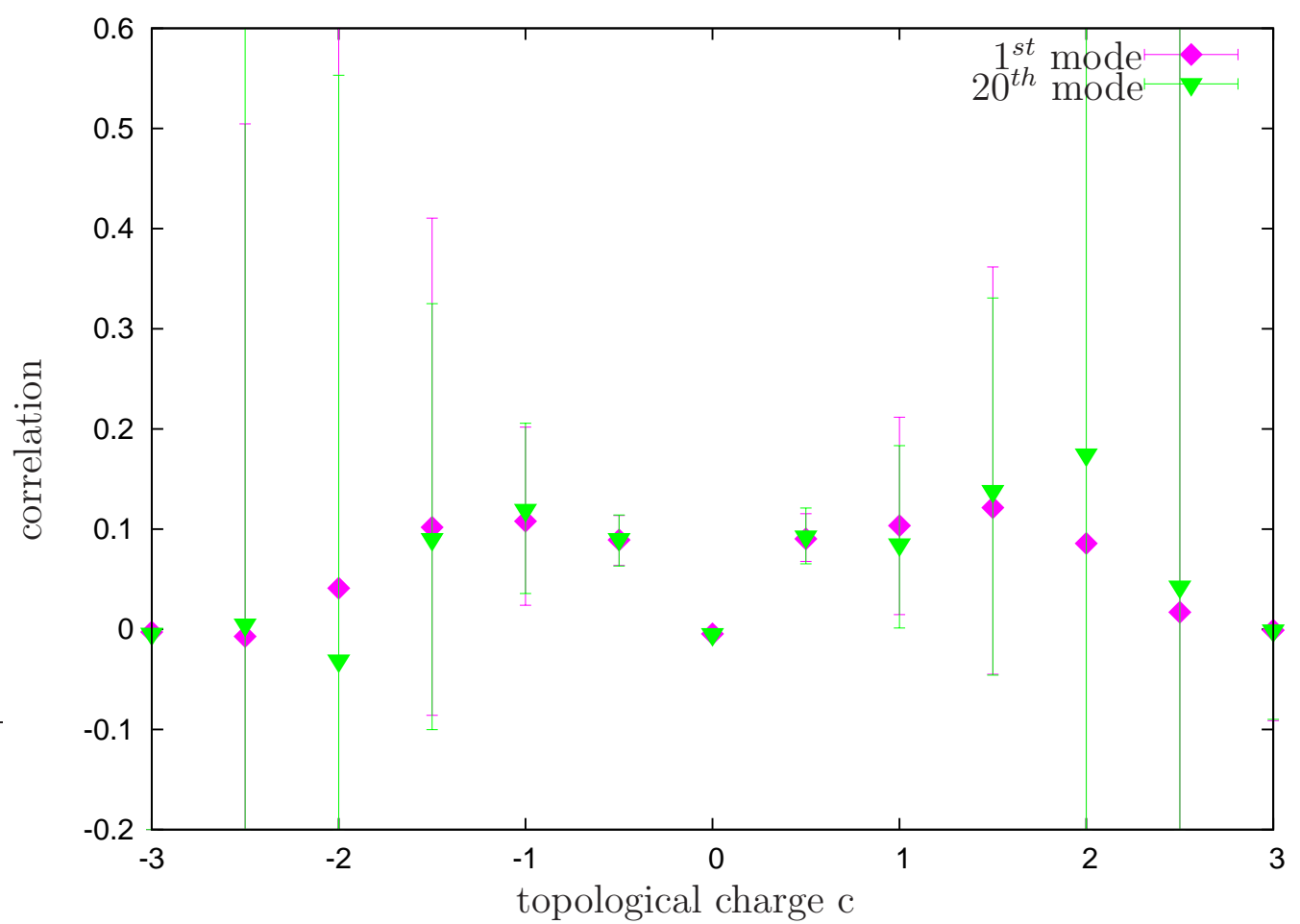

b)

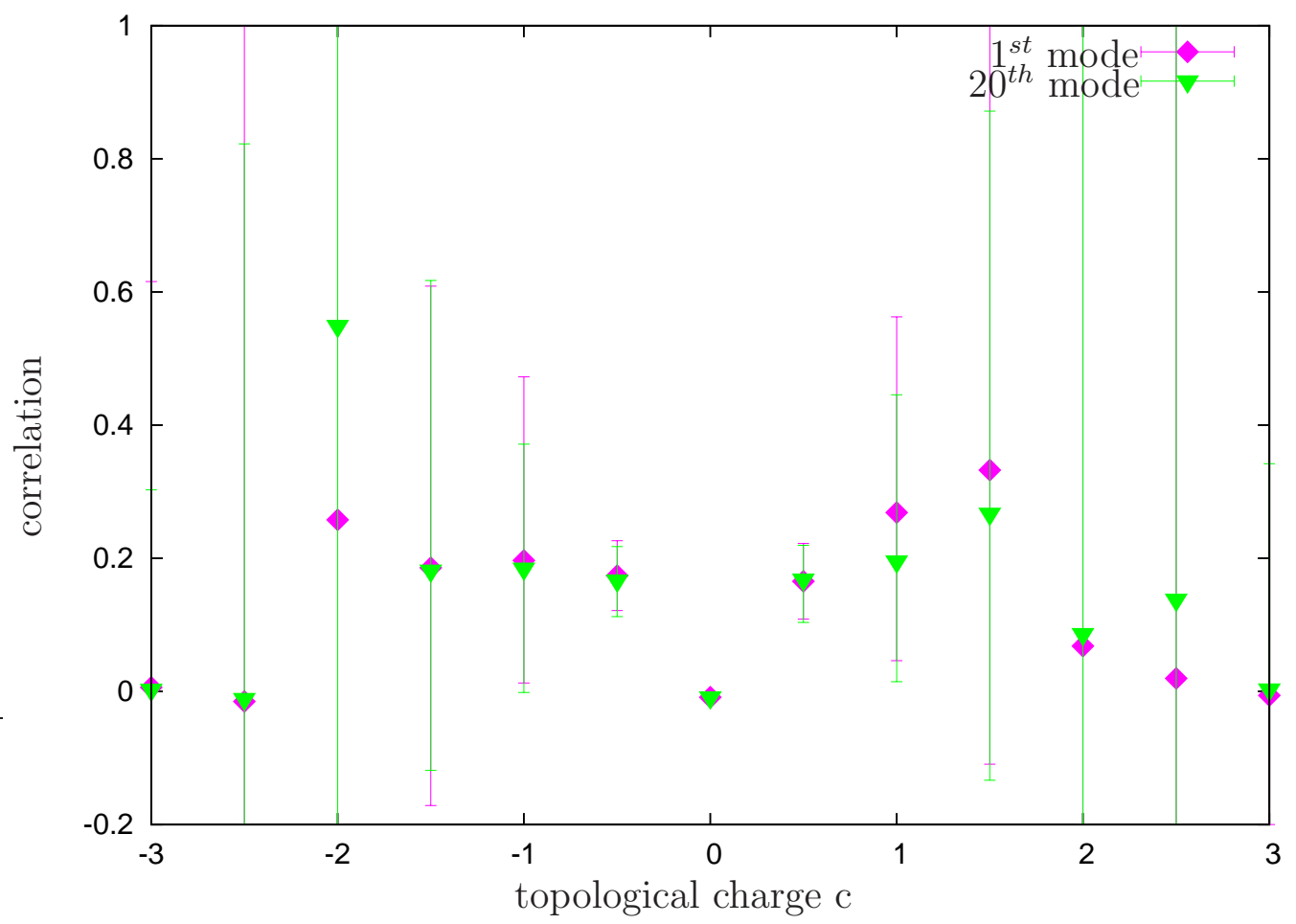

Figure 150: Correlation between topological charge and asqtad staggered eigenmodes on a $16^{4}$ lattice at $\beta=3.1$, a) full and b) projected configuration. 
a)

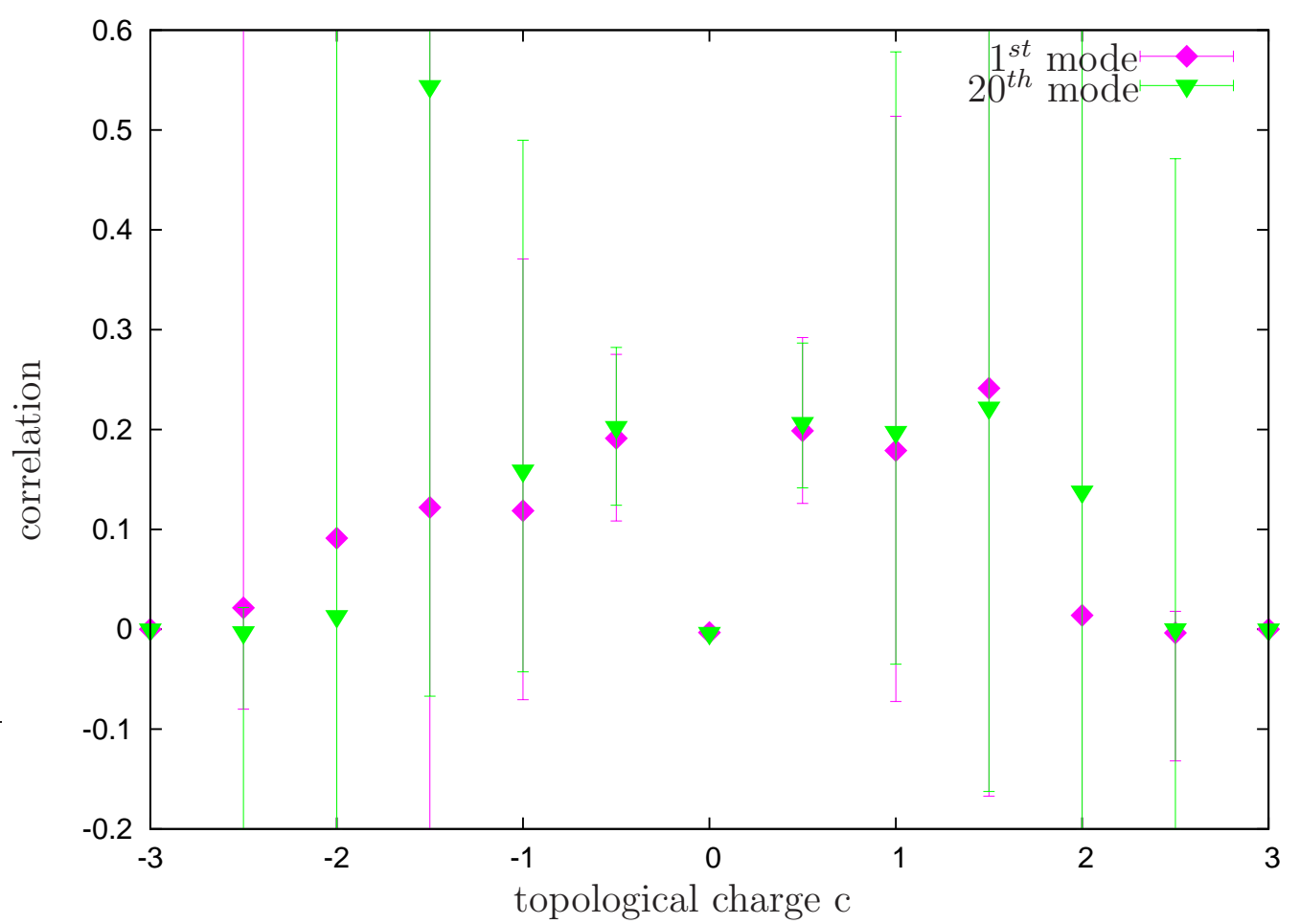

b)

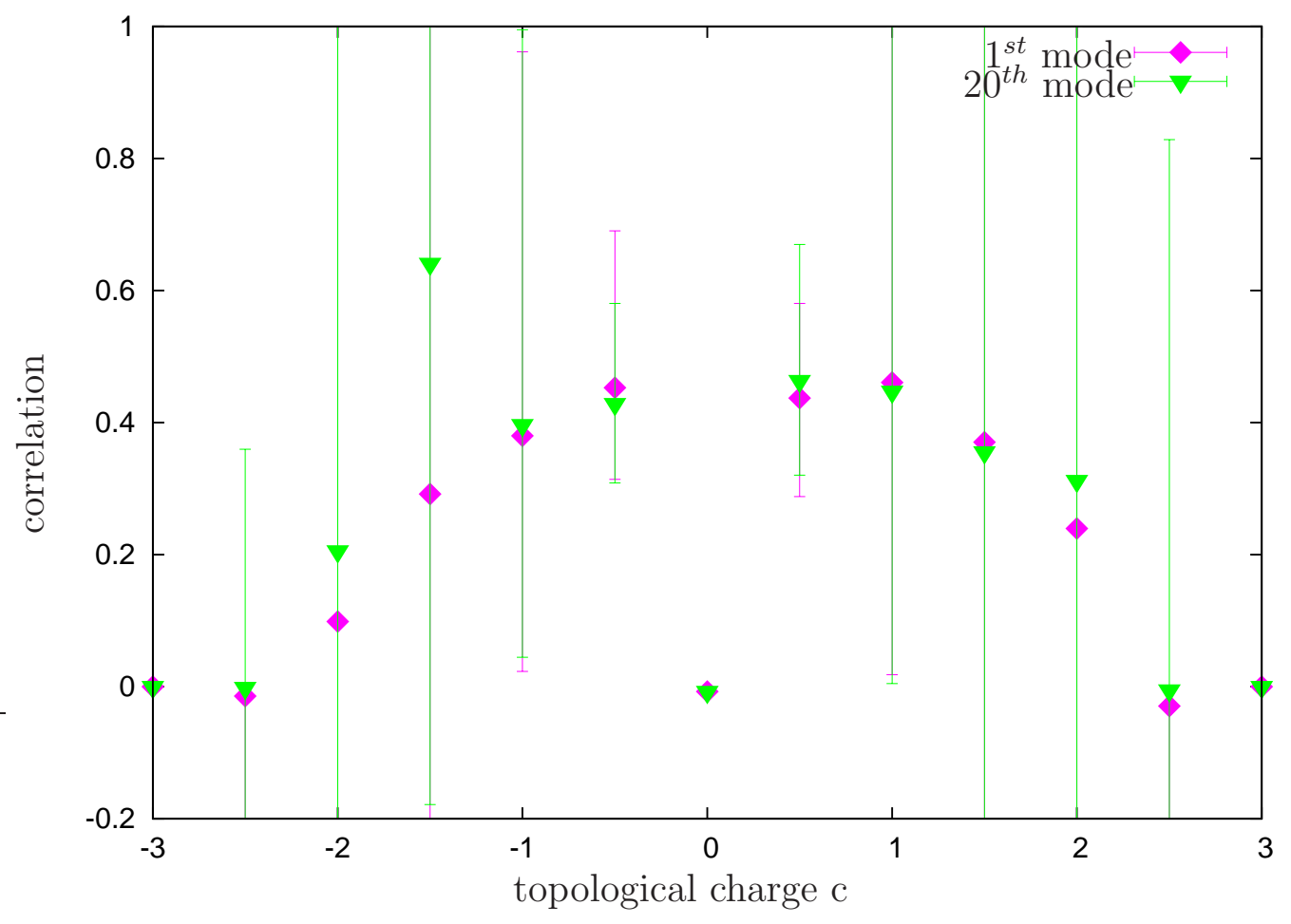

Figure 151: Correlation between topological charge and asqtad staggered eigenmodes on a $16^{4}$ lattice at $\beta=3.3$, a) full and b) projected configuration. 


\section{$C$ Plots and Figures}

a)

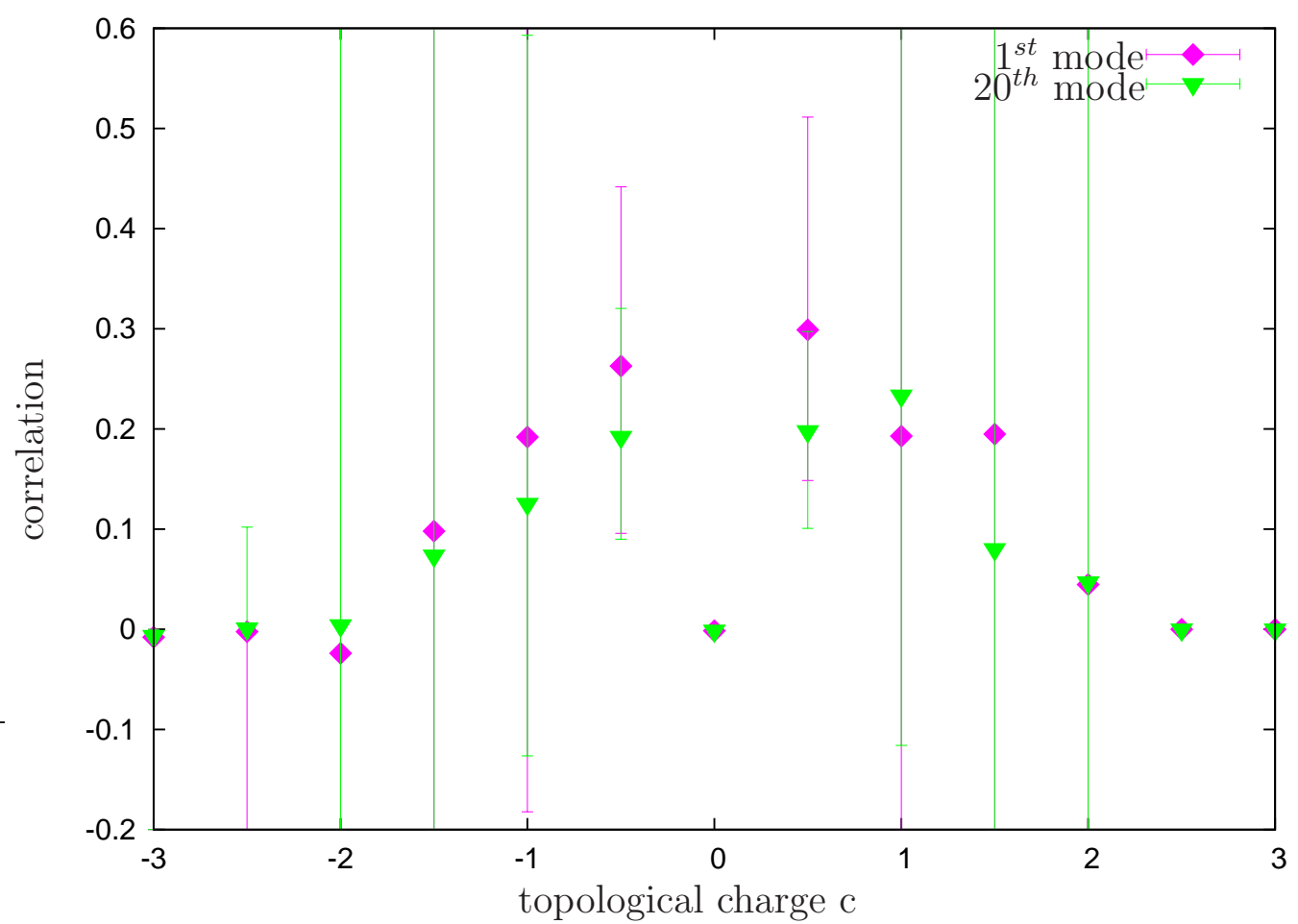

b)

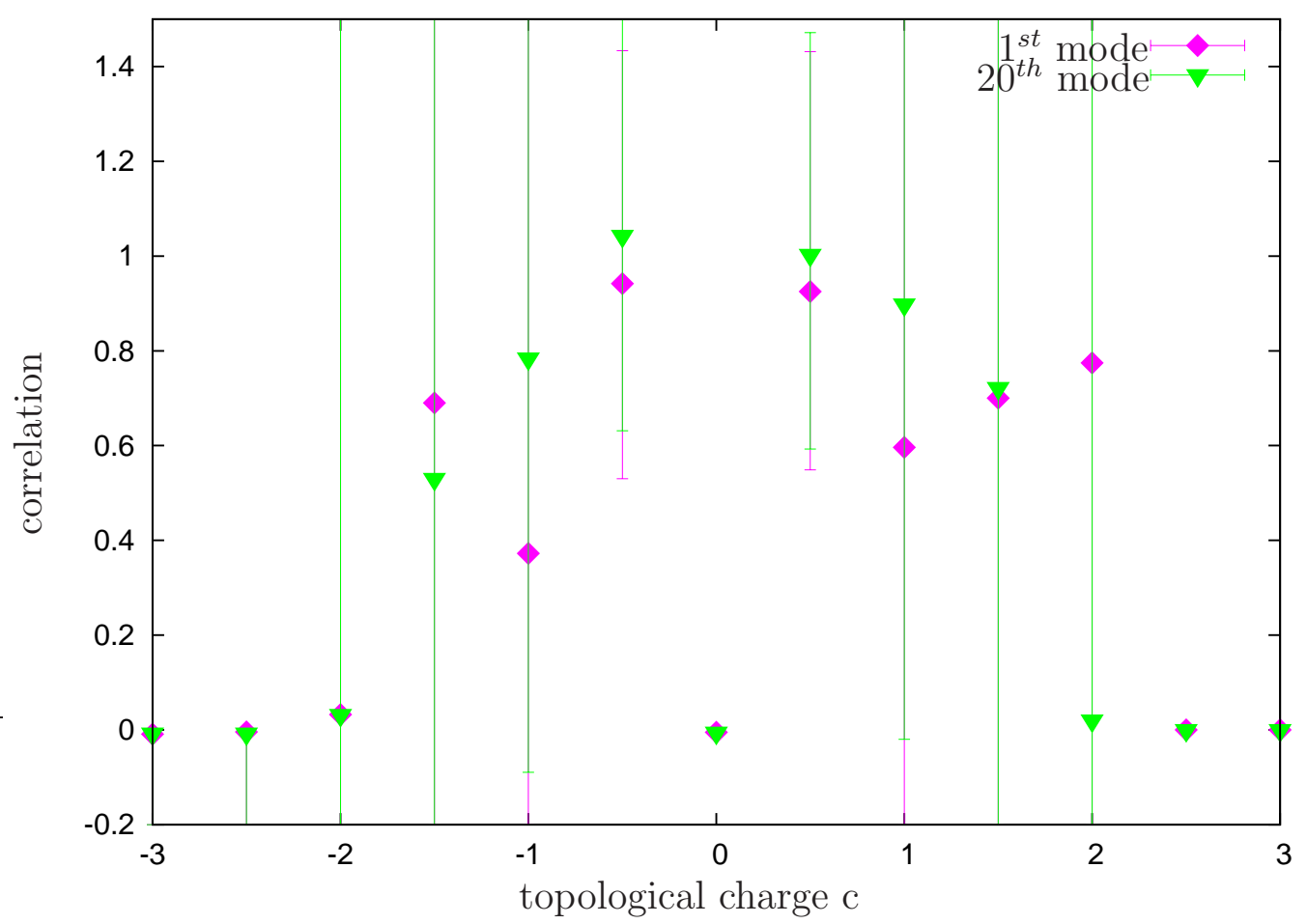

Figure 152: Correlation between topological charge and asqtad staggered eigenmodes on a $16^{4}$ lattice at $\beta=3.5$, a) full and b) projected configuration. 


\section{$C$ Plots and Figures}

a)

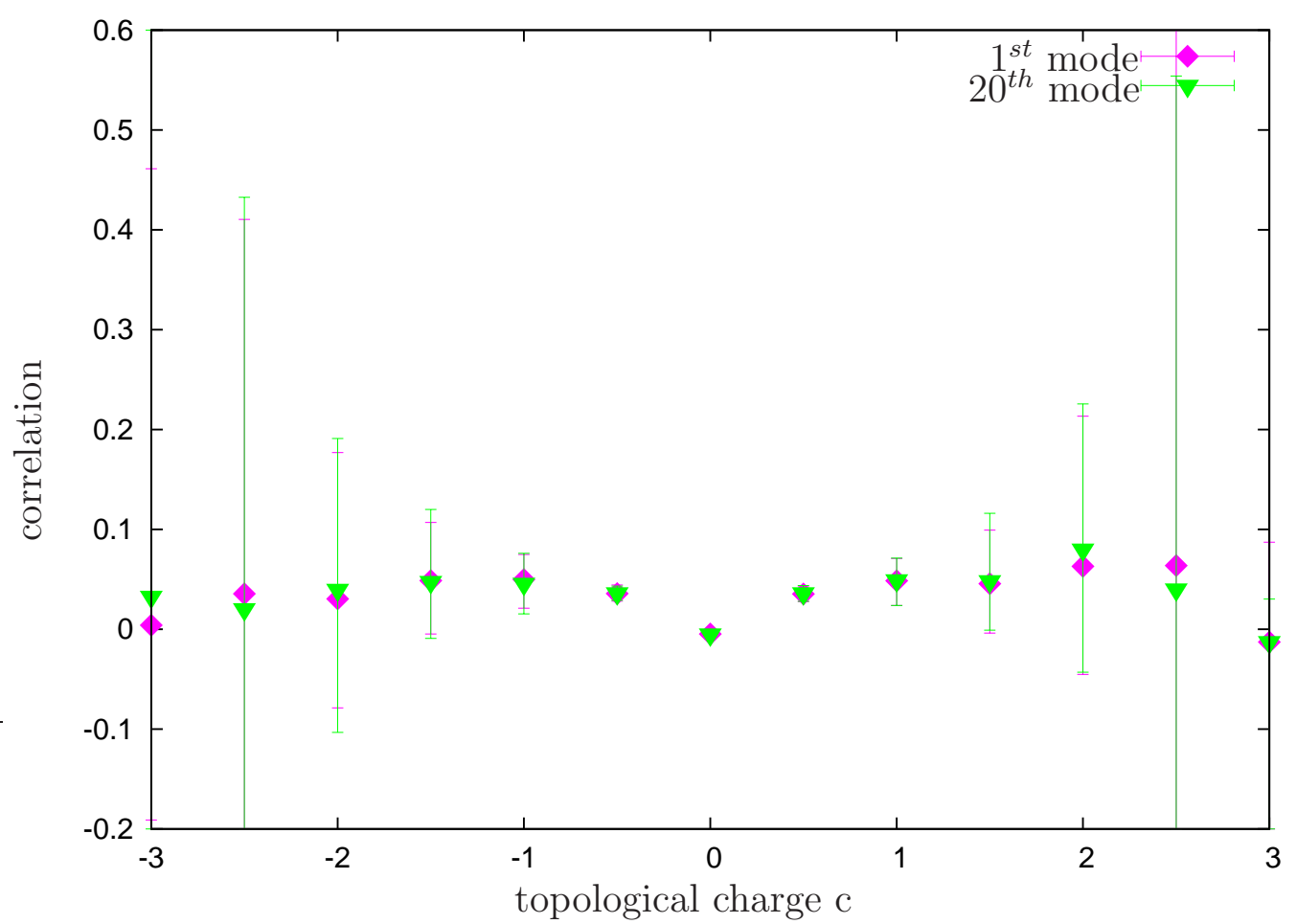

b)

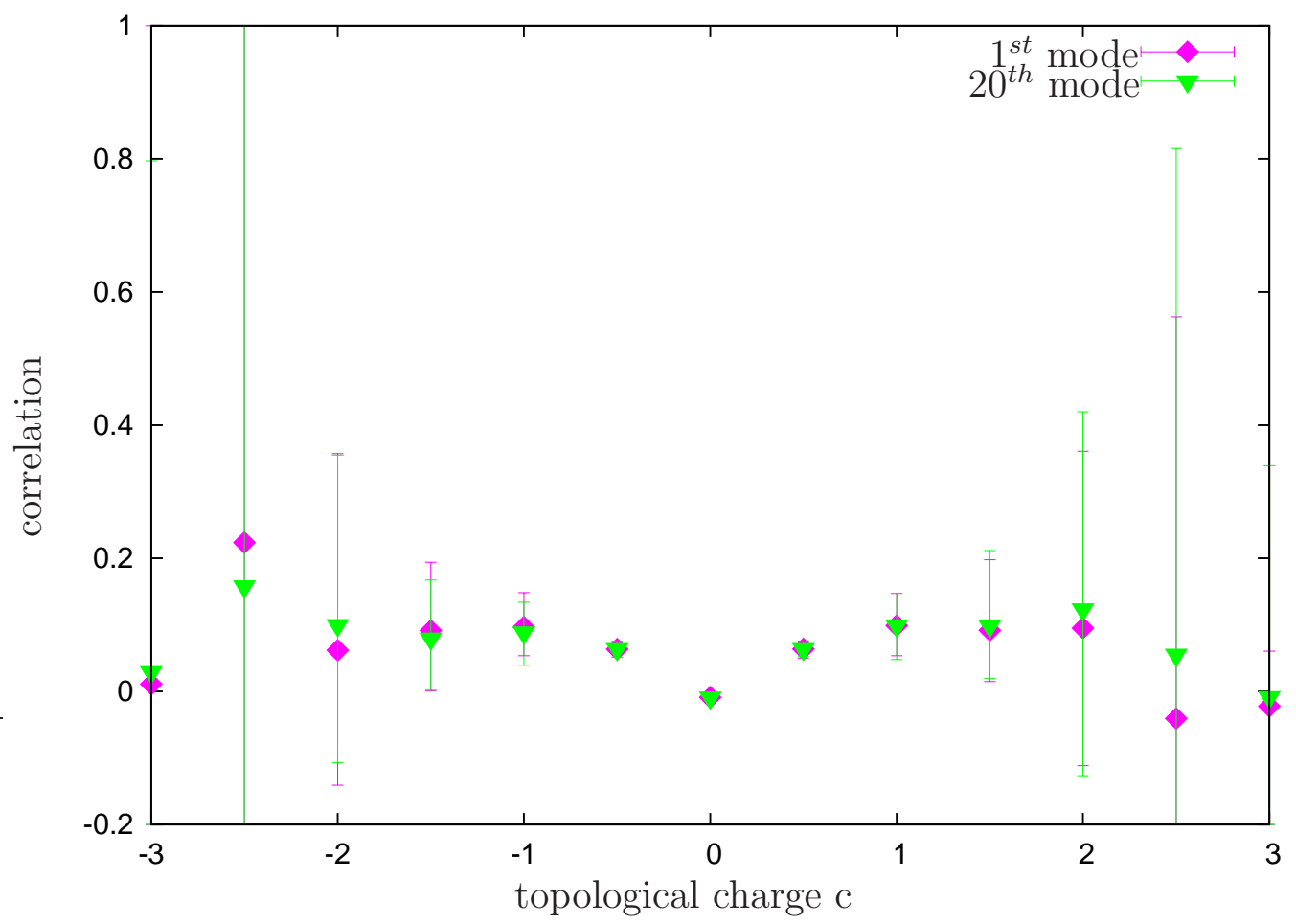

Figure 153: Correlation between topological charge and asqtad staggered eigenmodes on a $20^{4}$ lattice at $\beta=2.9$, a) full and b) projected configuration. 
a)

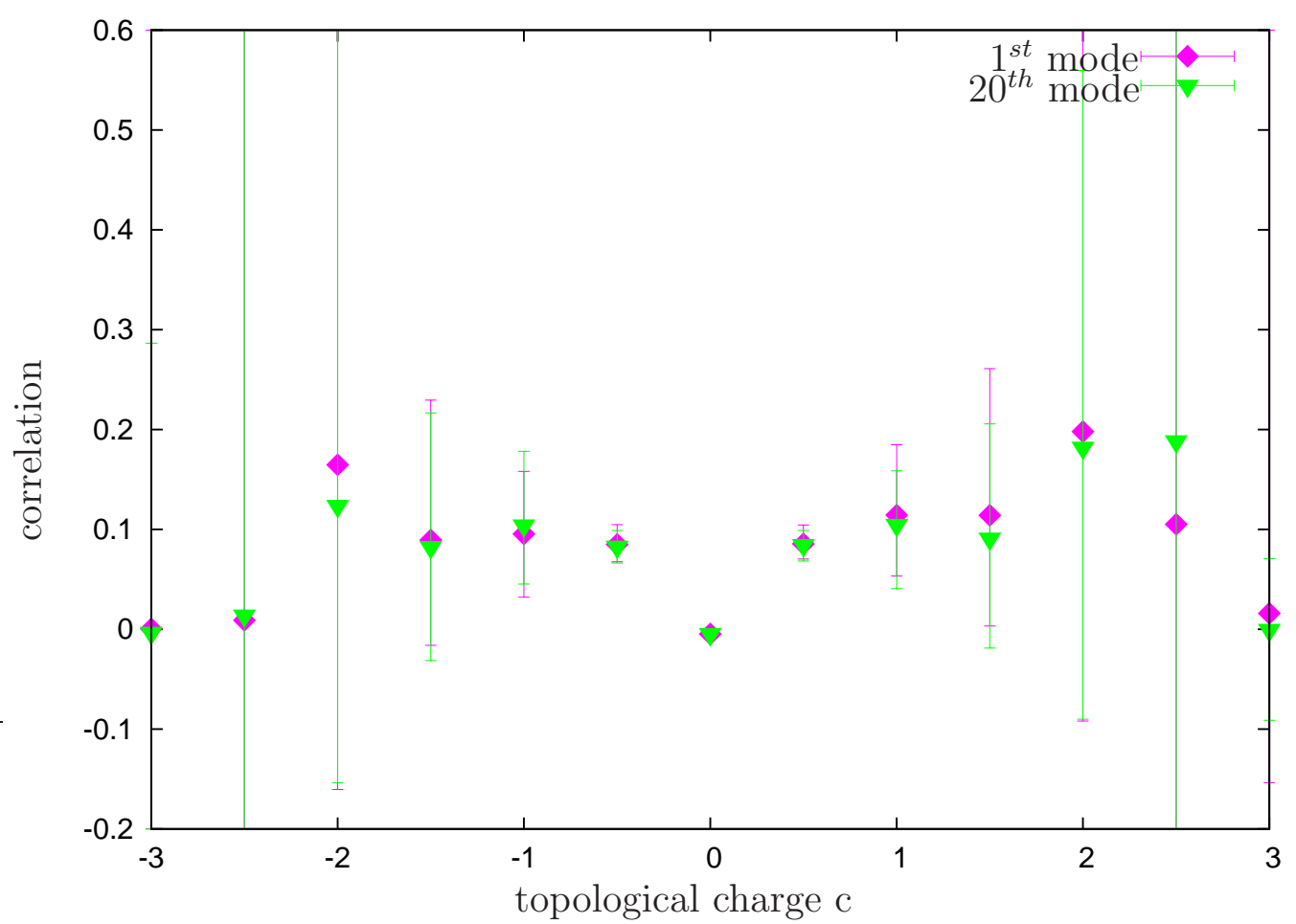

b)

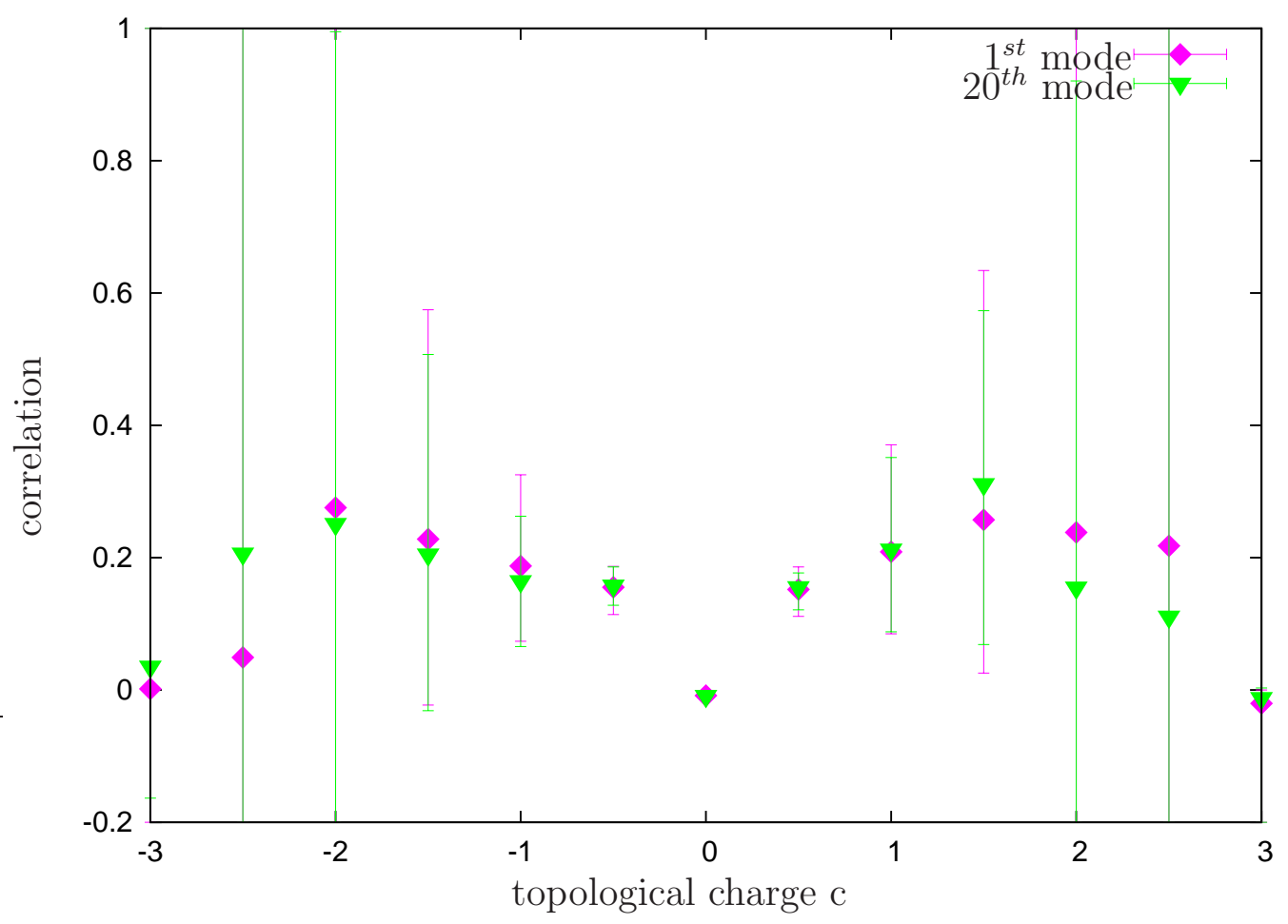

Figure 154: Correlation between topological charge and asqtad staggered eigenmodes on a $20^{4}$ lattice at $\beta=3.1$, a) full and b) projected configuration. 
a)

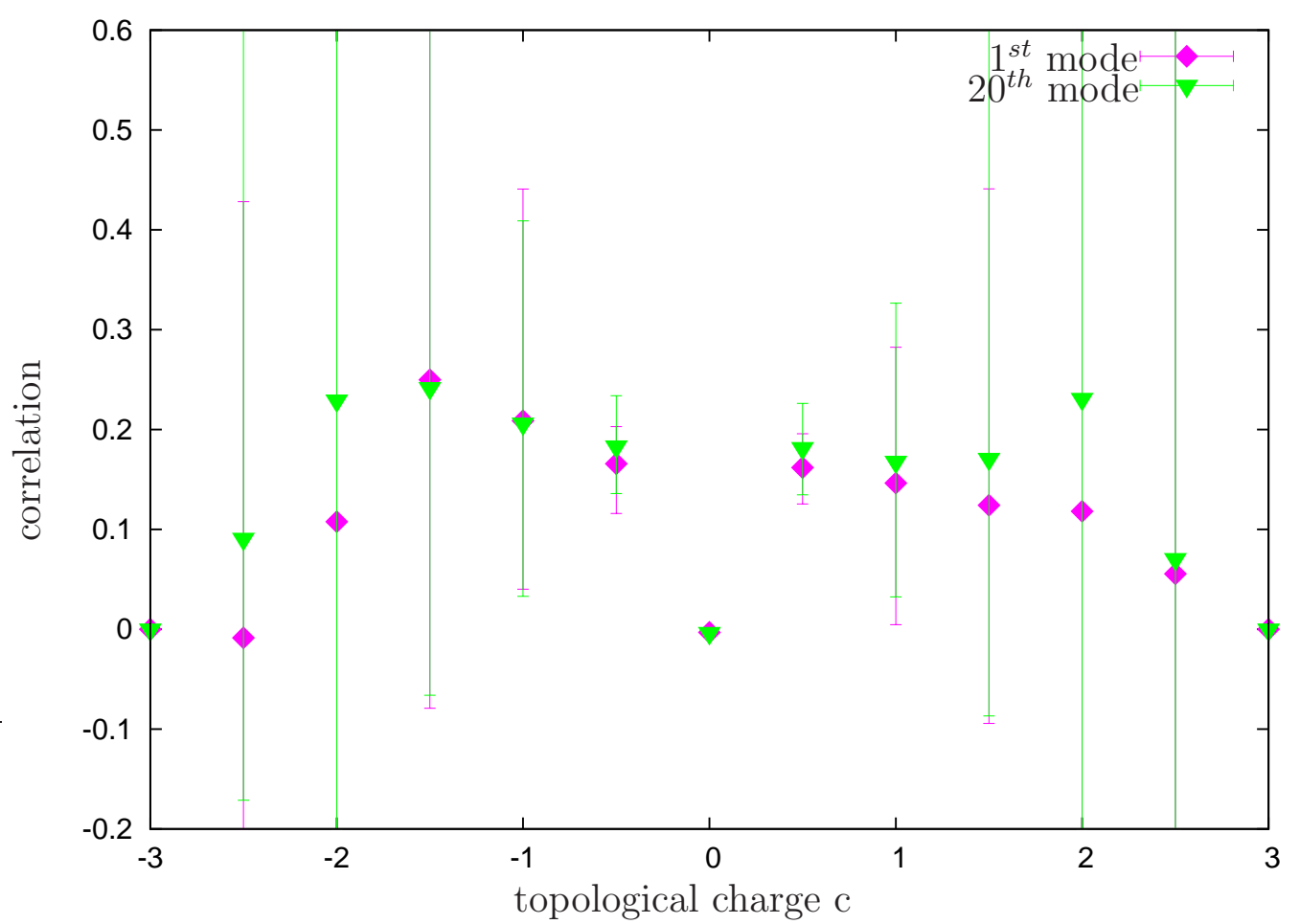

b)

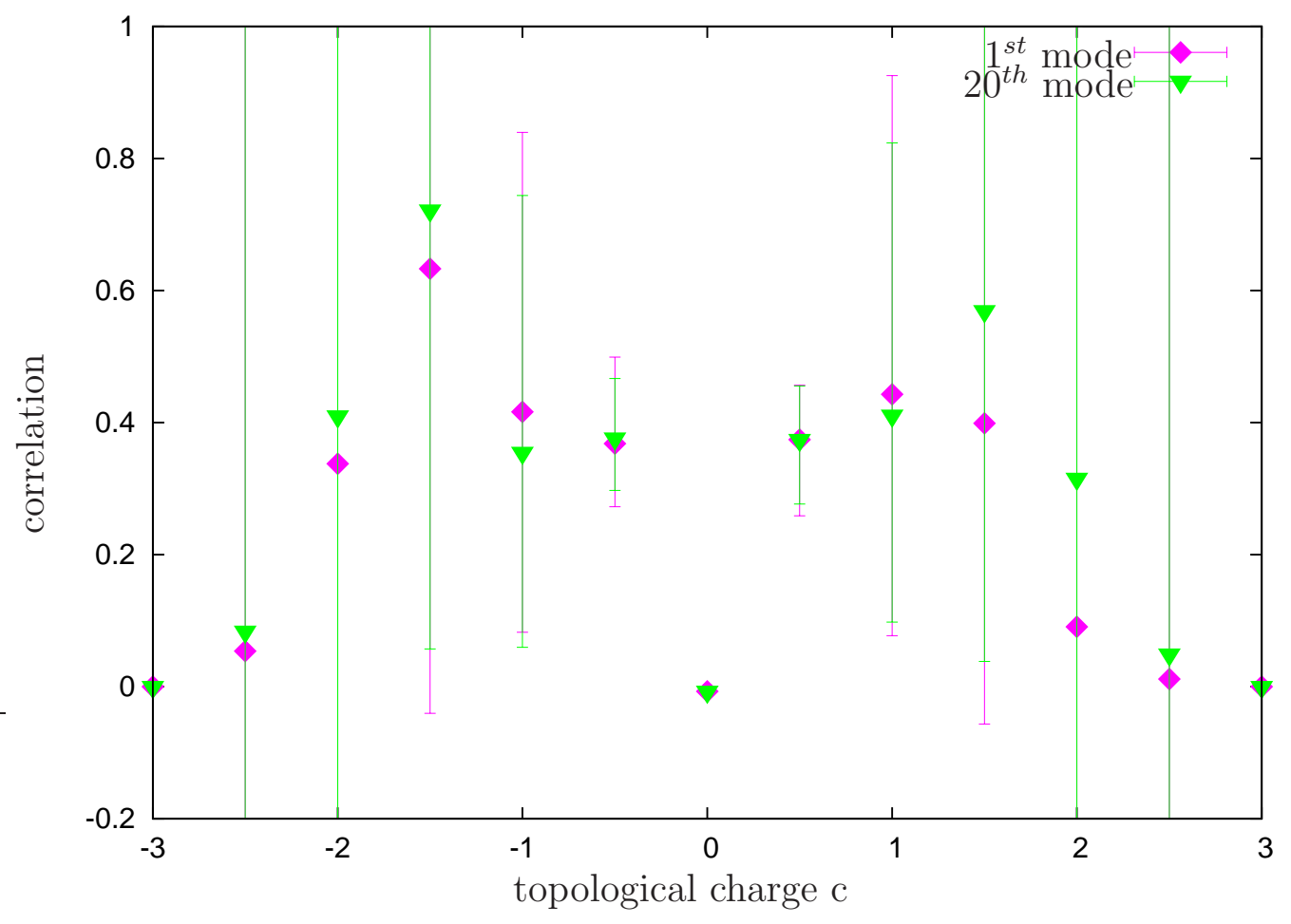

Figure 155: Correlation between topological charge and asqtad staggered eigenmodes on a $20^{4}$ lattice at $\beta=3.3$, a) full and b) projected configuration. 


\section{$C$ Plots and Figures}

a)

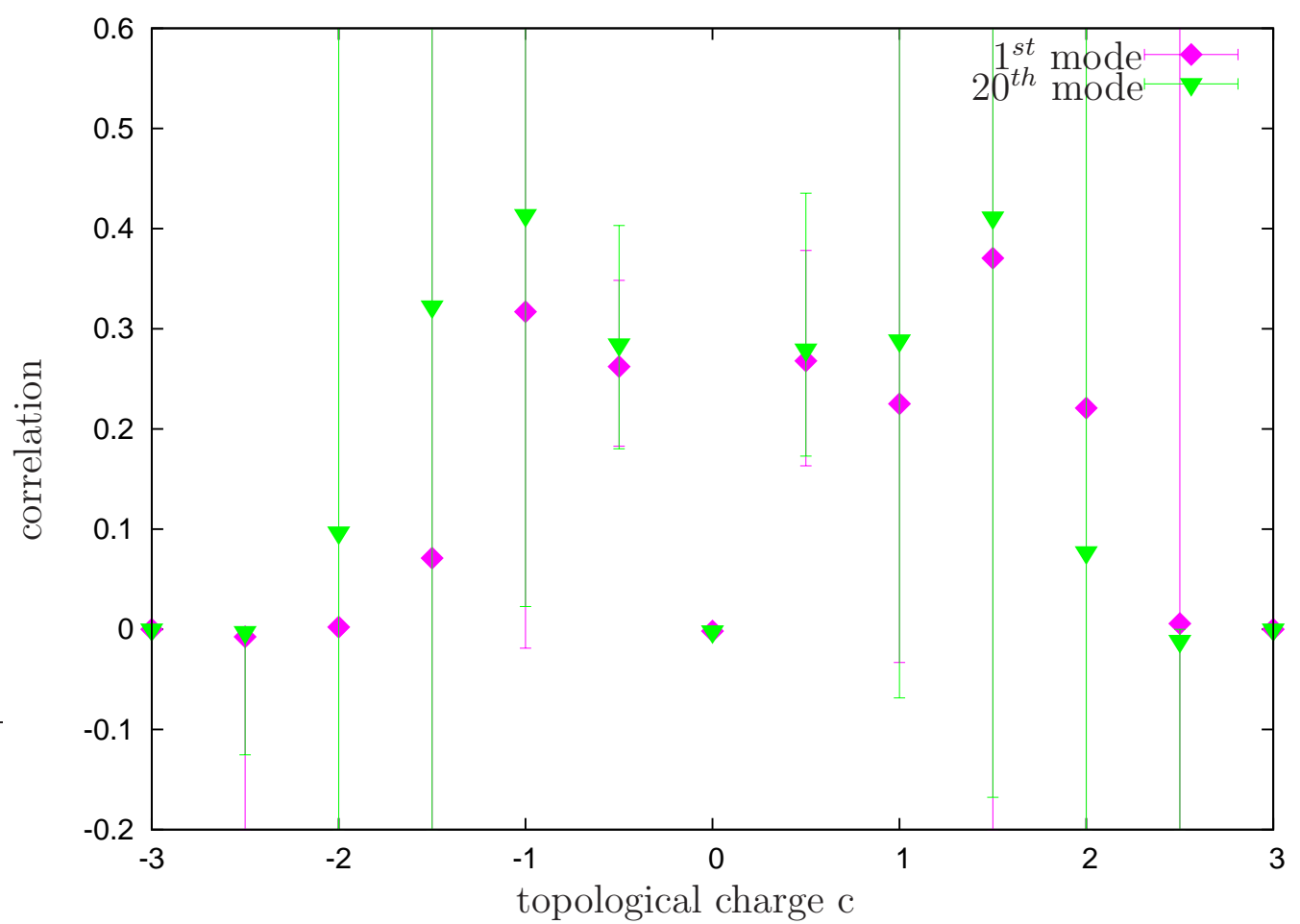

b)

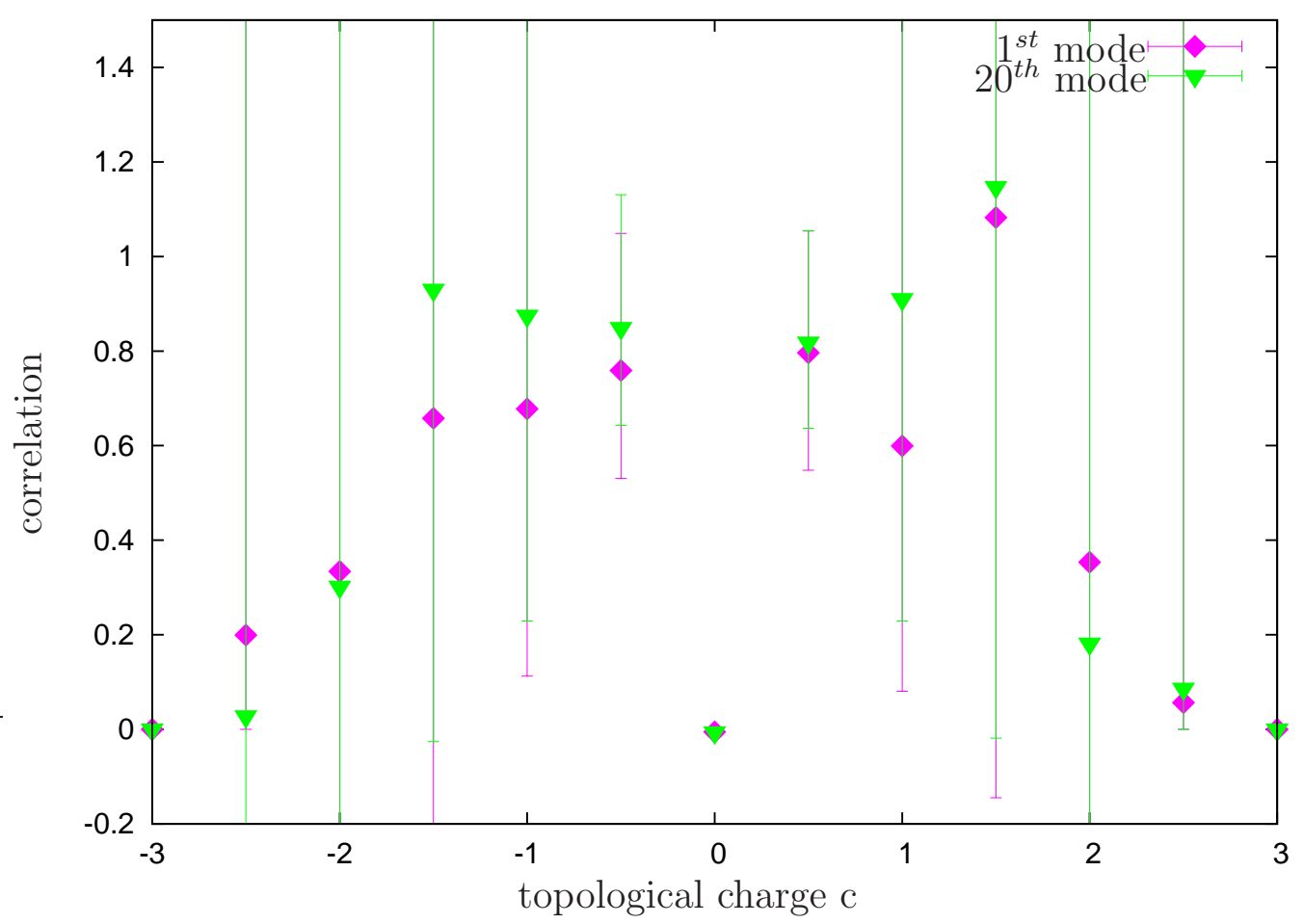

Figure 156: Correlation between topological charge and asqtad staggered eigenmodes on a $20^{4}$ lattice at $\beta=3.5$, a) full and b) projected configuration. 


\section{$C$ Plots and Figures}

a)

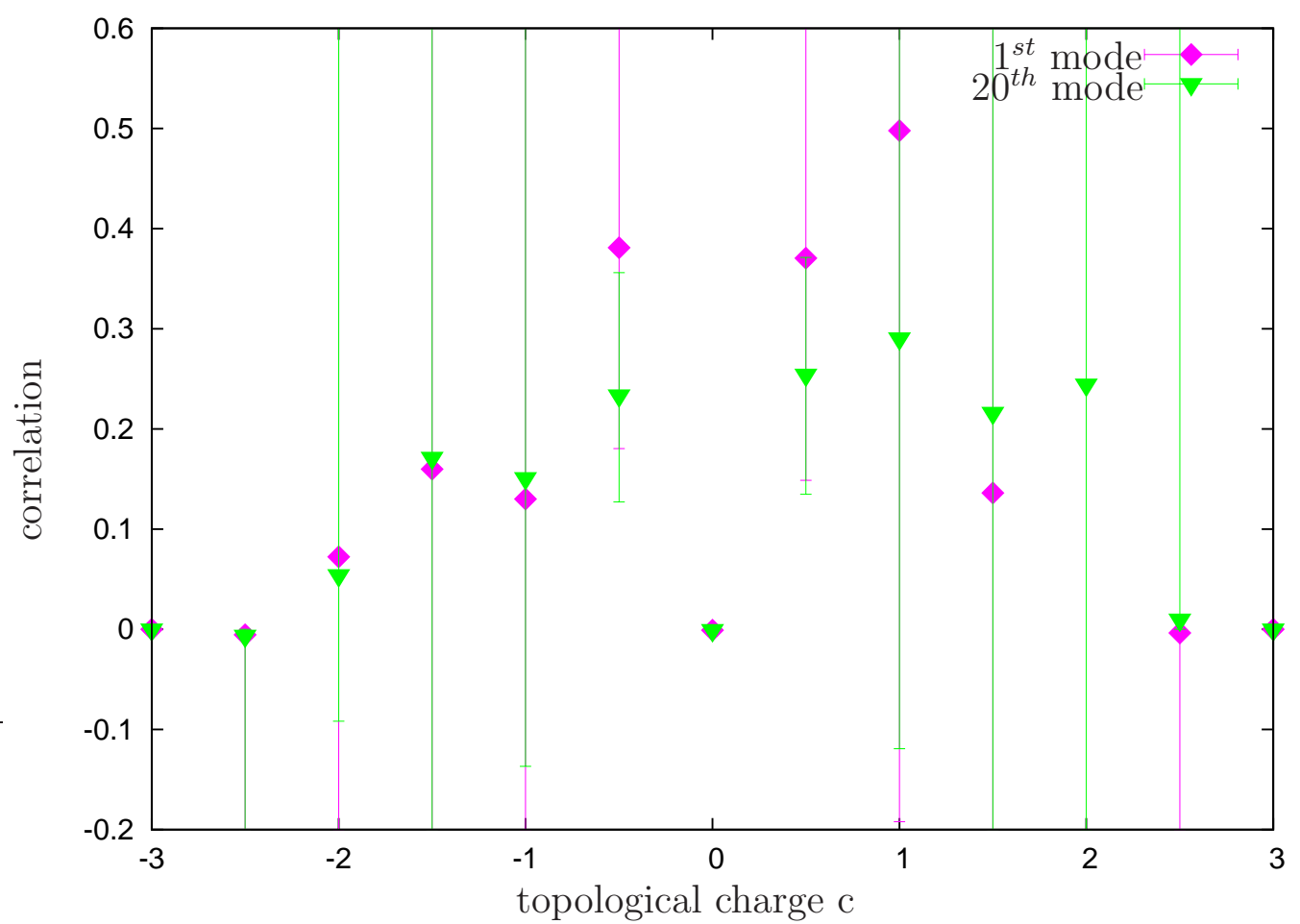

b)

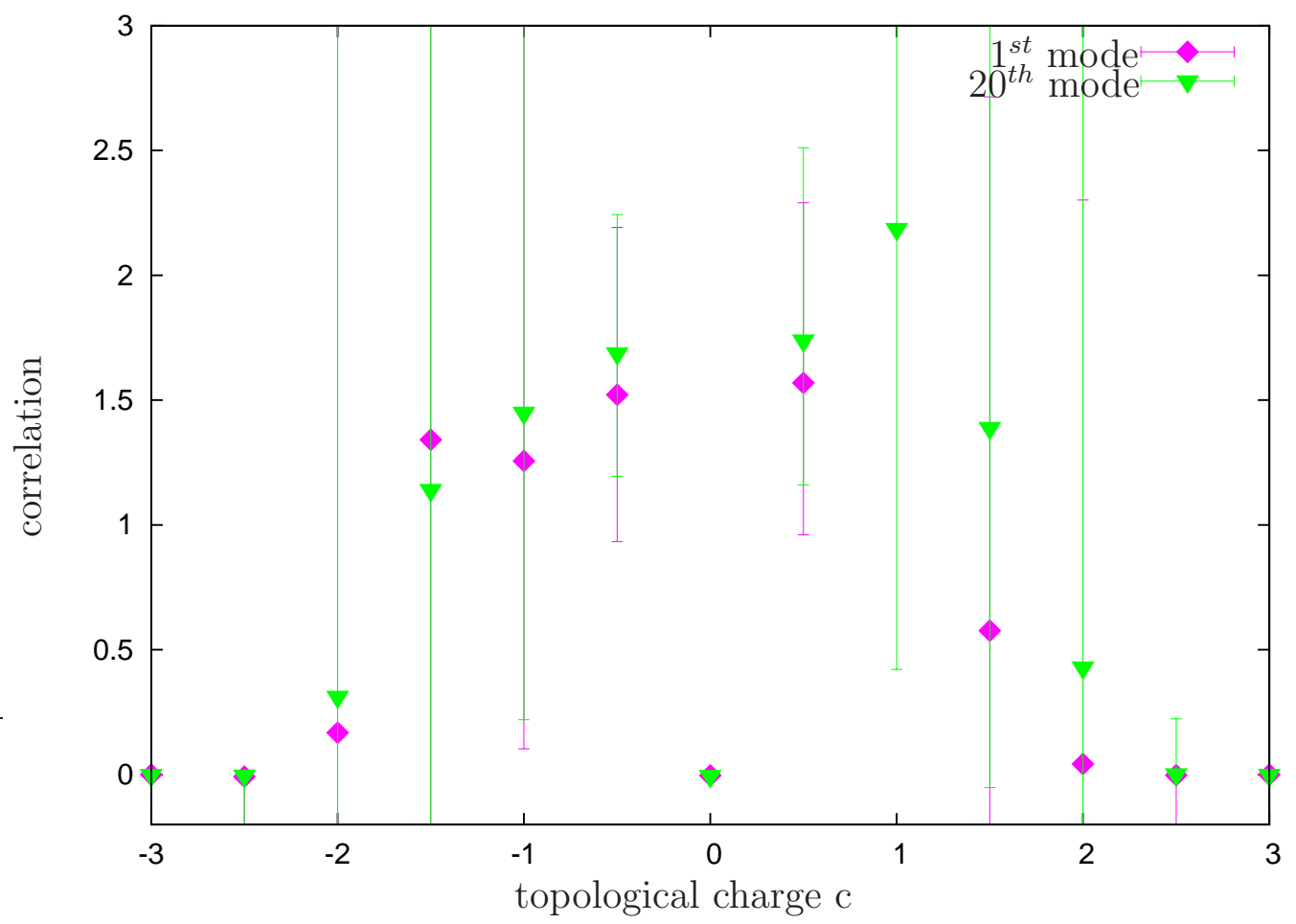

Figure 157: Correlation between topological charge and asqtad staggered eigenmodes on a $20^{4}$ lattice at $\beta=3.7$, a) full and b) projected configuration. 
a)

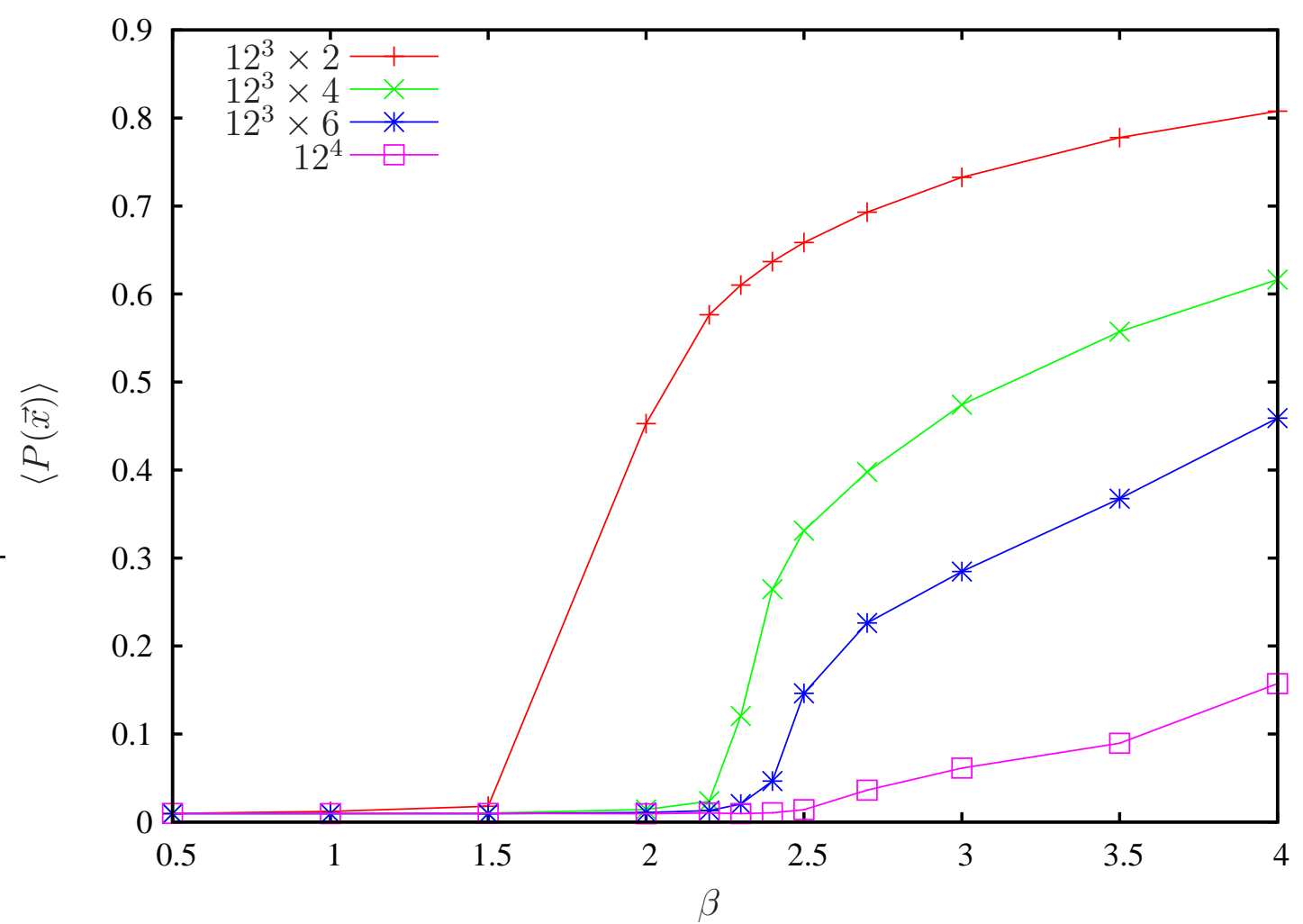

b)

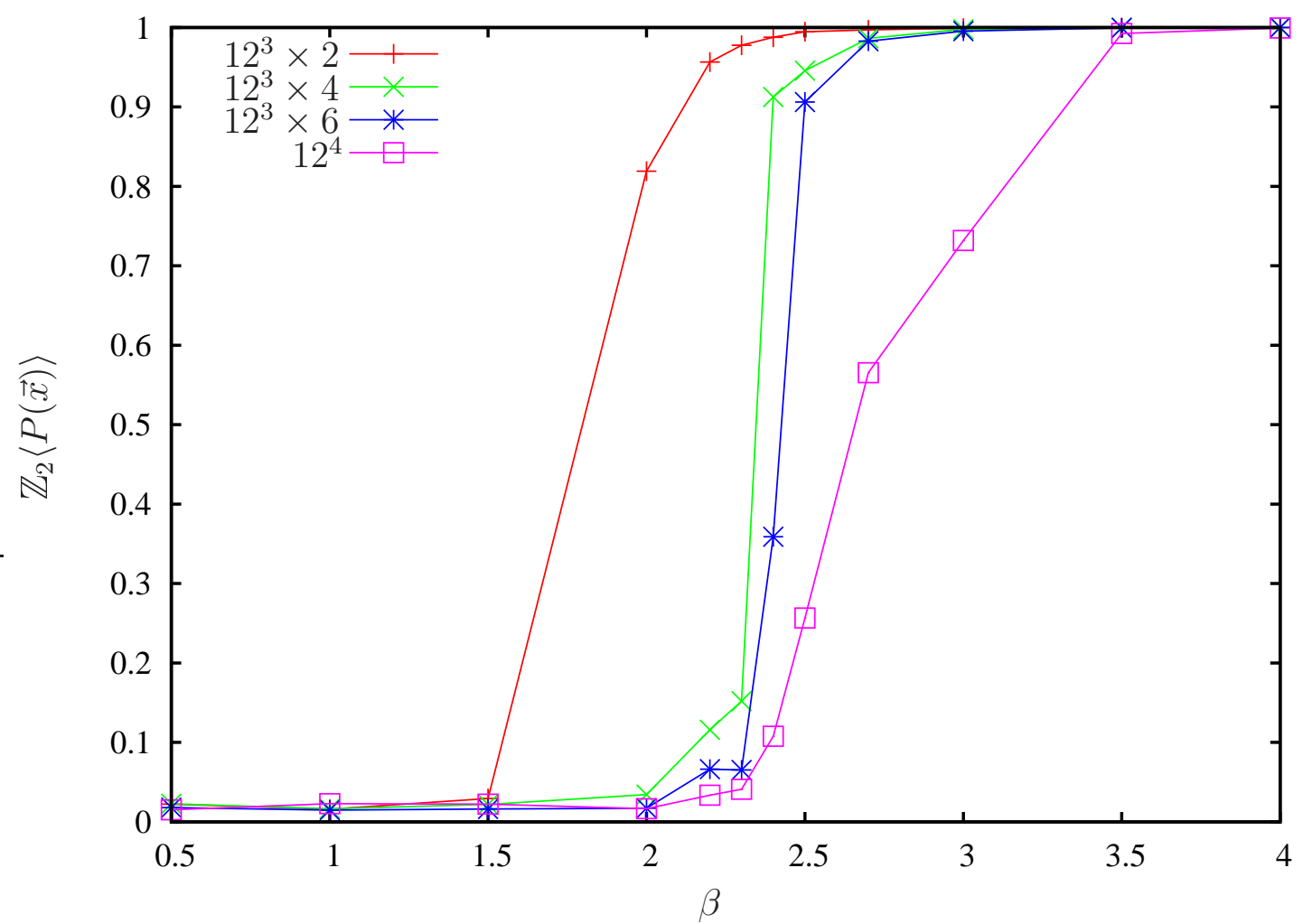

Figure 158: Polyakov loop phase transition of a) full and b) center-projected configurations for different lattice sizes with Wilson action. 
a)

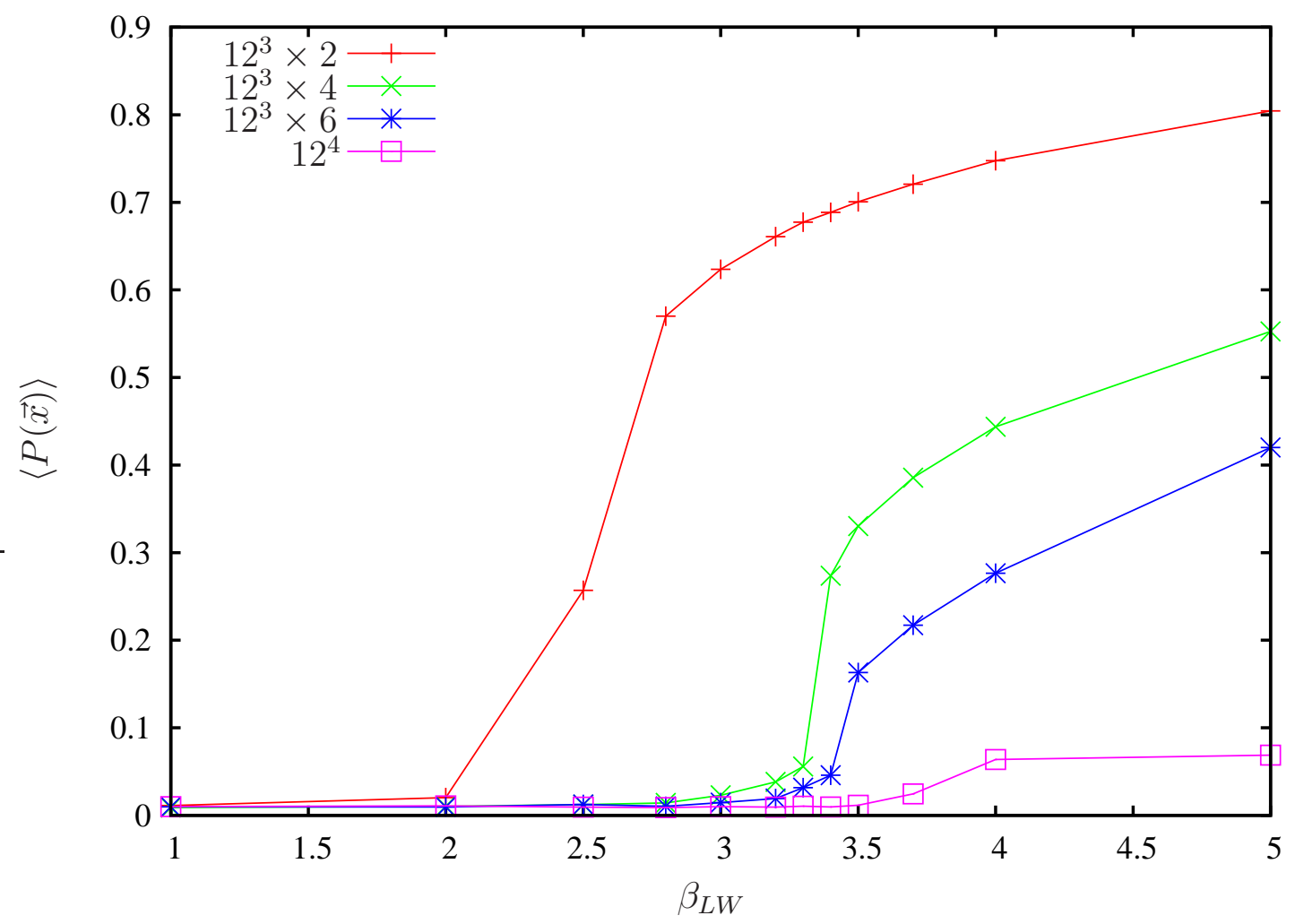

b)

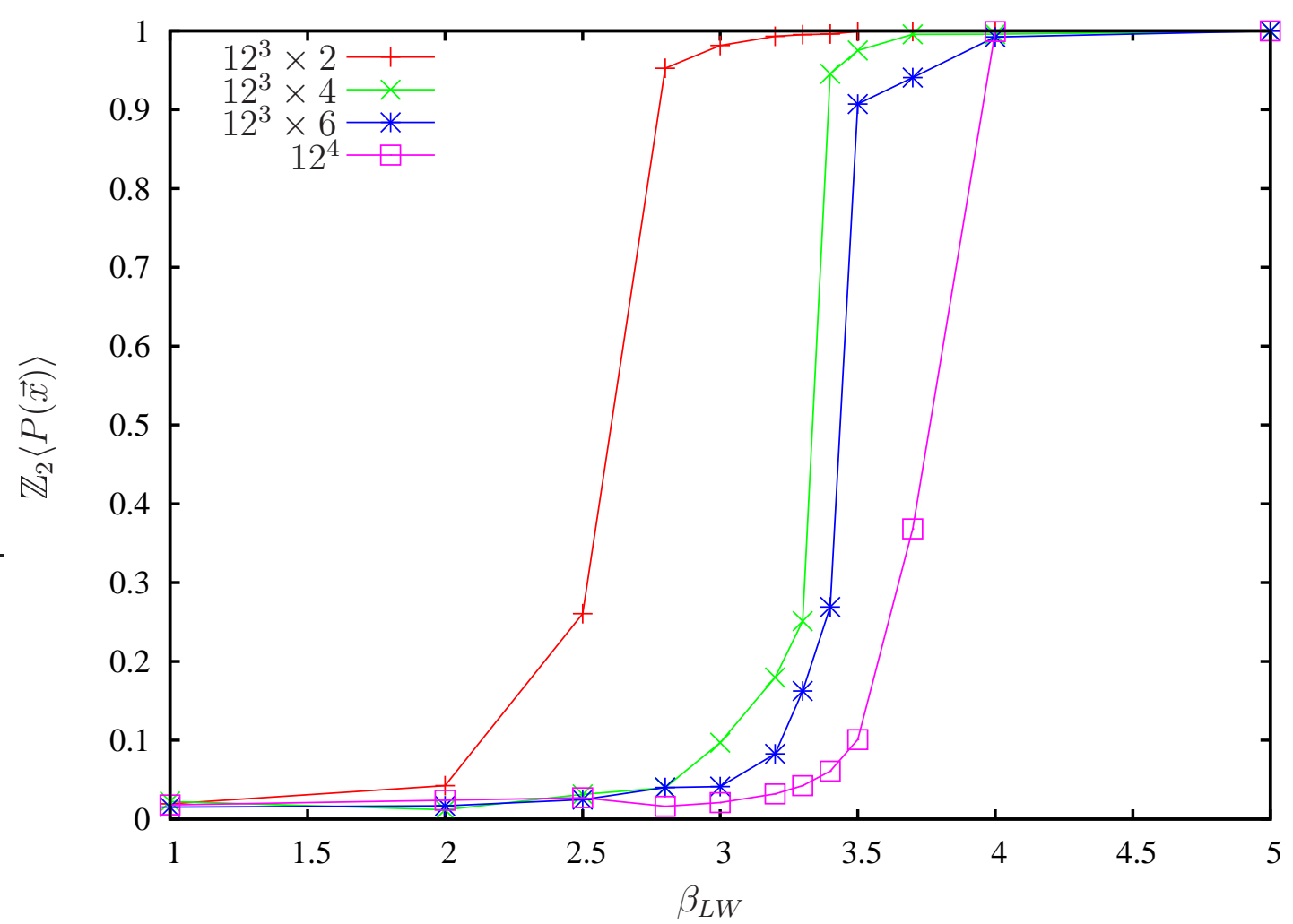

Figure 159: Polyakov loop phase transition of a) full and b) center-projected configurations for different lattice sizes with Lüscher-Weisz action. 
$C$ Plots and Figures

$12^{3} \times 2$

$12^{3} \times 4$

$12^{3} \times 6$

$12^{4}$
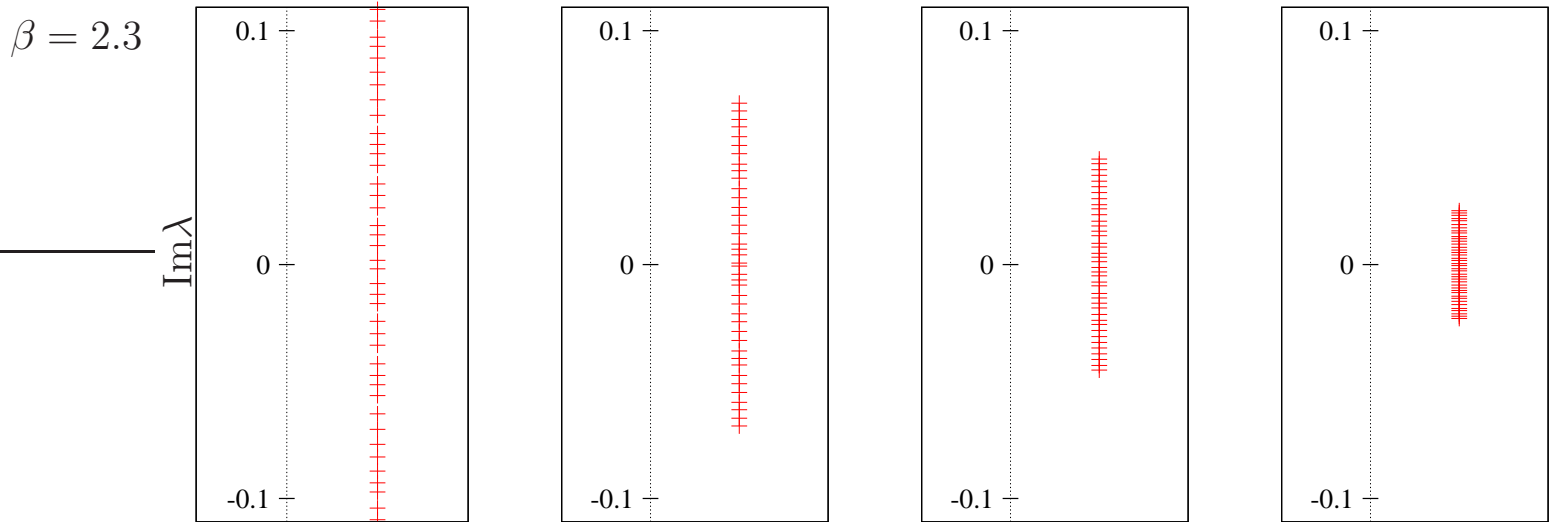

$\beta=2.5$
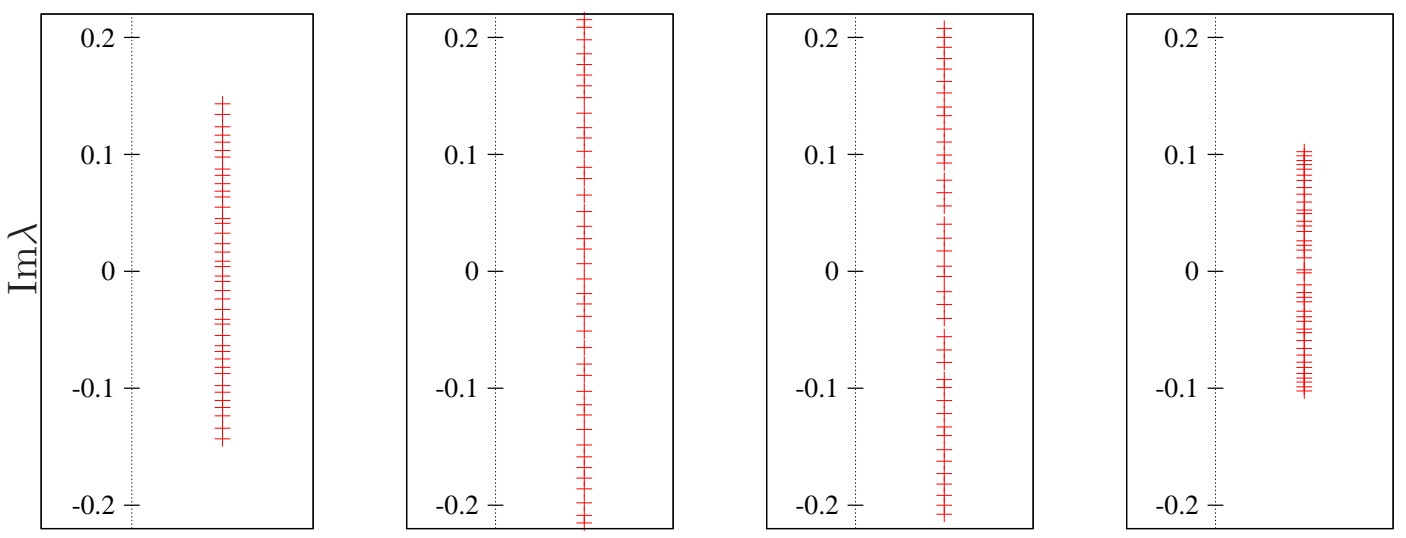

$\beta=2.7$
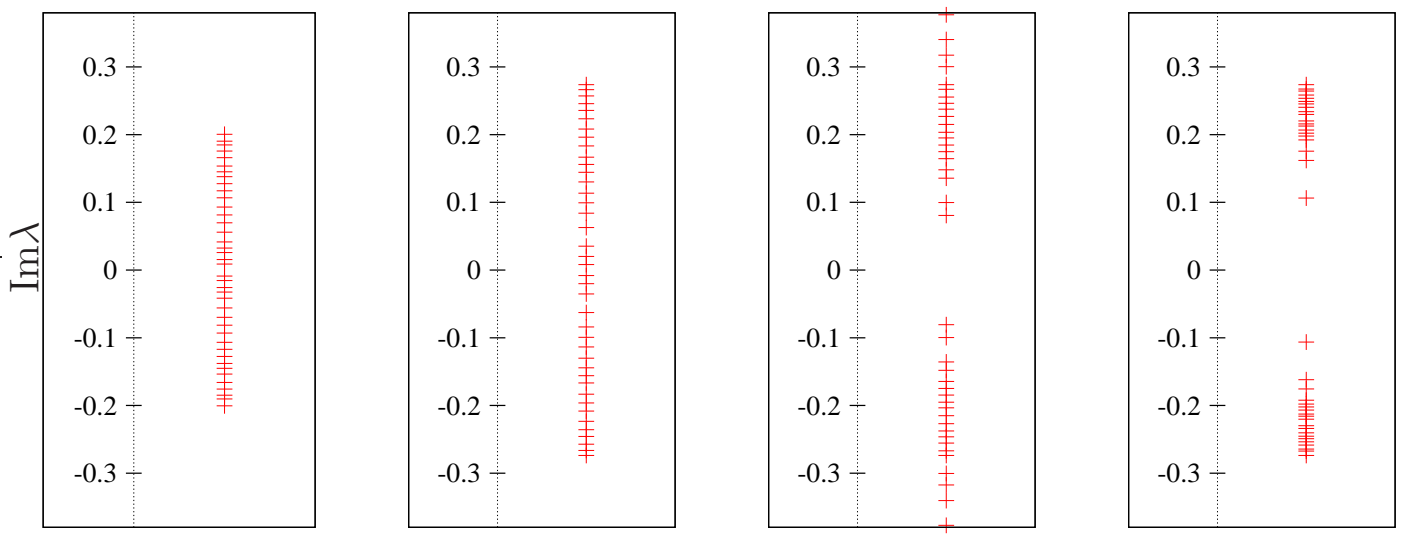

$\beta=3.0$
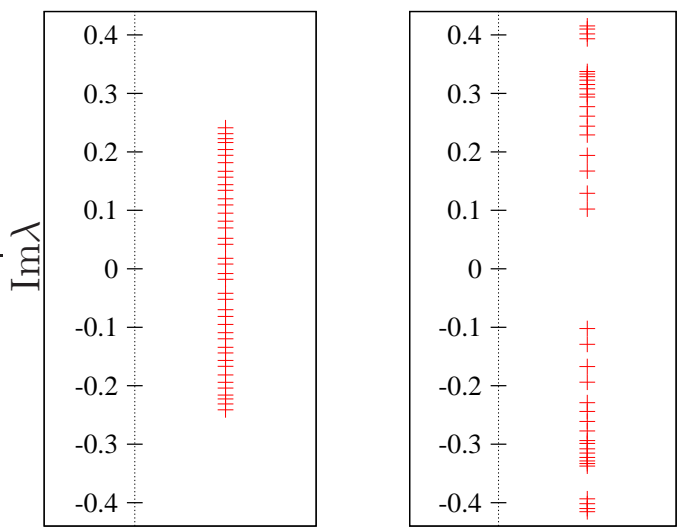

\begin{tabular}{|c|c|}
\hline 0.4 & 丰 \\
\hline $0.3 \div$ & 拝 \\
\hline $0.2 \div$ & 麦 \\
\hline $0.1-$ & \\
\hline 0 & \\
\hline-0.1 & \\
\hline-0.2 & 青 \\
\hline-0.3 & 表 \\
\hline-0.4 & \\
\hline
\end{tabular}

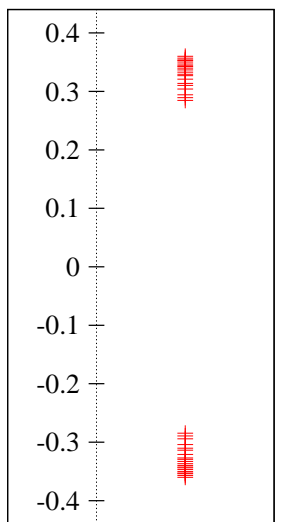

Figure 160: 20 lowest asqtad eigenvalues on center-projected configurations with Wilson action, periodic bc. 
$C$ Plots and Figures

$12^{3} \times 2$

$\beta=2.3$

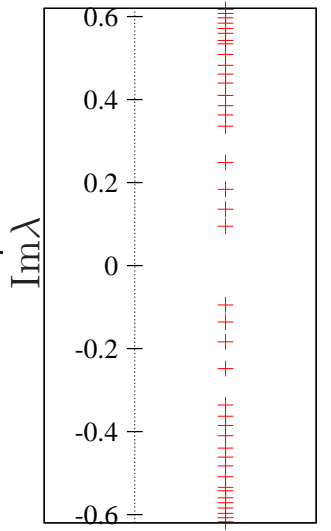

$\beta=2.5$

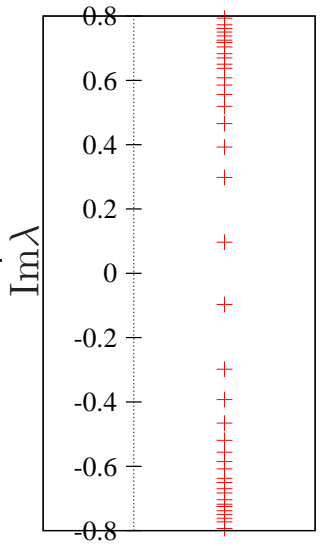

$\beta=2.7$

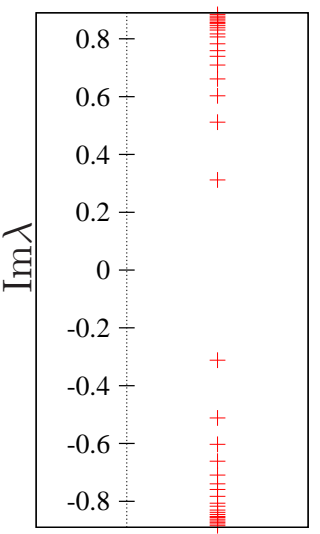

$\beta=3.0$

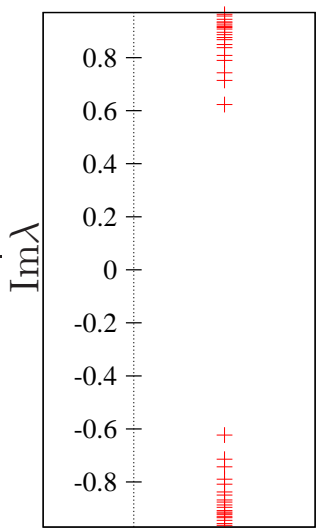

$12^{3} \times 4$
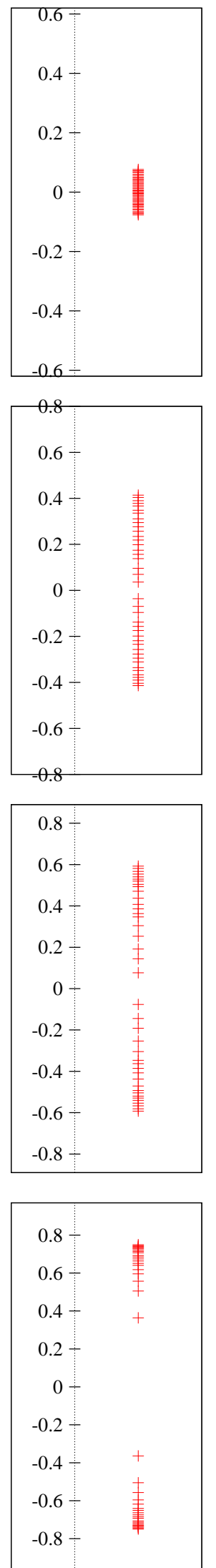

$12^{3} \times 6$
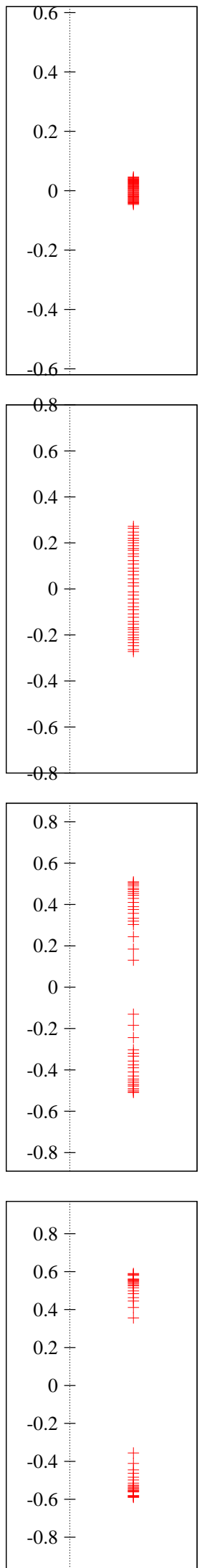

$12^{4}$
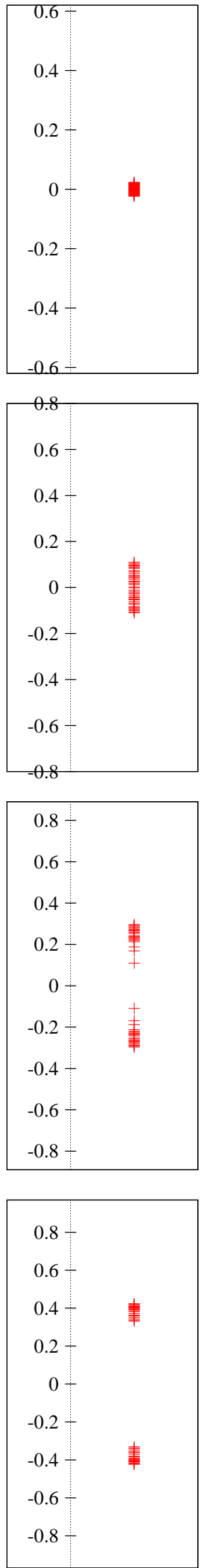

Figure 161: 20 lowest asqtad eigenvalues on center-projected configurations with Wilson action, anti periodic bc. 
$C$ Plots and Figures

$12^{3} \times 2$

$\beta=3.3$

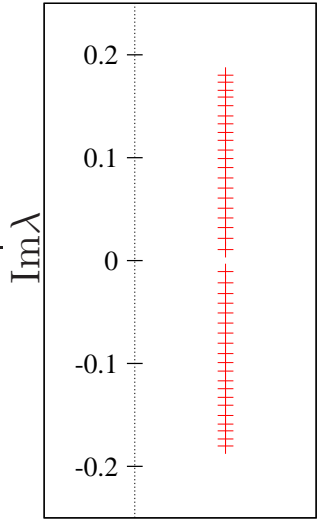

$\beta=3.5$

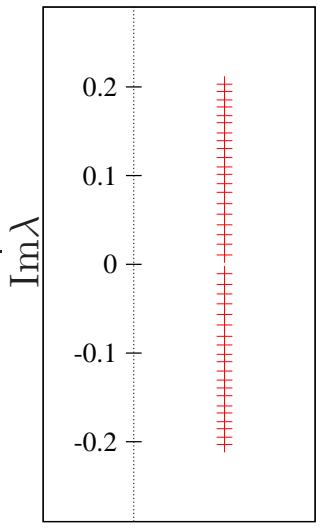

$\beta=3.7$

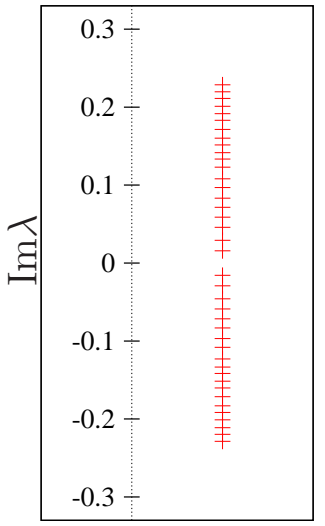

$\beta=4.0$

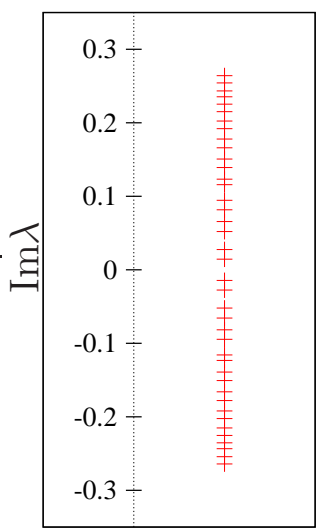

$12^{3} \times 4$
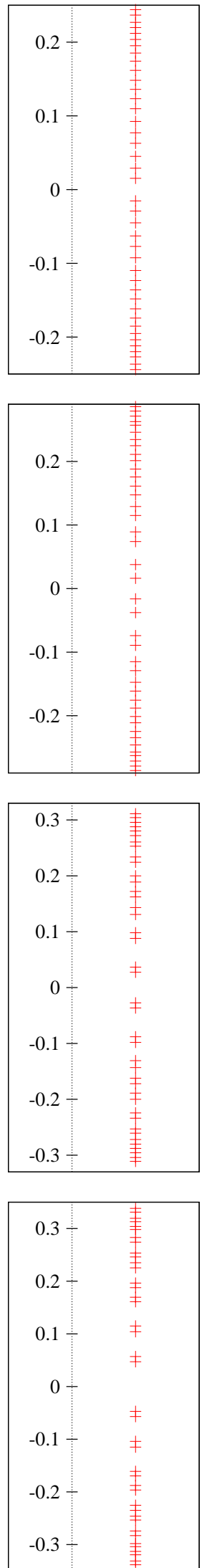

$12^{3} \times 6$
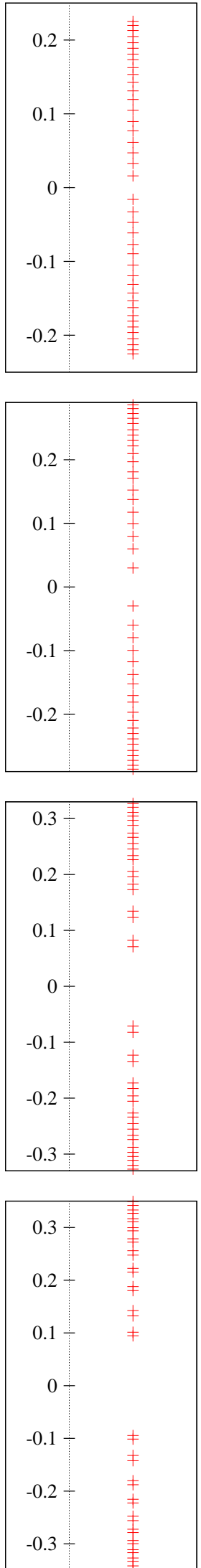

$12^{4}$
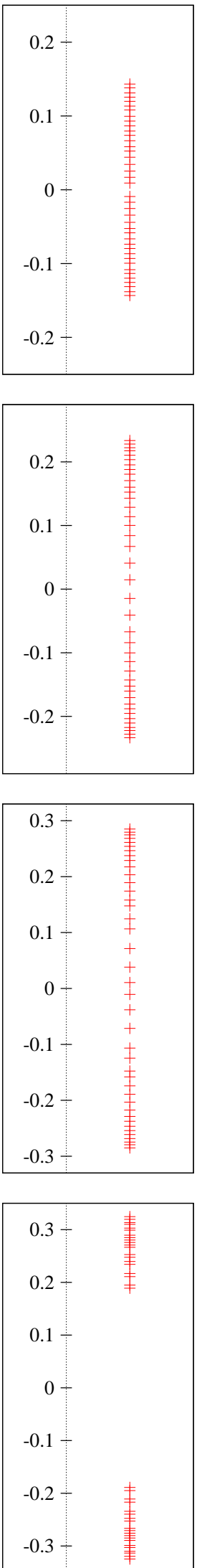

Figure 162: 20 lowest asqtad eigenvalues on full configurations with Lüscher-Weisz action, periodic bc. 
$C$ Plots and Figures

$12^{3} \times 2$

$\beta=3.3$

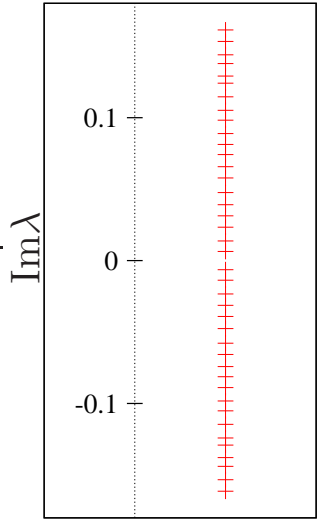

$\beta=3.5$

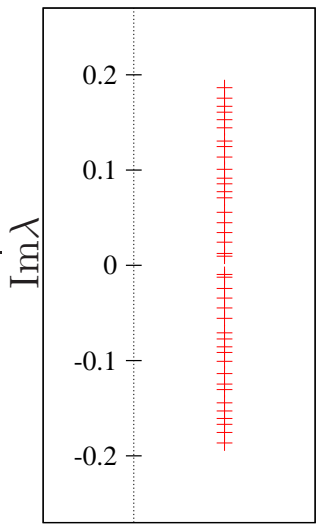

$\beta=3.7$

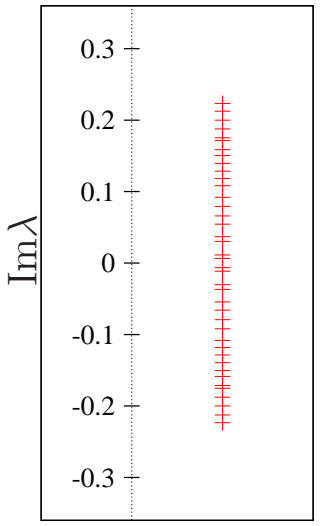

$\beta=4.0$

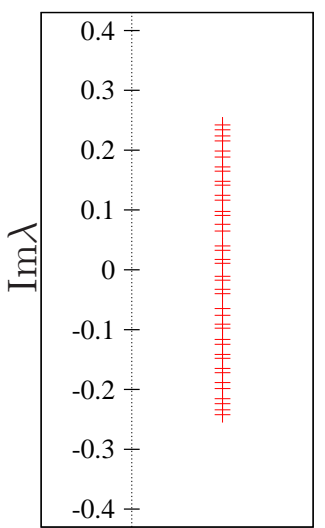

$12^{3} \times 4$
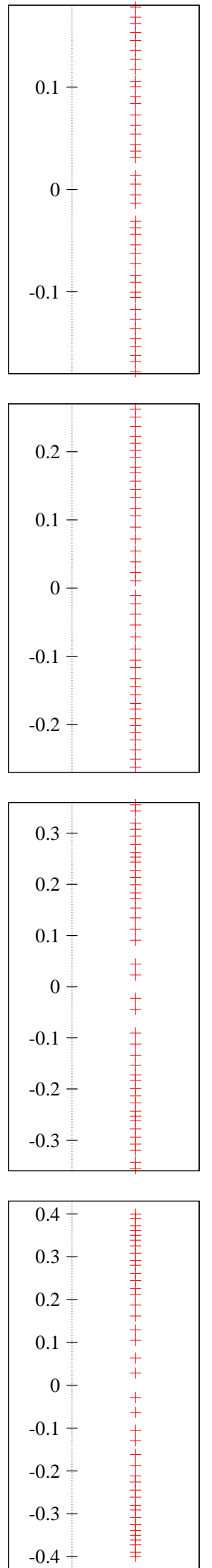

$12^{3} \times 6$
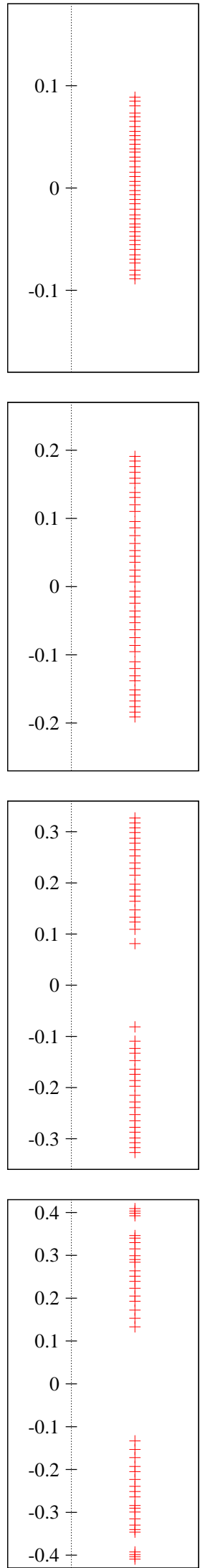

$12^{4}$
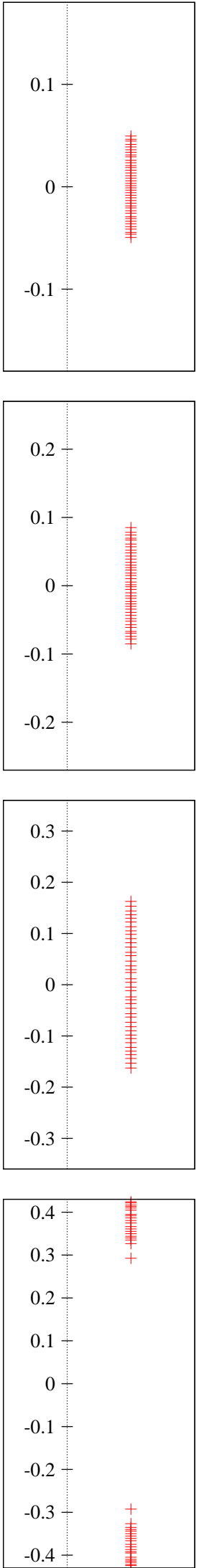

Figure 163: 20 lowest asqtad eigenvalues on center-projected configurations with Lüscher-Weisz action, pbc. 
$C$ Plots and Figures

$12^{3} \times 2$

$\beta=3.3$

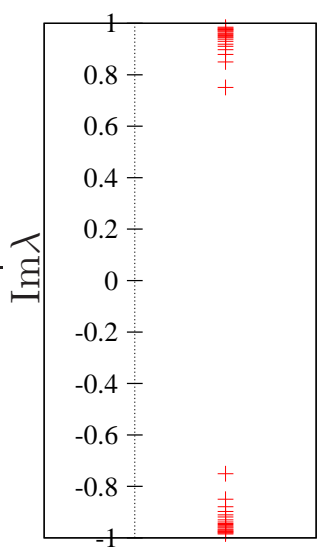

$\beta=3.5$

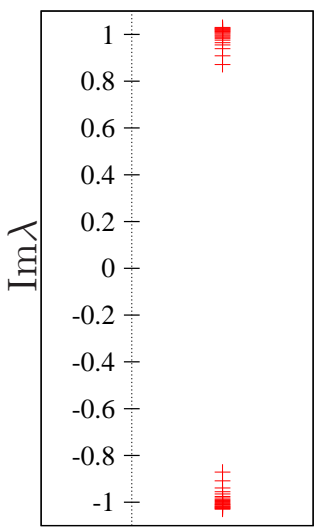

$\beta=3.7$

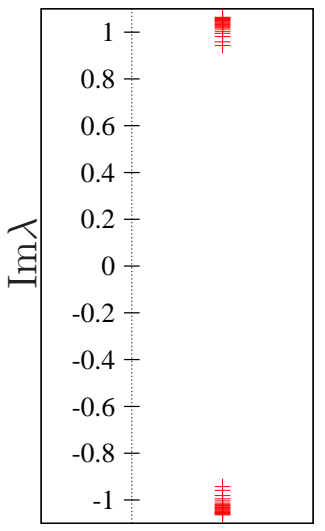

$\beta=4.0$

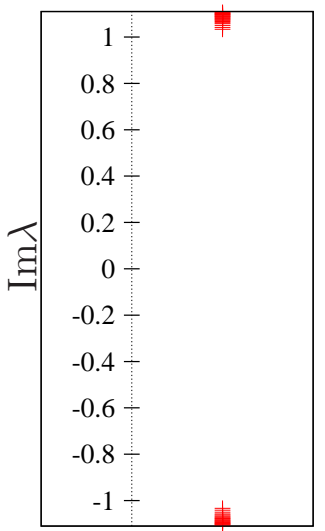

$12^{3} \times 4$
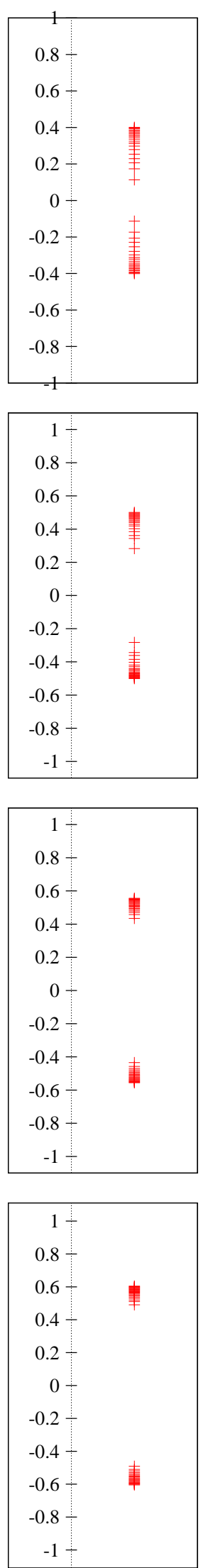

$12^{3} \times 6$
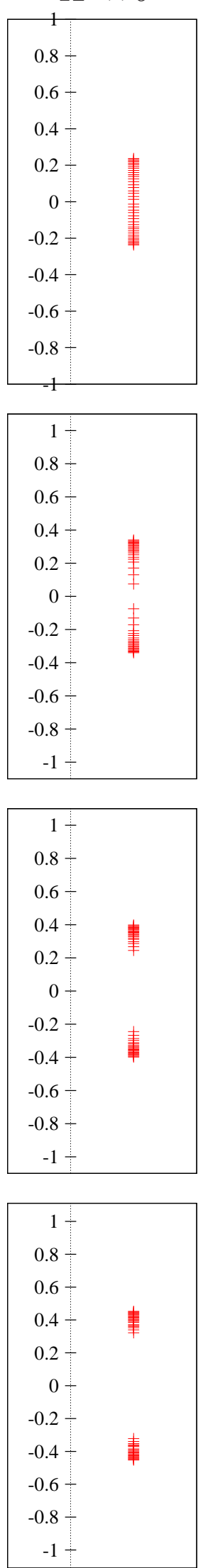

$12^{4}$
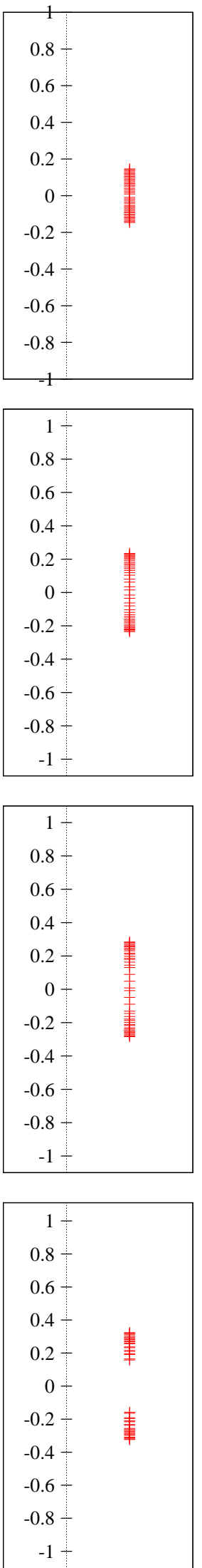

Figure 164: 20 lowest asqtad eigenvalues on full configurations with Lüscher-Weisz action, anti periodic bc. 
$C$ Plots and Figures

$12^{3} \times 2$

$\beta=3.3$

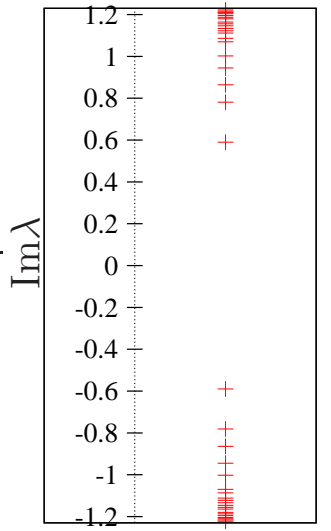

$\beta=3.5$

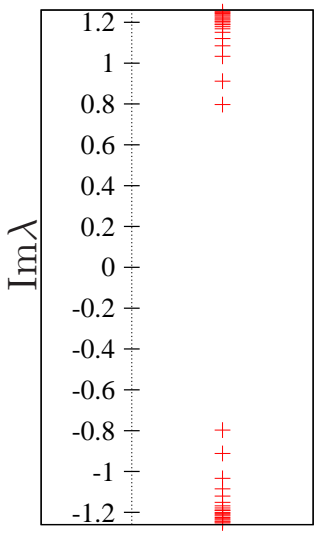

$\beta=3.7$

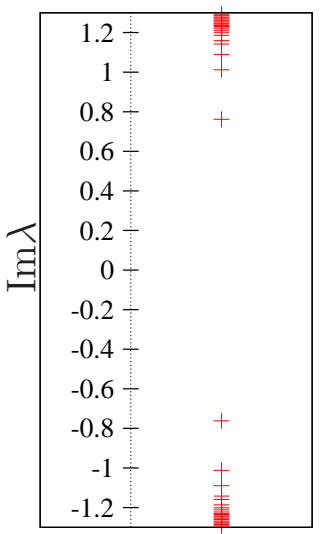

$\beta=4.0$

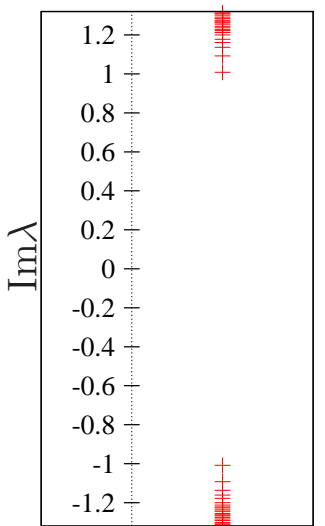

$12^{3} \times 4$
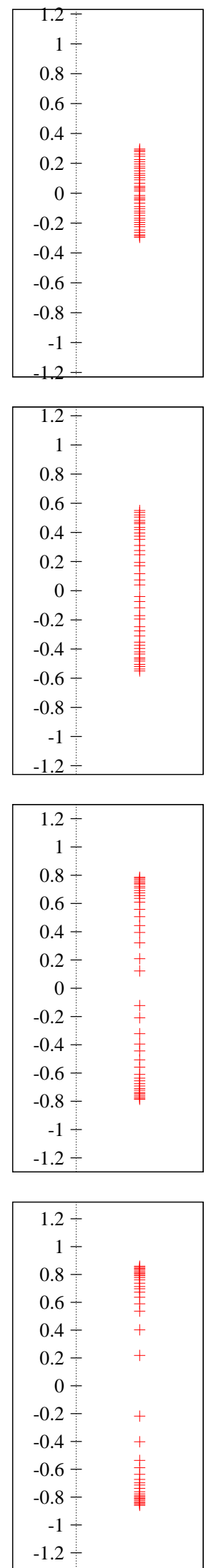

$12^{3} \times 6$
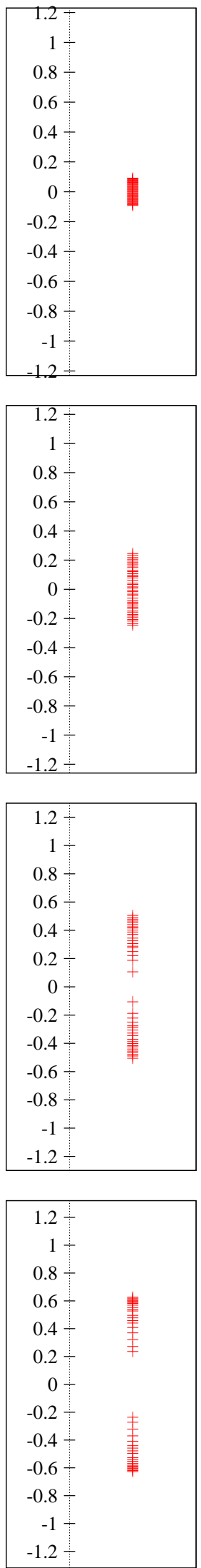

$12^{4}$
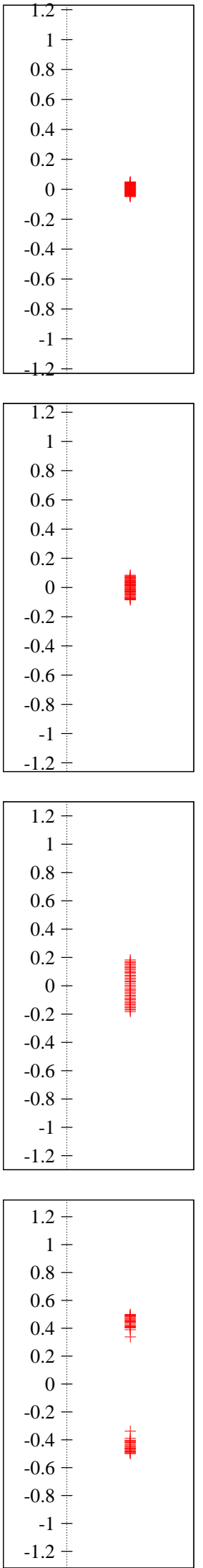

Figure 165: 20 lowest asqtad eigenvalues on center-projected configurations with Lüscher-Weisz action, apbc. 177 


\begin{tabular}{c|cccc} 
Correlation & Wilson action & anti periodic & Lüscher-Weisz & anti periodic \\
\hline$P(\vec{x}) /$ gap & 0.3417 & 0.1931 & -0.1326 & 0.3532 \\
$\mathbb{Z}_{2} P(\vec{x}) /$ gap & 5.0627 & 5.4658 & 1.5739 & 2.2587 \\
E-plaqs/gap & -0.1024 & -0.1605 & -0.0749 & -0.1347 \\
B-plaqs/gap & -0.1614 & -0.2383 & -0.1419 & -0.2091 \\
P-plaqs/gap & -0.2638 & -0.3988 & -0.2169 & -0.3439 \\
$P(\vec{x}) /$ dens & -0.1945 & -0.2337 & -0.0986 & -0.2577 \\
$\mathbb{Z}_{2} P(\vec{x}) /$ dens & -4.7398 & -4.8173 & -2.0382 & -3.1190 \\
E-plaqs/dens & 0.0993 & 0.0915 & 0.0680 & 0.0771 \\
B-plaqs/dens & 0.1279 & 0.0877 & 0.0758 & 0.0724 \\
P-plaqs/dens & 0.2273 & 0.1792 & 0.1438 & 0.1496
\end{tabular}

Table 10: Correlations $\langle X Y\rangle-\langle X\rangle\langle Y\rangle$ between size of the gap or eigenvalue density near zero and Polyakov loop $\left(P(\vec{x})\right.$ or $\left.\mathbb{Z}_{2} P(\vec{x})\right)$, number of electric, magnetic or total number of vortex-plaquettes. Size of gap correlates with Polyakov loops, eigenvalue density near zero correlates with vortex densities.

\begin{tabular}{c|cccc}
$\beta_{(L W)}$ & $12^{3} \times 2$ & $12^{3} \times 4$ & $12^{3} \times 6$ & $12^{4}$ \\
\hline 2.3 & 0.02609 & 0.01048 & 0.00356 & 0.00021 \\
2.5 & -0.00184 & 0.02874 & 0.01526 & 0.02273 \\
2.7 & 0.02763 & 0.03182 & 0.14122 & 4.54309 \\
3.0 & -0.00125 & 0.01400 & 0.06222 & 0.54893 \\
3.3 & 0.05257 & 0.08881 & 0.01149 & -0.00275 \\
3.5 & 0.01319 & 0.08002 & 0.09454 & -0.00124 \\
3.7 & 0.03192 & 0.08010 & 0.51254 & 0.07197 \\
4.0 & 0.00396 & 0.01612 & 0.12191 & 1.08357
\end{tabular}

Table 11: Correlation between size of the gap and Polyakov loop of center-projected configurations $\left(\mathbb{Z}_{2} P(\vec{x})\right)$, different $\beta_{(L W)} \mathrm{S}(\beta: 2.3-3.0 \rightarrow$ Wilson action, Fig. 161, $\beta_{L W}: 3.3, \ldots \rightarrow$ Lüscher-Weisz action, Fig. 165) and lattice sizes, anti periodic bc.

\begin{tabular}{c|cccc}
$\beta_{(L W)}$ & $12^{3} \times 2$ & $12^{3} \times 4$ & $12^{3} \times 6$ & $12^{4}$ \\
\hline 2.3 & 0.01973 & -0.00322 & 0.00456 & 0.02492 \\
2.5 & 0.00458 & 0.01675 & 0.00483 & 0.08774 \\
2.7 & $-8.6 \cdot 10^{-05}$ & 0.00642 & 0.00312 & 0.04077 \\
3.0 & 0.00925 & 0.00441 & -0.00666 & 0.01020 \\
3.3 & -0.00089 & 0.03704 & 0.02166 & 0.02135 \\
3.5 & 0.00416 & -0.00108 & 0.04210 & 0.01865 \\
3.7 & 0.00475 & 0.00867 & 0.02664 & 0.03028 \\
4.0 & 0.00431 & 0.00016 & 0.00898 & 0.00964
\end{tabular}

Table 12: Correlation between eigenvalue density near zero and vortex-density, different $\beta_{(L W)} \mathrm{S}\left(\beta: 2.3-3.0 \rightarrow\right.$ Wilson action, Fig. $160, \beta_{L W}: 3.3-4.0 \rightarrow$ LüscherWeisz action, Fig. 163) and lattice sizes, periodic bc. 

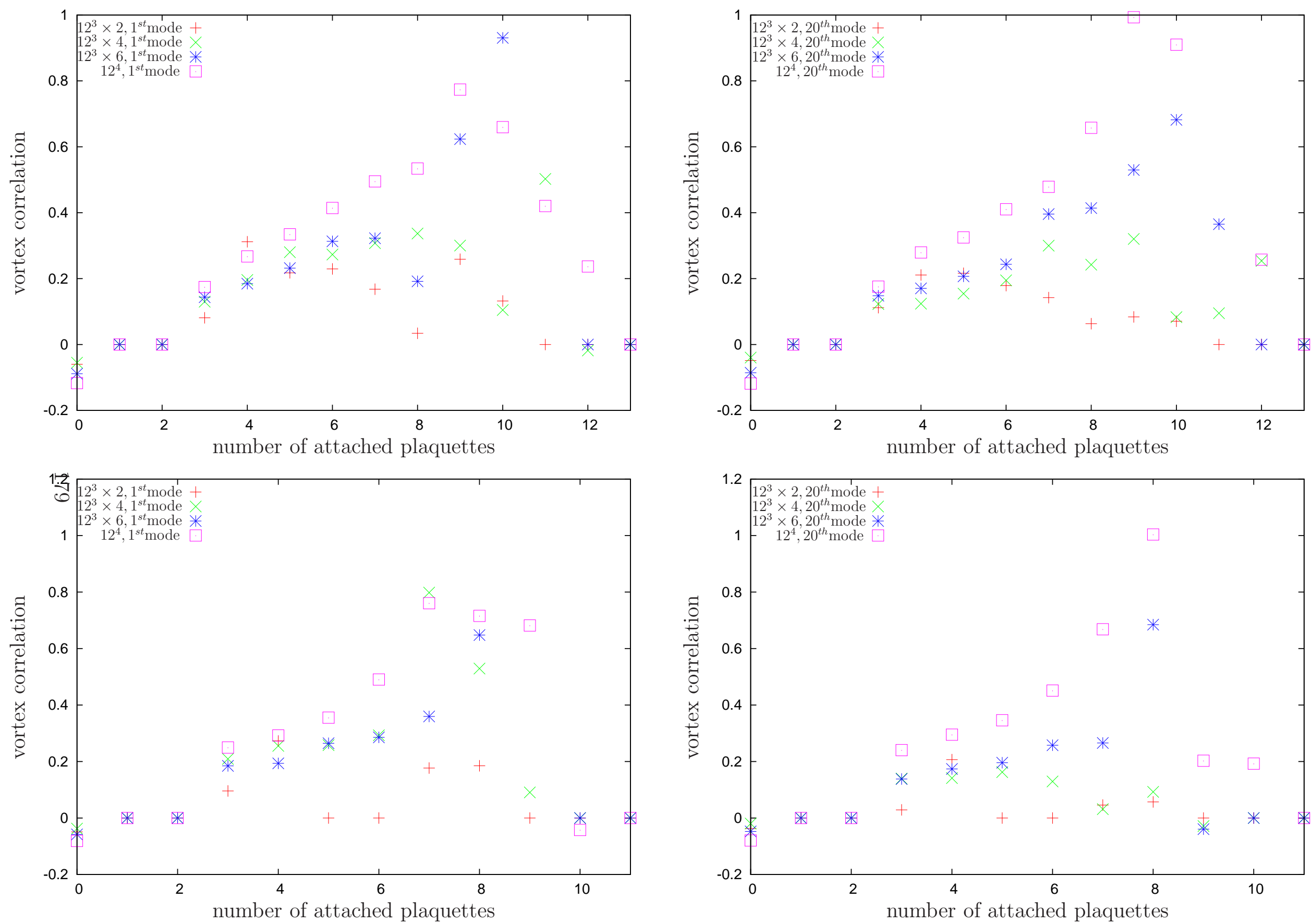

Figure 166: Correlation between eigenmodes and vortex surface, full configuration, $\beta_{L W}=3.3$ 

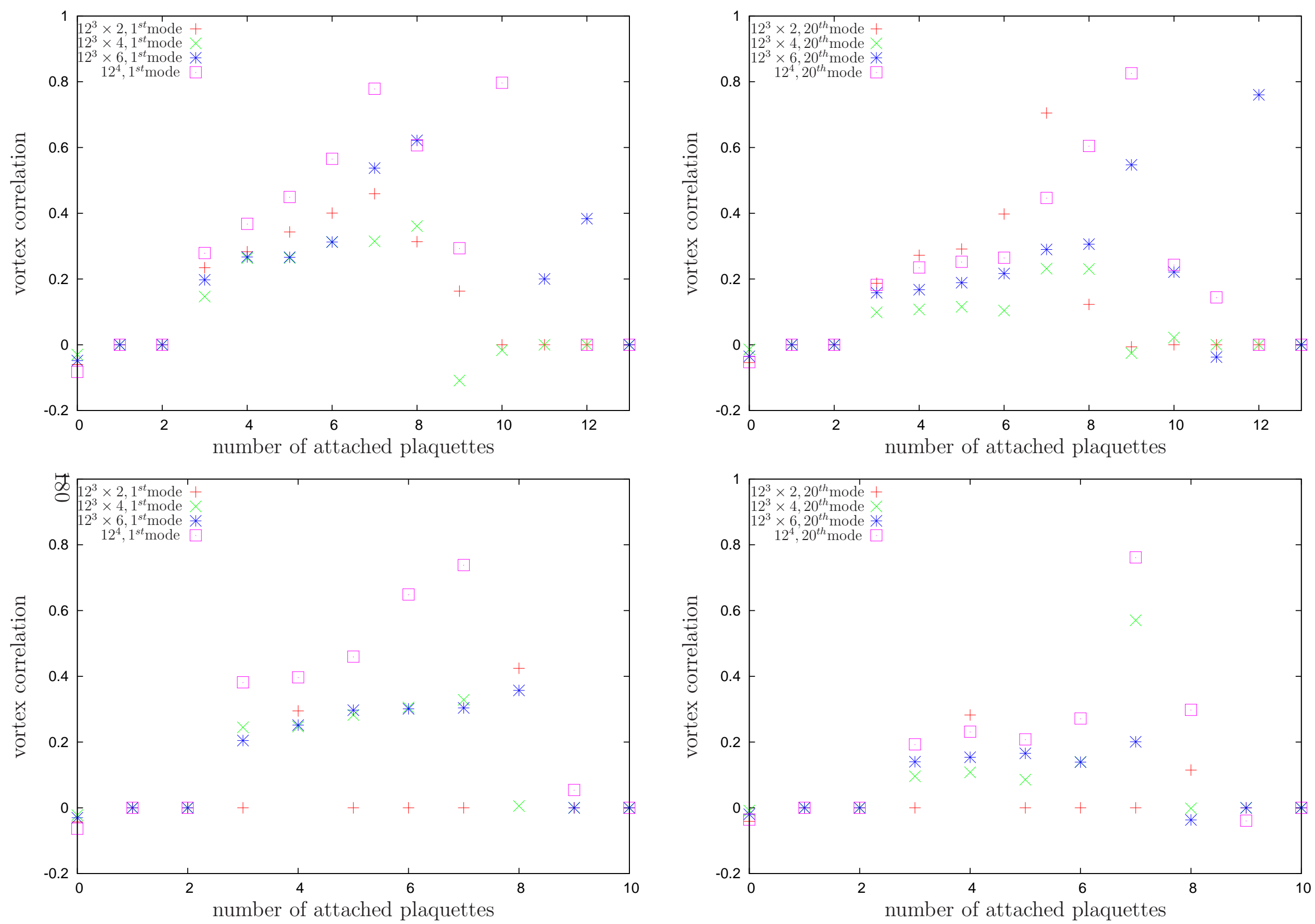

Figure 167: Correlation between eigenmodes and vortex surface, full configuration, $\beta_{L W}=3.5$ 

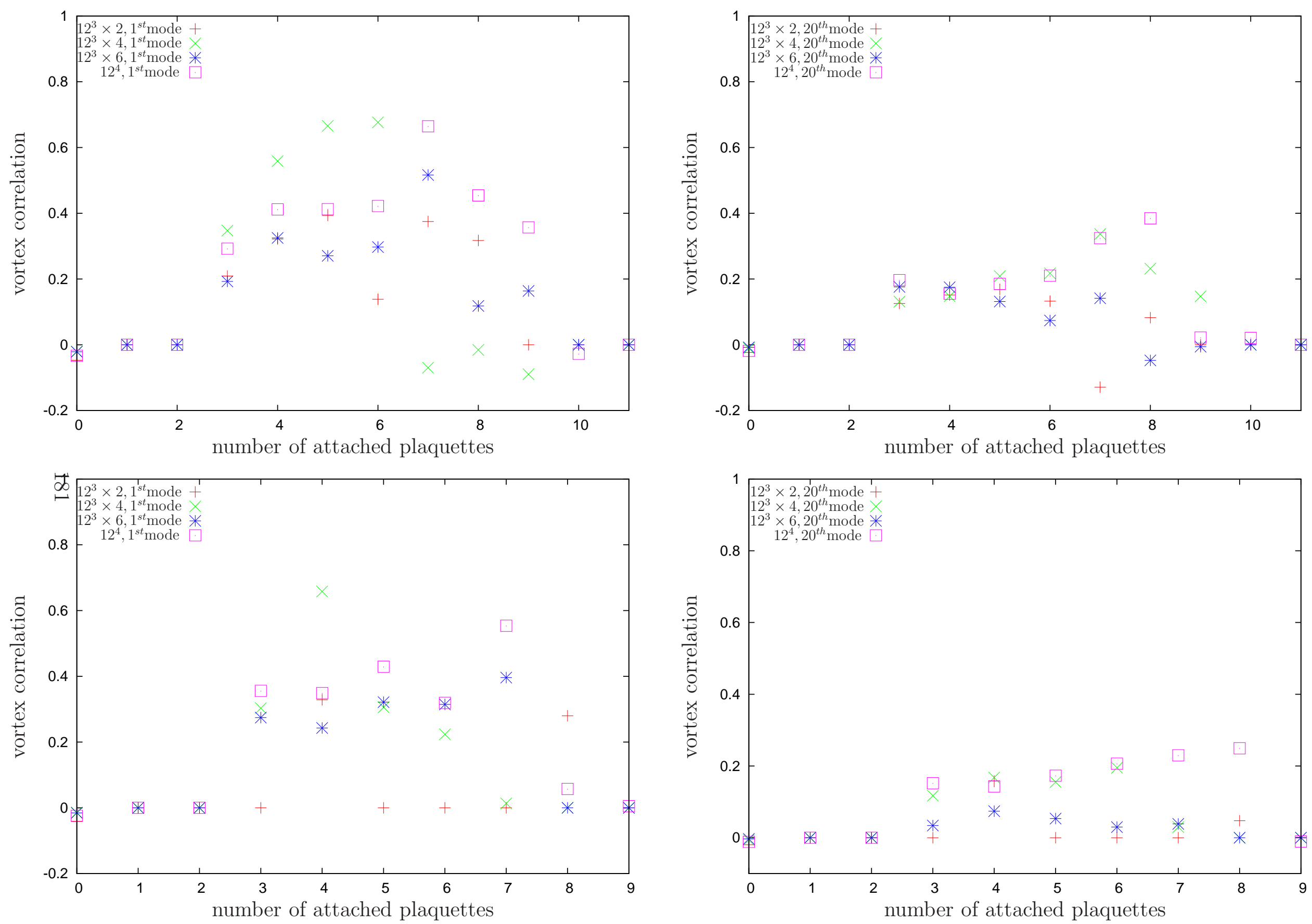

Figure 168: Correlation between eigenmodes and vortex surface, full configuration, $\beta_{L W}=3.7$ 

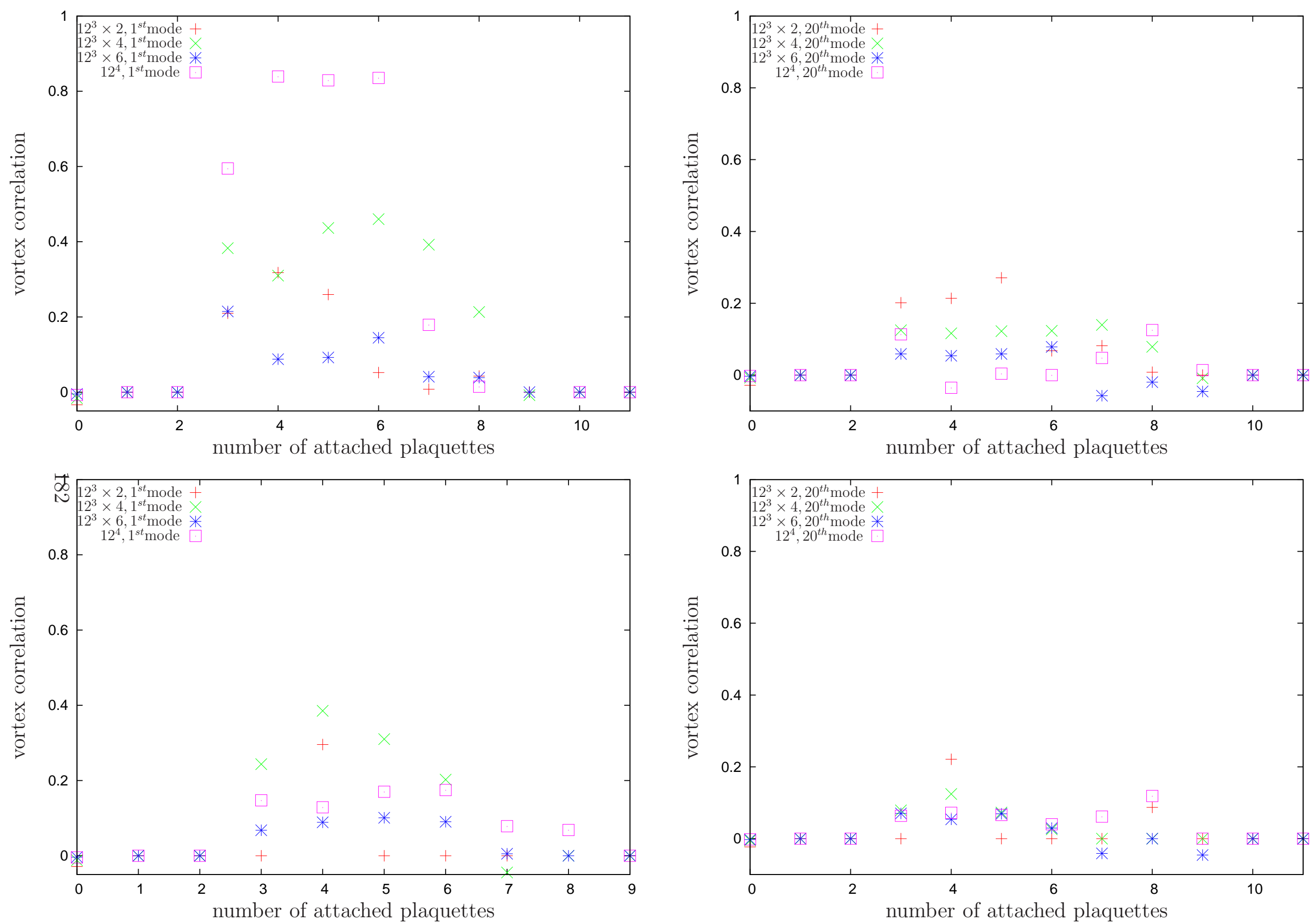

Figure 169: Correlation between eigenmodes and vortex surface, full configuration, $\beta_{L W}=4.0$ 

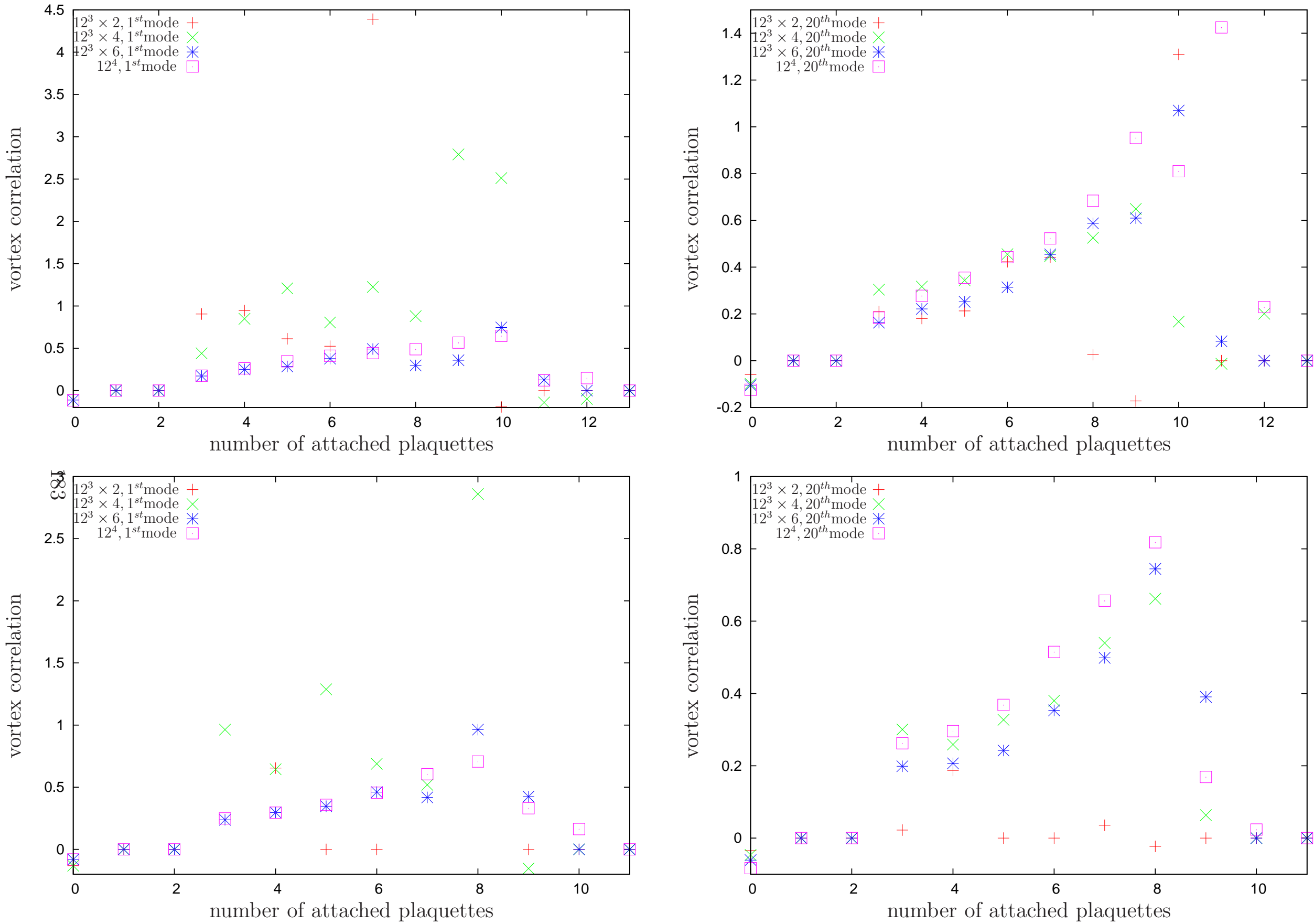

Figure 170: Correlation between eigenmodes and vortex surface, full configuration, anti periodic bc., $\beta_{L W}=3.3$ 

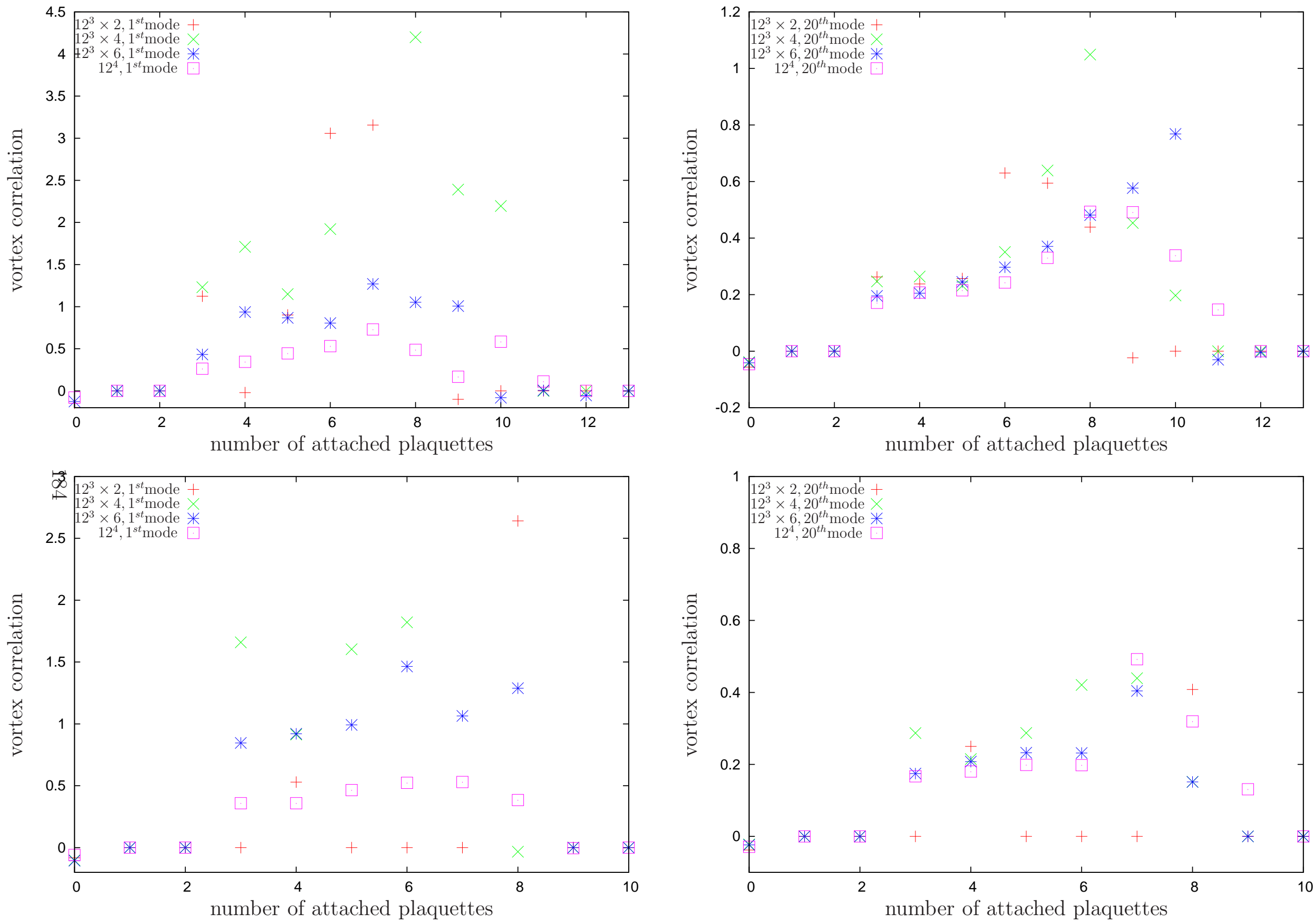

Figure 171: Correlation between eigenmodes and vortex surface, full configuration, anti periodic bc., $\beta_{L W}=3.5$ 

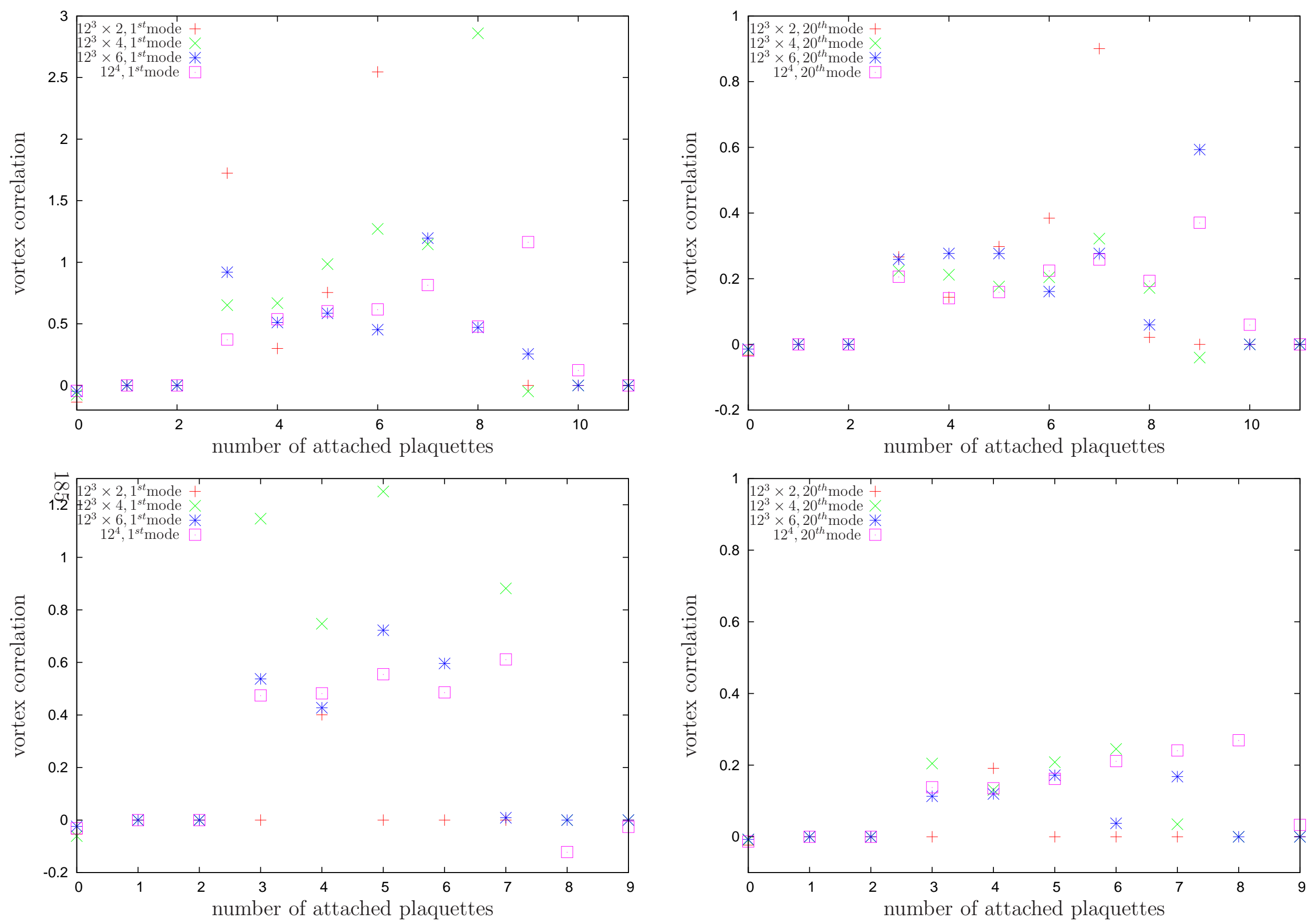

Figure 172: Correlation between eigenmodes and vortex surface, full configuration, anti periodic bc., $\beta_{L W}=3.7$ 

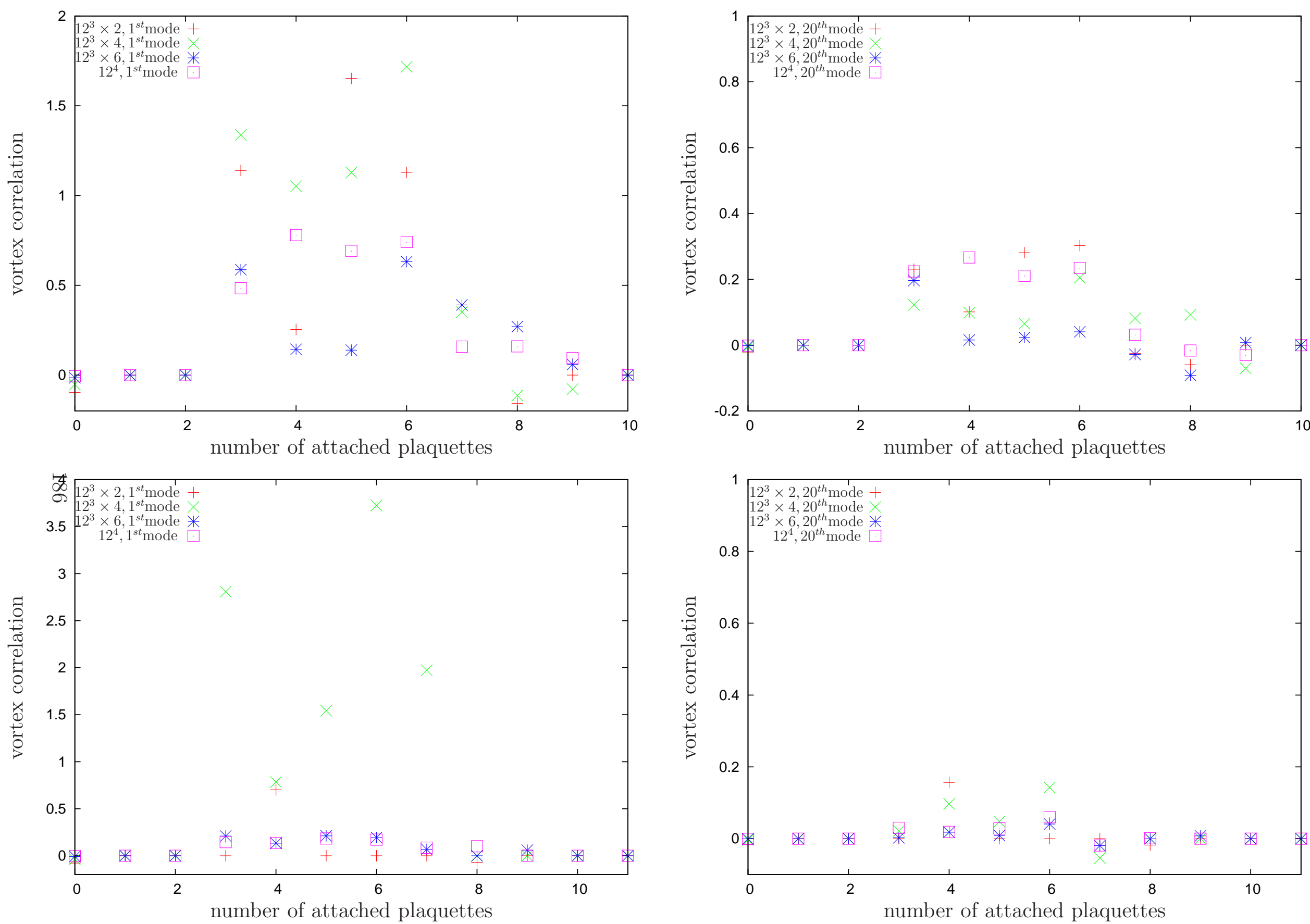

Figure 173: Correlation between eigenmodes and vortex surface, full configuration, anti periodic bc., $\beta_{L W}=4.0$ 

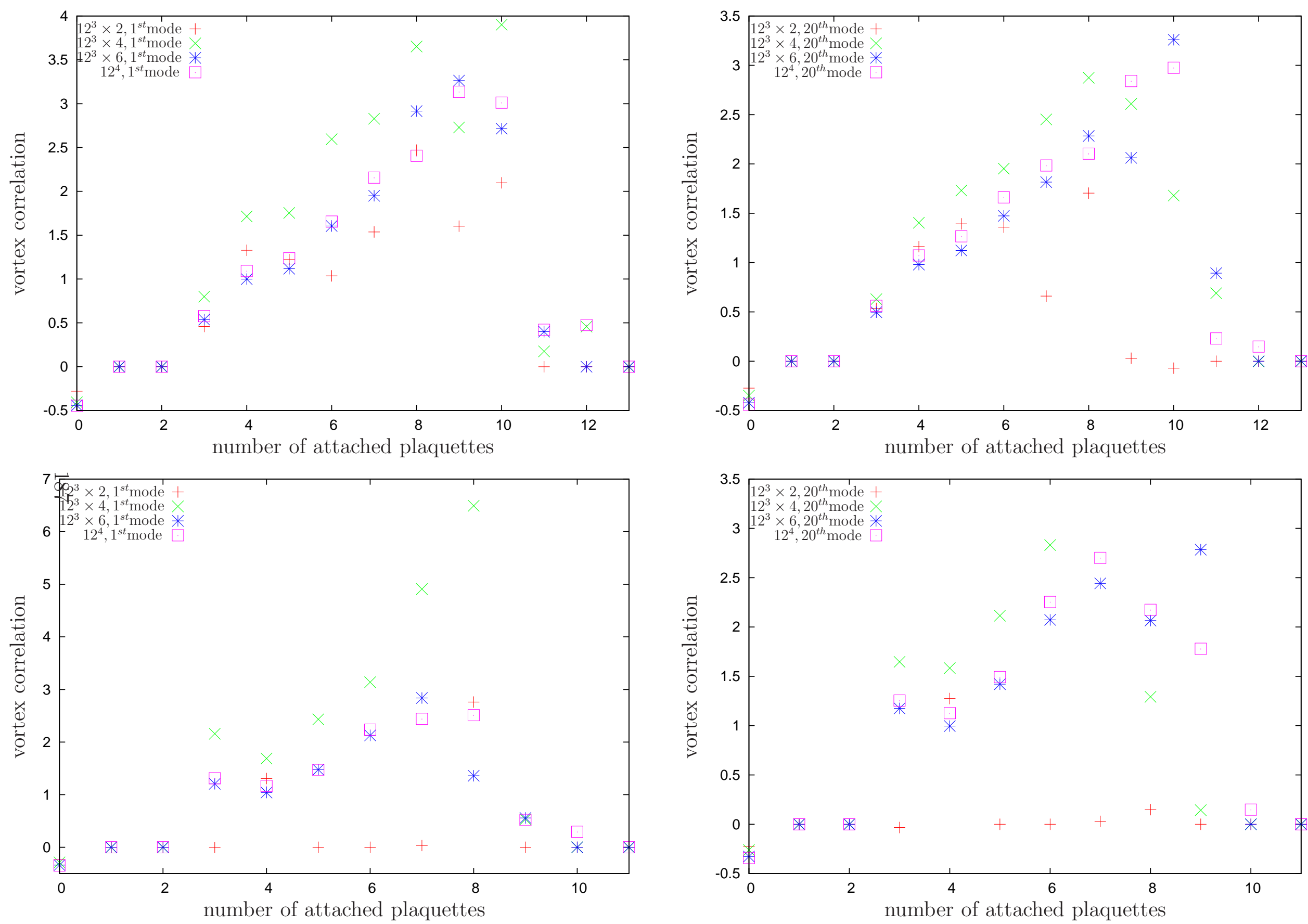

Figure 174: Correlation between eigenmodes and vortex surface, center-projected configuration, $\beta_{L W}=3.3$ 

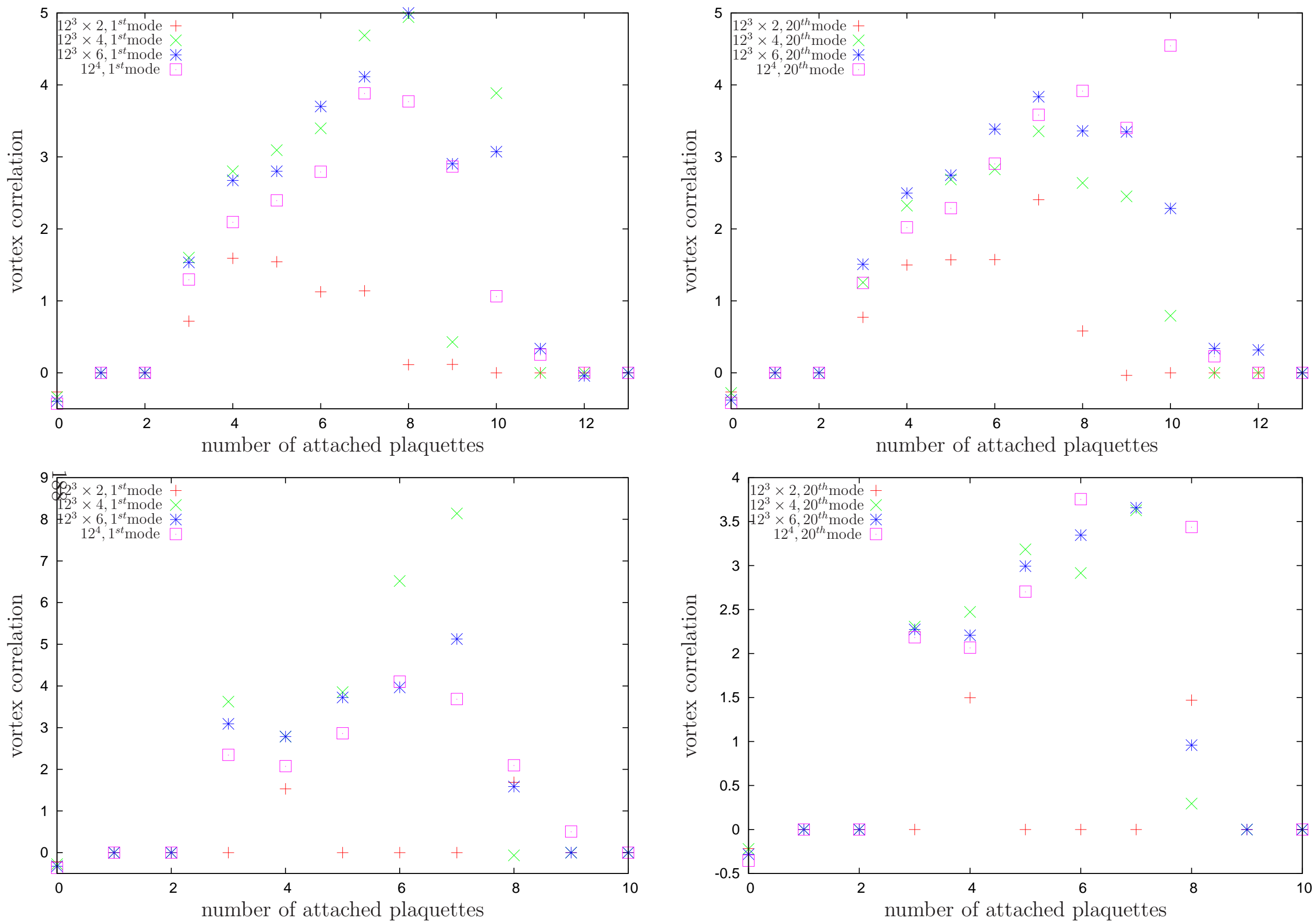

Figure 175: Correlation between eigenmodes and vortex surface, center-projected configuration, $\beta_{L W}=3.5$ 

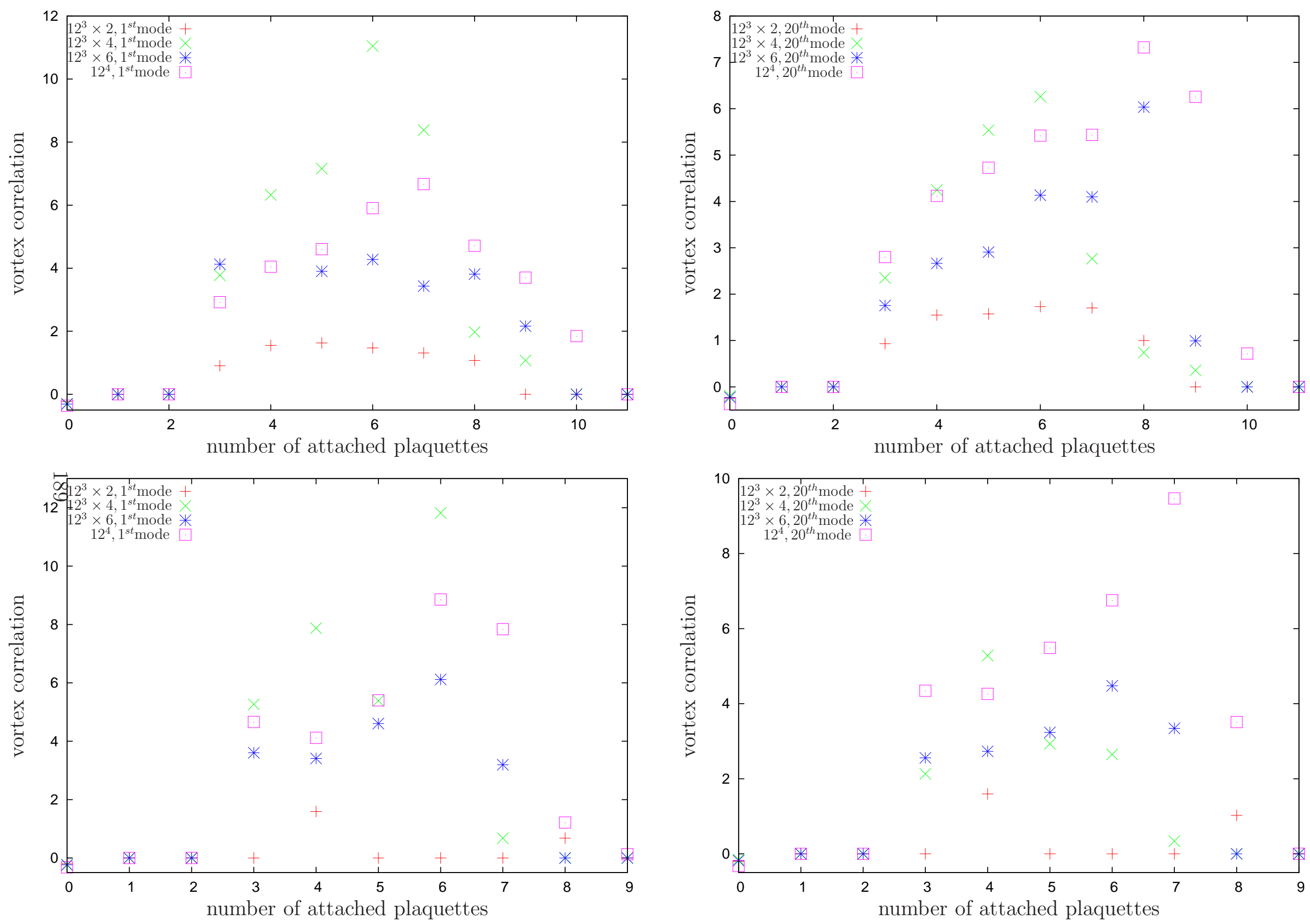

Figure 176: Correlation between eigenmodes and vortex surface, center-projected configuration, $\beta_{L W}=3.7$ 

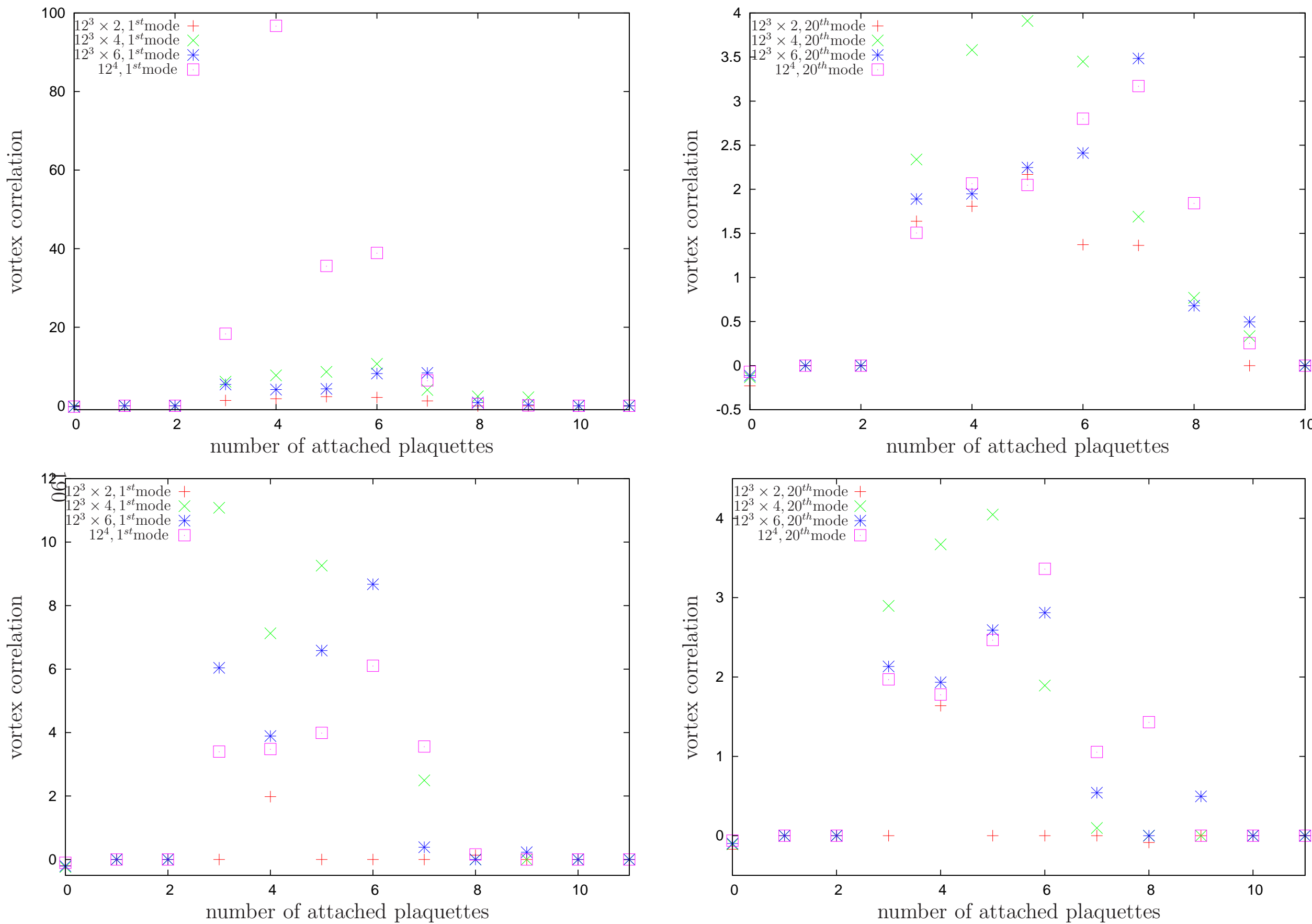

Figure 177: Correlation between eigenmodes and vortex surface, center-projected configuration, $\beta_{L W}=4.0$ 

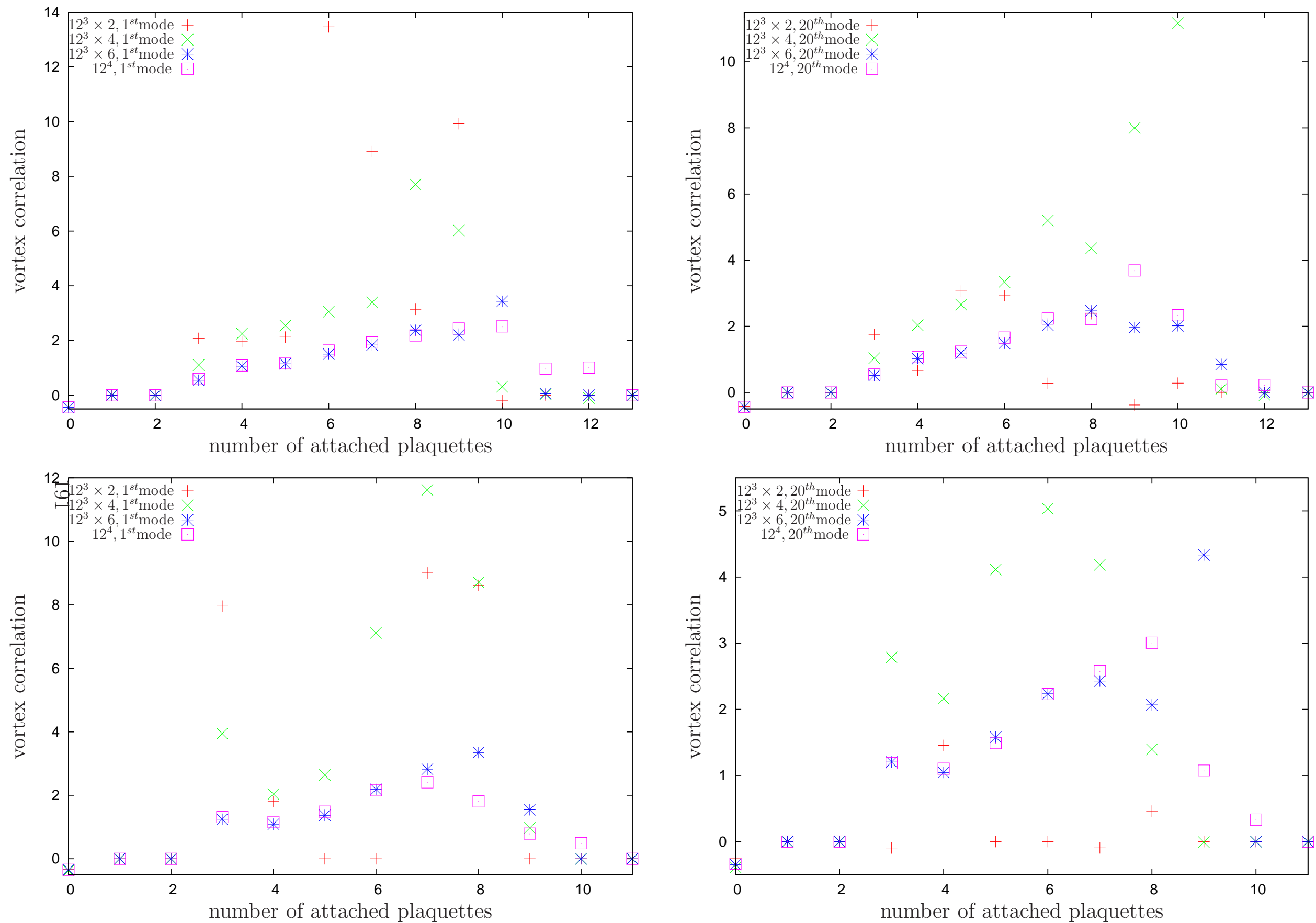

Figure 178: Correlation between eigenmodes and vortex surface, center-projected configuration, anti periodic bc., $\beta_{L W}=3.3$ 

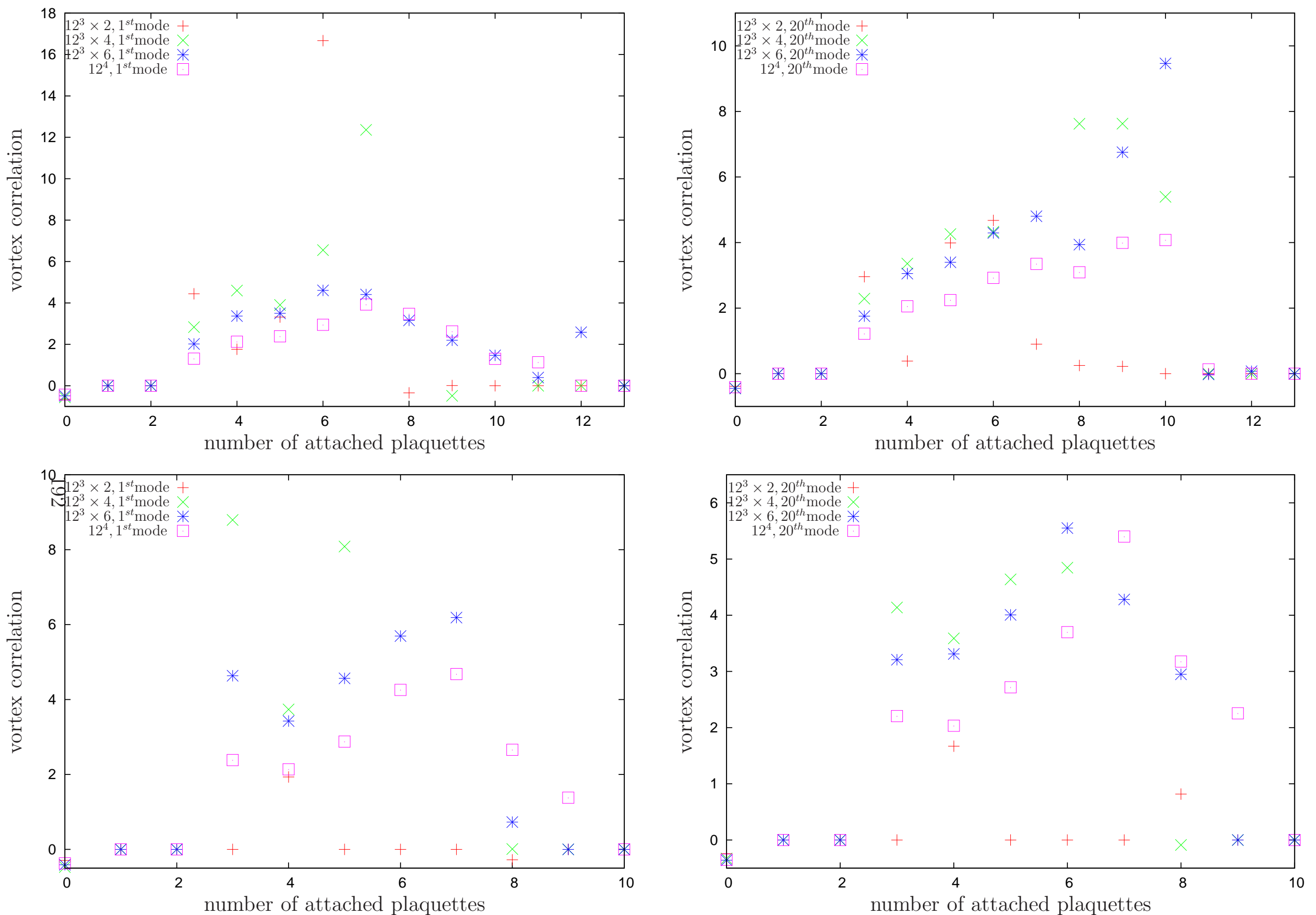

Figure 179: Correlation between eigenmodes and vortex surface, center-projected configuration, anti periodic bc., $\beta_{L W}=3.5$ 

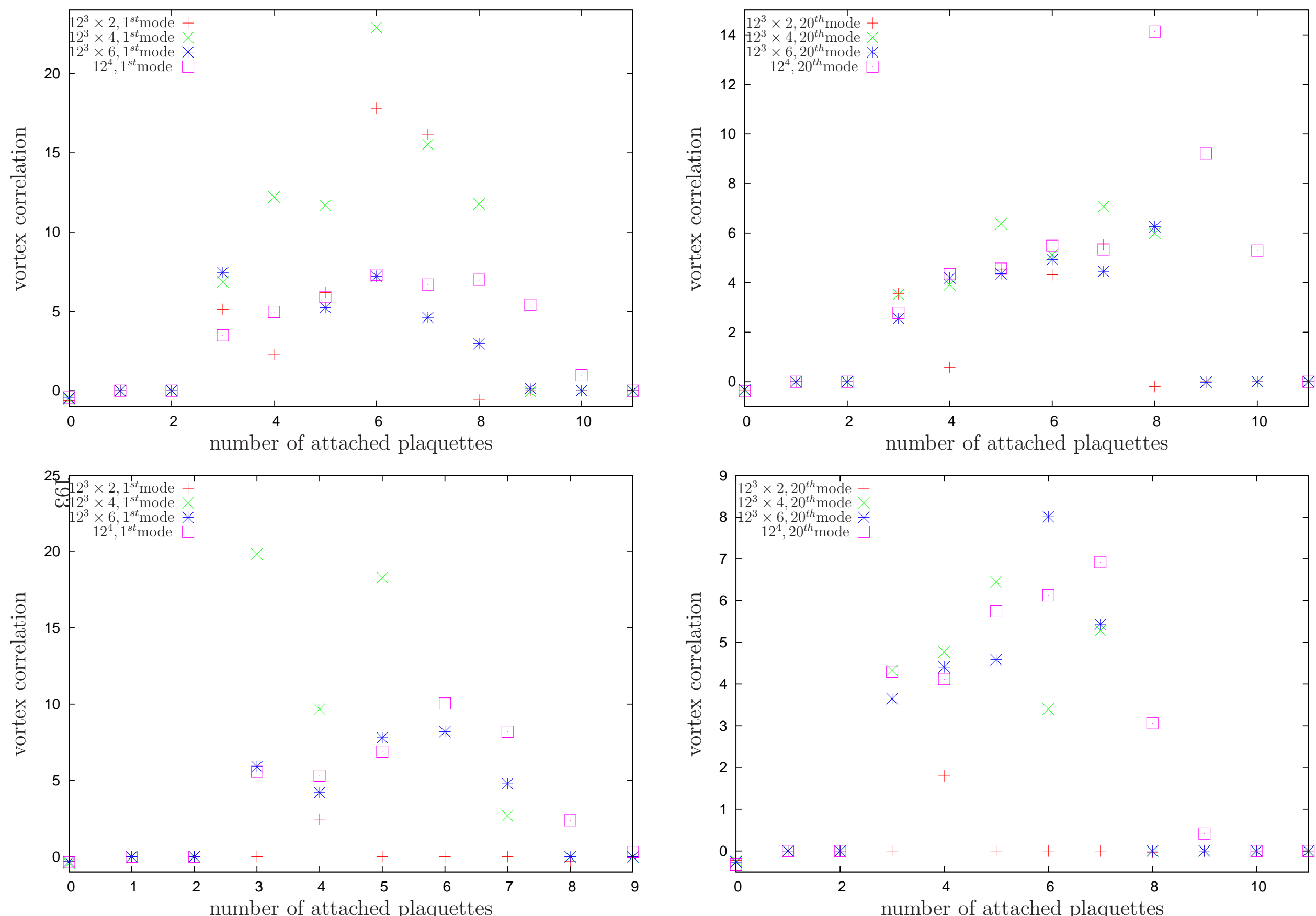

Figure 180: Correlation between eigenmodes and vortex surface, center-projected configuration, anti periodic bc., $\beta_{L W}=3.7$ 

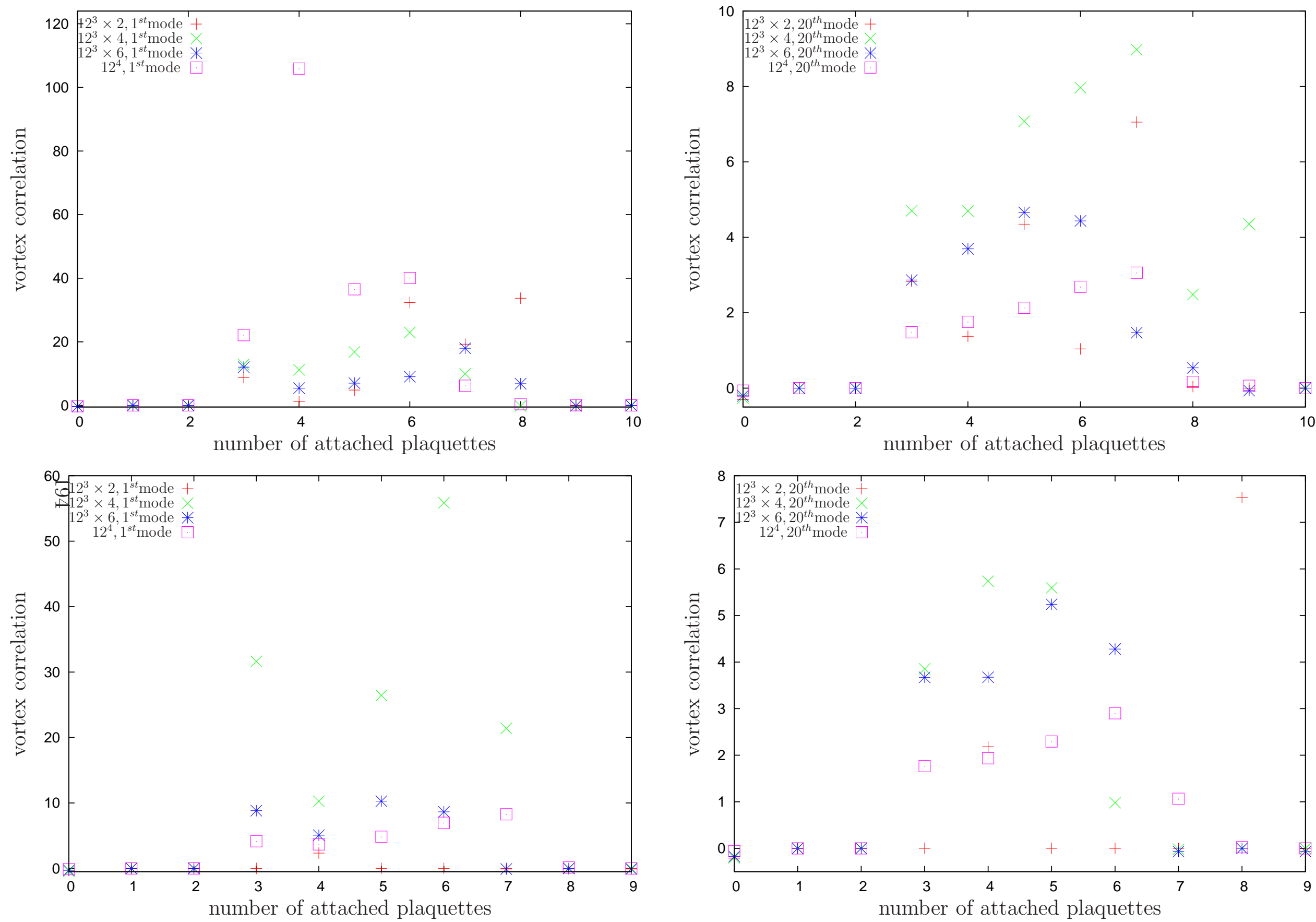

Figure 181: Correlation between eigenmodes and vortex surface, center-projected configuration, anti periodic bc., $\beta_{L W}=4.0$ 


\section{References}

[1] M. Creutz. Monte carlo study of quantized su(2) gauge theory. Phys. Rev., D21:2308-2315, 1980.

[2] G. S. Bali, K. Schilling, and C. Schlichter. Observing long color flux tubes in $\mathrm{su}(2)$ lattice gauge theory. Phys. Rev., D51:5165-5198, 1995.

[3] Thomas A. DeGrand, Anna Hasenfratz, and Tamas G. Kovacs. Topological structure in the su(2) vacuum. Nucl. Phys. Proc. Suppl., 63:528-530, 1998.

[4] Andreas S. Kronfeld, G. Schierholz, and U. J. Wiese. Topology and dynamics of the confinement mechanism. Nucl. Phys., B293:461, 1987.

[5] Tsuneo Suzuki and Ichiro Yotsuyanagi. A possible evidence for abelian dominance in quark confinement. Phys. Rev., D42:4257-4260, 1990.

[6] Del Debbio, L. and Faber, M. and Greensite, J. and Olejník, Š. Casimir scaling vs. abelian dominance in qcd string formation. Phys. Rev., D53:5891-5897, 1996.

[7] Gerard 't Hooft. On the phase transition towards permanent quark confinement. Nucl. Phys., B138:1, 1978.

[8] G. Mack and V. B. Petkova. Comparison of lattice gauge theories with gauge groups $\mathrm{z}(2)$ and $\mathrm{su}(2)$. Ann. Phys., 123:442, 1979.

[9] John M. Cornwall. Quark confinement and vortices in massive gauge invariant qcd. Nucl. Phys., B157:392, 1979.

[10] Del Debbio, L. and Faber, M. and Greensite, J. and Olejník, Š. Center dominance and $\mathrm{z}(2)$ vortices in su(2) lattice gauge theory. Phys. Rev., D55:2298-2306, 1997.

[11] Tamas G. Kovacs and E. T. Tomboulis. Vortices and confinement at weak coupling. Phys. Rev., D57:4054-4062, 1998.

[12] H. Reinhardt and M. Engelhardt. Center vortices in continuum yang-mills theory. In Wolfgang Lucha and Khin Maung Maung, editors, Quark Confinement and the Hadron Spectrum IV, pages 150-162. World Scientific, 2002.

[13] Robert G. Edwards, Urs M. Heller, and Rajamani Narayanan. A study of chiral symmetry in quenched qcd using the overlap-dirac operator. Phys. Rev., D59:094510, 1999.

[14] Philippe de Forcrand and Massimo D'Elia. On the relevance of center vortices to qcd. Phys. Rev. Lett., 82:4582-4585, 1999.

[15] C. Alexandrou, P. de Forcrand, and M. D'Elia. The role of center vortices in qcd. Nucl. Phys., A663:1031-1034, 2000. 
[16] Michael Engelhardt. Center vortex model for the infrared sector of yang-mills theory: Quenched dirac spectrum and chiral condensate. Nucl. Phys., B638:81-110, 2002.

[17] Lüscher, Martin. Exact chiral symmetry on the lattice and the ginsparg- wilson relation. Phys. Lett., B428:342-345, 1998.

[18] Rajamani Narayanan and Herbert Neuberger. A construction of lattice chiral gauge theories. Nucl. Phys., B443:305-385, 1995.

[19] M. F. Atiyah and I. M. Singer. The index of elliptic operators. 5. Annals Math., 93:139-149, 1971.

[20] Albert S. Schwarz. On regular solutions of euclidean yang-mills equations. Phys. Lett., B67:172-174, 1977.

[21] Lowell S. Brown, Robert D. Carlitz, and Choon-kyu Lee. Massless excitations in instanton fields. Phys. Rev., D16:417-422, 1977.

[22] Tom Banks and A. Casher. Chiral symmetry breaking in confining theories. Nucl. Phys., B169:103, 1980.

[23] P. de Forcrand and M. Pepe. Center vortices and monopoles without lattice gribov copies. Nucl. Phys., B598:557-577, 2001.

[24] K. Langfeld, H. Reinhardt, and A. Schfke. Center vortex properties in the laplace center gauge of su(2) yang-mills theory. Phys. Lett., B504:338-344, 2001.

[25] Faber, Manfried and Greensite, Jeff and Olejník, Štefan. Direct laplacian center gauge. JHEP, 11:053, 2001.

[26] Roman Bertle. The Vortex Model in Lattice QCD. Master's thesis, TU Wien, 2005.

[27] Del Debbio, L. and Faber, M. and Greensite, J. and Olejník, Š. Center dominance, center vortices, and confinement. 1997.

[28] J. Greensite. The confinement problem in lattice gauge theory. Prog. Part. Nucl. Phys., 51:1, 2003.

[29] John M. Cornwall. Nexus solitons in the center vortex picture of QCD. Phys. Rev., D58:105028, 1998.

[30] John M. Cornwall. Center vortices, nexuses, and fractional topological charge. Phys. Rev., D61:085012, 2000.

[31] M. Engelhardt and H. Reinhardt. Center vortex model for the infrared sector of yang-mills theory: Confinement and deconfinement. Nucl. Phys., B585:591-613, 2000 . 
[32] R. Bertle, M. Engelhardt, and M. Faber. Topological susceptibility of yang-mills center projection vortices. Phys. Rev., D64:074504, 2001.

[33] Faber, M. and Greensite, J. and Olejník, Š. Casimir scaling from center vortices: Towards an understanding of the adjoint string tension. Phys. Rev., D57:2603-2609, 1998.

[34] H. Reinhardt, O. Schroeder, T. Tok, and V. C. Zhukovsky. Quark zero modes in intersecting center vortex gauge fields. Phys. Rev., D66:085004, 2002.

[35] Stefan Solbrig et al. Topologically non-trivial field configurations: Interplay of vortices and dirac eigenmodes. PoS, LAT2005:301, 2005.

[36] Jochen Gattnar et al. Center vortices and dirac eigenmodes in su(2) lattice gauge theory. Nucl. Phys., B716:105-127, 2005.

[37] Kurt Langfeld, Hugo Reinhardt, and Oliver Tennert. Confinement and scaling of the vortex vacuum of su(2) lattice gauge theory. Phys. Lett., B419:317-321, 1998.

[38] Gerhard Mack and Hildegard Meyer. A disorder parameter that tests for confinement in gauge theories with quark fields. Nucl. Phys., B200:249, 1982.

[39] Hildegard Meyer. The vortex free energy in the screening phase of the $\mathrm{z}(2)$ higgs model. Nucl. Phys., B235:115, 1984.

[40] Roman Bertle and Manfried Faber. Vortices, confinement and higgs fields. pages 3-12, 2003.

[41] Kurt Langfeld. Lattice effective theory and the phase transition at finite densities. 2002 .

[42] Bertle, Roman and Faber, Manfried and Greensite, Jeff and Olejník, Štefan. Center dominance in su(2) gauge-higgs theory. Phys. Rev., D69:014007, 2004.

[43] K. Langfeld, O. Tennert, M. Engelhardt, and H. Reinhardt. Center vortices of yang-mills theory at finite temperatures. Phys. Lett., B452:301, 1999.

[44] M. N. Chernodub, M. I. Polikarpov, A. I. Veselov, and M. A. Zubkov. Aharonovbohm effect, center monopoles and center vortices in $\mathrm{su}(2)$ lattice gluodynamics. Nucl. Phys. Proc. Suppl., 73:575-577, 1999.

[45] Bertle, R. and Faber, M. and Greensite, J. and Olejník, Š. The structure of projected center vortices in lattice gauge theory. JHEP, 03:019, 1999.

[46] M. Engelhardt and H. Reinhardt. Center projection vortices in continuum yangmills theory. Nucl. Phys., B567:249, 2000.

[47] Herbert Neuberger. Exactly massless quarks on the lattice. Phys. Lett., B417:141$144,1998$. 
[48] Robert G. Edwards, Urs M. Heller, and Rajamani Narayanan. A study of practical implementations of the overlap-dirac operator in four dimensions. Nucl. Phys., B540:457-471, 1999.

[49] David H. Adams. On the continuum limit of fermionic topological charge in lattice gauge theory. J. Math. Phys., 42:5522-5533, 2001.

[50] Lüscher, Martin. Abelian chiral gauge theories on the lattice with exact gauge invariance. Nucl. Phys., B549:295-334, 1999.

[51] Herbert Neuberger. Bounds on the wilson dirac operator. Phys. Rev., D61:085015, 2000 .

[52] P. Di Vecchia, K. Fabricius, G. C. Rossi, and G. Veneziano. Preliminary evidence for u(1)-a breaking in qcd from lattice calculations. Nucl. Phys., B192:392, 1981.

[53] P. Di Vecchia, K. Fabricius, G. C. Rossi, and G. Veneziano. Numerical checks of the lattice definition independence of topological charge fluctuations. Phys. Lett., B108:323, 1982.

[54] H. Reinhardt. Resolution of gauss' law in yang-mills theory by gauge- invariant projection: Topology and magnetic monopoles. Nucl. Phys., B503:505-529, 1997.

[55] Pierre van Baal. Some results for $\mathrm{su}(\mathrm{n})$ gauge fields on the hypertorus. Commun. Math. Phys., 85:529, 1982.

[56] Herbert Neuberger. More about exactly massless quarks on the lattice. Phys. Lett., B427:353-355, 1998.

[57] Gerald Jordan. Dirac Eigenmodes of Centre Vortex Fields in SU(2) Lattice Gauge Theory. PhD thesis, TU Wien, 2005.

[58] Kit Yan Wong and R. M. Woloshyn. Topology and staggered fermion action improvement. Nucl. Phys. Proc. Suppl., 140:620-622, 2005.

[59] H. Leutwyler and A. Smilga. Spectrum of Dirac operator and role of winding number in QCD. Phys. Rev., D46:5607-5632, 1992.

[60] Aharon Casher. Chiral Symmetry Breaking in Quark Confining Theories. Phys. Lett., B83:395, 1979.

[61] Kostas Orginos and Doug Toussaint. Testing improved actions for dynamical kogutsusskind quarks. Phys. Rev., D59:014501, 1999.

[62] G. Peter Lepage. Flavor-symmetry restoration and Symanzik improvement for staggered quarks. Phys. Rev., D59:074502, 1999.

[63] Chulwoo Jung. Fermionic force term with the asqtad action. MILC notes, 2004. 
[64] E. M. Ilgenfritz et al. Exploring the structure of the quenched qcd vacuum with overlap fermions. Phys. Rev., D76:034506, 2007.

[65] M. I. Polikarpov, F. V. Gubarev, S. M. Morozov, and V. I. Zakharov. Localization of low lying eigenmodes for chirally symmetric dirac operator. PoS, LAT2005:143, 2006.

[66] C. Aubin et al. The scaling dimension of low lying dirac eigenmodes and of the topological charge density. Nucl. Phys. Proc. Suppl., 140:626-628, 2005.

[67] C. Bernard et al. More evidence of localization in the low-lying dirac spectrum. PoS, LAT2005:299, 2006.

[68] Greensite, J. and Olejník, Š. and Polikarpov, M. and Syritsyn, S. and Zakharov, V. Localized eigenmodes of covariant Laplacians in the Yang- Mills vacuum. Phys. Rev., D71:114507, 2005.

[69] Philippe de Forcrand. Localization properties of fermions and bosons. AIP Conf. Proc., 892:29-35, 2007.

[70] F. V. Gubarev, S. M. Morozov, M. I. Polikarpov, and V. I. Zakharov. Low lying eigenmodes localization for chirally symmetric Dirac operator. 2005.

[71] Yoshiaki Koma et al. Localization properties of the topological charge density and the low lying eigenmodes of overlap fermions. PoS, LAT2005:300, 2006.

[72] Volker Weinberg et al. The QCD vacuum probed by overlap fermions. PoS, LAT2006:078, 2006.

[73] Ernst-Michael Ilgenfritz et al. Localization of overlap modes and topological charge, vortices and monopoles in SU(3) LGT. PoS, LAT2007:311, 2007.

[74] A. V. Kovalenko, S. M. Morozov, M. I. Polikarpov, and V. I. Zakharov. On topological properties of vacuum defects in lattice yang-mills theories. Phys. Lett., B648:383-387, 2007.

[75] Ivan Hip. Interactive Visualization Package for 4D Lattice Field Theories. PoS, LAT2007:035, 2007.

[76] Greensite, J. and Langfeld, K. and Olejník, Š. and Reinhardt, H. and Tok, T. Color screening, Casimir scaling, and domain structure in $\mathrm{G}(2)$ and $\mathrm{SU}(\mathrm{N})$ gauge theories. Phys. Rev., D75:034501, 2007.

[77] Jordan, Gerald and Höllwieser, Roman and Faber, Manfried and Heller, Urs M. Tests of the lattice index theorem. Phys. Rev., D77:014515, 2008.

[78] Höllwieser, Roman and Faber, Manfried and Greensite, Jeff and Heller, Urs M. and Olejník, Štefan. Center Vortices and the Dirac Spectrum. Phys. Rev., D78:054508, 2008 . 
[79] M. Lüscher and P. Weisz. Computation of the Action for On-Shell Improved Lattice Gauge Theories at Weak Coupling. Phys. Lett., B158:250, 1985.

[80] G. I. Poulis. Scaling and confinement aspects of tadpole improved SU(2) lattice gauge theory and its Abelian projection. Phys. Rev., D56:161-173, 1997.

[81] Christof Gattringer, Roland Hoffmann, and Stefan Schaefer. Setting the scale for the Lüscher-Weisz action. Phys. Rev., D65:094503, 2002.

[82] Holger Bech Nielsen and M. Ninomiya. No go theorem for regularizing chiral fermions. Phys. Lett., B105:219, 1981.

[83] Holger Bech Nielsen and M. Ninomiya. Absence of neutrinos on a lattice. 2. intuitive topological proof. Nucl. Phys., B193:173, 1981.

[84] Paul H. Ginsparg and Kenneth G. Wilson. A remnant of chiral symmetry on the lattice. Phys. Rev. D, 25(10):2649-2657, May 1982.

[85] Robert G. Edwards, Urs M. Heller, Joe E. Kiskis, and Rajamani Narayanan. Topology and chiral symmetry in qcd with overlap fermions. 1999.

[86] H.J. Rothe. Lattice Gauge Theories - An Introduction. World Scientific Publishing Co. Pte. Ltd., 1952.

[87] Stephen L. Adler. An overrelaxation method for the Monte Carlo evaluation of the partition function for multiquadratic actions. Phys. Rev., D23:2901, 1981.

[88] Faber, M. and Greensite, J. and Olejník, Š. and Yamada, D. The vortex-finding property of maximal center (and other) gauges. JHEP, 12:012, 1999.

[89] Faber, Manfried and Greensite, Jeff and Olejník, Štefan. Remarks on the Gribov problem in direct maximal center gauge. Phys. Rev., D64:034511, 2001.

[90] http://www.wikipedia.org.

[91] http://mathworld.wolfram.com. 


\section{Curriculum Vitae}

\section{Roman HÖLLWIESER}

Am Brand 10, A-2833 Bromberg

romanhoellwieser@aon.at

General Information

Citizenship: Austria

Date of Birth: February 23, 1983

Birthplace: Wr.Neustadt (Lower Austria)

\section{Education}

1989-1993

1993-2001

Elematary School Bromberg

BRG Gröhrmühlgasse Wr.Neustadt

(Secondary education)

June 7, 2001

Final examination passed with distinction

2001-2002

Military Service at The Austrian International Operations Command in Götzendorf (Lower Austria), Public Relations, Journalism

since 2002

January 2004

Vienna University of Technology (TU Vienna), Major: Physics

2004-2005

First Diploma Examination

Ècole Polytèchnique Fèderal de Lausanne (EPFL), Switzerland

Exchange student in physics

since 2005 TU Vienna, Atomic Institute, Nuclear Physics

Diploma Thesis in Lattice Gauge Field Theory, Research Work

January 2007 Diploma Examination

since Feb. 2007 TU Vienna, Atomic Institute, Nuclear Physics

Master Thesis in Lattice Qunatum Chromodynamics, Research Work

\section{Work Experience}

July-August 1998 FERRO-BETONIT (civil engineering)

July-August 1999 ALPINE Bauges.m.b.H. (civil engineering)

July-August 2000 Zimmerei Franz STOCKER, 2833 Bromberg (construction engineering)

July $2003 \quad$ W.HAMBURGER AG, 2328 Pitten (papermill, shift work)

August $2003 \quad$ ALPINE MAYREDER Bau GmbH (civil engineering)

July-August 2004 TU Vienna, Atomic Institute, Nuclear Physics

since July 2005 TU Vienna, Atomic Institute, Nuclear Physics, Research Work

Mai-July 2006 Zimmerei Franz STOCKER, 2833 Bromberg (construction engineering)

\section{Activities}

Playing music (several instruments), Sports (many kinds of), Astronomy (star observing), Mountaineering (climbing), Snowboard touring, etc.

\section{Interests}

Astronomy, Informatics, Mathematics, (Theoretical) Physics, Philosophy \& Regligion

\section{Languages}

German (mother-tongue), English, French 


\section{List of Publications}

- Manfried Faber, Gerald Jordan and Roman Höllwieser:

Topology, Center Vortices, Confinement and Chiral Symmetry Breaking in SU(2) Lattice $Q C D$, Proceedings of the International School-seminar "New Physics and Quantum Chromodynamics at external Conditions", pp. 27-37, 3-6 May 2007, Dnipropetrovsk, Ukraine

- Gerald Jordan, Roman Höllwieser, Manfried Faber and Urs M. Heller: Surprises with the lattice index theorem, PoS LAT2007: 076 (2007)

- Gerald Jordan, Roman Höllwieser Manfried Faber and Urs M. Heller: Tests of the lattice index theorem, Phys. Rev. D77, 014515 (2008) [arXiv: hep-lat:0710.5445]

- Urs M. Heller, Roman Höllwieser, Manfried Faber, Jeff Greensite and Štefan Olejník: Center Vortex influence on the Dirac Spectrum, PoS LAT2008: 258 (2008)

- Roman Höllwieser, Manfried Faber, Jeff Greensite, Urs M. Heller and Štefan Olejník: Center Vortices and the Dirac Spectrum, Phys. Rev. D78, 054508 (2008) [arXiv: hep-lat:0805.1846]

- Roman Höllwieser, Manfried Faber, Jeff Greensite, Urs M. Heller and Štefan Olejník: Correlations between Center Vortices and low-lying Dirac eigenmodes, PoS Confinement8: 036 (2008) 